2. To: (Receiving Organization)

Distribution

5. Proj./Prog./Dept./Div.:

Spent Nuclear Fuels Project

8. Originator Remarks:

11. Receiver Remarks:

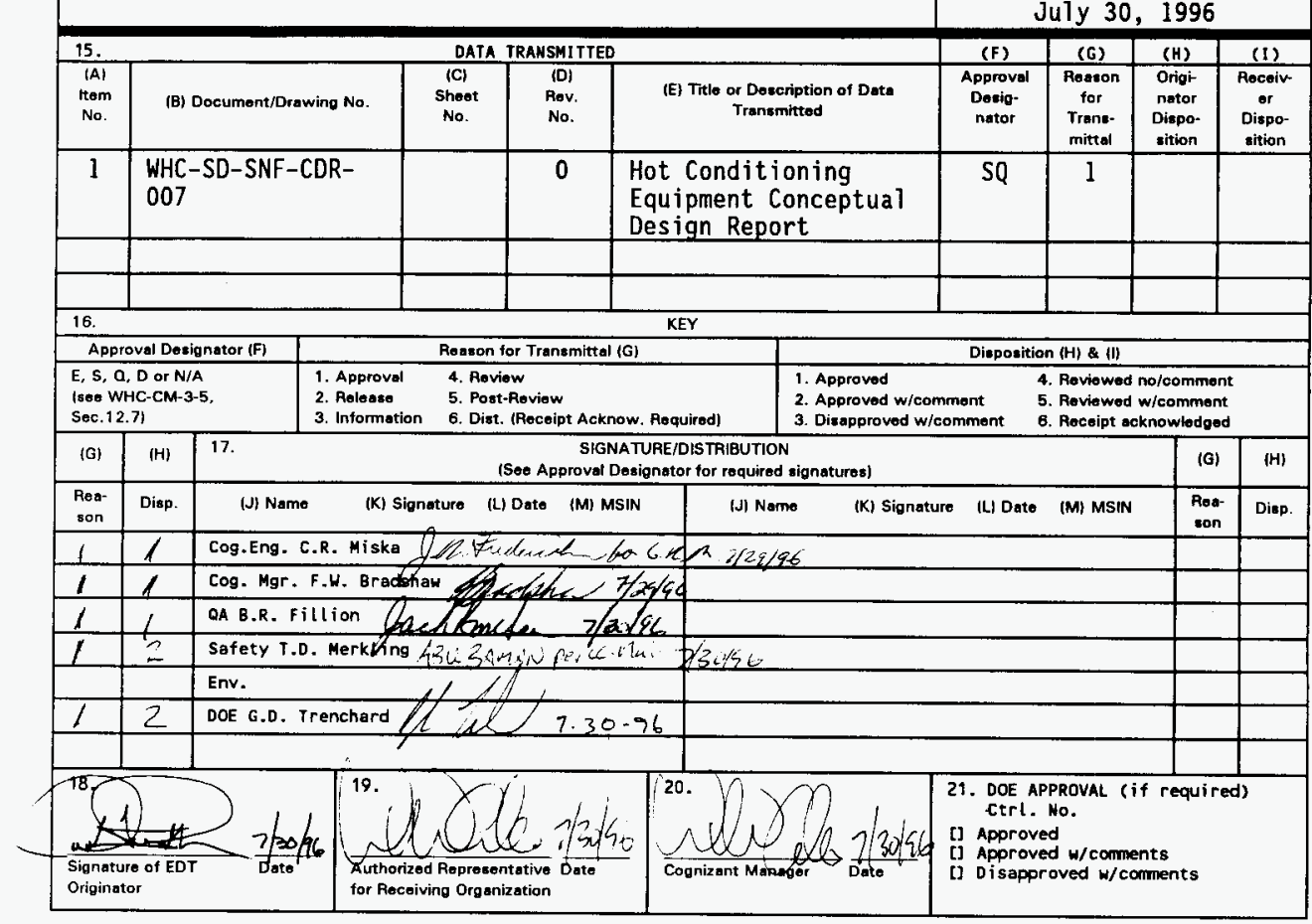

3. From: (Originating Organization) Spent Nuclear Fuels Project

6. Cog. Engr.:

C. R. Miska
4. Related EOT No.:

$\mathrm{N} / \mathrm{A}$

7. Purchase Order No.:

N/A

9. Equip./Component Ho.:

N/A

10. System/Btdg./Facility:

$N / A$

12. Major Assm. Owg. No.: N/A

13. Permit/Permit Application No.: $N / A$

14. Required Response Date: July 30,1996

BD-7400-172-2(04/94) GEF097 
WHC-SD-SNF-CDR-007, Rev. 0

\title{
Hot Conditioning Equipment Conceptual Design Report
}

\author{
Merrick and Associates \\ Westinghouse Hanford Company, Richland, WA 99352 \\ U.S. Department of Energy Contract DE-AC06-87RL10930 \\ EDT/ECN: $616136 \quad$ UC: 510 \\ Org Code: 2C400 Charge Code: LH002 \\ B\&R Code: EW3135040 Total Pages: 432
}

Key Hords: Spent Nuclear Fuel, N Reactor Spent Nuclear Fuel, Single Pass Reactor Spent Nuclear Fuel, Hot Conditioning System Equipment, Canister Storage Building, K-Basins, Cold Vacuum Drying System

Abstract: This report documents the conceptual design of the Hot Conditioning System Equipment. The Hot Conditioning System will consist of two separate designs: the Hot Conditioning System Equipment; and the Hot Conditioning System Annex. The Hot Conditioning System Equipment Design includes the equipment such as ovens, vacuum pumps, inert gas delivery systems, etc. necessary to condition spent nuclear fuel currently in storage in the K Basins of the Hanford Site. The Hot Conditioning System Annex consists of the facility to house the Hot conditioning System. The Hot Conditioning System will be housed in an annex to the Canister Storage Building. The Hot Conditioning System will consist of pits in the floor which contain ovens in which the spent nuclear will be conditioned prior to interim storage.

TRADEMARK DISCLAIMER. Reference herein to any specific commercial product, process, or service by trade name, trademark, manufacturer, or otherwise, does not necessarily constitute or imply its endorsement, recommendation, or favoring by the United States Government or any agency thereof or its contractors or subcontractors.

Printed in the United States of America. To obtain copies of this document, contact: WHC/BCS Document Control Services, P.O. Box 1970, Mailstop H6-08, Richland WA 99352, Phone (509) 372-2420; Fax $(509) 376-4989$

Companies whose information is photocopied and included in this document have been contacted to verify that the information is not copyrighted or company sensitive in anyway. Attached are 2 letters of permission to use that companies copyrighted information.
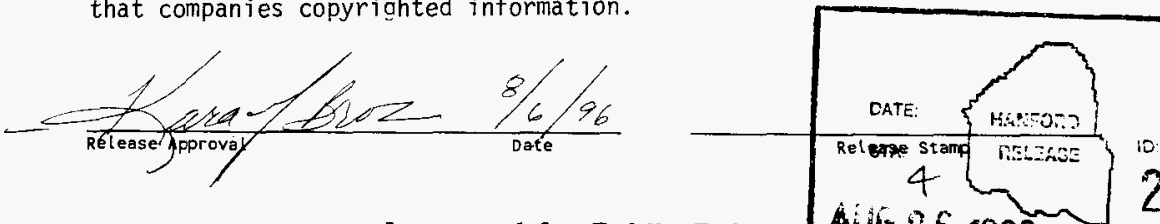

Approved for Public Release Auts 061996 
Westinghouse Hanford Compeny

P.A. Box 1970

Richland, un gisst

\section{APPLICATION FOR PERMISSION TO USE} YOUR COPYRIGHTED MATERIAL

TO: EGSG ROTRON

Dato; $8 / 1 / 96$

Permission is requested to reproduce the following copyrighted matarial from:

Egst ROTRON Cutsheets for The MD 101

Selectlona from text (apecify by date of lasue, page, paragraph, or fllustration; If destred, sttach a copy of the material In question):

The attached pages include a brief introduction to our document and the EGsG cutsheets our author sould like to include in his Conceptual Design Report.

Title of work or projeot in whlch this material will be included:

Hot Conditioning System Equipment -, K-Basin Spent Nuclear Fuel Project

Eatimatod publication date: $8 / 1 / 96$

Authar: Merrick and Assoc.

and

H.L. Hillis

Hestinghouse Hanford Co.

Publishar (if applicabla): N/A

If the copyrighted material is not to be used in a published work, please provide a briaf description of how it is to be uaed:

Name: Kara M. Broz

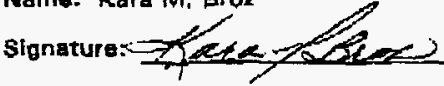

MSIN: R1-29

Date: $8 / 1 / 96$

Wastinghouse Hanford Company
Application approved by:

Nome:

VINCE Conte

Companr Name: EG: G RoTon

Signature:
Date:

$8 1 6 \longdiv { 9 6 }$

Signatory's Position:

(on behalf of) 


\section{TELECOPY \\ from \\ PALL ADVANCED SEPARATIONS SYSTEMS \\ Pall Trinity Micro Corporation 3643 Route 281, PO Box 2030 \\ Cortland, NY 13045 \\ Phone: (607) 753.6041 \\ Fax: (607) 753-8525}

\section{Pager Total (Including Cover Sheet)}

(If you do not receive this entire facuimile, ploase call (607) 753.6041 Ext. 1811)

TO: Kara Broz - Westinghouse Harford Company

Fax: (509) 373-7739

FROM: Paul Dittman

SUBJECT: Design Data Report

DATE: $\quad$ August 2, 1996

I am writing to inform you that it is acceptable for you to include Pall Corporation's Literature UAF-100 (Pall Cltramet /Air Filters/Systems Brochure) in your report that discusses the use of Pall filters for the subject of HCSE Specification.

You may also use Pall Proposal Number: BP7ACDR96-129PDI for Santa Fe Engineering, with the exception of the pricing and delivery sections.

If you have any questions on need further information, please do not hesitate to call me at (607) 753-6041 Ext. 1680.

Regards,

Pall Advanced Separations Systems

Daul Dittrman/grar

Paul Dittman

Senior Applications Engines

lotus notes/typing/dittman 
WHC-SD-SNF-CDR-C07, Revision 0

TABLE OF CONTENTS

TABLE OF CONTENTS

SECTION

PAGE

TABLE OF CONTENTS

ACRONYMS

iii

DEFINITIONS

ix

1.0 INTRODUCTION

2.0 PROCESS

2.1 HCSE MODEL OPERATION SEQUENCE

2.2 MAIN PROCESS SYSTEMS

2.3 COMBINED VENTILATION SYSTEM (CVS) CONFIGURATION

2.4 PROCESS CONTROL

2.5 OVEN ASSEMBLY DESCRIPTION

2.6 PROCESS MODULE

2.7 SERVICE MODULE

2.8 PROCESS ENCLOSURE DESCRIPTION

2.9 SOLID WASTE

3.0 FACILITIES/UTILITIES SUPPORT

3.1 HCSE LAYOUT

3.2 HCSE/HCSA INTERFACES

3.3 AUXILIARY FACILITIES

4.0 INTERFACES

4.1 HCSE INTERFACES

5.0 COST AND SCHEDULE

5.1 METHOD OF ACCOMPLISHMENT

5-1

5.2 SCHEDULE

$5-2$

5.3 SUMMARY COST ESTIMATE 
WHC-SD-SNF-CDR-007, Revision 0

\section{APPENDICES}
A. DRAWINGS
B. ALARA IMPLEMENTATION PLAN
C. SHIELDING AND RADIATION EXPOSURE ANALYSIS
D. OPERATIONS AND MAINTENANCE PHILOSOPHY
E. PERFORMANCE SPECIFICATIONS REPORT
F. COST ESTIMATE BACKUP
G. CALCULATIONS
H. VENDOR CUT SHEETS
I. CRITERIA EVALUATION
J. OPERABILITY AND MAINTAINABILITY ASSESSMENT
K. COMMENT DATABASE
L. USE OF COOLING WATER IN THE HOT CONDITIONING SYSTEM 
WHC-SD-SNF-CDR-007, Revision 0

\section{ACRONYMS}

\section{ACRONYMS}

acfm Actual cubic feet per minute

ACGIH American Conference of Governmental Industrial Hygienists

$\mathrm{ACl} \quad$ American Concrete Institute

Al Residual Gas Analyzer Indicator

ALARA As Low As Reasonably Achievable

ANS American National Standard

ANSI American National Standards Institute

ASHRAE American Society of Heating, Refrigeration, and Air Conditioning Engineers

ASME American Society of Mechanical Engineers

BFD Block Flow Diagram

BLO Blower

CAM Continuous Air Monitors

cc/sec Cubic centimeters per second

CDR Conceptual Design Report

CFR Code of Federal Regulations

$\mathrm{CH} \quad$ Chiller

CHW Chilled Water System

CLR Cooler

CM Control Manual

Co Cobalt

Cs Cesium

CSB Canister Storage Building

CVDM Cold Vacuum Drying Module

CVDS Cold Vacuum Drying System 
WHC-SD-SNF-CDR-007, Revision 0

\section{ACRONYMS}

CVS Combined Ventilation System

DBA Design Basis Accident

DE Design Earthquake

DNFSB Defense Nuclear Facilities Safety Board

DOE Department of Energy

DOP Dioctylphthalate aerosol

DOT Department of Transportation

EDE Effective Dose Equivalent

ERDA Energy Research \& Development Administration

ES\&H Environmental Protection, Safety and Health

EWS Engineering Work Station

F $\quad$ Filter

FI Flow Indicator

FIC Flow Indicating Control

FOB Free On Board

FQIC Totalizing Flow Indicating Control

FSF Fuel Stabilization Facility

$\mathrm{ft} / \mathrm{min} \quad$ Feet per minute

GDC General Design Criteria

GOCO Government Owned Contractor Operated

GOV Gas Operated Valve; flow, ball

gpm Gallons per minute

H Hydrogen

HC2 Hazard Category 2

HC3 Hazard Category 3 


\begin{tabular}{|c|c|}
\hline $\mathrm{HCL}$ & Electric Heater \\
\hline HCS & Hot Conditioning System \\
\hline HCSA & Hot Conditioning System Annex \\
\hline HCSE & Hot Conditioning System Equipment \\
\hline $\mathrm{He}$ & Helium \\
\hline HEPA & High Efficiency Particulate Air \\
\hline HLAN & Hanford Local Area Networks \\
\hline HSRCM & Hanford Site Radiological Control Manual \\
\hline HVAC & Heating, Ventilation, and Air Conditioning \\
\hline HVCE & Hot Vacuum Conditioning Equipment \\
\hline $\mathbf{I}$ & lodine \\
\hline I/O & Input/Output \\
\hline ICRP & International Commission on Radiological Protection \\
\hline IEEE & Institute of Electrical \& Electronics Engineers \\
\hline IFMSF & Irradiated Fissile Material Storage Facilities \\
\hline ISA & Instrument Society of America \\
\hline ISFSI & Independent Spent Fuel Storage Installation \\
\hline $\mathrm{kg}$ & Kilogram \\
\hline $\mathrm{Kr}$ & Krypton \\
\hline LAN & Local Area Network \\
\hline LCP & Local Control Panel \\
\hline Ipm & Liters per minute \\
\hline LPT & Liquid Penetrant Test \\
\hline MCO & Multi-canister Overpack \\
\hline MCS & MCO Cooling System \\
\hline
\end{tabular}


WHC-SD-SNF-CDR-007, Revision 0

\section{ACRONYMS}

$\begin{array}{ll}\text { Mg } & \text { Magnesium } \\ \text { MHM } & \text { MCO Handling Machine } \\ \text { MMI } & \text { Man-Machine Interface } \\ \text { MSHA } & \text { Mine Safety and Health Administration } \\ \text { N } & \text { Nitrogen } \\ \text { NFPA } & \text { National Fire Protection Association } \\ \text { NGS } & \text { Non-Government Standards } \\ \text { NIOSH } & \text { National Institute for Occupational Safety and Health } \\ \text { NQA } & \text { Nuclear Quality Assurance } \\ \text { NRC } & \text { Nuclear Regulatory Commission } \\ \text { O } & \text { Oxygen } \\ \text { OIS } & \text { Operator Interface Station } \\ \text { ORA } & \text { Operational Readiness Assessment } \\ \text { ORR } & \text { Operational Readiness Review } \\ \text { OSHA } & \text { Operational Safety and Health Administration } \\ \text { P\&ID } & \text { Piping and Instrument Diagram } \\ \text { PC } & \text { Personal Computer } \\ \text { PCS } & \text { Process Control System } \\ \text { PCV } & \text { Constant Pressure Regulator Valve } \\ \text { PE } & \text { Process Enclosure } \\ \text { PFD } & \text { Process Flow Diagram } \\ \text { PFR } & \text { Purifier } \\ \text { PG } & \text { Process Gas } \\ \text { PGS } & \text { Process Gas System } \\ \text { PHA } & \text { Preliminary Hazards Analysis } \\ & \end{array}$




\section{ACRONYMS}

\begin{tabular}{ll} 
PHS & Process Heating System \\
PI & Pressure Indicator \\
PIC & Pressure Indicating Control \\
PID & Proportional Integral Derivative \\
PLC & Programmable Logic Controller \\
PNL & Pacific Northwest Laboratory \\
psia & Pounds per square inch absolute \\
psig & Pounds per square inch gauge \\
PTFE & Teflon based solid lubricant \\
PV & Process Vent \\
QA & Quality Assurance \\
QAP & Quality Assurance Plan \\
RAS & Radiation Air Sampler \\
RCW & Revised Codes of Washington \\
SAR & Safety Analysis Report \\
SCADA & Supervisory Control and Data Acquisition \\
sCfm & Standard cubic feet per minute \\
SDD & System Design Description \\
SMACNA & Sheet Metal and Air Conditioning Contractors' National Association, Inc. \\
SMCS & System Monitoring and Control System \\
SNF & Spent Nuclear Fuel \\
SNFP & Spent Nuclear Fuel Project \\
SOV & Gas Operated Valve, solenoid \\
SSC & Structure, System and Component \\
SSF & Staging and Storage Facility \\
& \\
\hline
\end{tabular}


WHC-SD-SNF-CDR-007, Revision 0

\section{ACRONYMS}

STD Standard

TBD To Be Determined

TCP/IP Transmission Control Protocol/Intemet Protocol

TEDE Total Effective Dose Equivalent

TI Temperature Indicator

TIC Temperature Indicating Control

TSR Technical Safety Requirements

UL Underwriter's Laboratory

VAC Vacuum

VPS Vacuum Pumping System

WAC Waste Acceptance Criteria

WAC Washington State Administrative Codes

WBS Work Breakdown Structure

WHC Westinghouse Hanford Company

Xe Xenon 
WHC-SD-SNF-CDR-007, Revision 0

DEFINITIONS

\section{DEFINITIONS}

\section{Annular Space Cover}

A ring (that now will be 3-in thick to provide shielding) with a bellows to seal the top of the annular space between the Oven and the MCO. When not is use, the Annular Space Cover can be stored in the Process Enclosure or in a room next to the solid waste storage room. Since the cover will not normally become contaminated, it can be transferred in and out of the Process Enclosure by a heavy duty tray that slides goes in and out of the Process Enclosure. A fork lift can put it on the tray and the manipulator can place it on the MCO. This will require a larger manipulator with more lifting capability.

Cold Trap (a.k.a. “Cs/l Trap")

A water cooled unit that will be located in the Trench and that will be used to remove lodine, Cesium and particulate from the gases being circulated through or removed from the MCO. The water cooling will be accomplished by wrapping a tube around the Cold Trap body such that a double leak would be required for water to contact the process gases (i.e., the tubing would have to leak and the Cold Trap body would also have to leak.) The Cold Trap will be changed when the Process Enclosure is positioned over the Process Pit (and thus also over the Trench Cover). The Trench Cover will be raised and the manipulator will be used to disconnect the spent Cold Trap, blank the Cold Trap end connections, place the spent Cold Trap in a shielded waste drum, and install a new Cold Trap.

\section{Connection Valves}

The valves that will be used to connect the equipment on the Process Module to the interior of the MCO. There are two such valves on each MCO, one that is connected to a dip tube that extends to the bottom of the MCO and one that is connected to a sintered metal filter that is located at the top (interior) of the MCO. The exact configuration of the Connection Valves is TBD.

\section{Insulation Cover (a.k.a. "Insulated MCO Top Cover")}

The cover that is placed on top of the MCO after all of the connections are made. Its purpose is to limit the heat loss from the top of the MCO during the heating cycle. The cover will be handled in the same manner as the Annular Space Cover except that it will be lighter in weight. 
WHC-SD-SNF-CDR-007, Revision 0

\section{DEFINITIONS}

\section{MCO Process Heater}

The electrically heated unit that will be used to heat the air that is circulated around the MCO when it is in the Oven. This unit is currently conceived as an in-duct type heater that will be located in the Process Module.

\section{Oven (a.k.a. "furnace" and "dewar")}

The vacuum insulation around the Oven is also referred to as the Oven Jacket. The current concept of the Oven is a vacuum insulated sleeve in which the MCO is placed. The annular space between the sleeve (Oven) and the MCO is connected by vacuum insulated piping to the MCO Process Heater. During the heating cycle, air is circulated between the Process Heater and the Oven to heat the MCO. Collection rings are located at the top and bottom of the Oven to distribute and collect the heating gases.

\section{Port Covers}

The covers that are on the MCO to protect the Valve Connections when the MCO arrives at the Process Pit.

\section{Process Enclosure (a.k.a. "Cell" and "Hole Portable Operations Cell")}

A shielded enclosure that is equipped with a manipulator, HEPA filtration, operator work station, personnel entry door, waste drum bagport, welder, CCTV, lighting, shelves, hangers, air monitor and radiation monitors. The Process Enclosure will be moved from Process Pit to Process Pit by a cable that is manually attached to the Enclosure. It will have limited, manual, steering capability so that it can be positioned over a Process Pit. Potentially outlines of the Process Enclosure could be painted on the floor to allow sufficiently accurate placement. Its height will be less than 10 feet so the MHM top rail can pass over it without interference. If it is necessary to move it out of the center line of the Process Pits when the MHM is present, then a floor level transfer tray can be provided for this purpose. The Process Enclosure would be parked on the transfer tray and then the tray would transport it to the South and out of the path of the MHM.

Process Module (a.k.a. "Process Bay Skid")

This is skid where the bulk of the hot vacuum conditioning process equipment is located. There will be one Process Module per Process Pit. The Process Module would contain the vacuum pumps, heat exchangers, valving, instrumentation, and other hot conditioning process equipment that does not have to be located in the Process Pit or the Trench. 
WHC-SD-SNF-CDR-007, Revision 0

\section{DEFINITIONS}

Process Pit (a.k.a. "furnace pit" "vault")

This is a hole in the CSB Annex floor that will be approximately 4-ft diameter and 20 -ft deep. The oven will be located in the Process pit. It is anticipated that six Process Pits will be required with a space provided for a seventh to allow welding or active neutron interrogation of the MCO to determine residual water content.

Process Pit Cover (a.k.a. "Oven Top" and "6-in Thick Steel Covers")

A two piece unit consisting of the Process Pit Cover and the Shield Plug. The Process Pit Cover is hinged on one end and will be pivoted out of the way to allow access to the top of the MCO after the Process Enclosure has been positioned over the Process Pit. As currently envisioned, the Process Pit Cover will include a HEPA filter that is capable of allowing $150 \mathrm{cfm}$ of air to be drawn from the CSB Annex operating space to the Process Pit.

\section{Shield Plug}

A unit that is centered in the Process Pit Cover and that is removed by the MHM when a MCO is placed in the Process Pit. This unit is intended to look like a storage position plug to the MHM and thus be handled by it in an identical manner. The plug will probably have to be mechanically locked to the Process Pit Cover so that it will not fall out of the cover then the Process Pit Cover is raised. The details of this mechanical interlock are TBD.

Trench (a.k.a. "Process Trench")

A rectangular cross-section space that is located below floor level and that connects the Process Pit with the Process Module. The piping that connects the MCO and Oven with equipment that is located in the Process Module is located in the Trench.

\section{Trench Cover}

A removable shield that will be located over the Trench to provide shielding should radioactive constituents from the SNF plate out in the piping or be trapped in the Cold Trap.

\section{Vent Cover}

The cover that will be placed over the HEPA filtered MCO vent port. That port must be plugged during the hot conditioning process. " 
WHC-SD-SNF-CDR-007, Revision 0

\subsection{INTRODUCTION}

The K Basins Spent Nuclear Fuel Removal project has been undertaken for the purposes of decommissioning K Basins, stabilizing the SNF, and placing the SNF into dry storage. The project will retrieve Spent Nuclear Fuel (SNF) elements from their storage canisters in the basins, package the SNF into vessels called Multi-Canister Overpacks (MCOs), drain and vacuum dry the MCO contents, condition (degas) the MCO contents at high temperature, and place sealed MCOs into dry storage. This Conceptual Design Report addresses the processes and equipment systems required to perform the hot conditioning.

The mission of the Hot Conditioning System Equipment (HCSE) project is to degas and passivate the SNF that has been dried by the Cold Vacuum Drying System (CVDS), and that has been stored temporarily in the Canister Storage Building (CSB) awaiting availability of a hot conditioning process station. The hot conditioning will assure that the hydrogen production arising from uranium - water vapor reaction, water radiolysis, and uranium hydride breakdown during storage will be less than the design threshold so that the MCOs can be permanently sealed. The degassing is to be accomplished by baking the SNF at a temperature of $300^{\circ} \mathrm{C}$ and evacuating the MCO. This recipe will release chemically bound water (waters of hydration) and also the readily releasable fraction of the hydrogen that is contained in uranium hydride. The passivation of exposed uranium will be accomplished by oxidizing with a low concentration oxygen/inert gas blend. Performance requirements for the HCSE that contain the process parameters are found in Performance Specifications for the Spent Nuclear Fuel Hot Vacuum Conditioning Equipment (WHC-S-0460, Rev 0). The hot conditioning sequence of operations and the process system design are found in Chapter 2 below.

The hot conditioning equipment is to be installed in an annex to the CSB. The construction of the annex, Hot Conditioning System Annex (HCSA) is being provided by the CSB project. This arrangement will allow the MCO Handling Machine (MHM) to perform MCO interchange between the hot treatment process stations and the CSB storage vaults. The CSB storage vaults are vertically oriented, below grade, tubes that protect the SNF from external threats such as tornadoes, earthquakes, and industrial accidents that might be initiators for an SNF release and that also provide concrete and earthen radiation shielding. The MHM is designed to lift the MCO from a below grade tube and to place the MCO into a below grade tube. Therefore, the ovens used to heat the MCO in the HCSE must be located in below grade tubes to that they emulate a storage tube to the MHM. The design requirements that define the interfaces between the HCSE and the annex conceptually are given in Chapter 3.

The hazards associated with Hot Conditioning will be described in a Preliminary Hazards Analysis (PHA). The initial conclusion of the $\mathrm{PH}$ are that there are both process upset and natural phenomena hazards that could cause the primary confinement to fail resulting in significant radiological consequences. Therefore, the Hot Conditioning process will be designated as a Hazard Category 2 (HC2) system per the guidance of DOE STD 1027. 
WHC-SD-SNF-CDR-007, Revision 0

\subsection{INTRODUCTION}

The CSB building structure was not designed to act as an accidental nuclear material release confinement barrier. It does not have a nuclear grade ventilation system that will maintain a negative pressure and collect released material in HEPA filters, nor does it have "safety class" features that might be needed to mitigate a release from the HCSE. The HCSA will not provide any secondary confinement capability to support the HCSE since it is an extension of the CSB structure. The responsibility of providing secondary confinement and of creating the safety structures, systems, and components (SSCs) that mitigate the postulated accidental release from the Hot Conditioning process is assigned to the HCSE project. This means that HCSE will include structures to provide a secondary barrier at all steps of the process that is resistant to the action of Design Basis Accidents (DBAs) including natural phenomenon events, and that traps releases. The HCSE will also include a ventilation system with HEPA filters and stack to hold the secondary confinement volume at a negative pressure. Localized "hardening" will be included in the HCSE design to provide the protection required to assure that the secondary confinement functions during and after a DBA as required by DOE $6430.1 \mathrm{~A}$.

The project has agreed to a policy of "Nuclear Regulatory Commission (NRC) Equivalency" in addition to satisfying the DOE criteria. For the most part the DOE criteria are more restrictive than the equivalent NRC criteria. The major impact on the HCSE project is that the NRC requires that the DBAs include a tornado that is not deemed credible in the DOE criteria for the Hanford Site.

Radiation exposure management is a key issue in the HCSE design as well as it is throughout all phase of the K Basins SNF Removal project. Because the MCO is not contained in a shield cask, the radiation exposure would be quite large in the HCSE unless special design features are implemented. The design incorporates a combination of shielding, remote operations, and automation to absorb radiation, to provide distance between the source and operator, and to minimize operator exposure time. These actions are taken in accordance with the requirements of an ALARA Plan included in the Appendix B. The radiation exposure in the HCSE has been calculated using the CDR sequence of operations (see Chapter 2) and geometry, and radiation exposure maps for the bare MCO design provided by WHC analysts. The resulting dose estimates are reported in Appendix $C$ of this CDR.

The designs and supporting analysis presented in the CDR have been selected using the following reasonableness judgments which the designers believe does lead to a design that will support the specific acceptance criteria whenever they become available.

1. The geometry of the CSB, HCSA, and MHM supports a row of HCSE process stations spaced according to the requirement to avoid interference with the MHM. Seven HCSE stations can fit reasonably in this space. 
WHC-SD-SNF-CDR-007, Revision 0

\subsection{INTRODUCTION}

2. The majority of the times in the process sequence can be reasonably estimated including the heat-up and cool-down times. Although the heat-up and cool-down times are quite model dependent (flows are complex), the conservative results can be selected to assure that the design is robust enough.

3. The evacuation time is unpredictable. However, the vacuum pump selected for the CVDS where off gas rates (water evaporation) are high is a reasonable choice for the HCSE. The time estimates for the CVDS evacuation (which are highly uncertain because of unknown sludge content) have been assumed to be reasonable for the HCSE since the amount of off gas mass removed in the CVDS will certainly exceed the amount of gas drawn off by the CVDS.

4. The worst credible accidental release scenario will be limited by the amount of SNF powder that a burst of gas can carry out of the MCO. This amount is dependent on the mass of gas in the release and is not affected by excess powder in the MCO. Therefore the HCSE safety systems designs are not affected by the amount of powder in the MCO.

5. Six (6) HCSE stations were selected because process time is expected to be similar to the CVDS processing time.

The design has been selected to be a robust design that fits within the constraints of the MHM access area. If the process times turn out to be shorter than anticipated then the Hot Conditioning of the SNF will be completed early. Conversely, if the time is longer than anticipated then the Hot Conditioning will take longer than specified.

A cost estimate has been developed for the HCSE. The details of the estimate are presented in Appendix F. 
WHC-SD-SNF-CDR-007, Revision 0

\subsection{PROCESS}

\subsection{HCSE MODEL OPERATING SEQUENCE}

A model operating sequence has been developed and depicted in an Overall Block Flow Diagram (BFD) (See Drawing SK-2-300411). Rough time estimates have been assigned to each block in the sequence for the purposes of estimating equipment quantities, equipment utilization, and radiation exposure estimates. The sequence assumes the following general description of the HCSE configuration:

Treatment will take place in ovens that are located in process pits below the facility floor. An oven will essentially be a thermos bottle placed within the process pit. The MCO will be placed inside the oven. A cover ring will be placed so that hot air blown through the annular space between the $\mathrm{MCO}$ and the oven interior wall is not lost into the process pit. Heating will be accomplished by blowing hot air through the oven. An insulation cover will be placed over the MCO top. The process pit and oven assembly is shown in Drawing SK-2-400417.

There will be six (6) ovens in the HCSE. The number of oven stations has been determined by calculating the process duration for a single oven using the conceptual sequence of operations as a basis, and by imposing a total annual process demand of $200 \mathrm{MCOs}$.

Transactions where an MCO is either inserted into, or pulled from, an oven will be accomplished by the MCO Handling Machine (MHM) which is part of the Canister Storage Building (CSB). The HCSE will be housed in an annex of the CSB that is accessible to the MHM and that is referred to as the HCSA.

The process pits will have top covers that serve as secondary confinement and radiation shields. These covers will be different from the storage hole plugs in the CSB because the oven will have a larger diameter than the storage holes. The design incorporates a two part cover ( plug within a cover) so that the plug will have the same diameter as the CSB storage hole plugs. This will allow the MHM to make the transactions while maintaining the double confinement of the SNF using the same procedure already planned in the MHM and CSB designs.

There will be a process equipment module associated with each oven. This module will contain a heater and fan that will supply hot $\left(300^{\circ} \mathrm{C}\right)$ air to the oven. The MCO will be heated externally by this air. The process module will contain a vacuum pumping system and an inert gas purge system to handle the gas within the MCO. The oven insulation will be a vacuum jacket that is supported by a vacuum pump in one of the process modules. A service module will contain an exhaust fan and 
WHC-SD-SNF-CDR-007, Revision 0

\subsection{PROCESS}

air cleaning equipment for the air in the process pits and trenches. The exhausts will pass through air cleaning equipment before being discharged from the facility through a stack.

Process lines that connect the process module and the oven will run below floor level in a trench. The hot air lines will be insulated. The trench cover will be thick steel plate to shield radiation arising from condensation of volatile radioactive materials in the lines. The lines have a "cold trap" or "adsorber trap" contained within the trench.

The radiation exposure associated with making and breaking the MCO connections manually and with changing the trap is anticipated to be unacceptable. Furthermore, the connections will be made a few feet below the floor level where manual reach will be awkward. A portable process enclosure will be provided. This enclosure will be parked above the oven when needed. It will offer some shielding and it will contain a hoist and tele-operated manipulator. The hoist will be used to handie the process pit cover and the manipulators will be used to make and break the MCO connections. It has been assumed that the MCO has been designed to support remotely manipulated connections and valves. The enclosure will be ventilated so that it provides secondary confinement while the MCO top is exposed and while the MCO ports are manipulated.

The final closure of the MCO will be made by welding a cover piece on the MCO while the MCO is in the oven after the hot conditioning has been completed. This scheme has not been defined by the MCO design project. It is assumed that the weld will be similar to the multiple pass weld that holds the MCO top plug to the body and that the procedure for the two welds will be approximately the same (root pass, root pass LPT, 5 cover passes, LPT last pass). The new cover will be designed to mate with the MHM.

Given these assumptions the overall sequence is given on Drawing SK- 2-300411. The sequence description is:

\begin{tabular}{|c|c|c|}
\hline ID & Name & Description \\
\hline 1.0 & Select Oven & $\begin{array}{l}\text { Choose an oven to receive the MCO. Inspect } \\
\text { the process module, instruments, gas } \\
\text { supplies, etc. Verify Readiness. }\end{array}$ \\
\hline
\end{tabular}


WHC-SD-SNF-CDR-007, Revision 0

\subsection{PROCESS}

\begin{tabular}{|c|c|c|}
\hline ID & Name & Description \\
\hline 2.0 & Move-in MHM & $\begin{array}{l}\text { Position the MHM over the chosen process } \\
\text { pit. }\end{array}$ \\
\hline 3.0 & Lower MCO & $\begin{array}{l}\text { The MHM executes a sequence where it pulls } \\
\text { the center piece from the process pit cover, } \\
\text { lowers the MCO into the oven, and replaces } \\
\text { the process pit cover piece. }\end{array}$ \\
\hline 4.0 & Remove MHM & $\begin{array}{l}\text { The MHM is undocked and moved out of the } \\
\text { area. }\end{array}$ \\
\hline 5.0 & $\begin{array}{l}\text { Move-in Process } \\
\text { Enclosure }\end{array}$ & $\begin{array}{l}\text { The process enclosure is moved from its } \\
\text { parking place into its operating position above } \\
\text { the process pit. }\end{array}$ \\
\hline 6.0 & $\begin{array}{l}\text { Connect Process } \\
\text { Enclosure }\end{array}$ & $\begin{array}{l}\text { Make the electrical and instrumentation } \\
\text { connections that operate the process } \\
\text { enclosure equipment. }\end{array}$ \\
\hline 7.0 & Open Process Pit & $\begin{array}{l}\text { Actuate the process pit cover opening } \\
\text { mechanism and expose the oven. }\end{array}$ \\
\hline 8.0 & Place Annulus Cover & $\begin{array}{l}\text { Get the annulus cover from its storage } \\
\text { location in the enclosure and place it on the } \\
\text { oven top using the manipulator. }\end{array}$ \\
\hline 9.0 & $\begin{array}{l}\text { Attach Annulus } \\
\text { Cover }\end{array}$ & $\begin{array}{l}\text { Use manipulator and drill motor driven socket } \\
\text { to tighten the bolts. }\end{array}$ \\
\hline 10.0 & Remove Port Covers & $\begin{array}{l}\text { Use the manipulator and drill motor driven } \\
\text { socket to loosen port cover bolts. Use } \\
\text { manipulator and suction device to remove the } \\
\text { covers. }\end{array}$ \\
\hline 11.0 & Install Vent Cover & $\begin{array}{l}\text { Use the manipulator to get a cover from its } \\
\text { storage location in the enclosure and place it } \\
\text { in the vent port. Use the manipulator and drill } \\
\text { motor driven socket to tighten the bolts. }\end{array}$ \\
\hline 12.0 & Install Valves & $\begin{array}{l}\text { Use the manipulator to pick the valves from } \\
\text { their storage ports and plug them into the } \\
\text { MCO ports. Use the manipulator and drill } \\
\text { motor driven socket to tighten the bolts. }\end{array}$ \\
\hline 13.0 & Open Valves & $\begin{array}{l}\text { Use the manipulator and drill motor driven } \\
\text { sockets to drive the valve operator screws. }\end{array}$ \\
\hline
\end{tabular}


WHC-SD-SNF-CDR-007, Revision 0

\subsection{PROCESS}

\begin{tabular}{|c|c|l|}
\hline ID & Name & \multicolumn{1}{|c|}{ Description } \\
\hline 14.0 & Evacuate MCO & $\begin{array}{l}\text { Remove old gas from the MCO using the } \\
\text { system vacuum pump. }\end{array}$ \\
\hline 15.0 & Fill MCO With He & Fill the MCO with helium. \\
\hline 16.0 & Leak Check Ports & $\begin{array}{l}\text { Use the manipulator to move the the sniffer } \\
\text { hose attached to a helium leak detector (part } \\
\text { of the process enclosure equipment) around } \\
\text { the MCO ports. }\end{array}$ \\
\hline 17.0 & Pass Leak Test? & $\begin{array}{l}\text { Determine if corrective action is required } \\
\text { before proceeding. }\end{array}$ \\
\hline 18.0 & Close Bad Valve & $\begin{array}{l}\text { Use the manipulator and drill motor driven } \\
\text { sockets to close the leaking port. }\end{array}$ \\
\hline 19.0 & Disconnect Valve & $\begin{array}{l}\text { Use the manipulator and drill motor driven } \\
\text { socket to loosen the valve attachment bolts. } \\
\text { Use the manipulator to pull the valve out of } \\
\text { the port. }\end{array}$ \\
\hline 20.0 & Clean Port & $\begin{array}{l}\text { Use the manipulator and drill motor driven } \\
\text { cloth pad to clean port. }\end{array}$ \\
\hline 21.0 & Install Reserve Valve & $\begin{array}{l}\text { Use the manipulator to re-install the valve or } \\
\text { to install a reserve valve. Use the manipulator } \\
\text { and drill motor driven socket to tighten the } \\
\text { bolts. Open the valve using the manipulator } \\
\text { held drill motor and sockets. }\end{array}$ \\
\hline 22.0 & Install Insulation & $\begin{array}{l}\text { Use the manipulator to get the insulation } \\
\text { cover from its storage location in the } \\
\text { enclosure and place it on top of the MCO. }\end{array}$ \\
\hline 24.0 & Close Process pit & $\begin{array}{l}\text { Actuate the process pit cover operating } \\
\text { mechanism. }\end{array}$ \\
\hline Cycle & $\begin{array}{l}\text { Hot air flows through the oven. The air } \\
\text { temperature versus time profile will be } \\
\text { controlled by the system PLC. The helium } \\
\text { atmosphere will be circulated and analyzed } \\
\text { continuously. A pressure actuated valve will } \\
\text { relieve gas as the pressure builds due to } \\
\text { heating. Periodic evacuation and helium refill } \\
\text { will be triggered by build-up of water or } \\
\text { hydrogen content. }\end{array}$ \\
\hline & Peat-up \\
\hline 20
\end{tabular}


WHC-SD-SNF-CDR-007, Revision 0

\subsection{PROCESS}

\begin{tabular}{|c|c|c|}
\hline ID & Name & Description \\
\hline 25.0 & $\begin{array}{l}\text { Survey Process } \\
\text { Enclosure }\end{array}$ & $\begin{array}{l}\text { Use the manipulators to swipe selected spots } \\
\text { in the enclosure. Pass bottled swipes out of } \\
\text { the enclosure for counting. }\end{array}$ \\
\hline 26.0 & $\begin{array}{c}\text { Disconnect Process } \\
\text { Enclosure }\end{array}$ & $\begin{array}{l}\text { Disconnect the electrical and instrumentation } \\
\text { services. }\end{array}$ \\
\hline 27.0 & $\begin{array}{l}\text { Remove Process } \\
\text { Enclosure }\end{array}$ & Retum the enclosure to its parking place. \\
\hline 28.0 & Purge Vacuum Cycle & $\begin{array}{l}\text { When the conditioning temperature has been } \\
\text { reached the MCO will undergo a sequence of } \\
\text { evacuation and purge cycles. }\end{array}$ \\
\hline 29.0 & $\begin{array}{l}\text { Perform Acceptance } \\
\text { Test }\end{array}$ & $\begin{array}{l}\text { An acceptance test (unknown at this time) will } \\
\text { be performed to determine that the hot } \\
\text { conditioning has met acceptance criteria } \\
\text { (undetermined at this time). }\end{array}$ \\
\hline 30.0 & Accept? & Determine if more conditioning is needed. \\
\hline 31.0 & $\begin{array}{l}\text { Additional Purge } \\
\text { Vacuum Cycling }\end{array}$ & $\begin{array}{l}\text { Acceptance was not achieved. Continue the } \\
\text { purge vacuum cycle. }\end{array}$ \\
\hline 32.0 & Cool For Passivation & $\begin{array}{l}\text { Circulate cooled air through the oven. The air } \\
\text { temperature versus time function will be } \\
\text { controlled by the system PLC. Stop when the } \\
\text { passivation temperature is achieved. }\end{array}$ \\
\hline 33.0 & Evacuate MCO & Remove the conditioning gas from the MCO. \\
\hline 34.0 & Passivation Gas Fill & Fill the MCO with inert gas/oxygen blend. \\
\hline 35.0 & Passivation Period & $\begin{array}{l}\text { Allow uranium oxidation to proceed. Add } \\
\text { make-up gas to replenish oxygen } \\
\text { concentration as needed. }\end{array}$ \\
\hline 36.0 & Cool Down & $\begin{array}{l}\text { Circulate cooled air through the oven. The air } \\
\text { temperature versus time function will be } \\
\text { controlled by the system PLC. }\end{array}$ \\
\hline 37.0 & Evacuate MCO & Remove the passivation gas from the MCO. \\
\hline 38.0 & Fill With $\mathrm{He}$ & Fill the MCO with Helium. \\
\hline 39.0 & $\begin{array}{l}\text { Move-in Process } \\
\text { Enclosure }\end{array}$ & $\begin{array}{l}\text { The process enclosure is moved from its } \\
\text { parking place into its operating position above } \\
\text { the process pit. }\end{array}$ \\
\hline
\end{tabular}


WHC-SD-SNF-CDR-007, Revision 0

\subsection{PROCESS}

\begin{tabular}{|c|c|c|}
\hline ID & Name & Description \\
\hline 40.0 & $\begin{array}{l}\text { Connect Process } \\
\text { Enclosure }\end{array}$ & $\begin{array}{l}\text { Make the electrical and instrumentation } \\
\text { connections that operate the process } \\
\text { enclosure equipment. }\end{array}$ \\
\hline 41.0 & Open Process Pit & $\begin{array}{l}\text { Actuate the process pit cover opening } \\
\text { mechanism and expose the oven. }\end{array}$ \\
\hline 42.0 & Remove Insul. Cover & $\begin{array}{l}\text { Use the manipulator to grab the insulation } \\
\text { cover and return it to its storage location in the } \\
\text { process enclosure. }\end{array}$ \\
\hline 43.0 & Close MCO Valves & $\begin{array}{l}\text { Use the manipulator and drill motor driven } \\
\text { sockets to close the valves. }\end{array}$ \\
\hline 44.0 & $\begin{array}{l}\text { Place Weld-On } \\
\text { Cover }\end{array}$ & $\begin{array}{l}\text { Use the manipulator to get the weld-on cover } \\
\text { from its storage location in the process } \\
\text { enclosure. Place it over the MCO. }\end{array}$ \\
\hline 45.0 & Install Welder & $\begin{array}{l}\text { Use the hoist and manipulator to get the } \\
\text { automatic welder from its storage location in } \\
\text { the process enclosure. Install the welder on } \\
\text { the top of the MCO. }\end{array}$ \\
\hline 46.0 & Weld Root Pass & Turn on the welder. Make the root pass. \\
\hline 47.0 & Visual inspect & $\begin{array}{l}\text { Inspect the weld using a television camera on } \\
\text { the manipulator arm. }\end{array}$ \\
\hline 48.0 & Clean Weld & $\begin{array}{l}\text { Clean the weld using a rotary stainless steel } \\
\text { wire brush and the manipulator held drill } \\
\text { motor. }\end{array}$ \\
\hline 49.0 & Repair & Is weld repair required? \\
\hline 50.0 & Grind & $\begin{array}{l}\text { Grind out the defect area using a small grinder } \\
\text { (dremel tool) held by the manipulator. }\end{array}$ \\
\hline 51.0 & Repair Weid & Use the welder to reweld the ground out area. \\
\hline 52.0 & Apply Dye & $\begin{array}{l}\text { Apply liquid penetrant dye using a specially } \\
\text { designed applicator held by the manipulator. }\end{array}$ \\
\hline 53.0 & Soak & $\begin{array}{l}\text { Wait the prescribed time for the dye to seep } \\
\text { into weld defects. }\end{array}$ \\
\hline 54.0 & Remove Dye & $\begin{array}{l}\text { Using a specially designed applicator apply } \\
\text { the cleaning fluid and wash off the dye. Finish } \\
\text { with a cleaning fluid soaked rotary buffing pad } \\
\text { driven by the manipulator held drill motor. }\end{array}$ \\
\hline
\end{tabular}


WHC-SD-SNF-CDR-007, Revision 0

\subsection{PROCESS}

\begin{tabular}{|c|c|c|}
\hline ID & Name & Description \\
\hline 55.0 & Apply Developer & $\begin{array}{l}\text { Apply the developer using a specially design } \\
\text { applicator and the manipulator. }\end{array}$ \\
\hline 56.0 & Bleed & $\begin{array}{l}\text { Wait the prescribed time for the dye to "blot } \\
\text { out" of weld defects. }\end{array}$ \\
\hline 57.0 & Inspect & $\begin{array}{l}\text { Inspect the developer coated weld using the } \\
\text { manipulator mounted camera. }\end{array}$ \\
\hline 58.0 & Clean & $\begin{array}{l}\text { Using a specially designed applicator apply } \\
\text { the cleaning fluid and wash off the developer. } \\
\text { Finish with a cleaning fluid soaked rotary } \\
\text { buffing pad driven by the manipulator held drill } \\
\text { motor }\end{array}$ \\
\hline 59.0 & Repair & Is weld repair required? \\
\hline 60.0 & Grind & $\begin{array}{l}\text { Grind out the defect area using a small grinder } \\
\text { (dremel tool) held by the manipulator. }\end{array}$ \\
\hline 61.0 & Repair Weld & Use the welder to reweld the ground out area. \\
\hline 62.0 & Weld Pass 1 & Turn on the welder. Make the weld pass. \\
\hline 63.0 & Visual Inspect & $\begin{array}{l}\text { Inspect the weld using a television camera on } \\
\text { the manipulator arm. }\end{array}$ \\
\hline 64.0 & Repair & Is weld repair required? \\
\hline 65.0 & Grind & $\begin{array}{l}\text { Grind out the defect area using a small grinder } \\
\text { (dremel tool) held by the manipulator. }\end{array}$ \\
\hline 66.0 & Repair Weld & Use the welder to reweld the ground out area. \\
\hline 67.0 & Weld Pass 2 & Tum on the welder. Make the weld pass. \\
\hline 68.0 & Visual Inspect & $\begin{array}{l}\text { Inspect the weld using a television camera on } \\
\text { the manipulator arm. }\end{array}$ \\
\hline 69.0 & Repair & Is weld repair required? \\
\hline 70.0 & Grind & $\begin{array}{l}\text { Grind out the defect area using a small grinder } \\
\text { (dremel tool) held by the manipulator. }\end{array}$ \\
\hline 71.0 & Repair Weld & Use the welder to reweld the ground out area. \\
\hline 72.0 & Weld Pass 3 & Turn on the welder. Make the weld pass. \\
\hline 73.0 & Visual Inspect & $\begin{array}{l}\text { Inspect the weld using a television camera on } \\
\text { the manipulator arm. }\end{array}$ \\
\hline 74.0 & Repair & Is weld repair required? \\
\hline
\end{tabular}


WHC-SD-SNF-CDR-007, Revision 0

\subsection{PROCESS}

\begin{tabular}{|c|c|c|}
\hline ID & Name & Description \\
\hline 75.0 & Grind & $\begin{array}{l}\text { Grind out the defect area using a small grinder } \\
\text { (dremel tool) held by the manipulator. }\end{array}$ \\
\hline 76.0 & Repair Weld & Use the welder to reweld the ground out area. \\
\hline 77.0 & Weld Pass 4 & Tum on the welder. Make the weld pass. \\
\hline 78.0 & Visual Inspect & $\begin{array}{l}\text { Inspect the weld using a television camera on } \\
\text { the manipulator arm. }\end{array}$ \\
\hline 79.0 & Repair & Is weld repair required? \\
\hline 80.0 & Grind & $\begin{array}{l}\text { Grind out the defect area using a small grinder } \\
\text { (dremel tool) held by the manipulator. }\end{array}$ \\
\hline 81.0 & Repair Weld & Use the welder to reweld the ground out area. \\
\hline 82.0 & Weid Pass 5 & Tum on the welder. Make the weld pass. \\
\hline 83.0 & Visual Inspect & $\begin{array}{l}\text { Inspect the weld using a television camera on } \\
\text { the manipulator arm. }\end{array}$ \\
\hline 84.0 & Repair & Is weld repair required? \\
\hline 85.0 & Grind & $\begin{array}{l}\text { Grind out the defect area using a small grinder } \\
\text { (dremel tool) held by the manipulator. }\end{array}$ \\
\hline 86.0 & Repair Weld & Use the welder to reweld the ground out area. \\
\hline 87.0 & Apply Dye & $\begin{array}{l}\text { Apply liquid penetrant dye using a specially } \\
\text { designed applicator held by the manipulator. }\end{array}$ \\
\hline 88.0 & Soak & $\begin{array}{l}\text { Wait the prescribed time for the dye to seep } \\
\text { into weld defects. }\end{array}$ \\
\hline 89.0 & Remove Dye & $\begin{array}{l}\text { Using a specially designed applicator apply } \\
\text { the cleaning fluid and wash off the dye. Finish } \\
\text { with a cleaning fluid soaked rotary buffing pad } \\
\text { driven by the manipulator held drill motor. }\end{array}$ \\
\hline 90.0 & Apply Developer & $\begin{array}{l}\text { Apply the developer using a specially design } \\
\text { applicator and the manipulator. }\end{array}$ \\
\hline 91.0 & Bleed & $\begin{array}{l}\text { Wait the prescribed time for the dye to "blot } \\
\text { out" of weld defects. }\end{array}$ \\
\hline 92.0 & Inspect & $\begin{array}{l}\text { Inspect the developer coated weld using the } \\
\text { manipulator mounted camera. }\end{array}$ \\
\hline
\end{tabular}


WHC-SD-SNF-CDR-007, Revision 0

\subsection{PROCESS}

\begin{tabular}{|c|c|c|}
\hline ID & Name & Description \\
\hline 93.0 & Clean & $\begin{array}{l}\text { Using a specially designed applicator apply } \\
\text { the cleaning fluid and wash off the developer. } \\
\text { Finish with a cleaning fluid soaked rotary } \\
\text { buffing pad driven by the manipulator held drill } \\
\text { motor }\end{array}$ \\
\hline 94.0 & Repair & Is weld repair required? \\
\hline 95.0 & Grind & $\begin{array}{l}\text { Grind out the defect area using a small grinder } \\
\text { (dremel tool) held by the manipulator. }\end{array}$ \\
\hline 96.0 & Repair Weld & Use the welder to reweld the ground out area. \\
\hline 97.0 & Remove Trap & $\begin{array}{l}\text { Use the manipulator to disconnect the trap } \\
\text { and set it aside. }\end{array}$ \\
\hline 98.0 & Install New Trap & $\begin{array}{l}\text { Use the manipulator to get the new trap from } \\
\text { its storage location in the enclosure. Make the } \\
\text { new trap connections using the manipulator. }\end{array}$ \\
\hline 99.0 & $\begin{array}{l}\text { Connect Waste } \\
\text { Drum }\end{array}$ & $\begin{array}{l}\text { Use a cart to place a shielded waste drum } \\
\text { below the process enclosure bag port. } \\
\text { Connect the drum and port. }\end{array}$ \\
\hline 100.0 & Remove Top Shield & $\begin{array}{l}\text { Open the bagport door. Lower a hoist into the } \\
\text { drum and pull the top of the shield into the } \\
\text { enclosure. Set it out of the way. }\end{array}$ \\
\hline 101.0 & Dispose Hot Trap & $\begin{array}{l}\text { Use the manipulator to place the trap into the } \\
\text { drum. }\end{array}$ \\
\hline 102.0 & Insert Top Shield & $\begin{array}{l}\text { Use the hoist to replace the shield to in the } \\
\text { drum. }\end{array}$ \\
\hline 103.0 & Tie Bag & Tie off and cut the bag. \\
\hline 104.0 & Close Drum & $\begin{array}{l}\text { Place the top on the drum and attach it. Move } \\
\text { the drum cart to the waste management area. }\end{array}$ \\
\hline 105.0 & Undo Annulus Cover & $\begin{array}{l}\text { Use the manipulator and drill motor driven } \\
\text { socket to release the annulus cover bolts. }\end{array}$ \\
\hline 106.0 & $\begin{array}{l}\text { Remove Annulus } \\
\text { Cover }\end{array}$ & $\begin{array}{l}\text { Use the manipulator to remove the annulus } \\
\text { cover and place it into its storage location in } \\
\text { the enclosure. }\end{array}$ \\
\hline 107.0 & Close Process Pit & $\begin{array}{l}\text { Actuate the process pit cover mechanism to } \\
\text { close the cover. }\end{array}$ \\
\hline
\end{tabular}


WHC-SD-SNF-CDR-007, Revision 0

\subsection{PROCESS}

\begin{tabular}{|c|c|l|}
\hline ID & Name & \multicolumn{1}{|c|}{ Description } \\
\hline 108.0 & $\begin{array}{c}\text { Survey Process } \\
\text { Enclosure }\end{array}$ & $\begin{array}{l}\text { Use the manipulators to swipe selected spots } \\
\text { in the enclosure. Pass bottled swipes out of } \\
\text { the enclosure for counting. }\end{array}$ \\
\hline 109.0 & $\begin{array}{c}\text { Disconnect Process } \\
\text { Enclosure }\end{array}$ & $\begin{array}{l}\text { Disconnect the electrical and instrumentation } \\
\text { services. }\end{array}$ \\
\hline 110.0 & $\begin{array}{c}\text { Remove Process } \\
\text { Enclosure }\end{array}$ & Return the enclosure to its parking place. \\
\hline 111.0 & $\begin{array}{c}\text { Move-in MHM } \\
112.0\end{array}$ & $\begin{array}{l}\text { Position the MHM over the chosen process } \\
\text { pit. }\end{array}$ \\
\hline 113.0 & Rower MCO & $\begin{array}{l}\text { The MHM executes a sequence where it pulls } \\
\text { the center piece from the process pit cover, } \\
\text { lowers the MCO into the oven, and replaces } \\
\text { the process pit cover piece. }\end{array}$ \\
\hline 114.0 & $\begin{array}{c}\text { Prepare For Next } \\
\text { MCO }\end{array}$ & $\begin{array}{l}\text { The MHM is undocked and moved out of the } \\
\text { area. }\end{array}$ \\
$\begin{array}{l}\text { Perform activities such as supplies } \\
\text { preparation to prepare for the system for its } \\
\text { next use. }\end{array}$ \\
\hline
\end{tabular}

This sequence was used to model the HCSE throughput using an industrial modeling program (WITNESS Release 7.0). This model uses a random number generator to simulate real events (such as which branch paths are followed at decision points in the sequence). The calculation details are given in Appendix $\mathbf{G}$. A run of 10 MCOs indicated that the average process time for an MCO is $101 \mathrm{hrs}$. This suggests that 505 oven hours are required in average week to produce the necessary average of $5 \mathrm{MCOs}$ per week. Assuming a 7 day, 3 shift, operating schedule each week an average of 3.0 ovens will be occupied $100 \%$ of the time. Reasonable allowance for statistical fluctuations, maintenance, and so forth suggests that six stations is appropriate for processing $400 \mathrm{MCOs}$ in two years. 
WHC-SD-SNF-CDR-007, Revision 0

\subsection{PROCESS}

\subsection{MAIN PROCESS SYSTEMS}

The hot conditioning process is schematically depicted by the Process Flow Diagram (SK-2-300412), which has the Material \& Energy Balance on sheet 2 and the Piping and Instrument Diagrams (SK-2-300413) of which there are 5 sheets. The hot condition process is comprised of the Vacuum and Gas Pumping System, the MCO Heating System, and the MCO Cooling system.

\subsubsection{Vacuum \& Gas Pumping Systems}

\subsubsection{System Function}

The vacuum system function is to evacuate the $\mathrm{MCO}$ and connected process piping in order to remove:

A. Chemically bound water;

B. Hydrogen when the hydrides of uranium are decomposed, and

C. Oxygen following the hot conditioning sequence prior to cool-down of the MCO.

The gas pumping system provides for circulation of the helium heat transfer gas during steps in the process where the MCO and its contents are being either heated or cooled.

\subsubsection{System Design Requirements}

The vacuum system must be able to remove gaseous fission products during the treatment of the SNF as well as support the conditions necessary for optimum heat transfer during heating and cooling of the MCO. Operation at 13 psia helium pressure is the base case for this conceptual design. However the process is capable of operation at elevated pressure up to 24.7 psia should that be required to achieve the desired heat transfer rate.

\subsection{Vacuum Operations}

The system, shall be capable of evacuation of the MCO during the following three steps in the HVCS operation:

A. The initial evacuation of the MCO, as received from cold conditioning, prior to system filling with fresh helium to the prescribed pressure, 
WHC-SD-SNF-CDR-007, Revision 0

\subsection{PROCESS}

B. Evacuation at elevated temperature for removal of the decomposition products of uranium hydrides and chemically bound water, and

C. The evacuation of residual oxygen and gaseous fission products following the hot conditioning process.

The vacuum system provides a means of system evacuation in preparation for filling with helium prior to heat transfer operations. A pressure of approximately 10 torr is sought prior to the introduction of a helium gas charge. This provides removal of over 98 percent of the constituents of the previous MCO atmosphere.

The system also provides an MCO vacuum of 5 torr which is conducive to the disassociation of chemically bound water and uranium hydrides at temperatures of $300^{\circ} \mathrm{C}$ and above (see Figure 2.2-1 for the Van't Hoff curve for the uraniumhydrogen system). The Van't Hoff curve shows that the vapor pressure of hydrogen over uranium hydride is about $28 \mathrm{Torr}$ at $300^{\circ} \mathrm{C}$. The vacuum system is held at this condition for approximately $\mathbf{4 8}$ hours, sufficient time to allow for water release, hydride decomposition and for hydrogen diffusion to the gas space of the MCO.

During the lower temperature partial oxidation phase of the hot conditioning process, low concentrations of oxygen are introduced into the MCO in an inert carrier gas stream. This low concentration of oxygen consumes highly reactive sites on the fuel inside the MCO, reducing the chemical reactivity of the damaged fuel matrix that may be present in the MCO. Not the reactive sites consumed by this partial oxidation may include small fuel fragments, high surface area uranium hydride particles, and high surface area uranium metal particles created by the thermal decomposition of uranium hydride. During oxide formation, gaseous fission products diffuse through the oxide and are released to the gas phase. These fission products, which are radioactive, are removed in a product purifier by either sorption, reaction, or condensation. Equipment for gas purification is part of the vacuum system.

During vacuum operations as well as during operations with gas circulation, fission products are removed from the gas phase.

\subsection{Gas Circulation}

Helium gas is charged to the MCO void space to promote heat transfer from the MCO wall to the fuel elements. The rate of this heat transfer may be the limiting factor which determines the time for MCO heating and cooling. To increase the likelihood of heat transfer operations taking place at a rate which is conducive to achieving a faster throughput, forced convective heat transfer from the MCO wall to the fuel will be used. The characterization of this heat transfer operation is quite 
WHC-SD-SNF-CDR-007, Revision 0

\subsection{PROCESS}

definitive design, advanced thermal analysis using finite element techniques will be use to predict the composite internal heat transfer coefficient for MCO to fuel heat transfer. This conceptual design provides a vacuum system that is capable of heating the MCO and SNF with recirculating helium at pressures ranging from 13 psia to 24.7 psia. The baseline pressure for the CDR is 13 psia.

Helium circulation is employed in all heat transfer operations, so that heat can be added or removed from by transfer in external heat exchangers. Fission products are removed in a cold trap during gas circulation.

Table 2.2-1 provides key design parameters for the Vacuum System.

\begin{tabular}{|l|c|c|}
\hline \multicolumn{1}{|c|}{ Parameter } & Units & Specified Rating \\
\hline Evacuation prior to helium fill & torr & 10 \\
\hline Design pressure range & psia & 0.1 to 24.7 \\
\hline Material of construction & NA & 304 or 316 SS \\
\hline Maximum design temp. for: & & \\
\hline vacuum pump & ${ }^{\circ} \mathrm{C}$ & 40 \\
\hline circulation blower & ${ }^{\circ} \mathrm{C}$ & \\
\hline heat exchangers & ${ }^{\circ} \mathrm{C}$ & 375 \\
\hline Operating temperature for: & & \\
\hline vacuum pump & ${ }^{\circ} \mathrm{C}$ & 25 \\
\hline circulation blower & ${ }^{\circ} \mathrm{C}$ & 100 \\
\hline heat exchangers & ${ }^{\circ} \mathrm{C}$ & 40 to 300 \\
\hline Time at vacuum: & & \\
\hline pre He fill prior to heat-up & min. & 15 \\
\hline hydride removal & hrs. & 48 \\
\hline $\begin{array}{l}\text { pre He fill following } \mathrm{O}_{2} \\
\text { passivation }\end{array}$ & min. & 15 \\
\hline Time at He circulation & & \\
\hline heating to $300^{\circ} \mathrm{C}$ & hrs. & 12 \\
\hline cooling to $150^{\circ} \mathrm{C}$ & hrs & 12 \\
\hline He circulation rate & ACFM & $<10$ \\
\hline
\end{tabular}

Table 2.2-1 Vacuum \& Gas Pumping System Design Specifications 
WHC-SD-SNF-CDR-007, Revision 0

\subsection{PROCESS}

Figure 2.2-1

\section{Van't-Hoff Curve}

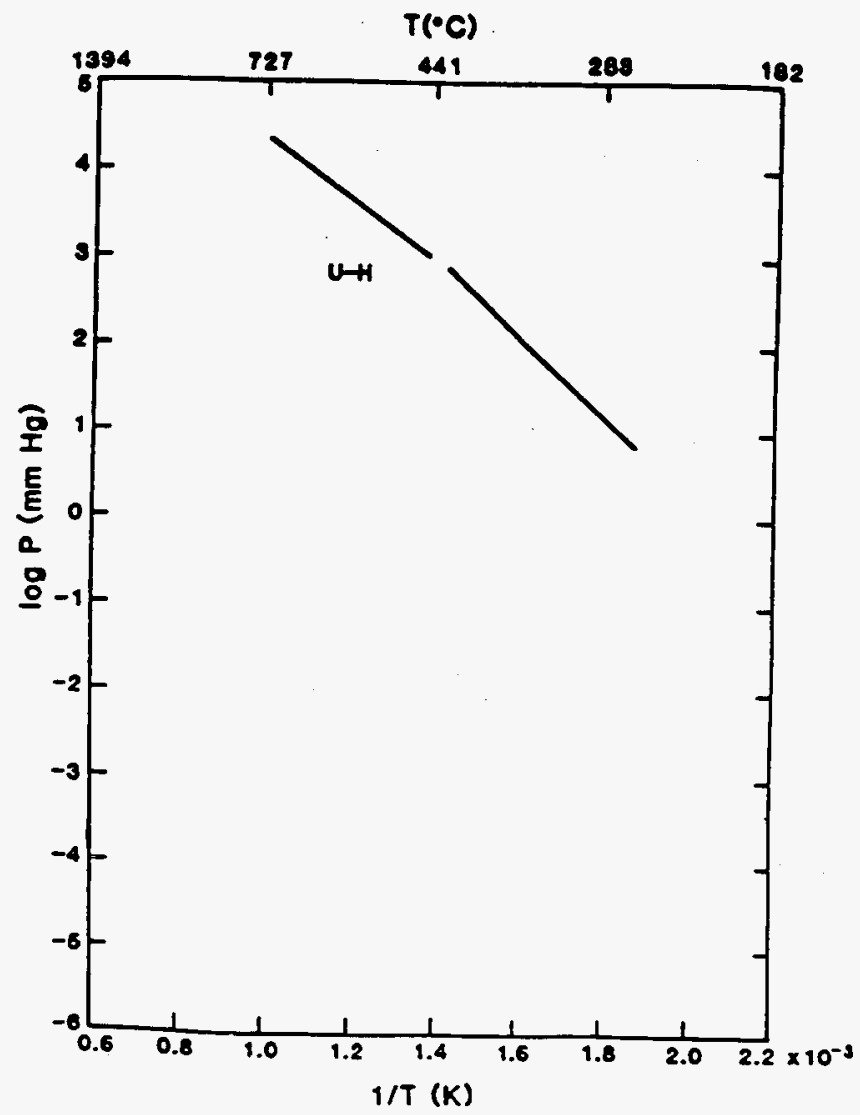


WHC-SD-SNF-CDR-007, Revision 0

\subsection{PROCESS}

The vacuum and gas pumping system will interface with the following other process systems:
A. the MCO heating system
B. the MCO cooling system
C. the process gas system
D. the process module system
E. the combined ventilation system
F. the HVCS control system

\subsubsection{Equipment Arrangement and Response to Design Requirements}

Equipment required for the HCSE is identified on the Piping and Instrument Diagrams (SK-2-300413). The following major equipment components comprise the MCO vacuum pumping system on each of the six process modules or in each of the six MCO trenches:
A. VPS-VAC-1104, the scroll-type vacuum pump.
B. VPS-BLO-1106, the Rotron recirculation blower.
C. VPS-PFR-1101, the purifier/cooler/filter containing copper gauze and activated charcoal.
D. VPS-CLR-1103, the cooler, a plate-type heat exchanger.
E. VPS-HCL-1105, the electrical heater.
F. VPS-F-1102, VPS-F-1107 HEPA filters.

\subsection{Gas pumps}

Two gas pumps are used in the vacuum system, one a vacuum pump and the other a circulating blower. Vacuum pump VPS-VAC-1104 is a scroll-type pump, capable of pumping 500 liters per minute at inlet pressures above 1 torr with reduced 
WHC-SD-SNF-CDR-007, Revision 0

\subsection{PROCESS}

pumping rates at higher vacuum levels. When properly installed and operated, this pump has an expected life of 6,000 hours before minor maintenance is required for shaft bearing replacement and of 12,000 hours before requiring major maintenance. With an MCO total process cycle time of 150 hours and an expectation of up to 68 MCO process events per bay over the life of the campaign, the vacuum pump will have to be either idled during portions of cycle, operated to an estimated 10,000 hours without performing the minor maintenance suggested by the manufacturer, or replaced once during the life of the MCO hot vacuum conditioning program. Because the level of vacuum required of VPS-VAC-1104 is moderate in comparison to its capability, it is likely that the pump will be operated without minor maintenance for the required period without serious impact to ongoing operations. Once a vacuum pump is taken out of service, it will be discarded as radioactive waste.

Helium circulation blower VPS-BLO-1106 is a magnetically coupled regenerative unit capable of operating under vacuum or at pressure up to $10 \mathrm{psig}$. The unit is constructed for intrinsically safe operation. The magnetic drive provides freedom from seal leakage worries.

\subsection{Heat Exchangers}

Proper operation of VPS-VAC-1104 requires precooling during the 48-hour vacuum phase of chemically bound water and hydride removal which takes place at or above $300^{\circ} \mathrm{C}$. To accomplish this, two stages of cooling are provided. The first stage is the cold trap which serves as the vent purifier VPS-PFR-1101. This unit reduces temperature from a nominal inlet temperature of $300^{\circ} \mathrm{C}$ to $100^{\circ} \mathrm{C}$. Further cooling is provided by a plate-type heat exchanger VPS-CLR-1103 which achieves an intended outlet temperature of $38^{\circ} \mathrm{C}$. Both exchangers are cooled with streams of exhaust air from the ventilation the MCO pit before it is passed to the central process ventilation system for HEPA filtration and discharge through the stack.

The exchangers of the vacuum system are designed to accommodate heat transfer operations with helium atmospheres from 13 to 24.7 psia.

\subsection{Cold Trap \& Gas Purification Equipment}

Cold trap VPS-PFR-1101 contains copper gauze to react with iodine fission product, chilling to capture cesium fission product and activated carbon for both cesium and iodine secondary capture. The cold trap may become contaminated with high level fission products and thus is installed in the MCO trench where it will be serviced by the process enclosure. It is anticipated that this unit may have to be changed for each MCO which is processed. Included in the purifier is a HEPA filter. 
WHC-SD-SNF-CDR-007, Revision 0

\subsection{PROCESS}

\subsection{Instrumentation and Control Requirements}

The module control configuration and logic are shown on the P\&IDs and are discussed in the Operating Sequence Section. Programmable Logic Controllers, one at each process module, are used to assure proper operation and reliability. Each communicates with each instrument element via local connection and with the control center via one line on which signals are multiplexed. Several interlocks are provided to assure the proper sequence is followed. While direct operator intervention for many operating steps is available to override control logic, the areas where serious safety implications are involved cannot be overridden without the approval from a second authority. Data logging as well as process control is available.

\subsubsection{MCO Heating System}

\subsubsection{System Function}

The MCO heating system heats the MCO and its fuel contents to a processing temperature of above $300^{\circ} \mathrm{C}$ within the required cycle time. Heating is indirect, via hot air circulation around the MCO and circulation of the helium contained in the MCO through an external electric heater. The system must be able to heat the $\mathrm{MCO}$ at a rate which does not excesd $50^{\circ} \mathrm{C} / \mathrm{hr}$. It must also function through a wide range of internal helium pressures. Heating is to be electrically powered.

The system shall be designed so that it has little if any impact upon the air balance in the HCSA.

\subsubsection{System Design Requirements}

There are several restrictions on the heating of the MCO as specified by WHC-S0460, revision A and summarized in Table 2.2-2. 
WHC-SD-SNF-CDR-007, Revision 0

\subsection{PROCESS}

\begin{tabular}{|l|c|c|}
\hline \multicolumn{1}{|c|}{ Parameter } & Units & Specified Rating \\
\hline Maximum MCO temperature & ${ }^{\circ} \mathrm{C}$ & 375 \\
\hline Minimum MCO temperature & ${ }^{\circ} \mathrm{C}$ & 300 \\
\hline Maximum MCO heating rate & ${ }^{\circ} \mathrm{C} / \mathrm{hr}$ & 50 \\
\hline $\begin{array}{l}\text { Maximum temperature } \\
\text { change over MCO }\end{array}$ & ${ }^{\circ} \mathrm{C}$ & 100 \\
\hline Duration for MCO heating & $\mathrm{hrs}$ & $\sim 12$ \\
\hline
\end{tabular}

Table 2.2-2 MCO Heating System Design Specifications

\subsubsection{Equipment Arrangement}

It was originally conceived that the MCO would be heated with clamping band-type electrical resistance heaters. Upon further consideration of this method it became apparent that it would be difficult to assure even heating and the ability to stay within the $50^{\circ} \mathrm{C} / \mathrm{hr}$ limitation. Also, the mechanical complexity of direct contact conductive heat transfer was significantly greater than alternative options using convective transfer. On this basis the determination was made to proceed with forced convection for heating of the outer shell with supplemental heating of the circulating helium stream via circulation through an electrical resistance heating element.

\subsection{Heating of the MCO Shell}

Heating the MCO with forced hot air is a two stage process. The shell is heated and the hot shell transfers its heat to the internal SNF. Transfer from the shell to the SNF occurs principally through convection of the internal gas. Helium is chosen for the heat transfer fluid because, next to hydrogen it has the highest thermal conductivity and unlike hydrogen it is safe to handle, at all concentrations.

Primary heating of the MCO was chosen to be via forced air convective heat transfer. Air is circulated and heat is applied at each pass with an electrically powered unit called the MCO Process Heater. The clearance between the oven wall and the outer surface of the MCO is one inch. The air flow rate is 750 acfm which is sufficient to produce turbulence in the annular space. 
WHC-SD-SNF-CDR-007, Revision 0

\subsection{PROCESS}

Calculation of the MCO outside and inside heat transfer coefficients indicated that heat transfer from the shell to the fuel would be the limiting factor with subatmospheric internal helium pressure. Heat transfer coefficients are shown in the following equation:

$$
\begin{array}{ll} 
& Q=U * A *\left(T_{1}-T_{2}\right) \\
\text { where: } & Q=\text { heating rate in Btu/hour } \\
& U=\text { heat transfer coefficient in Btu/hour * sq. ft. * oF } \\
A=\text { area of heat transfer in sq. } \mathrm{ft} . \\
\left(T_{1}-T_{2}\right)=\text { temperature difference between the heat } \\
\text { source and the heat sink in }{ }^{\circ} \mathrm{F}
\end{array}
$$

The outside coefficient was calculated, using the Colburn $\mathrm{j}$ factor , and found to be 5. The inside coefficient was calculated from a modified Nusselt number for convection in annular spaces and found to be 1.77 which was discounted to 1.0 due to the complex internal geometry. These two transfer coefficients were used in calculating both heating and cooling temperalure profiles. At sub-atmospheric pressure there is less than a 10 percent contribution to heating from the heat applied to the circulating helium. This contribution would increase if a pressure of 24.7 psia were used for the circulating helium.

The temperature profile for a MCO helium pressure of 13 psia is shown as Figure 2.2-2. It does not account for the minor contribution by heating of the circulating helium, but does allow for internal heating due to nuclear decay. It shows that heating to above $300^{\circ} \mathrm{C}$ may be accomplished in under 14 hours from a starting temperature of $40^{\circ} \mathrm{C}$. The actual starting temperature is expected to be about $140^{\circ} \mathrm{C}$ since the precooling step that was initially thought to be required is not necessary. Thus the heat up time will be about 10 hours. When the SNF removal project was originally conceived it was though that connections to the MCO would be made manually so the MCO would have to be cooled from its equilibrium storage temperature to $40^{\circ} \mathrm{C}$. All remote operations from the Process Enclosure are now planned so the connections can be made at $140^{\circ} \mathrm{C}$.

As shown on the heating temperature profile, incrementally changing the temperature of the heating air is necessary to assure that the MCO heating rate does not exceed the $50^{\circ} \mathrm{C}$ per hour specification. The control is accomplished via a programmed ramping function. Feedback to the controller is obtained by monitoring the temperature of the circulating helium gas. The ramping rate can be revised based on experience with the first MCOs that are processed. 
WHC-SD-SNF-CDR-007, Revision 0

\subsection{PROCESS}

\subsubsection{MCO Cooling System}

\subsubsection{MCO Cooling System Design Description}

\subsection{System Function}

The MCO cooling system is to cool the MCO from $300^{\circ} \mathrm{C}$ to $150^{\circ} \mathrm{C}$ within an approximately 12 hour period, while not exceeding the 50-degree per hour specification.

\subsection{System Design Requirements}

The design requirements are presented in the table below:

\begin{tabular}{|l|c|c|}
\hline \multicolumn{1}{|c|}{ Parameter } & Units & Specified Rating \\
\hline Maximum MCO cooling rate & ${ }^{\circ} \mathrm{C} / \mathrm{hr}$ & 50 \\
\hline $\begin{array}{l}\text { Maximum temperature } \\
\text { change over MCO }\end{array}$ & ${ }^{\circ} \mathrm{C}$ & 100 \\
\hline Duration for MCO cooling & $\mathrm{hrs}$ & 12 \\
\hline
\end{tabular}

Table 2.2-3 MCO Cooling System Design Specifications 
WHC-SD-SNF-CDR-007, Revision 0

Figure 2.2-2

Heating Temperature Profile

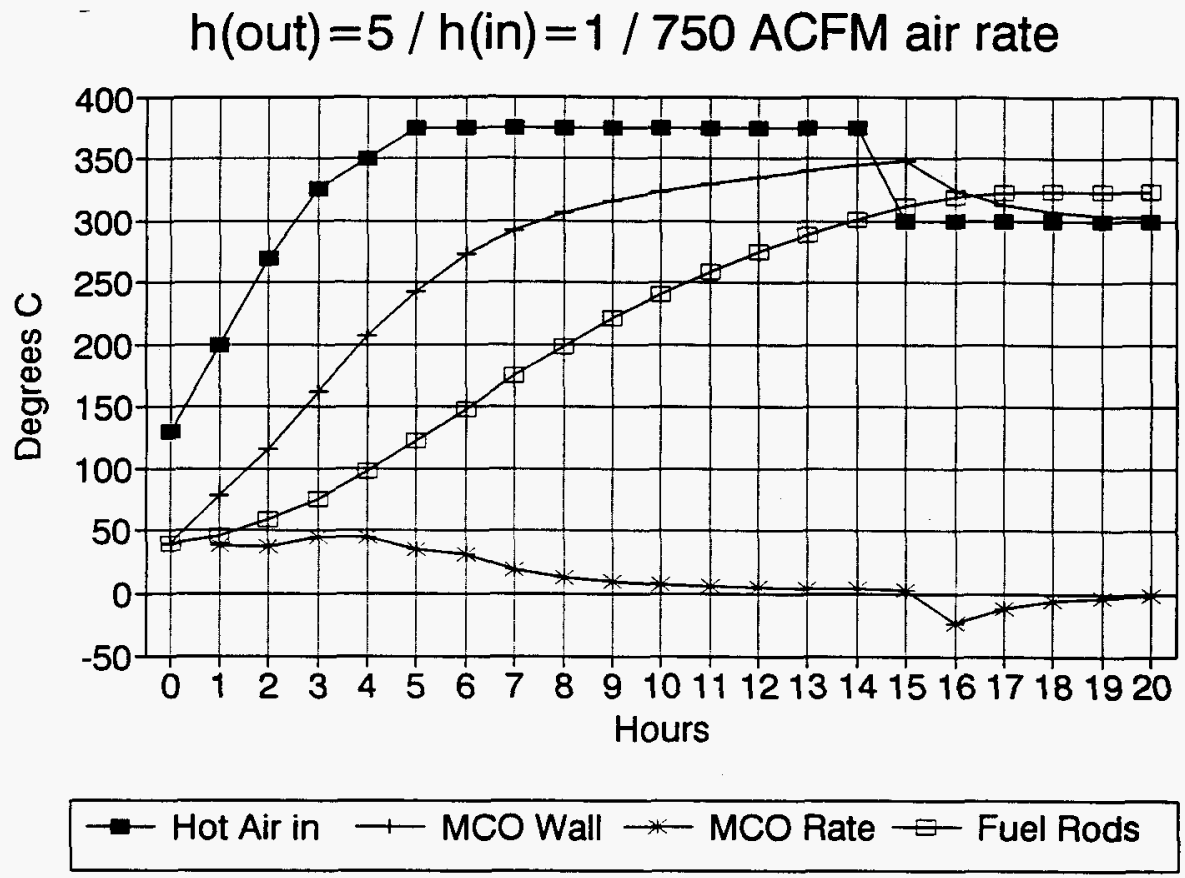


WHC-SD-SNF-CDR-007, Revision 0

\subsection{PROCESS}

\subsection{Equipment Arrangement}

\subsection{MCO Shell Cooling}

Air that circulates through the oven is cooled by exchanger MCS-CLR-1153. The cooled air is recirculated back to the MCO. MCS-CLR-1153 may be supplied with chilled water coolant from either or both of the central chillers CHW-CH-2023 and $\mathrm{CHW}-\mathrm{CH}-2024$. The temperature of the air exiting the cooling system is programmed to allow MCO cooling without exceeding the $50^{\circ} \mathrm{C}$ per hour temperature change specification.

The largest influence on MCO cooling will be the interior film coefficient. The base case design assumes an interior film coefficient on/when the internal helium atmosphere is 13 psia.

Cooling air is circulated to the MCO shell at a minimum temperature of $40^{\circ} \mathrm{C}$. Should a lower temperature be desired, it is easily achieved.

The cooling temperature profile is shown as Figure 2.2-3 on the following page.

\subsection{Helium Circulation Cooling}

Cooling of the circulating helium stream takes place in two stages. The cooling is primarily used to allow low temperature operation of the vacuum pump and circulating blower. The first stage cold trap, VPS-PFR-1101, provides a degree of purification as well as provides cooling to $100^{\circ} \mathrm{C}$ or lower so that VPS-BLO-1106 may safely operate within its temperature limit.

The second stage of cooling allows the scroll vacuum pump, VPS-VAC-1104 to operate below its maximum inlet temperature of $40^{\circ} \mathrm{C}$. Chilled water cools the circulating helium to $25^{\circ} \mathrm{C}$ before its introduction to the vacuum pump.

During the MCO heating cycle, the cooled helium is electrically reheated by VPS$\mathrm{HCL}-1105$ prior to its introduction back into the MCO, however in the cooling cycle it is introduced at the temperature at which it discharges from VPS-BLO-1106. The temperature rise through this blower is nominally $2^{\circ} \mathrm{C}$. 
WHC-SD-SNF-CDR-007, Revision 0

\subsection{PROCESS}

Figure 2.2-3

Cooling Temperature Profile

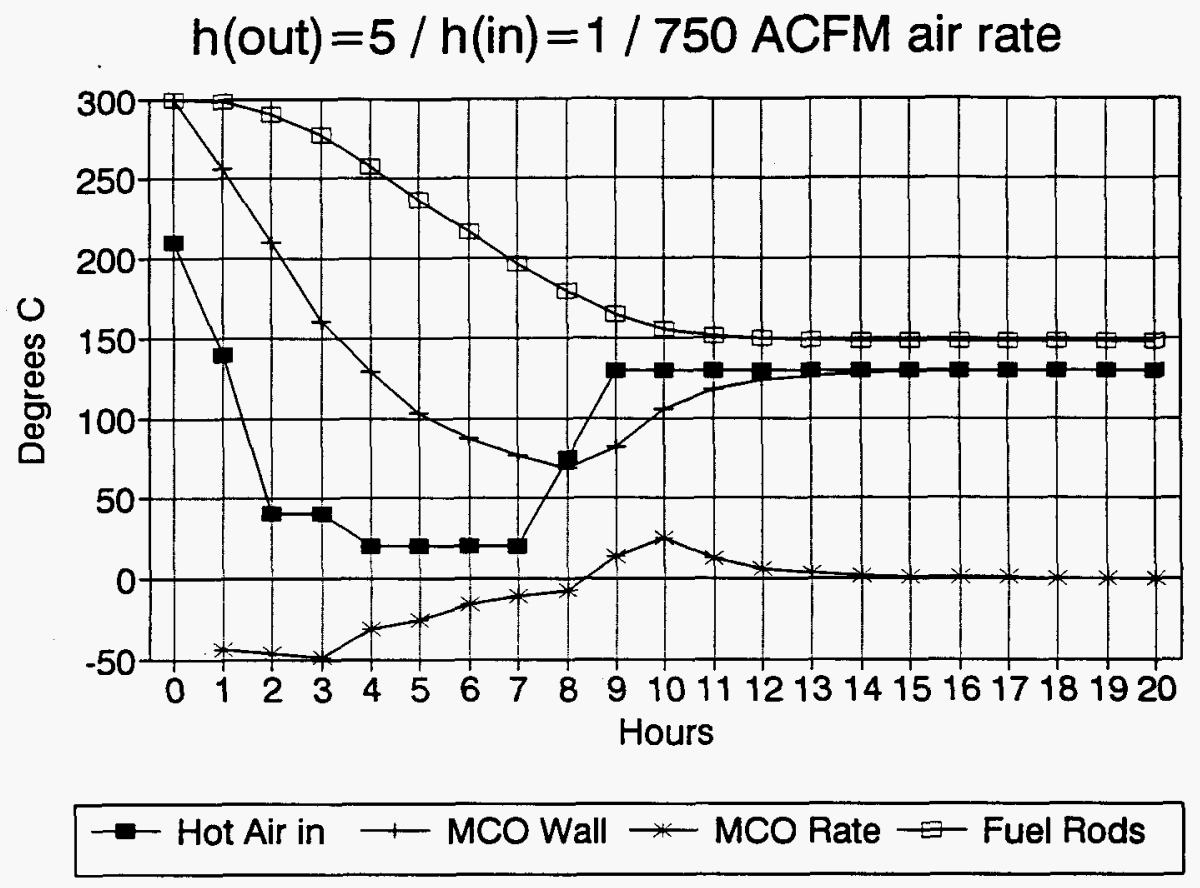


WHC-SD-SNF-CDR-007, Revision 0

\subsection{PROCESS}

\subsection{Instrumentation and Control Requirements}

Ramped temperature control is also used for MCO cooling for staying within the rate of temperature change specification. This is pre-programmed in the units PLC. Suggested ramping temperatures are shown on the temperature profile of the following page.

Control interlocks assure that heater PHS-HCL-1152 remains off during the cooling cycle.

\subsubsection{Sequence of Operation}

The sequence of operation for the HCSE is as follows. Please refer to P\&ID drawing SK-2-300413 sheet 2, Appendix A to follow these steps.

A. MCO vault ventilation air flow is initiated by selecting and starting either Process Vent Blower (PV-BLO-2043 or PV-BLO-2046), if not already running, and drawing air through the MCO vault and the vault trench, and checking that the flow is about 1000 cfm on Fl-1174;

B. The MCO is placed into the oven by the MHM and the process enclosure is positioned over the oven.

C. The block valve for the Oven (PHS-OVEN-1154) is opened if not already open, holding a vacuum of less than $10^{-4}$ torr measured on PIC-1181. The HCS Oven Vacuum System, VAC-VAC-3041,3042, should be functional;

D. The MCO is connected to process gas piping by swinging the connection valves and quick disconnects into place over the valve ports and fastening remotely. Anytime the MCO valves and quick disconnects are being manipulated, vacuum should be applied by running the MCO Vacuum Pump (VPS-VAC-1104), closing GOV-1112,1120 and opening GOV-1105,1121. Once the quick disconnect is secured, the valves are opened followed by immediate evacuation using MCO Vacuum Pump. The MCO Vault Cover is re-seated. During the evacuation, the MCO Vacuum Pump is exhausted through PV-1109, GOV-1112,1120,1121 are closed, GOV-1105 is opened and GOV-1136 is opened to allow a small process gas (He or $\mathrm{N}_{2}$ ) purge. Once a vacuum of 10 torr (PI-1102) is achieved, PV-1109 is closed, GOV1105 is closed, MCO Vacuum Pump stopped, GOV-1120 is opened, GOV1133 is opened and process gas is added through FIC-1134 at $280 \mathrm{lpm}$ until PIC-1115 reaches $\sim-0.1$ psig; 
WHC-SD-SNF-CDR-007, Revision 0

E. The MCO process heating system is changed from "STARTUP" to "HEATING" mode by closing GOV-1155, maintaining about a $750 \mathrm{cfm}$ Process Heating Loop flowrate with FIC-1153 and the Process Heating Loop Blower (PHS-BLO-1151) operating. TIC-1154, which now controls the MCO Process Heater, (PHS-HCL-1152), is setting FIC-1122 to "AUTO" (process gas added only for pressure control and water hydrogen dilution) with a setpoint determined by a prescribed temperature rise profile;

F. Circulating helium is heated using the MCO Circulating Heater (PG-HCL-1105). Temperalure exiting the heater is controlled by TIC-1119 according to a prescribed temperature rise profile. The MCO exit gas is passed through a Cold Trap (VPS-PFR-1101), where the gas is cooled to $\sim 100^{\circ} \mathrm{C}$ to protect downstream equipment and to adsorb volatile components such as $\mathrm{H}_{2} \mathrm{O}$, iodine, cesium, etc.;

G. Pressure and composition control of the MCO process gas loop is accomplished during heat-up by maintaining PI-1115 at about $\sim 0.1$ psig by bleeding MCO gas through SOV-1118, and by adding purge helium through either FI-1136 (preset to $\sim 0.9 \mathrm{lpm}$ ) or through FIC-1134 at higher rates. Helium purge may be needed to control $\mathrm{H}_{2} \mathrm{O}, \mathrm{O}_{2}$ or $\mathrm{H}_{2}$ levels as measured by $\mathrm{Al}-1117$ residual gas analyzer;

$\mathrm{H}$. Once the $\mathrm{MCO}$ core has reached approximately $300^{\circ} \mathrm{C}$, as indicated by the temperature of the MCO exit gas, TI-1101, and the patterned heat transfer calculations, the helium is evacuated from the system to a pressure of $\sim 5$ torr using the MCO Vacuum Pump with GOV-1112,1120,1121 closed and GOV-1105 opened. FI-1136 is used to bleed helium in at a rate of $0.9 \mathrm{lpm}$ (10 acfm MCO internal gas rate). The MCO is maintained at $\sim 5$ torr and $300-350^{\circ} \mathrm{C}$ for a period of 48 hours. $\mathrm{H}_{2}$ and $\mathrm{H}_{2} \mathrm{O}$ are monitored using Al-1117. When $\mathrm{H}_{2}$ and $\mathrm{H}_{2}$ generation ceases, it is assumed that all hydrides anc chemically bound water have been decomposed.

I. During withdrawal of the water and hydrogen with the vacuum pump, several fission products may be extracted. These could include gaseous $\mathrm{Cs}^{137}$ and $\mathrm{I}^{129}$ and $\mathrm{Kr}^{8} 5$ as well as isotopes of magnesium and cobalt which are in particulate form. $\mathrm{Cs}^{137}$ and $\mathrm{L}^{129}$ are adsorbed in the Cold Trap. The $\mathrm{Kr}^{85}$ passes through to the stack via the HEPA filters. Particulate $\mathrm{Mg}$ and $\mathrm{Co}$ are captured in the HEPA filter (VPS-F-1102). 
WHC-SD-SNF-CDR-007, Revision 0

\subsection{PROCESS}

J. The MCO heating and cooling system is changed to "COOLING" mode with a TIC-1154 setpoint of $300^{\circ} \mathrm{C}$ and GOV-1155 opened. A prescribed cool down rate is followed until the MCO core temperature is reduced to $150^{\circ} \mathrm{C}$ (TI-1101). The MCO process gas loop is returned to loop flow during this time, and the process system pressure adjusted to $\sim 0.1$ psig, PIC-1115, using purge helium as above. The MCO Process Heater, is disabled during the cool down period;

K. Oxygen sufficient to bring the composition of the MCO process gas to $\sim 2 \%$ is through an open GOV-1142 and FQIC-1141. The oxygen totalizer will close when the calculated amount of oxygen has been added, or the analyzer, $\mathrm{Al}-1117$, indicates $>2.5 \% \mathrm{O}_{2}$ or the system pressure, PIC-1115, has risen to a greater gas pressure that calculations permit. Once the oxygen has been introduced, the process gas loop is circulated at $150^{\circ} \mathrm{C}$ until the oxygen level drops below $0.5 \%$ (the system pressure should record a calculated pressure drop in rough agreement). The process is repeated until the oxygen level fails to decrease appreciably below $2 \%$. At this point the system is held for an additional 12 hours with the oxygen concentration continuously monitored;

L. Following the 12 hour passivation period, the oxygen is displaced by helium by purging as above and evacuating the MCO to a pressure $<50$ torr, and refilling with helium. Once $\mathrm{O}_{2}$ levels have been verified to below $0.1 \%$, process is complete.

The MCO vault cover is removed. The MCO valves and quick disconnects are disconnected.

M. The MCO ports are covered and seal-welded according to procedures;

N. The welds are inspected.

O. The top of the MCO is surveyed and decontaminated as necessary prior to transfer to a storage position in the CSB;

P. The cold trap will need to be periodically replaced according to procedures between MCO runs. The replacement schedule will depend upon the quantity of material that is collected in the trap as determined by radiation readings. 
WHC-SD-SNF-CDR-007, Revision 0

\subsection{PROCESS}

\subsubsection{DESCRIPTION OF SAFETY SYSTEMS}

Overall, the PLC control system will provide a high degree of non-passive control and coordination. Interlocks will include these important loops:

A. All operations will be structured to allow PLC computer control of sequencing, valve positioning and other important parameter setting. Operators will interact with the system by changing the sequencing mode to various allowed states such as "IDLE", "STARTUP", "HEATUP", "VACUUM_PURGE", "COOLDOWN", "PASSIVATE", etc. Within each of these states, are allowed valve positions, controller states, process conditions, etc. If these conditions are not correct progress through the process is held. The control system will normally function without operator intervention. If operators wish, they can override most PLC functions. Exceptions are required interlocks.

B. All operations will be tied into the utilities status. If utility systems including the oven vacuum, process vent, process gases and cooling water systems are malfunctioning, operation of the HCSE system will be limited to essential operations.

C. Oxygen addition during passivation is set up with several layers of protection. The first is that the addition controller, FQIC-1131 is a totalizing controller which feeds only a pre-calculated amount of oxygen into known conditions, the second is the protection of the gas analyzer, Al-1117, and the third is the secondary check of rising system gas pressure beyond the bounds of a $2 \%$ pressure rise. Any violation of these checks will cause the isolation valve, GOV-1142 to close and the output mode of the controller, FQIC-1131 to change to "MANUAL" with an output of 0.0 .

D. To avoid excessive dust migration within the MCO, the flow rate of circulating gas is limited to $10 \mathrm{scfm}$ at ambient pressures and $10 \mathrm{acfm}$ at low pressures. This is accomplished using FIC-1114 to control the flowrate (calculated flow intemal to MCO).

E. MCO gas composition control is accomplished by using analyzer, Al-1117, to sense high levels of hydrogen or water. When these gas levels reach a threshold, purge gas is added using either FIC-1134 or FI-1136. Excess, pressure is then bled off through SOV-1118. The MCO could also be evacuated and refilled at this point. 
WHC-SD-SNF-CDR-007, Revision 0

\subsection{PROCESS}

F. In the event of an electrical or instrument air failure, $\mathrm{FI}-1136$ will purge the MCO system with process gas up to a pressure of 0.5 psig. This protects the situation when a full vacuum is being held on a hot MCO and the control systems fail. If a leak were to develop, air would begin leaking into the MCO leading to rapid oxidation reaction. This system prevents this by purging the MCO with inert gas.

G. In the event that the internal MCO filter becomes plugged, a blowback system exists to clear the blockage. An accumulator holds sufficient gas inventory to supply a pulse of inert gas through GOV1132. In order to prevent gas bypassing or passage of dust into the draw tube, valves GOV$1105,1112,1120,1121$ will be closed during blowback operations.

H. Fl-1136 is a rotameter set at $0.9 \mathrm{lpm}$. This rate is equivalent to $10 \mathrm{acfm}$ through the $\mathrm{MCO}$ at 5 torr and $300^{\circ} \mathrm{C}$.

I. The MCO heating system uses flow control, FIC-1153, in the circulation loop. This allows for a wider range of heating and cooling situations to be met. Additionally, the heating system used temperature control, TIC-1154 to control the heater input while holding TV-1154 wide open. The cooling system uses TIC-1154 to disable the heater while modulating cooling temperature with TV-1154.

J. Pressure control of the heating system is by bleeding pressures over 0.1 psig, PCV-1159, into the process vent system (usually during startup and during heating cycles) and by allowing air into the system through PCV-1156 set at -0.1 psig. Air inlet will be required during cooling and when air leakage from between the MCO/OVEN annulus into the vault is excessive. 
WHC-SD-SNF-CDR-007, Revision 0

\subsection{PROCESS}

\subsection{COMBINED VENTILATION SYSTEM (CVS) CONFIGURATION}

The conceptual design for the HCSE exhaust gas system consists of a HEPA filter intake, ducted manifold for up to six bays, isolation nuclear grade dampers, two stage testable HEPA filter plenum with pre-filter, variable speed drive exhaust fan, static pressure senors, and exhaust stack.

\subsubsection{Reference Standards}

\section{ERDA 76-21}

ACGIH

ASHRAE

SMACNA

NFPA

ASME

40CFR61

\subsubsection{System Design Parameters}

The CVS shall:

Minimize particulate hold up by use of smooth duct transitions and maintaining a minimum duct velocity of $2000 \mathrm{fpm}$.

Minimize contamination spread and prevent the backflow of gases and particulate.

Provide a constant stack flow for proper stack discharge velocity and for stack monitoring.

Provide sufficient volume for dilution of the process gas system to $25 \%$ of the lower explosion limit for hydrogen.

Limit particulate discharge through the use of a two stage HEPA filter plenum.

\subsubsection{Process Description}

The CVS is designed to exhaust six process pit/irench spaces and the process gas exhaust from six MCOs. Air is drawn through a HEPA filter into the process pit space at a nominal rate of 150 CFM per process pit. The HEPA filter serves as an isolation, backdraft and filtering device. The air moves through the process pit and trench space to the exhaust duct inlet manifold, through an isolation damper, pre- 
WHC-SD-SNF-CDR-007, Revision 0

\subsection{PROCESS}

filter and two stages of HEPA filters with isolation dampers, exhaust fan, and finally exits the HCSA through a dedicated stack. Twenty CFM of process exhaust gas from each of the six MCOs may enter the process exhaust stream at any time. Variable speed drives on the exhaust fan motors, with control based on pressure sensors will adjust air flow to keep the exhaust flow in the stack constant.

Motorized dampers on the exhaust from each process pit will be modulated using pressure sensors with integral controllers to maintain a nominal 150 CFM flow rate.

\subsubsection{Equipment description}

\subsubsection{Exhaust Fan}

Backwardly inclined, class III centrifugal fan, standard application for hot gases to 200 degrees $F$, intrinsically safe construction, with belt drive and variable frequency motor controller.

\subsubsection{HEPA Filter Housing}

Type 304 stainless steel, bag in/out type, transition on both ends, assembled in the direction of air flow as follows: prefilter section, test section, first stage HEPA filter section, test section, second stage HEPA filter section, and final test section.

\subsubsection{HEPA Filters}

$24 \times 24 \times 11.5$ inches thick, UL Class 1, 99.97\% efficiency per DOP test, and stainless steel frame.

\subsubsection{Prefilter}

$24 \times 24 \times 6$ inches thick, UL Class 1, 65\% efficiency per ASHRAE standard 52-76.

\subsubsection{Isolation Dampers}

ANSI class 150 butterfly valves with motorized actuators and will meet the requirements of ASME N 509-1989, leakage class I.

\subsubsection{Centrifugal Pumps}

Nominal 24 GPM each. 
WHC-SD-SNF-CDR-007, Revision 0

\subsection{PROCESS}

\section{$2.4 \quad$ PROCESS CONTROL}

\subsubsection{Control System Design}

The System Monitoring and Control System (SMCS) design is based on integrated Programmable Logic Controllers (PLC's), Personal Computers (PC's), and ManMachine Interfaces (MMI's). The SMCS Architecture is based on Supervisory control and Data Acquisition (SCADA) type of control system. The SMCS will be capable of handling all process controls, data acquisition, reporting, and archiving. Process control, supervisory, and Management Data Communications will be handled via standard ethemet with Transmission Control Protocol/Internet Protocol (TCP/P). TCP/IP is the standard communications protocol used by the Internet and Hanford Local Area Networks (HLAN).

The recommended hardware are IBM compatible PC's with a $200 \mathrm{MHZ}$ Pentium Pro, 64 MB ram, 2.5 GB of hard drive primary, 2.5 GB hard drive backup, 3.5" floppy drive, 6x CD-ROM drive, 21" SVGA monitor. A Laser Printer will be required for report printing. The operating system platform will be on the Windows NT. Each PC will have a dynamic graphical display of process parameters and other system variables. The MMI will animate the process variables to show process states such as an open valve or flow through a pipe. There will be a total of 3 PC's, one Operator Interface Stations (OIS), one Process Enclosure (PE) Local Control Panel (LCP), Operator, and one Engineering Work Station (EWS).

\subsubsection{Programmable Logic Controllers}

The process control will be handled by the PLC processors which will be located in a panel that will be installed in the control room. Each Process Module and the Service Module will have remote I/O stations that communicate to the PLC. The control system provides redundant PLC controllers which will be located in a control room PLC panel. The primary processor maintains independent control of the process and monitoring. The hot standby processor maintains a mirror of the primary PLC program and a real time data image table via a fiber optic link. Should an upset occur within the primary processor, the hot standby will immediately take control within a few milliseconds. The availability of real time data and quick response time by the hot standby will allow for a bumpless transfer of control. Also provided are redundant communications cabling to the remote V/O racks in each bay/skid.

All Local Process Modules and Service Module and skid I/O points will be field terminated at the respective remote $1 / O$ panels. The system HVAC, Chiller, Air 
WHC-SD-SNF-CDR-007, Revision 0

\subsection{PROCESS}

Monitor, and other system I/O will be field terminated at the control room PLC I/O. The PE will have a remote I/O rack which will communicate to the PLC processor via radio modem. The radio modem will take advantage of Spread-Spectrum transmission technology.

\subsubsection{PLC Programming Interface}

Logic diagrams and PLC programming will be configured by graphical methods using AutoCAD with pre-designed blocks having pre-configured attributes. Examples of logic and control blocks are, but not limited to; AND, OR, Analog I/O, Sequential Step, PID, and Digital I/O Blocks. A commercially available software package will be used to compile the AutoCAD drawing into a function block which can be downioaded to the PLC. There will be no PLC relay ladder logic to program. As such, when the logic changes, the drawing follows suit and the program recompiled. The program will have a supporting $1 / O$ data base and program debugging tools. Run-Time simulations and PID Control Loop Tuning tools will be available.

\subsubsection{Man-Machine Interface}

The OIS will handle concurrent processing of the Modules. The OIS and the EWS will have the capability of handling full operation of all six Process Modules and all other system controls including, but not limited to, archiving, reporting and alarming. The EWS will normally show the System HVAC process and system alarming. Generating alarm, event, graphic, and analysis reports will normally be an EWS task. The EWS will be used by the Operations System Engineer to facilitate run-time and enhancement changes to the MMI and PLC.

\subsubsection{Communications}

Communications will be through an ethernet network using Microsoft (MS) TCP/IP protocol. This ensures compatibility for communications to Hanford Local Area Network (HLAN). Communications from the PLC to the PC's will be via a separate LAN to isolate the process communications from the management and HLAN system. Printing communications will be handled through the ethernet network.

The PMC will communicate to the PLC using a spread spectrum radio modem. The interface to the control system network will be standard ethernet with TCP/IP protocol. The radio modem communications design will facilitate control, interlocking, and alarming requirements from the PMC while maintaining mobility. 
WHC-SD-SNF-CDR-007, Revision 0

\subsection{PROCESS}

\subsection{OVEN ASSEMBLY DESCRIPTION}

The MCOs will be heated in six(6) ovens which will be installed in process pits that will be located below the HCSA floor level. As indicated in the P\&IDs (SK-2300413), the MCO will be heated by forced hot air that is blown through the oven. The heater and blower will be located on the process module and the pipes that carry the circulating air will run from the process module through a below grade trench to the oven. The general arrangement of the oven in the pit, the trench, and the connecting piping is shown on SK-2-300422.

The oven will be an insulated stainless steel cylinder with an insulation jacket. The proposed insulation is vacuum. In other words, the oven will be constructed as a thermos bottle. The outer vessel will be stainless steel and super insulation will be attached to the inside surface of the outer vessel. The space between the inner and outer vessel will be constantly evacuated by a vacuum pump so that gas release promoted by the heating of the vessel will be drawn away. Two variations of the concept that are under consideration are given Drawings SK-2-300417 and SK-2300418. The selection will be made once the MCO neck design has been settled.

This basic design concept was selected for a number of reasons:

A. The rate of temperature rise of the MCO is limited to $50^{\circ} \mathrm{C}$ per hour. The heat transfer from the MCO into the SNF is slow because the transfer is effected by a nearly static gas. Therefore the MCO wall is easily heated. Radiation from heaters could easily overwhelm the heating rate limitation, whereas heat transfer from forced air is easy to control. Furthermore, there would be no simple way to measure the MCO surface temperature if it was exposed to radiation from heaters. Measurement of the inlet and outlet air temperatures in the proposed design is simple and is a good measure of the MCO temperature because heat transfer is effected by convection and gas conduction.

B. There are no heaters and no electrical distribution in the oven. This means that there is no opportunity to have a heater failure that would take the oven out of service or cause it to be pulled from the pit for repair.

C. Vacuum insulation was chosen because it is effective. It makes the oven easily constructable as a single stainless steel assembly. It is the lightest insulation choice which will make the assembly easier to install. And, most importantly it has few contamination traps and smooth and hard surfaces that can be decontaminated. 
WHC-SD-SNF-CDR-007, Revision 0

\subsection{PROCESS}

In both design alternatives, the hot air enters into a ring contained in the upper portion of the vacuum jacket. This ring distributes air to three downcomer pipes that carry the hot air to an insertion ring located in the bottom of the vacuum jacket. The distribution ring allows the air to enter into the oven uniformly around the base of the MCO through perforations into the oven interior. The downcomer pipes contain bellows expansion sections and are mounted to allow for vertical motion.

The MCO concept where the vessel has straight walls from top to bottom is shown on Drawing SK-2-300417. The MCO rests on a ring which transfers the load to a ring between the inner and outer oven vessels. The oven outer vessel rests on an impact limiter that transfers the load to the pit foundation. The impact limiter, which is for the purposes of protecting the MCO in the event of an accidental drop, will be the same one used in the CSB storage vaults. Both the ring between the MCO and the oven and the one in the vacuum jacket will be perforated to allow for airflow. The air will flow upward through the annulus between the MCO and the oven. Heat transfer calculations (see Appendix G) indicate that a 1 " annulus with an airfiow of $750 \mathrm{CFM}$ will be appropriate. When the air reaches the top of the annulus it is blocked by an annulus cover piece that will be installed after the MCO is lowered into the oven. The cover side wall will be a bellows to allow for differential expansion between the MCO and the oven. The top plate of the cover will be $3^{\prime \prime}$ thick to create some shielding for the radiation stream coming up through the annulus. It will also provide enough weight to hold it against the MCO top as the oven grows faster than the MCO. The air will flow through perforations into a collection ring located in the top of the vacuum jacket. The collection ring feeds the return pipe to the process module. The air circulation pipes run within a vacuum jacket in the trench. The outer vessel of the oven will have a bellows to allow for thermal expansion of the oven. This bellows will have a restraint so that the oven can be lifted during installation. The top ring of the oven that holds the inner an outer vessels of the vacuum jacket will be thick stainless steel in order to shield the radiation stream rising from the $\mathrm{MCO}$.

Air flowing through the top of the oven pit and through the trench will be diverted from the MCO top by an insulation cover so that the MCO top temperature is not significantly cooler than the body.

The oven concept shown on Drawing SK-2-300418 assumes an MCO design where the upper part of the MCO has a small flare. In this case, the MCO flare rests on a beveled flange on the top of the oven. There is no support at the bottom of the MCO. The weight is carried though the inner vessel of the oven to a ring in the vacuum jacket base and then to the impact limiter. There is no need for a special 
WHC-SD-SNF-CDR-007, Revision 0

\subsection{PROCESS}

annulus cover. Otherwise the design is the same. With this design the thermal growth of the MCO is accommodated by the space between the MCO bottom and the oven.

The final closure of the MCO will be accomplished by welding a cover over the MCO top. This weld will be made while the MCO is in the HCSE oven after the hot conditioning process has been completed. The geometry of the cover, weld, and welding machine have not been defined at this time. This means that the relative vertical dimensions of the oven top and the MCO top cannot be defined at this time and assembly concept sketches cannot be developed for the welder/oven interface. Resolution of this interface will be required to complete the detailed design of the oven. 
WHC-SD-SNF-CDR-007, Revision 0

\subsection{PROCESS}

\subsection{PROCESS MODULE}

\subsubsection{Process Module Description}

The process module contains most of the equipment shown on the piping and instrument drawing sheet \#1. With the exception of central and combined service equipment, the MCO, its oven and the cold trap, all equipment servicing an MCO is part of this module.

Module equipment includes:
A. Vacuum pump (VPS-VAC-1104).
B. Recirculation blower (VPS-BLO-1106).
C. The vacuum system cooler (VPS-CLR-1103).
D. The vacuum system heater (VPS-HCL-1105).
E. The MCO cooling system cooler (MCS-CLR-1153).
F. The MCO heating system heater (PHS-HCL-1152).
G. The MCO heating system circulating blower (PHS-BLO-1151).
H. The process vent HEPA filter (PV-F-1171).

I. A control panel for the module which includes input/output interfaces to a central programmable logic controller.

The module measures six feet by nine feet in the plan dimension and is eight feet high. Some equipment items are vertically instalied to conserve space. Except for the module's vacuum pump, rotating mechanical equipment is mounted on one side for accessibility and ease of maintenance. The skid is placed in the CSB annex with ample accessibility to all its sides. It is located behind the service crane's area of influence so as not to be of concern. 
WHC-SD-SNF-CDR-007, Revision 0

\subsection{PROCESS}

Process piping to and from the module proceeds in the trench to the MCO. Service utilities are run to the module above grade.

Structural elements for its fabrication are standard AISC shapes. Deck plating covers the service area. It is intended that the process module be fabricated and assembled in a local shop and then shipped to the site where it is to be leveled and affixed to steel plates imbedded in the CSB annex floor.

The module requires operator attention only for maintenance. All controls are accessed from a remote central control station which is capable of monitoring and controlling each of the six process modules plus the central utilities.

The operation of the process module equipment is described in Section 2.2. Cut sheets for the equipment are available in Appendix $\mathrm{H}$.

While each module, trench and vault unit have three HEPA filters, only one is installed on the process module. That filter provides suction air to its associated MCO. Installation at the MCO was considered but abandoned when it was apparent that the filter would have to be installed at grade elevation and would thus be subject to contamination from the adjacent floor area. Its installation on the process module allows longer life and ease in servicing the filter. The piping from the filter to the MCO vault is accommodated in the trench.

HEPA filters VPS-F-1102 and VPS-F-1107 are the bag-out type and are pressure rated to 100 psig. This rating increases the cost of these units considerably. It is made necessary because of the current uncertainty about heat transfer rates in the sub-atmospheric MCO. Should this issue become resolved prior to detailed design and pressure operation not be required, these filters can be simplified considerably with a commensurate reduction in module cost. At this time they are shown installed in the trench.

\subsubsection{Equipment Description}

\subsubsection{Vacuum Pump Selection (VPS-VAC-1104)}

This section discusses the basis for selection of the scroll type vacuum pump for the HCS project. The starting point for this discussion is the alternatives study performed for the Cold Vacuum Drying System (CVDS) project. That study 
WHC-SD-SNF-CDR-007, Revision 0

\title{
2.0 PROCESS
}

evaluated eight alternatives involving different pump combinations. The rotary scroll pump was selected on the basis of operational simplicity, cleanliness and the wide pressure range covered by a single stage of pumping. A staged Roots blower was the second choice. The intent of the current study is to expand upon the earlier work by focusing on germane operating conditions in the CVDS and determining if changes in these conditions are required for the Hot Vacuum Conditioning System (HVCS) project are required and if so, are the changes detrimental to the pump. Germane operating conditions which could compromise the reliability of a rotary scroll pump are:

\author{
Elevated Temperatures \\ Presence of Particulates \\ Water Vapor Condensing Within the Pump \\ Materials Incompatibility
}

During the CVDS development testing program, the scroll pump's ability to operate for long periods while pumping water vapor at temperatures in excess of $100 \mathrm{C}$ will be demonstrated. The concern is that bearings within the pump may fail at the elevated temperature. Discussions with the manufacturer's representative identified that the stated operating limit of the pump $(40 \mathrm{C})$ is a limiting condition only when combined with high vacuum at the pump inlet. Under these conditions, cooling of the pump bearings relies mainly on conductive heat transfer to the pump body. When pumping at higher pressures the situation is relieved due to convective transfer on the bearings directly. As part of the CVDS, a pump will be tested to demonstrate long term pumping of water vapor at $100 \circ \mathrm{C}$ or above to verify that pump life is not compromised. If this demonstration is assumed to be successful, utilization of the pump for the HVCS process does not present any increase in severity as regards the operating temperature.

Particulates are a concem for a rotary scroll pump because of the close tolerances within the interleaved scrolls which could bind if subjected to particulates in the gas stream. Filters will be installed upstream of the pump inlet to protect the pumping surfaces. Additionally, the scroll pump that was selected for the CVDS has Teflon tip seals that are more tolerant of particulate than an all metal design. If necessary, the pump can be repaired should damage due to particulate be incurred. Routine replacement of some pump parts is recommended at 6,000 and 12,000 hour intervals. The development testing program will demonstrate pump life under particulate conditions that are comparable to the actual pumping environment. 
WHC-SD-SNF-CDR-007, Revision 0

\subsection{PROCESS}

For the CVDS application, a heater was provided immediately upstream of the pump to ensure that the pump inlet temperature was high enough to preclude condensation of water vapor as the pressure increased within the pump housing. Since the pressure at the pump exhaust is atmospheric, condensation can be avoided by maintaining the pump inlet temperature above $100 \mathrm{C}$. As for the HVCS process, that concem is reversed. With cask temperatures of up to $350 \mathrm{C}$ and very small quantities of residual water after cold vacuum drying, the possibility of water condensing within the pump is remote. The water which is pumped will mix with helium purge gas so that, after compression, the relative humidity is still low.

The wetted surfaces of the scroll pump are hard coat anodized aluminum and a Teflon compound. The gases being pumped, helium, hydrogen, water vapor, and trace quantities of tritium, air and fission gases are all compatible with these materials. Bearings are housed in a separate chamber and are lubricated with inorganic PTFE. The gases being pumped during hot vacuum conditioning should have no detrimental effect on the pump. Radioactive gases and particulate that may be released by oxidation of the SNF during the HVCS process ( $I, C s, M g$ ) will be removed before they reach the pump.

It is recommended that the rotary scroll pump that was selected for the CVDS process be used for the water HVCS process. No changes in operating conditions have been identified that would be deleterious to pump operation. If it is assumed that pump performance will be satisfactory during development testing for the CVDS project, then the transition to the HVCS process presents no risk relative to pumping performance. As in the CVDS, the fall back pump for HVCS is a multistage Roots pump.

\subsubsection{Recirculation Blower (VPS-BLO-1106)}

The recirculation blower is a magnetically coupled regenerative-type blower which circulates helium through the $\mathrm{MCO}$ and through external treatment in the trench and through heat exchange units on the process module. It has been specified to handle 13 to 25 psia of helium pressure. The blower has no seal and consequently no leakage. The pump can deliver 20 ACFM of helium against a 4-inch WC static head 
WHC-SD-SNF-CDR-007, Revision 0

\subsection{PROCESS}

\subsubsection{Vacuum System Cooler (VPS-CLR-1103)}

The cooler is a plate-type unit. Its duty is highly dependent upon the pressure in the helium recirculation system; the duty increases with increasing pressure.

The system is sized at a duty of 2,000 Btu per hour at pressure service.

\subsubsection{Vacuum System Heater (VPS-HCL-1105)}

As with the cooler, the duty of the heater increases significantly with increasing helium pressure. The heater is electrically powered and is sized at $3 \mathrm{~kW}$.

\subsubsection{MCO System Cooler (MCS-CLR-1153)}

The cooler is an air to water heat exchanger. It is sized at 83,000 Btu per hour to remove the appropriate amount of heat during the MCO cooling cycle.

\subsubsection{MCO Heating System Heater (PHS-HCL-1152)}

The heater is an electrically powered $25 \mathrm{~kW}$ unit. It is normally operating at less than half of its capacity during the major portion of the MCO heat up process.

\subsubsection{MCO Heating System Circulating Blower (PHS-BLO-1151)}

The blower is designed to work at the high temperature (375 C) that will be encountered in heat up. It is powered by a 5-horsepower motor which draws its maximum load at ambient temperature during startup.

\subsubsection{Process Vent HEPA Filter (PV-F-1171)}

This filter draws air from the building in the area of the process module and after passing it through the HEPA, pipes it to the MCO vault. Installation of this filter on the process module avoids problems which are inherent in mounting this filter at the MCO at floor level. 


\subsection{PROCESS}

\subsection{SERVICE MODULE}

The service module contains all utility systems which are common to each of the process modules, with the exception of the chiller system, the process gas system and the vent stack, all of which are outside the CSB. These outside systems are described in Section 3.3 as auxiliary facilities.

The service module is located at the mezzanine level in the southwest comer of the HCSA. It contains the following components:
A. Chilled water pumps, expansion tank and heat exchanger;
B. Cooling water pumps, expansion tank and heat exchanger;
C. Combined ventilation system blowers and HEPA filter;
D. MCO oven vacuum pumps; and
E. Instrument air system

The equipment, piping and instrumentation that are in the service module are identified on the P\&IDs (SK-2-300413).

\subsubsection{Chilled Water Systems}

The chilled water system provides cooling for the MCO cool down cycle and for protection of gas circulation and vacuum pumps during other portions of the HCSE operation.

In order to achieve adequate confinement from the radioactive systems which require cooling, a chiller system is used which provides three levels of isolation from the process heat load. The first level exchanges heat directly with the Freon(8) loop of the package chillers and the atmosphere. Inside the HCSA the Freone loop exchanges heat with a chilled water loop that provides cooling for the water loop. The cooled water loop extends to each of the modules and exchanges heat directly with the process heat exchangers. This multiple level system provides multiple 
WHC-SD-SNF-CDR-007, Revision 0

\subsection{PROCESS}

barriers to prevent radioactive material from contaminating either the chilled water loop or the Freon loop. Redundant pumps piped in parallel will be provided on the water side of each heat exchanger in order to provide operating flexibility during maintenance periods.

The chiller equipment is described as follows:

Compressor/Condensing Unit: Scroll compressor, air-cooled condensing unit, single circuit with 2 steps of capacity. Nominal 25 ton capacity at $105^{\circ} \mathrm{F}$. Supply with 25 ton evaporator chiller.

Centrifugal Pumps: Nominal 24 GPM each.

\subsubsection{HCSE Process Vent System}

The process vent system discharges to the combined ventilation system as described in Section 2.3. Process exhaust gases include those vented from pressure relief valves and discharge from the vacuum pumps. the pressure relief valve vents and vacuum pump exhaust are located at the process modules. A collection manifold extends from the combined ventilation system on the service module to each of the process modules. All of the process ventilation gases pass through HEPA filters that are located in the Trench before they are vented to the final set of HEPA filters in the combined ventilation system.

\subsubsection{MCO Oven Insulating Vacuum System}

Insulation for the $\mathrm{MCO}$ ovens is provided by a vacuum jacket that is designed into each oven. This vacuum jacket is evacuated by a central MCO Oven Insulating Vacuum system. The function of the MCO Oven Insulating Vacuum System is to maintain a vacuum of $10^{-4}$ torr in the oven jackets and in the jackets of the process piping that is vacuum insulated. Two vacuum pumps, operating in series, serve all MCO ovens. The fore pump is the same scroll pump unit used on each process module. It is used to bring the vacuum to the operating range of the turbomolecular pump. The turbomolecular maintains the vacuum system at approximately $10^{-4}$ torr. Installed spares are not provided for these vacuum pumps. The pumps discharge to the combined ventilation system. 
WHC-SD-SNF-CDR-007, Revision 0

\subsection{PROCESS}

\subsubsection{Instrument Air System}

The instrument air system consists of a 45 CFM compressor, an air receiver, and a molecular sieve dryer. The compressor is powered by a 10 horsepower electric motor and produces air to the 200 gallon ASME-stamped receiver at 100 psi. Inline between the receiver and the dryer is a deoiler. The air dryer has two molecular sieve columns, one which is on-line while the other is regenerating. Regeneration is accomplished on a timed cycle. 
WHC-SD-SNF-CDR-007, Revision 0

\subsection{PROCESS}

\subsection{PROCESS ENCLOSURE DESCRIPTION}

Each HCSE oven will be imbedded in a process pit so that the top of an oven will be a few feet below the facility floor. It is necessary to access the top of the MCO to make and break connections, to assemble and disassemble oven top pieces, to change the Cs/l trap, and to weld the final cover over the MCO. This access requires that the process pit cover (shield) be opened and that a means of manipulating items inside the process pit be provided.

A portable process enclosure is to be used while working in the open process pit. When not in use the enclosure will be parked out of the way. When access is required to an oven the enclosure will moved over the process and lowered so that it seals against the facility floor. An actuator located within the enclosure will be used to open the process pit cover. An operator using a tele-operated manipulator arm and a variety of tools and fixtures carried in the enclosure will perform a list of operations to either prepare for conditioning or to tear down after conditioning is complete as detailed in the HCSE Model Operating Sequence (see Section 2.1)

The enclosure performs the following basic functions:

A. Secondary Confinement: It acts as the secondary radioactive material confinement structure when the process pit cover is open. It will prevent accidental releases from the MCO during connect/disconnect operations from spreading into the HCSA.

B. Radiation Exposure Management: It removes the operator a reasonable distance from the radiation area immediately above the process pit and it provides shielding in front of the operator.

C. Remote Operations Tool Chest: It provides manipulation capability needed for a number of specific activities and it provides the structure required to mount the manipulators, hoists, and auxiliary tools and equipment. 
WHC-SD-SNF-CDR-007, Revision 0

\subsection{PROCESS}

The process steps to be performed with the process enclosure are:

\begin{tabular}{|c|c|c|}
\hline ID & Name & Description \\
\hline 5.0 & $\begin{array}{l}\text { Move-in Process } \\
\text { Enclosure }\end{array}$ & $\begin{array}{l}\text { The process enclosure is moved from } \\
\text { its parking place into its operating } \\
\text { position above the process pit. }\end{array}$ \\
\hline 6.0 & Connect Process Cell & $\begin{array}{l}\text { Make the electrical and } \\
\text { instrumentation connections that } \\
\text { operate the process enclosure } \\
\text { equipment. }\end{array}$ \\
\hline 7.0 & Open Process Pit & $\begin{array}{l}\text { Actuate the process pit cover opening } \\
\text { mechanism and expose the oven. }\end{array}$ \\
\hline 8.0 & Place Annulus Cover & $\begin{array}{l}\text { Get the annulus cover from its storage } \\
\text { location in the enclosure and place it } \\
\text { on the oven top using the manipulator. }\end{array}$ \\
\hline 9.0 & Attach Annulus Cover & $\begin{array}{l}\text { Use manipulator and drill motor driven } \\
\text { socket to tighten the bolts. }\end{array}$ \\
\hline 10.0 & Remove Port Covers & $\begin{array}{l}\text { Use the manipulator and drill motor } \\
\text { driven socket to loosen port cover } \\
\text { bolts. Use manipulator and suction } \\
\text { device to remove the covers. }\end{array}$ \\
\hline 11.0 & Install Vent Cover & $\begin{array}{l}\text { Use the manipulator to get a cover } \\
\text { from its storage location in the } \\
\text { enclosure and place it in the vent port. } \\
\text { Use the manipulator and drill motor } \\
\text { driven socket to tighten the bolts. }\end{array}$ \\
\hline 12.0 & Install Valves & $\begin{array}{l}\text { Use the manipulator to pick the valves } \\
\text { from their storage ports and plug them } \\
\text { into the MCO ports. Use the } \\
\text { manipulator and drill motor driven } \\
\text { socket to tighten the bolts. }\end{array}$ \\
\hline 13.0 & Open Valves & $\begin{array}{l}\text { Use the manipulator and drill motor } \\
\text { driven sockets to drive the valve } \\
\text { operator screws. }\end{array}$ \\
\hline 14.0 & Evacuate MCO & $\begin{array}{l}\text { Remove old gas from the MCO using } \\
\text { the system vacuum pump. }\end{array}$ \\
\hline 15.0 & Fill MCO With $\mathrm{He}$ & Fill the MCO with helium. \\
\hline
\end{tabular}


WHC-SD-SNF-CDR-007, Revision 0

\subsection{PROCESS}

\begin{tabular}{|c|c|c|}
\hline 16.0 & Leak Check Ports & $\begin{array}{l}\text { Use the manipulator to move the the } \\
\text { sniffer hose attached to a helium leak } \\
\text { detector (part of the process } \\
\text { enclosure equipment) around the } \\
\text { MCO ports. }\end{array}$ \\
\hline 17.0 & Pass Leak Test? & $\begin{array}{l}\text { Determine if corrective action is } \\
\text { required before proceeding. }\end{array}$ \\
\hline 18.0 & Close Bad Valve & $\begin{array}{l}\text { Use the manipulator and drill motor } \\
\text { driven sockets to close the leaking } \\
\text { port. }\end{array}$ \\
\hline 19.0 & Disconnect Valve & $\begin{array}{l}\text { Use the manipulator and drill motor } \\
\text { driven socket to loosen the valve } \\
\text { attachment bolts. Use the manipulator } \\
\text { to pull the valve out of the port. }\end{array}$ \\
\hline 20.0 & Clean Port & $\begin{array}{l}\text { Use the manipulator and drill motor } \\
\text { driven cloth pad to clean port. }\end{array}$ \\
\hline 21.0 & Install Reserve Valve & $\begin{array}{l}\text { Use the manipulator to re-install the } \\
\text { valve or to install a reserve valve. Use } \\
\text { the manipulator and drill motor driven } \\
\text { socket to tighten the bolts. Open the } \\
\text { valve using the manipulator held drill } \\
\text { motor and sockets. }\end{array}$ \\
\hline 22.0 & Install Insulation Cover & $\begin{array}{l}\text { Use the manipulator to get the } \\
\text { insulation cover from its storage } \\
\text { location in the enclosure and place it } \\
\text { on top of the MCO. }\end{array}$ \\
\hline 23.0 & Close Process Pit & $\begin{array}{l}\text { Actuate the process pit cover } \\
\text { operating mechanism. }\end{array}$ \\
\hline 25.0 & Survey Process Cell & $\begin{array}{l}\text { Use the manipulators to swipe } \\
\text { selected spots in the cell. Pass bottled } \\
\text { swipes out of the enclosure for } \\
\text { counting. }\end{array}$ \\
\hline 26.0 & Disconnect Process Cell & $\begin{array}{l}\text { Disconnect the electrical and } \\
\text { instrumentation services. }\end{array}$ \\
\hline 27.0 & Remove Process Cell & $\begin{array}{l}\text { Return the enclosure to its parking } \\
\text { place. }\end{array}$ \\
\hline
\end{tabular}


WHC-SD-SNF-CDR-007, Revision 0

\subsection{PROCESS}

\begin{tabular}{|c|c|c|}
\hline 39.0 & Move-in Process Cell & $\begin{array}{l}\text { The process enclosures moved from } \\
\text { its parking place into its operating } \\
\text { position above the process pit. }\end{array}$ \\
\hline 40.0 & Connect Process Cell & $\begin{array}{l}\text { Make the electrical and } \\
\text { instrumentation connections that } \\
\text { operate the process enclosure } \\
\text { equipment. }\end{array}$ \\
\hline 41.0 & Open Process Pit & $\begin{array}{l}\text { Actuate the process pit cover opening } \\
\text { mechanism and expose the oven. }\end{array}$ \\
\hline 42.0 & Remove Insul. Cover & $\begin{array}{l}\text { Use the manipulator to grab the } \\
\text { insulation cover and return it to its } \\
\text { storage location in the process cell. }\end{array}$ \\
\hline 43.0 & Close MCO Valves & $\begin{array}{l}\text { Use the manipulator and drill motor } \\
\text { driven sockets to close the valves. }\end{array}$ \\
\hline 44.0 & Place Weld-On Cover & $\begin{array}{l}\text { Use the manipulator to get the weld- } \\
\text { on cover from its storage location in } \\
\text { the process cell. Place it over the } \\
\text { MCO. }\end{array}$ \\
\hline 45.0 & Install Welder & $\begin{array}{l}\text { Use the hoist and manipulator to get } \\
\text { the automatic welder from its storage } \\
\text { location in the process cell. Install the } \\
\text { welder on the top of the MCO. }\end{array}$ \\
\hline 46.0 & Weld Root Pass & $\begin{array}{l}\text { Turn on the welder. Make the root } \\
\text { pass. }\end{array}$ \\
\hline 47.0 & Visual Inspect & $\begin{array}{l}\text { Inspect the weld using a television } \\
\text { camera on the manipulator arm. }\end{array}$ \\
\hline 48.0 & Clean Weld & $\begin{array}{l}\text { Clean the weld using a rotary } \\
\text { stainless steel wire brush and the } \\
\text { manipulator held drill motor. }\end{array}$ \\
\hline 49.0 & Repair & Is weld repair required? \\
\hline 50.0 & Grind & $\begin{array}{l}\text { Grind out the defect area using a } \\
\text { small grinder (dremel tool) held by the } \\
\text { manipulator. }\end{array}$ \\
\hline 51.0 & Repair & $\begin{array}{l}\text { Use the welder to reweld the ground } \\
\text { out area. }\end{array}$ \\
\hline
\end{tabular}


WHC-SD-SNF-CDR-007, Revision 0

\subsection{PROCESS}

\begin{tabular}{|c|c|c|}
\hline 52.0 & Apply Dye & $\begin{array}{l}\text { Apply liquid penetrant Dye using a } \\
\text { specially designed applicator held by } \\
\text { the manipulator. }\end{array}$ \\
\hline 53.0 & Soak & $\begin{array}{l}\text { Wait the prescribed time for the Dye } \\
\text { to seep into weld defects. }\end{array}$ \\
\hline 54.0 & Remove Dye & $\begin{array}{l}\text { Using a specially designed applicator } \\
\text { apply the cleaning fluid and wash off } \\
\text { the Dye. Finish with a cleaning fluid } \\
\text { soaked rotary buffing pad driven by } \\
\text { the manipulator held drill motor. }\end{array}$ \\
\hline 55.0 & Apply Developer & $\begin{array}{l}\text { Apply the developer using a specially } \\
\text { design applicator and the manipulator. }\end{array}$ \\
\hline 56.0 & Bleed & $\begin{array}{l}\text { Wait the prescribed time for the Dye } \\
\text { to "blot out" of weld defects. }\end{array}$ \\
\hline 57.0 & Inspect & $\begin{array}{l}\text { Inspect the developer coated weld } \\
\text { using the manipulator mounted } \\
\text { camera. }\end{array}$ \\
\hline 58.0 & Clean & $\begin{array}{l}\text { Using a specially designed applicator } \\
\text { apply the cleaning fluid and wash off } \\
\text { the developer. Finish with a cleaning } \\
\text { fluid soaked rotary buffing pad driven } \\
\text { by the manipulator held drill motor }\end{array}$ \\
\hline 59.0 & Repair & Is weld repair required? \\
\hline 60.0 & Grind & $\begin{array}{l}\text { Grind out the defect area using a } \\
\text { small grinder (dremel tool) held by the } \\
\text { manipulator. }\end{array}$ \\
\hline 61.0 & Repair Weld & $\begin{array}{l}\text { Use the welder to reweld the ground } \\
\text { out area. }\end{array}$ \\
\hline 62.0 & Weld Pass 1 & $\begin{array}{l}\text { Tum on the welder. Make the weld } \\
\text { pass. }\end{array}$ \\
\hline 63.0 & Visual Inspect & $\begin{array}{l}\text { Inspect the weld using a television } \\
\text { camera on the manipulator arm. }\end{array}$ \\
\hline 64.0 & Repair & Is weld repair required? \\
\hline 65.0 & Grind & $\begin{array}{l}\text { Grind out the defect area using a } \\
\text { small grinder (dremel tool) held by the } \\
\text { manipulator. }\end{array}$ \\
\hline
\end{tabular}


WHC-SD-SNF-CDR-007, Revision 0

\subsection{PROCESS}

\begin{tabular}{|c|c|c|}
\hline 66.0 & Repair Weld & $\begin{array}{l}\text { Use the welder to reweld the ground } \\
\text { out area. }\end{array}$ \\
\hline 67.0 & Weld Pass 2 & $\begin{array}{l}\text { Turn on the welder. Make the weld } \\
\text { pass. }\end{array}$ \\
\hline 68.0 & Visual inspect & $\begin{array}{l}\text { Inspect the weld using a television } \\
\text { camera on the manipulator arm. }\end{array}$ \\
\hline 69.0 & Repair & Is weld repair required? \\
\hline 70.0 & Grind & $\begin{array}{l}\text { Grind out the defect area using a } \\
\text { small grinder (dremel tool) held by the } \\
\text { manipulator. }\end{array}$ \\
\hline 71.0 & Repair Weld & $\begin{array}{l}\text { Use the welder to reweld the ground } \\
\text { out area. }\end{array}$ \\
\hline 72.0 & Weld Pass 3 & $\begin{array}{l}\text { Turn on the welder. Make the weld } \\
\text { pass. }\end{array}$ \\
\hline 73.0 & Visual Inspect & $\begin{array}{l}\text { Inspect the weld using a television } \\
\text { camera on the manipulator arm. }\end{array}$ \\
\hline 74.0 & Repair & Is weld repair required? \\
\hline 75.0 & Grind & $\begin{array}{l}\text { Grind out the defect area using a } \\
\text { small grinder (dremel tool) held by the } \\
\text { manipulator. }\end{array}$ \\
\hline 76.0 & Repair Weld & $\begin{array}{l}\text { Use the welder to reweld the ground } \\
\text { out area. }\end{array}$ \\
\hline 77.0 & Weld Pass 4 & $\begin{array}{l}\text { Turn on the welder. Make the weld } \\
\text { pass. }\end{array}$ \\
\hline 78.0 & Visual Inspect & $\begin{array}{l}\text { Inspect the weld using a television } \\
\text { camera on the manipulator arm. }\end{array}$ \\
\hline 79.0 & Repair & Is weld repair required? \\
\hline 80.0 & Grind & $\begin{array}{l}\text { Grind out the defect area using a } \\
\text { small grinder (dremel tool) held by the } \\
\text { manipulator. }\end{array}$ \\
\hline 81.0 & Repair Weld & $\begin{array}{l}\text { Use the welder to reweld the ground } \\
\text { out area. }\end{array}$ \\
\hline 82.0 & Weld Pass 5 & $\begin{array}{l}\text { Turn on the welder. Make the weld } \\
\text { pass. }\end{array}$ \\
\hline 83.0 & Visual Inspect & $\begin{array}{l}\text { Inspect the weld using a television } \\
\text { camera on the manipulator arm. }\end{array}$ \\
\hline
\end{tabular}


WHC-SD-SNF-CDR-007, Revision 0

\subsection{PROCESS}

\begin{tabular}{|c|c|l|}
\hline 84.0 & Repair & Is weld repair required? \\
\hline 85.0 & Grind & $\begin{array}{l}\text { Grind out the defect area using a } \\
\text { small grinder (dremel tool) held by the } \\
\text { manipulator. }\end{array}$ \\
\hline 86.0 & Repair Weld & $\begin{array}{l}\text { Use the welder to reweld the ground } \\
\text { out area. }\end{array}$ \\
\hline 87.0 & Apply Dye & $\begin{array}{l}\text { Apply liquid penetrant Dye using a } \\
\text { specially designed applicator held by } \\
\text { the manipulator. }\end{array}$ \\
\hline 88.0 & Soak & $\begin{array}{l}\text { Wait the prescribed time for the Dye } \\
\text { to seep into weld defects. }\end{array}$ \\
\hline 89.0 & Remove Dye & $\begin{array}{l}\text { Using a specially designed applicator } \\
\text { apply the cleaning fluid and wash off } \\
\text { the Dye. Finish with a cleaning fluid } \\
\text { soaked rotary buffing pad driven by } \\
\text { the manipulator held drill motor. }\end{array}$ \\
\hline 90.0 & Apply Developer & $\begin{array}{l}\text { Apply the developer using a specially } \\
\text { design applicator and the manipulator. }\end{array}$ \\
\hline 91.0 & Bleed & $\begin{array}{l}\text { Wait the prescribed time for the Dye } \\
\text { to "blot out" of weld defects. }\end{array}$ \\
\hline 92.0 & Inspect & $\begin{array}{l}\text { Inspect the developer coated weld } \\
\text { using the manipulator mounted } \\
\text { camera. }\end{array}$ \\
\hline 93.0 & Repair & $\begin{array}{l}\text { Using a specially designed applicator } \\
\text { apply the cleaning fluid and wash off } \\
\text { the developer. Finish with a cleaning } \\
\text { fluid soaked rotary buffing pad driven } \\
\text { by the manipulator held drill motor }\end{array}$ \\
\hline 95.0 & Grind & $\begin{array}{l}\text { Grind out the defect area using a } \\
\text { small grinder (dremel tool) held by the } \\
\text { manipulator. }\end{array}$ \\
\hline 97.0 & Remove Trap & $\begin{array}{l}\text { Use the welder to reweld the ground } \\
\text { out area. }\end{array}$ \\
\hline $\begin{array}{l}\text { Use the manipulator to disconnect the } \\
\text { trap and set it aside. }\end{array}$ \\
\hline 9
\end{tabular}


WHC-SD-SNF-CDR-007, Revision 0

\subsection{PROCESS}

\begin{tabular}{|c|c|c|}
\hline 98.0 & Install New Trap & $\begin{array}{l}\text { Use the manipulator to get the new } \\
\text { trap from its storage location in the } \\
\text { cell. Make the new trap connections } \\
\text { using the manipulator. }\end{array}$ \\
\hline 99.0 & Connect Waste Drum & $\begin{array}{l}\text { Use a cart to place a shielded waste } \\
\text { drum below the process enclosure } \\
\text { bag port. Connect the drum and port. }\end{array}$ \\
\hline 100.0 & Remove Top Shield & $\begin{array}{l}\text { Open the bagport door. Lower a hoist } \\
\text { into the drum and pull the top of the } \\
\text { shield into the cell. Set it out of the } \\
\text { way. }\end{array}$ \\
\hline 101.0 & Dispose Hot Trap & $\begin{array}{l}\text { Use the manipulator to place the trap } \\
\text { into the drum. }\end{array}$ \\
\hline 102.0 & Insert Top Shield & $\begin{array}{l}\text { Use the hoist to replace the shield to } \\
\text { in the drum. }\end{array}$ \\
\hline 103.0 & Tie Bag & Tie off and cut the bag. \\
\hline 104.0 & Close Drum & $\begin{array}{l}\text { Place the top on the drum and attach } \\
\text { it. Move the drum cart to the waste } \\
\text { management area. }\end{array}$ \\
\hline 105.0 & Undo Annulus Cover & $\begin{array}{l}\text { Use the manipulator and drill motor } \\
\text { driven socket to release the annulus } \\
\text { cover bolts. }\end{array}$ \\
\hline 106.0 & Remove Annulus Cover & $\begin{array}{l}\text { Use the manipulator to remove the } \\
\text { annulus cover and place it into its } \\
\text { storage location in the cell. }\end{array}$ \\
\hline 107.0 & Close Process Pit & $\begin{array}{l}\text { Actuate the process pit cover } \\
\text { mechanism to close the cover. }\end{array}$ \\
\hline 108.0 & Survey Process Cell & $\begin{array}{l}\text { Use the manipulators to swipe } \\
\text { selected spots in the cell. Pass bottled } \\
\text { swipes out of the enclosure for } \\
\text { counting. }\end{array}$ \\
\hline 109.0 & Disconnect Process Cell & $\begin{array}{l}\text { Disconnect the electrical and } \\
\text { instrumentation services. }\end{array}$ \\
\hline 110.0 & Remove Process Cell & $\begin{array}{l}\text { Return the enclosure to its parking } \\
\text { place. }\end{array}$ \\
\hline
\end{tabular}


WHC-SD-SNF-CDR-007, Revision 0

\subsection{PROCESS}

In order to accomplish these process steps the enclosure must have the following functions and capabilities:

\begin{tabular}{|c|c|c|}
\hline $\begin{array}{l}\text { Function } \\
\text { ID }\end{array}$ & $\begin{array}{c}\text { Affected } \\
\text { Sequence } \\
\text { Steps }\end{array}$ & Description \\
\hline F1 & $5,27,39,110$ & $\begin{array}{l}\text { The enclosure must be movable with } x, y \\
\text { axis motion. It must be able to move over } \\
\text { each of the HCSE process pits and to a } \\
\text { storage location where it does not interfere } \\
\text { with the MHM. It may be either self } \\
\text { motivated or towed. }\end{array}$ \\
\hline F2 & $6,26,40,109$ & $\begin{array}{l}\text { The enclosure must have vertical motion } \\
\text { so that it can be lifted while traveling and } \\
\text { lowered to rest on the floor when over a } \\
\text { process pit. An elastomer seal is required } \\
\text { between the enclosure base perimeter and } \\
\text { the floor. }\end{array}$ \\
\hline F3 & $7,23,41,107$ & $\begin{array}{l}\text { The enclosure must contain a mechanism } \\
\text { for opening and closing the process pit } \\
\text { cover. The cover weight estimate is } 5,000 \\
\text { lbs. }\end{array}$ \\
\hline F4 & $8,9,105,106$ & $\begin{array}{l}\text { The enclosure must have a position for } \\
\text { storing one annulus space cover. }\end{array}$ \\
\hline F5 & 10,11 & $\begin{array}{l}\text { The enclosure must have positions for } \\
\text { storing three port covers. }\end{array}$ \\
\hline F6 & $\begin{array}{c}8,9,10,11,12,13 \\
16,18,19,20,21 \\
22,25,42,43,44 \\
45,47,48,50,52 \\
54,55,57,58,60 \\
63,65,68,70 \\
73,75,78,80,83 \\
85,87,89,90,92 \\
93,95,97,98,101 \\
105,106,108 \\
\end{array}$ & $\begin{array}{l}\text { The enclosure must have dextrous tele- } \\
\text { operated hydraulic manipulator with force } \\
\text { feedback controls. The manipulator must } \\
\text { be able to reach the MCO top surface, the } \\
\text { trap, the drum output port, and the various } \\
\text { tool and accessory storage locations in the } \\
\text { cell. The largest and heaviest item that it } \\
\text { must be able to lift and place will be the } \\
\text { annulus space cover. It must be able to } \\
\text { hold a wide variety of tools. }\end{array}$ \\
\hline
\end{tabular}


WHC-SD-SNF-CDR-007, Revision 0

\subsection{PROCESS}

\begin{tabular}{|c|c|c|}
\hline $\mathrm{F} 7$ & $\begin{array}{c}9,10,11,12,13 \\
18,19,21,43,105\end{array}$ & $\begin{array}{l}\text { A drill motor and socket attachments are } \\
\text { needed to drive various bolts. There must } \\
\text { be a manipulator friendly system and there } \\
\text { must be a storage rack in the enclosure for } \\
\text { these items. }\end{array}$ \\
\hline F8 & 16 & $\begin{array}{l}\text { A helium leak detector equipped for } \\
\text { operating in the sniffing mode must be } \\
\text { mounted to the cell. The manipulator must } \\
\text { be able to direct the sniffer hose around } \\
\text { the MCO port connections. }\end{array}$ \\
\hline F9 & $\begin{array}{c}20,48,54,58,89 \\
93\end{array}$ & $\begin{array}{l}\text { A rotary buffing pad that can be attached to } \\
\text { the drill motor is required in the tool } \\
\text { storage rack. }\end{array}$ \\
\hline F10 & 48 & $\begin{array}{l}\text { A rotary stainless steel wire brush that can } \\
\text { be attached to the drill motor is required in } \\
\text { the tool storage rack. }\end{array}$ \\
\hline F11 & $\begin{array}{c}50,60,65,70,75 \\
80,85,95\end{array}$ & $\begin{array}{l}\text { A small grinder (dremel tool) that can be } \\
\text { manipulator held is required in the tool } \\
\text { rack. }\end{array}$ \\
\hline$F 12$ & $\begin{array}{c}47,48,50,51,52, \\
54,55,57,58,60 \\
61,63,65,66,68, \\
70,71,73,75,76, \\
78, \\
80,81,83,85,86, \\
87,89,90,92,93, \\
95,96 \\
\end{array}$ & $\begin{array}{l}\text { A manipulator mounted television camera } \\
\text { is required to perform visual inspection of } \\
\text { the weld and the liquid penetrant } \\
\text { developer. A monitor is required at the } \\
\text { operator station. }\end{array}$ \\
\hline F13 & $7,23,41,107$ & $\begin{array}{l}\text { A process pit cover actuator is required to } \\
\text { open and close the process pit. }\end{array}$ \\
\hline F14 & 52,87 & $\begin{array}{l}\text { A liquid penetrant dye applicator that can } \\
\text { be remotely handled with a manipulator is } \\
\text { required in the cell. }\end{array}$ \\
\hline F15 & 55,90 & $\begin{array}{l}\text { A liquid penetrant developer applicator that } \\
\text { can be remotely handled with a } \\
\text { manipulator is required in the cell. }\end{array}$ \\
\hline$F 16$ & 99,103 & $\begin{array}{l}\text { A waste drum bag port connection is } \\
\text { required. }\end{array}$ \\
\hline
\end{tabular}


WHC-SD-SNF-CDR-007, Revision 0

\subsection{PROCESS}

\begin{tabular}{|c|c|l|}
\hline F17 & 100,102 & $\begin{array}{l}\text { A hoist to pull the shield from the waste } \\
\text { drum is required. }\end{array}$ \\
\hline F18 & $\begin{array}{c}45,46,51,61,62, \\
66,67,71,72,76, \\
77,81,82,86,96\end{array}$ & $\begin{array}{l}\text { An automatic tube welder and fixture is } \\
\text { required. The head and fixture must be } \\
\text { stored in the enclosure and the } \\
\text { manipulator must be able to place and } \\
\text { attach it. the power supply should be } \\
\text { mounted on the outside of the cell. }\end{array}$ \\
\hline F19 & & $\begin{array}{l}\text { Television cameras that can see the top of } \\
\text { the MCO and into the waste drum are } \\
\text { required. The operator station monitor must } \\
\text { be able to display multiple cameras. }\end{array}$ \\
\hline F20 & 22,42 & $\begin{array}{l}\text { The enclosure must be able to store an } \\
\text { insulation cover. }\end{array}$ \\
\hline F21 & 98 & $\begin{array}{l}\text { The enclosure must be able to store a new } \\
\text { trap. }\end{array}$ \\
\hline F22 & & $\begin{array}{l}\text { The enclosure must have a pass through } \\
\text { door that allows the insulation cover, } \\
\text { annulus cover, trap, and various } \\
\text { miscellaneous supplies to be passed. }\end{array}$ \\
\hline F23 & $\begin{array}{l}\text { The enclosure must have shielding built } \\
\text { into the walls and ceiling as determined by } \\
\text { the radiation exposure analysis. }\end{array}$ \\
\hline F24 & $\begin{array}{l}\text { The enclosure must have an operator } \\
\text { station with a shielded viewing window, TV } \\
\text { monitor, manipulator control, motor control, } \\
\text { valve control, welder control, and } \\
\text { communication with the process control } \\
\text { PLC. }\end{array}$ \\
\hline
\end{tabular}

A process enclosure concept that incorporates these functions is shown on Drawing SK-2-300420. The enclosure will be a floorless steel plate enclosure with a corner operator station and a recess for the drum bag port. The viewing station will have a leaded Lexan window. The enclosure will contain a hydraulic manipulator similar to the Schilling Titan VII depicted. The drum bagport system will be the Central Research system. The enclosure vertical motion can be accomplished with manually pumped hydraulic jacks. The enclosure rides on an open frame supported on four wheels. There is limited turning capability for positioning the cell. The 
WHC-SD-SNF-CDR-007, Revision 0

\subsection{PROCESS}

enclosure will be moved along the process pit centerline by towing with a hand operated electric tow motor. A battery charging station for the tow motor will be provided at the enclosure parking station. As indicated on the HCSE floor plan the parking space will be to the (Southeast) of the process modules. A shuttle table will move the enclosure from the process pit centerline to the parking location. 
WHC-SD-SNF-CDR-007, Revision 0

\subsection{PROCESS}

\subsection{SOLID WASTE}

The solid waste handling system and requirements will be designed in accordance with the requirements outline in WAC 173-303, Dangerous Waste Regulations and WHC EP 0063, Waste Acceptance Criteria. This system shall provide the equipment and local storage necessary to prepare all solid waste produced by the Hot Conditioning Equipment System (HCSE) for transfer to the appropriate storage and or treatment facility.

The sources of solid routine waste generated by the HCSE fall into two categories. The first is waste generated as a direct result of operating the conditioning equipment (i.e. HEPA filters, iodine traps, and filters used by the radioactive monitoring equipment) and the second is from general cleaning and inspection (i.e. welding rods, kim wipes, swipe tests, etc.). If a accidental breach occurs additional solid waste would be generated from the decontamination operations such as absorbent materials, swipe test papers, kim wipes, etc.

The amount of solid waste expected to contain potentially low levels of contamination has been estimated as follows:

A. Contaminated HEPA Filters (adjacent to the exhaust stack)

Changed on average 2 times yearly

B. Contaminated HEPA Filters (inlet to the process trench)

Changed on average 4 times yearly

C. Getter and Charcoal Beds (HVAC), if required

Changed on average 2 times yearly

D. Filters Incorporated in the Radiation Monitors

Changed on average Monthly

E. Filters Used by the RAS Monitors

Changed on average Weekly 
WHC-SD-SNF-CDR-007, Revision 0

\subsection{PROCESS}

F. Process Filters VPS-PFR-1101 and VPS-F-1107

Changed on average Monthly

G. Vacuum Pump Filters

Changed on average

Monthly

H. Spent Welding Rods

Approximately $1 / 2$ lbs per MCO

I. Kim Wipes for General Cleaning

Approximately 15 per MCO

J. Swipe Papers

Approximately 10 per MCO

K. Soiled Protective Clothing Approximately 1 per Operator per month

L. Dye Penetrant Testing Materials

Approximately $1 / 4 \mathrm{lbs}$ per MCO

The amount of solid waste expected to contain potentially moderate to high levels of contamination has been estimated as follows:

A. lodine/Cesium Traps

Approximately 1 per $\mathrm{MCO}$

Failed equipment can potentially fall into both low and high levels of contamination. The process equipment that is used to pump the gases and moisture from the MCO (vacuum pumps and blowers), the piping used to convey these gases, and the valves and fittings in this portion of the system have the potential to become moderately contaminated if the MCO internal filter fails. Other equipment such as radiation monitors, gas supply system components, and heating elements do not have a potential to become moderately or highly contaminated. An analysis of the average expected lifetime of the process equipment will be performed as part of the 
WHC-SD-SNF-CDR-007, Revision 0

\subsection{PROCESS}

detailed design effort to estimate the frequency of equipment failure and thus the amount of waste generated as a result of the failure.

The Hot Conditioning System Annex will be provided with appropriate storage containers for both low level.waste and moderately contaminated wastes. Failed equipment will be disposed of appropriately based on a radiation survey. The waste storage area will also provide functions for characterizing the waste materials before shipment to storage (i.e., burial ground, WRAP, etc.). 
WHC-SD-SNF-CDR-007, Revision 0

\subsection{FACILITIES/UTILITIES SUPPORT}

\subsection{HCSE LAYOUT}

\subsubsection{Functions and Requirements}

The function of the HCSE layout is to configure the process equipment and other components to best support maintenance and operation of the system. This includes providing; sufficient access to each component, optimizing the flow of process materials, and matching the requirements for interfacing with the MHM. A secondary requirement is to configure the system to be compatible with future SNF storage in a vault that could be constructed on the south end of the Annex.

\subsubsection{HCSE Layout Description}

The HCSA will be located on the south end of the CSB. The layout of the process pits, trenches, process modules, service module and solid waste storage area are shown on Drawing No. SK-2-300421.

The six process pits and the spare are aligned in a row to facilitate access by the process enclosure. The pits must also be accessed by the MHM so they are located between the MHM rails. Clearance distances for the MHM are maintained from the MHM rails and between the centerline of the pits and the edge of the process modules. The distance between pits is mainly driven by the access area required around the process modules. This distance is larger than the distance between CSB storage holes so it is compatible with the MHM space requirements.

The trenches join the process pits with the process modules where the bulk of the process equipment is located. The trenches are required because a flat floor is required for all areas serviced by the MHM. It is anticipated that the trenches will be 24-in wide and 51-in deep. The length is dictated by the MHM clearance requirements.

The process modules will be 6 -ft wide by 9 -ft long by 8 -ft. high. They are laid out with a 4-ft wide aisle all around to provide access for maintenance. A module is not shown for the spare process pit but space is provided for it. The process modules are located as close to the process pits as allowed by the MHM to minimize the size of the HCSA.

The service module will be in the southeast corner of the CSB annex at floor level. An aisle way is provided all around the service module to allow access for maintenance. 
WHC-SD-SNF-CDR-007, Revision 0

\subsection{FACILITIES/UTILITIES SUPPORT}

The bottom surface of the MHM bridge will be 9 feet off the floor. This will allow the bridge to clear the process enclosure. The process enclosure will be parked over an MCO pit when it is not in use.

All of the HCSE components that will be located outside of the HCSA are classified as auxiliary facilities. These include the ventilation stack, the refrigerant condenser for the chilled water system, the gas bottles, and the tube trailers. 


\begin{tabular}{|c|c|c|}
\hline INTERFACE & HCSE & HCSA \\
\hline General Arrangement - Oven & $\begin{array}{l}\text { Ovens including self-contained } \\
\text { insulation \& shielding, will be } \\
\text { installed in process pit by HCSE. }\end{array}$ & No HCSA design team involvement. \\
\hline $\begin{array}{l}\text { General Arrangement - Process } \\
\text { Module }\end{array}$ & $\begin{array}{l}\text { Process Modules will be installed. } \\
\text { Size to fit within 6-ft wide by 9-ft } \\
\text { long by } 8 \text {-ft 6-in high space. }\end{array}$ & No HCSA design team involvernent. \\
\hline $\begin{array}{l}\text { General Arrangement - Process } \\
\text { Module Mounting Plates }\end{array}$ & $\begin{array}{l}\text { HCSE to provide installation details } \\
\text { to weld process modules to } \\
\text { mounting plates. }\end{array}$ & $\begin{array}{l}\text { Mounting piates are to be cast in HCSA } \\
\text { floor to support Process Modules. Plates } \\
\text { to be located as shown on HCSE layout } \\
\text { Dwg No. SK-2-300430 Sheet } 1 \text {. }\end{array}$ \\
\hline General Arrangement - Trench & $\begin{array}{l}\text { HCSE to provide all supports, } \\
\text { conduits, piping, insulation, } \\
\text { instrumentation and equipment that } \\
\text { will be located in trenches. }\end{array}$ & $\begin{array}{l}\text { Trenches to be formed in HCSA floor and } \\
\text { located as shown on HCSE layout Dwg } \\
\text { No. SK-2-300430, Sheets } 1 \text { and } 2 \text {. } \\
\text { Dimensions will be standard for } 7 \\
\text { Trenches. }\end{array}$ \\
\hline $\begin{array}{l}\begin{array}{l}\text { General Arrangement - Trench } \\
\text { Cover }\end{array} \\
\end{array}$ & $\begin{array}{l}\text { Trench cover to be } 6 \text {-in thick with } \\
\text { metal edges and sealing surfaces. }\end{array}$ & No HCSA design team involvement. \\
\hline $\begin{array}{l}\text { General Arrangement - Process } \\
\text { Pit }\end{array}$ & $\begin{array}{l}\text { HCSE to provide design details for } \\
\text { installing ovens and associated } \\
\text { piping and instrumentation in the } \\
\text { process pits. }\end{array}$ & $\begin{array}{l}\text { Seven identical process pits to be formed } \\
\text { in HCSA floor and located per HCSE } \\
\text { layout Dwg No. SK-2-300340, Sheets } \\
1 \text { and } 2 \text {. }\end{array}$ \\
\hline $\begin{array}{l}\text { General Arrangement - Process } \\
\text { Pit Cover }\end{array}$ & $\begin{array}{l}\text { Process pit cover to be } 6 \text {-in thick } \\
\text { with metal edges and sealing } \\
\text { surfaces. }\end{array}$ & No HCSA design team involvement. \\
\hline $\begin{array}{l}\text { General Arrangement - Process } \\
\text { Pit Impact Limiters }\end{array}$ & $\begin{array}{l}\text { HCSE to design impact limiters that } \\
\text { are essentially identical to overpack } \\
\text { storage hole impact limiters, } \mathrm{H}-2- \\
120142 \text {. }\end{array}$ & No HCSA design team involvement. \\
\hline $\begin{array}{l}\text { General Arrangement - Service } \\
\text { Module }\end{array}$ & $\begin{array}{l}\text { Service Module will be installed by } \\
\text { HCSE. }\end{array}$ & lesign team \\
\hline $\begin{array}{l}\text { General Arrangement - Process } \\
\text { Enclosure } \\
\end{array}$ & $\begin{array}{l}\text { Process Enclosure will be installed } \\
\text { by HCSE. }\end{array}$ & No HCSA design team involvement. \\
\hline General Arrangement - Floor & $\begin{array}{l}\text { HCSE design team to develop } \\
\text { equipment and module loads and to } \\
\text { determine locations for Process } \\
\text { Pits, Trenches and Mounting Plates }\end{array}$ & $\begin{array}{l}\text { HCSA to develop drawings, specifications } \\
\text { and design analysis for HCSA floor. }\end{array}$ \\
\hline Process Piping & $\begin{array}{l}\text { Process piping and piping supports } \\
\text { in process pit, trench and on } \\
\text { process skids to be provided by } \\
\text { HCSE. }\end{array}$ & No HCSA design team involvement. \\
\hline Process HVAC & $\begin{array}{l}\text { HCSE to provide all ventilation } \\
\text { systems required to support HCSE } \\
\text { including stack, ductwork and } \\
\text { monitoring equipment and solid } \\
\text { waste handling area ventilation. } \\
\end{array}$ & $\begin{array}{l}\text { HCSE system will draw approximately } \\
1500 \mathrm{cfm} \text { from HVSA. Heat load will be } \\
\text { from air convection cooling of electric } \\
\text { motors (TBD) Btu/hr. }\end{array}$ \\
\hline Building HVAC & HCSE design team involvement. & $\begin{array}{l}\text { HCSA to develop drawings, specifications } \\
\text { and design analysis for annex HVAC } \\
\text { system. }\end{array}$ \\
\hline
\end{tabular}


WHC-SD-SNF-CDR-007, REV. 0

\begin{tabular}{|c|c|c|}
\hline INTERFACE & HCSE & HCSA \\
\hline Electrical Power - Normal & $\begin{array}{l}\text { HCSE to provide all electrical gear } \\
\text { required to interface the } 480 \text { vac } \\
\text { supply with the connected loads. }\end{array}$ & $\begin{array}{l}480 \text { vac, 3-phase provided to } 8 \text { boxes } \\
\text { located on south HCSA wall (7 process } \\
\text { modules, } 1 \text { service module) in close } \\
\text { proximity to modules as shown on HCSE } \\
\text { layout Dwg No. SK-2-300421, Sheet } 1.40 \\
\text { kW connected load per box. Total } \\
\text { demand load is } 175 \mathrm{~kW} \text {. }\end{array}$ \\
\hline Electrical Power - Uninterruptible & $\begin{array}{l}\text { HCSE to provide dedicated } \\
\text { uninterruptible power to all } \\
\text { equipment that requires it. }\end{array}$ & No HCSA design team involvement. \\
\hline Electrical Power - Standby & Not required & Not required \\
\hline Grounding Systems & $\begin{array}{l}\text { HCSE design team to connect to } \\
\text { grounding systems from process } \\
\text { equipment and instrumentation. }\end{array}$ & $\begin{array}{l}\text { Two grounding systems, one for power } \\
\text { and one for sensitive instruments. Access } \\
\text { points to be provided in proximity to } \\
\text { modules. On south end of HCSA. }\end{array}$ \\
\hline Lighting & No HCSE design team involvement. & $\begin{array}{l}\text { HCSA to provide general building lighting } \\
\text { and outdoor lighting. }\end{array}$ \\
\hline Process Gases & $\begin{array}{l}\text { HCSE to provide any required } \\
\text { specialty gases and the pads, tanks, } \\
\text { piping, instrumentation, wall } \\
\text { penetrations, etc. required to store } \\
\text { and distribute them. }\end{array}$ & No HCSA design team involvement \\
\hline Instrument Air & $\begin{array}{l}\text { HCSE to provide any instrument air } \\
\text { required for the HCSE process } \\
\text { including distribution piping. }\end{array}$ & No HCSA design team involvement \\
\hline $\begin{array}{l}\text { Instrumentation \& Control - } \\
\text { Process Modules and Service } \\
\text { Module }\end{array}$ & $\begin{array}{l}\text { HCSE to provide hardware and } \\
\text { software required at both ends of } \\
\text { the Ethernet cables. }\end{array}$ & $\begin{array}{l}\text { HCSA design team to provide one conduit } \\
\text { per module from HCSA control room to } \\
\text { proximity of modules as shown on HCSE } \\
\text { layout Dwg No. SK-2-300321, Sheet } 1 \text {. } \\
\text { Conduit to contain two Ethernet cables per } \\
\text { IEEE } 802.3 \text {, cable type (TBD). }\end{array}$ \\
\hline Process Cooling System & $\begin{array}{l}\text { HCSE to provide process cooling } \\
\text { system including outside air } \\
\text { exchanger, wall penetrations, } \\
\text { transfer fluid piping, pumps, } \\
\text { instrumentation and other heat } \\
\text { exchange equipment. } \\
\end{array}$ & No HCSA design team involvement \\
\hline $\begin{array}{l}\text { Safety Signals such as Stack } \\
\text { Monitor and Radiation Monitoring }\end{array}$ & $\begin{array}{l}\text { HCSE provides monitors and signal } \\
\text { generators and conductors, cables } \\
\text { and conduits to transmit signals to } \\
\text { common point in proximity of south } \\
\text { side of Service Module. HCSE to } \\
\text { provide hardware and software in } \\
\text { control room to process signals. }\end{array}$ & $\begin{array}{l}\text { HCSA to provide cable with } 50 \text { conductors } \\
\text { to hard wire safety signals to CSB control } \\
\text { room from south wall in proximity of } \\
\text { service module. }\end{array}$ \\
\hline MHM Interface & $\begin{array}{l}\text { HCSE to provide process pit cover } \\
\text { with MCO access cover that } \\
\text { emulates storage hole cover. }\end{array}$ & No HCSA design team involvement. \\
\hline
\end{tabular}


WHC-SD-SNF-CDR-007, REV. 0

\begin{tabular}{|c|c|c|}
\hline INTERFACE & HCSE & HCSA \\
\hline Water & $\begin{array}{l}\text { Not required, water not allowed in } \\
\text { storage vaults and combustibles not } \\
\text { present in HCSE. If water is used in } \\
\text { closed loop cooling systems it will } \\
\text { be trucked in. Spills will be } \\
\text { contained by HCSE and mopped } \\
\text { up. }\end{array}$ & No HCSA design team involvement. \\
\hline Occupancy & No HCSE design team involvement. & $\begin{array}{l}\text { CSB change room and lavatory facilities to } \\
\text { accommodate } 4 \text { additional people per } \\
\text { shift, } 21 \text { shifts per woek to supplement } \\
\text { CSB staff who will also operate and } \\
\text { maintain the HCSE. }\end{array}$ \\
\hline Equipment installation & $\begin{array}{l}\text { HCSE responsible for installing all } \\
\text { hot conditioning systems and } \\
\text { equipment. }\end{array}$ & $\begin{array}{l}\text { Building access to accommodate the } \\
\text { largest HCSE unit which will be the } \\
\text { Process Enclosure, SK-2-300420. }\end{array}$ \\
\hline Sewer & No HCSE design team involvement. & HCSA to provide if necessary \\
\hline Access Roads & No HCSE design team involvement. & $\begin{array}{l}\text { HCSA to provide roads and truck apron on } \\
\text { west side of annex. }\end{array}$ \\
\hline HCSE Ventilation Stack & $\begin{array}{l}\text { HCSE to provide ductwork, wall } \\
\text { penetrations, stack monitors, } \\
\text { support structure and ventilation } \\
\text { stack for HCSE exhaust gases. }\end{array}$ & No HCSA design team involvement \\
\hline Staging Areas & $\begin{array}{l}\text { HCSE to provide any staging areas } \\
\text { required for solid waste, service } \\
\text { equipment, samples and other } \\
\text { miscellaneous items. }\end{array}$ & No HCSA design team involvement \\
\hline Auxiliary Crane & $\begin{array}{l}\text { Not required for HCSE process or } \\
\text { equipment, fork lifts can handle all } \\
\text { transfer duties. }\end{array}$ & Not required. \\
\hline MHM Rails & No HCSE design team involvement. & $\begin{array}{l}\text { HCSA design team to extend MHM rails } \\
\text { from CSB to annex. }\end{array}$ \\
\hline Doors & $\begin{array}{l}\text { HCSE to design equipment and } \\
\text { modules to fit through doors for } \\
\text { initial installation. }\end{array}$ & $\begin{array}{l}\text { HCSA design team to provide all access } \\
\text { doors to HCSA. Door on west wall of } \\
\text { annex must be large enough to allow the } \\
\text { Process Enclosure (SK-2-300420) to pass } \\
\text { through. }\end{array}$ \\
\hline $\begin{array}{l}\text { Communications, Fire Alarms, } \\
\text { Security Alarms }\end{array}$ & No HCSE design team involvement. & $\begin{array}{l}\text { HCSA design team to provide all } \\
\text { communication systems, fire alarm } \\
\text { connections and interties to the site } \\
\text { security system. }\end{array}$ \\
\hline
\end{tabular}


WHC-SD-SNF-CDR-007, Revision 0

\subsection{FACILITIES/UTILITIES SUPPORT}

\subsection{HCSEJHCSA INTERFACES}

The HCSE project is being developed as a project separate from the HCSA of the CSB that will house it. This is because the HCSA interacts structurally with the CSB and services must be extended from the CSB, while the HCSE is a process equipment development activity that is not dependent on the detailed characteristics of the HCSA building. The HCSE will be stand-alone process systems designed to operate as independently from the HCSA as possible. The HCSA will be a building with properties that are similar to the CSB. It will be open to the CSB storage area to allow the MCO Handling Machine (MHM) to move MCOs between the storage vaults and the HCSE. Therefore, the criteria for the CSB regarding design features such as structure, ventilation, radiation monitoring, and so forth that define the nuclear facility characteristics of the building apply to the HCSA. The existing CSB design and partially complete construction assume criteria that do not require the building envelope to act as a nuclear material release confinement structure. In principle, the HCSE will be a miniature nuclear system with confinement and safety class SSCs housed within the HCSA shell. Generally the HCSA will be a minimal building shell and the HCSE will provide its own amenities after the HCSA is completed. This approach allows the HCSA to catch up with the CSB so that it can be built as part of completing the CSB construction while the HCSE progresses through its design and safety analysis activities without impacting the construction of the CSB. The HCSA must provide a number of services that are to be connected to the HCSE. In addition, the HCSE will generate loads such as heat and weight on the HCSA. The HCSE also requires service by the MHM in order to make MCO input/output transactions. This discussion describes the basic interfaces.

The HCSE/HCSA interfaces and responsibilities are given in Table 3.2-1.

\subsubsection{General Arrangement}

The general arrangement of the HCSE is given by Drawing SK-2-300421 (Building Layout). The HCSA project will be responsible for providing the process pits (without covers), trenches (without covers), floor, mounting pads, and other features needed to obtain this arrangement.

\subsubsection{Process Station Arrangement}

A process station consists of the process pit, oven, and process trench. The process station plan and elevation views are given in Drawing SK-2-300422. The HCSA project will be responsible for providing the vaults (without covers), trenches (without covers), mounting pads, and other structural features required to attain this 
WHC-SD-SNF-CDR-007, Revision 0

\subsection{FACILITIES/UTILITIES SUPPORT}

arrangement. The HCSA project will perform the structural design, such as concrete specification, while preserving the critical dimensions called out on the Drawings.

\subsubsection{Oven Interface}

The oven will be a jacketed vessel with a smooth stainless steel skin. The process pit in which it will reside shall have cast concrete walls and bottom. The interior dimensions shall be as given on Drawing SK-2-300422. The concrete surfaces shall be sealed with an epoxy paint. The oven outer skin temperature will not exceed $50^{\circ} \mathrm{C}$. The empty oven weight will not exceed 5,000 lbs. The weight of the loaded oven will not exceed 25,000 lbs. The estimated weight of the vault cover is 5,000 Ibs. The foundation shall support these loads. The $20,000 \mathrm{lb}$ MCO may be accidentally dropped onto the bottom of the oven from a height of $14 \mathrm{ft}$. The hole assembly will contain the same shock absorber utilized in the CSB overpack storage holes. The HCSE project shall provide these shock absorbers. The foundation design shall be able to absorb the drop load without sustaining structural failure.

The HCSE will provide and install the oven, process pit cover, process trench cover, other items contained within the process pit and process trench, the process enclosure, and the process module.

\subsubsection{Process Piping Interface}

The HCSA project shall provide a trench with interior dimensions as given on Drawing SK-2-300430. The trench floor and sides shall be cast concrete sealed with epoxy paint. The exterior temperature of the pipes in the trench will not exceed $50^{\circ} \mathrm{C}$. Mounting hardware for the pipes and conduit will be attached using concrete anchors to be located at the time of installation by the HCSE project. The estimated weight of the piping is $75 \mathrm{lbs}$ per foot. The trench design shall support a cover that will weigh approximately 500 lbs per foot.

The HCSE project shall supply all piping, conduit, supports, and trench covers.

\subsubsection{HVAC Interface}

The HCSA is not required to provide any nuclear material confinement capability. Nuclear material confinement will be provided within the HCSE.

The HCSE will contain its own exhaust fans, ducts, and nuclear grade filtration systems. Intake air will be drawn from the HCSA volume, through HEPA filters (provided by the HCSE) into potentially contaminated pit space, through the potentially contaminated vault and process trench, through an HCSE provided 
WHC-SD-SNF-CDR-007, Revision 0

\subsection{FACILITIES/UTILITIES SUPPORT}

HEPA filter, through an HCSE provided fan, and out a stack provided by the HCSE. This scheme assures a negative pressure in the potentially contaminated pit and process trench. Hardening of the HVAC system to protect it from adverse effects arising from Design Basis Accidents (DBAs) and its safety features will be the responsibility of the HCSE project.

The HCSE will design and construct its own exhaust stack for the potentially contaminated exhaust generated by the HCSE process. Penetration in the preexisting HCSA structure for the stack, stack, pipe, stack foundation, stack support, stack feed ducting, and so forth will be part of the HCSE scope. The air volume drawn from the HCSA will range from 1000 to 1500 CFM.

The estimated heat load of the HCSE equipment that will be discharged to the HCSA will be (TBD) BTU. This heat will be from the process modules where motors and heat exchangers will be located.

The HCSE will provide safety interlock signals, such as HVAC flow loss, to be used in the CSB control room when monitoring the facility.

\subsubsection{Electrical Interface}

\subsubsection{Normal Power}

The HCA shall provide 480 VAC three phase power for use by the HCSE. The power shall be provided to eight (8) disconnect switches (one for each process module, including the spare and one for service module). The HCSA provided power will terminate in these eight boxes to be located on the HCSA wall near each of the process modules. The HCSE will be responsible for transformation and distribution of power within the HCSE systems.

The power requirements are:

A. Process Module Supply: Average Demand $=(T B D)$ Amps, Peak Demand $=$ (TBD) Amps

B. Central Service Supply: Average Demand $=(T B D)$ Amps, Peak Demand $=$ (TBD) Amps

\subsubsection{Grounding}

Two grounding systems shall be provided. An isolated ground that is nol connected to power circuits shall be provided for use by sensitive instruments. The HCSA shall provide ground and isolated ground connections near each process module. 
WHC-SD-SNF-CDR-007, Revision 0

\subsection{FACILITIES/UTILITIES SUPPORT}

\subsubsection{Process Gas Interface}

The HCSE will require a source of Helium and Inert Gas/Oxygen Blend. These are to be provided from tube trailers parked immediately outside the HCSA. The HCSA project shall provide aprons for truck access. The HCSE will be responsible for penetrating the HCSA wall and for distribution of the gases within the facility.

\subsubsection{I8.C Interface}

Each skid will contain its own PLC and data collection capability. The HCSA will provide cabling only to support Ethernet connection of the HCSE processors (located on the process modules) and the process monitoring station to be located in the CSB control room. All computing equipment, control logic, sensors, etc. are the responsibility of the HCSE.

Safety signals, such as stack monitor signals, fire detectors, and radiation monitors, will be hard wired to the CSB control room. The HCSA shall provide 50 conductors for these signais that will be collected on the central services skid. The patterns, "rules of thumb", and instrument models selected in the CSB design shall be followed by the HCSE project. CSB data collection and facility monitoring equipment will accept the safety system detector signals.

The HCSE will provide all radiation monitoring. The HCSE will provide the stack monitoring system.

The HCSA will provide security systems components such as TV cameras. The HCSA shall provide cabling between a collection box located in the HCSA and the CSB control room. The HCSA shall coordinate provision of TV monitors and security alarm monitoring equipment in the CSB control room.

\subsubsection{MHM Interface}

The HCSE will design the process pit cover so that it simulates the top of a storage hole in the CSB. The MHM will not have to take any special actions when loading or unloading the HCSE ovens.

The MHM collision avoidance system must allow the MHM to load or unload an oven when the process enclosure is located over another oven. The box beam of the MHM will be located at 9-ft above the floor to allow this. 
WHC-SD-SNF-CDR-007, Revision 0

\subsection{FACILITIES/UTILITIES SUPPORT}

\subsubsection{Water}

No process water connection will be required by the HCSE. The chilled water system will be filled from a portable water tank.

No fire water will be required in the HCSE.

\subsubsection{Occupancy}

Occupancy will be 4 additional people per shift to the CSB operating staff working three shifts per day and 7 days per week. CSB facilities such as change rooms and bathrooms must be able to accommodate this staff in addition to CSB operations staff.

\subsubsection{Equipment Installation Interface}

The HCSA shall provide an equipment access door with an opening large enough for the process enclosure to pass through. The maximum estimated module weight is (TBD)) lbs. Equipment movement and placement will be accomplished using forklifts. No overhead crane will be required in the HCSA. The process skids will be mounted to imbedded steel floor plates. Attachment will be made by welding the equipment skid steel to the floor plates. The floor plates are located on Drawing SK2-300430. The HCSE will be responsible for installing the equipment.

\subsubsection{HCA Structural Requirements}

The HCSA will be a structure designed to meet the same load, earthquake, wind, missile, criteria as the CSB to which it is attached. The HCSE shall provide local secondary confinement that shall meet the necessary safety class/safety significant criteria.

\subsection{AUXILIARY FACILITIES}

Auxiliary facilities are defined as those that are located outside of the HCSA building. These include the tube trailers and gas bottles for the process gas system, the stack for the combined ventilation system and the refrigerant to air portion of the chilled water system. 
WHC-SD-SNF-CDR-007, Revision 0

\subsection{FACILITIES/UTILITIES SUPPORT}

\subsubsection{Process Gas System}

\subsubsection{Function and Requirements}

The process gas system includes items necessary for the storage, pressure reduction and manifolding of all process gasses for the HCSE. The system should function on demand from each of the individual process modules.

\subsubsection{Process Gas System Description}

The equipment and instrumentation used in the process gas system is identified on the P\&IDs, SK-2-300413. The following major equipment components comprise the process gas system:

The process gas system is installed on a pad located outside of the HCSA. The system consists of the following gases:
A. Helium
B. Nitrogen
C. Oxygen/inert gas blend

Nitrogen and helium are supplied by tube trailers while oxygen is supplied at premixed concentrations in cylinders. Oxygen and an inert gas wili be preblended to an oxygen concentration of 2 percent for fuel oxidation. Pressure from each station is reduced to 5 psi above the internal pressure of the MCO prior to introduction to the process area.

\subsubsection{Stack}

\subsubsection{Functions and Requirements}

The function of the ventilation stack is to exhaust the HEPA filtered effluent from the combined ventilation system to the atmosphere. The stack is to be provided with an isokinitic sampling system as described in Section 2.3 and shall be $1-1 / 2$ times the HCSA height. Effluent from the stack is to have a minimum exit velocity of 3000 $\mathrm{ft} / \mathrm{min}$. 
WHC-SD-SNF-CDR-007, Revision 0

\subsection{FACILITIES/UTILITIES SUPPORT}

\subsubsection{Stack Description}

The stack is anticipated to be $90-\mathrm{ft}$ high with an internal diameter of 8-inches. It will be construction of steel and will be located on concrete pad. Guy wires will be attached as appropriate to stabilize it such that it will withstand the postulated DBAs.

\subsubsection{Water Chiller}

\subsubsection{Functions and Requirements}

The function of the water chiller system is to provide cooling to the process equipment. The requirements for the system are shown in Section 2.7 since parts of the system are located in the service module. The system is also discussed as part of the auxiliary facilities because the refrigerant condenser will be located on a pad outside of the HCSA to allow air cooling.

\subsubsection{Water Chiller Description}

The refrigerant condenser will be a 25 -ton unit designed for exterior air cooling. A concrete pad will be provided for mounting of the condenser. Electrical power for the condenser will be supplied from the HCSE and refrigerant piping will connect the condenser with the service module. 


\subsection{INTERFACES}

\subsection{HCSE INTERFACES}

The HCSE will interact primarily with three subprojects. These are the HCSA, the MHM, and the MCO. The HCSA interfaces are required to define the facility and services that must be provided to house the HCSE. They are described in the Section 3.2 of the facility chapter. The MHM interface is required because the MHM will perform the MCO input / output transactions with the HCSE. The MCO interfaces are defined by the physical mating of the MCO and the oven assembly, by the need to have a scheme for connecting the HCSE to the MCO, by the need to make the final closure of the MCO in the HCSE ovens, and by the operating temperature and pressure design limitations of the MCO. The interfaces are given Table $4.0-1$ below.

\begin{tabular}{|c|c|c|c|}
\hline $\begin{array}{l}\text { Interface } \\
\text { Number }\end{array}$ & Name & Description & Requirement \\
\hline MCO1 & $\begin{array}{c}\text { MCO Body } \\
\text { Size }\end{array}$ & $\begin{array}{l}\text { The MCO diameter and } \\
\text { height are important for } \\
\text { determining the oven } \\
\text { dimensions. }\end{array}$ & $\begin{array}{l}\text { Dimensions must be } \\
\text { constant after oven } \\
\text { detailed design is complete } \\
\text { or after HCSA design is } \\
\text { complete. }\end{array}$ \\
\hline $\mathrm{MCO} 2$ & $\begin{array}{c}\text { MCO Neck } \\
\text { Shape }\end{array}$ & $\begin{array}{l}\text { The MCO neck shape } \\
\text { is not finalized. If the } \\
\text { flared design is selected } \\
\text { the MCO will hang from } \\
\text { a beveled flange and no } \\
\text { annulus cover will be } \\
\text { required. If the MCO } \\
\text { neck is straight, the } \\
\text { MCO will rest on its } \\
\text { base and an annulus } \\
\text { cover will be required. }\end{array}$ & $\begin{array}{l}\text { If flare design is selected - } \\
\text { flare dimensions and oven } \\
\text { flange dimensions must } \\
\text { agree. If the straight body } \\
\text { design is selected the top } \\
\text { surface must allow the } \\
\text { annulus cover to sit } \\
\text { without interference. }\end{array}$ \\
\hline MCO3 & MCO Weight & $\begin{array}{l}\text { The MCO weight must } \\
\text { be constant after oven } \\
\text { detail design is } \\
\text { complete. The oven } \\
\text { structure and shock } \\
\text { absorber are dependent } \\
\text { upon the weight. }\end{array}$ & None \\
\hline $\mathrm{MCO} 4$ & $\begin{array}{l}\text { Draw Tube } \\
\text { and Head } \\
\text { Space Port } \\
\text { Cover }\end{array}$ & $\begin{array}{l}\text { The ports are covered } \\
\text { by a bolted identical } \\
\text { bolted plates which } \\
\text { must be removed for } \\
\text { access to MCO } \\
\text { connectors. }\end{array}$ & $\begin{array}{l}\text { Bolts must be hexhead } \\
\text { type. Bolts must be } \\
\text { captured and spring loaded } \\
\text { so that they cannot fall out } \\
\text { and so that they pop-up } \\
\text { when disengaged. }\end{array}$ \\
\hline
\end{tabular}


WHC-SD-SNF-CDR-007, Revision 0

\subsection{INTERFACES}

\begin{tabular}{|c|c|c|c|}
\hline $\begin{array}{c}\text { Interface } \\
\text { Number }\end{array}$ & Name & Description & Requirement \\
\hline MCO5 & $\begin{array}{l}\text { Draw Tube } \\
\text { and Head } \\
\text { Space } \\
\text { Connections }\end{array}$ & $\begin{array}{l}\text { The connectors allow } \\
\text { the HCSE process lines } \\
\text { to be connected to the } \\
\text { MCO. }\end{array}$ & $\begin{array}{l}\text { Connectors must be } \\
\text { remotely (manipulator and } \\
\text { tools) operable. } \\
\text { Connectors should have a } \\
\text { flow cross sectional area } \\
\text { as close to } 0.8 \text { sq. in. as } \\
\text { possible. Connector seals } \\
\text { must be capable of } \\
\text { operating at } 350^{\circ} \mathrm{C} \text {. } \\
\text { Connector must be helium } \\
\text { leak tight. Connector must } \\
\text { be tolerant to dirt (SNF } \\
\text { corrosion dust). The } \\
\text { connector must be helium } \\
\text { leak tight measured with a } \\
\text { helium sniffer (max leak } \\
\text { rate } 10 \mathrm{O}-06 \text { std. cc/sec at } \\
\text { 100PSI). }\end{array}$ \\
\hline MCO6 & $\begin{array}{l}\text { Final Closure } \\
\text { Design }\end{array}$ & $\begin{array}{l}\text { The final closure of the } \\
\text { MCO will be a cover } \\
\text { welded in the HCSE. }\end{array}$ & $\begin{array}{l}\text { Weld must be } \\
\text { circumferential so that it } \\
\text { can be accomplished with } \\
\text { the same welding head/ } \\
\text { fixture as used in the } \\
\text { CVDS. Weld procedure } \\
\text { using ultra-sonic inspection } \\
\text { is preferable to LPT } \\
\text { because remote execution } \\
\text { is easier. The cover must } \\
\text { include features to allow } \\
\text { the MHM to lift the MCO } \\
\text { out of the oven. }\end{array}$ \\
\hline $\mathrm{MCO} 7$ & $\begin{array}{l}\text { Temperature } \\
\text { Restraints }\end{array}$ & $\begin{array}{l}\text { The MCO has design } \\
\text { ratings for maximum } \\
\text { operating temperature, } \\
\text { rate of temperature rise, } \\
\text { and point to point } \\
\text { temperature difference. }\end{array}$ & $\begin{array}{l}\text { The limits are } 325^{\circ} \mathrm{C}, 50^{\circ} \mathrm{C} \\
\text { between any two points, } \\
\text { and } 50^{\circ} \mathrm{C} \text { per hour. }\end{array}$ \\
\hline
\end{tabular}


WHC-SD-SNF-CDR-007, Revision 0

\subsection{INTERFACES}

\begin{tabular}{|c|c|c|c|}
\hline $\begin{array}{c}\text { Interface } \\
\text { Number }\end{array}$ & Name & Description & Requirement \\
\hline MCO7 & $\begin{array}{l}\text { Operating } \\
\text { Pressure }\end{array}$ & $\begin{array}{l}\text { The MCO pressure is } \\
\text { limited by its design } \\
\text { pressure and its rupture } \\
\text { disk. }\end{array}$ & $\begin{array}{l}\text { The HCSE desires to have } \\
\text { the ability to operate at } 25 \\
\text { psia at } 300^{\circ} \mathrm{C} \text { so that it has } \\
\text { the flexibility to improve } \\
\text { the heat transfer rate if it } \\
\text { proves to be necessary to } \\
\text { meet processing time } \\
\text { budgets. }\end{array}$ \\
\hline MCO8 & $\begin{array}{l}\text { Head Space } \\
\text { Vent Filter }\end{array}$ & $\begin{array}{l}\text { Dust entrained in the } \\
\text { helium flow must be } \\
\text { filtered inside the MCO. }\end{array}$ & $\begin{array}{l}\text { Micron type filter. Capable } \\
\text { of operating at } 300^{\circ} \mathrm{C} \text {. }\end{array}$ \\
\hline MHM1 & $\begin{array}{l}\text { MCO } \\
\text { Alignment }\end{array}$ & $\begin{array}{l}\text { The angular position of } \\
\text { the port pattern on the } \\
\text { top of the MCO cannot } \\
\text { vary form MCO to MCO } \\
\text { because the HCSE } \\
\text { process connections } \\
\text { have a limited range of } \\
\text { motion. }\end{array}$ & $\begin{array}{l}\text { No more than }+/-15 \\
\text { degrees. }\end{array}$ \\
\hline MHM2 & Plug Docking & $\begin{array}{l}\text { The MHM will locate } \\
\text { above the process pit } \\
\text { center plug. Its grapple } \\
\text { will lock onto the plug } \\
\text { and pull it. After the } \\
\text { sequence is completed } \\
\text { it will replace the plug. }\end{array}$ & $\begin{array}{l}\text { Plug top must be a replica } \\
\text { of the CSB storage vault } \\
\text { covers that the MHM is } \\
\text { capable of handling. }\end{array}$ \\
\hline MHM3 & $\begin{array}{l}\text { MHM - } \\
\text { Process } \\
\text { Enclosure } \\
\text { Collision } \\
\text { Avoidance }\end{array}$ & $\begin{array}{l}\text { The MHM may require } \\
\text { access to one oven } \\
\text { while the process } \\
\text { enclosure is over the } \\
\text { other. The MHM bridge } \\
\text { and enclosure cannot } \\
\text { collide. }\end{array}$ & $\begin{array}{l}\text { Can be administratively } \\
\text { controlled. }\end{array}$ \\
\hline MHM4 & $\begin{array}{l}\text { MHM } \\
\text { Lockout }\end{array}$ & $\begin{array}{l}\text { The MHM should not be } \\
\text { able to access a hot } \\
\text { oven by error. }\end{array}$ & $\begin{array}{l}\text { HCSE to provide an } \\
\text { actuator that indicates } \\
\text { visually to MHM operator } \\
\text { that the oven is active. } \\
\text { HCSE to provide actuator } \\
\text { that prevents MHM grapple } \\
\text { from attaching to plug. }\end{array}$ \\
\hline
\end{tabular}


WHC-SD-SNF-CDR-007, Revision 0

\subsection{COST AND SCHEDULE}

\subsection{METHOD OF ACCOMPLISHMENT}

The HCSE project will be a joint effort between the DOE as the owner, WHC as the operator, Merrick as the architectengineer, and a yet to be determined prime construction contractor. The responsibilities of each organization are as follows.

\section{Department of Eneray}

DOE will furnish overall project coordination, review all conceptual design and definitive design documents and based on their review authorize the definitive design, construction and operation of the HCSE. DOE administers the WHC prime contract to operate the Hanford facilities.

\section{Westinghouse Hanford Company}

WHC will provide design criteria and engineering management services during the conceptual design, definitive design and construction project phases. WHC will review all design documents and based on this review recommend to DOE that the definitive design, construction and operation of the HCSE be authorized. WHC will award and administer the architect-engineer and prime construction contracts. If it is determined that government furnished equipment is required, WHC will be responsible for procuring this equipment and providing it to the prime construction contractor. Following construction of the HCSE, WHC will be responsible for HCSE operations.

\section{Merrick \& Company}

Merrick is under contract to WHC to perform all conceptual engineering services. Engineering required to develop the definitive design packages and construction support engineering are options under this contract. Merrick will be responsible for identifying and recommending to WHC items that should be classified as government furnished equipment. Merrick administers the subcontracts of the speciality design firms that support the architect-engineering work.

\section{Prime Construction Contracter}

A prime construction contractor will be selected and placed under contract to WHC to construct the HCSE. This contractor will be responsible for procuring materials and supplies and constructing the HCSE in accordance with the definitive design packages. The contractor will also be responsible for installing any government furnished equipment and administering the subcontracts of the speciality firms that will support the construction activities. 
WHC-SD-SNF-CDR-007, Revision 0

\subsection{COST AND SCHEDULE}

\subsection{SCHEDULE}

The key milestones in the HCSE design and construction schedule are as follows.

Conceptual Design Complete

Authorization to Proceed with Definitive Design

Definitive Design Complete

Authorization to Proceed with Construction

Construction Complete

Beneficial Occupancy
July 31, 1993

August 1, 1996

March 25, 1997

October 2, 1997

June 30,1998

September 30, 1998

\subsection{SUMMARY COST ESTIMATE}

The project cost estimate has been developed by the individual designers and utilizes a factored approach where the installed cost is based upon the cost of the equipment. The costs for the process modules and the central service module have been developed with the following criteria:

A. The cost estimate is developed for the pressure system, operating at an internal helium pressure of 25 psia. This was done because this option is the more expensive of the two cases. For an atmospheric system, deduct $\$ 60,000$ from the overall cost.

B. All installed costs are factored to equipment cost. All equipment cost is obtained from a vendor unless otherwise noted on the equipment list.

C. Cost factors are taken from the method of Miller (Perry, Table 25-51) for battery-limit installations. As skids will be factory assembled, the lower range of factors is normally used. In the event of significant complexity, the factor is adjusted to the higher end of the range. When extraordinary costs are involved for support equipment, as in the case of specific instrumentation, these items will be costed separately.

D. The structural estimate is applied once for each skid assembly.

E. Installed cost assumes FOB Richland, WA. A local skid fabricator is assumed. 
WHC-SD-SNF-CDR-007, Revision 0

\subsection{COST AND SCHEDULE}

Contingency, Engineering, Project Management, Insurance, etc. are factored to Construction Cost Estimate.

The auxiliary area estimate was developed from Means Construction Cost Estimator. The cost estimate for the Process Enclosure was developed from the experience of Paul Smith on like projects. Engineering cost and contingency are factored to the constructed cost of the equipment.

Backup information used to develop the estimate is found in the Appendix F.

The summary of the construction cost estimate is shown in Table 5.3 below:

Table 5.3

Project Cost Estimate

(all costs in thousand \$)

$\begin{array}{lrrr} & \begin{array}{r}\text { Item } \\ \text { Cost }\end{array} & \begin{array}{r}\text { No. } \\ \text { Items }\end{array} & \begin{array}{r}\text { Total } \\ \text { Cost }\end{array} \\ \text { Construction Costs } & 300 & 6 & 2,318 \\ \text { Process Module } & 150 & 1 & 328 \\ \text { Service Module } & 550 & 1 & 620 \\ \text { Process Enclosure } & 130 & 1 & 622 \\ \text { Auxiliary Facilities } & 210 & 6 & 1,635 \\ \begin{array}{l}\text { Process Pit Compnts } \\ \text { Subtotal Const. Cost }\end{array} & & & 5,523 \\ \text { Project Management } & & & 442 \\ \text { (8\%) } & & & \\ \text { Eng. Design \& Insp. } & & & 1,215 \\ \text { (22\%) } & & & 110 \\ \text { Bond \& Insurance (2\%) } & & & 1,657 \\ \text { Contingency (30\%) } & & & 8,947 \\ \text { TOTAL PROJECT } & & & \end{array}$


WHC-SD-SNF-CDR-007, Revision 0

\title{
APPENDICES
}

\section{APPENDIX A}

\author{
DRAWINGS
}




\section{HOT CONDITIONING \\ SYSTEM EQUIPMENT}

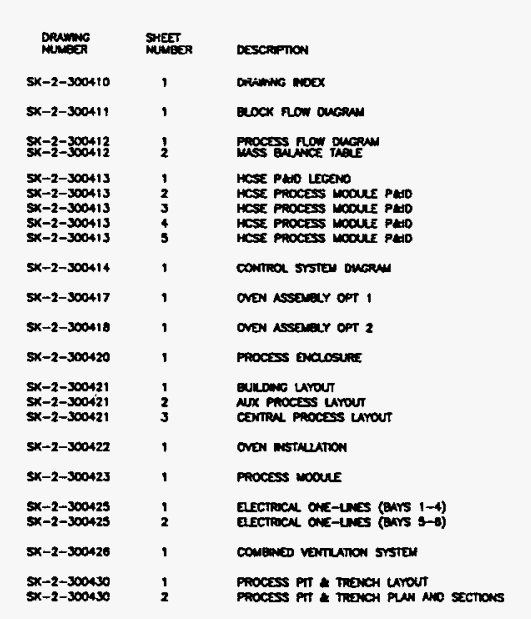




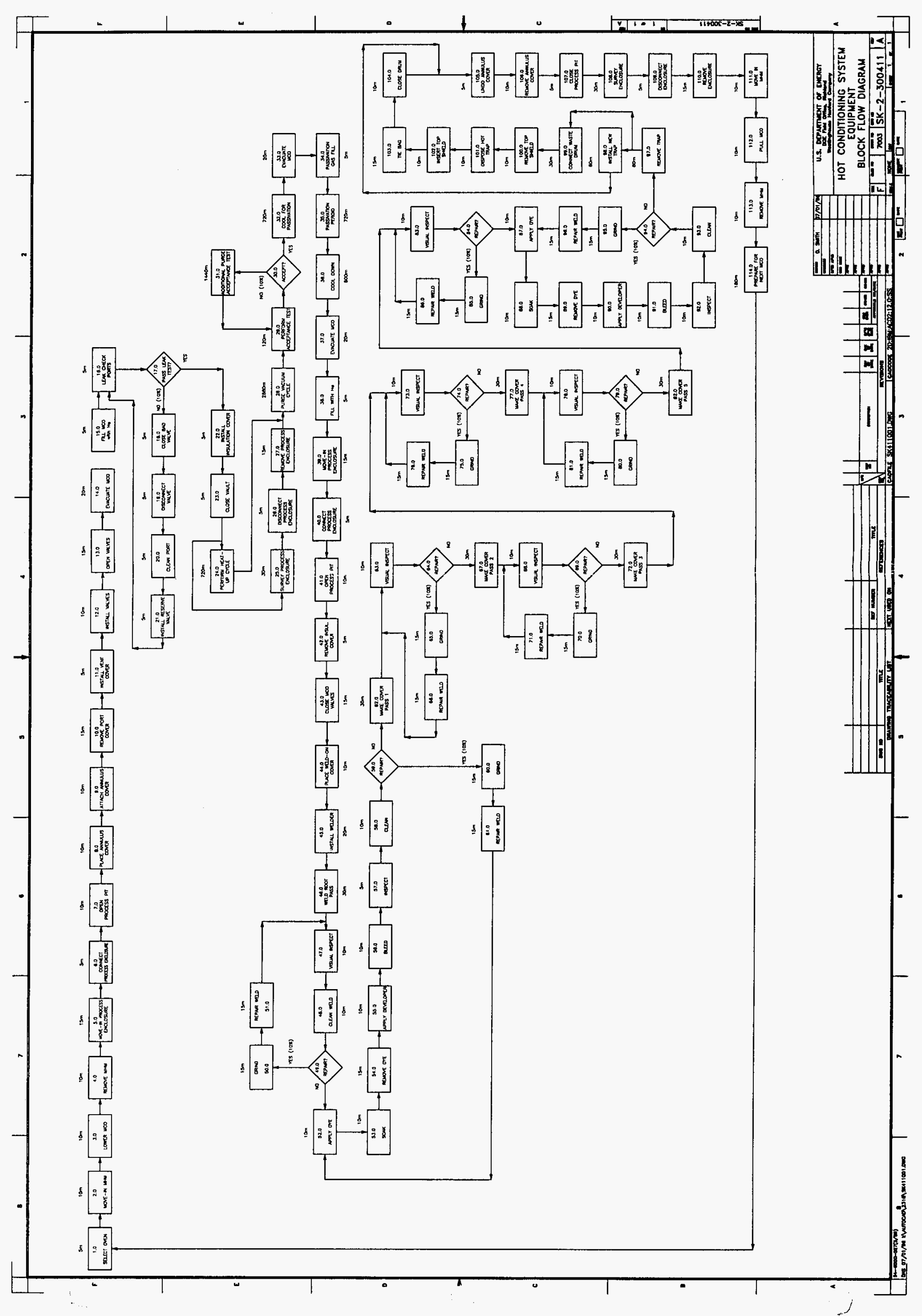




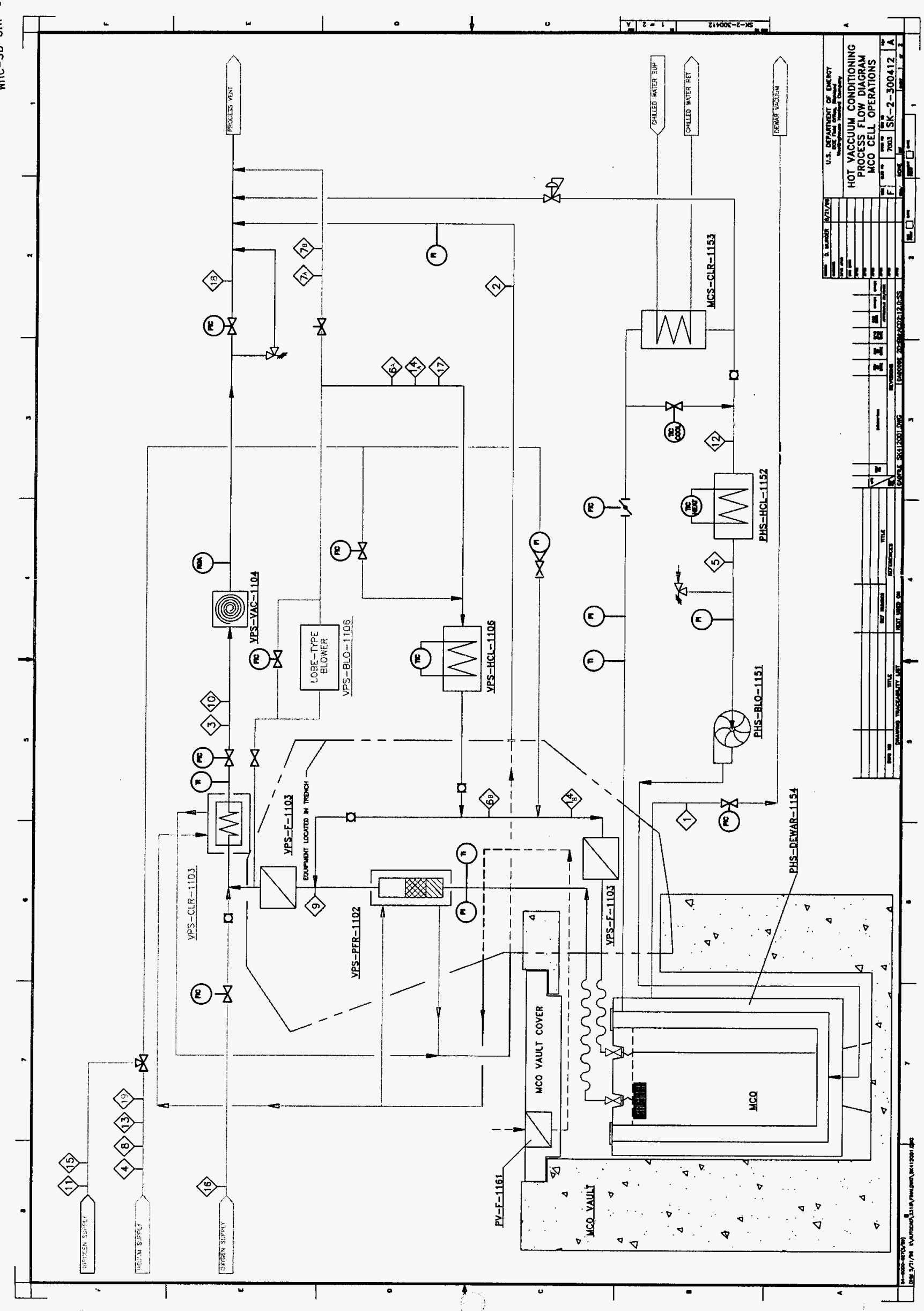




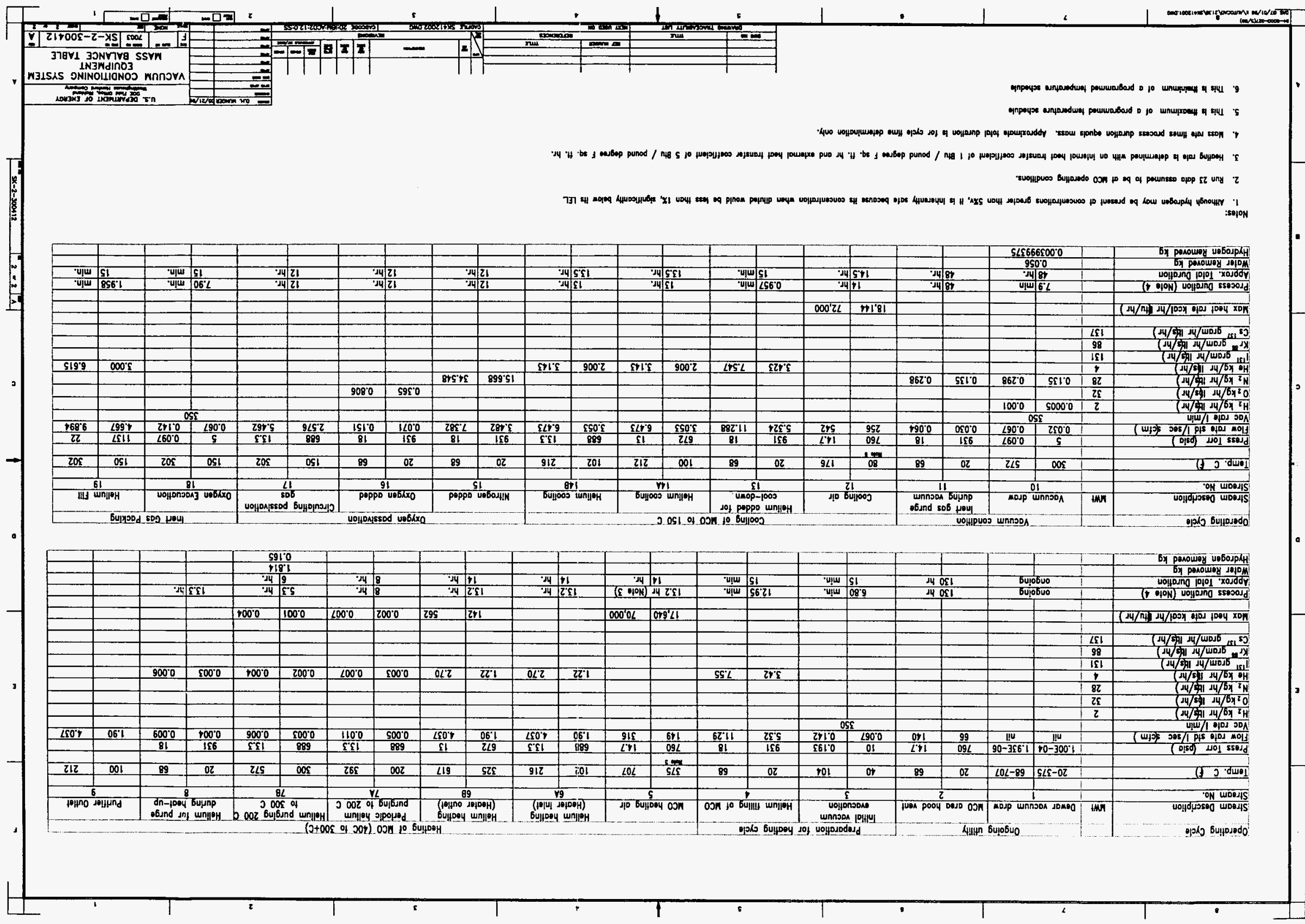




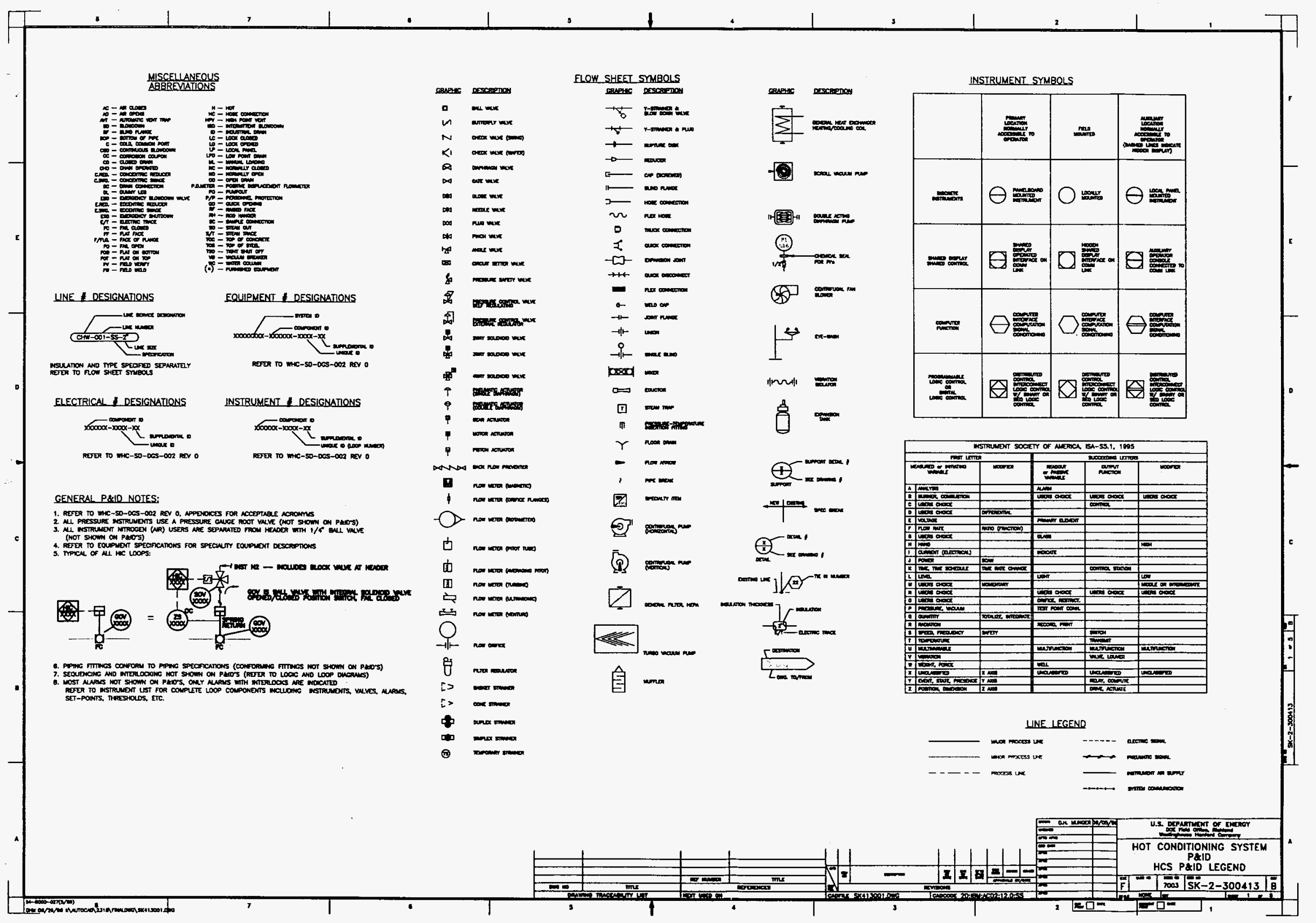




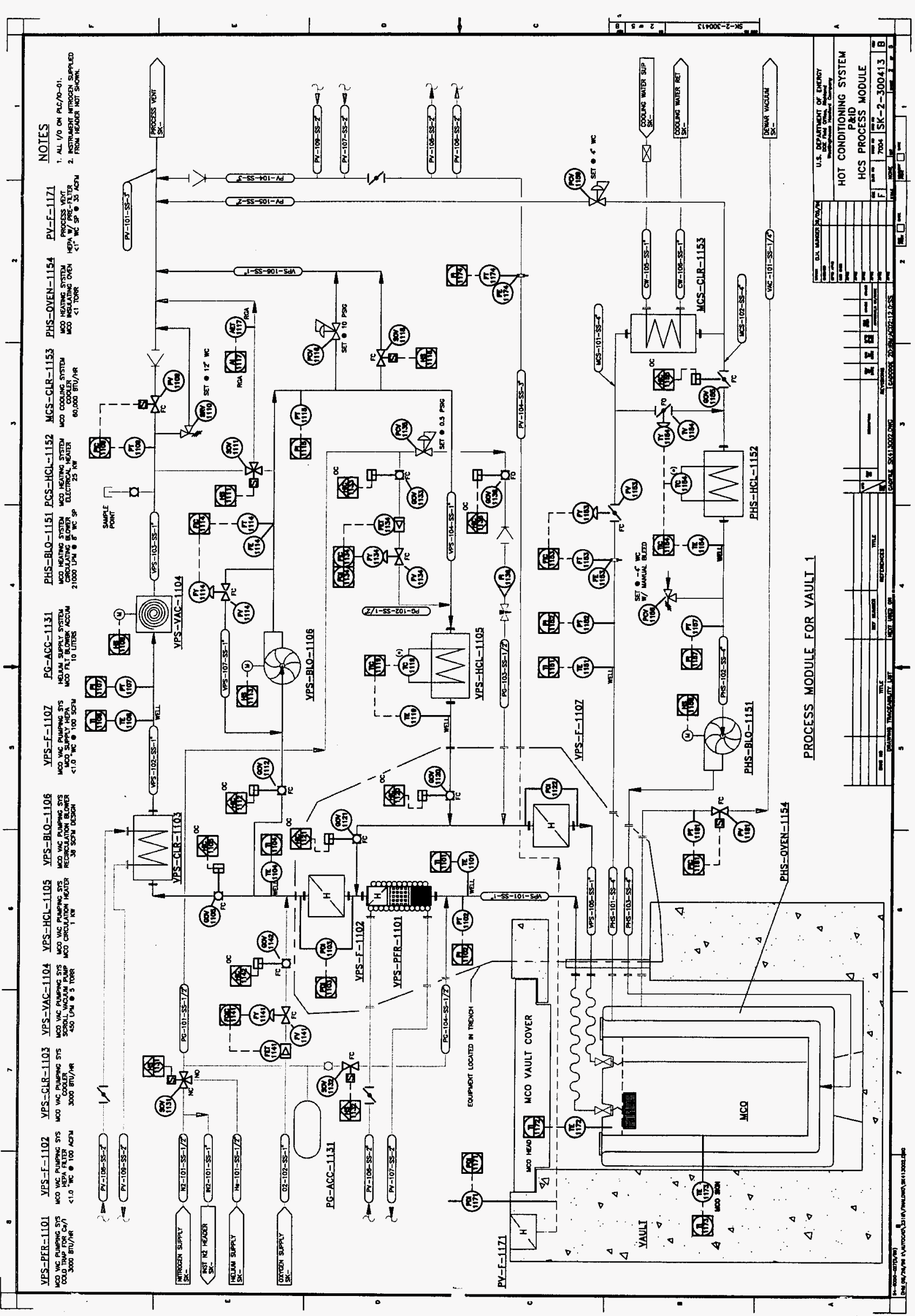









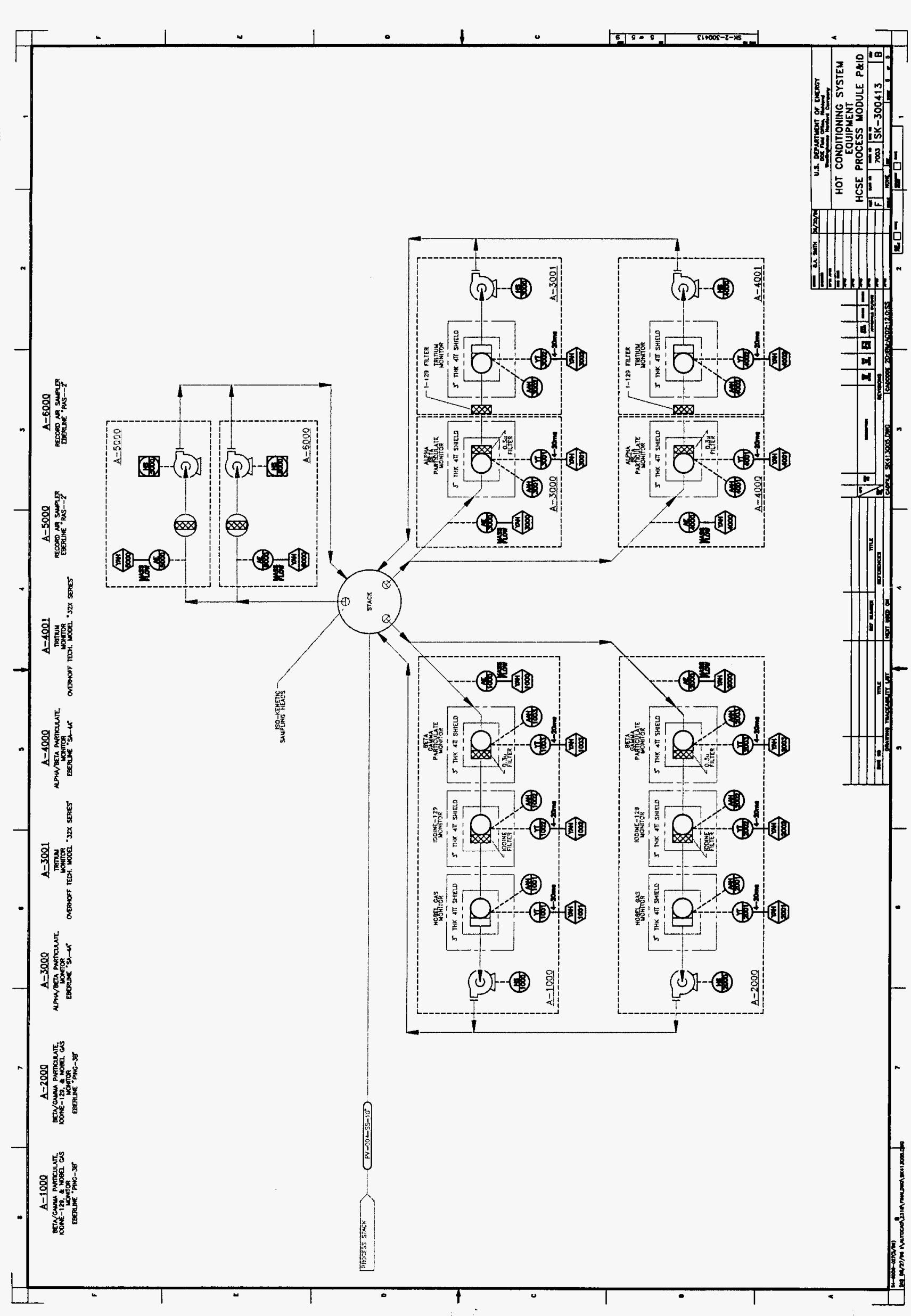




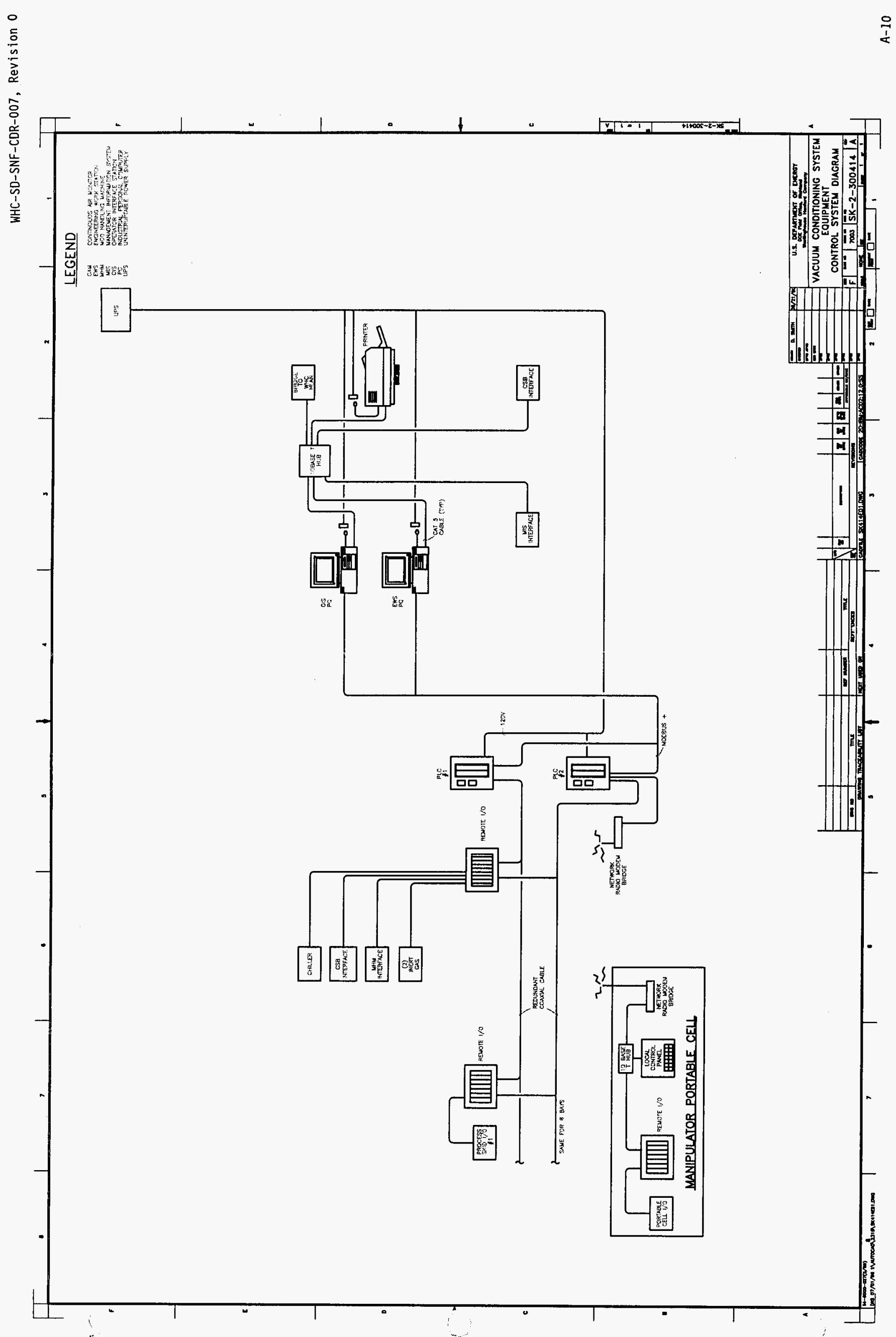



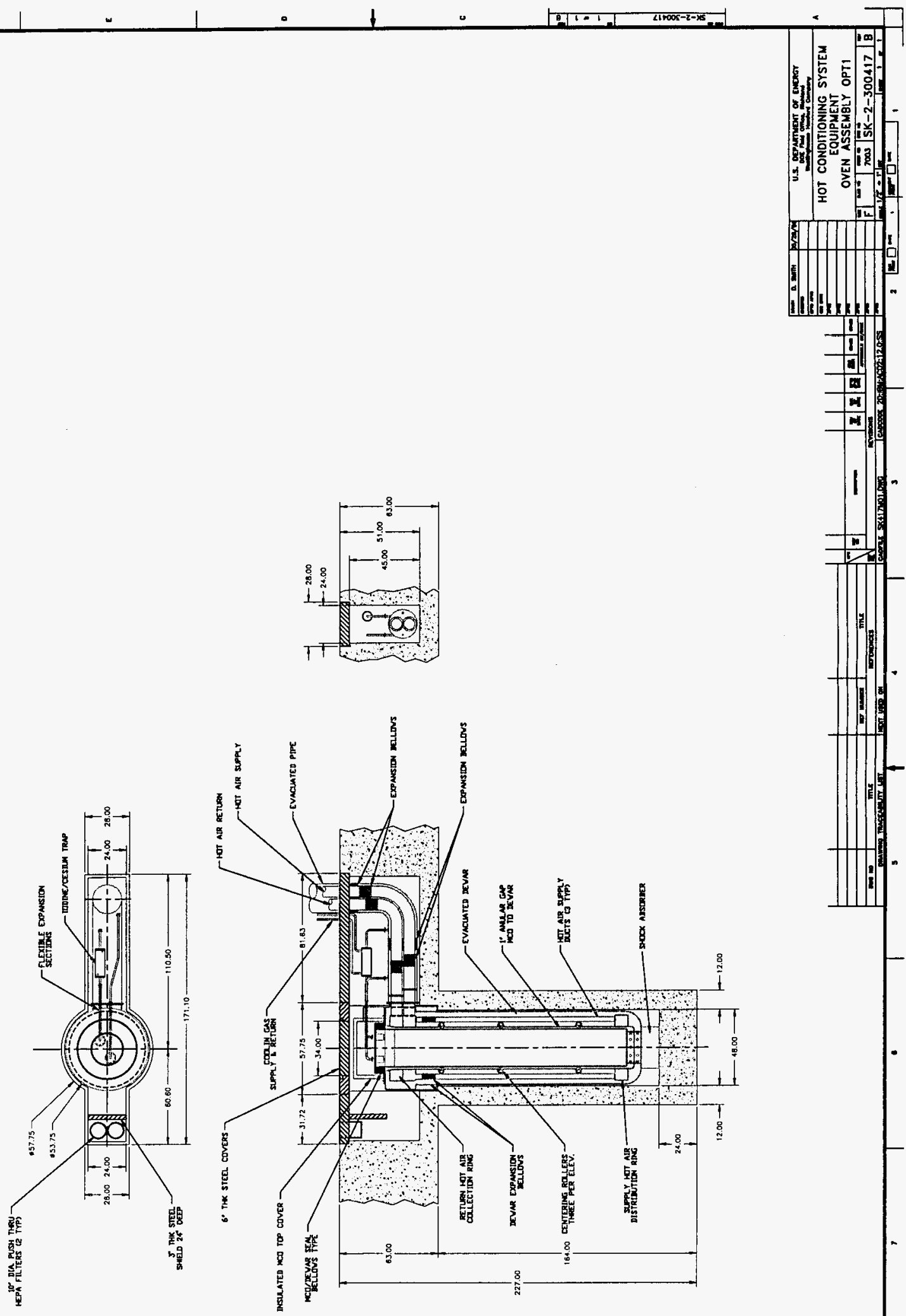


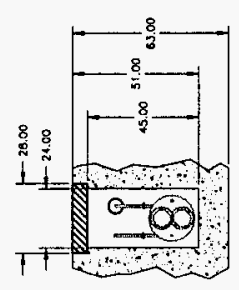

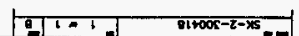
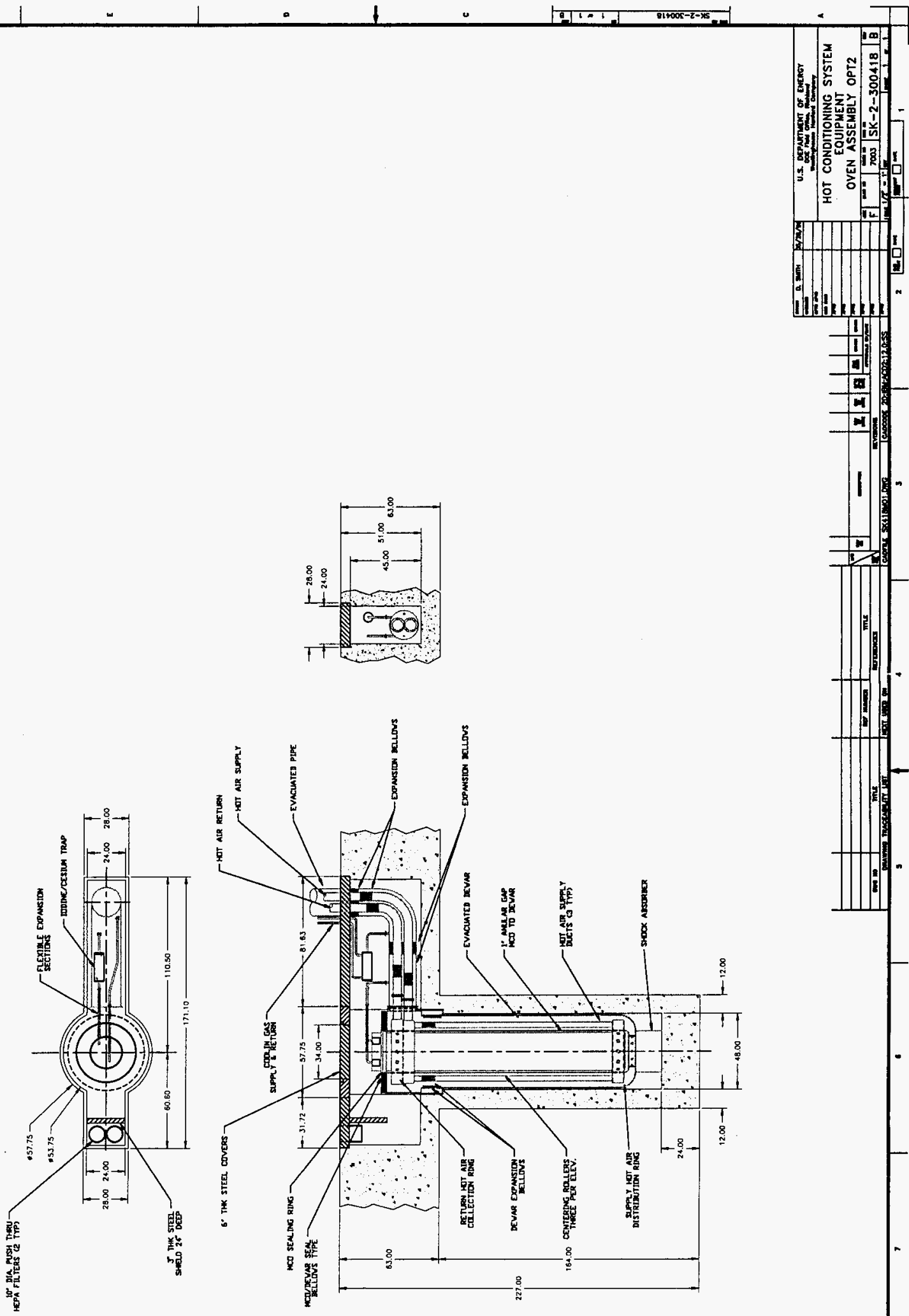


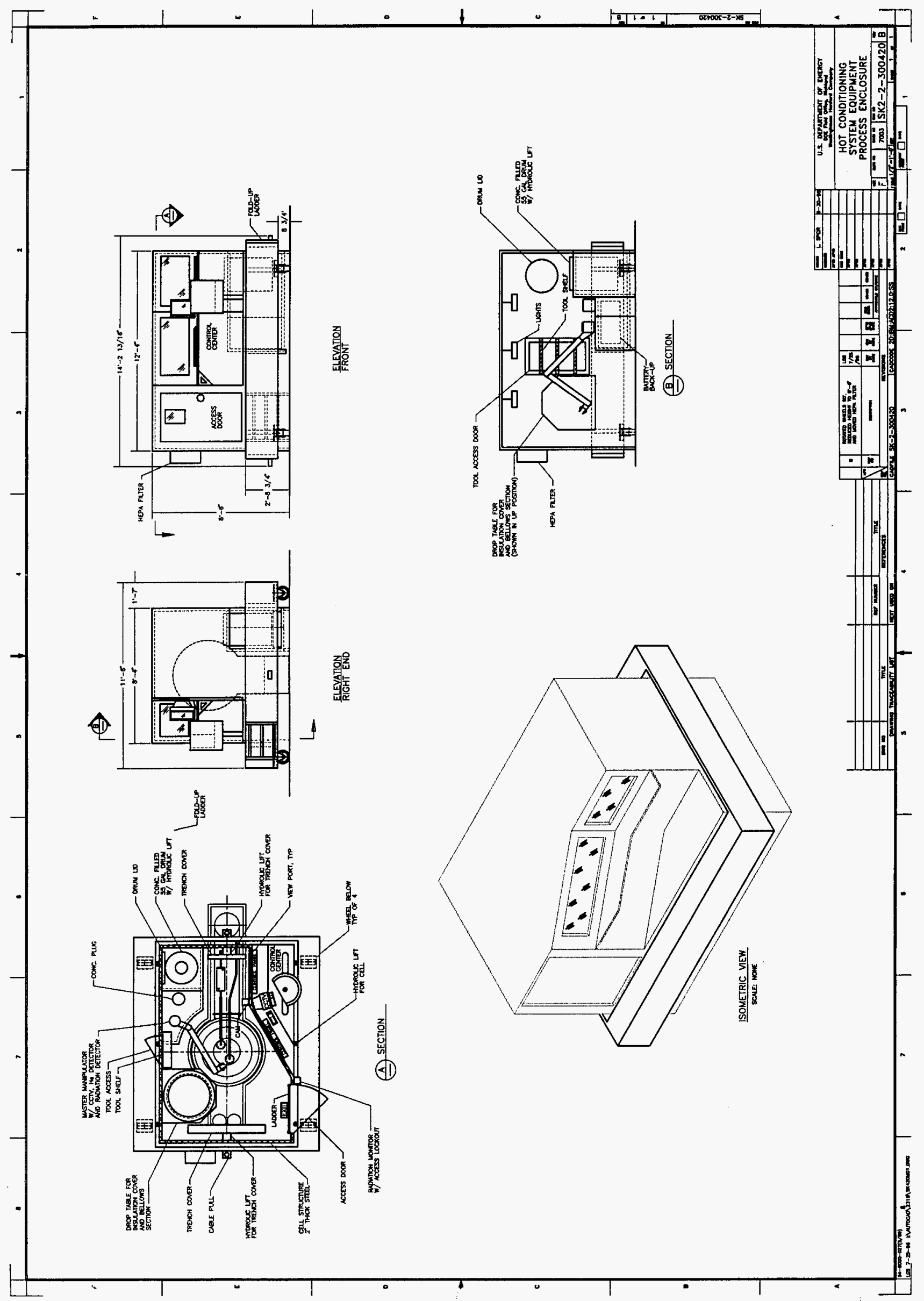



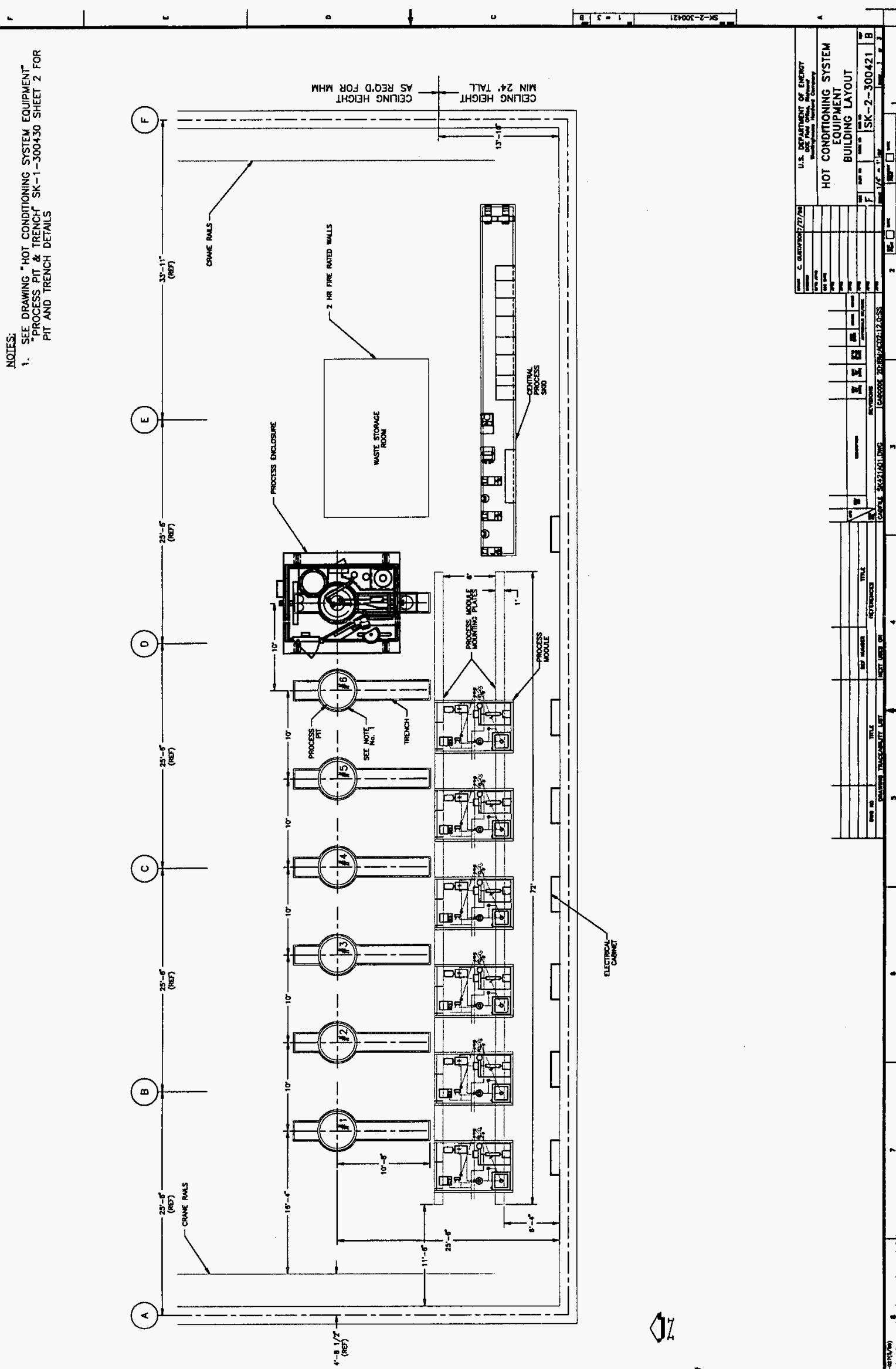

DI 


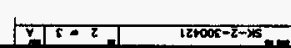

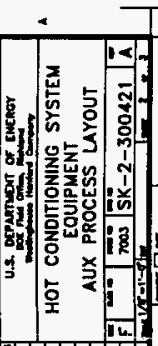

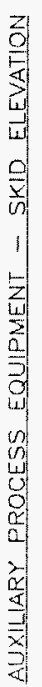

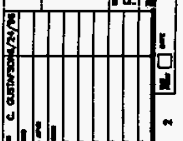

instertse

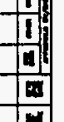

m

4

1

$\int \begin{array}{ll}0 & 0 \\ 0 & 0 \\ 0 \\ 0 \\ 0 \\ 2 \\ 0 \\ 0 \\ 0 \\ 0 \\ 0\end{array}$

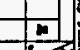

$+$

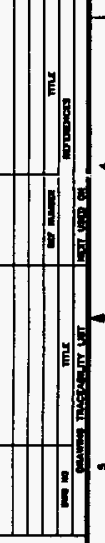

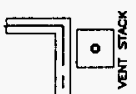

图

点
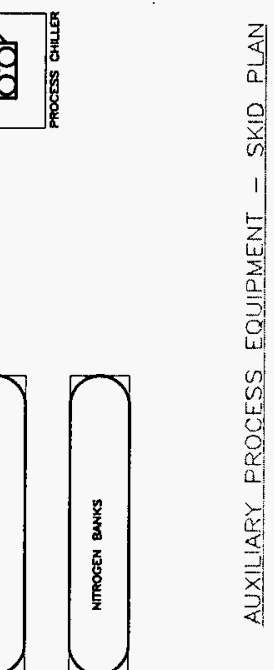

网

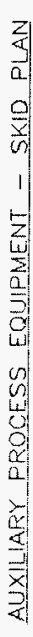




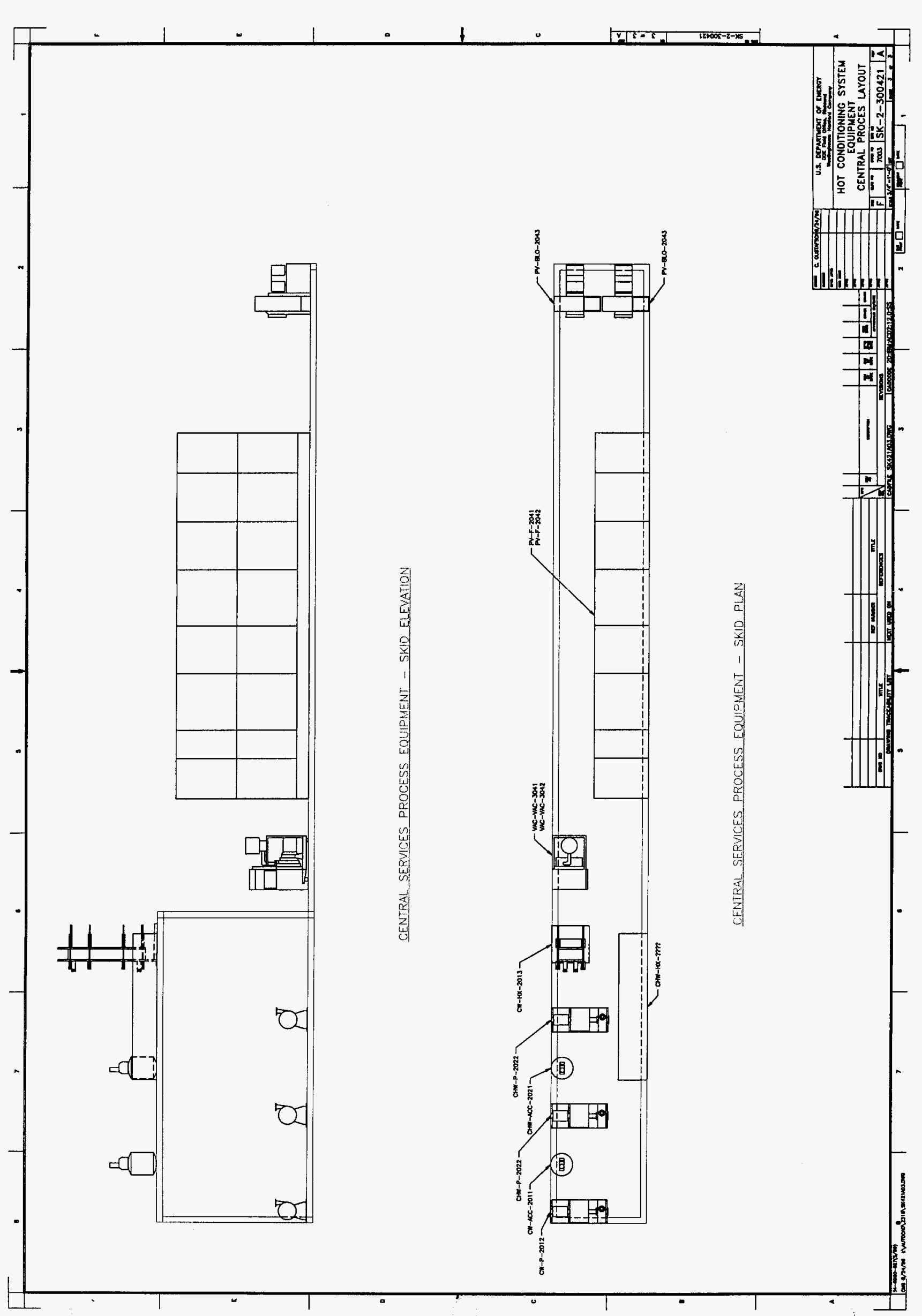




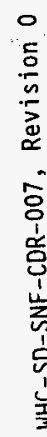

!

P...
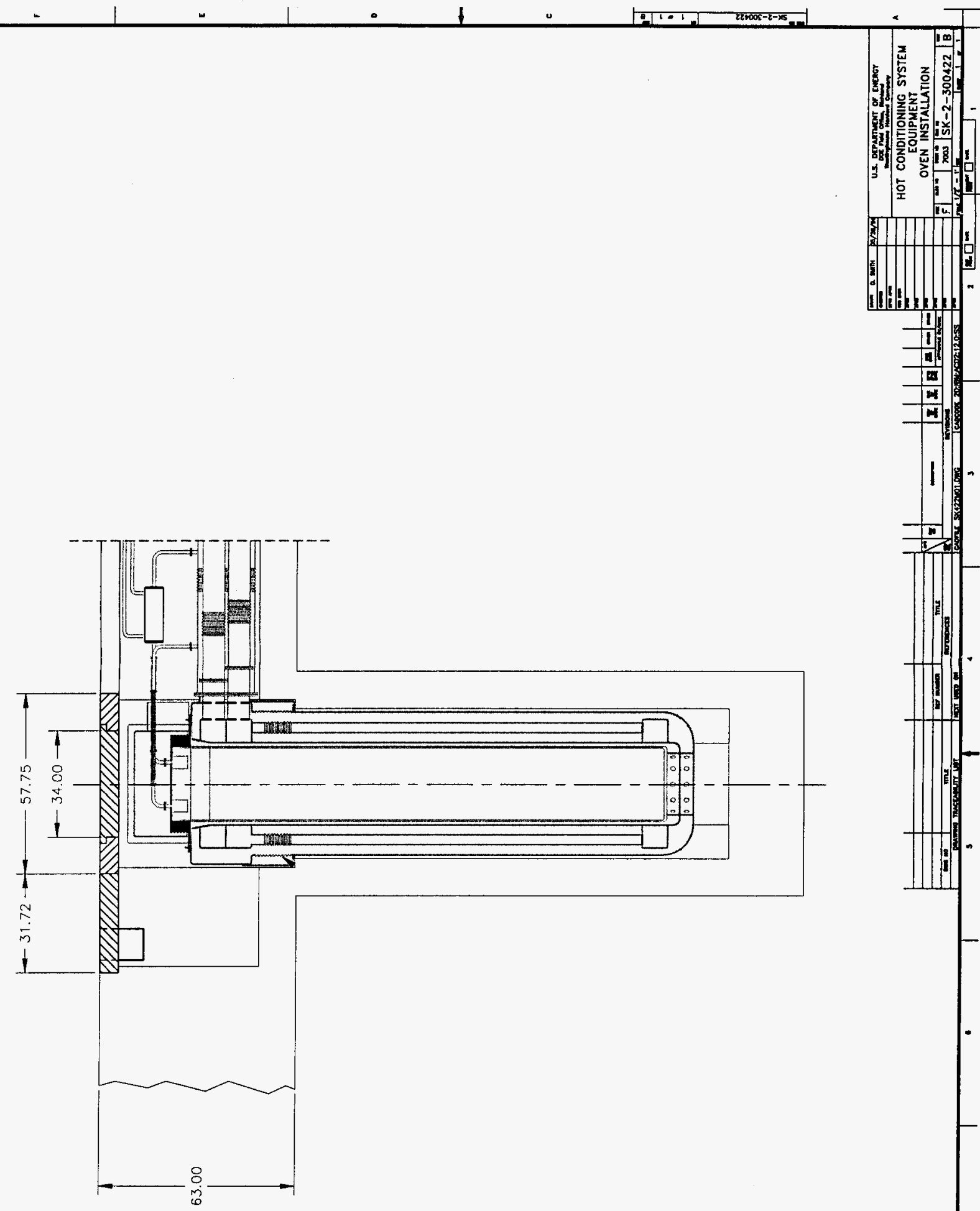


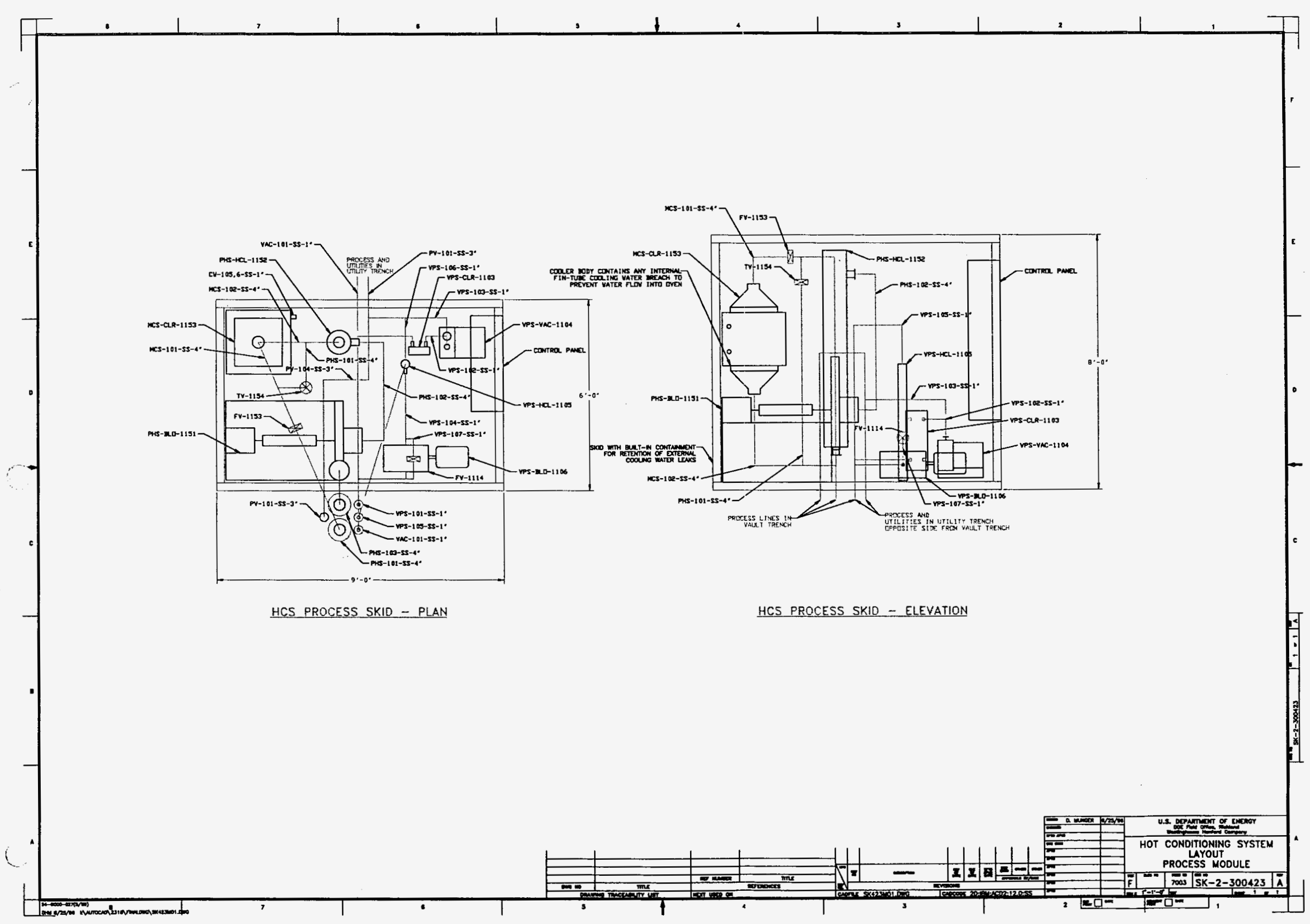




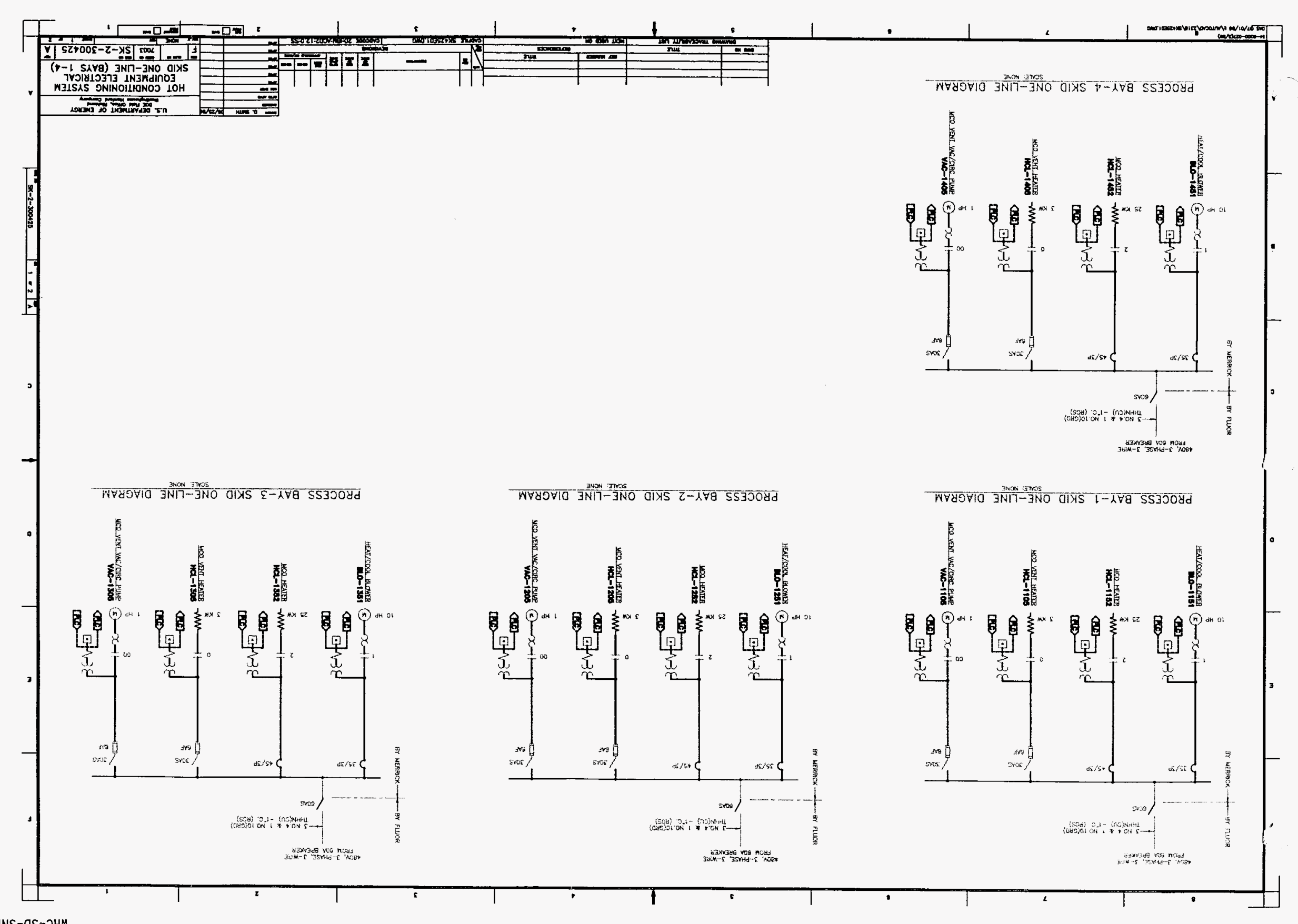




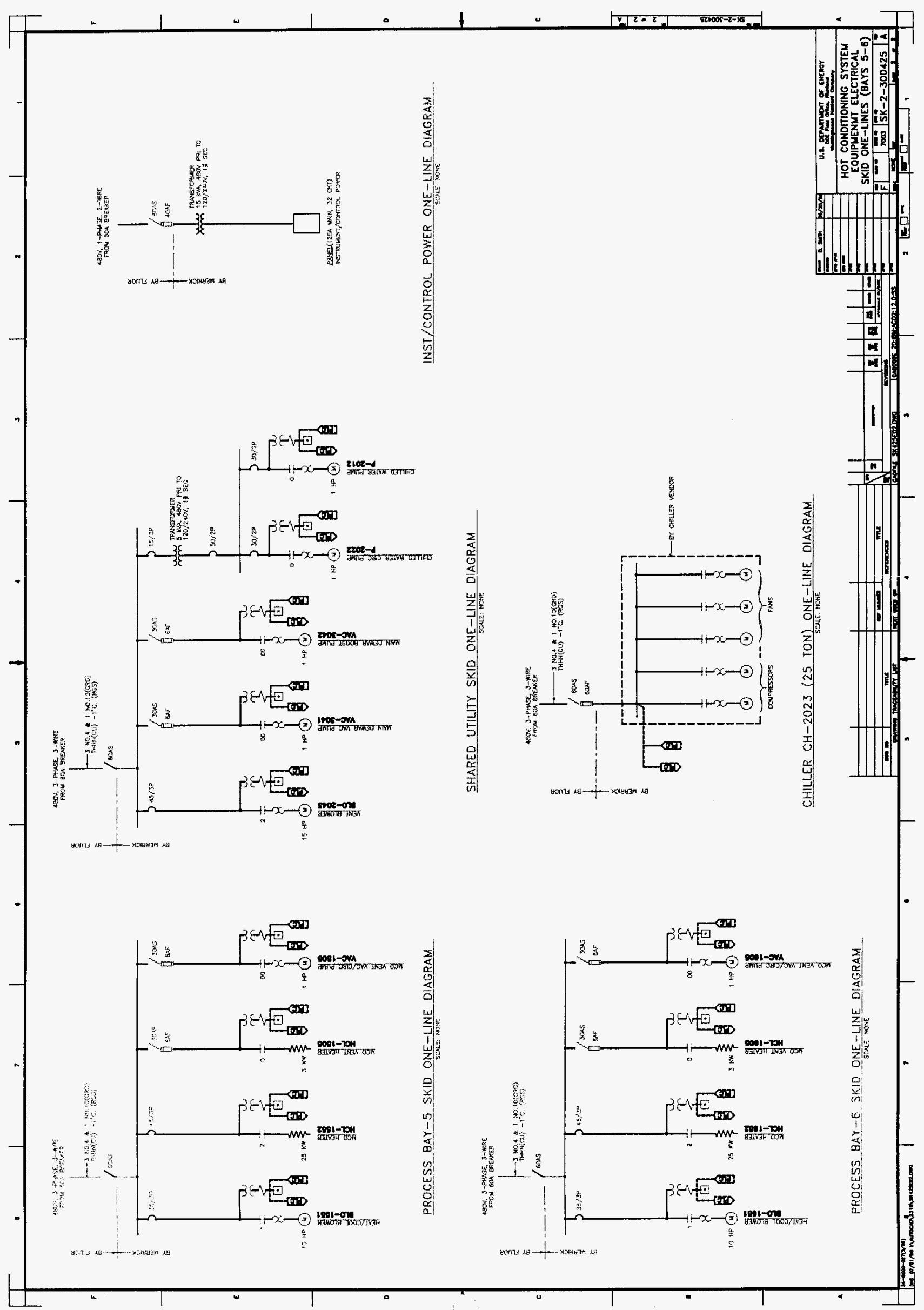




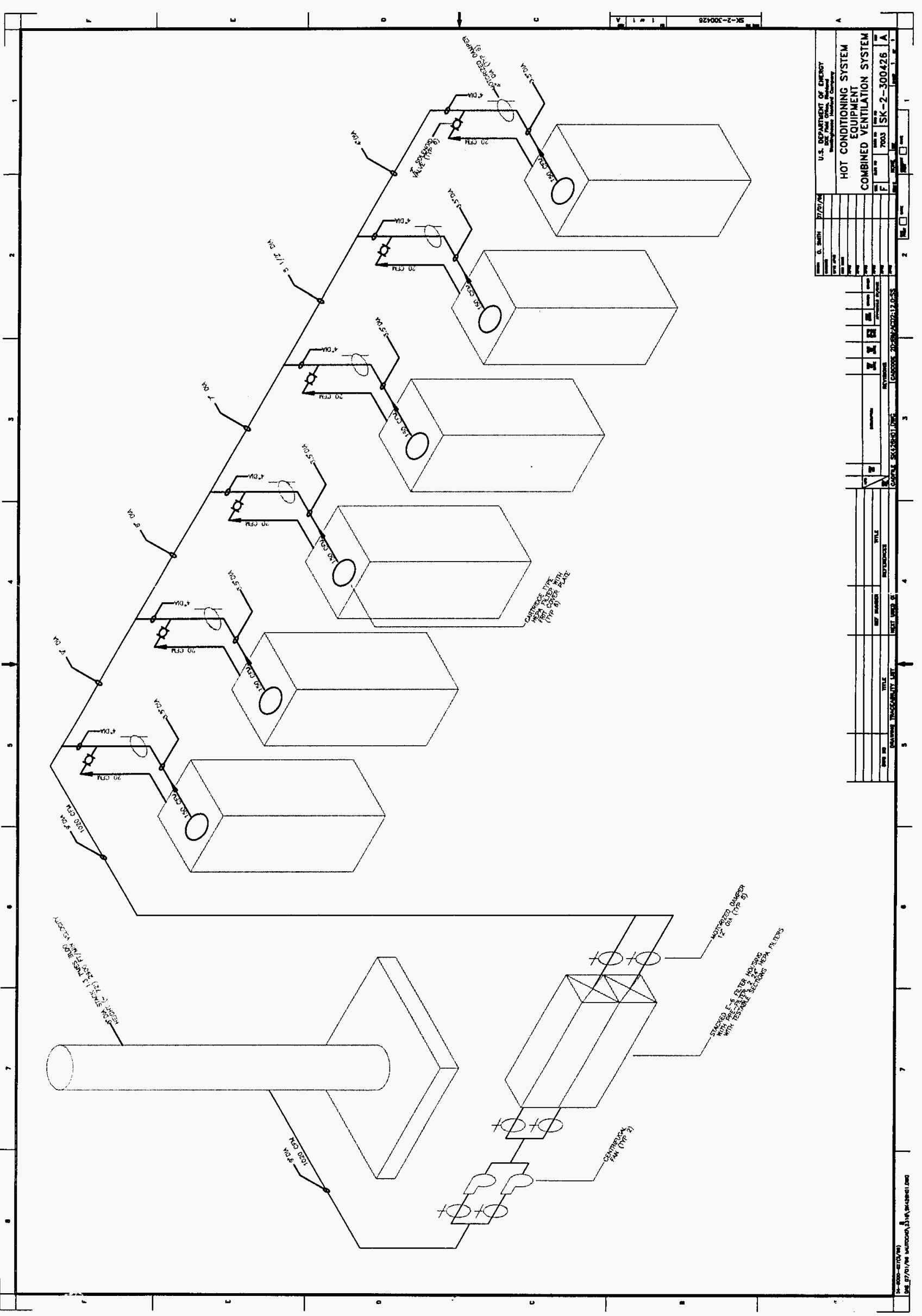




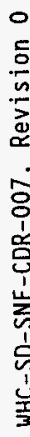
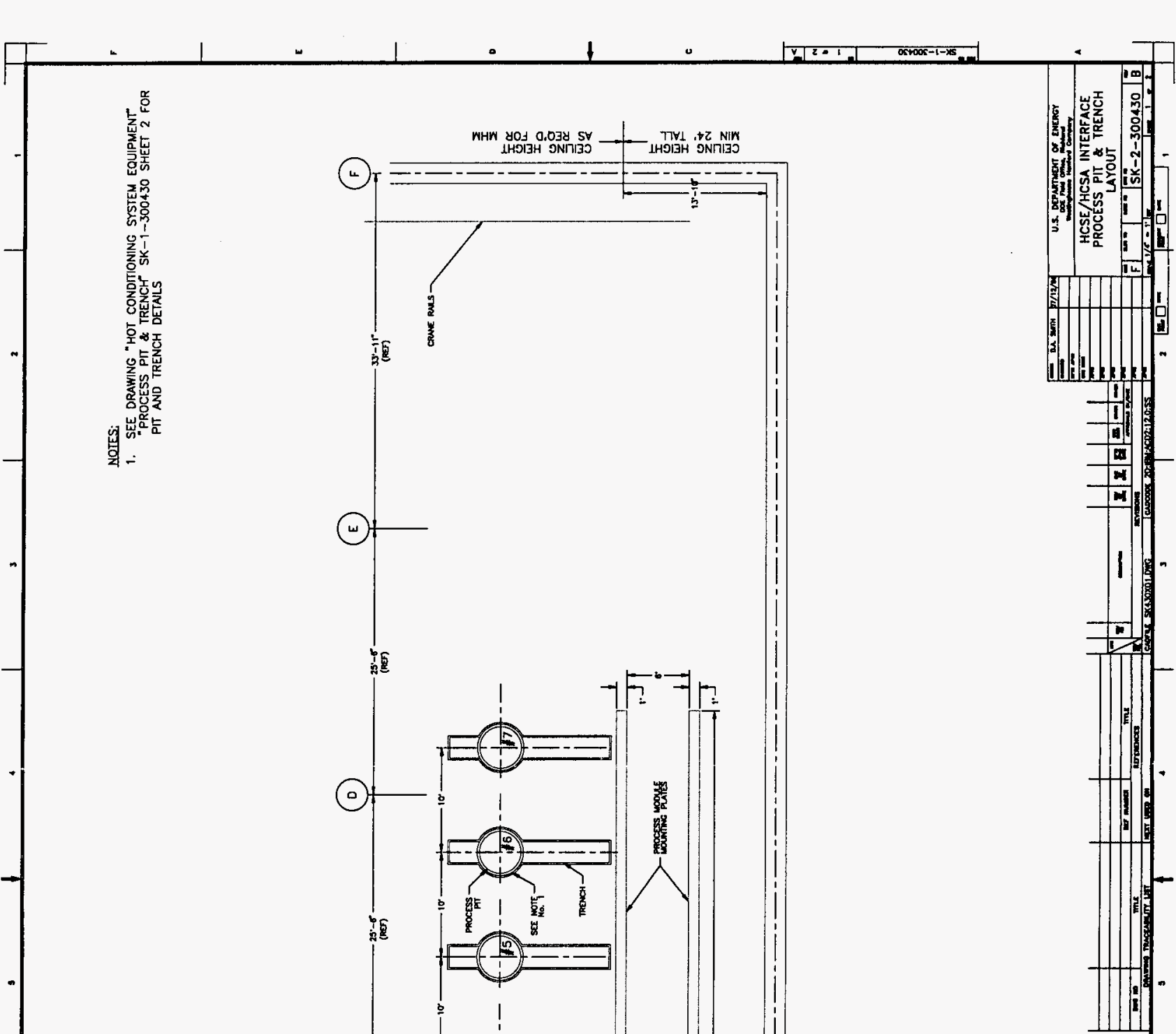

(ㅇ) 


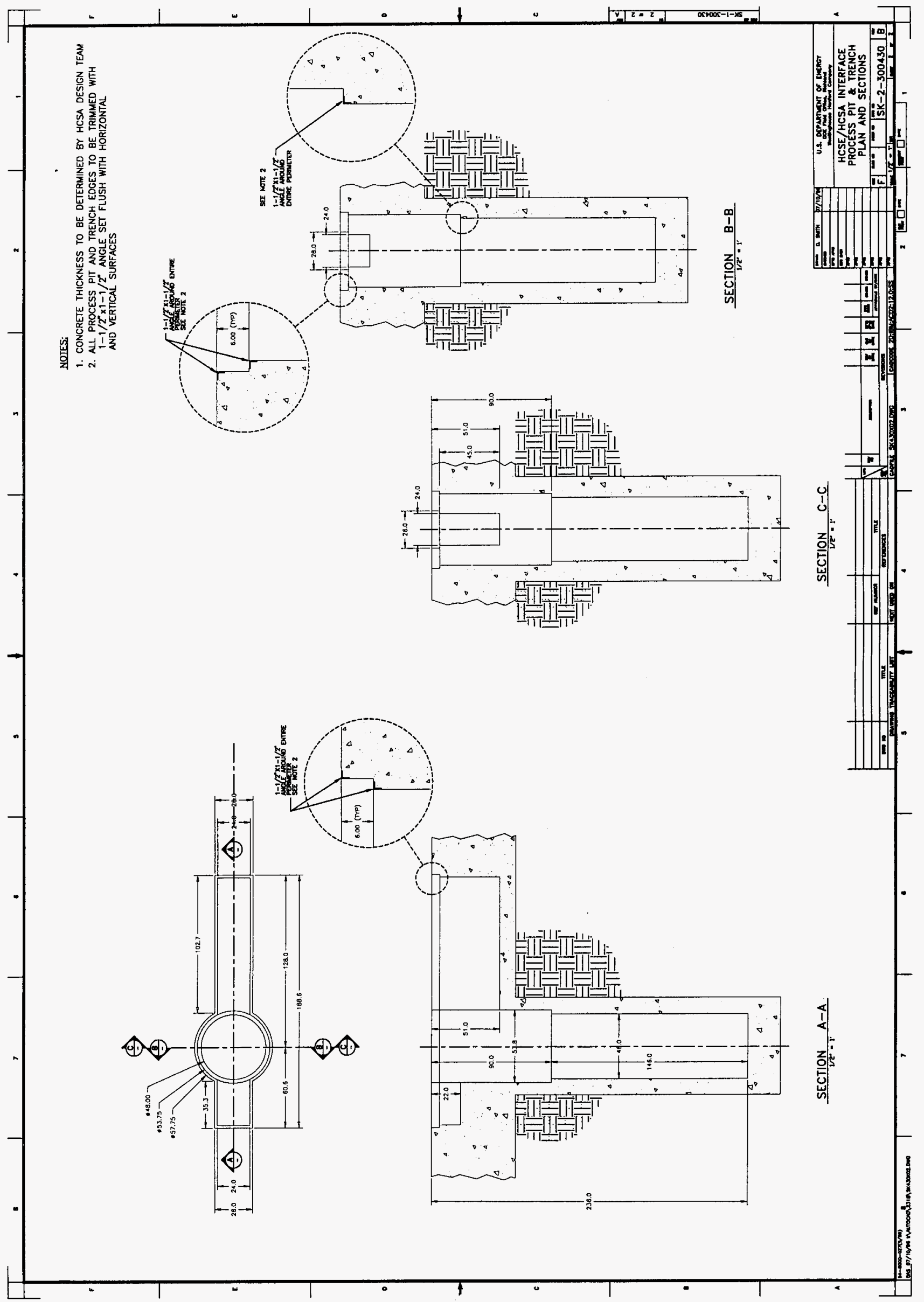


WHC-SD-SNF-CDR-007, Revision 0

\section{APPENDICES}

\section{APPENDIX B}

\section{ALARA IMPLEMENTATION PLAN}




\author{
ALARA IMPLEMENTATION PLAN \\ for the \\ HOT CONDITIONING SYSTEM EQUIPMENT \\ of the \\ K-BASIN SPENT NUCLEAR FUEL STORAGE PROJECT
}

\title{
1.0 INTRODUCTION
}

Approximately 2100 tons of SNF are stored in the K-Basins. The DOE has agreed to an accelerated schedule to move this material from the basins into interim dry storage. The project requires the construction of a process facility, called the Cold Vacuum Drying Module (CVDM), to dewater and dry the fuel outside of the basin buildings, before shipment to a distant storage facility. The SNF elements will be placed within a container called the Multiple Canister Overpack (MCO) in the basin. The MCO, which will be full of basin water as well as fuel, will be placed in a shielded shipping cask on a transport trailer at the basin. This assembly will be transported to the CVDM where it will be drained and dried by a vacuum process. When processing is complete in the CVDM, the MCO and cask are transported to the Canister Storage Building (CSB) where the $\mathrm{MCO}$ is placed in interim storage to await further drying by the Hot Conditioning System Equipment (HCSE). The HCSE is located in an annex to the CSB and is served by the CSB MCO Handling Machine (MHM).

There are two important radiation sources associated with the hot vacuum drying process. The principle source will be the MCO filled with spent nuclear fuel. The shielding integral to the MHM and oven will reduce the dose to relatively low levels in the area that the workers will occupy during the hot vacuum drying process. The second source will be the process equipment. Fission products from leaking fuel and activation products will accumulate in the process equipment, creating a source. Controls will be required to protect staff from inhaling or spreading releasable contamination that could be released when MCO connections are made or broken during the hot vacuum drying process.

The federal law governing design objectives for radiation exposure for this type of work is 10 CFR 835, Occupational Radiation Protection. This is the code that establishes radiation protection standards for the conduct of Department of Energy activities. The annual limit for total effective dose equivalent (TEDE) is 5 rems. Administrative limits are imposed by DOE Notice 441.1, Radiological Protection for DOE Activities. A guide to useful practices for achieving the objective of the ALARA process is DOE publication PNL-6577, "Health Physics Manual of Good Practices for Reducing Radiation Exposure to Levels that are As Low As Reasonably Achievable (ALARA)". The purpose of this plan is to 
WHC-SD-SNF-CDR-007, Revision 0

\section{APPENDIX B - ALARA IMPLEMENTATION PLAN}

provide the framework for a design and operations planning program which assures that the ALARA requirements will be met.

This ALARA Implementation plan provides guidance during the design phase for estimating the expected radiation doses and for selecting appropriate remote or automated operations and shielding features for the Hot Conditioning System Equipment. It also provides input for decision making while writing operational procedures.

\subsection{ALARA PRINCIPLES}

The following documents serve as references for ALARA considerations:

10 CFR 835

ASME NQA-1

DOE Notice 441.1

DOE Order 6430.1A PNL 6577

WHC-IP-1043

WHC-CM-4-29

WHC-CM-4-46

WHC-CM-7-5

WHC-SD-GN-30011

DOE/EH-0256T

HSRCM-1
Occupational Radiation Protection

Quality Assurance Program Requirements for Nuclear

Facilities

Radiological Protection for DOE Activities

General Design Criteria

Health Physics Manual of Good Practices for Reducing

Radiation Exposure to Levels that are As Low As

Reasonably Achievable (ALARA)

WHC Occupational ALARA Program

Nuclear Criticality Safety

Nonreactor Facility Safety Analysis Manual

Environmental Compliance

Radiological Design Guide

DOE Radiological Control Manual

Hanford Site Radiological Control Manual

In combination, the DOE Orders and Guidelines generally require the following radiological design criteria:

A. Individual worker dose shall be ALARA and should be less than 500 mrem per year.

B. Control of contamination should be achieved by containment of radioactive material.

C. Efficiency of maintenance, decontamination, operations, and decommissioning shall be maximized.

The basic principles that are to be adhered to in the design of the Hot Vacuum Conditioning Equipment are to: 1) determine the major contributors to the dose 
WHC-SD-SNF-CDR-007, Revision 0

\section{APPENDIX B - ALARA IMPLEMENTATION PLAN}

and examine methods for making the process more efficient, 2) provide shielding to reduce the dose, 3 ) examine the cost-effectiveness of using robotics, and 4) examine the cost-effectiveness of using remote controls where possible. These determinations should include assessment of normal operations exposure and maintenance/repair exposure.

\subsection{HOT VACUUM DRYING EQUIPMENT MODULE DESCRIPTION}

\section{A. Process Description}

The purpose of the Hot Treatment Process is to dry the SNF by driving off water vapor trapped in pores that may remain after Cold Vacuum Drying has been completed and releasing chemically bound water (water of hydration). In addition to completing the drying, the process will break down uranium hydride and release the hydrogen. After drying has been completed the exposed uranium surfaces will be oxidized by a controlled reaction. These processes are designed to remove hydrogen, eliminate further hydrogen production during storage, and reduce the reactivity of the uranium surfaces prior to storage.

The process involves heating the SNF to $300^{\circ} \mathrm{C}$ with a Helium atmosphere, then evacuating the vessel to a pressure of 5 torr. The SNF is then cooled somewhat and a Helium / Oxygen mixture is added to oxidize the exposed uranium. When the process is completed a cover is placed over the MCO ports. The cover is welded in place.

The hot treatment process will be executed in an extension of the Canister Storage Building. This building covers an array of subterranean holes into which the Multiple Canister Overpacks (MCOs) are placed for storage. Removal of the MCO from the shipping cask that it arrives in and input / output transactions with the storage holes is effected with a special remotely operated machine (within a shield cask) called the MCO Handling Machine (MHM). The Hot Treatment Process ovens will be located in subterranean holes similar to the storage holes. The MHM will get an MCO from storage, insert it into an oven for treatment, and return it to storage after hot treatment is completed.

The key elements of a Hot Treatment Process station will be:

1. An oven will be located below the facility floor level in a hole. The oven will hold an MCO. Hot air circulated between the oven vessel 
WHC-SD-SNF-CDR-007, Revision 0

wall and the MCO outer surface will heat the MCO to $300^{\circ} \mathrm{C}$. The bottom of the oven will be approximately $16 \mathrm{ft}$ below grade. The top of the MCO will be about 30" below grade. The hole will be covered by a thick steel plate which will be selected to control radiation exposure rate above the oven.

2. A process skid containing the hot air source, the vacuum pump and miscellaneous items will be located on the floor a few feet distant from the oven.

3. Hot air and process lines running between the furnace and the process equipment skid will be in a trench below floor level. The trench will be covered with steel to provide shieiding from radioactivity which may be caught in in-line traps and filters built into the MCO exhaust line. These devices will be located in the trench. Their purpose is to prevent contamination of the process equipment.

4. The space above the oven within the hole will be ventilated through the trench. Thus the covers for the hole and trench will form the secondary confinement barrier for the process.

5. A portable process enclosure will be provided. This enclosure will be stationed over the oven after the MHM has departed in order to prepare for the Hot Treatment Process and will also be stationed over the oven to remove process connections after the process is completed. The process enciosure will also be used to change and package hot traps that are in the trench. The process enclosure will contain tele-operated manipulators so that the operator using CCTV guidance can reach down into the oven hole to place fixtures, make MCO valve connections, and place insulation cover pieces. The enclosure will contain the hoist required to lift the oven hole lid and place it out of the way. The enclosure will also include equipment such as a helium leak detector, a welder, drumout port, etc. needed to complete the process. The thickness of the cell walls and the selection of window materials and thickness will be determined by radiation exposure analysis. An important function of the enclosure is to provide secondary confinement for the process when the oven hole top is off. The air drawn through the trench will create a negative pressure and airflow in the enclosure when it is parked over the oven. 
WHC-SD-SNF-CDR-007, Revision 0

\section{APPENDIX B - ALARA IMPLEMENTATION PLAN}

It is expected that six (6) of the HTP stations will be required to meet the throughput requirements.

The overall sequence of operations is given in block flow diagram form on drawing SK-2-300411. A summary of the sequence is :

1. The MHM moves in, opens the hole, places the MCO into the oven, closes the hole, and leaves.

2. The process enclosure is moved in and parked over the hole.

3. The hole cover is pulled and the manipulator is used to install the top of oven parts and the MCO process connections.

4. The hole cover is replaced and the process enclosure is moved away.

5. The treatment process is run and controlled from the CSB control room via computer.

6. The process enclosure is returned after the process is complete.

7. The hole is opened, the process connections and oven parts are removed, the cover is placed and welded, the traps are replaced if necessary and packaged for waste shipment, and the hole cover is replaced.

8. The process enclosure is removed.

9. The MHM moves in, pulls the MCO, and places it in storage.

B. Hot Vacuum Drying Equipment Radiation Hazards

There are two important radiation sources associated with the hot vacuum drying process. The principle source will be the MCO filled with spent nuclear fuel. The shielding integral to the MHM and oven will reduce the dose to relatively low levels in the area that the workers will occupy during the hot vacuum drying process. The second source will be the process equipment. Fission products from leaking fuel and activation products will accumulate in the process equipment, creating a source. This is anticipated to be significantly below the radiation levels at the top of the MCO during the connect/disconnect operations. 
WHC-SD-SNF-CDR-007, Revision 0

\section{APPENDIX B - ALARA IMPLEMENTATION PLAN}

\subsection{RADIATION SAFETY DESIGN}

DOE Order 5480.11 paragraph 9.j addresses Design and Control. The means for maintaining exposures ALARA are to be through physical controls confinement, ventilation, remote handling and shielding. Administrative and procedural controls provide supplemental means of controlling exposure and maintaining exposures ALARA.

In the design phase, the following objectives shall be applied:

A. Optimization. Optimization principles, as discussed in ICRP Publication 37 , shall be utilized in developing and justifying design and physical controls.

B. External Exposure should not exceed $2.5 \mathrm{mrem} / \mathrm{hr}$ on the average.

C. Internal Exposure should be limited to zero under normal operating conditions.

D. Maintenance. Ease of maintenance, decontamination and decommissioning shall be considered in the facility design and selection of materials.

Radiation Exposure shall be monitored, recorded and controlled in accordance with the Hanford Site Radiological Control Manual, HSRCM-1.

1. Normal Operations Design

Each process step will be analyzed as part of the analysis. Each step will be characterized by:

- The position of the operator(s)

- The dose rate contribution from the MCO

- The dose rate contribution from the process equipment

- The amount of operator time anticipated

- The total operator dose attributable to performing the step

- A description of potential ingestion paths

- A description of mitigating features incorporated in the design or recommended

The total dose for the process will be estimated by summing the expected dose calculated for each step of the process. 
WHC-SD-SNF-CDR-007, Revision 0

The dose rates at various distances from the $M C O$ as well as dose rates for spots of lesser shielding (MCO nozzle locations) have been previously calculated. If the dose rate for any specific location has not been determined by the provided calculations, these will need to be calculated using a conservative model for the source.

The dose to any given worker by the HCSE will be determined by the staff size, the extent of cross training allowing for rotation of tasks, and the rotation among shifts. An operating staff plan will be developed during detailed design. Radiation exposure considerations may be used in developing the plan in order to determine how tasks will be grouped for training and assignment. Local and temporary shielding features will be planned and implemented so that the staff is not increased (beyond the amount that would be planned if there were no radiation exposure limitation) for the purposes of spreading the dose.

Airborne release may occur when MCO connections are made or broken. The process enclosure and ventilation design features will be used to capture any releases that may occur.

2. Maintenance Design

The process flow diagrams and equipment design will be reviewed on a component by component basis during the definitive design to assess likely modes of failure and the implied maintenance/repair activities. Routine maintenance activities will be addressed as well. Each of the anticipated activities will be described, exposure rates will be estimated, and activity durations will be estimated. The combination of these estimates will yield a maintenance exposure estimate for the most likely occurrences. Design features such as temporary shielding, decontamination, or mechanical assistance/ special tools incorporated in the design to reduce the activity time will be described. Control of airborne and releasable contamination will be planned for the maintenance activities. Local or temporary ventilation may be incorporated in the design.

3. Radiation Monitoring Instrumentation

Radiation monitoring and alarm instrumentation will be located at key locations around the process. The design will include source specific radiation detectors to monitor build-up of material in the 
WHC-SD-SNF-CDR-007, Revision 0

\section{APPENDIX B - ALARA IMPLEMENTATION PLAN}

system (such as on the filters). These will be used to determine when system cleaning or filter changes are required. The design will include ionization detectors in the area where staff is working to monitor the radiation field and to warn of unacceptable dose rate. The design will include Continuous Air Monitors (CAMs) capable of detecting airborne particles that emit alpha or beta radiation. These will be located at strategic points near potential release points.

\subsection{LESSONS LEARNED PROGRAM}

One of the greatest potentials for reducing dose is the application of lessons learned from operational experience. The lessons leamed from Duke Power Company's Oconee Nuclear Station during the process of loading and storing casks of spent nuclear fuel into the Independent Spent Fuel Storage Installation (ISFSI) on-site at the station will be considered. Other commercial nuclear facilities such as Arkansas Nuclear One, Palisades, and Surry that may have lessons learned will be investigated in the design phase of this project. The experience at these plants is likely to show system modification and optimization actions that have reduced radiation exposure. The crews performing the operation for the first casks will be debriefed immediately after the operation to determine what may be done to improve the efficiency of the process. The ideas from these crews will provide the information necessary to modify equipment and procedures to improve efficiency and thus reduce dose. A video tape of the process will be made. It will be reviewed for additional improvements by others not directly involved in the actual process. Video taping later cold vacuum drying processes after modifications have been implemented will also aid in training additional workers. 
WHC-SD-SNF-CDR-007, Revision 0

APPENDICES 
WHC-SD-SNF-CDR-007, Revision 0

\title{
APPENDIX C - SHIELDING AND RADIATION EXPOSURE ANALYSIS
}

\author{
SHIELDING AND RADIATION EXPOSURE ANALYSIS \\ for the \\ HOT CONDITIONING SYSTEM EQUIPMENT \\ of the
}

K-BASIN SPENT NUCLEAR FUEL STORAGE PROJECT

\subsection{INTRODUCTION}

The purpose of this analysis is to evaluate the radiation exposure received by workers during each step of the hot vacuum drying process, and to determine the total cumulative exposure (person-rem) for the process. The results of this analysis will be used during the detailed design phase of the Hot Conditioning System Equipment (HCSE) to reduce exposure where appropriate, in keeping with the ALARA (As Low As Reasonably Achievable) principle.

The radiological design of the HCSE will incorporate the criteria specified in the applicable orders, regulations, and publications listed in the Performance Specification for the Spent Nuclear Fuel Hot Vacuum Conditioning Equipment, WHC-S-0460, Rev. A. Also, the facility will be designed in accordance with the following documents: HSRCM-1, Hanford Site Radiological Control Manual; WHC-SC-GN30011, Radiological Design Guide; 10CFR835, Occupational Radiation Protection; DOE Order 6430.1A, General Design Criteria; and the ALARA Implementation Plan for the K-Basin Spent Fuel Storage Project.

This analysis will be updated during the detailed design of the HCSE to ensure that the radiological design criteria for the facility are met.

\subsection{SUMMARY}

The total exposure received from processing one MCO in the HCSE is approximately 80 person-mrem ( 0.080 person-rem). This number is based on the average shielding source term and assumes one inch of steel shielding integral to the hot cell enclosure.

\subsection{DISCUSSION}

\subsection{Methodology}

All activities to be performed are listed in Table 1. These activities are from the Hot Conditioning System Equipment Block Flow Diagram, SK-2- 
WHC-SD-SNF-CDR-007, Revision 0

\section{APPENDIX C - SHIELDING AND RADIATION EXPOSURE ANALYSIS}

300-411, Reference 1. Each activity was evaluated to determine if the location at which the activity is to be performed would result in any radiation exposure to the personnel performing that specific activity. If it was determined that there would be exposure from that activity, the location and dose rate associated with that location was listed in Table 1 for that activity. The estimated dose rate for each location was calculated from source term information from Reference 2 . The workforce and time required to perform each exposure activity was estimated and the frequency of each activity was determined from the Block Flow Diagram. These values are listed in Table 2. The exposure (person-mrem) for each activity was then calculated and those were summed to determine the total exposure from processing one cask.

\subsection{Assumptions}

It is conservatively assumed that $100 \%$ of the duration for each activity is spent in the radiation field.

One inch of steel shielding is assumed to be incorporated in the process enclosure design.

Tasks performed over the top of the Multi-Canister Overpack (MCO) are performed with manipulators inside the process enclosure. This is required because the streaming dose rate directly over the annular space between the cask and MCO is estimated to be on the order of 1500 $\mathrm{mrem} / \mathrm{hr}$.

\subsection{Results}

The exposures for each process lask are given in Tables 1 and 2.

The total exposure received from processing one MCO in the HCSE is approximately 80 person-mrem ( 0.080 person-rem). This number is based on the average shielding source term and assumes one inch of steel shielding integral to the not cell enclosure.

The dose budget for the HCSE is 49.0 person-rem (Reference 3). The total dose budget for welding in the K-Basins Spent Nuclear Fuel Project is 30.0 person-rem (Reference 3). Assuming the welding budget can be split between the Cold and Hot Conditioning Projects, the total available dose budget for HCSE is 64.0 person-rem. Assuming a total of 400 MCOs, the total exposure for HCSE is approximately 32.0 person-rem. While this is well within the dose budget, it does not include exposure from airborne activity in the CSB. This exposure issue must be evaluated 
WHC-SD-SNF-CDR-007, Revision 0

\section{APPENDIX C - SHIELDING AND RADIATION EXPOSURE ANALYSIS}

and included in the exposure analysis during the definitive design phase of the HCSE.

While the calculated total exposure is within the dose budget, it is likely now as low as reasonably achievable (ALARA) when considering that the oven shielding design is not optimized due to the unresolved interface details with the MCO design. Also, the current analysis indicates that the $0.25 \mathrm{mrem} / \mathrm{hr}$ general area dose rate criteria for the floor area is not met. Additional shielding will be incorporated into the oven design and the process enclosure design during the definitive design to ensure that dose rate criteria are met and that exposures are ALARA.

\subsection{REFERENCES}

1) Drawing SK-2-300411.

2) 3/14/96 Memo from R.A. Schwarz to J.S. Lan regarding Neutron and Photon Source for Safeguards.

3) 4/30/96 Memo from J.A. Swenson, Manager, Project Integration regarding Individual Design Project Target Cumulative Dose Values for the Spent Nuclear Fuel Project. 
WESTINGHOUSE HANFORD COMPANY

Hot Conditioning Syatem Equipment

Contract AMC6-SWV310416, Teak 17
WHC-SD-SNF-CDR-007, REV. 0

Table 1
MERRICK COMPANY

Advenced Techrobody Sector

Proinet No. 30013218

Exposure Locations and Dose Rates

\begin{tabular}{|c|c|c|c|}
\hline Action & $\begin{array}{l}\text { Exposur } \\
\text { Activity? }\end{array}$ & $\begin{array}{l}\text { Exposure } \\
\text { Location }\end{array}$ & $\begin{array}{l}\text { Dose Rate } \\
\text { (mrem/hr) }\end{array}$ \\
\hline Select Oven & No & N/A & N/A \\
\hline Move in the MHM & Yes & Oven Deck Area & 2.0 \\
\hline Lower MCO into Oven & Yes & Óven Deck Area & 2.0 \\
\hline Remove the MHM & Yes & Oven Deck Area & 2.0 \\
\hline Move in Process Enclosure & Yes & Oven Deck Area & 2.0 \\
\hline Connect Process Enclosure & Yes & Oven Deck Area & 2.0 \\
\hline Open Process Pit & Yes & Process Enclosure Control Station & 5.0 \\
\hline Place Annulus Cover & Yes & Process Enclosure Control Station & 5.0 \\
\hline Attach Annulus Cover & Yes & Process Enciosure Control Station & 5.0 \\
\hline Remove Port Cover & Yes & Process Enclosure Control Station & 5.0 \\
\hline Install Vent Cover & Yes & Process Enclosure Control Station & 5.0 \\
\hline Install Vałves & Yes & Process Enclosure Control Station & 5.0 \\
\hline Open Valves & Yes & Process Enclosure Control Station & 5.0 \\
\hline Evacuate $\mathrm{MCO}$ & No & N/A & N/A \\
\hline Fill MCO with Helium & No & N/A & N/A \\
\hline Leak Check Ports & Yes & Process Enclosure Control Station & 5.0 \\
\hline Close Bad Valve & Yes & Process Enclosure Control Station & 5.0 \\
\hline Disconnect Valve & Yes & Process Enclosure Control Station & 5.0 \\
\hline Clean Port & Yes & Process Enclosure Control Station & 5.0 \\
\hline Instail Reserve Valve & Yes & Process Enciosure Control Station & 5.0 \\
\hline Install Insulation Cover & Yes & Process Enclosure Control Station & 5.0 \\
\hline Close Process Pit & Yes & Process Enclosure Control Station & 5.0 \\
\hline Perform Heat-up Cycle & No & N/A & N/A \\
\hline Survey Process Enclosure & Yes & Process Enclosure Control Station & 5.0 \\
\hline Disconnect Process Enclosure & Yes & Oven Deck Area & 2.0 \\
\hline Remove Process Enclosure & Yes & Oven Deck Area & 2.0 \\
\hline PurgeNacuum Cycle & No & N/A & NAA \\
\hline Perform Acceptence Test & No & N/A & N/A \\
\hline Cool for Pasivation & No & IN/A & NA \\
\hline Evacuate MCO & No & N/A & N/A \\
\hline Fill with Passivation Gas & No & N/A & N/A \\
\hline Passivation Period & No & N/A & N/A \\
\hline Cool Down & No & N/A & N/A \\
\hline Evacuate MCO & No & N/A & N/A \\
\hline Fill MCO with Helium & No & N/A & N/A \\
\hline Move in Process Enclosure & Yes & Oven Deck Area & 2.0 \\
\hline Connect Process Enclosure & Yes & Oven Deck Area & 2.0 \\
\hline Open Process Pit & Yes & Process Enclosure Control Station & 5.0 \\
\hline Remove Insuliation Cover & Yes & Process Enclosure Control Station & 5.0 \\
\hline Close Valves & Yes & Process Enclosure Control Station & 5.0 \\
\hline Disconnect Valves & Yes & Process Enclosure Control Station & 5.0 \\
\hline Place Weld-on Cover & Yes & Process Enclosure Control Station & 5.0 \\
\hline Install Welder & Yes & Process Enclosure Control Station & 5.0 \\
\hline Weld Root Pass & No & N/A & N/A \\
\hline Visual Inspection & Yes & Process Enclosure Control Station & 5.0 \\
\hline Clean Weld & Yes & Process Enclosure Control Station & 5.0 \\
\hline
\end{tabular}


WESTINGHOUSE HANFORD COMPANY

Hot Conditioning Sytem Equipment

Contract imMc6-SWW-310416, Tack 17
WHC-SD-SNF-CDR-007, REV. 0

Table 1
MERRICK COMPANY

Advenced Technolody Sector Project No. 30013218

Exposure Locations and Dose Rates

\begin{tabular}{|c|c|c|c|}
\hline Action & $\begin{array}{l}\text { Exposure } \\
\text { Activity? }\end{array}$ & $\begin{array}{l}\text { Exposure } \\
\text { Location }\end{array}$ & $\begin{array}{l}\text { Dose Rate } \\
\text { (mrem/hr) }\end{array}$ \\
\hline Grind & Yes & Process Enclosure Control Station & $\overline{5.0}$ \\
\hline Repair Weld & Yes & Process Enclosure Control Station & 5.0 \\
\hline Apply Dye & Yes & Process Enclosure Control Station & 5.0 \\
\hline Soak & No & $\mathrm{N} / \mathrm{A}$ & N/A \\
\hline Remove Dye & Yes & Process Enclosure Control Station & 5.0 \\
\hline Apply Developer & Yes & Process Enclosure Control Station & 5.0 \\
\hline Bleed & No & N/A & N/A \\
\hline Inspect & Yes & Process Enclosure Control Station & 5.0 \\
\hline Clean & Yes & Process Enclosure Control Station & 5.0 \\
\hline Grind & Yes & Process Enclosure Control Station & 5.0 \\
\hline Repair Weld & Yes & Process Enclosure Control Station & 5.0 \\
\hline Make Cover Pass 1 & No & NA & N/A \\
\hline Visual Inspection & Yes & Process Enclosure Control Station & 5.0 \\
\hline Grind & Yes & Process Enclosure Control Station & 5.0 \\
\hline Repair Weld & Yes & Process Enclosure Control Station & 5.0 \\
\hline Make Cover Pass 2 & No & N/A & NA \\
\hline Visual Inspection & Yes & Process Enclosure Control Station & 5.0 \\
\hline Grind & Yes & Process Enclosure Control Station & 5.0 \\
\hline Repair Weld & Yes & Process Enclosure Control Station & 5.0 \\
\hline Make Cover Pass 3 & No & $\mathrm{N} / \mathrm{A}$ & N/A \\
\hline Visual Inspection & Yes & Process Enclosure Control Station & 5.0 \\
\hline Grind & Yes & Process Enclosure Control Station & 5.0 \\
\hline Repair Weld & Yes & Process Enclosure Control Station & 5.0 \\
\hline Make Cover Pass 4 & No & N/A & N/A \\
\hline Visual Inspection & Yes & Process Enclosure Control Station & 5.0 \\
\hline Grind & Yes & Process Enclosure Control Station & 5.0 \\
\hline Repair Weld & Yes & Process Enclosure Control Station & 5.0 \\
\hline Apply Dye & Yes & Process Enclosure Control Station & 5.0 \\
\hline Soak & No & N/A & N/A \\
\hline Remove Dye & Yes & Process Enclosure Control Station & 5.0 \\
\hline Apply Developer & Yes & Process Enclosure Control Station & 5.0 \\
\hline Bloed & No & N/A & N/A \\
\hline inspect & Yes & Process Enclosure Control Station & 5.0 \\
\hline Clean & Yes & Process Enclosure Control Station & 5.0 \\
\hline Grind & Yes & Process Enclosure Control Station & 5.0 \\
\hline Repair Weld & Yes & Process Enclosure Control Station & 5.0 \\
\hline Remove Welder & Yes & Process Enclosure Control Station & 5.0 \\
\hline Remove Trap & Yes & Process Enclosure Control Station & 5.0 \\
\hline Install New Trap & Yes & Process Enclosure Control Station & 5.0 \\
\hline Connect Waste Drum & Yes & Process Enclosure Control Station & 5.0 \\
\hline Remove Top Shield & Yes & Process Enclosure Control Station & 5.0 \\
\hline Dispose Hot Trap & Yes & Process Enclosure Control Station & 5.0 \\
\hline Insert Top Shield & Yes & Process Enclosure Control Station & 5.0 \\
\hline Tie Bag & Yes & Process Enclosure Control Station & 5.0 \\
\hline Close Drum & Yes & Process Enclosure Control Station & 5.0 \\
\hline Undo Annulus Cover & Yes & Process Enclosure Control Station & 5.0 \\
\hline
\end{tabular}


WESTINGHOUSE HANFORD COMPANY

Hot Conditioning Syatem Equipment

Contrect \#MC6-SWV-310416, Tack 17
WHC-SD-SNF-CDR-007, REV. 0

\section{Table 1}

MERRICK COMPANY

Advanced Tectinobody Sector

Project No. 30013218

\section{Exposure Locations and Dose Rates}

\begin{tabular}{|c|c|c|c|}
\hline Action & $\begin{array}{l}\text { Exposure } \\
\text { Activity? }\end{array}$ & $\begin{array}{l}\text { Exposure } \\
\text { Location }\end{array}$ & \begin{tabular}{|l|} 
Dose Rate \\
(mrem/hr)
\end{tabular} \\
\hline Remove Annulus Cover & Yes & Process Enclosure Control Station & 5.0 \\
\hline Close Process Pit & Yes & Process Enclosure Control Station & 5.0 \\
\hline Survey Process Enclosure & Yes & Process Enclosure Control Station & 5.0 \\
\hline Disconnect Process Enclosure & Yes & Oven Deck Area & 2.0 \\
\hline Remove Process Enclosure & Yes & Oven Deck Area & $2 . \overline{0}$ \\
\hline Move in the MHM & Yes & Oven Deck Area & 2.0 \\
\hline Pull the MCO & Yes & Oven Deck Area & 2.0 \\
\hline Remove the MHM & Yes & Oven Deck Area & 2.0 \\
\hline Prepare for next MCO & No & N/A & N/A \\
\hline
\end{tabular}


Table 2

Exposure per MCO

\begin{tabular}{|c|c|c|c|c|c|c|}
\hline Action & $\begin{array}{l}\text { Exposure } \\
\text { Activity? } \\
\end{array}$ & Workforce & $\begin{array}{c}\text { Duration } \\
\text { (min.) }\end{array}$ & $\begin{array}{l}\text { Frequency } \\
\text { (per Cask) }\end{array}$ & $\begin{array}{l}\text { Dose Rate } \\
\text { (mrem/hr) }\end{array}$ & \begin{tabular}{|c|} 
Exposure \\
(person mrem) \\
\end{tabular} \\
\hline Select Oven & No & N/A & $\mathbf{N} / \mathbf{A}$ & N/A & N/A & - \\
\hline Move in the MHM & Yes & 2 & 10 & 1.0 & 2.0 & 0.7 \\
\hline Lower MCO into Oven & Yes & 2 & 10 & 1.0 & 2.0 & 0.7 \\
\hline Remove the MHM & Yes & 2 & 10 & 1.0 & 2.0 & 0.7 \\
\hline Move in Process Enclosure & Yes & 2 & 15 & 1.0 & 2.0 & 1.0 \\
\hline Connect Process Enclosure & Yes & 2 & 5 & 1.0 & 2.0 & 0.3 \\
\hline Open Process Pil & Yes & 1 & 10 & 1.0 & 5.0 & 0.8 \\
\hline Place Annulus Cover & Yes & 1 & 10 & 1.0 & 5.0 & 0.8 \\
\hline Attach Annulus Cover & Yes & 1 & 10 & 1.0 & 5.0 & 0.8 \\
\hline Remove Port Cover & Yes & 1 & 15 & 1.0 & 5.0 & 1.3 \\
\hline Install Vent Cover & Yes & 1 & 5 & 1.0 & 5.0 & 0.4 \\
\hline Install Valves & Yes & 1 & 10 & 1.0 & 5.0 & 0.8 \\
\hline Open Vahes & Yes & 1 & 15 & 1.0 & 5.0 & 1.3 \\
\hline Evacuate MCO & No & N/A & N/A & N/A & N/A & - \\
\hline Fill MCO with Helium & No & N/A & N/A & N/A & N/A & - \\
\hline Leak Check Ports & Yes & 1 & 15 & 1.1 & 5.0 & 1.4 \\
\hline Close Bad Valve & Yes & 1 & 5 & 0.1 & 5.0 & 0.0 \\
\hline Disconnect Valve & Yes & 1 & 5 & 0.1 & 5.0 & 0.0 \\
\hline Clean Port & Yes & 1 & 5 & 0.1 & 5.0 & 0.0 \\
\hline Install Reserve Valve & Yes & 1 & 5 & 0.1 & 5.0 & 0.0 \\
\hline Install Insulation Cover & Yes & 1 & 5 & 1.0 & 5.0 & 0.4 \\
\hline Close Process Pit & Yes & 1 & 5 & 1.0 & 5.0 & 0.4 \\
\hline Perform Heat-up Cycle & No & N/A & N/A & N/A & N/A & - \\
\hline Survey Process Enclosure & Yes & 2 & 30 & 1.0 & 5.0 & 5.0 \\
\hline Disconnect Process Enciosure & Yes & 2 & 5 & 1.0 & 2.0 & 0.3 \\
\hline Remove Process Enclosure & Yes & 2 & 15 & 1.0 & 2.0 & 1.0 \\
\hline PurgeNacuum Cycle & No & N/A & N/A & N/A & N/A & - \\
\hline Perform Acceptance Test & No & N/A & N/A & N/A & N/A & - \\
\hline Cool for Passivation & No & N/A & N/A & N/A & N/A & - \\
\hline Evacuate MCO & No & N/A & N/A & N/A & N/A & - \\
\hline Fill with Passivation Gas & No & N/A & N/A & N/A & N/A & - \\
\hline Passivation Period & No & N/A & N/A & N/A & N/A & - \\
\hline Cool Down & No & N/A & N/A & N/A & N/A & - \\
\hline Evacuate MCO & No & N/A & N/A & N/A & N/A & - \\
\hline Fill MCO with Helium & No & N/A & N/A & N/A & N/A & - \\
\hline Move in Process Enclosure & Yes & 2 & 15 & 1.0 & 2.0 & 1.0 \\
\hline Connect Process Enclosure & Yes & 2 & 5 & 1.0 & 2.0 & 0.3 \\
\hline Open Process Pit & Yes & 1 & 10 & 1.0 & 5.0 & 0.8 \\
\hline Remove Insulation Cover & Yes & 1 & 5 & 1.0 & 5.0 & 0.4 \\
\hline Close Valves & Yes & 1 & 15 & 1.0 & 5.0 & 1.3 \\
\hline Disconnect Valves & Yes & 1 & 10 & 1.0 & 5.0 & 0.8 \\
\hline Place Weld-on Cover & Yes & 1 & 10 & 1.0 & 5.0 & 0.8 \\
\hline Install Welder & Yes & 1 & 20 & 1.0 & 5.0 & 1.7 \\
\hline Weld Root Pass & No & N/A & N/A & N/A & N/A & - \\
\hline Visual Inspection & Yes & 1 & 10 & 1.1 & 5.0 & 0.9 \\
\hline Clean Weld & Yes & 1 & 10 & 1.1 & 5.0 & 0.8 \\
\hline
\end{tabular}


Table 2

Exposure per MCO

\begin{tabular}{|c|c|c|c|c|c|c|}
\hline Action & \begin{tabular}{|l|} 
Exposure \\
Activity? \\
\end{tabular} & Workforce & $\begin{array}{l}\text { Duration } \\
\text { (min.) }\end{array}$ & $\begin{array}{l}\text { Frequency } \\
\text { (per Cask) }\end{array}$ & $\begin{array}{l}\text { Dose Rate } \\
\text { (mrem/hr) }\end{array}$ & \begin{tabular}{|c|} 
Exposure \\
(person mrem \\
\end{tabular} \\
\hline Grind & Yes & 1 & 15 & 0.1 & 5.0 & 0.1 \\
\hline Repair Weld & Yes & 1 & 15 & 0.1 & 5.0 & 0.1 \\
\hline Apply Dye & Yes & 1 & 10 & 1.1 & 5.0 & 0.9 \\
\hline Soak & No & N/A & $\mathbf{N} / \mathbf{A}$ & N/A & N/A & - \\
\hline Remove Dye & Yes & 1 & 15 & 1.1 & 5.0 & 1.4 \\
\hline Apply Developer & Yes & 1 & 10 & 1.1 & 5.0 & 0.9 \\
\hline Bleed & No & $\mathrm{N} / \mathrm{A}$ & N/A & N/A & N/A & - \\
\hline Inspect & Yes & 1 & 5 & 1.1 & 5.0 & 0.5 \\
\hline Clean & Yes & 1 & 10 & 1.1 & 5.0 & 0.9 \\
\hline Grind & Yes & 1 & 15 & 0.1 & 5.0 & 0.1 \\
\hline Repair Weld & Yes & 1. & 15 & 0.1 & 5.0 & 0.1 \\
\hline Make Cover Pass 1 & No & N/A & N/A & N/A & $\mathbf{N} / \mathbf{A}$ & - \\
\hline Visual Inspection & Yes & 1 & 10 & 1.1 & 5.0 & 0.9 \\
\hline Grind & Yes & 1 & 15 & 1.1 & 5.0 & 1.4 \\
\hline Repair Weld & Yes & 1 & 15 & 1.1 & 5.0 & 1.4 \\
\hline Make Cover Pass 2 & No & N/A & N/A & N/A & N/A & - \\
\hline Visual Inspection & Yes & 1 & 10 & 1.1 & 5.0 & 0.9 \\
\hline Grind & Yes & 1 & 15 & 1.1 & 5.0 & 1.4 \\
\hline Repair Weld & Yes & 1 & 15 & 1.1 & 5.0 & 1.4 \\
\hline Make Cover Pass 3 & No & N/A & N/A & N/A & N/A & - \\
\hline Visual Inspection & Yes & 1 & 10 & 1.1 & 5.0 & 0.9 \\
\hline Grind & Yes & 1 & 15 & 1.1 & 5.0 & 1.4 \\
\hline Repair Weld & Yes & 1 & 15 & 1.1 & 5.0 & 1.4 \\
\hline Make Cover Pass 4 & No & N/A & N/A & N/A & $N / A$ & - \\
\hline Visual Inspection & Yes & 1 & 10 & 1.1 & 5.0 & 0.9 \\
\hline Grind & Yes & 1 & 15 & 1.1 & 5.0 & 1.4 \\
\hline Repair Weld & Yes & 1 & 15 & 1.1 & 5.0 & 1.4 \\
\hline Apply Dye & Yes & 1 & 10 & 1.1 & 5.0 & 0.9 \\
\hline Soak & No & N/A & N/A & N/A & N/A & - \\
\hline Remove Dye & Yes & 1 & 15 & 1.1 & 5.0 & 1.4 \\
\hline Apply Developer & Yes & 1 & 10 & 1.1 & 5.0 & 0.9 \\
\hline Bleed & No & $N / A$ & N/A & N/A & N/A & - \\
\hline Inspect & Yes & 1 & 5 & 1.1 & 5.0 & 0.5 \\
\hline Clean & Yes & 1 & 10 & 1.1 & 5.0 & 0.9 \\
\hline Grind & Yes & 1 & 15 & 0.1 & 5.0 & 0.1 \\
\hline Repair Weld & Yes & 1 & 15 & 0.1 & 5.0 & 0.1 \\
\hline Remove Welder & Yes & 1 & 15 & 1.0 & 5.0 & 1.3 \\
\hline Remove Trap & Yes & 1 & 60 & 1.0 & 5.0 & 5.0 \\
\hline Install New Trap & Yes & 1 & 60 & 1.0 & 5.0 & 5.0 \\
\hline Connect Waste Drum & Yes & 1 & 30 & 1.0 & 5.0 & 2.5 \\
\hline Remove Top Shield & Yes & 1 & 10 & 1.0 & 5.0 & 0.8 \\
\hline Dispose Hot Trap & Yes & 1 & 10 & 1.0 & 5.0 & 0.8 \\
\hline Insert Top Shield & Yes & 1 & 10 & 1.0 & 5.0 & 0.8 \\
\hline Tie Bag & Yes & 1 & 15 & 1.0 & 5.0 & 1.3 \\
\hline Close Drum & Yes & 1 & 10 & 1.0 & 5.0 & 0.8 \\
\hline Undo Annulus Cover & Yes & 1 & 10 & 1.0 & 5.0 & 0.8 \\
\hline
\end{tabular}


Table 2

\section{Exposure per MCO}

\begin{tabular}{|l|c|c|c|c|c|c|}
\hline Action & $\begin{array}{c}\text { Exposure } \\
\text { Activity? }\end{array}$ & Workforce & $\begin{array}{c}\text { Duration } \\
\text { (min.) }\end{array}$ & $\begin{array}{c}\text { Frequency } \\
\text { (per Cask) }\end{array}$ & $\begin{array}{c}\text { Dose Rate } \\
\text { (mrem/hr) }\end{array}$ & Exposure \\
\hline \hline Remove Annulus Cover & Yes & 1 & 5 & 1.0 & 5.0 & 0.4 \\
\hline Close Process Pit & Yes & 1 & 5 & 1.0 & 5.0 & 0.4 \\
\hline Survey Process Enclosure & Yes & 2 & 30 & 1.0 & 5.0 & 5.0 \\
\hline Disconnect Process Enclosure & Yes & 2 & 5 & 1.0 & 2.0 & 0.3 \\
\hline Remove Process Enclosure & Yes & 2 & 15 & 1.0 & 2.0 & 1.0 \\
\hline Move in the MHM & Yes & 2 & 10 & 1.0 & 2.0 & 0.7 \\
\hline Puil the MCO & Yes & 2 & 10 & 1.0 & 2.0 & 0.7 \\
\hline Remove the MHM & Yes & 2 & 10 & 1.0 & 2.0 & 0.7 \\
\hline Prepare for next MCO & No & N/A & N/A & N/A & N/A & - \\
\hline \hline
\end{tabular}


WHC-SD-SNF-CDR-007, Revision 0

\section{APPENDICES}

\section{APPENDIX D}

\section{OPERATIONS AND MAINTENANCE PHILOSOPHY}




\author{
OPERATIONS AND MAINTENANCE PHILOSOPHY \\ for the \\ HOT CONDITIONING SYSTEM EQUIPMENT \\ of the \\ K-BASIN SPENT NUCLEAR FUEL STORAGE PROJECT
}

\title{
1.0 INTRODUCTION
}

The purpose of this document is to set forth operating and maintenance philosophies to guide the Hot Conditioning System Equipment (HCSE) design and operations planning process. In the modern DOE nuclear facility project environment, the transition from the engineering/construction phase of the project to operations is critical to project success. It is essential that this transition be planned, that safety documentation including a SAR and TSRs are complete when needed, that operations testing procedures be written, that staff is hired and trained before while construction is ongoing so that they are ready to take the reigns when the facility is ready, and that an appropriate Operations Readiness verification process as required by DOE 5480.31 and guided by DOE STD-3011 is completed smoothly. This philosophy document is a first step in the process of planning for operations.

The operations and maintenance philosophy for the HCSE should reflect the following facts about the facility:

A. The HCSE is located in the Canister Storage Building (CSB). The facility is a nuclear facility. It must be operated with the rigor and controls normally associated with a nuclear facility. Only qualified, trained radiation workers shall be allowed into the facility. Workers will wear protective clothing when required (routine operational activities will not require protective clothing). There will be personnel radiation monitoring programs. Areas of potential contamination or radiation fields will be specially controlled, etc.

B. The radiation dose of a given worker will be administratively controlled per HSRCM-1.

C. The active processing life of the equipment is scheduled to be 2 years with a 10 year design life for the equipment and a 75 year design life for the HCSE annex to the CSB. 


\section{APPENDIX D - OPERATIONS AND MAINTENANCE PHILOSOPHY}

D. The project is schedule driven. Payment is determined by meeting workoff milestones. Lost time for responding to abnormal drying conditions or results, and for repairing essential components must be minimized. The design team needs to attempt to plan for the most likely upsets and incorporate "work arounds" into the design process.

\subsection{INDEPENDENCE OF OPERATIONS IN THE HCSE}

The HCSE will be designed to have independent process ovens that will allow for the drying of more than one MCO simultaneously. Each oven will have its own ventilation system, its own drying system, and its own control station in the CSB control room. Redundancy of key components should be a design strategy where a central service feeds all ovens (chilled water for example) so that its failure could potentially take the entire facility out of service for a duration greater than a single shift. The decision driver during design of the facility regarding provision of redundant components should be consideration of lost process time.

\subsection{EQUIPMENT SELECTION AND DEVELOPMENT}

The drying process equipment will be a collection of common commercial components arranged on a skid, connected by pipes and conduits. The equipment should be selected for operations in a radiation field and a contaminated environment. Typical features of equipment suitable for this type of service are:

A. Minimize the use of rubbers, plastics, and polymers that degrade as a result of radiation damage.

B. Sealed equipment, with secondary seals, where contaminated leakage may occur.

C. Avoid equipment that requires oil changes or greasing.

D. Smooth, impervious surfaces that allow for decontamination.

E. Easy replacement ( as opposed to repair in place) - flange or union connections, clamp hold-downs, electrical and signal cable plugs, etc.

F. Sealed bagging schemes should be utilized where regular change-outs will be required - MCO connectors, filters. 


\section{APPENDIX D - OPERATIONS AND MAINTENANCE PHILOSOPHY}

G. A high degree of reliability should be required. Mean time between failure data should be requested from potential component vendors. The goal will be to assure that all components have at least a $90 \%$ likelihood of operating for the design life without a failure.

H. Components potentially requiring maintenance or replacement in the oven should be designed for remote manipulator replacement, if possible. Contact maintenance of this equipment would only be possible after removal of the MCO and shielding or removal of accumulated dose contributors such as contaminated process lines, cold traps, and filters.

The equipment arrangement on the skids should allow for easy accesses to components. Clearances should be checked during the detail design to assure that reasonable paths for component access and removal exist, that allowance has been made for bags and tents that may be used to limit the spread of contamination when a component is being used, and that lifting aids can access the maintenance activity when a component may be too heavy to be lifted manually. Items requiring frequent access, such as filters should be located conveniently near an outer edge of the skid. Shielding that may surround some components (filters for example) should be modular and should be easily moved or swung out of the way (hinged).

Equipment should be selected to minimize manual operation or verification. $A$ primary design goal is to minimize the presence of staff in the processing area where they may be exposed to radiation. The operation and status monitoring of the drying system and components should be accomplished from a remote control room. Sensor failure and repair is a potential negative impact that must be considered as the design is developed.

The process equipment system should contain radiation monitors for points in the system where radioactive material may accumulate, such as filters. These monitors should be used to determine when filters are to be changed.

An important goal is to limit the spread of radioactive contamination to the maximum extent possible. Ideally all contamination would be trapped in the MCO or within the first few feet of process line immediately above the MCO. Filters are to be installed to accomplish this goal. Contamination may be released and spread when MCO connections are made and broken. A connection design that incorporates an operations within a bag technique are to be implemented to contain potential releases. The process enclosure will contain any contaminated air that may be present in the area. 


\section{APPENDIX D - OPERATIONS AND MAINTENANCE PHILOSOPHY}

Ventilation system HEPA filters that may become contaminated shall include a bag-in/bag-out filter changing system that will allow filter changes without release of contamination and without shutting down the ventilation system. HEPA filters will be equipped with DOP test capability so that their efficiency can be certified while in operation.

\subsection{RADIATION SAFETY AND EXPOSURE CONTROL}

The administrative radiation exposure limitation for the HCSE will be per HSRCM-1. Preliminary exposure estimates suggest that this can be achieved through a combination of shielding near the MCO integral to the oven assembly, and shielding provided by the process pit covers and the process enclosure. Detailed operations recipes need to be written for each activity to assess whether it is reasonable to reduce exposure estimates. The operations staff must be thoroughly cross trained so that they can be rotated among all the jobs in the facility. Also, the operations scheduling must distribute the steps where doses are received among all shifts and days uniformly (in other words an MCO received on Friday evening may be held until Saturday afternoon so that the dose loading of all staff is uniform).

In order to promote cross training of staff so that they can perform several functions, the design should attempt to minimize actions requiring decisions or actions by the staff. The use of Process Logic Controller software and simplified user friendly control screens are techniques to be used to reduce dependence on specialized training. The implementation of a human factors engineering program in the detailed design (as required by DOE 6430.1A) can also contribute to increasing operator flexibility.

The following radiation safety features will be provided in the HCSE to aid in operating the facility:

\subsection{Emergency Respiratory Protection}

The facility shall be capable of providing as emergency devices only respiratory protection equipment that has been specifically certified or had certification extended for emergency use by NIOSH/MSHA. 10 CFR 20.17030

\subsection{Radiological Alarm System}

Radiological alarm systems shall be provided in accessible work areas as appropriate to warn operating personnel of radiation and airborne radioactive material concentrations above a given set point and of 
concentrations of radioactive material in effluent above control limits. 10 CFR 72.126(b)

Radiation alarm systems shall be designed with provisions for calibration and testing their operability.

10 CFR 72.126(a), (b)

Set points shall be established to ensure that exposures are kept below the allowable limits.

10 CFR 72.126(a), (b)

\subsection{Direct Radiation Monitoring}

Areas containing radioactive materials shall be provided with systems for measuring the direct radiation levels in and around these areas.

10 CFR 72.126(c)(2)

The facility shall provide capability to monitor the extemal surfaces of a package known to contain radioactive material for radioactive

contamination and radiation levels if the package is labeled as containing radioactive material; or has evidence of potential contamination, such as packages that are crushed, wet, or damaged.

10 CFR 20.1906(b)

\subsection{Effluent Monitoring}

The facility shall monitor the amount of radionuclides in effluents during normal and off-normal conditions.

10 CFR $72.126(c)(1)$

Systems designed to monitor the release of radioactive materials shall have means to calibrate and test their operability.

10 CFR 72.126(d)

\subsection{Airbome Radioactive Material Control}

The design of the facility shall use, to the extent practicable, process or other engineering controls (e.g., containment or ventilation) to control the concentrations of radioactive material in air.

10 CFR 20.1701

When it is not practical to apply process or other engineering controls to control the concentrations of radioactive material in the air to values 


\section{APPENDIX D - OPERATIONS AND MAINTENANCE PHILOSOPHY}

below those that define an airborne radioactivity area, the design of the facility shall, consistent with maintaining the total effective dose equivalent ALARA, have the capability to increase monitoring and limit intakes by: control of access, limitation of exposure times, use of respiratory protection equipment, or other controls.

10 CFR 20.1702

\subsection{Personnel Dosimeters}

The facility shall provide capability for processing and evaluating all personnel dosimeters that require processing to determine the radiation dose, and that are used by the facility to comply with 10 CFR 20.1201 or with conditions specified in the facility license. this capability does not apply to direct and indirect reading pocket ionization chambers and those dosimeters used to measure the dose to the extremities.

10 CFR 20.1501(c)(1), (2)

\subsection{Shielding and Protective Clothing}

Normally Occupied Areas. The shielding design basis shall limit the maximum exposure to an individual worker to one-fifth of the annual occupational external exposure limits. Within this design basis, personnel exposures must be maintained ALARA. Specifically, the shielding must be designed with the objective of limiting the total EDE (Effective Dose Equivalent) to less than one rem per year to workers, based on their predicted exposure time in the normally occupied area. The EDE is the sum of all contributing external penetrating radiation (gamma and neutron). In addition, appropriate shielding must be installed, if necessary, to minimize non penetrating external radiation exposures to the skin and lens of the eye of the worker. In most cases, the confinement barrier or process equipment provides this shielding. DOE Order $6430.11300-6.2$

Intermittently Occupied Areas. Shielding and other radiation protection measures shall be provided for areas requiring intermittent access, such as for preventive maintenance, component changes, adjustment of systems and equipment, and so forth, so that the projected dose rates based on occupancy, time, and frequency of exposure do not exceed one rem per year.

DOE Order 6430.1 1300-6.2 


\section{APPENDIX D - OPERATIONS AND MAINTENANCE PHILOSOPHY}

Concrete radiation shielding design shall comply with ANS 6.4 and $\mathrm{ACl}$ 349 where it provides a critical confinement or structural function.

ANSI/ANS 6.4 Sec. 1

$\mathrm{ACl} 349$ Pt. 1 Sec. 1.1

DOE Order $6430.11300-6.2$

Concrete radiation shielding design shall consider the material specifications of ANS 6.4.2 where it provides a critical confinement or structural function.

ANSI/ANS-6.4.2 Sec. 1

DOE Order $6430.11300-6.2$

ACl 318 shall be considered for radiation shields, used for other than critical confinement or structural function, since ACl 318 is appropriate and provides adequate strength for design earthquake (DE) loads.

ANSI/ACI-318 Pt. $1 \mathrm{Sec}$. 1.1.1

DOE Order $6430.11300-6.2$

Penetrations. Straight line penetration of shield walls shall be avoided to prevent radiation streaming.

DOE Order 6430.1 1300-6.2

\subsection{Remote Shield Operation}

Remote shielded operation (i.e., with remote handling equipment such as remote manipulators) shall be considered where it is anticipated that exposures to hands and forearms would otherwise approach the dose guidance in Section 3.2.2 or where contaminated puncture wounds could occur.

DOE Order $6430.11300-6.3$

\subsection{Posting}

The facility shall be posted with radiation area, contamination area, and airborne contamination area signs in accordance with federal law.

10 CFR 20.1902(a), (b), (c), (d)

\subsection{MAINTENANCE PHILOSOPHY}

Generally, and to the maximum extent possible, equipment should be selected that will not require routine maintenance during the processing lifetime of the HCSE. Equipment with a high degree of reliability should be chosen to reduce the likelihood of breakage. Policy such as requesting warranties and reviewing 
failure data should be instituted when selecting equipment in order to improve reliability. When repair is needed it should be accomplished by replacement of defective components (ie, pump) instead of repair in place procedures (ie. Pump bearing rebuild). Equipment system designers should recommend a spare components list to be kept on site and should attempt to select components that can be obtained from manufacturers in $\mathbf{2 4}$ hours should an emergency arise.

Maintenance or repair of contaminated items is a particularly hazardous activity because it is not a routine operation that can be included in the training program and because it involves opening a path between the contaminated equipment interior cavity and inhabited space. Provision must be made in the design anticipating the use of bags and tents for contamination control. Provision should also be made for temporary shielding and for lifting assistance.

\subsection{OPERATIONS READINESS VERIFICATION}

DOE 5480.31 requires that a new nuclear facility undergo an Operations Readiness verification process (either an ORR or ORA depending on the nature of the facility and its associated hazards). The extent of the verification process is to be determined by the nuclear safety issues associated with the facility. The facility will be considered an $\mathrm{HC}-2$ facility with low potential for severe accidents. There are three operations program activities that must be accomplished prior to initiating the verification process. First, an Operations Readiness plan must be written and approved by whatever review agency (DOE - HQ, DOE - RL, WHC) is required to certify readiness. Second, test procedures must be prepared so that the verification can be conducted. Third, staff must be trained so that they can demonstrate readiness.

The engineering team should support this process as follows:

A. Write System Design Descriptions (SDDs) with thorough operating recipes included.

B. Write the Operations Readiness Plan draft.

C. Write the test procedures.

Writing these documents in conjunction with the design development/construction will help to assure that the design has the appropriate features to support the facility operations. 


\subsection{OPERATIONS SUPPORT}

The HCSE will share operations support functions with the CSB. 
WHC-SD-SNF-CDR-007, Revision 0

APPENDICES

\section{APPENDIX E}

\section{PERFORMANCE SPECIFICATIONS REPORT}




\section{UNDERSTANDING OF K-BASIN SPENT NUCLEAR FUEL HOT VACUUM CONDITIONING EQUIPMENT PERFORMANCE SPECIFICATIONS REPORT}

\subsection{INTRODUCTION}

The objectives of the Department of Energy (DOE) at Hanford include cleanup of the site, protecting the health and safety of workers and the public, and protecting the environment. The cleanup will be accomplished in accordance with the Hanford Federal Facility Agreement and Consent Order (the Tri Party Agreement), and in compliance with applicable Federal, State, local laws, and American Indian Treaty Rights. The Hanford cleanup must also comply with DOE policies and directives.

The Hol Vacuum Conditioning Equipment (HVCE) Subproject is an important step in the cleanup of Spent Nuclear Fuel (SNF). In order for the HVCE to safely and efficiently accomplish its functions, the equipment designer must have a complete understanding of the functions and performance requirements of the equipment. The Performance Specification for the Spent Nuclear Fuel Hot Vacuum Conditioning Equipment, WHC-S-0460 Rev. A, March 11, 1996, states the intended functional design and performance specifications for the HVCE. These specifications provide the basis for the design, operation, and monitoring of the process and equipment. The Performance Specification also identifies federal, state, and local regulations that may be applicable to the design basis for the HVCE.

The scope of this evaluation is to: (a) demonstrate Merrick's understanding of the performance specifications for the HVCE that must be reflected in the equipment design and (b) demonstrate Merrick's understanding of regulations that impact design of the HVCE.

\subsection{IDENTIFICATION OF FUNCTIONS AND PERFORMANCE SPECIFICATIONS}

Hot vacuum conditioning of SNF is the last of several processes to be performed to package the spent fuel for transportation, interim dry storage, and eventual final disposition. The HVCE provides the system to remove chemically bound water (by heat and vacuum) from SNF, to condition the fuel (decompose uranium hydrides), and to passivate metal fuel (oxidize exposed uranium metal surfaces). The HVCE, as conceived, is multiple modular structures located in the Canister Storage Building. The HVCE consists of a Process Station (heating coils, shielding thermal 
WHC-SD-SNF-CDR-007, Revision 0

\section{APPENDIX E - PERFORMANCE SPECIFICATIONS REPORT}

insulation), a cooling system, measurement and control instrumentation, a Process Module (vacuum pumps, HEPAs, fans, instrumentation), a Combined Ventilation System for process off-gas management, and a Solid Waste Handling System. In the following paragraphs, each conceptual operating phase is described, on the bases of phase duration, process steps, required subsystems, functions of each subsystem, and design points to be controlled.

Receipt. The first operating phase consists of receiving Multi-Canister Overpacks (MCO) from the staging area and placing an MCO into a Process Station (PS). The duration of the receipt operating phase is $\mathbf{8}$ hours. The system components involved in this operating phase are: a) the multi-canister overpack, which provides primary containment of radionuclides. Allowable external contamination levels for the MCO are not defined in the Perfomance Specifications: b) The MCO Handling Machine (MHM), which provides secondary containment of radionuclides and shielding of direct radiation. Allowable external contamination levels for the MHM are not defined in the Performance Specifications; shielding performance must result in general room $\beta / \gamma$ doses $<0.5 \mathrm{mrem} / \mathrm{hr}$ and contact $\beta / \gamma$ doses $<10 \mathrm{mrem} / \mathrm{hr}$. The MHM also provides the capability to move and manipulate the MCO; however, this capability is not within the scope of this task; c) The MHM Process Station (PS) holds the MCO during processing and provides the support structure for the heating coils and thermal insulation, discussed in the Heatup operating phase; and d) The Process Control System (PCS), which has four design points for the operating phase - MCO temperature $<140^{\circ} \mathrm{C}$ as staged or $<75^{\circ} \mathrm{C}$ at receipt, initial $\mathrm{H}_{2}$ concentration $<100$ volume $\%$, initial $\mathrm{O}_{2}$ concentration $<5$ volume $\%$, MCO internal pressure approximately 14.7 psia (vented) or $<150$ psia (as received).

Cool Down for Connection. The second operating phase consists of cooling each received MCO sufficiently to permit initial connection of process piping to the MCO. The duration of this operating phase is 8 hours. The system components involved in this operating phase are: a) The MCO Cooling System (MCS), which draws ambient HEPA-filtered air from the CSB across the outside of the MCO. The initial MCO temperature is $5140^{\circ} \mathrm{C}$ and the design point for the final MCO temperature is $40^{\circ} \mathrm{C}$ (measured at the MCO head, which is the point of greatest thermal mass). The MCS nominal design cooling rate is approximately $10^{\circ} \mathrm{C} /$ hour, with a maximum cooling rate of $<50^{\circ} \mathrm{C} /$ hour, and a maximum temperature difference between the MCO head and sidewall of $100^{\circ} \mathrm{C}$; b) The Combined Ventilation System (CVS), which receives, filters (redundant HEPAs) and discharges the MCS air flows.

Hookup and Purge. This operating phase consists of connecting the inert and oxidizing gas supplies, connecting the conditioning off gas lines, connecting the vacuum lines, and preparing the Process Heating System. The total phase duration 
WHC-SD-SNF-CDR-007, Revision 0

\section{APPENDIX E - PERFORMANCE SPECIFICATIONS REPORT}

is 8 hours. The system components involved in this phase are: a)Process Gas System, supplying inert and oxygen gases; and b) The Vacuum Pumping System.

Heat Up. The next operating phase is heat up of the SNF in an MCO. The duration of this operating phase is 12 hours. The system components involved in this phase are: a) The Process Heating System (PHS), which heats, controls and maintains the temperature of the $\mathrm{MCO}$. The final fuel temperature is $300^{\circ} \mathrm{C}$ within a range of $+50^{\circ} \mathrm{C},-0^{\circ} \mathrm{C}$. The fuel heating rate is $20^{\circ} \mathrm{C}$ hour (nominal), $50^{\circ} \mathrm{C} /$ hour (maximum). The maximum temperature difference between separate points on the MCO is $100^{\circ} \mathrm{C}$; b) The Process Gas System (PGS), supplying an inert gas purge at up to 10 SCFM, maintaining a maximum hydrogen concentration of 2 volume $\%$, a nominal oxygen concentration of zero, and a maximum oxygen concentration of 5 volume \%; and c) the Vacuum Pumping System (VPS), which maintains an MCO internal pressure of approximately 13 PSIA during this phase.

Drving and $\mathrm{UH}_{3}$ Decomposition. This operating phase holds the fuel at a high temperature and low pressure for an extended time, thus removing water, decomposing uranium hydrides, and removing the resultant hydrogen. The duration of this phase is 48 hours. The system components involved in this phase are: a) The PHS, which maintains an MCO set point of $350^{\circ} \mathrm{C}$, an $\mathrm{MCO}$ maximum of $375^{\circ} \mathrm{C}$, and a fuel temperature of $\left.300+50,-0^{\circ} \mathrm{C} ; b\right)$ The PGS, which maintains a purge gas flow rate of up to 10 ACFM, a maximum hydrogen concentration of 2 volume $\%$, a nominal oxygen concentration of zero, and a maximum oxygen concentration of 5 volume \%; and c) The VPS, which maintains a final pressure of $0.1 \mathrm{PSIA}$ and a final hydrogen partial pressure of $0.05 \mathrm{PSI}$.

Cool Down Prior to Partial Oxidation. This operating phase accomplishes a partial cooldown and partial repressurization of the MCO, to prepare for the next operating phase of Partial Oxidation. The duration of this phase is 12 hours. The system components necessary for this phase are: a) The MCS, which cools the MCO from $300^{\circ} \mathrm{C}$ to $150^{\circ} \mathrm{C}$ at a maximum cooling rate of $50^{\circ} \mathrm{C} /$ hour. The maximum temperature difference allowable between any two points on the $\mathrm{MCO}$ is $100^{\circ} \mathrm{C}$; b) The PGS, with a purge rate of up to 10 ACFM, to maintain a maximum hydrogen concentration of 2 volume \% (nominal zero) and a maximum oxygen concentration of 5 volume \% (nominal zero); and c) The VPS, which achieves and maintains a final MCO intemal pressure of approximately 13 PSIA (the rate of pressure change from 0.1 to 13 PSIA is not specified).

Partial Oxidation. This operating phase passivates exposed surfaces of uranium metal by formation of oxides. The duration of this phase is 12 hours. The system components involved are: a) The MCS, only if required to prevent the final fuel temperature from exceeding $250^{\circ} \mathrm{C}$, as the oxidation reaction heats the fuel; $b$ ) The 
WHC-SD-SNF-CDR-007, Revision 0

\section{APPENDIX E - PERFORMANCE SPECIFICATIONS REPORT}

PGS, with a gas flow rate of $u$ to 10 SCFM, to maintain a maximum hydrogen concentration of 2 volume $\%$ and to achieve a final oxygen concentration of up to approximately 20 volume \%; and c) The VPS, to maintain an MCO internal pressure of approximately 13 PSIA.

Final Cool Down. This operating phase cools the MCO containing conditioned and passivated SNF to near ambient temperatures, which allow the final processing phase, Backfill, Disconnect, Seal, to be safely accomplished. The duration of this phase is 10 hours. The involved system components are: a)The MCS, which cools the MCO from $\leq 250^{\circ} \mathrm{C}$ to $40^{\circ} \mathrm{C}$, at a cooling rate of $10^{\circ} \mathrm{C} /$ hour nominal $\left(50^{\circ} \mathrm{C} /\right.$ hour maximum, with a maximum MCO temperature delta of $100^{\circ} \mathrm{C}$; and b) the PGS, which provides purge gas to achieve final oxygen concentration $<5$ volume $\%$ and final hydrogen concentration of $<2$ volume $\%$.

Backfill. Disconnect. Seal. This is the last operating phase of the SNF Hot Vacuum Conditioning and Passivation process. The functions of this step are to backfill the MCO void volume with an inert gas (helium at 99.995 volume \% purity), to disconnect the MCO from process connections, and to weld all MCO openings closed. The estimated duration of this phase is 24 hours. The system component involved in this phase is the PGS, which supplies the backfill helium.

\subsection{IDENTIFICATION OF REGULATORY REQUIREMENTS}

The Performance Specification outlines the HVCE functions and traces the relationship to the Spent Nuclear Fuel Project (SNFP) through the Work Breakdown Structure (WBS) and organizational block diagrams. The technical requirements and descriptions are organized by WBS number and the associated requirement references are listed.

The requirements listed include Federal, State, and local laws and regulations as well as Westinghouse Hanford Company (WHC) rules and regulations. The functions and requirements in the Performance Specification report implement the requirements set forth in the Spent Nuclear Fuel Program Project Management Plan, which requires management, development, and maintenance of baseline documentation.

The requirements listed in the Performance Specification report mainly consist of the Code of Federal Regulations (CFRs), DOE Orders, Washington State Administrative Codes (WACs) including Revised Codes of Washington (RCW), WHC control manuals (WHC-CM), and other miscellaneous documents. 
WHC-SD-SNF-CDR-007, Revision 0

\section{APPENDIX E - PERFORMANCE SPECIFICATIONS REPORT}

The Code of Federal Regulations is a codification of applicable regulations promulgated by an issuing Federal Agency and published in the Federal Register. The Code is divided into 50 titles which represent broad areas subject to Federal Regulations. Each title is divided into chapters which usually bear the name of the issuing agency. Each chapter is further subdivided into several parts to address specific regulations. Historically, CFRs have applied to commercial nuclear reactor facilities but not to Govemment Owned Contractor Operated (GOCO) sites such as Hanford. The Federal Government desires to provide adequate protection of the public from radioactive material releases associated with normal operations and accident/natural phenomena. To this end, the Congress has legislated that this is generally the responsibility of the DOE where DOE facilities are involved and generally the responsibility of the NRC where commercial facilities are involved. The Congress has further established the DNFSB as the watch dog over the DOE. 10 CFR 30.12 indicates that prime contractors of the DOE are exempt from the regulations to the extent that the contractor working under his prime contract manufactures, produces, transfers, receives, acquires, owns, possesses, or uses byproduct material for the performance of work for the DOE at a GOCO site. The wording very specifically says prime contractors because such contractors operate DOE owned facilities on the DOE's behalf. This exemption applies to the HVCE. The DOE established in the K-Basin Spent Nuclear Fuel Project - Regulatory Policy, dated August 4, 1995, the requirement for the SNFP facilities to achieve "nuclear safety equivalency" comparable to NRC licensed facilities. An evaluation was performed to identify any additional requirements that were needed to supplement the existing and applicable DOE requirements to establish nuclear safety equivalency with the NRC licensed facilities. The additional requirements were consolidated into 29 items. All items are to be implemented per the NRC regulations with the exception of the design earthquake which will be implemented in a manner that established equivalence in safety, as opposed to direct equivalence to the regulation.

The DOE Orders establish policies, guidelines, and requirements for the safe operation and maintenance of DOE owned facilities. Historically, these orders have been considered to be the governing regulations, and not the CFRs, for the DOE GOCO sites. In addition to DOE orders, several supplemental guidance documents, which are specific to Hanford, are also listed in the Performance Specification report.

The Washington Administrative Codes (WACs) and Revised Codes of Washington (RCWs) are compiled by the various agencies of the State of Washington. WACs and RCWs are normally considered to be additional requirements to Federal guidelines rather than as replacements. However, WACs promulgate similar 
WHC-SD-SNF-CDR-007, Revision 0

\section{APPENDIX E - PERFORMANCE SPECIFICATIONS REPORT}

requirements as CFRs for many instances. Hence, compliance with either Federal or State regulations is normally considered adequate. Since 10 CFR 30.12 exempts the HVCE from the CFRs, in this case it is the State regulations that apply.

The goals of WHC are to make the Hanford site a model for environmental management, demonstrate and apply advanced and innovative technologies for cleanup, and restore the land for productive use. To accomplish these goals, WHC has created several control manuals (CMs). The purpose of these control manuals is to establish various compliance requirements and guidelines in conjunction with applicable DOE Orders, and Federal, State, and local laws and regulations.

\subsection{CLASSIFICATION OF REQUIREMENTS}

DOE Order 6430.1A (General Design Criteria) gives the main requirements needed for the design. The quality assurance requirements are given in 10 CFR Part 830.120. These and other applicable requirements are listed in Appendices $A$ and B. Requirements that do not have any significant direct impact, but may affect the design process, are listed in Appendix $C$. These requirements are maintained for reference. Although these requirements may not have direct influence on the actual design of the Hot Vacuum Conditioning Equipment, each requirement may impact the design process. The extent of impact will be determined later.

\subsection{DESCRIPTION OF APPLICABLE REQUIREMENTS}

Five requirements listed in Appendix $A$ are judged to be applicable for the Hot Vacuum Conditioning Equipment design. Of these five, DOE 6430.1A and $10 \mathrm{CFR}$ Part 830.120 are the two significant documents. Detailed descriptions of each of the five requirements are given below.

\section{A. $\quad 10$ CFR Part 830.120, Quality Assurance}

The ten criteria of 10 CFR Part 830.120 prescribe a comprehensive management system for DOE work. The quality management program described in 10 CFR Part 830.120 provides a results-oriented management system that focuses on the customer while providing a method for the organization to become more efficient through constant process improvement. The ten criteria are as follows: 
WHC-SD-SNF-CDR-007, Revision 0

APPENDIX E - PERFORMANCE SPECIFICATIONS REPORT

1. Program: The contractor shall develop and maintain a written Quality Assurance Plan (QAP). The QAP shall describe the organizational structure, functional responsibilities, levels of authority, and interfaces for those managing, performing, and assessing adequacy of work. The QAP shall describe the contractors management system, including planning, scheduling, and cost control considerations.

2. Personne/ Training and Qualifications: Personnel shall be trained and qualified to ensure that they are capable of performing their assigned work. Personnel shall be provided continuing training to ensure that job proficiency is maintained.

3. Quality Improvement: The contractor shall establish and implement processes to detect and prevent quality problems and to ensure quality improvement. Items and processes that do not meet established requirements shall be identified, controlled, and corrected. Correction shall include identifying the causes of problems and preventing recurrence. Item reliability, process implementation, and other quality-related information shall be reviewed and the data analyzed to identify items and processes needing improvement.

4. Documents and Records: Documents shall be prepared, reviewed, approved, issued, used, and revised to prescribe processes, specify requirements, or establish design. Records shall be specified, prepared, reviewed, approved, and maintained.

5. Work Processes: Work shall be performed to establish technical standards and administrative controls. Work shall be performed under controlled conditions using approved instructions, procedures, or other appropriate means. Items shall be identified and controlled to ensure their proper use. Items shall be maintained to prevent their damage, loss, or deterioration. Equipment used for process monitoring or data collection shall be calibrated and maintained.

6. Desian: Items and processes shall be designed using sound engineering/scientific principles and appropriate standards. Design work, including changes, shall incorporate applicable requirements and design bases. Design interfaces shall be identified and controlled. The adequacy of design products shall be verified or validated by individuals or groups other than those who performed the work. Verification and validation work shall be completed before approval and implementation of the change. 
WHC-SD-SNF-CDR-007, Revision 0

\section{APPENDIX E - PERFORMANCE SPECIFICATIONS REPORT}

7. Procurement: Procured items and services shall meet established requirements and perform as specified. Prospective suppliers shall be evaluated and selected on the basis of specified criteria. The contractor shall ensure that approved suppliers can continue to provide acceptable items and services.

8. Inspection and Acceptance Testing: Inspection and acceptance testing of specified items and processes shall be conducted using established acceptance and performance criteria. Equipment used for inspections and tests shall be calibrated and maintained.

9. Management Assessment: Management at all levels shall periodically assess the integrated quality assurance program and its performance. Problems that hinder the organization from achieving its objectives shall be identified and corrected.

10. Independent Assessment: Planned and periodic independent assessments shall be conducted to measure items' quality and process effectiveness and to promote improvement. The organization performing independent assessments shall have sufficient authority and freedom from the line organization to carry out its responsibilities. Persons conducting independent assessments shall be technically qualified and knowledgeable in the areas assessed.

Merrick will develop a QAP that is in accordance with these criteria and that shows how the criteria will be satisfied. A QA matrix will also be developed to provide a cross walk between the criteria and the Merrick quality assurance, administrative, and design procedures.

\section{B. DOE 6430.1A General Design Criteria}

This DOE Order provides General Design Criteria (GDC) for various engineering disciplines. This GDC is mandatory for all DOE related work and provides minimal acceptable design requirements for DOE Facilities. Although DOE facilities are not mandated to follow state, county, and other requirements, it is recommended to interface with the non-DOE authorities to accommodate their intent as much as possible. The GDC contains 16 numerical divisions devoted to major building systems or design specialities. According to DOE instructions, GDC must be used in the planning, design, and development of specifications for facilities, including the development of site and equipment-specific designs. Provided the GDC is not adequate, 
WHC-SD-SNF-CDR-007, Revision 0

APPENDIX E - PERFORMANCE SPECIFICATIONS REPORT

supplemental standards may be used. The ASME B31.3 Chemical Plant and Petroleum Refinery Piping standard and the Industrial Vacuum Practices are considered to be supplemental standards that will be used for the HVCE design.

The following sections of the GDC are considered applicable for the Hot Vacuum Conditioning Equipment Design. The criteria evaluation document developed for the Hot Vacuum Conditioning Equipment goes into the applicability of the GDC in more extensive detail.

\section{Division 1. General Requirements}

Section 0111-99.0.1, Non-Reactor Nuclear Facilities

Paragraphs 1, 4, 5, and 6 on Page 1-97,

Paragraphs 2 and 3 on Page 1-98.

Section 0111-99.0.2, Tornado of Extreme Wind

Portions of this section are applicable.

Section 0111-99.0.4, Earthquakes

The entire section is applicable.

Section 0111-99.0.7, Explosion, Internal Pressurization, Criticality, and Other DBA Causes.

Section 0140 on Quality Assurance.

Division 13. Special Facilities

Section 1300-1, Coverage and Objectives

Many sub-sections are applicable.

Section 1300-2, Safety Analysis

Section 1300-3.2, Safety Class Items

Most of this section's contents are applicable. This section also proposes the use of ASME Boiler and Pressure Vessel Codes wherever they are applicable.

Section 1300-3.3, Single Failure Criterion and Redundancy

Section 1300-3.4, Equipment Environment Considerations

Most of the requirements specified in the three subsections are applicable.

Section 1300-3.5, Maintenance

Second paragraph of this section is especially applicable.

Section 1300-3.6, Testing

Portions of first and second paragraphs are applicable. 
WHC-SD-SNF-CDR-007, Revision 0

\section{APPENDIX E - PERFORMANCE SPECIFICATIONS REPORT}

Section 1300-4, Nuclear Criticality Safety

This section describes the procedures to assess as early as possible if the potential for nuclear criticality exists. Several portions of this section may be applicable.

Section 1320 Irradiated Fissile Material Storage Facilities

This section describes irradiated fissile material storage facilities (ISMSF). Portion of subsections 1320-1, -2 , and -3 are applicable.

C. WHC-CM-4-2 Quality Assurance Manual

This manual describes the requirements that apply to WHC Divisions and Departments which prescribe, perform, or verify activities affecting quality including operations, decommissioning and decontamination, and environmental activities. This manual emphasizes that quality assurance controls must be applied to any items and activities performed under WHC Management. The manual consists of 19 sections with each section dedicated to a certain aspect of quality control.

This is a compulsory requirement for each operation involved in the SNF transfer from K-Basins. Requirements of this manual in conjunction with 10 CFR Part $\mathbf{8 3 0 . 1 2 0}$ should provide more than adequate quality control.

\section{WHC-CM-6-1 Standard Engineering Practices}

This manual establishes the engineering practices which ensure that uniform methods are in place for performing all tasks under WHC Control. These procedures provide methodologies for tasks such as design review, configuration management, change control, specification preparation, and review and approval requirements. The use of this document is strongly recommended on all engineering, development, and project tasks which culminate in a document design and/or deliverable hardware end items.

Of several sections in this manual, Section 4 on Design Verification Requirements is the most applicable for Hot Vacuum Conditioning Equipment Design. This section provides methods and procedures governing design verification measures to verify the design adequacy. This requirement and DOE Order 6430.1A provide comprehensive guidelines for the design task. 
WHC-SD-SNF-CDR-007, Revision 0

\section{APPENDIX E - PERFORMANCE SPECIFICATIONS REPORT}

\section{E. NRC Regulations}

The DOE established in the K-Basin Spent Nuclear Fuel Project - Regulatory Policy, dated August 4,1995, the requirement for the SNFP facilities to achieve "nuclear safety equivalency" comparable to NRC licensed facilities. An evaluation was performed to identify any additional requirements that were needed to supplement the existing and applicable DOE requirements to establish nuclear safety equivalency with the NRC licensed facilities. The additional requirements were consolidated into 29 items. All items are to be implemented per the NRC regulations with the exception of the design earthquake which will be implemented in a manner that established equivalence in safety, as opposed to direct equivalence to the regulation.

\subsection{DESCRIPTION OF REFERENCE REQUIREMENTS}

The requirements listed in Appendix $\mathrm{C}$ will be used as reference documents. These requirements have no direct impact on the design, but may affect the design methodologies.

10 CFR 20 and 10 CFR 71 are promulgated by the Nuclear Regulatory Commission (NRC). It should be noted that DOE nuclear facilities are not required to comply with NRC regulations. DOE, however, use the NRC requirements as a source of guidance and good practice for the SNF transfer program.

10 CFR 20, Standards for Protection Against Radiation, establishes standards for individual protection from ionizing radiation resulting from activities conducted under licenses issued by NRC. These regulations quantify the permissible radiation doses to various body parts of an individual. The prescribed dose limits do not apply to doses due to background radiation and radiation received as part of a medical therapy or due to voluntary participation in medical research programs.

This regulation does not have direct impact on the Hot Vacuum Conditioning Equipment Design. However, this regulation will be maintained for reference as dose rates may have to be considered in the design process.

10 CFR 71, Packaging and Transportation of Radioactive Material, is also an NRC Regulation which establishes requirements for packaging, preparation for shipment, and transportation of licensed material. This regulation also describes procedures and standards for NRC approval of packaging and shipping procedures for fissile material. The packaging and shipping aspects are also subject to other NRC 
WHC-SD-SNF-CDR-007, Revision 0

\section{APPENDIX E - PERFORMANCE SPECIFICATIONS REPORT}

regulations and requirements of other agencies, such as the Department of Transportation.

This regulation does not have direct bearing on the Hot Vacuum Conditioning Equipment Design. However, these regulations may have to be consulted to verify that the Hot Vacuum Conditioning Equipment Design poses no problems for packaging and transportation.

\section{A. DOE Regulations}

10 CFR 835, Occupational Radiation Protection, is a DOE Regulation which is very similar to 10 CFR 20 . 10 CFR 835 promulgates radiation protection standards, limits, and program requirements for protecting individuals from ionizing radiation resulting from the conduct of DOE activities. Activities conducted under NRC license are not covered by this regulation. Like 10 CFR 20, this requirement is also considered as a reference requirement.

\section{B. OSHA Requirements}

29 CFR 1910, Occupational Safety and Health Standard, is promulgated by the Occupational Safety and Health Administration of the Department of Labor. The intent of this directive is to carry out OSHA requirements which have been found to be National Consensus Standards or Established Standards. All routine industrial operations are covered under this requirement. From a simple administrative function such as maintaining worker's exposure and medical surveillance records to complex operations such as handling hazardous materials are covered by 29 CFR 1910. Minimum environmental controls required for a work area, provisions of proper personal protective equipment, fire protection requirements, noise control, and providing medical and first aid during emergencies are also covered under this requirement. These regulations may be exempt if it is determined that these standards do not improve health and safety for specially designated employees.

These is no direct impact of these regulations on the Hot Vacuum Conditioning Equipment Design, but the design may have to be accomplished such that the Hot Vacuum Conditioning Equipment usage adheres to 29 CFR 1910. Hence, this regulation is maintained for reference. 
WHC-SD-SNF-CDR-007, Revision 0

\section{APPENDIX E - PERFORMANCE SPECIFICATIONS REPORT}

\section{DOE 1300.2A Department of Energy Technical Standards Programs}

This DOE Order sets forth policies and responsibilities for development and application of technical standards under DOE controlled programs. This order stipulates that any applicable Non-Government Standards (NGSs) must be used and the usage must be properly documented. This Order also states that all DOE facilities, programs, and projects will use NGSs in their design, construction, testing, modification, operation, decommissioning, decontamination, and remediation when such standards are adequate. If the existing Federal Standards is more conservative than NGSs, DOE requirements will be used. Uses of all standards in the design have to be properly documented.

This DOE Order is a general guideline for using the Technical Standards. This document is kept on the reference list.

D. DOE 1540.2 Hazardous Material Packaging for Transport Administrative Procedures

This Order establishes administrative procedures for the certification and use of packaging methods for radioactive and hazardous materials. The administrative procedures also summarize the actions associated with the review and approval of packaging for the transportation of radioactive and hazardous materials. This Order details the review and approval procedure that provides a basis for DOE to assure DOT, NRC, and other agencies that regulations are compiled with and the entire process is relevant to that of NRC.

This DOE Order has no direct impact on the Hot Vacuum Conditioning Equipment Design. As the packaging process has very little effect on the Hot Vacuum Conditioning Equipment Design, it is unlikely that this Order will be used during the design process. However, DOE 1540-2 is retained for reference.

E. DOE 1540.3A Base Technology for Radioactive Material Transportation Packaging Systems

DOE 1540-A is a culmination of DOE Orders 1330.1D, 1540.1A, 15402, and 5480.3; 10 CFR 71 and 49 CFR 171 through 179; Atomic Energy Act; and the Department of Energy Organization Act of 1977. This Order establishes DOE policies and responsibilities for coordinating and planning base 
WHC-SD-SNF-CDR-007, Revision 0

\section{APPENDIX E - PERFORMANCE SPECIFICATIONS REPORT}

technology for radioactive material, transportation packaging systems. Radioactive material is defined as any material having a specific activity greater than 0.002 microcuries per gram.

This DOE Order is more of an administrative document than a design document. Consequently, this Order will have very little impact on either the Hot Vacuum Conditioning Equipment Design or the design process. However, this document is maintained for reference purposes.

\section{F. DOE 5480.4 Environmental Protection, Safety, and Health}

This DOE Order provides listing of requirements for the application of the mandatory Environmental Protection, Safety, and Health (ES\&H) Standards applicable to all DOE Operations. This Order must be followed during design, construction, modification, and decommissioning. All permanent and temporary facilities must comply with this Order. The requirements listed in this DOE Order are divided into the following three categories:

1. Mandatery ES\&H Standards (Statutory Requirements): Those standards that are mandatory as a result of Non-DOE Federal or State Statutes are addressed in this category. None of these standards are applicable to Hot Vacuum Conditioning Equipment Design.

2. Mandatory ES\&H Standards (Policy Requirements): Standards that are mandatory because of DOE Policies are listed under this category. Subsection F which addresses Nuclear Safety may be used for guidance for Hot Vacuum Conditioning Equipment Design. However, these standards are mainly oriented for nuclear reactors.

3. Reference ES\&H Standards: Those standards that are not mandatory but are useful to refer to as good practice and for general ES\&H purposes. None are directly applicable for Hot Vacuum Conditioning Equipment Design.

This DOE Order does not appear to have any direct impact on the Hot Vacuum Conditioning Equipment Design. However, this document will be retained as reference. 
WHC-SD-SNF-CDR-007, Revision 0

\section{APPENDIX E - PERFORMANCE SPECIFICATIONS REPORT}

G. Fiscal Year 1995, Hanford Mission Plan, Volume 1, Site Guidance, September 1994

The Hanford Mission is to cleanup the site, develop new technologies, and to help the economic diversification of the region. The transfer of K-Basin fuel is a part of the cleanup mission. This document has no impact on either HVCE design or the design process. However, this document is the basic report in which all Hanford cleanup programs are described. This document is maintained on the reference list.

H. WHC-CM-2-14 Hazardous Material Packaging and Shipping

This WHC Manual describes requirements and procedures for packaging and shipping hazardous wastes both on-site and off-site of Hanford. This manual has been prepared by WHC based on several CFRs, DOE Orders, WACs, and other pertinent documents including WHC-CMs. In addition to other requirements, instructions of this requirement have to be followed only when the SNF is categorized as hazardous material.

This requirement has no direct impact on the Hot Vacuum Conditioning Equipment Design. This document is maintained for reference.

\section{WHC-CM-3-5 Document Control and Records Management Manual}

This manual was developed to provide requirements and procedures for maintaining the information that is produced for any project. The information could be as paper, punched cards, magnetic tapes, computer disks, etc. The main objective of this program is to preserve records that may be useful and of future value in a proper manner.

There is no impact of this requirement on the Hot Vacuum Conditioning Equipment Design. However, all the paper work and computer formatted data will be transferred to WHC for up keeping the records. This manual is retained for reference purposes only. 
WHC-SD-SNF-CDR-007, Revision 0

\section{ADDENDUM 1 \\ APPLICABLE REQUIREMENTS FOR THE VACUUM DRYING MODULE SUBPROJECT DESIGN}

1. 10 CFR Part 830.120, Quality Assurance

2. DOE 6430.1A, General Design Criteria

3. WHC-CM-4-2, Quality Assurance Manual

4. WHC-CM-6-1, Standard Engineering Practices

5. WHC-SD-SNF-DB-003, Rev 1, Additional NRC Requirements 
WHC-SD-SNF-CDR-007, Revision 0

\section{APPENDIX E - PERFORMANCE SPECIFICATIONS REPORT}

\section{ADDENDUM 2}

\section{MISCELLANEOUS APPLICABLE DOCUMENTS}

1. DNFSB letter of May 26, 1994, to Secretary O'Leary recommending expediting the SNF removal from K-Basins to an interim storage until an option is chosen for ultimate disposal.

2. J. E. Lytle letter to T. P. Grumbly, Approval of Path Forward for N-Reactor Spent Fuel Interim Storage, DOE EM-36, 9 November 1994, regarding the expeditious transfer of N-Reactor SNF to interim storage, but still within the applicable regulatory requirements.

3. SNF Project K-Basins Path Forward Acquisition Strategy (WHC-SP-1144, December 1994 draft) which describes the following four key interconnecting subsystems:

- $\quad$ Staging and Storage Facility (SSF)

- Multi-canister Over packs (MCO)

- Transportation System

- Fuel Stabilization Facility (FSF)

4. U. S. Department of Energy (DOE) October 1994, Spent Nuclear Fuel Program Requirements Document (SNF-RD-PM-001), Revision 1, describing the management approach to integrate, coordinate, and optimize activities necessary for conditioning, handling, and transporting SNF to interim storage and preparing SNF for final disposal.

5. WHC-EP-009, WHC Reference Guide Acronyms and Abbreviations, Vol. 1, part 1.

6. WHC-IP-0117, Procedure Development Specification Manual.

7. WHC-SP-0708, Westinghouse Hanford Company Conduct of Operation. 
WHC-SD-SNF-CDR-007, Revision 0

\section{APPENDIX E - PERFORMANCE SPECIFICATIONS REPORT}

\section{ADDENDUM 3 \\ REQUIREMENTS FOR REFERENCE}

1. 10 CFR 20, Standards for Protection Against Radiation

2. 10 CFR 71, Packaging and Transportation of Radioactive Material

3. 10 CFR 835, Occupational Radiation Protection Standards

4. 20 CFR 1910, Occupational Safety and Health Act of 1976

5. DOE 1300.2A, Department of Energy Technical Standards Program

6. DOE 1540.2, Hazardous Material Packaging for Transportation

7. DOE 1540.3A, Base Technology for Radioactive Material Transportation Packaging Systems

8. DOE 5480.4, Environmental Protection, Safety, and Health Protection

9. Fiscal Year 1995 Hanford Mission Plan, Volume 1, Site Guidance, September 1994

10. MRP 5.20, Packaging and Transportation of Hazardous Material

11. DOE 5480.28, Natural Phenomena Hazards Mitigation

12. Spent Nuclear Fuel Project, K-Basins Path Forward Acquisition Strategy, WHC-SP-1144, December 1994 (Draft)

13. WHC-CM-2-14, Hazardous Material Packaging and Shipping

14. WHC-CM-3-5, Document Control and Records Management Manual 
WHC-SD-SNF-CDR-007, Revision 0

\section{APPENDICES}

APPENDIX F

\section{COST ESTIMATE BACKUP}




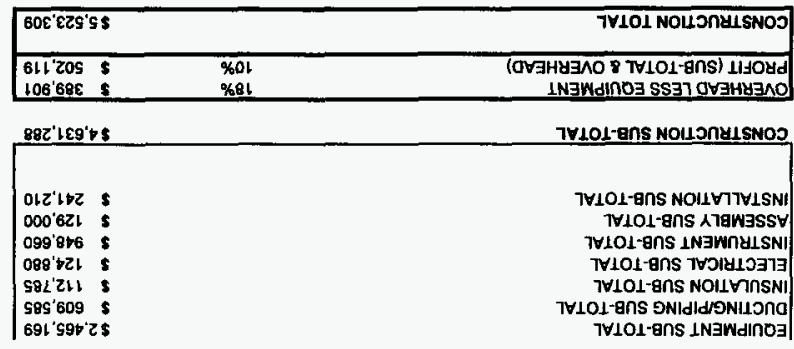

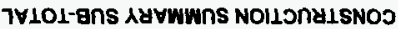

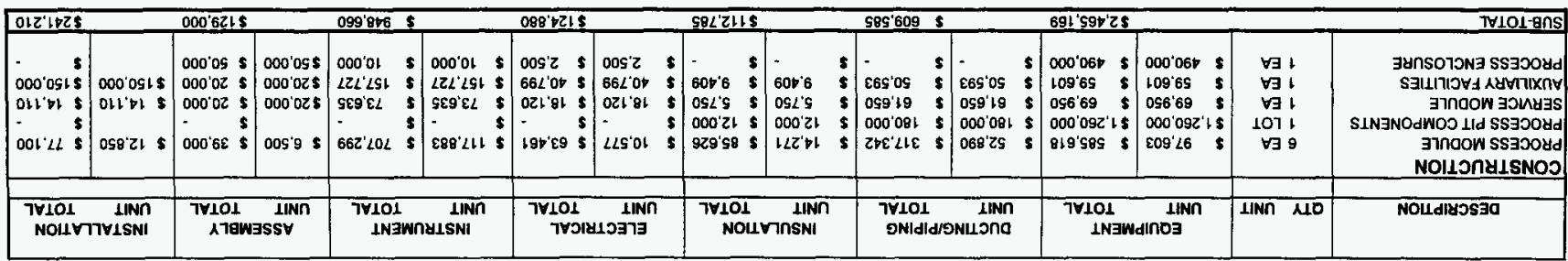

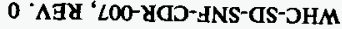


WHC-SD-SNF-CDR-007, REV. 0

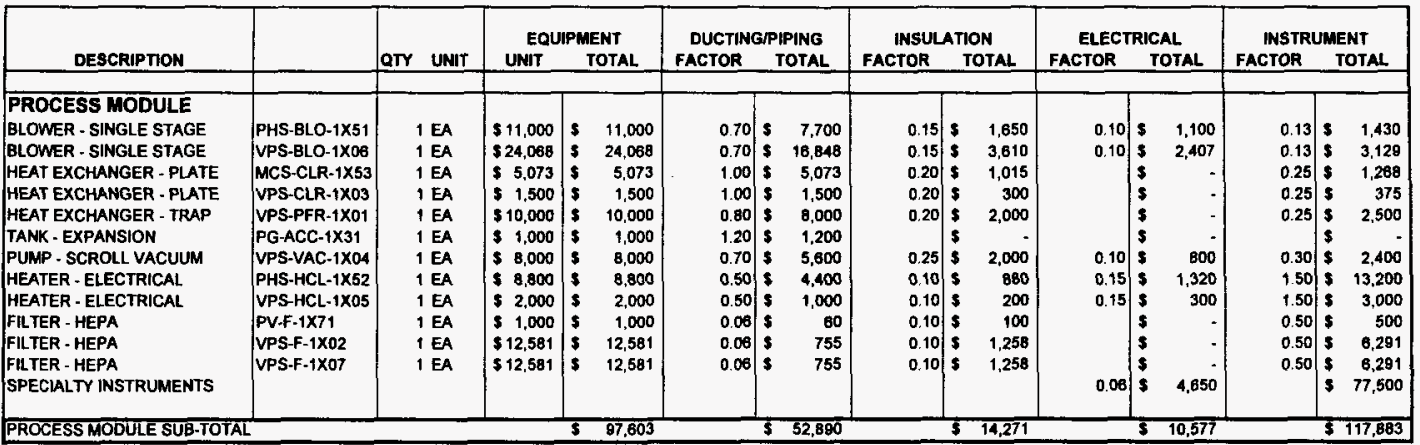

\section{PROCESS MODULE SUB-TOTAL SUMMARY}

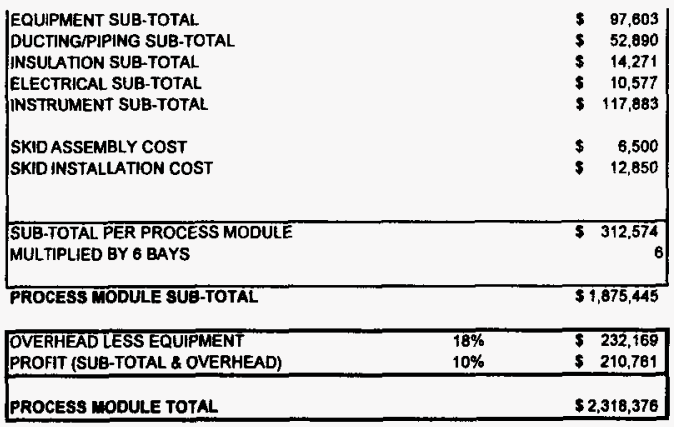


WHC-SD-SNF-CDR-007, REV. 0

\begin{tabular}{|c|c|c|c|c|c|c|c|c|c|c|c|}
\hline \multirow[b]{2}{*}{ DESCRIPTION } & \multirow[b]{2}{*}{ UNIT } & \multicolumn{2}{|c|}{ EQUIPMENT } & \multicolumn{2}{|c|}{ DUCTINGIPIPING } & \multicolumn{2}{|c|}{ INSULATION } & \multicolumn{2}{|c|}{ ELECTRICAL } & \multicolumn{2}{|c|}{ INSTRUMENT } \\
\hline & & UNIT & TOTAL & UNIT & TOTAL & UNIT & TOTAL & UNIT & TOTAL & UNIT & TOTAL \\
\hline $\begin{array}{l}\text { PROCESS PIT COMPONENTS } \\
\text { OVEN } \\
\text { PROCESS PIT COVER } \\
\text { TRENCH COVER }\end{array}$ & $\begin{array}{l}6 \text { EA } \\
7 \text { EA } \\
7 \text { EA }\end{array}$ & $\begin{array}{l}\$ 140,000 \\
\$ 35,000 \\
\$ 25,000\end{array}$ & $\begin{array}{ll}\$ & 840,000 \\
\$ & 245,000 \\
\$ & 175,000\end{array}$ & $\$ 30,000$ & $\$ 180,000$ & $\$ 2,000$ & $\$ 12,000$ & & & & \\
\hline PROCESS PIT COMPONENTS SUB-T & TAL & & $\$ 1,260,000$ & & $\$ 180,000$ & & $\$ 12,000$ & & $\$$ & & $\$$ \\
\hline
\end{tabular}

\section{PROCESS PIT COMPONENTS SUB-TOTAL SUMMARY}

\begin{tabular}{|lrr|} 
EQUIPMENT SUB-TOTAL & $\$ 1,260,000$ \\
DUCTINGIPIPING SUB-TOTAL & $\$ 180,000$ \\
INSULATION SUB-TOTAL & $\$ 12,000$ \\
ELECTRICAL SUB-TOTAL & $\$$ & - \\
INSTRUMENT SUB-TOTAL & $\$$ & - \\
\hline
\end{tabular}

PROCESS PIT COMPONENTS SUB-TOTAL $\$ 1,452,000$

\begin{tabular}{|lrr|}
\hline OVERHEAD LESS EQUIPMENT & $18 \%$ & $\$ 34,560$ \\
PROFIT (SUB-TOTAL \& OVERHEAD) & $10 \%$ & $\$ 148,656$ \\
\hline & & $\$ 1,635,216$ \\
\hline
\end{tabular}


WHC-SD-SNF-CDR-007, REV. 0

\begin{tabular}{|c|c|c|c|c|c|c|c|c|c|c|c|c|}
\hline \multirow[b]{2}{*}{ DESCRIPTION } & & \multirow[b]{2}{*}{ QTY UNIT } & \multicolumn{2}{|c|}{ EQUIPMENT } & \multicolumn{2}{|c|}{ DUCTINGIPIPING } & \multicolumn{2}{|c|}{ INSULATION } & \multicolumn{2}{|c|}{ ELECTRICAL } & \multicolumn{2}{|c|}{ INSTRUMENT } \\
\hline & & & UNIT & TOTAL & FACTOR & TOTAL & FACTOR & TOTAL & FACTOR & TOTAL & FACTOR & TOTAL \\
\hline SERVICE MODULE & & & & & & & & & & & & \\
\hline PUMP - TURBO VACUUM & VAC-VAC-3041 & $1 \mathrm{EA}$ & $\$ 14,350$ & $\$ 14,350$ & 2.00 & $\$ 28,700$ & & $\$$ & 0.10 & $\$ 1,435$ & 0.30 & $\$ 4,305$ \\
\hline PUMP - SCROLL ROUGHER & VAC-VAC-3042 & $1 \mathrm{EA}$ & $\$ 8,100$ & $\$ 8,100$ & 2.00 & $\$ 16,200$ & & & 0.10 & $\$ 810$ & 0.30 & $\$ 2,430$ \\
\hline FILTER - HEPA TRAIN & PV-F-2041,2,4,5 & 4 EA & $\$ 6,875$ & $\$ 27,500$ & 0.10 & $\$ 2,750$ & 0.10 & $\$ 2,750$ & 0.05 & $\$ 1,375$ & 0.52 & $\$ 14,300$ \\
\hline BLOWER - SINGLE STAGE & PV-BLO-2043,6 & $2 \mathrm{EA}$ & $\$ 10,000$ & $\$ 20,000$ & 0.70 & $\$ 14,000$ & 0.15 & $\$ 3,000$ & 0.10 & $\$ 2,000$ & 0.13 & $\$ 2,600$ \\
\hline JPECAAL WSIRUMENIS & & & & & & & & & 0.25 & $\$ 12,500$ & & $\$ 50,000$ \\
\hline SERVICE MODULE SUB-TO & & & & $\$ 69,950$ & & $\$ 61,650$ & & $\$ 5,750$ & & $\$ 18,120$ & & $\$ 73,635$ \\
\hline
\end{tabular}

\section{SERVICE MODULE SUB-TOTAL SUMMARY}

\begin{tabular}{|c|c|c|}
\hline EQUIPMENT SUB-TOTAL & & $\$ 69,950$ \\
\hline DUCTING/PIPING SUB-TOTAL & & \$ 61,650 \\
\hline INSULATION SUB-TOTAL & & $\$ \quad 5,750$ \\
\hline ELECTRICAL SUB-TOTAL & & $\$ 18,120$ \\
\hline INSTRUMENT SUB-TOTAL. & & $\$ 73,635$ \\
\hline SKID ASSEMBLY COST & & $\$ 20,000$ \\
\hline SKID INSTALLATION COST & & $\$ 14,110$ \\
\hline SERVICE MODULE SUB-TOTAL & & $\$ 263,215$ \\
\hline $\begin{array}{l}\text { OVERHEAD LESS EQUIPMENT } \\
\text { PROFIT (SUB-TOTAL \& OVERHEAD) }\end{array}$ & $18 \%$ & $\$ 34,788$ \\
\hline SERVICE MODULE TOTAL. & & $\$ 327,803$ \\
\hline
\end{tabular}


WHC-SD-SNF-CDR-007, REV. 0

\begin{tabular}{|c|c|c|c|c|c|c|c|c|c|c|c|c|}
\hline DESCRIPTION & & QTY UNIT & \multicolumn{2}{|c|}{ EQUIPMENT } & \multicolumn{2}{|c|}{ DUCTINGIPIPING } & \multicolumn{2}{|c|}{ INSULATION } & \multicolumn{2}{|c|}{ ELECTRICAL } & \multicolumn{2}{|c|}{ INSTRUMENT } \\
\hline $\begin{array}{l}\text { CHILLER - SPLIT SYSTEM } \\
\text { TANK - EXPANSION } \\
\text { PUMP - CHILLED WATER } \\
\text { PUMP - CHILLED WATER } \\
\text { TANK - EXPANSION } \\
\text { HEAT EXCHANGER - GLYCOL } \\
\text { PUMP - COOLING WATER } \\
\text { PUMP - COOLING WATER } \\
\text { PROCESS STACK } \\
\text { STACK MONITORING }\end{array}$ & $\begin{array}{l}\text { CHW-CH-2026 } \\
\text { CHW-ACC-2021 } \\
\text { CHW-P-2022 } \\
\text { CHW-P-2023 } \\
\text { CW-ACC-2011 } \\
\text { CW-HX-2014 } \\
\text { CW-P-2012 } \\
\text { CW-P-2013 }\end{array}$ & $\begin{array}{l}1 \text { EA } \\
1 \text { EA } \\
1 \text { EA } \\
1 \text { EA } \\
1 \text { EA } \\
1 \text { EA } \\
1 \text { EA } \\
1 \text { EA } \\
1 \text { EA }\end{array}$ & $\begin{array}{l}\$ 38,540 \\
\$ 1,629 \\
\$ 2,000 \\
\$ 2,000 \\
\$ 1,629 \\
\$ 3,103 \\
\$ 1,600 \\
\$ 1,600 \\
\$ 7,500\end{array}$ & $\begin{array}{rr}\$ & 38,540 \\
\$ & 1,629 \\
\$ & 2,000 \\
\$ & 2,000 \\
\$ & 1,629 \\
\$ & 3,103 \\
\$ & 1,600 \\
\$ & 1,600 \\
\$ & 7,500\end{array}$ & $\begin{array}{l}1.00 \\
1.20 \\
0.70 \\
0.70 \\
1.20 \\
1.00 \\
0.70 \\
0.70\end{array}$ & $\begin{array}{rr}\$ 38,540 \\
\$ 1,955 \\
\$ 1,400 \\
\$ 1,400 \\
\$ 1,955 \\
\$ 3,103 \\
\$ 1,120 \\
\$ 1,120 \\
\$ & -\end{array}$ & $\begin{array}{l}0.20 \\
0.15 \\
0.15 \\
0.20 \\
0.15 \\
0.15\end{array}$ & $\begin{array}{rr}\$ 7,708 \\
\$ & - \\
\$ & 300 \\
\$ & 300 \\
\$ & - \\
\$ & 621 \\
\$ & 240 \\
\$ & 240 \\
\$ & -\end{array}$ & $\begin{array}{l}0.10 \\
0.10 \\
0.10 \\
\\
0.10 \\
0.10 \\
0.25\end{array}$ & $\begin{array}{rr}\$ & 3,854 \\
\$ & - \\
\$ & 200 \\
\$ & 200 \\
\$ & - \\
\$ & - \\
\$ & 160 \\
\$ & 160 \\
\$ & - \\
\$ 36,225\end{array}$ & $\begin{array}{l}0.25 \\
0.50 \\
0.50 \\
0.25 \\
0.13 \\
0.13\end{array}$ & $\begin{array}{rr}\$ & 9,635 \\
\$ & - \\
\$ & 1,000 \\
\$ & 1,000 \\
\$ & - \\
\$ & 776 \\
\$ & 208 \\
\$ & 208 \\
\$ & \\
\$ 144,900\end{array}$ \\
\hline AUXILIARY FACILITIES SUB & & & & 559,601 & & $\$ 50,593$ & & $\$ 9,409$ & & $\$ 40,799$ & & $\$ 157,727$ \\
\hline
\end{tabular}

\section{AUXILIARY FACILITIES SUB-TOTAL SUMMARY}

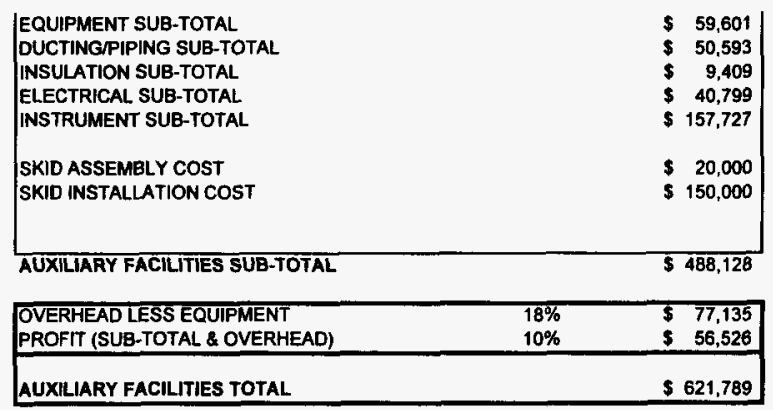


WHC-SD-SNF-CDR-007, REV. 0

\begin{tabular}{|c|c|c|c|c|c|}
\hline DESCRIPTION & $\begin{array}{c}\begin{array}{c}\text { EQUIPMENT } \\
\text { UNIT }\end{array} \\
\end{array}$ & $\begin{array}{c}\text { DUCTING/PIPING } \\
\text { TOTAL }\end{array}$ & $\begin{array}{c}\text { INSULATION } \\
\text { TOTAL }\end{array}$ & $\begin{array}{c}\text { ELECTRICAL } \\
\text { TOTAL } \\
\end{array}$ & $\begin{array}{c}\text { INSTRUMENT } \\
\text { TOTAL } \\
\end{array}$ \\
\hline $\begin{array}{l}\text { PROCESS ENCLOSURE } \\
\text { TITAN VII MANIPULATOR } \\
\text { HELIUM LEAK DETECTOR } \\
\text { WELDER WITH SUPPLY } \\
\text { DRUM BAG STATION } \\
\text { TOW MOTOR } \\
\text { ENCLOSURE } \\
\text { CONTROLS }\end{array}$ & $\begin{array}{rr}\$ & 250,000 \\
\$ & 20,000 \\
\$ & 15,000 \\
\$ & 45,000 \\
\$ & 10,000 \\
\$ & 150,000\end{array}$ & & & 2,500 & 10,000 \\
\hline PROCESSENCLOSURE SUB-TOTAL & 490,000 & $\$$ & $\$$ & 2,500 & 10,000 \\
\hline
\end{tabular}

\section{PROCESS ENCLOSURE SUB-TOTAL}

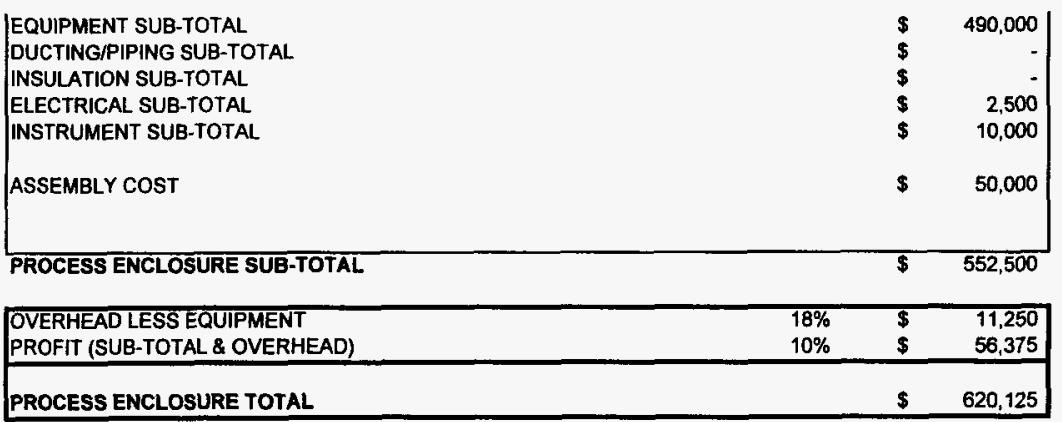




\begin{tabular}{|c|c|c|c|c|c|c|}
\hline \multicolumn{3}{|c|}{ TAG \# } & ITEM & EQUIPMENT SPECIFICATIONS & ELECTRICAL LOAD/SERVICE & EQUIPMENT COST \\
\hline A & 1000 & & Beta/Gamma Particulate Monitor & & $115 \mathrm{VAC} / 60 \mathrm{~Hz} / 15 \mathrm{~A}$ & $\$ 54,700$ \\
\hline A & 2000 & & Beat/Gamma Particulate Monitor & & $115 \mathrm{VAC} / 60 \mathrm{Kz} / 15 \mathrm{~A}$ & $\$ 54,700$ \\
\hline A & 3000 & & Alpha/Beta Particulate Monitor & & $120 / 240 \mathrm{VAC} / 50 / 60 \mathrm{~Hz}$ & $\$ 5,000$ \\
\hline A & 3000 & & Tritium Monitor & & $150 / 230 \mathrm{VAC} / 50 / 60 \mathrm{~Hz}$ & $\$ 6,000$ \\
\hline A & 4000 & & Alpha/Beta Particulate Monitor & & $120 / 240 \mathrm{VAC} / 50 / 60 \mathrm{~Hz}$ & $\$ 5,000$ \\
\hline A & 4001 & & Tritium, Monitor & & $150 / 230 \mathrm{VAC} / 50 / 60 \mathrm{~Hz}$ & $\$ 6,000$ \\
\hline A & 5000 & & Record Air Sampler & & $1 / 4 \mathrm{hp} / 115 \mathrm{~V} / 6 \mathrm{OHz} / 5 \mathrm{~A}$ & $\$ 1,250$ \\
\hline A & 6000 & & Record Air Sampler & & $1 / 4 \mathrm{hp} / 115 \mathrm{~V} / 6 \mathrm{OHz} / 5 \mathrm{~A}$ & $\$ 1.250$ \\
\hline $\mathrm{CHW}$ & $A C C$ & 2021 & Expansion Tank & 72 liter capacity & None & $\$ 1,629$ \\
\hline $\mathrm{CHW}$ & $\mathrm{CH}$ & 2026 & Chiller & 25 ton & $30 \mathrm{KW} / 440 \mathrm{~V} / 3$ phase & $\$ 38,450$ \\
\hline CHW & $\mathbf{P}$ & 2022 & Chilled Water Circ.Pump & $15 \mathrm{gpm} @ 40 \mathrm{ft}$ TDH & $1 / 2 \mathrm{hp} / 20 \mathrm{~V} / 1$ phase & $\$ 2,000$ \\
\hline $\mathrm{CHW}$ & $\mathbf{P}$ & 2023 & Chilled Water Circ.Pump & $15 \mathrm{gpm} @ 40 \mathrm{ft} \mathrm{TDH}$ & $1 / 2 \mathrm{hp} / 20 \mathrm{~V} / 1$ phase & $\$ 2,000$ \\
\hline $\mathrm{CW}$ & $A C C$ & 2011 & Expansion Tank & 72 liter capacity & None & $\$ 1,629$ \\
\hline $\mathrm{CW}$ & $H X$ & 2014 & Glycol/CW Exchr & $240,000 \mathrm{BTU} / \mathrm{hr}$ & None & $\$ 3,103$ \\
\hline CW & $P$ & 2012 & CW Pump & $50 \mathrm{gpm} @ 115 \mathrm{ft}$ TDH & $3 \mathrm{hp} / 230 \mathrm{~V} / 1$ phase & $\$ 1,582$ \\
\hline CW & $\mathbf{P}$ & 2013 & CW Pump & $50 \mathrm{gpm} @ 115 \mathrm{ft}$ TDH & $3 \mathrm{hp} / 230 \mathrm{~V} / 1$ phase & $\$ 1,582$ \\
\hline MCS & CLR & 1153 & MCO Chiller Exchanger & 94,000 BTU/hr & None & $\$ 5,073$ \\
\hline MCS & CLR & 1253 & MCO Chiller Exchanger & $94,000 \mathrm{BTU} / \mathrm{hr}$ & None & $\$ 5,073$ \\
\hline MCS & CLR & 1353 & MCO Chiller Exchanger & $94,000 \mathrm{BTU} / \mathrm{hr}$ & None & $\$ 5,073$ \\
\hline MCS & CLR & 1453 & MCO Chiller Exchanger & $94,000 \mathrm{BTU} / \mathrm{hr}$ & None & $\$ 5,073$ \\
\hline MCS & CLR & 1553 & MCO Chiller Exchanger & $94,000 \mathrm{BTU} / \mathrm{hr}$ & None & $\$ 5,073$ \\
\hline MCS & CLR & 1653 & MCO Chiller Exchanger & $94,000 \mathrm{BTU} / \mathrm{hr}$ & None & $\$ 5,073$ \\
\hline PG & $A C C$ & 1131 & Fitter Blowback Reservoir & 10 liters & None & $\$ 1,000$ \\
\hline PG & ACC & 1231 & Filter Blowback Reservoir & 10 liters & None & $\$ 1,000$ \\
\hline PG & ACC & 1331 & Filter Blowback Reservoir & 10 liters & None & $\$ 1,000$ \\
\hline PG & ACC & 1431 & Filter Blowback Reservoir & 10 liters & None & $\$ 1,000$ \\
\hline$P G$ & $\mathrm{ACC}$ & 1531 & Filter Blowback Reservoir & 10 liters & None & $\$ 1,000$ \\
\hline$P G$ & $\mathrm{ACC}$ & 1631 & Filter Blowback Reservoir & 10 liters & None & $\$ 1,000$ \\
\hline PHS & BLO & $\$ 151$ & HeatCool Blower & 750 ACFM@ 8 in sp - 375 deg. C & $5 \mathrm{HP} / 240 \mathrm{~V} / 1$ phase & $\$ \$ 1,000$ \\
\hline PHS & BLO & 1251 & Heat/Cool Blower & 750 ACFM@ 8 in sp - 375 deg. C & $5 \mathrm{HP} / 240 \mathrm{~V} / 1$ phase & $\$ 11,000$ \\
\hline PHS & BLO & 1351 & Heat/Cool Blower & 750 ACFM@ 8 in sp - 375 deg. C & $5 \mathrm{HP} / 240 \mathrm{~V} / 1$ phase & $\$ 11,000$ \\
\hline PHS & BLO & 1451 & Heat/Cool Blower & 750 ACFM@ 8 in sp - 375 deg. C & $5 \mathrm{HP} / 240 \mathrm{~V} / 1$ phase & $\$ 11,000$ \\
\hline PHS & BLO & 1551 & Heat/Cool Blower & 750 ACFM@ 8 in sp - 375 deg. C & $5 \mathrm{HP} / 240 \mathrm{~V} / 1$ phase & $\$ 11,000$ \\
\hline PHS & BLO & 1651 & Heat/Cool Blower & 750 ACFM@ 8 in sp - 375 deg. C & $5 \mathrm{HP} / 240 \mathrm{~V} / 1$ phase & $\$ 11,000$ \\
\hline PHS & OVEN & 1154 & MCO Oven & & None & $\$ 140,000$ \\
\hline PHS & OVEN & 1254 & MCo Oven & & None & $\$ 140,000$ \\
\hline PHS & OVEN & 1354 & MCO Oven & & None & $\$ 140,000$ \\
\hline PHS & OVEN & 1454 & MCO Oven & & None & $\$ 140,000$ \\
\hline PHS & OVEN & 1554 & MCO Oven & & None & $\$ 140,000$ \\
\hline PHS & OVEN & 1654 & MCo Oven & & None & $\$ 140,000$ \\
\hline PHS & $\mathrm{HCL}$ & 1152 & MCO Heating Element & $60 \mathrm{~W}^{\prime} \mathrm{in} \wedge 2$ & $25 \mathrm{KW} / 240 \mathrm{~V} / 1$ phase & $\$ 8,800$ \\
\hline
\end{tabular}




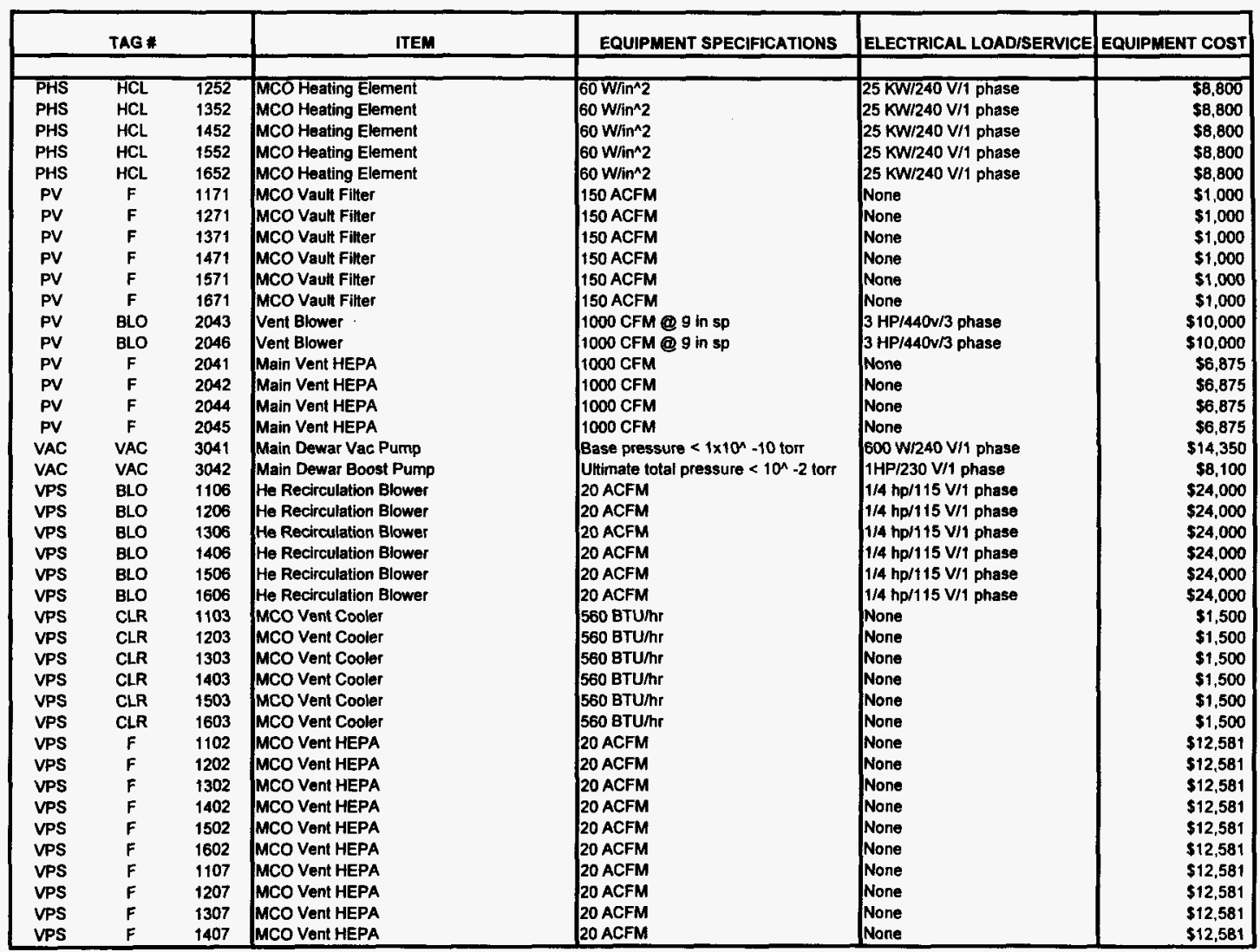


WHC-SD-SNF-CDR-007, REV. 0

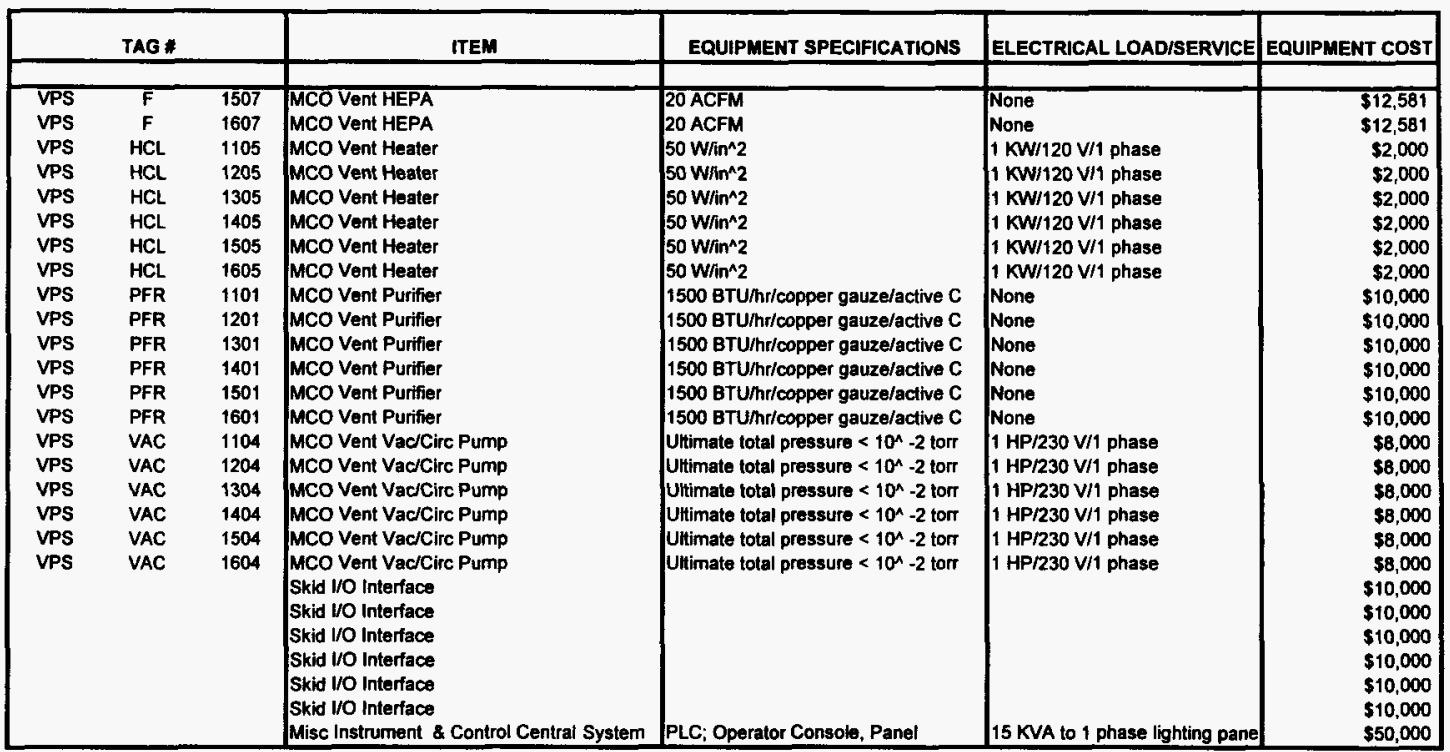


WESTINGHOUSE HANFORD COMPANY

Hot Conditioning Syatem Equipment

Contract \#MW6-SWV-310416, Tack 17
MERRICK \& COMPANY

Advanced Technology Sector Project No. 30012318

WHC-SD-SNF-CDR-007, Revision 0

\section{APPENDICES}

APPENDIX G

CALCULATIONS

G- $j$ 


\section{Appendix G \\ CALCULATIONS}

\section{CALCULATION LOG}

\begin{tabular}{|l|l|l|r|c|}
\hline $\begin{array}{c}\text { Calc } \\
\text { No. }\end{array}$ & $\begin{array}{c}\text { Rev. } \\
\text { No. }\end{array}$ & \multicolumn{1}{|c|}{ Description } & Date & Page \\
\hline HVC-1 & Initial & General Comments & $5 / 20 / 96$ & G-1 \\
\hline HVC-2 & Initial & MCO Heat Transfer Coefficients & $5 / 7 / 96$ & G-2 \\
\hline HVC-3 & Initial & MCO Heating Temperature Profile & $5 / 13 / 96$ & G-8 \\
\hline HVC-4 & Initial & MCO Cooling Temperature Profile & $5 / 13 / 96$ & G-16 \\
\hline HVC-5 & Initial & Material Balance Calculations & $6 / 12 / 96$ & G-24 \\
\hline HVC-6 & Initial & Temperature Diff. on MCO during Cool-Down & $6 / 25 / 96$ & G-40 \\
\hline HVC-7 & Initial & Heat Exchanger Duty - All Units & $6 / 24 / 96$ & G-47 \\
\hline HVC-8 & Initial & WTTNESS Model Run & $7 / 25 / 96$ & G-51 \\
\hline
\end{tabular}



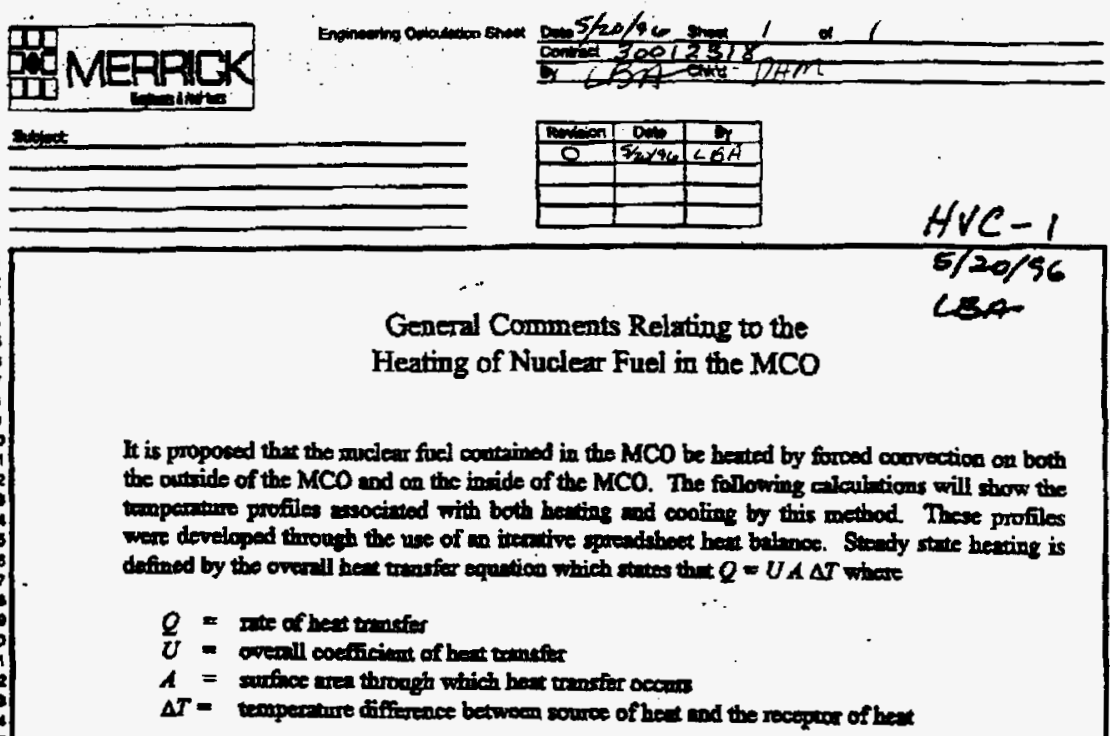

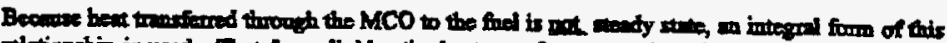

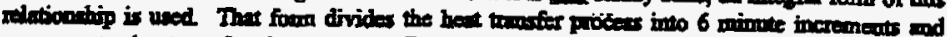

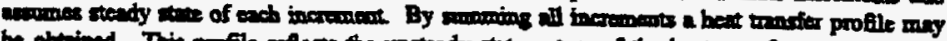

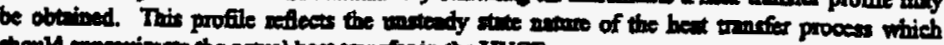
chould sproximate the motul bert rensiex in the FVCE prosess.

References:

Kem, D. Q.. Process Hear Tranafer. New Yot" MoOmw Fill, 1950.

Hundbook of Chemistry and Physict. 37th ed. Clevelend: Chemical Rabber Publishing $\mathrm{Co}$, 1955.

Perry, R H. Chentical Engineers Fandbook th ed. New Yotk: MeGraw Fill, 1984.

MoCube and Swith. Unit Operations of Chented Erginuerting. Now Yort. MoGnw fall, 1956:

Ryerson. Steel and Aluminum Data Boak

MCO imerface Characteristscs. Rev of Jan 18, 1996.

Crane Technieal Poper 410 - Flow of Fuids. Chicaso: Crene Co, 1965.

$$
G-1
$$



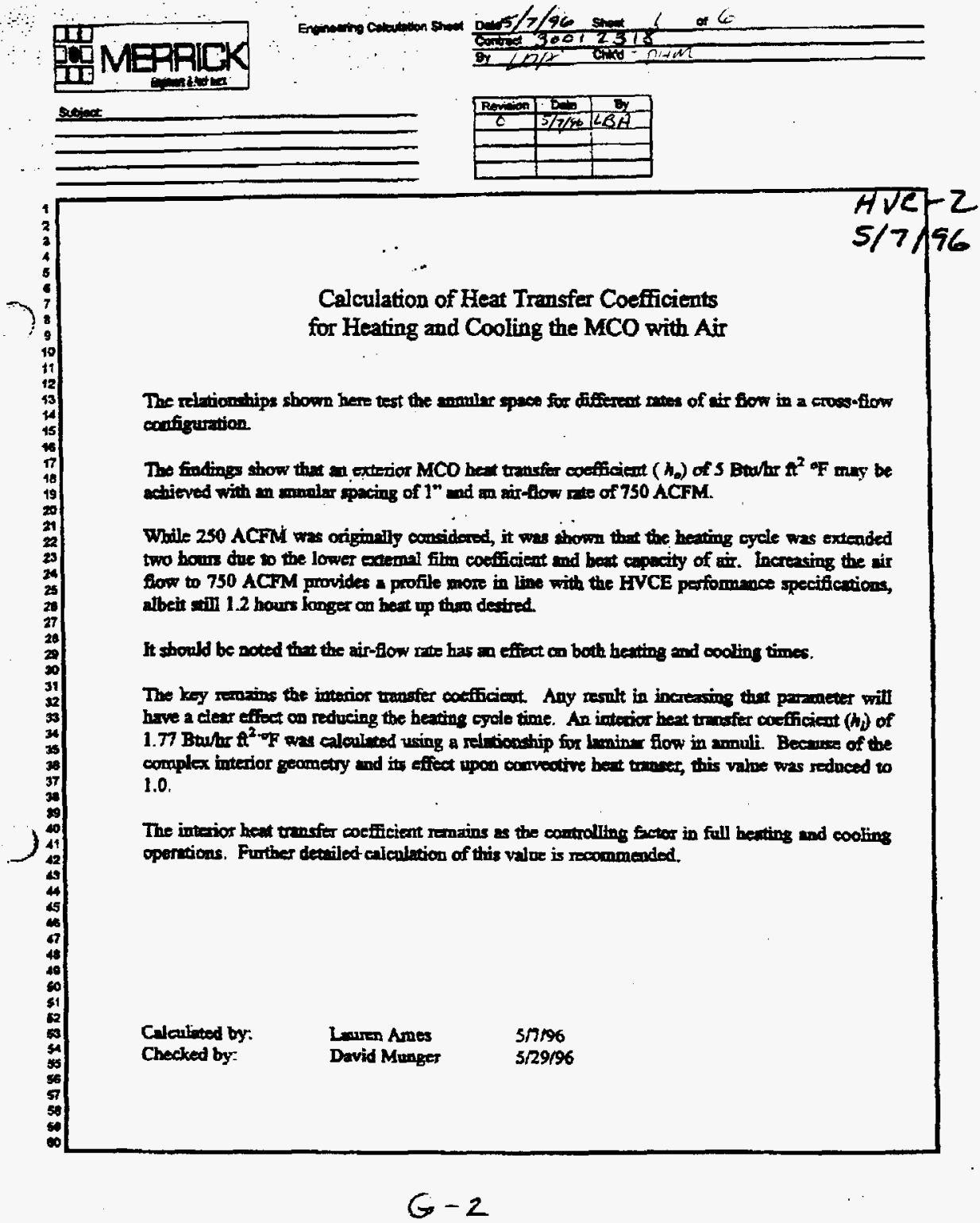


\section{Calculation of Interior Heat Transfer Coefficient for MCO to Fuel Heating}

The relationships used here are taken from Perry, pages 10-13 and 10-14, as well as the sections dealing with convection appearing in the other referenced texts on heat transfer. Because the area of the fuel is so much greater than the area of the interior surface of the $\mathrm{MCO}$, the rate of transfer from the MCO to the helium controls, not the rate of heat transfer from the helium to the fuel

Try forced convection with 10 SCFM purge rate:

$\begin{array}{lll}\text { MCO Volume Empty } & = & 272 \mathrm{gal} \\ \text { MCO Volume Loaded } & = & 134 \mathrm{gal} \\ \text { Void Volume } & = & 138 \mathrm{gal}\end{array}$

For heat transfer calcs, assume this is annular volume:

Cross section rods $=\frac{134 \text { gal } .}{7.48 \mathrm{gal}} \mathrm{ft}^{3} / 160 / 12 \mathrm{ft}=1.34 \mathrm{ft}^{2}$

$$
\begin{aligned}
& \pi r^{2}=1.34 \mathrm{ft}^{2} \quad r=0.65 \mathrm{ft} \quad D_{1}=1.31 \mathrm{ft} \quad D=24.5-1 \\
& =23.5 / 12 \\
& =1.96 \mathrm{ft}
\end{aligned}
$$

$N_{R e}=\frac{D_{e q} J \rho}{\mu} \quad D_{e q}=\frac{D_{2}^{2}-D_{1}^{2}}{D_{1}}=\frac{1.96^{2}-1.31^{2}}{1.31}=1.62 \mathrm{Ft} . \quad$ (Kern, p. 105)

at $170^{\circ} \mathrm{C}$ mean temp

$V=10 \mathrm{SCM} x\left(\frac{273+170}{273}\right)=16.2 \mathrm{ACFM}$

Natural convection calculations:

$$
\begin{aligned}
\text { Properties at mean temp.: } & =\frac{300-40}{2}+40 \\
& =170^{\circ} \mathrm{C} \\
& =338^{\circ} \mathrm{F}
\end{aligned}
$$




$$
\begin{aligned}
& N_{G R}=\frac{D^{3} q \rho^{2} \beta \Delta \mathrm{ti}}{\mu^{2}} \\
& D=2 \mathrm{ft} \\
& g=32.17 \times 3600^{2}=\mathrm{ft} / \mathrm{hr}^{2} \\
& \rho=\frac{4 \#}{359 \mathrm{ft}^{3}} \times \frac{492}{798}=0.0069 \\
& \beta=\frac{1}{T}\left(\text { ideal gas) }=\frac{1}{460+338}=0.0013\right. \\
& \Delta t_{\max }=375-40=335^{\circ} \mathrm{C}=603^{\circ} \mathrm{F}(\mathrm{max}) \\
& \Delta t_{\min }=375-300=75^{\circ} \mathrm{C}=135^{\circ} \mathrm{F}(\mathrm{min}) \\
& \mu 338^{F}=0.025 \mathrm{eN}=2.42 \times 0.25=0.0605 \mathrm{lb} / \mathrm{ft}-\mathrm{hr} \quad \text { (McCabe and Smith) } \\
& N_{G R}=\frac{(2)^{3} \times 32.17 \times 3600^{2} \times(0.0069)^{2} \times 0.0013 \times 603}{(0.0605)^{2}} \\
& N_{G R}=34 \times 10^{6} \\
& N_{\rho r}=0.761 \\
& \left.\quad 170^{\circ} C^{2} \quad \text { (Perry, } \mathrm{p} .3-247\right) \\
& 443^{\circ} \mathrm{K}
\end{aligned}
$$

$k_{H e} @ 170^{\circ} \mathrm{C}=19.3 \times 10^{-2}$ watts $/$ meter ${ }^{\circ} \mathrm{K}$

(Perry, p. 3-247)

$k_{H e}\left(170^{\circ} \mathrm{C}=0.193 \frac{\text { watt }}{\text { meter }^{\circ} K} \times \frac{3.413 \mathrm{Btu}}{\text { watt hr }} \times \frac{1 \text { meter }}{3.281 \mathrm{ft}} \times \frac{{ }^{\circ} \mathrm{K}}{1.8^{\circ} \mathrm{F}}\right.$

$$
=0.112 \mathrm{Btu} / \mathrm{hr} \mathrm{ft}^{\circ} \mathrm{F}
$$

$$
\begin{aligned}
\text { Annular area } & =\pi\left(r_{2}^{2}-r^{2}\right) \\
& =\pi\left[\left(\frac{1.96}{2}\right)^{2}-(1.31 / 2)^{2}\right] \\
& =1.67 \mathrm{ft}^{2}
\end{aligned}
$$




$$
\begin{aligned}
V e l & =\frac{16.2 \mathrm{ft}^{3}}{\min } \times \frac{1 \mathrm{~min}}{60 \mathrm{sec}} \times \frac{1}{1.67 \mathrm{ft}^{2}}=0.16 \mathrm{ft} / \mathrm{sec} . \\
\rho & =0.0069 \# / \mathrm{ft}^{3}(\mathrm{from} \mathrm{p.} \mathrm{1)} \\
\mu & =0.0605 \# / \mathrm{ft} \mathrm{hr} x \frac{1 \mathrm{hr} .}{3600 \mathrm{sec} .}=1.68 \times 10^{-5} \# / \mathrm{ft} / \mathrm{sec} . \\
N_{R e} & =\frac{1.62 \mathrm{ft} x \frac{0.16 \mathrm{ft}}{\mathrm{sec} .} \times \frac{0.0069 \#}{1.68 \times 10^{-5} \# / \mathrm{ft} .-\mathrm{sec} .}}{N_{N U}}=1.02\left(N_{P r}\right)^{0.5} \times\left(N_{R e}\right)^{0.45} \times\left(\frac{D_{a j}}{L}\right)^{0.4}\left(\frac{D_{2}}{D_{1}}\right)^{0.8}\left(\frac{\mu_{3}}{\mu_{1}}\right)^{0.14}\left(N_{E R}\right)^{0.05}
\end{aligned}
$$

for heat transfer of laminar flow in annuli (Perry, p. 10-15)

$$
\begin{aligned}
N_{P R} & =0.761 \quad N_{R E}=106.4 \quad N_{G R}=34 \times 10^{6} \\
\frac{D_{e}}{L} & =\frac{1.62}{160 / 12}=0.12 \quad \frac{\mu_{3}}{\mu_{1}}=1 \\
\frac{D_{2}}{D_{1}} & =\frac{1.96}{1.31}=1.50 \\
N_{N U} & =1.02(0.761)^{-5}(106.4)^{0.45}(0.12)^{0.4}(1.50)^{0.8}\left(34 \times 10^{6}\right)^{0.05} \\
& =10.25 \\
N_{N U} & =\frac{h_{i} L}{k} \quad k=0.112 \mathrm{Btu} / \mathrm{hr} \mathrm{ft}^{\circ} \mathrm{F} \\
L & =\text { distance across annulus }=D_{2}-D_{1}=1.96-1.31 \\
& =0.65 \mathrm{ft.} . \\
h_{i} & =\frac{\left(N_{u}\right) k}{L}=\frac{10.25 \times 0.112 \mathrm{Btu}}{0.65 \mathrm{ft} . \quad \mathrm{hr} / \mathrm{ft}^{\circ} \mathrm{F}} \\
& =1.77 \mathrm{Btu} / \mathrm{ft}^{2} \mathrm{ht.}{ }^{\circ} \mathrm{F}
\end{aligned}
$$

Because the interior of the MCO is complex and the helium must travel through fuel holding baskets, then through more than one layer of fuel rods, and because the flow (10 SCFM) upon which the calculation is based on is on the high side, the calculated coefficient of 1.77 is being discounted to $1.0 \mathrm{Btu} / \mathrm{ft}^{2} \mathrm{hr}{ }^{\circ} \mathrm{F}$. This value will be used in all subsequently derived heating and cooling temperature profiles for the MCO. 


\section{Calculation of Outside Film Coefficient for Forced Circulation}

It will be assumed that heat will be transferred via cross-flow convection in the annulus. These segmented baffles will be used to reverse flow and force cross flow.

The first iteration will calculate the outside film coefficient $\left(h_{o}\right)$ at an air flow of $250 \mathrm{ACFM}$.

Assume spacing between the outer wall of the MCO and the inner wall of the heat-transfer plenum of $I$ inch

$$
J_{H}=\frac{h_{o} D_{e}}{k}\left(\frac{C H}{k}\right)^{-1 / 3}
$$

where

$$
\begin{aligned}
& D_{e y}=4 x \text { hydraulic radius }=\frac{D_{2}^{2}-D_{1}^{2}}{D_{1}}=\frac{26^{2}-24^{2}}{24} \\
& \text { properties } \\
& \text { for average of inlet and outlet temp. } \\
& C_{\mathrm{p}} \quad=\text { for air at } 300^{\circ} \mathrm{C}\left(572^{\circ} \mathrm{F}\right)=.26 \mathrm{Btu} / \#^{\circ} \mathrm{F} \\
& k=0.0228 \mathrm{Btu}-\mathrm{Ft} / \mathrm{Ft}^{2} \mathrm{Hr}^{\circ} \mathrm{F} \\
& \mu \quad=0.028 \mathrm{e}_{\rho} \times 2.42=0.06816 / \mathrm{Ft} . \mathrm{hr}
\end{aligned}
$$

$$
\begin{aligned}
\frac{C_{\mathrm{P} \mu}}{k} & =\frac{0.26 \times 0.68}{0.0228}=.775 \\
J_{H} & =\frac{h_{o} \times 0.33}{0.0228}(0.775)^{-1 / 3} \quad J_{H}=15.8 h_{o}
\end{aligned}
$$

$$
N_{R e}=\frac{D_{e} G_{s}}{\mu}
$$$$
G_{s}=\frac{250 \times \frac{273}{200+273} \times \frac{29}{359} \times 60=699.35 \# / \mathrm{hr}}{(13 / 12)^{2} \pi-1 \pi}
$$

$$
=1282 \# / \mathrm{ft}^{2}
$$

$N_{R E}=\frac{0.33 \times 1282}{0.068}=6221$

$$
J_{H}=44 \quad h_{o}=44 / 15.8=2.8
$$

Suggest trebling the air rate rather than reducing the annular space

$$
6-6
$$




$$
\begin{aligned}
G_{s} & =3,846 \# / \mathrm{hr} \mathrm{ft}^{2} \\
N_{R e} & =\frac{0.33 \times 3846}{.0668}=19,000 \\
J_{H} & =80 \\
h_{o} & =80 / 15.8=5 \mathrm{BTU} /{ }^{\circ} \mathrm{F} \mathrm{hr} \mathrm{ft} t_{2}
\end{aligned}
$$

By reducing the annular area a factor of $3 @ 250 \mathrm{CFM}$ the same $h_{o}$ may be achieved:

$$
\begin{aligned}
& (13 / 12)^{2} \pi-1 \pi=0.55 \mathrm{ft}^{2} \\
& A_{1}=0.55 / 3=0.18 \\
& \pi r_{2}^{2}=0.18+1 \pi \quad r_{2}=1.03=0.34 \\
& \text { or } 5 / 16^{\prime \prime} \text { annular space }
\end{aligned}
$$

This is too small. Use 750 ACFM with 1 -inch annular spacing.

Note temperature profiles attached showing the effect of each condition.

$$
6-7
$$


WHC-SD-SNF-CDR-007, REV. 0

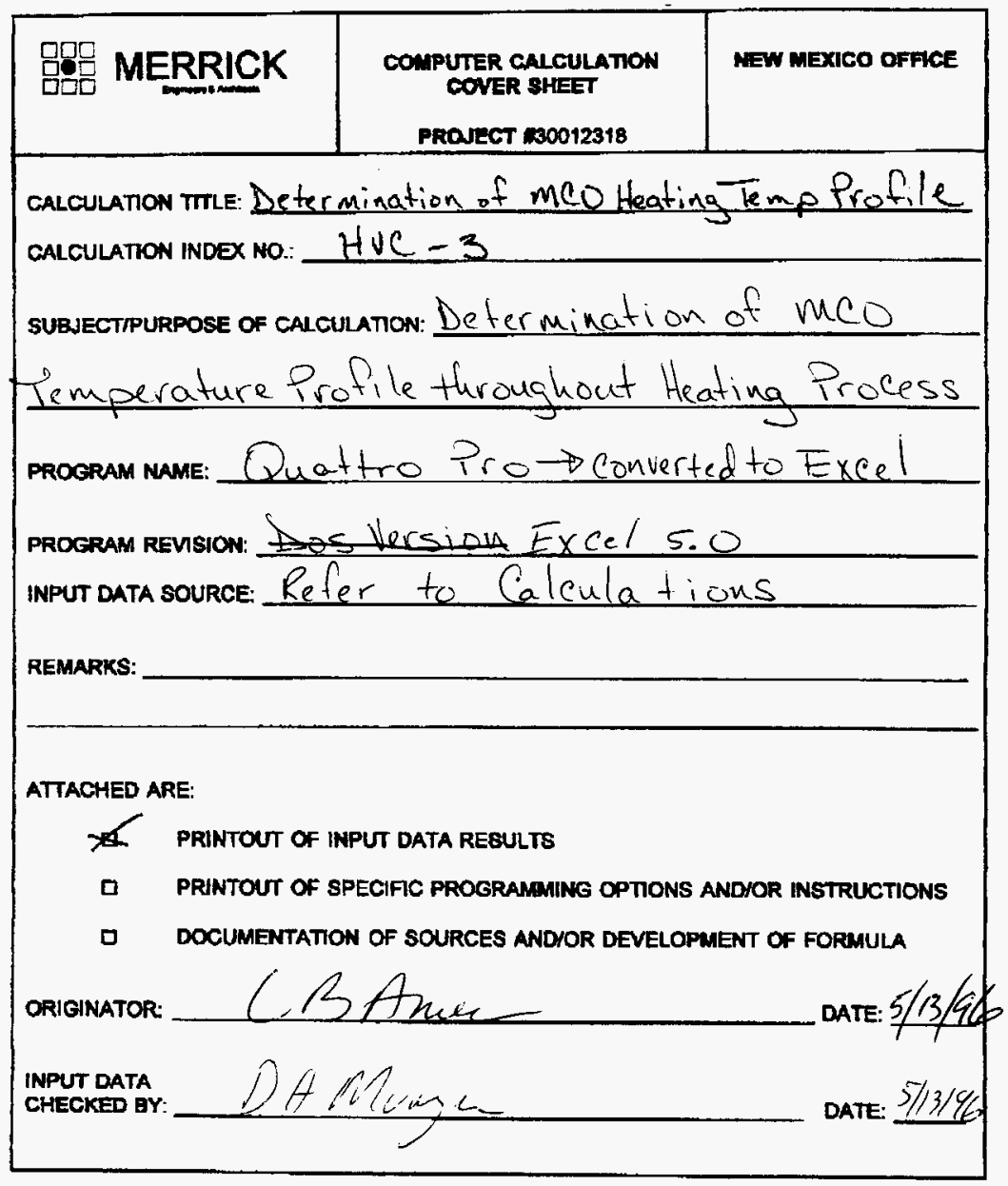

$6-8$ 
he heating profile consists of a series of heat balances on six minute increments which are integrated over time to describe the temperature profiles of interest. The calculation allows for heat from the oven and heat from the nuclear activity of the fuel.

At MCO operating pressures near atmospheric the heat input from external heating of circulating helium is minimal and is not considered in this calculation. As the internal pressure is increased, heat added to the circulating stream can be significant and this calculation would underestimate the rise in temperature for the following reasons:

1. the internal heat transfer coefficient would increase significantly,

2. conduction through the helium would be significant and would add to heat transfer from forced convection, and

3. the mass flow of the helium through an external heat source would provide a significant boost to fuel heating.

The calculations forming the temperature profile are made as follows. All English units are converted to metric in a table which is the basis for the graphical information. Spreadsheet formulas are shown in bold.

Hot air in ( $\left.{ }^{\circ} \mathrm{F}\right)$

This value is manually set at each hourly increment.

End time (hours)

Time is stepped off in 0.1 hour increments from 0 to 20 hours.

Delta temperature air to $\mathrm{MCO}\left({ }^{\circ} \mathrm{F}\right)$

This is the difference between the average hot air temperature and the wall temperature of the MCO.

(hot air temp in + hot air temp out)/2 - MCO wall temp

Net heat into $\mathrm{MCO}(\mathrm{Btu} / \mathrm{hr})$

This is the heat into the MCO from the oven less the heat from the MCO to the fuel external heat transfer coefficient $\mathrm{x}$ external surface area of $\mathrm{MCO}$ x delta temperature air to $\mathrm{MCO}$ - heat from MCO

Hot air temperature out $\left({ }^{\circ} \mathrm{F}\right)$

This is the hot air into the oven less the temperature resulting from heat transferred to the MCO

$I F$ (air temp in -(net heat into MCO + net heat from MCO - nuclear heat) / heat capacity of air $x$ mass flow rate of air) < MCO wall temperature THEN MCO wall temperature ELSE (net heat in theat from MCO - nuclear heat) / heat capacity of air $x$ mass now rate of air)

MCO wall temperature ( $\left.{ }^{\circ} \mathrm{F}\right)$

net heat in $\mathrm{x}$ (end time this period - end time previous period) / (mass MCO x specific heat MCO) + previous wall temperature

Temperature of fuel rods $\left({ }^{\circ} \mathrm{F}\right)$

previous temperature of fuel rods + (heat from MCO $x$ (end time this period - end time previous period) $/$ (mass of fuel + specific heat of fuel)

Heat from MCO (Btu/hr)

This is the heat from the MCO to the fuel plus the heat generated from the nuclear activity of the fuel (MCO wall temperature - fuel rod temperature) + nuclear energy from rods

alculations by Lauren Ames on HANHEAT3.WQ1 5/B/96

reviewed by David Munger

$6-9$ 


\begin{tabular}{|c|c|c|c|c|c|c|c|}
\hline \multicolumn{8}{|c|}{ HANHEAT3.WQ1 of $5 / 7 / 96--$ FOR CALCULATION OF HEATING TEMP PROFILE } \\
\hline \multicolumn{8}{|c|}{ Santa Fe Engineering, Ltd. - LBA } \\
\hline & & & & & & & \\
\hline \multicolumn{8}{|c|}{ hl $(B t u / l b * f t 2 * F * h r)$} \\
\hline to $\mathrm{MCO}$ ou & 5 & & \multicolumn{5}{|c|}{ AIR TEMP DATA ENTRY TAKES PLACE } \\
\hline AI (ft2) of & 92 & & \multicolumn{2}{|c|}{ IN COLUMN $\mathrm{N}$} & & & \\
\hline & & & & & & & \\
\hline \multicolumn{8}{|c|}{ h2 (Btu/1b*ft2*F*hr) } \\
\hline thru MCO & 1 & & & & & & \\
\hline A2 (ft2) of & 83 & & & & & & \\
\hline$M * C$ (air in & 397.8 & [assume: & S $750 \mathrm{ACFN}$ & & & & \\
\hline$m 1 * c 1(M C O \quad i$ & 471.6 & & & & & & \\
\hline $\mathrm{m} 2 * \mathrm{c} 2$ (fuel & 451 & & & & & & \\
\hline & & & & & & & \\
\hline $\mathrm{T} 1=$ air tem & programr & med as $\mathbf{s}$ & hown & & & & \\
\hline \multicolumn{8}{|c|}{$\mathrm{T} 2=$ air temp out (F) } \\
\hline \multicolumn{8}{|c|}{ tl $=$ MCo wall temp at } \\
\hline \multicolumn{8}{|c|}{ increment end (F) } \\
\hline \\
\hline \multicolumn{8}{|c|}{ increment end (F) } \\
\hline & & & & & & & \\
\hline \multicolumn{8}{|c|}{$\mathrm{Q}=$ internal rad heat } \\
\hline \multirow{2}{*}{ generate } & 835 & & & & & & \\
\hline & & & & Hot & & & \\
\hline Hot air & End & Delta & Net Heat & air & $\mathrm{MCO}$ & Fuel & Heat \\
\hline in & Time & temp. & into $\mathrm{MCO}$ & out & wall & rods & from $\mathrm{MCO}$ \\
\hline (T1) & (hrs) & air/MCO & (Btu/hr) & (T2) & $(t 1)$ & $(t 2)$ & (Btu/hr) \\
\hline & & & & & & & \\
\hline 266 & 0.0 & 162 & & & 104 & 104 & \\
\hline 266 & 0.1 & 65 & 74,520 & 104 & 120 & 104 & 4,161 \\
\hline 266 & 0.2 & 106 & 25,830 & 197 & 125 & 105 & 4,539 \\
\hline 266 & 0.3 & 73 & 44,281 & 149 & 135 & 106 & 5,235 \\
\hline 266 & 0.4 & 86 & 28,196 & 188 & 141 & 107 & 5,635 \\
\hline 266 & 0.5 & 71 & 34,115 & 172 & 148 & 108 & 6,132 \\
\hline 266 & 0.6 & 75 & 26,581 & 190 & 154 & 110 & 6,487 \\
\hline 266 & 0.7 & 67 & 27,784 & 186 & 159 & 111 & 6,856 \\
\hline 266 & 0.8 & 66 & 23,795 & 195 & 164 & 113 & 7,149 \\
\hline 266 & 0.9 & 62 & 23,319 & 196 & 169 & 114 & 7,428 \\
\hline 392 & 1.0 & 123 & 20,880 & 201 & 174 & 116 & 7,659 \\
\hline 392 & 1.1 & 99 & 48,869 & 174 & 184 & 118 & 8,378 \\
\hline 392 & 1.2 & 96 & 37,036 & 184 & 192 & 119 & 8,875 \\
\hline 392 & 1.3 & 92 & 35,309 & 192 & 200 & 121 & 9,333 \\
\hline 392 & 1.4 & 89 & 33,213 & 200 & 207 & 123 & 9,746 \\
\hline 392 & 1.5 & 86 & 31,283 & 207 & 213 & 126 & 10,117 \\
\hline 392 & 1.6 & 83 & 29,480 & 213 & 219 & 128 & 10,450 \\
\hline 392 & 1.7 & 80 & 27,797 & 219 & 225 & 130 & 10,747 \\
\hline 392 & 1.8 & 78 & 26,227 & 225 & 231 & 133 & 11,011 \\
\hline
\end{tabular}


WHC-SD-SNF-CDR-007, REV. 0

\begin{tabular}{|c|c|c|c|c|c|c|c|}
\hline & & & & Hot & & & \\
\hline Hot air & End & Delta & Net Heat & air & $\mathrm{MCO}$ & Fuel & Heat \\
\hline in & Time & temp. & into $\mathrm{MCO}$ & out & wall & rods & from MCO \\
\hline (T1) & (hrs) & $\operatorname{air} / \mathrm{MCO}$ & (Btu/hr) & (T2) & $(t 1)$ & $(t 2)$ & (Btu $/ \mathrm{hr}$ ) \\
\hline 392 & 1.9 & 75 & 24,761 & 231 & 236 & 135 & 11,244 \\
\hline 518 & 2.0 & 136 & 23,391 & 236 & 241 & 138 & $\frac{1,244}{11,449}$ \\
\hline 518 & 2.1 & 128 & 51,093 & 241 & 252 & 140 & 12,137 \\
\hline 518 & 2.2 & 123 & 46,561 & 252 & 262 & 143 & 12,733 \\
\hline 518 & 2.3 & 119 & 43,915 & 262 & 271 & 146 & 13,272 \\
\hline 518 & 2.4 & 115 & 41,364 & 271 & 280 & 149 & 13,756 \\
\hline 518 & 2.5 & 111 & 38,987 & $28 \overline{0}$ & 288 & 152 & 14,189 \\
\hline 518 & 2.6 & 107 & 36,769 & 288 & 296 & 155 & 14,575 \\
\hline 518 & 2.7 & 104 & 34,698 & 296 & 303 & 158 & 14,917 \\
\hline 518 & 2.8 & 100 & 32,764 & 303 & 310 & 161 & 15,219 \\
\hline 518 & 2.9 & 97 & 30,959 & 310 & 317 & 165 & 15,484 \\
\hline 617 & 3.0 & 144 & 29,272 & 317 & 323 & 168 & 15,714 \\
\hline 617 & 3.1 & 136 & 50,466 & 323 & 334 & 172 & 16,313 \\
\hline 617 & 3.2 & 132 & 46,372 & 334 & 344 & 175 & 16,829 \\
\hline 617 & 3.3 & 127 & 43,795 & 344 & 353 & 179 & 17,290 \\
\hline 617 & 3.4 & 123 & 41,323 & 353 & 362 & 183 & 17.699 \\
\hline 617 & 3.5 & 119 & 39,020 & 362 & 370 & 187 & 18,060 \\
\hline 617 & 3.6 & 116 & 36,868 & 370 & 378 & 191 & 18,377 \\
\hline 617 & 3.7 & 112 & 34,858 & 378 & 385 & 195 & 18,652 \\
\hline 617 & 3.8 & 109 & 32,981 & 385 & 392 & 199 & 18,889 \\
\hline 617 & 3.9 & 106 & 31,227 & 392 & 399 & 203 & 19,091 \\
\hline 662 & 4.0 & 125 & 29,587 & 399 & 405 & 207 & 19,261 \\
\hline 662 & 4.1 & 120 & 38,405 & 405 & 413 & 212 & 19,582 \\
\hline 662 & 4.2 & 117 & 35,781 & 413 & 421 & 216 & 19,851 \\
\hline 662 & 4.3 & 113 & 33,894 & 421 & 428 & 220 & 20,082 \\
\hline 662 & 4.4 & 110 & 32,102 & 428 & 435 & 225 & 20,278 \\
\hline 662 & 4.5 & 107 & 30,428 & 435 & 441 & 229 & 20,440 \\
\hline 662 & 4.6 & 104 & 28,864 & 441 & 447 & 234 & 20,572 \\
\hline 662 & 4.7 & 102 & 27,400 & 447 & 453 & 238 & 20,676 \\
\hline 662 & 4.8 & 99 & 26,032 & 453 & 459 & 243 & 20,753 \\
\hline 662 & 4.9 & 96 & 24,751 & 459 & 464 & 248 & 20,807 \\
\hline 707 & 5.0 & 117 & 23,553 & 464 & 469 & 252 & 20,839 \\
\hline 707 & 5.1 & 112 & 32,781 & 469 & 476 & 257 & 21,032 \\
\hline 707 & 5.2 & 109 & 30,539 & 476 & 482 & 261 & 21,182 \\
\hline 707 & 5.3 & 106 & 29,008 & 482 & 488 & 266 & 21,303 \\
\hline 707 & 5.4 & 103 & 27,548 & 488 & 494 & 271 & 21,396 \\
\hline 707 & 5.5 & 101 & 26,183 & 494 & 500 & 276 & 21,463 \\
\hline 707 & 5.6 & 98 & 24,905 & 500 & 505 & 280 & 21,506 \\
\hline 707 & 5.7 & 96 & 23,710 & 505 & 510 & 285 & 21,528 \\
\hline 707 & 5.8 & 94 & 22,590 & 510 & 515 & 290 & 21,529 \\
\hline 707 & 5.9 & 91 & 21,542 & 515 & 520 & 295 & 21,512 \\
\hline 707 & 6.0 & 89 & 20,559 & 520 & 524 & 299 & 21,478 \\
\hline 707 & 6.1 & 87 & 19,639 & 524 & 528 & 304 & 21,428 \\
\hline
\end{tabular}


WHC-SD-SNF-CDR-007, REV, 0

\begin{tabular}{|c|c|c|c|c|c|c|c|}
\hline & & & & Hot & & & \\
\hline Hot air & End & Delta & Net Heat & air & MCO & Fuel & Heat \\
\hline in & Time & temp. & into $\mathrm{MCO}$ & out & waII & rods & from $\mathrm{Mco}$ \\
\hline (TI) & (hrs) & $\operatorname{air} / \mathrm{MCO}$ & (Btu/hr) & $(T 2)$ & $(t-1)$ & $(t 2)$ & (Btu/hr) \\
\hline & & & & & & & \\
\hline 707 & 6.2 & 86 & 18,775 & 528 & 532 & 309 & 21,364 \\
\hline 707 & 6.3 & 84 & 17,966 & 532 & 536 & 314 & 21,287 \\
\hline 707 & 6.4 & 82 & 17,206 & 536 & 539 & 318 & 21,199 \\
\hline 707 & 6.5 & 80 & 16,493 & 539 & 543 & 323 & 21,099 \\
\hline 707 & 6.6 & 79 & 15,823 & 543 & 546 & 328 & 20,989 \\
\hline 707 & 6.7 & 77 & 15,194 & 546 & 550 & 332 & 20,870 \\
\hline 707 & 6.8 & 76 & 14,602 & 550 & 553 & 337 & 20,743 \\
\hline 707 & 6.9 & 74 & 14,046 & 553 & 556 & 342 & 20,608 \\
\hline 707 & 7.0 & 73 & 13,523 & 556 & 558 & 346 & 20,467 \\
\hline 707 & 7.1 & 71 & 13,030 & 558 & 561 & 351 & 20,320 \\
\hline 707 & 7.2 & 70 & 12,566 & 561 & 564 & 355 & $20, \overline{167}$ \\
\hline 707 & 7.3 & 69 & 12,129 & 564 & 566 & $36 \overline{0}$ & 20,009 \\
\hline 707 & 7.4 & 68 & 11,716 & 566 & 569 & 364 & 19,847 \\
\hline 707 & 7.5 & 67 & 11,327 & 569 & 571 & 369 & 19,681 \\
\hline 707 & 7.6 & 65 & 10,959 & 571 & 574 & 373 & 19,512 \\
\hline 707 & 7.7 & 64 & 10,612 & 574 & 576 & 377 & 19,340 \\
\hline 707 & 7.8 & 63 & 10,284 & 576 & 578 & 382 & 19,165 \\
\hline 707 & 7.9 & 62 & 9,973 & 578 & 580 & 386 & 18,988 \\
\hline 707 & 8.0 & 61 & 9,679 & 580 & 582 & 390 & 18,808 \\
\hline 707 & 8.1 & 60 & 9,400 & 582 & 584 & 394 & 18,628 \\
\hline 707 & 8.2 & 59 & 9,136 & 584 & 586 & 398 & 18,446 \\
\hline 707 & 8.3 & 59 & 8,886 & 586 & 588 & $40 \overline{2}$ & 18,263 \\
\hline 707 & 8.4 & 58 & 8,648 & 588 & 590 & 406 & 18,079 \\
\hline 707 & 8.5 & 57 & 8,421 & 590 & 592 & 410 & 17,894 \\
\hline 707 & 8.6 & 56 & 8,206 & 592 & 593 & 414 & 17,709 \\
\hline 707 & 8.7 & 55 & 8,001 & 593 & 595 & 418 & 17,524 \\
\hline 707 & 8.8 & 54 & 7,806 & 595 & 597 & 422 & 17,339 \\
\hline 707 & 8.9 & 53 & 7,620 & 597 & 598 & 426 & 17,154 \\
\hline 707 & 9.0 & 53 & 7,443 & 598 & 600 & 430 & 16,969 \\
\hline 707 & 9.1 & 52 & 7,273 & 600 & 602 & 434 & 16,785 \\
\hline 707 & 9.2 & 51 & 7,111 & 602 & 603 & 437 & 16,601 \\
\hline 707 & 9.3 & 50 & 6,956 & 603 & 605 & 441 & 16,418 \\
\hline 707 & 9.4 & 50 & 6,807 & 605 & 606 & 445 & 16,236 \\
\hline 707 & 9.5 & 49 & 6,665 & 606 & 607 & 448 & 16,054 \\
\hline 707 & 9.6 & 48 & 6,528 & 607 & 609 & 452 & 15,874 \\
\hline 707 & 9.7 & 48 & 6,397 & 609 & 610 & 455 & 15,694 \\
\hline 707 & 9.8 & 47 & 6,271 & 610 & 611 & 459 & 15,516 \\
\hline 707 & 9.9 & 46 & 6,150 & 611 & 613 & 462 & 15,339 \\
\hline 707 & 10.0 & 46 & 6,033 & 613 & 614 & 466 & 15,162 \\
\hline 707 & 10.1 & 45 & 5,921 & 614 & 615 & 469 & 14,988 \\
\hline 707 & 10.2 & 45 & 5,812 & 615 & 617 & 472 & 14,814 \\
\hline 707 & 10.3 & 44 & 5,708 & 617 & 618 & 476 & 14,642 \\
\hline 707 & 10.4 & 43 & 5,606 & 618 & 619 & 479 & 14,471 \\
\hline
\end{tabular}


WHC-SD-SNF-CDR-007, REV. 0

\begin{tabular}{|c|c|c|c|c|c|c|c|}
\hline & & & & Hot & & & \\
\hline Hot air & End & Delta & Net Heat & air & $\mathrm{MCO}$ & Fuel & Heat \\
\hline in & Time & temp. & into $\mathrm{MCO}$ & out & wall & rods & from $\mathrm{MCO}$ \\
\hline (TI) & (hrs) & $\mathrm{air} / \mathrm{MCO}$ & (Btu/hr) & $(\mathrm{T} 2)$ & $(t 1)$ & $(t 2)$ & (Btu/hr) \\
\hline 707 & 10.5 & 43 & 5,509 & 619 & 620 & 482 & 14,302 \\
\hline 707 & 10.6 & 42 & 5,414 & 620 & 621 & 485 & 14,134 \\
\hline 707 & 10.7 & 42 & 5,323 & 621 & 622 & 488 & 13,967 \\
\hline 707 & 10.8 & 41 & 5,234 & 622 & 623 & 492 & 13,802 \\
\hline 707 & 10.9 & 41 & 5,148 & 623 & 625 & 495 & 13,639 \\
\hline 707 & 11.0 & 40 & 5,065 & 625 & 626 & 498 & 13,477 \\
\hline 707 & 11.1 & 40 & 4,983 & 626 & 627 & 501 & 13,317 \\
\hline 707 & 11.2 & 39 & 4,905 & 627 & 628 & 504 & 13,158 \\
\hline 707 & 11.3 & 39 & 4,828 & 628 & 629 & 506 & 13,001 \\
\hline 707 & 11.4 & 38 & 4,754 & 629 & 630 & 509 & 12,845 \\
\hline 707 & 11.5 & 38 & 4,681 & 630 & 631 & 512 & 12,691 \\
\hline 707 & 11.6 & 37 & 4,610 & 631 & 632 & 515 & 12,539 \\
\hline 707 & 11.7 & 37 & 4,541 & 632 & 633 & 518 & 12,388 \\
\hline 707 & 11.8 & 36 & 4,474 & 633 & 634 & 521 & 12,239 \\
\hline 707 & 11.9 & 36 & 4,408 & 634 & 635 & 523 & 12,091 \\
\hline 707 & 12.0 & 35 & 4,344 & 635 & 636 & 526 & 11,945 \\
\hline 707 & 12.1 & 35 & 4,282 & 636 & 636 & 529 & 11,801 \\
\hline 707 & 12.2 & 34 & 4,220 & 636 & 637 & 531 & 11,658 \\
\hline 707 & 12.3 & 34 & 4,160 & 637 & 638 & 534 & 11,516 \\
\hline 707 & 12.4 & 34 & 4,102 & 638 & 639 & 536 & 11,377 \\
\hline 707 & 12.5 & 33 & 4,044 & 639 & 640 & 539 & 11,238 \\
\hline 707 & 12.6 & 33 & 3,988 & 640 & 641 & 541 & 11,102 \\
\hline 707 & 12.7 & 32 & 3,933 & 641 & 642 & 544 & 10,967 \\
\hline 707 & 12.8 & 32 & 3,879 & 642 & 642 & 546 & 10,833 \\
\hline 707 & 12.9 & 31 & 3,826 & 642 & 643 & 549 & 10,701 \\
\hline 707 & 13.0 & 31 & 3,774 & 643 & 644 & 551 & 10,571 \\
\hline 707 & 13.1 & 31 & 3,723 & 644 & 645 & 553 & 10,442 \\
\hline 707 & 13.2 & 30 & 3,673 & 645 & 646 & 556 & 10,314 \\
\hline 707 & 13.3 & 30 & 3,624 & 646 & 646 & 558 & 10,188 \\
\hline 707 & 13.4 & 30 & 3,575 & 646 & 647 & 560 & 10,063 \\
\hline 707 & 13.5 & 29 & 3,528 & 647 & 648 & 562 & 9,940 \\
\hline 707 & 13.6 & 29 & 3,481 & 648 & 649 & 565 & 9,819 \\
\hline 707 & 13.7 & 28 & 3,435 & 649 & 649 & 567 & 9,698 \\
\hline 707 & 13.8 & 28 & 3,390 & 649 & 650 & 569 & 9,580 \\
\hline 707 & 13.9 & 28 & 3,346 & 650 & 651 & 571 & 9,462 \\
\hline 707 & 14.0 & 27 & 3,302 & 651 & 651 & 573 & 9,346 \\
\hline 707 & 14.1 & 27 & 3,260 & 651 & 652 & 575 & 9,232 \\
\hline 707 & 14.2 & 27 & 3,217 & 652 & 653 & 577 & 9,118 \\
\hline 707 & 14.3 & 26 & 3,176 & 653 & 654 & 579 & 9,006 \\
\hline 707 & 14.4 & 26 & 3,135 & 654 & 654 & 581 & 8,896 \\
\hline 707 & 14.5 & 26 & 3,094 & 654 & 655 & 583 & 8,787 \\
\hline 707 & 14.6 & 25 & 3,055 & 655 & 656 & 585 & 8,679 \\
\hline 707 & 14.7 & 25 & 3,016 & $65 \overline{6}$ & 656 & 587 & 8,572 \\
\hline
\end{tabular}


WHC-SD-SNF-CDR- $\infty 7$, REV. 0

\begin{tabular}{|c|c|c|c|c|c|c|c|}
\hline & & & & Hot & & & \\
\hline Hot air & End & Deltal & Net Heat & air & $\mathrm{MCO}$ & Fuel & Heat \\
\hline in & Time & temp. & into MCO & out & wall & rods & from MCD \\
\hline$(\mathrm{T} 1)$ & (hrs) & air/MCO & $(\mathrm{Btu} / \mathrm{hr})$ & $(\mathrm{T} 2)$ & (t1) & (t2) & (Btu/hr) \\
\hline & & & & & & & \\
\hline 707 & 14.8 & 25 & 2,977 & 656 & 657 & 589 & 8,467 \\
\hline 707 & 14.9 & 24 & 2,939 & 657 & 657 & 591 & 8,363 \\
\hline 572 & 15.0 & -43 & 2,902 & 657 & 658 & 593 & 8,260 \\
\hline 572 & 15.1 & -37 & $(28,185)$ & 658 & 652 & 595 & 7,612 \\
\hline 572 & 15.2 & -35 & $(24,646)$ & 652 & 647 & 596 & 7,038 \\
\hline 572 & 15.3 & -33 & $(23,043)$ & 647 & 642 & 598 & 6,503 \\
\hline 572 & 15.4 & -30 & $(21,462)$ & 642 & 637 & 599 & 6,005 \\
\hline 572 & 15.5 & -28 & $(19,995)$ & 637 & 633 & 601 & 5,543 \\
\hline 572 & 15.6 & -27 & $(18,629)$ & 633 & 629 & 602 & 5,113 \\
\hline 572 & 15.7 & -25 & $(17,357)$ & 629 & 626 & 603 & 4,713 \\
\hline 572 & 15.8 & -23 & $(16,173)$ & 626 & 622 & 604 & 4,342 \\
\hline 572 & 15.9 & -22 & $(15,071)$ & 622 & 619 & 605 & 3,997 \\
\hline 572 & 16.0 & -20 & $(14,045)$ & 619 & 616 & 606 & 3,676 \\
\hline 572 & 16.1 & -19 & $(13,089)$ & 616 & 613 & 607 & 3,378 \\
\hline 572 & 16.2 & -18 & $(12,199)$ & 613 & 611 & 608 & 3,101 \\
\hline 572 & 16.3 & -17 & $(11,371)$ & 611 & 608 & 608 & 2,844 \\
\hline 572 & 16.4 & -16 & $(10,599)$ & 608 & 606 & 609 & 2,605 \\
\hline 572 & 16.5 & -15 & $(9,881)$ & 606 & 604 & 609 & 2,383 \\
\hline 572 & 16.6 & -14 & $(9,212)$ & 604 & 602 & 610 & 2,177 \\
\hline 572 & 16.7 & -13 & $(8,590)$ & 602 & 600 & 610 & 1,986 \\
\hline 572 & 16.8 & -12 & $(8,010)$ & 600 & 598 & 611 & 1,809 \\
\hline 572 & 16.9 & -12 & $(7,470)$ & 598 & 597 & 611 & 1,644 \\
\hline 572 & 17.0 & -11 & $(6,967)$ & 597 & 595 & 612 & 1,491 \\
\hline 572 & 17.1 & -10 & $(6,499)$ & 595 & 594 & 612 & 1,349 \\
\hline 572 & 17.2 & -10 & $(6.063)$ & 594 & 593 & 612 & 1,218 \\
\hline 572 & 17.3 & -9 & $(5,657)$ & 593 & 591 & 613 & 1,096 \\
\hline 572 & 17.4 & -9 & $(5,279)$ & 591 & 590 & 613 & 983 \\
\hline 572 & 17.5 & -8 & $(4,927)$ & 590 & 589 & 613 & 878 \\
\hline 572 & 17.6 & -8 & $(4,599)$ & 589 & 588 & 613 & 781 \\
\hline 572 & 17.7 & -7 & $(4,294)$ & 588 & 587 & 613 & 691 \\
\hline 572 & 17.8 & -7 & $(4,009)$ & 587 & 586 & 614 & 607 \\
\hline 572 & 17.9 & -6 & $(3,744)$ & 586 & 586 & 614 & 530 \\
\hline 572 & 18.0 & -6 & $(3,498)$ & 586 & 585 & 614 & 459 \\
\hline 572 & 18.1 & -6 & $(3,268)$ & 585 & 584 & 614 & 393 \\
\hline 572 & 18.2 & -5 & $(3,054)$ & 584 & 584 & 614 & 332 \\
\hline 572 & 18.3 & -5 & $(2,854)$ & 584 & 583 & 614 & 276 \\
\hline 572 & 18.4 & -5 & $(2,668)$ & 583 & 582 & 614 & 224 \\
\hline 572 & 18.5 & -5 & $(2,495)$ & 582 & 582 & 614 & 176 \\
\hline 572 & 18.6 & -4 & $(2,334)$ & 582 & 581 & 614 & 131 \\
\hline 572 & 18.7 & -4 & $(2,184)$ & 581 & 581 & 614 & 91 \\
\hline 572 & 18.8 & -4 & $(2,044)$ & 581 & 581 & 614 & 53 \\
\hline 572 & 18.9 & -4 & $(1,913)$ & 581 & 580 & 614 & 18 \\
\hline 572 & 19.0 & -4 & $(1,792)$ & 580 & 580 & 614 & (14) \\
\hline
\end{tabular}


WHC-SD-SNF-CDR-007, REV. 0

\begin{tabular}{|r|r|r|r|r|r|r|r|}
\hline & & & Hot & & & \\
\hline Hot air & End & Delta & Net Heat & air & MCO & Fuel & Heat \\
\hline in & Time & temp. & into MCO & out & wall & rods & from MCO \\
\hline (T1) & $(\mathrm{hrs})$ & air/MCO & (Btu/hr) & $(\mathrm{T2})$ & $(\mathrm{t} 1)$ & $(\mathrm{t2})$ & $(\mathrm{Btu} / \mathrm{hr})$ \\
\hline & & & & & & & \\
\hline 572 & 19.1 & -4 & $(1,678)$ & 580 & 579 & 614 & $(43)$ \\
\hline 572 & 19.2 & -3 & $(1,573)$ & 579 & 579 & 614 & $(70)$ \\
\hline 572 & 19.3 & -3 & $(1,474)$ & 579 & 579 & 614 & $(94)$ \\
\hline 572 & 19.4 & -3 & $(1,382)$ & 579 & 578 & 614 & $(117)$ \\
\hline 572 & 19.5 & -3 & $(1,297)$ & 578 & 578 & 614 & $(138)$ \\
\hline 572 & 19.6 & -3 & $(1,217)$ & 578 & 578 & 614 & $(157)$ \\
\hline 572 & 19.7 & -3 & $(1,143)$ & 578 & 578 & 614 & $(174)$ \\
\hline 572 & 19.8 & -3 & $(1,073)$ & 578 & 577 & 614 & $(190)$ \\
\hline 572 & 19.9 & -3 & $(1,009)$ & 577 & 577 & 614 & $(204)$ \\
\hline 572 & 20.0 & -2 & $(949)$ & 577 & 577 & 614 & $(217)$ \\
\hline
\end{tabular}


WHC-SD-SNF-CDR-007, REV. 0

哭哭 MERRICK

COMPUTER CALCULATION COVER SHEET

PROJECT $\$ 30012318$

CALCULATON THE: Determination of me o Cooling temp Profile CALCULATION INDEX NO: HJC-4

SUBJECTPURPOSE OF CALCULATON: Determination of

MCO Temperature Profile throughout Cooling Process

program name: Quatre Pro $\rightarrow$ converted to Excel

PROGRAM REVISION:

inPUT data SOURCE: Refer to Calculations

REMARKS:

ATTACHED ARE:

\. PRINTOUT OF INPUT DATA RESULTS

D PRINTOUT OF SPECIFIC PROGRAMMING OPTIONS ANDNOR INSTRUCTIONS

D DOCUMENTATION OF SOURCES ANDIOR DEVELOPMENT OF FORMULA

ORIGINATOR:

CAthie

DATE: $5 / 13 / 5$ \&

INPUT DATA

Dthivgen

DATE: 5113/44

$G-16$ 
The cooling profile consists of a series of heat balances on six minute increments which are integrated over time to describe the temperature profiles of interest. The calculation allows for heat translier to the oven from the heat capacity of the MCO and its contents and heat from the nuclear activity of the fuel. The model is the same as for MCO heating except that negative heating rates indicate a reverse direction of heat flow from the MCO system to the cooler air from the oven.

At MCO operating pressures near atmospheric the heat removal from external cooling of circulating helium is minimal and is not considered in this calculation. As the intemal pressure is increased, heat removed by the circulating stream can be significant and this calculation would underestimate the rise in temperature for the following reasons:

1. the internal heat transfer coefficient would increase significantly,

2. conduction through the helium would be significant and would add to heat transfer from forced convection, and

3. the mass flow of the helium through an extemal heat source would provide a significant boost to fuel cooling.

The calculations forming the temperature profile are made as follows. All English units are converted to metric in a table which is the basis for the graphical information. Spreadsheet formulas are shown in bold.

Cool air in ( $\left.{ }^{\circ} \mathrm{F}\right)$

This value is manually set at each hourly increment.

End time (hours)

Time is stepped off in 0.1 hour increments from 0 to 20 hours.

Delta temperature air to $\mathrm{MCO}\left({ }^{\circ} \mathrm{F}\right)$

This is the difference between the average cool air temperature and the wall temperature of the MCO. (cool air temp in + cool air temp out)/2 - MCO wall temp

Net heat to MCO (Btu/hr)

This is the heat to the MCO to the oven less the heat from the MCO from the fuel. It is a negative value. external heat transfer coefficient $x$ external surface area of MCO $x$ delta temperature air to MCO heat from $\mathrm{MCO}$

Cool air temperature out $\left({ }^{\circ} \mathrm{F}\right)$

This is the cool air into the oven less the temperature resulting from heat transferred from the MCO IF(air temp in - (net heat to MCO + net heat from MCO - nuclear heat) I heat capacity of air x mass flow rate of air) < MCO wall temperature THEN (net heat in + heat from MCO - nuclear heat) / heat capacity of air $x$ mass flow rate of air) ELSE MCO wall temperature

MCO wall temperature ( $\left.{ }^{\circ} \mathrm{F}\right)$

net heat in $x$ (end time this period - end time previous period) / (mass MCO x specific heat MCO) + previous wall temperature

Temperature of fuel rods $\left({ }^{\circ} \mathrm{F}\right)$

previous temperature of fuel rods + (heat from MCO $\times$ (end time this period - end time previous period) / (mass of fuel + specific heat of fuel)

Heat from MCO (Btu/hr)

This is the heat from the MCO to the fuel plus the heat generated from the nuclear activity of the fuel. It is a negative value.

(MCO wall temperature - fuel rod temperature) + nuclear energy from rods

calculations by Lauren Ames on HANHEAT4.WQ1 5/3/96reviewed by David Munger

$$
G-17
$$




\begin{tabular}{|c|c|c|c|c|c|c|c|}
\hline HANHEAT 4.WQI & of $5 / 7$ & $96--F$ & GOR CALCUIA & ON OF & COOLII & TEMP & PROFILE \\
\hline Santa Fe Eng & ineering & g, Ltd. & - LBA & & & & \\
\hline & & & & & & & \\
\hline h1 (Btu/1b*f & $t 2 * F * h r)$ & & & & & & \\
\hline to MCO o & 5 & & AIR TEMP D & TA ENT & RY TAKE & PLACE & \\
\hline$A 1$ (ft 2 ) of & 92 & & IN COLUMN & & & & \\
\hline & & & & & & & \\
\hline h2 (Btu/lb*f & $t 2 * \mathrm{~F} * \mathrm{hr}$ & & & & & & \\
\hline thru $\mathrm{MCO}$ & 1 & & & & & & \\
\hline A2 (ft2) of & 83 & & & & & & \\
\hline & & & S 750 ACFM? & & & & \\
\hline Cp air (in & $\begin{array}{r}562 \\
471.6\end{array}$ & [assumes & S 750 ACFM] & & & & \\
\hline $\mathrm{ml}$-cl $(\mathrm{MCO}$ & 471.6 & & & & & & \\
\hline $\mathrm{m} 2 * \mathrm{c} 2$ (fuel & 451 & & & & & & \\
\hline $\mathrm{TI}=\operatorname{air}$ te & programm & ned as sl & hown & & & & \\
\hline $\mathrm{T} 2=\mathrm{air}$ ten & ap out (I & & & & & & \\
\hline$t_{1}=\mathrm{MCO}$ wal & 11 temp & at & & & & & \\
\hline increme & ent end & (F) & & & & & \\
\hline $\mathrm{t} 2=$ fuel ro & demp & at & & & & & \\
\hline increme & ent end & (F) & & & & & \\
\hline & & & & & & & \\
\hline$Q=$ internal & rad hea & & & & & & \\
\hline generat & 835 & & & & & & \\
\hline & & & & Cold & & & \\
\hline Cold air & End & Delta & Heat & air & MCO & Fuel & Heat \\
\hline in & Time & temp. & from MCO & out & wall & rods & from Fuel \\
\hline (T1) & (hrs) & a ir $/ \mathrm{MCO}$ & (Btu/hr) & (T2) & (tI) & $(t 2)$ & (Btu/hr) \\
\hline & & & & & & & \\
\hline 410 & 0.0 & -367 & & 0 & 572 & 572 & \\
\hline 410 & 0.1 & -107 & $(49,221)$ & 499 & 562 & 572 & 1,984 \\
\hline 410 & 0.2 & -94 & $(51,204)$ & 504 & 551 & 572 & 1,046 \\
\hline 410 & 0.3 & -90 & $(44,077)$ & 493 & 541 & 573 & 251 \\
\hline 410 & 0.4 & -83 & $(41,574)$ & 490 & 533 & 573 & (485) \\
\hline 410 & 0.5 & -78 & $(37,505)$ & 484 & 525 & 573 & $(1,137)$ \\
\hline 410 & 0.6 & -72 & $(34,595)$ & 480 & 517 & 572 & $(1,724)$ \\
\hline 410 & 0.7 & -68 & $(31,583)$ & 475 & 511 & 572 & $(2,249)$ \\
\hline 410 & 0.8 & -64 & $(28,996)$ & 472 & 504 & 571 & $(2,718)$ \\
\hline 410 & 0.9 & -60 & $(26,566)$ & 468 & 499 & 571 & $(3,135)$ \\
\hline 284 & 1.0 & -119 & $(24,381)$ & 465 & 494 & 570 & $(3,506)$ \\
\hline 284 & 1.1 & -94 & $(51,353)$ & 494 & 483 & 569 & $(4,346)$ \\
\hline 284 & 1.2 & -91 & $(38,855)$ & 483 & 474 & 568 & $(4,950)$ \\
\hline 284 & 1.3 & -87 & $(36,966)$ & 474 & 467 & 567 & $(5,509)$ \\
\hline 284 & 1.4 & -84 & $(34,695)$ & 467 & 459 & 566 & $(6,018)$ \\
\hline 284 & 1.5 & -81 & $(32,605)$ & 459 & 452 & 565 & $(6,481)$ \\
\hline 284 & 1.6 & -78 & $(30,654)$ & 452 & 446 & 563 & $(6,902)$ \\
\hline 284 & 1.7 & -75 & $(28,834)$ & 446 & 440 & 562 & $(7,282)$ \\
\hline 284 & 1.8 & -72 & $(27,136)$ & 440 & 434 & 560 & $(7,626)$ \\
\hline
\end{tabular}


WHC-SD-SNF-CDR-007, REV. 0

\begin{tabular}{|c|c|c|c|c|c|c|c|}
\hline & & & & cold & & & \\
\hline Cold air & End & Delta & Heat & air & $\mathrm{MCO}$ & Fuel & Heat \\
\hline in & Time & temp. & from $\mathrm{MCO}$ & out & wall & rods & from Fuel \\
\hline (T1) & (hrs) & $\mathrm{air} / \mathrm{MCO}$ & (Btu/hr) & (T2) & $(t I)$ & $(t 2)$ & (Btu/hr) \\
\hline 284 & 1.9 & -70 & $(25,551)$ & 434 & 429 & 559 & $(7,935)$ \\
\hline 104 & 2.0 & -157 & $(24,073)$ & 429 & 423 & 557 & $(8,213)$ \\
\hline 104 & 2.1 & -146 & $(64,094)$ & 423 & 410 & 555 & $(9,190)$ \\
\hline 104 & 2.2 & -141 & $(58,039)$ & 410 & 398 & 553 & $(10,042)$ \\
\hline 104 & 2.3 & -135 & $(54,651)$ & 398 & 386 & 551 & $(10,819)$ \\
\hline 104 & 2.4 & -130 & $(51,374)$ & 386 & 375 & 548 & $(11,524)$ \\
\hline 104 & 2.5 & -125 & $(48,323)$ & 375 & 365 & 546 & $(12,162)$ \\
\hline 104 & 2.6 & -121 & $(45,477)$ & 365 & 355 & 543 & $(12,739)$ \\
\hline 104 & 2.7 & -117 & $(42,821)$ & 355 & 346 & 540 & $(13,258)$ \\
\hline 104 & 2.8 & -113 & $(40,343)$ & 346 & 338 & 537 & $(13,724)$ \\
\hline 104 & 2.9 & -109 & $(38,031)$ & 338 & 330 & 534 & $(14,141)$ \\
\hline 104 & 3.0 & -105 & $(35,872)$ & 330 & 322 & 531 & $(14,512)$ \\
\hline 104 & 3.1 & -102 & $(33,857)$ & 322 & 315 & 528 & $(14,841)$ \\
\hline 104 & 3.2 & -99 & $(31,975)$ & 315 & 308 & 525 & $(15,130)$ \\
\hline 104 & 3.3 & -96 & $(30,218)$ & 308 & 302 & 521 & $(15,384)$ \\
\hline 104 & 3.4 & -93 & $(28,576)$ & 302 & 295 & 518 & $(15,604)$ \\
\hline 104 & 3.5 & -90 & $(27,043)$ & 295 & 290 & 514 & $(15,792)$ \\
\hline 104 & 3.6 & -87 & $(25,610)$ & 290 & 284 & 511 & $(15,952)$ \\
\hline 104 & 3.7 & -85 & $(24,271)$ & 284 & 279 & 507 & $(16,086)$ \\
\hline 104 & 3.8 & -83 & $(23,019)$ & 279 & 274 & 504 & $(16,195)$ \\
\hline 104 & 3.9 & -81 & $(21,848)$ & 274 & 270 & 500 & $(16,282)$ \\
\hline 68 & 4.0 & -96 & $(20,753)$ & 270 & 265 & 497 & $(16,347)$ \\
\hline 68 & 4.1 & -93 & $(28,009)$ & 265 & 259 & 493 & $(16,539)$ \\
\hline 68 & 4.2 & -90 & $(26,097)$ & 259 & 254 & 489 & $(16,694)$ \\
\hline 68 & 4.3 & -88 & $(24,762)$ & 254 & 249 & 486 & $(16,823)$ \\
\hline 68 & 4.4 & -85 & $(23,491)$ & 249 & 244 & 482 & $(16,927)$ \\
\hline 68 & 4.5 & -83 & $(22,304)$ & 244 & 239 & 478 & $(17,008)$ \\
\hline 68 & 4.6 & -81 & $(21,193)$ & 239 & 234 & 474 & $(17,068)$ \\
\hline 68 & 4.7 & -79 & $(20,154)$ & 234 & 230 & 471 & $(17,108)$ \\
\hline 68 & 4.8 & -77 & $(19,181)$ & 230 & 226 & 467 & $(17,131)$ \\
\hline 68 & 4.9 & -75 & $(18,270)$ & 226 & 222 & 463 & $(17,137)$ \\
\hline 68 & 5.0 & -73 & $(17,417)$ & 222 & 218 & 459 & $(17,128)$ \\
\hline 68 & 5.1 & -72 & $(16,618)$ & 218 & 215 & 455 & $(17,106)$ \\
\hline 68 & 5.2 & -70 & $(15,869)$ & 215 & 212 & 452 & $(17,070)$ \\
\hline 68 & 5.3 & -69 & $(15,167)$ & 212 & 208 & 448 & $(17,023)$ \\
\hline 68 & 5.4 & -67 & $(14,509)$ & 208 & 205 & 444 & $(16,965)$ \\
\hline 68 & 5.5 & -66 & $(13,892)$ & 205 & 202 & 440 & $(16,897)$ \\
\hline 68 & 5.6 & $-6 \overline{4}$ & $(13,312)$ & 202 & 199 & 436 & $(16,821)$ \\
\hline 68 & 5.7 & -63 & $(12,768)$ & 199 & 197 & 433 & $(16,736)$ \\
\hline 68 & 5.8 & -62 & $(12,256)$ & 197 & 194 & 429 & $(16,643)$ \\
\hline 68 & 5.9 & -61 & $(11,776)$ & 194 & 192 & 425 & $(16,544)$ \\
\hline 68 & 6.0 & -59 & $(11,324)$ & 192 & 189 & 422 & $(16,439)$ \\
\hline 68 & 6.1 & -58 & $(10,899)$ & 189 & 187 & 418 & $(16,329)$ \\
\hline
\end{tabular}


WHC-SD-SNF-CDR-007, REV. 0

\begin{tabular}{|c|c|c|c|c|c|c|c|}
\hline & & & & Cold & & & \\
\hline Cold air & End & Delta & Heat & $a i x$ & MCO & Fuel & Heat \\
\hline in & Time & temp. & from $\mathrm{MCO}$ & out & wall & rods & from Fuel \\
\hline (T1) & (hrs) & air/MCo & (Btu/hr) & (T2) & $(t 1)$ & $(t 2)$ & (Btu/hr) \\
\hline & & & & & & & \\
\hline 68 & 6.2 & -57 & $(10,499)$ & 187 & 185 & 414 & $(16,213)$ \\
\hline 68 & 6.3 & -56 & $(10,122)$ & 185 & 183 & 411 & $(16,093)$ \\
\hline 68 & 6.4 & -55 & $(9,767)$ & 183 & 181 & 407 & $(15,968)$ \\
\hline 68 & 6.5 & -54 & $(9,432)$ & 181 & 179 & 404 & $(15,840)$ \\
\hline 68 & 6.6 & -53 & $(9,116)$ & 179 & 177 & 400 & $(15,709)$ \\
\hline 68 & 6.7 & -52 & $(8,818)$ & 177 & 175 & 397 & $(15.575)$ \\
\hline 68 & 6.8 & -52 & $(8,537)$ & 175 & 173 & 393 & $(15,439)$ \\
\hline 68 & 6.9 & -51 & $(8,271)$ & 173 & 171 & 390 & $(15,300)$ \\
\hline 68 & 7.0 & -50 & $(8,019)$ & 171 & 169 & 386 & $(15,160)$ \\
\hline 68 & 7.1 & -49 & $(7,780)$ & 169 & 168 & 383 & $(15,018)$ \\
\hline 68 & 7.2 & -48 & $(7,555)$ & 168 & 166 & 380 & $(14,875)$ \\
\hline 68 & 7.3 & -48 & $(7,341)$ & 166 & 165 & 376 & $(14,730)$ \\
\hline 68 & 7.4 & -47 & $(7,138)$ & 165 & 163 & 373 & $(14,584)$ \\
\hline 68 & 7.5 & -46 & $(6,945)$ & 163 & 162 & 370 & $(14,438)$ \\
\hline 68 & 7.6 & -45 & $(6,762)$ & 162 & 160 & 367 & $(14,292)$ \\
\hline 68 & 7.7 & -45 & $(6,588)$ & 160 & 159 & 364 & $(14,145)$ \\
\hline 68 & 7.8 & -44 & $(6,422)$ & 159 & 157 & 360 & $(13,997)$ \\
\hline 68 & 7.9 & -43 & $(6,264)$ & 157 & 156 & 357 & $(13,850)$ \\
\hline 167 & 8.0 & 7 & $(6,114)$ & 156 & 155 & 354 & $(13,703)$ \\
\hline 167 & 8.1 & 3 & 16,800 & 155 & 158 & 351 & $(13,155)$ \\
\hline 167 & 8.2 & 1 & 14,315 & 158 & 161 & 348 & $(12,661)$ \\
\hline 167 & 8.3 & 0 & 13,244 & 161 & 164 & 345 & $(12,195)$ \\
\hline 167 & 8.4 & -1 & 12,184 & 164 & 167 & 343 & $(11,756)$ \\
\hline 167 & 8.5 & -2 & 11,203 & 167 & 169 & 340 & $(11,342)$ \\
\hline 167 & 8.6 & -3 & 10,291 & 169 & 171 & 338 & $(10,952)$ \\
\hline 167 & 8.7 & -4 & 9,444 & 171 & 173 & 335 & $(10,585)$ \\
\hline 167 & 8.8 & -5 & 8,657 & 173 & 175 & 333 & $(10,237)$ \\
\hline 167 & 8.9 & -6 & 7,926 & 175 & 177 & $33 I$ & $(9,910)$ \\
\hline $26 \overline{6}$ & 9.0 & 43 & 7,247 & 177 & 178 & 328 & $(9,600)$ \\
\hline 266 & 9.1 & 38 & 29,387 & 178 & 185 & 326 & $(8,906)$ \\
\hline 266 & 9.2 & 35 & 26,180 & 185 & 190 & 324 & $(8,281)$ \\
\hline 266 & 9.3 & 33 & 24,435 & 190 & 195 & 322 & $(7,699)$ \\
\hline 266 & 9.4 & 30 & 22,746 & 195 & 200 & 321 & $(7,157)$ \\
\hline 266 & 9.5 & 28 & 21,177 & 200 & 205 & 319 & $(6,652)$ \\
\hline 266 & 9.6 & 26 & 19,716 & 205 & 209 & 318 & $(6,183)$ \\
\hline 266 & 9.7 & 25 & 18,356 & 209 & 213 & 316 & $(5,746)$ \\
\hline 266 & 9.8 & 23 & 17,091 & 213 & 216 & 315 & $(5,339)$ \\
\hline 266 & 9.9 & 21 & 15,912 & 216 & 220 & 314 & $(4,961)$ \\
\hline 266 & 10.0 & 20 & 14,815 & 220 & 223 & 313 & $(4,609)$ \\
\hline 266 & 10.1 & 19 & 13,794 & 223 & 226 & 312 & $(4,281)$ \\
\hline 266 & 10.2 & 17 & 12,844 & 226 & 229 & 311 & $(3,977)$ \\
\hline 266 & 10.3 & 16 & 11,959 & 229 & 231 & 310 & $(3,693)$ \\
\hline 266 & 10.4 & 15 & 11,135 & 231 & 233 & 309 & $(3,429)$ \\
\hline
\end{tabular}


WHC-SD-SNF-CDR-007, REV. 0

\begin{tabular}{|c|c|c|c|c|c|c|c|}
\hline & & & & Cold & & & \\
\hline cold air & End & Delta & Heat & air & $\mathrm{MCO}$ & Fuel & Heat \\
\hline in & Time & temp. & from MCo & out & wall & rods & from Fuel \\
\hline (T1) & (hrs) & air/MCo & (Btu/hr) & (T2) & $(t 1)$ & $(t 2)$ & (Btu/hr) \\
\hline 266 & 10.5 & 14 & 10,368 & 233 & 236 & 308 & $(3) 183)$ \\
\hline 266 & 10.6 & 13 & 9,654 & 236 & 238 & 308 & $\frac{(3,183)}{(2,955)}$ \\
\hline 266 & 10.7 & 12 & 8,990 & 238 & 240 & 307 & $(2,742)$ \\
\hline 266 & 10.8 & 11 & 8,371 & 240 & 241 & 306 & $(2,545)$ \\
\hline 266 & 10.9 & 11 & 7,795 & 241 & 243 & 306 & $(2,361)$ \\
\hline 266 & 11.0 & 10 & 7,259 & 243 & 245 & 305 & $(2,189)$ \\
\hline 266 & 11.1 & 9 & 6,760 & 245 & 246 & 305 & $(2,030)$ \\
\hline 266 & 11.2 & 9 & 6,296 & 246 & 247 & 304 & $(1,882)$ \\
\hline 266 & 11.3 & 8 & 5,863 & 247 & 249 & 304 & $(1,744)$ \\
\hline 266 & 11.4 & 8 & 5,460 & 249 & 250 & 304 & $(1,616)$ \\
\hline 266 & 11.5 & 7 & 5,086 & 250 & 251 & 303 & $(1,497)$ \\
\hline 266 & 11.6 & 7 & 4,737 & 251 & 252 & 303 & $(1,386)$ \\
\hline 266 & 11.7 & 6 & 4,412 & 252 & 253 & 303 & $(1,283)$ \\
\hline 266 & 11.8 & 6 & 4,109 & 253 & 254 & 302 & $(1,187)$ \\
\hline 266 & 11.9 & 5 & 3,828 & 254 & 254 & 302 & $(1,097)$ \\
\hline 266 & 12.0 & 5 & 3,565 & 254 & 255 & 302 & $(1,015)$ \\
\hline 266 & 12.1 & 5 & 3,321 & 255 & 256 & 302 & $(937)$ \\
\hline $26 \overline{6}$ & 12.2 & 4 & 3,094 & 256 & 257 & 301 & $(866)$ \\
\hline 266 & 12.3 & 4 & 2,883 & 257 & 257 & 301 & (799) \\
\hline 266 & 12.4 & 4 & 2,686 & 257 & 258 & 301 & $(737)$ \\
\hline 266 & 12.5 & 4 & 2,502 & 258 & 258 & 301 & $(679)$ \\
\hline 266 & 12.6 & 3 & 2,332 & 258 & 259 & 301 & $(626)$ \\
\hline 266 & 12.7 & 3 & 2,173 & 259 & 259 & 301 & $(576)$ \\
\hline 266 & 12.8 & 3 & 2,025 & 259 & 260 & 300 & $(530)$ \\
\hline 266 & 12.9 & 3 & 1,887 & 260 & 260 & 300 & $(487)$ \\
\hline 266 & 13.0 & 3 & 1,759 & 260 & 260 & 300 & $(447)$ \\
\hline 266 & 13.1 & 2 & 1,639 & 260 & 261 & 300 & $(410)$ \\
\hline 266 & 13.2 & 2 & 1,528 & 261 & 261 & 300 & $(376)$ \\
\hline 266 & 13.3 & 2 & 1,425 & 261 & 261 & 300 & (344) \\
\hline 266 & 13.4 & 2 & 1,328 & 261 & 262 & 300 & (314) \\
\hline 266 & 13.5 & 2 & 1,238 & 262 & 262 & 300 & $(286)$ \\
\hline 266 & 13.6 & 2 & 1,155 & 262 & 262 & 300 & (261) \\
\hline 266 & 13.7 & 2 & 1,077 & 262 & 262 & 300 & $(237)$ \\
\hline 266 & 13.8 & 2 & 1,004 & 262 & 263 & 300 & (215) \\
\hline 266 & 13.9 & 1 & 937 & 263 & 263 & 300 & (194) \\
\hline 266 & 14.0 & 1 & 874 & 263 & 263 & 299 & $(175)$ \\
\hline 266 & 14.1 & 1 & 816 & 263 & 263 & 299 & (158) \\
\hline 266 & 14.2 & 1 & 761 & 263 & 263 & 299 & (142) \\
\hline 266 & 14.3 & 1 & 710 & 263 & 264 & 299 & $(126)$ \\
\hline 266 & 14.4 & 1 & 663 & 264 & 264 & 299 & (112) \\
\hline 266 & 14.5 & 1 & 619 & 264 & 264 & 299 & $(100)$ \\
\hline 266 & 14.6 & 1 & 578 & 264 & 264 & 299 & $(88)$ \\
\hline 266 & 14.7 & 1 & 540 & 264 & 264 & 299 & $(76)$ \\
\hline
\end{tabular}


WHC-SD-SNF-CDR-007, REV. 0

\begin{tabular}{|c|c|c|c|c|c|c|c|}
\hline & & & & Cold & & & \\
\hline Cold air & End & Delta & Heat & air & $\mathrm{MCO}$ & Fuel & Heat \\
\hline in & Time & temp. & from MCO & out & wall & rods & from Fuel \\
\hline (T1) & (hrs) & $\mathrm{air} / \mathrm{MCO}$ & (Btu/hr) & (T2) & $(t 1)$ & $(t 2)$ & (Btu/hr) \\
\hline & & & & & & & \\
\hline 266 & $\frac{14.9}{15.0}$ & $\frac{1}{1}$ & 471 & 264 & 264 & 299 & $(57)$ \\
\hline$\frac{266}{266}$ & $\frac{15.0}{15.1}$ & $\frac{1}{1}$ & $\frac{440}{411}$ & 264 & 264 & 299 & $(48)$ \\
\hline $\begin{array}{l}266 \\
266 \\
\end{array}$ & $\frac{15.1}{15.2}$ & $\frac{1}{1}$ & $\frac{411}{385}$ & 264 & 264 & 299 & $(40)$ \\
\hline$\frac{266}{266}$ & $\frac{15 \cdot 2}{15 \cdot 3}$ & $\frac{1}{1}$ & $\frac{385}{360}$ & 264 & 264 & 299 & $(32)$ \\
\hline 266 & $\frac{15.3}{15.4}$ & $\frac{1}{1}$ & $\frac{360}{336}$ & 264 & 265 & 299 & (25) \\
\hline$-\frac{266}{266}$ & $\frac{15.4}{15.5}$ & $\frac{1}{1}$ & $\frac{336}{315}$ & 265 & 265 & 299 & (19) \\
\hline $\begin{array}{r}266 \\
266 \\
\end{array}$ & $\frac{15.5}{15.6}$ & $\frac{1}{1}$ & $\frac{315}{295}$ & 265 & 265 & 299 & (13) \\
\hline 266 & $\begin{array}{l}15.6 \\
15.7\end{array}$ & $\frac{1}{1}$ & $\frac{295}{276}$ & 265 & 265 & 299 & (8) \\
\hline$\frac{266}{266}$ & $\frac{15.7}{15.8}$ & $\frac{1}{1}$ & $\begin{array}{l}276 \\
258\end{array}$ & 265 & 265 & 299 & (3) \\
\hline 266 & $\frac{15.8}{15.9}$ & 1 & $\frac{258}{242}$ & 265 & 265 & 299 & 2 \\
\hline$\frac{266}{266}$ & $\frac{15.9}{16.0}$ & 0 & $\begin{array}{l}242 \\
227\end{array}$ & 265 & 265 & 299 & 6 \\
\hline $\begin{array}{l}266 \\
266 \\
\end{array}$ & $\frac{16.0}{16.1}$ & 0 & 227 & 265 & 265 & 299 & 10 \\
\hline 266 & 16.2 & 0 & $\begin{array}{l}213 \\
199 \\
\end{array}$ & 265 & 265 & 299 & 14 \\
\hline 266 & 16.3 & 0 & $\begin{array}{l}199 \\
187\end{array}$ & 265 & 265 & 299 & 17 \\
\hline 266 & 16.4 & 0 & $\begin{array}{l}187 \\
175 \\
\end{array}$ & 265 & 265 & 299 & 20 \\
\hline 266 & 16.5 & 0 & $\frac{175}{165}$ & 265 & 265 & 299 & 23 \\
\hline 266 & 16.6 & 0 & $\frac{165}{155}$ & 265 & 265 & 299 & 25 \\
\hline 266 & 16.7 & 0 & 155 & 265 & 265 & 299 & 27 \\
\hline 266 & 16.8 & 0 & $\frac{145}{137}$ & 265 & 265 & 299 & 29 \\
\hline 266 & 16.9 & 0 & $\begin{array}{l}137 \\
129\end{array}$ & 265 & 265 & 299 & 31 \\
\hline 266 & 17.0 & 0 & $\begin{array}{l}129 \\
121\end{array}$ & 265 & 265 & 299 & 33 \\
\hline 266 & 17.1 & 0 & $\frac{121}{114}$ & 265 & 265 & 299 & 35 \\
\hline 266 & 17.2 & 0 & $\begin{array}{r}114 \\
107\end{array}$ & 265 & 265 & 299 & 36 \\
\hline 266 & 17.3 & 0 & $\begin{array}{l}107 \\
101\end{array}$ & 265 & 265 & 299 & 37 \\
\hline 266 & 17.4 & 0 & $\frac{101}{96}$ & 265 & 265 & 299 & 38 \\
\hline 266 & 17.5 & 0 & $\begin{array}{l}96 \\
90 \\
\end{array}$ & 265 & 265 & 299 & 39 \\
\hline 266 & 17.6 & 0 & $\frac{90}{85}$ & 265 & 265 & 299 & 40 \\
\hline 266 & 17.7 & 0 & 85 & 265 & 265 & 299 & 41 \\
\hline 266 & 17.8 & 0 & $\frac{81}{76}$ & 265 & 265 & 299 & 41 \\
\hline 266 & 17.9 & 0 & $\begin{array}{l}76 \\
72\end{array}$ & 265 & 265 & 299 & 42 \\
\hline 266 & 18.0 & 0 & $\begin{array}{l}72 \\
68 \\
\end{array}$ & 265 & 266 & 299 & 43 \\
\hline 266 & 18.1 & 0 & $\begin{array}{r}68 \\
65 \\
\end{array}$ & 266 & 266 & 299 & 43 \\
\hline 266 & 18.2 & 0 & $\frac{65}{61}$ & 266 & 266 & 299 & 43 \\
\hline 266 & 18.3 & 0 & $\frac{61}{58}$ & 266 & 266 & 299 & 44 \\
\hline 266 & 18.4 & 0 & $\begin{array}{l}58 \\
55 \\
\end{array}$ & 266 & 266 & 299 & 44 \\
\hline 266 & 18.5 & 0 & 53 & 266 & 266 & 299 & 44 \\
\hline 266 & 18.6 & 0 & 50 & $\begin{array}{l}266 \\
266\end{array}$ & 266 & 299 & 44 \\
\hline 266 & 18.7 & 0 & 48 & $\begin{array}{r}266 \\
266\end{array}$ & 266 & 299 & 44 \\
\hline 266 & 18.8 & 0 & 45 & $\frac{266}{26}$ & 266 & 299 & 44 \\
\hline 266 & 18.9 & 0 & 43 & $\frac{266}{266}$ & 266 & 299 & 44 \\
\hline 266 & 19.0 & 0 & 41 & $\frac{266}{266}$ & 266 & 299 & 44 \\
\hline
\end{tabular}


WHC-SD:SNF-CDR-007, REV. 0

\begin{tabular}{|c|c|c|c|c|c|c|c|}
\hline & & & & $\operatorname{cold}$ & & & \\
\hline cold air & End & Delta & Heat & air & $\mathrm{MCO}$ & Fuel & Heat \\
\hline in & Time & temp. & from $\mathrm{MCO}$ & out & wall & rods & from Fuel \\
\hline (T1) & (hrs) & air/MCO & (Btu/hr) & $(T 2)$ & $(t I)$ & $(t 2)$ & (Btu/hr) \\
\hline & & & & & & & \\
\hline 266 & 19.1 & 0 & 40 & 266 & 266 & 299 & 44 \\
\hline 266 & 19.2 & 0 & 38 & 266 & 266 & 299 & 44 \\
\hline 266 & 19.3 & 0 & 36 & 266 & 266 & 299 & 44 \\
\hline 266 & 19.4 & 0 & 35 & 266 & 266 & 299 & 43 \\
\hline 266 & 19.5 & 0 & 33 & 266 & $26 \overline{6}$ & 299 & 43 \\
\hline 266 & 19.6 & 0 & 32 & 266 & 266 & 299 & 43 \\
\hline 266 & 19.7 & 0 & 31 & 266 & 266 & 300 & 43 \\
\hline 266 & 19.8 & 0 & 29 & 266 & 266 & 300 & 42 \\
\hline 266 & 19.9 & 0 & 28 & 266 & 266 & 300 & 42 \\
\hline 266 & 20.0 & 0 & 27 & 266 & 266 & 300 & 42 \\
\hline
\end{tabular}


WHC-SD-SNF-CDR-007, REV. 0

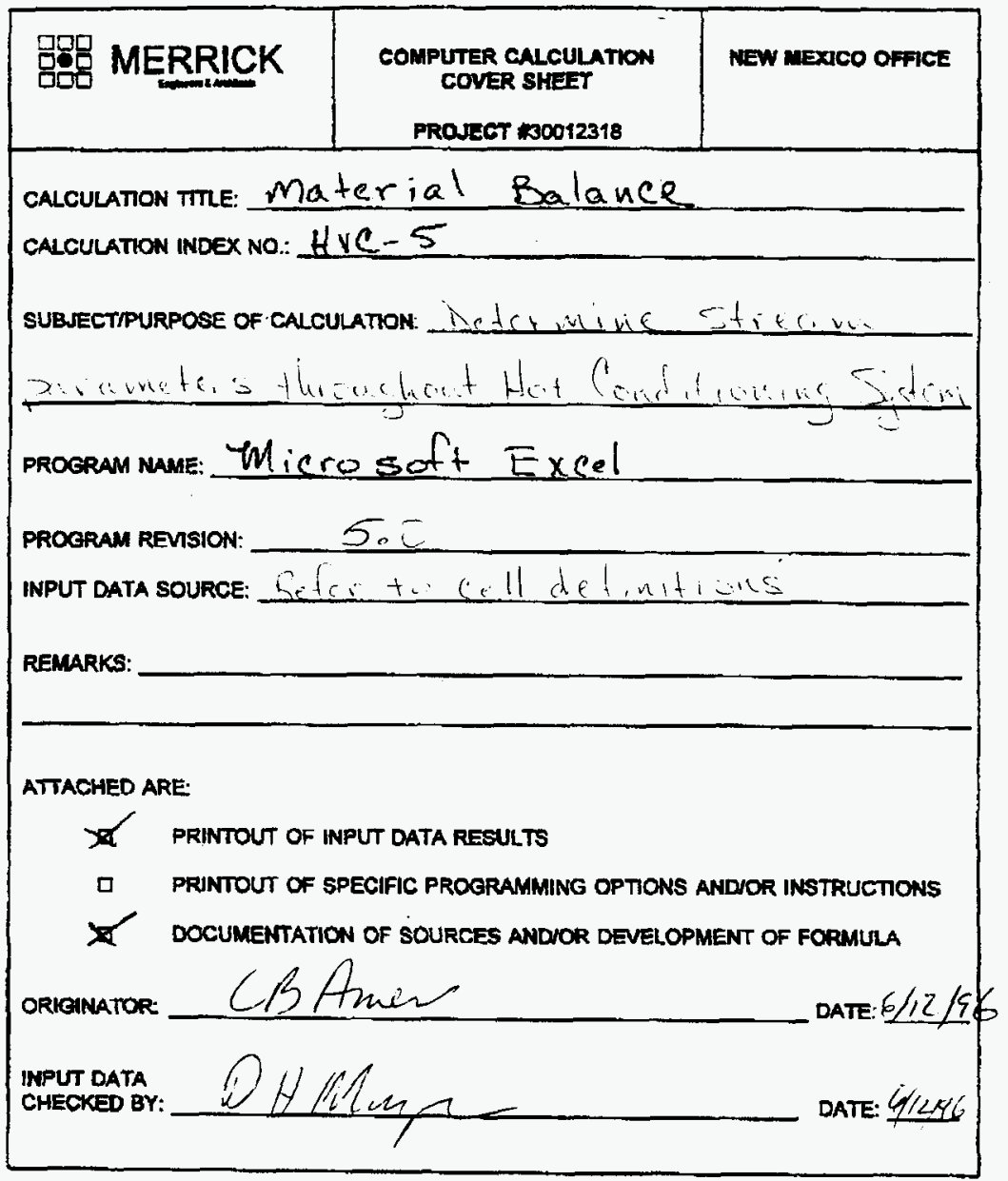




\section{Material Balance Calculations}

The following pages contain printout from the MS Excel spreadsheet used to prepare the material balance. The cell columns and rows are labeled so that they may be referenced to the calculations shown in the following notes section. Some manual calculations on vacuum pumping speed follow the Excel output.

prepared by L.B. Ames $6 / 12 / 96$ 


\begin{tabular}{|c|c|c|c|c|c|c|c|c|c|c|}
\hline & A & $\mathbf{B}$ & $\mathbf{C}$ & D & $\mathbf{E}$ & $\mathbf{F}$ & $\mathbf{G}$ & $\mathrm{H}$ & I & $\mathrm{J}$ \\
\hline 2 & Operating Cycle & & \multicolumn{4}{|c|}{ Ongoing utility } & \multicolumn{4}{|c|}{ Preparation for heating cycle } \\
\hline 3 & Stream Description & $\mathrm{MWt}$ & \multicolumn{2}{|c|}{ Dewar vacuum draw } & \multicolumn{2}{|c|}{ MCO area hood vent } & \multicolumn{2}{|c|}{$\begin{array}{l}\text { Initial vacuum } \\
\text { evacuation }\end{array}$} & \multicolumn{2}{|c|}{ Helium filling of $\mathrm{MCO}$} \\
\hline 4 & Stream No. & & \multicolumn{2}{|c|}{1} & \multicolumn{2}{|c|}{2} & \multicolumn{2}{|c|}{3} & \multicolumn{2}{|c|}{4} \\
\hline 5 & & & & & & & & & & \\
\hline 6 & T̈ernp.C (F) & & $20-375$ & $68-707$ & 20 & 68 & 40 & 104 & 20 & 68 \\
\hline 7 & & & & & & & & & & \\
\hline 8 & Press Torr (psia) & & $1.00 \mathrm{E}-04$ & 1.93E-06 & 760 & 14.7 & 10 & 0.193 & 931 & 18 \\
\hline 9 & Flow rate std $1 / \mathrm{sec}$ (scfm) & & nil & nil & 66 & 140 & 0.067 & 0.142 & 5.32 & 11.29 \\
\hline 10 & Vac rate U/min & & & & & & \multicolumn{2}{|c|}{350} & & \\
\hline 11 & $\mathrm{H}_{2} \mathrm{~kg} / \mathrm{hr}(\mathrm{bs} / \mathrm{hr})$ & 2 & & & & & & $=$ & & \\
\hline 12 & $\mathrm{O}_{2} \mathrm{~kg} / \mathrm{hr}(\mathrm{bs} / \mathrm{hr})$ & 32 & & & & & & & & \\
\hline 13 & $\mathrm{~N}_{2} \mathrm{~kg} / \mathrm{hr}(\mathrm{lbs} / \mathrm{hr})$ & 28 & & & & & & & & \\
\hline 14 & He kg/hr (lbs/hr) & 4 & & & & & & & 3.42 & 7.55 \\
\hline 15 & $f^{13 \pi}$ gramhr (lbs/hr) & 131 & & & & & & & & \\
\hline 16 & $\mathrm{Kr}^{\mathrm{ab}}$ gram/hr (lbs/hr) & 86 & & & & & & & & \\
\hline 17 & $\mathrm{Cs}^{137}$ gram/hr (lbs/hr) & 137 & & & & & & & & \\
\hline 18 & & & & & & & & & & \\
\hline 19 & Max heat rate kcal/hr $(B t u / h r)$ & & & & & & & & & \\
\hline 20 & & & & & & & & & & \\
\hline 21 & Process Duration (Note 4 ) & & ongc & oing & & & 6.80 & $\min$. & 12.95 & min. \\
\hline 22 & Approx. Total Duration & & ongc & jing & & & 15 & $\min$ & 15 & min. \\
\hline 23. & Water Removed kg & & & & & & & & & \\
\hline 24 & Hydrogen Removed kg & & & & & & & & & \\
\hline 25 & & & & & & & & & & \\
\hline 26 & & & & & & & & & & \\
\hline 27 & & & & & & & & & & \\
\hline 28 & & & & & & & & & & \\
\hline 29 & & & & & & & & & & \\
\hline 30 & & & & & & & & & & \\
\hline 31 & & & & & & & & & & \\
\hline 32 & & & & & & & & & & \\
\hline 33 & & & & & & & & & & \\
\hline 34 & & & & & & & & & & \\
\hline 35 & & & & & & & & & & \\
\hline 36 & & & & & & & & & & \\
\hline 37 & & & & & & & & & & \\
\hline 38 & & & & & & & & & & \\
\hline 39 & & & & & & & & & & \\
\hline 40 & MCO volume (liter) $=$ & & 507.19 & & & & & & & \\
\hline 41 & External MCO piping (liter) = & & 60.56 & & & & & & & \\
\hline 42 & Cyl. gas fill rate $(a / m)=$ & & 280 & & & & & & & \\
\hline 43 & Cyt gas fill pressure (psia) $=$ & & 18 & & & & & & & \\
\hline 44 & $\begin{array}{l}\text { Gas constant (liter }{ }^{*} \text { torr }{ }^{*}\left[\mathrm{KT}^{1}{ }^{-}\right. \\
\left.[\mathrm{gmol}]^{-1}\right)=\end{array}$ & & 62.361 & & & & & & & \\
\hline 45 & $\begin{array}{l}\text { System operating pressure } \\
\text { (psia) }\end{array}$ & & 13 & & & & & & & \\
\hline 46 & Specific heat of helium (cal/gC) & & & & & & & & & \\
\hline 47 & 1 atm $(100-300 \mathrm{C})$ & & 0.519 & & & & & & & \\
\hline 48 & 5 atm $(100-300 \mathrm{C})$ & & 0.519 & & & & & & & \\
\hline 49 & MCO gas Circulation rate (ACFM) & & 10 & & & & & & & \\
\hline
\end{tabular}




\begin{tabular}{|c|c|c|c|c|c|c|c|c|c|c|}
\hline & A & $\mathbf{B}$ & $\mathbf{K}$ & $\mathbf{L}$ & $\mathbf{M}$ & $\mathbf{N}$ & 0 & $\mathbf{P}$ & $\mathbf{Q}$ & $\overline{\mathbf{R}}$ \\
\hline 2 & Operating Cycle & & \multicolumn{8}{|c|}{ Heating of $\mathrm{MCO}(40 \mathrm{C}$ to 3} \\
\hline 3 & Stream Description & MWt & \multicolumn{2}{|c|}{ MCO heating air } & \multicolumn{2}{|c|}{$\begin{array}{l}\text { Helium heating } \\
\text { (Heater inlet) }\end{array}$} & \multicolumn{2}{|c|}{$\begin{array}{l}\text { Helium heating } \\
\text { (Heater outlet) }\end{array}$} & \multicolumn{2}{|c|}{$\begin{array}{l}\text { Periodic helium } \\
\text { purging to } 200 \mathrm{C}\end{array}$} \\
\hline 4 & Stream No. & & \multicolumn{2}{|c|}{5} & \multicolumn{2}{|c|}{$6 \mathrm{~A}$} & \multicolumn{2}{|c|}{68} & \multicolumn{2}{|c|}{$7 A$} \\
\hline 5 & & & & & & & & & & \\
\hline 6 & Ternp. C (F) & & 375 & 707 & 102 & 216 & 325 & 617 & 200 & 392 \\
\hline 7 & & & theces & & & & & & & \\
\hline 8 & Press Torr (psia) & & 760 & 14.7 & 688 & 13.3 & 672 & 13 & 688 & 13.3 \\
\hline 9 & Flow rate std V/sec (scfm) & & 149 & 316 & 1.90 & 4.037 & 1.90 & 4.037 & 0.005 & 0.011 \\
\hline 10 & Vac rate U/min & & & & & & & & & \\
\hline 11 & $\mathrm{H}_{2} \mathrm{~kg} / \mathrm{hr}(\mathrm{bs} / \mathrm{hr})$ & 2 & & & & & & & & \\
\hline 12 & $\mathrm{O}_{2} \mathrm{~kg} / \mathrm{hr}(/ \mathrm{bs} / \mathrm{hr})$ & 32 & & & & & & & & \\
\hline 13 & $\mathrm{~N}_{2} \mathrm{~kg} / \mathrm{hr}(\mathrm{bs} / \mathrm{hr})$ & 28 & & & & & & & & \\
\hline 14 & He kg/hr $(\mathrm{lbs} / \mathrm{hr})$ & 4 & & & 1.22 & 2.70 & 1.22 & 2.70 & 0.003 & 0.007 \\
\hline 15 & $f^{134}$ gram/hr (lbs/hr) & 131 & & & & & & & & \\
\hline 16 & $\mathrm{Kr}^{20}$ gram/hr (lbs/hr) & 86 & & & & & & & & \\
\hline 17 & $\mathrm{Cs}^{137}$ gram/hr (lbs/hr) & 137 & & & & & & & & \\
\hline 18 & & & & & & & & & & \\
\hline 19 & Max heat rate $\mathrm{kcal} / \mathrm{hr}(\mathrm{Btu} / \mathrm{hr})$ & & 17,640 & 70,000 & & & 142 & 562 & 0.002 & 0.007 \\
\hline 20 & & & & & & & & & & \\
\hline 21 & Process Duration (Note 4 ) & & $13.2 \mathrm{hr}$ & Note 3) & 13.2. & $\mathrm{hr}$. & 13.2 & hr. & 8 & hr. \\
\hline 22 & Approx. Total Duration & & $14 \mid$ & hr. & 14 & hr. & 14 & hr & 8 & hi. \\
\hline 23 & Water Removed kg & & & & & & & & & \\
\hline 24 & Hydrogen Removed kg & & & & & & & & & \\
\hline 25 & & & & & & & & & & \\
\hline 26 & & & & & & & & & & \\
\hline 27 & & & & & & & & & & \\
\hline 28 & & & & & & & & & & \\
\hline 29 & & & & & & & & & & \\
\hline 30 & & & & & & & & & & \\
\hline 31 & & & & & & & & & & \\
\hline 32 & & & & & & & & & & \\
\hline 33 & & & & & & & & & & \\
\hline 34 & & & & & & & & & & \\
\hline 35 & & & & & & & & & & \\
\hline 36 & & & & & & & & & & \\
\hline 37 & & & & & & & & & & \\
\hline 38 & & & & & & & & & & \\
\hline 39 & & & & & & & & & & \\
\hline 40 & MCO volume (liter) = & & & & & & & $g$ moles Q & $10 \mathrm{C}=$ & 19.54967 \\
\hline 41 & External MCO piping (liter) = & & & & & & & $g$ moles Q & $00 \mathrm{C}=$ & 12.93667 \\
\hline 42 & Cyl. gas fill rate $(a / m)=$ & & & & & & & 9 moles 6 & $00 \mathrm{C}=$ & 10.6790 \\
\hline 43 & Cyl gas fill pressure (psia) $=$ & & & & & & & & & \\
\hline 44 & $\begin{array}{l}\text { Gas constant (liter " torr }{ }^{*}\left[\mathrm{KT}^{\top} \text {. }\right. \\
\left.\left(\mathrm{gmo}^{-1}\right]^{-1}\right)=\end{array}$ & & & & & & & & & \\
\hline 45 & $\begin{array}{l}\text { System operating pressure } \\
\text { (psia) }\end{array}$ & & & & & & & & & \\
\hline 46 & Specific heat of helium (cal/gC) & & & & & & & & & \\
\hline 47 & 1 atm $(100-300 \mathrm{C})$ & & & & & & & & & \\
\hline 48 & 5 atm $(100-300 \mathrm{C})$ & & & & & & & & & \\
\hline 49 & MCO gas Circulation rate (ACFM) & & & & & & & & & \\
\hline
\end{tabular}




\begin{tabular}{|c|c|c|c|c|c|c|c|c|}
\hline & A & $\mathbf{B}$ & $\mathbf{s}$ & $T$ & $\mathbf{U}$ & $\mathbf{v}$ & $\mathbf{w}$ & $\bar{x}$ \\
\hline 2 & Operating Cycle & & \multicolumn{6}{|l|}{$0+c)$} \\
\hline 3 & Stream Description & MWt & \multicolumn{2}{|c|}{$\begin{array}{c}\text { Helium purging } 200 \mathrm{C} \\
\text { to } 300 \mathrm{C}\end{array}$} & \multicolumn{2}{|c|}{$\begin{array}{l}\text { Helium for purge } \\
\text { during heat-up }\end{array}$} & \multicolumn{2}{|c|}{ Purifier Outlet } \\
\hline 4 & Stream No. & & \multicolumn{2}{|c|}{78} & \multicolumn{2}{|c|}{8} & \multicolumn{2}{|c|}{9} \\
\hline 5 & & & & & & & & \\
\hline 6 & Temp. C (F) & & 300 & 572 & 20 & 68 & 100 & 212 \\
\hline 7 & & & & & & & & \\
\hline 8 & Press Torr (psia) & & 688 & 13.3 & 931 & 18 & & \\
\hline 9 & Flow rate std $\mathrm{V} / \mathrm{sec}$ (schm) & & 0.003 & 0.006 & 0.004 & 0.009 & 1.90 & 4.037 \\
\hline 10 & Vac rate $\mathrm{U} / \mathrm{min}$ & & & & & & & \\
\hline 11 & $\mathrm{H}_{2} \mathrm{~kg} / \mathrm{hr}(\mathrm{lbs} / \mathrm{hr})$ & 2 & & & & & & \\
\hline 12 & $\mathrm{O}_{2} \mathrm{~kg} / \mathrm{hr}(\mathrm{bs} / \mathrm{hr})$ & 32 & & & & & & \\
\hline$\overline{13}$ & $\mathrm{~N}_{2} \mathrm{~kg} / \mathrm{hr}(\mathrm{bs} / \mathrm{hr})$ & 28 & & & & & & \\
\hline 14 & He kg/hr (Ihs/hr) & 4 & 0.002 & 0.004 & 0.003 & 0.006 & & \\
\hline 15 & $1^{131}$ gram/hr ( $(\mathrm{bs} / \mathrm{hr})$ & 131 & & & & & & \\
\hline 16 & $\mathrm{Kr}^{\mathrm{M}} \mathrm{gram} / \mathrm{hr}(\mathrm{lbs} / \mathrm{hr})$ & 86 & & & & & & \\
\hline 17 & $\mathrm{Cs}^{137}$ gram/hr (10s/hr) & 137 & & & & & & \\
\hline 18 & & & & & & & & \\
\hline 19 & Max heat rate kcal/hr (Btu/hr) & & 0.001 & 0.004 & & & & \\
\hline 20 & & & & & & & & \\
\hline 21 & Process Duration (Note 4 ) & & 5.3 & hr. & 13.3 & hr. & & \\
\hline 22 & Approx. Total Duration & & 6 & $\mathrm{hr}$. & & & & \\
\hline 23 & Water Removed kg & & 1.8 & & & & & \\
\hline 24 & Hydrogen Removed kg & & 0.1 & & & & & \\
\hline 25 & & & & & & & & \\
\hline 26 & & & & & & & & \\
\hline 27 & & & & & & & & \\
\hline 28 & & & & & & & & \\
\hline 29 & & & & & & & & \\
\hline 30 & & & & & & & & \\
\hline 31 & & & & & & & & \\
\hline 32 & & & & & & & & \\
\hline 33 & & & & & & & & \\
\hline 34 & & & & & & & & \\
\hline 35 & & & & & & & & \\
\hline 36 & & & & & & & & \\
\hline 37 & & & & & & & & \\
\hline 38 & & & & & & & & \\
\hline 39 & & & & & & & & \\
\hline 40 & MCO volume (liter) $=$ & & & & & & & \\
\hline 41 & External MCO piping (liter) $=$ & & & & & & & \\
\hline 42 & Cyt. gas fill rate $(a l / m)=$ & & & & & & & \\
\hline 43 & Cyl gas fill pressure (psia) $=$ & & & & & & & \\
\hline 44 & $\begin{array}{l}\text { Gas constant (ifter * tort * }\left[\mathrm{KT}^{\prime} \text {. }\right. \\
\left.[\mathrm{gmol}]^{-1}\right)=\end{array}$ & & & & & & & \\
\hline 45 & $\begin{array}{l}\text { System operating pressure } \\
\text { (psia) }\end{array}$ & & & & & & & \\
\hline 46 & Specific heat of helium (cal $/ \mathrm{gC}$ ) & & & & & & & \\
\hline 47 & $1 \mathrm{~atm}(100-300 \mathrm{C})$ & & & & & & & \\
\hline 48 & $5 \operatorname{atm}(100-300 \mathrm{C})$ & & & & & & & \\
\hline 49 & MCO gas Circulation rate (ACFM) & & 1 & & & & & \\
\hline
\end{tabular}




\begin{tabular}{|c|c|c|c|c|c|c|c|c|c|c|}
\hline & A & $\mathbf{B}$ & $\mathbf{Y}$ & 2 & $\mathbf{A A}$ & $\mathbf{A B}$ & $A C$ & $\mathbf{A D}$ & $\mathbf{A E}$ & $\mathbf{A F}$ \\
\hline 2 & Operating Cycle & & \multicolumn{4}{|c|}{ Vacuum condition } & \multicolumn{4}{|c|}{ Cooling of } \\
\hline 3 & Stream Description & MWt & \multicolumn{2}{|c|}{ Vacuum draw } & \multicolumn{2}{|c|}{$\begin{array}{c}\text { Inert gas purge during } \\
\text { vacuum }\end{array}$} & \multicolumn{2}{|c|}{ Cooling air } & \multicolumn{2}{|c|}{$\begin{array}{l}\text { Helium added for cool- } \\
\text { down }\end{array}$} \\
\hline 4 & Stream No. & & \multicolumn{2}{|c|}{10} & \multicolumn{2}{|c|}{11} & \multicolumn{2}{|c|}{12} & \multicolumn{2}{|c|}{13} \\
\hline $\mathbf{5}$ & & & & & & & & & & \\
\hline 6 & Temp. C $(F)$ & & 300 & 572 & 20 & 68 & 80 & 176 & 20 & 68 \\
\hline 7 & & & & & & & Nold 6 & & & \\
\hline 8 & Press Torr (osia) & & 5 & 0.097 & 931 & 18 & 760 & 14.7 & 931 & 18 \\
\hline 9 & Flow rate std l/sec (scim) & & 0.032 & 0.067 & 0.030 & 0.064 & 256 & 542 & 5.324 & 11.288 \\
\hline 10 & Vac rate U/min & & \multicolumn{2}{|c|}{350} & & & & & & \\
\hline 11 & $\mathrm{H}_{2} \mathrm{~kg} / \mathrm{hr}(\mathrm{lbs} / \mathrm{hr})$ & 2 & 0.0005 & 0.001 & & & & & & \\
\hline 12 & $\mathrm{O}_{2} \mathrm{~kg} / \mathrm{hr}(\mathrm{bs} / \mathrm{hr})$ & 32 & & & & & & & & \\
\hline 13 & $\mathrm{~N}_{2} \mathrm{~kg} / \mathrm{hr}(\mathrm{bs} / \mathrm{hr})$ & 28 & 0.135 & 0.298 & 0.135 & 0.298 & & & & \\
\hline 14 & He kg/hr (1bs/hr) & 4 & & & & & & & 3.423 & 7.547 \\
\hline 15 & $\mathrm{f}^{134} \mathrm{gram} / \mathrm{hr}(\mathrm{bs} / \mathrm{hr})$ & 131 & & & & & & & & \\
\hline 16 & $\mathrm{Kr}^{\text {in }} \mathrm{gram} / \mathrm{hr}$ (lbs/hr) & 86 & & & & & & & & \\
\hline 17 & $\mathrm{Cs}^{137}$ gram/hr (/hs/hr) & 137 & & & & & & & & \\
\hline \multicolumn{11}{|c|}{18} \\
\hline 19 & Max heat rate kcal/hr (Btw/hr) & & & & & & 18,144 & 72,000 & & \\
\hline \multicolumn{11}{|l|}{20} \\
\hline 21 & Process Duration (Note 4 ) & & 7.9 & $\min$ & 48 & hr. & $14 \mid$ & hr. & 0.957 & min. \\
\hline 22 & Approx. Total Duration & & 48 & hr. & 48 & hr. & 14.5 & hr. & 15 & $\min$. \\
\hline 23 & Water Removed kg & & \multicolumn{2}{|c|}{0.056} & & & & & & \\
\hline 24 & Hydrogen Removed kg & & \multicolumn{2}{|c|}{0.003999375} & & & & & & \\
\hline \multicolumn{11}{|l|}{25} \\
\hline 26 & & & Notes: & & & & & & & \\
\hline 27 & & & \multicolumn{8}{|c|}{ 1. Although hydrogen may be present at concentrations greater than $5 \% \mathrm{v}$, it is inherentiy safe beca } \\
\hline 28 & & & & & & & & & & \\
\hline 29 & & & \multicolumn{5}{|c|}{ 2. Run 23 data assumed to be at MCO operating conditions. } & & & \\
\hline 30 & & & & & & & & & & \\
\hline 31 & & & \multicolumn{8}{|c|}{ 3. Heating rate is determined with an internal heat transfer coeficient of I Btu / pound degree F sq. } \\
\hline 32 & & & & & & & & & & \\
\hline 33 & & & 4. Mass ra & le times pro & ess duration & equals mass. & Approximat & to total dure & in is for cycle & le time deter \\
\hline 34 & & & & 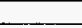 & \begin{tabular}{|l|l|} 
& 0
\end{tabular} & & & & & \\
\hline 35 & & & 5. This is t & he maximur & of a program & imod tempera & ture schedul & & & \\
\hline 36 & & & & & & & & & & \\
\hline 37 & & & 6. This is th & he minimun & of a programr & med tempera & ure schedule & & & \\
\hline 38 & & & & & & & & & & \\
\hline 39 & & & & & & & & & & \\
\hline 40 & MCO volume (liter) = & & & & & & & & & \\
\hline 41 & External MCO piping (liter) = & & & & & & & & & \\
\hline 42 & Cyl. gas fill rate $(\mathrm{al} / \mathrm{m})=$ & & & & & & & & & \\
\hline 43 & Cyl gas fill pressure (psia) = & & & & & & & & & \\
\hline 44 & $\begin{array}{l}\text { Gas constant (liter }{ }^{*} \text { torr }{ }^{*}\left[\mathrm{KJ}^{\top}\right. \\
{\left[\mathrm{gmol}^{-1}\right)=}\end{array}$ & & & & & & & & & \\
\hline 45 & $\begin{array}{l}\text { System operating pressure } \\
\text { (psia) }\end{array}$ & & & & & & & & & \\
\hline 46 & Specific heat of helium (cal/gC) & & & & & & & & & \\
\hline 47 & 1 atm $(100-300 \mathrm{C})$ & & & & & & & & & \\
\hline 48 & $5 \mathrm{~atm}(100-300 \mathrm{C})$ & & & & & & & & & \\
\hline 49 & MCO gas Circulation rate (ACFM) & & & & & & & & & \\
\hline
\end{tabular}




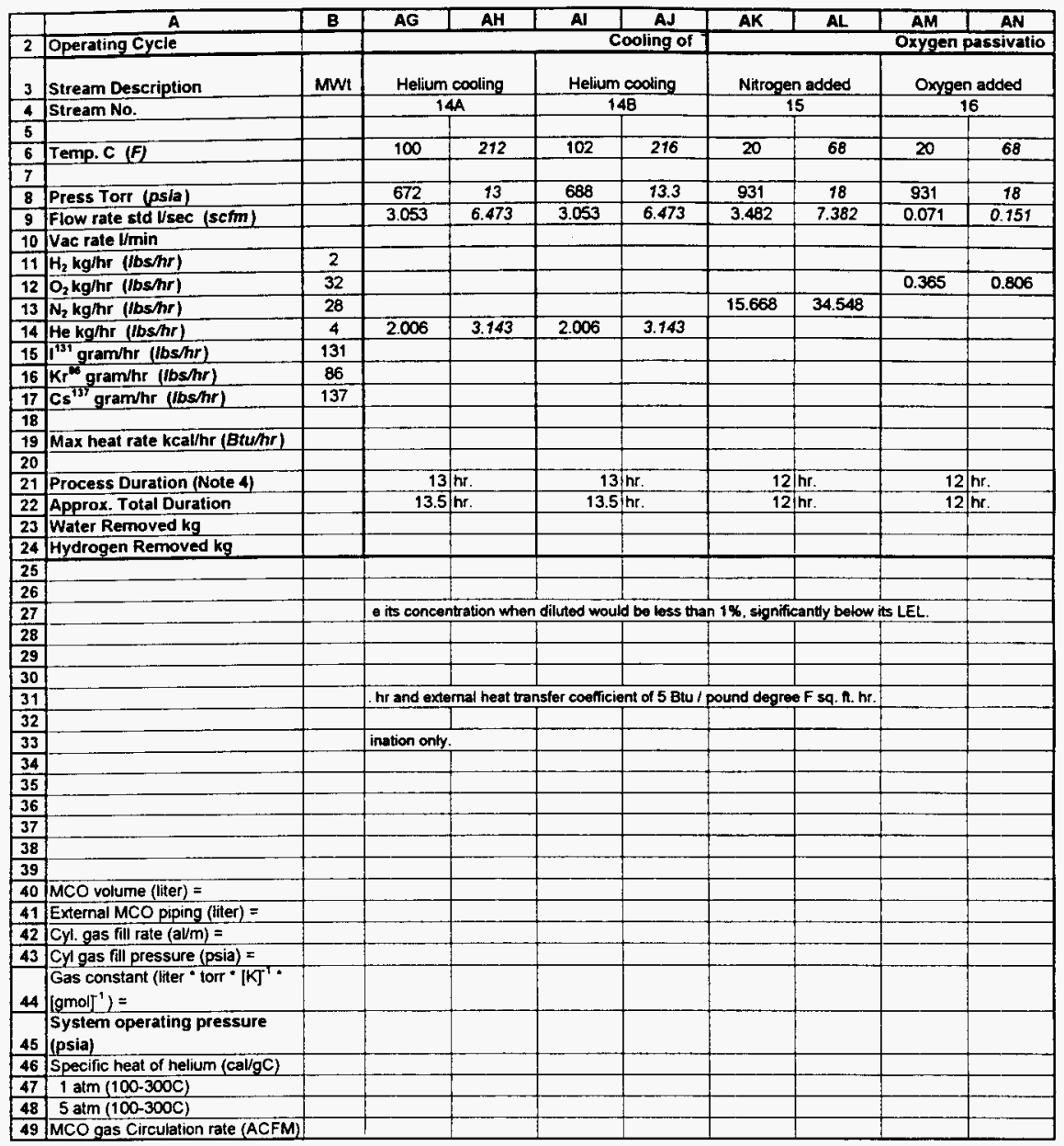




\begin{tabular}{|c|c|c|c|c|c|c|c|c|}
\hline & A & $\mathbf{B}$ & $\overline{A O}$ & AP & $\mathrm{AQ}$ & $\overline{A R}$ & AS & $\overline{A T}$ \\
\hline 2 & Operating Cycle & & & & \multicolumn{4}{|c|}{ Inert Gas Packing } \\
\hline 3 & Stream Description & MWt & \multicolumn{2}{|c|}{$\begin{array}{l}\text { Circulating passivation } \\
\text { gas } \\
\end{array}$} & Oxygen Ev & vacuation & \multicolumn{2}{|c|}{ Heium Fill } \\
\hline 4 & Stream No. & & \multicolumn{2}{|c|}{17} & \multicolumn{2}{|c|}{18} & \multicolumn{2}{|l|}{19} \\
\hline 5 & & & & & & & & \\
\hline 6 & Temp, C $(F)$ & & 150 & 302 & 150 & 302 & 150 & 302 \\
\hline 7 & & & & & & & & \\
\hline 8 & Press Torr (psh) & & 688 & 13.3 & 5 & 0.097 & 1137 & 22 \\
\hline 9 & Flow rate std U/sec (scfin) & & 2.576 & 5.462 & 0.067 & 0.142 & 4.667 & 9.894 \\
\hline 10 & Vac rate Vmin & & & & \multicolumn{2}{|c|}{350} & & \\
\hline 11 & $\mathrm{H}_{2} \mathrm{~kg} / \mathrm{hr}(\mathrm{lbs} / \mathrm{hr})$ & 2 & & & & & & \\
\hline 12 & $\mathrm{O}_{2} \mathrm{~kg} / \mathrm{hr}(\mathrm{lbs} / \mathrm{hr})$ & 32 & & & & & & \\
\hline 13 & $\mathrm{~N}_{2} \mathrm{~kg} / \mathrm{hr}(\mathrm{bs} / \mathrm{hr})$ & 28 & & & & & & \\
\hline 14 & He kg/hr $(\mathrm{bs} / \mathrm{hr})$ & 4 & & & & & 3.000 & 6.615 \\
\hline 15 & $1^{131} \mathrm{gtam} / \mathrm{hr}(\overline{b s} / \mathrm{hr})$ & 131 & & & & & & \\
\hline 16 & $\mathrm{Kr}^{\mathrm{r}} \mathrm{gram} / \mathrm{hr}(\mathrm{bs} / \mathrm{hr})$ & 86 & & & & & & \\
\hline 17 & $\mathrm{Cs}^{137}$ gram/hr $(\omega b s / \mathrm{hr})$ & 137 & & & & & & \\
\hline 18 & & & & & & & & \\
\hline 19 & Max heat rate kcal/hr (Btu/hr) & & & & & & & \\
\hline 20 & & & & & & & & \\
\hline 21 & Process Duration (Note 4 ) & & $12 / \overline{1}$ & hr. & 7.90 & $\min$. & 1.956 & min. \\
\hline 22 & Approx. Total Duration & & 12 r & hr. & 15 & $\min$. & 15 & $\min$. \\
\hline 23 & Water Removed kg & & & & & & & \\
\hline 24 & Hydrogen Removed $\mathrm{kg}$ & & & & & & & \\
\hline 25 & & & & & & & & \\
\hline 26 & & & & & & & & \\
\hline 27 & & & & & & & & \\
\hline 28 & & & & & & & & \\
\hline 29 & & & & & & & & \\
\hline 30 & & & & & & & & \\
\hline 31 & & & & & & & & \\
\hline 32 & & & & & & & & \\
\hline 33 & & & & & & & & \\
\hline 34 & & & & & & & & \\
\hline 35 & & & & & & & & \\
\hline 36 & & & & & & & & \\
\hline 37 & & & & & & & & \\
\hline 38 & & & & & & & & \\
\hline 39 & & & & & & & & \\
\hline 40 & MCO volume (liter) $=$ & & & & & & & \\
\hline 41 & External MCO piping (liter) = & & & & & & & \\
\hline 42 & Cyl. gas fill rate $(\mathrm{al} / \mathrm{m})=$ & & & & & & & \\
\hline 43 & Cyl gas fil pressure (psia) = & & & & & & & \\
\hline 44 & $\begin{array}{l}\text { Gas constant (iter }{ }^{*} \text { torr }{ }^{\cdot}[\mathrm{K}]^{-1}- \\
\left(\mathrm{gmol}^{-1}\right)=\end{array}$ & & & & & & & \\
\hline 45 & $\begin{array}{l}\text { System operating pressure } \\
\text { (psia) }\end{array}$ & & & & & & & \\
\hline 46 & Specific heat of helium (cal/gC) & & & & & & & \\
\hline 47 & $1 \operatorname{atm}(100-300 \mathrm{C})$ & & & & & & & \\
\hline 48 & 5 atm $(100-300 \mathrm{C})$ & & & & & & & \\
\hline 491 & MCO gas Circulation rate (ACFM) & & & & & & & \\
\hline
\end{tabular}


Cell: $\mathrm{C6}$

Note: Range of allowable heating and cooling temperature

Cell: $\mathbf{G 6}$

Note: Assumes receipt at 40 degrees C

Cell: 16

Note: Assumes ambient temperature at start of filling.

Cell: $\mathrm{K} 6$

Note: Set at the maximum permissable temperature for the MCO shell. This temperature is programmed to ramp up to this value. See MCO Heating Temperature Profile for suggesting temperature vs. time for this stream.

Cell: M6

Note: Set based upon blower inlet temperature specifications plus 2 degrees rise for heat of compression.

Cell: W6

Note: Set by inlet temperature restriction to circulation blower

Cell: $Y 6$

Note: per specification

Cell: $\mathrm{C8}$

Note: Vacuum for effective thermal resistance.

Celt: GB

Note: Set per WHC-S-0460 rev. A of 3/11/96

Cell: 18

Note: Fills to this pressure for maximum internal heat transfer.

Cell: M8

Note: Filts to this pressure for maximum internal heat transfer.

Cell: P8

Note: Assumes 0.5 psi drop across helium heater

Cell: Y8

Note: per specification

Cell: E9

Note: Equivalent to $150 \mathrm{ACFM}$

$=150^{*}(459.7+32) /\left((459.7+68)^{*} 60^{*} 0.0353\right)$

Cell: $\mathbf{6 9}$

Note: $=G 9^{*}(10 / 760)^{*}(273 /(273+40))^{*}(1 / 60)$

Cell: 19

Note: Based upon flow set at 280 liters/min at 120 psia. $=\$ C \$ 41^{*}(\$ C \$ 42 / 14.7)^{*}(273 /(15+273)) / 60$

Cell: $K 9$

Note: $=750^{*}(273 /(273+K 5))^{*} 28.3 / 60$

Cell: $M 9$

Note: $=08$

Cell: NG

Note: Maximum internal helium flow based upon Performance Specification WHC-S-0460, rev A

Cell: P9

Note: Maximum internal helium flow based upon Performance Specification WHC-S-0460, rev A $=\$ C \# 48^{*}(07 / 760)^{*}(273 /(273+05))$

Page 7

$G-32$ 
Cell: 09

Note: $=(\$ R \$ 41-\$ R \$ 42)^{\bullet 22.4 /\left(Q 20^{\bullet} 3600\right)}$

Cell: $\$ 9$

Note: $=(\$ R \$ 42+\$ R \$ 43)^{*} 22.4 /(\$ 20 * 3600)$

Cell: U9

Note: $=\left(08^{*} 020+S 8^{*} S 20\right) / U 20$

Cell: W9

Note: $=M B$

Cell: $Y 9$

Note: Set based upon the capacity of the vacuum purnp at operating conditions.

Cell: AA9

Note: $=A A 12 * 1000^{*}(22.4 / 812) / 3600$

Cell: AE9

Note: Based upon flow set at 280 liters/min at 120 psia.

Cell: AH9

Note: Maximum internal helium flow based upon Performance Specification WHC-S-0460, rev A $=\$ C \$ 50^{*}(A G 7 / 760)^{*}(273 /(273+A G 5))$

Cell: A19

Note: $=A G 8$

Cell: AJ9

Note: Maximum intemal helium fow based upon Performance Specification WHC-S-0460. reV A

Cell: AK9

Note: Based upon flow set at 280 liters/min at 120 psia. $=A M 8^{*}(98 / 2)$

Cell: AM9

Note: $=(A M 11 / 3.6)^{*} 22.4 / \$ B \$ 11$

Cell: AP9

Note: $=\$ C \$ 50^{*}(A P 7 / 14.7)^{*}(460 /(460+A P 5))$

Cell: AQ9

Note: $=A Q 9^{*}(10 / 760)^{*}(273 /(273+40))^{\bullet}(1 / 60)$

Cell: AS9

Note: $=22.4^{*}\left(\$ A S \$ 7^{*}(\$ C \$ 41+\$ C \$ 42) /\left(\$ C \$ 45^{*}(\$ A S \$ 5+273)\right)\right) /($ AS20*60)

Cell: Y१ 1

Note: Five volume percent of nitrogen purge per Run 23 . $\left.=Y 8^{*} 0.05 * 3600 / 22.4\right)^{*} 2 / 1000$

Cell: AM12

Note: Based upon $37 \mathrm{~kg}$ of UO2 formed over a 12 hour period.

Cell: $Y 13$

Note: $=\left(1.8^{*} 60 / 22.4\right)^{*} \$ B \$ 12 / 1000$

Cell: AA13

Note: $=Y 12$

Cell: AK\3

Note: Based upon being a 98\%v diluent for enough oxygen to produce $37 \mathrm{~kg}$ UO2 over 12 hour period

Page 8

$$
G-33
$$


Cell: 114

Note: $=(4 / 22.4)^{* 18 * 3600 / 1000 ~}$

Cell: $\mathbf{S 1 4}$

Note: $=\$ 8^{*} 3600^{\circ} 0.004 / 22.4$

Cell: U14

Note: $=\left(Q 13^{*} Q 20+513^{*} S 20\right) / U 2 O$

Cell: AE14

Note: $=\left(A E B^{*} 3600 / 22.4\right)^{* 0.001} 1^{\bullet B 13}$

Cell: AG14

Nole: $=A G 8 * 3.68^{*}(B 13 / 22.4)$

Cell: Al14

Note: $=$ AG13

Cell: AS14

Note: $=0.004^{*}\left(\$ A S \$ 7^{*}(\$ C \$ 41+\$ C \$ 42) /\left(\$ C \$ 45^{*}(\$ A S \$ 5+273)\right)\right) /($ AS20/60)

Cell: L19

Note: This is taken from the temperature profile on spreadsheet HANHEAT3.WQ1

Cell: 019

Note: $=Q^{13^{*}} \mathrm{C} 48$

Cell: $\$ 19$

Note: $=S 13^{*} \mathrm{C} 48$

Cell: AC19

Note: Taken from Cooling Temperature Profite spreadsheet HANHEAT4.WQ1

Cell: G21

Note: computed from pumping speed formula from Perry page 5-34 with calcs enclosed in appendix

Cell: 121

Note: $=\left(\left((\$ C \$ 39+\$ C \$ 40)^{*}(115 / 14.7)^{*}(273 /(15+273))\right) / 18\right) / 60$

Cell: 021

Note: Taken from MCO Temperature Profile for heating. Assumes starting heat up at $40 \mathrm{C}$

Cell: $\mathbf{S 2 1}$

Note: Taken from MCO Temperature Profile for heat up.

Cell: Y21

Note: time for vacuum evacuation only

Cell: AA21

Note: per specification

Cell: AE21

Note: $=\left((C 41+C 42) /\left(A E 8^{*}((273+Y 5) / 273)\right) *(14.7 / C 46)\right) / 60$

Cell: AQ21

Note: from pumping speed formula of Perry

Cell: AS21

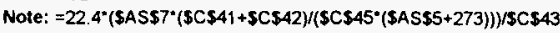

$$
\begin{aligned}
& \text { Page } 9 \\
& \qquad x-34
\end{aligned}
$$


Cell: 523

Note: Assumes all hydrate water charged with fuel is removed during heat up between $200 \mathrm{C}$ and $300 \mathrm{C}$. The interface listing give no more than 4 lbs of this type of water 
Calculation

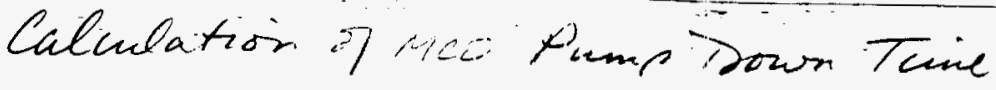

asounce $100 f^{\prime}$ (encialein') " sch 40 pipe with $160 "$ " "2" sec 40 connected to if. Use method of perry $5-32$.

Reference pressure $=1 \mathrm{tb} / \mathrm{A} \mathrm{t}^{2}$

$$
\begin{aligned}
& 14.7 \times 144=2117 \frac{16}{77^{2}} \text { ser atmosphe } \\
& \frac{2117}{760}=2.79 \frac{16}{f+t^{2}} \text { tow } \\
& \frac{16}{7+t^{2}}=0.36 \text { tow } \quad T_{1}=150^{\circ} \mathrm{C}=762^{\circ} \mathrm{R} \\
& T_{2}=40^{\circ} \mathrm{C}=564^{\circ} \mathrm{r} \\
& \rho_{1}=\frac{M}{R T}=\frac{4}{1545 \times 762}
\end{aligned}
$$$$
D_{1}=3.40 \times 10^{-6} \mathrm{lb} / \mathrm{ft}^{3}
$$$$
\rho_{2}=4.59 \times 10^{-6} \mathrm{lt} / \mathrm{ft}^{3}
$$$$
w=\text { wight sate of flow }
$$

at 0.36 tow pump dewplaccomst is $350 \mathrm{l} / \mathrm{mm}$

$$
\begin{aligned}
& w_{1}=3.4 \times 10^{-6} \frac{16}{7 t^{3}} \times 350 \frac{\mathrm{k}}{\mathrm{min}} \times \frac{1 \mathrm{~min}}{604 \mathrm{c}} \times \frac{1 \mathrm{At}^{3}}{28.36} \\
& =7.00 \times 10^{-7} \mathrm{Ht} / \mathrm{sec} \\
& w_{2}=\frac{4.59 \times 10^{-6}}{3.40 \times 10^{-6}} \times 7.00 \times 10^{-7}=9.45 \times 10^{-7} \frac{1 \mathrm{~b}}{\mathrm{sec}} \\
& q^{\prime}=w / \rho \\
& q_{1}^{\prime}=7 \times 10^{-7} \frac{1 t}{\sec } \times \frac{1}{3.4 \times 10^{-6} 1 t b}=0.21 t^{3} t^{3} / \mathrm{sec} \\
& q_{2}^{\prime}=9.45 \times 10^{-7} \frac{1 \mathrm{~b}}{\mathrm{scc}} \times \frac{1}{4.59 \times 10^{-6} \frac{\mathrm{H}^{2}}{110}}=0.21 \mathrm{ft}^{3} / \mathrm{sec}
\end{aligned}
$$

$G-36$ 
$5.0 \times 5 \cdot h$

$=0 \times 5 \cdot h$

s. $O \cdot b \cdot p$

$\underline{5-0, \times b i}$

in prapo
$2 .-1 \times p \cdot 9$

$0,0.1 \times 11$

$1-0,+1 ' \varepsilon$

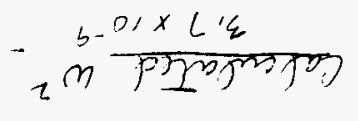

0900

120029

$1003^{\circ}$

2दाच

$L=2$

$=$

ofsel $6900 \cdot 0=$ ofsd $\frac{h+1}{1}=\mathrm{d}$ tf $\varepsilon^{\prime} \varepsilon_{1}=21 / 091=m 2 / 17$ $+\phi o_{\theta 1}=m 17$

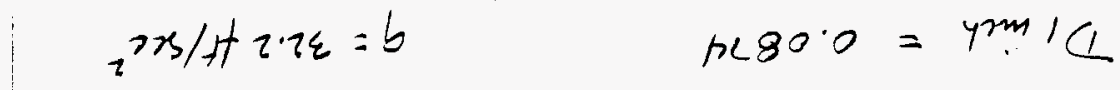
8150.0 = n? $2 / 10$

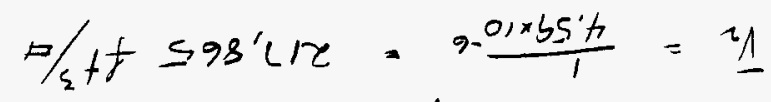
F/ $/ \xi+811$ hbz $=\frac{901 \times h \cdot \xi}{T}=\frac{d d}{1}=\frac{41}{\varepsilon+7}=11$ ${ }_{1-} 01 \times 5 \cdot h=z^{t} \quad 11200 \cdot 0=t \quad 0^{2} / 1$ uef $5-01 \times 9 \cdot \varepsilon={ }_{2} t \quad 900 \cdot 0=\forall$ rint"

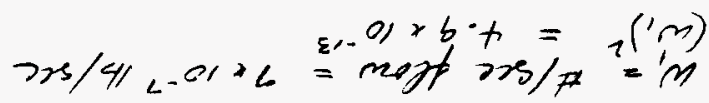

(hz-o $\alpha$ olf Tang as) $9.0+20$

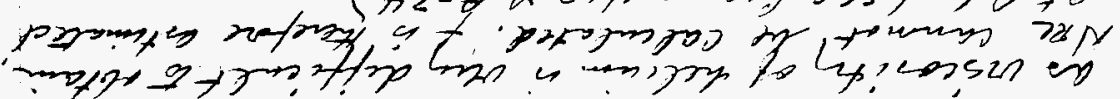

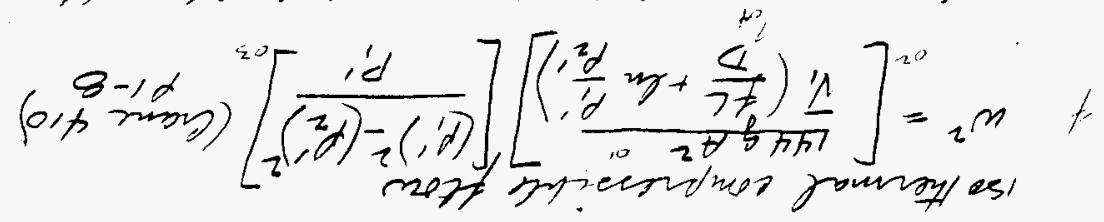

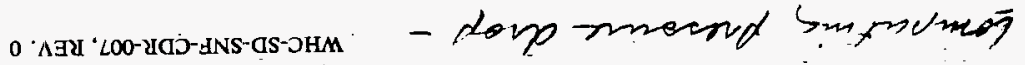

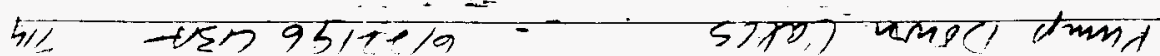


Hump down calls

$6 / 22 / 46 \mathrm{LBA} / 1 / 6$

$\Delta P$ for $1^{\prime \prime}=0.0001$ pisa

WHC-SD-SNF-CDR-007, REV. 0

$\Delta \rho$ for $1 / 2$

$\frac{\operatorname{Trm} P_{2}}{.005}$

.006

.0065
$\frac{\operatorname{Cal} a \cot d \mathrm{w}^{2}}{1.63 \times 10^{-11}}$

$7.68 \times 10^{-12}$

$3.5 \times 10^{-12}$ $\frac{\text { Actual } \omega^{2}}{4.5 \times 10^{-13}}$

$\Delta P$ for $1 / 2^{\prime \prime}=0.0001$ sta

$\sum \Delta \omega^{\prime}=0.0002$ spa

Flow nate \& is at 1 pst

$$
\begin{aligned}
& c=\frac{q}{\Delta p}=0.21 / .0002=1050 \mathrm{ft}^{3} / \mathrm{sec} \\
& 1 / s_{0}=1 / s_{p}+1 / c \quad s_{p}=350 \frac{\mathrm{m}}{\mathrm{mm}} \times \frac{\mathrm{At}^{+}}{28.31} \times \frac{\mathrm{min}}{6054} \\
& 1 / s_{0}=1 / s_{r}+1 / c=4.85 \quad=0.2061 \\
& s_{0}=0.206
\end{aligned}
$$

Pump down time -

$$
\begin{aligned}
& \theta=\left(V_{t} / s_{0}\right) \ln \left[\left(p_{1}-p_{2}\right) /\left(p_{2}-p_{0}\right)\right] \\
& V_{t}=507.19 l+60.56 l=567.75 l=20.06 \mathrm{ft}^{3} \\
& S_{0}=0.206 f^{+3} / \mathrm{sec} \\
& P_{1}=131 \mathrm{~b} / \mathrm{in}^{2}=1,872 \mathrm{lt} / \mathrm{ft}^{2} \\
& P_{2}=5 \text { tour }=5 / 6.36=13.91 \mathrm{~b} / \mathrm{ft}^{2} \\
& P_{0}=6 \times 10^{-3} \text { tor }=6 \times 10^{-3} \operatorname{ton} \times \frac{116 / 4 t^{2}}{0.36 \text { tot }} \\
& =1.67 \times 10^{-2} 1 \mathrm{t} / \mathrm{ft} \mathrm{f}^{2} \\
& E=\frac{20.06}{c .206} \ln \left[(1872-13.9) /\left(13.9-1.67 \times 10^{-2}\right)\right] \\
& =476 \mathrm{gec}=7.9 \mathrm{~mm} \text { do pan. tim } 13 \text { aria } \\
& G-38
\end{aligned}
$$


$b \varepsilon-D$

$$
\begin{aligned}
& \text { man } 8 \cdot 9=\cos 807=
\end{aligned}
$$

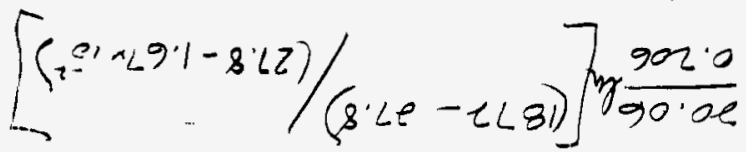

$$
\begin{aligned}
& \text { mot } 01 \text { of dmed }
\end{aligned}
$$


WHC-SD-SNF-CDR-007, REV. 0

HVC-6

LBA

$7 / 26 / 96$

\section{CALCULATIONS TO DEMONSTRATE THE TEMPERATURE DIFFERENCE ON THE MCO DURING COOL-DOWN}

Since issuing the 90 percent CDR, we have received calculations from Q-Metrics which indicate a temperature difference of approximately $150^{\circ} \mathrm{C}$ across the MCO when taking approximately 24 hours to heat the fuel to a bulk temperature of $300^{\circ} \mathrm{C}$. This calculation was performed using finite element analysis. It supersedes the calculation submitted with the $90 \%$ CDR.

It should be kept in mind that the $\mathrm{MCO}$ can be operated within its $100^{\circ} \mathrm{C}$ temperature difference specification by changing the temperature profile, taking longer to either heat or cool. We will constrain the profile with both the $50^{\circ} \mathrm{C}$ rate spec and the $100^{\circ} \mathrm{C}$ gradient spec in the final MCO temperature patterned profile and intend to coordinate these changes with Q-Matrix through WHC. 
Q-Metrics, Inc.

P.O. $\$ 0 \times 3016$

Woodinville, WA $98072-3016$

Prone: (206) 915-8590/Fax: (206) 48i-5953

FAX TIRANSMUTTAL RAIESSAGE SHEET

Date/Time: Z/2/96

No. of Pages (Inciuding eover): 2

Deliver To: Curt Mlska/WHC

Fax Phone: 1509$) \quad 376-3252$

Project: Spent Fuel Thermal Support

Subject: MCO Wall Temperature Transient For Heatuo/Cool Down

\section{Message:}

The attached figure illustrates the estimated wall temperatures along the MCO length during the heat up and cool down cycles of the hot conditioning process. The analysis is based on the following assumptions:

1) Merrick's proposed approach of using $750 \mathrm{cfm}$ of air in a 1-inch annulus around the MCO. Flow procesds from bottom to top.

2) Five layers of Mark IV fuel baskets with maximum decay heat. No chemical reaction heating is assumed for this analysis.

3) MCO design with the weided head. No special head region heaters employed.

The eurves presented on the figure are daflned as follows:

TPLUG - average temperature of MCO closure plug

T3 - inlet air temperature

TA270 - temperature of MCO sidewall at bottom

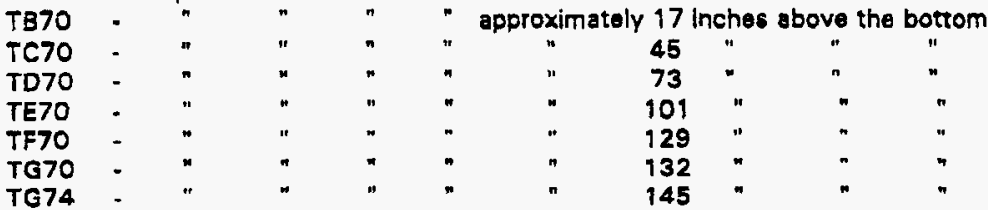

As seen from the figure, the MCO wall temperature gredient ls expected to be relativaly low except in the head region. Without some sort of supplemental heating or cooling. the closure flug will lag the average MCO wall temperature by up to $100^{\circ} \mathrm{C}$ during heat up and $75^{\circ} \mathrm{C}$ during cool down.

Let me know if you have any questions. 
FROM : Panasonic FAX SYSTEM.

PAONE NO. :

Jul. 98 1996 24:05PM F2 WHC-SD-SNF-CDR-007, REV: 0

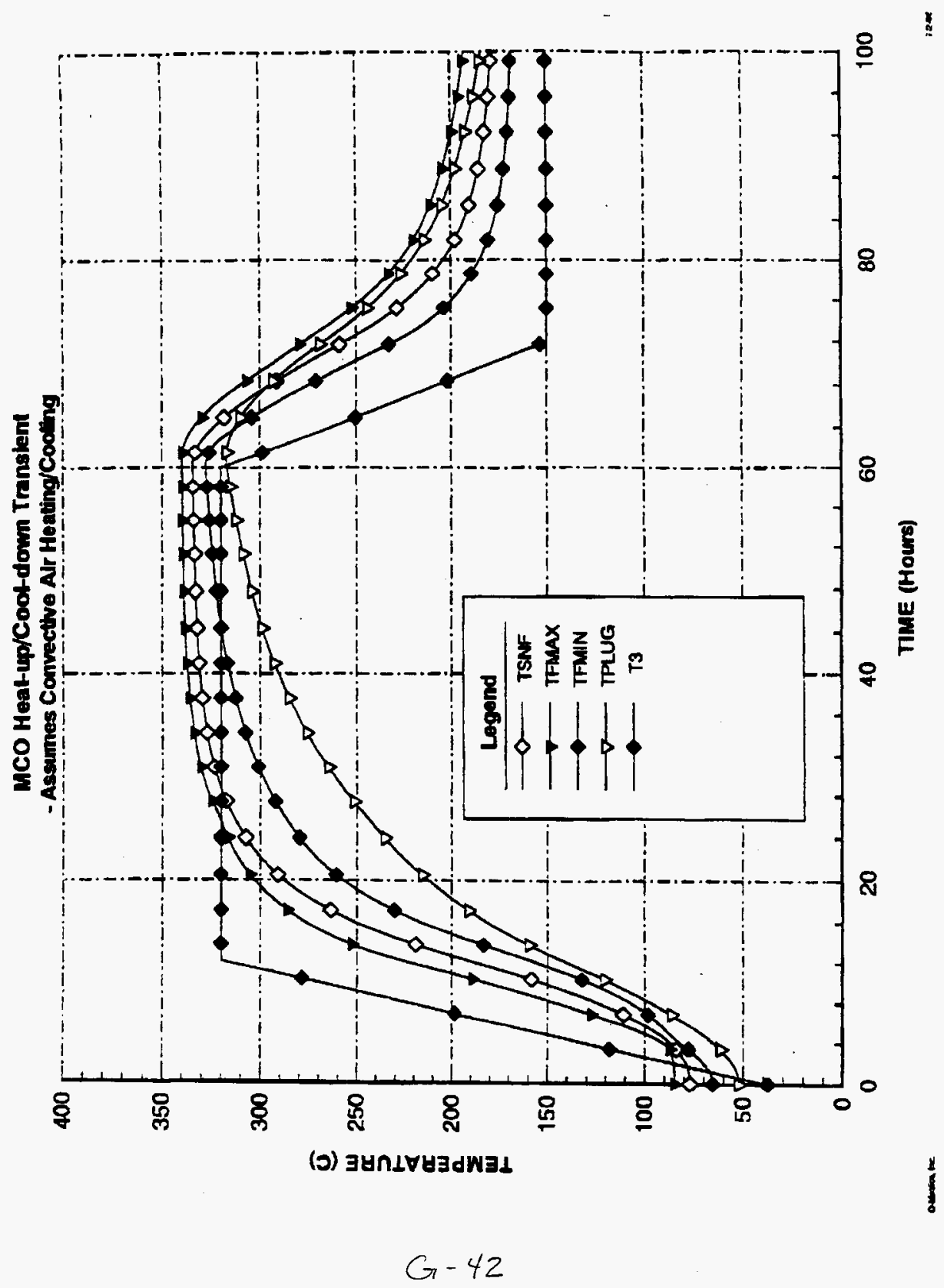




\section{Q-Metrics}

Q-Metrics, Inc.

P.0. Box 3016

Woodinville. WA $98072-3016$

Plone: (206) 915-8590/Fax: (206) 48i-5953

FAX TRANSMUTIAL MESSAGE SHEET

Date/Time: $7 / 2 / 96$

No. of Pages (including eover): 2

16 Deliver To: Curt Miska/WHC

Fax Phone: 1509 ) 376-3252

Project: Spent Euel Thermal Supoert
Voice \#: $376-7103$

Project \#:E014N-KBasin

\section{Subject: MCO Wall Temperature Transient For Heatup/Cool Down}

\section{Message:}

The attached figure illustrates the estimated wall temperatures along the MCO length during the heat up and cool down cycles of the hot conditioning pracess. The analysis is based on the following assumptions:

1) Merrick's proposed approach of using $750 \mathrm{cfm}$ of air in a 1+inch annulus around the MCO. Flow proseads from bottom to top.

2! Five layers of Mark IV fuel baskets with maximum decay heat. No chemical reaction heating ls assumed for this analysis.

3) MCO design with the welded head. No special head region heaters employed.

The curves presented on the figure are dafined as follows:

TPLUG - average remperature of MCO closure plug

T3 - iniet air temperature

TA270 - temperazure of MCO aldowall at bottom

TB70 - " " " approximately 17 inches above the bottom

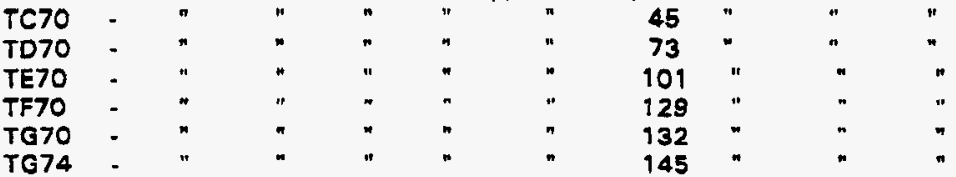

As seen from the figure, the MCO wall temperature gradient is expected to be relatively low except in the head region. Without some sort of supplemental heating or cooling, the closure plug will lag the average MCO wall temperature by up to $100^{\circ} \mathrm{C}$ during heat up and $75^{\circ} \mathrm{C}$ during cool down.

Let me know if you have any questions. 


\section{Q-Metrics}

Q-Metrics, Inc.

P.O. Box 3016

Wondinville, WA $98072-3016$

Phore: (206) 915-8590/Fax: (206) 481-5953

\section{FAX TRANSMITTAL M NESSAGE SHEET}

Date/Time: 7/8/96

No. of Pages (including cover): 2

Deliver To: Cur Miske/ WHC

Fax Phone: (509) 376-3252

Voice \#: $376-7103$

Project: Soent Fuel Thermal Supoort Project \#:E014N-KBasin

Subject: Mark IV Fuel Temo. Iransient For Heatup/Cool Down

\section{Message:}

The attached figure lliustrates the estimated average (TSNF), maximum (TFMAX), and minimum (TFMIN) funl element temperatures during the heat up and cool down eycles of the hot conditioning process. The plot is a companion to the plot faxed to you on $7 / 2$. In addition to the analysis assumptions described in that fax. the following pertinent facts need to be added:

1) no credit is taken for heating and cooltng via the feed gases.

2) Onfy "clean" Mark IV fuel Is sssumed. A thermal model with sludge coated fuel and the latest fuel basket design is being created and checked out.

Let me know if you have any questions. 


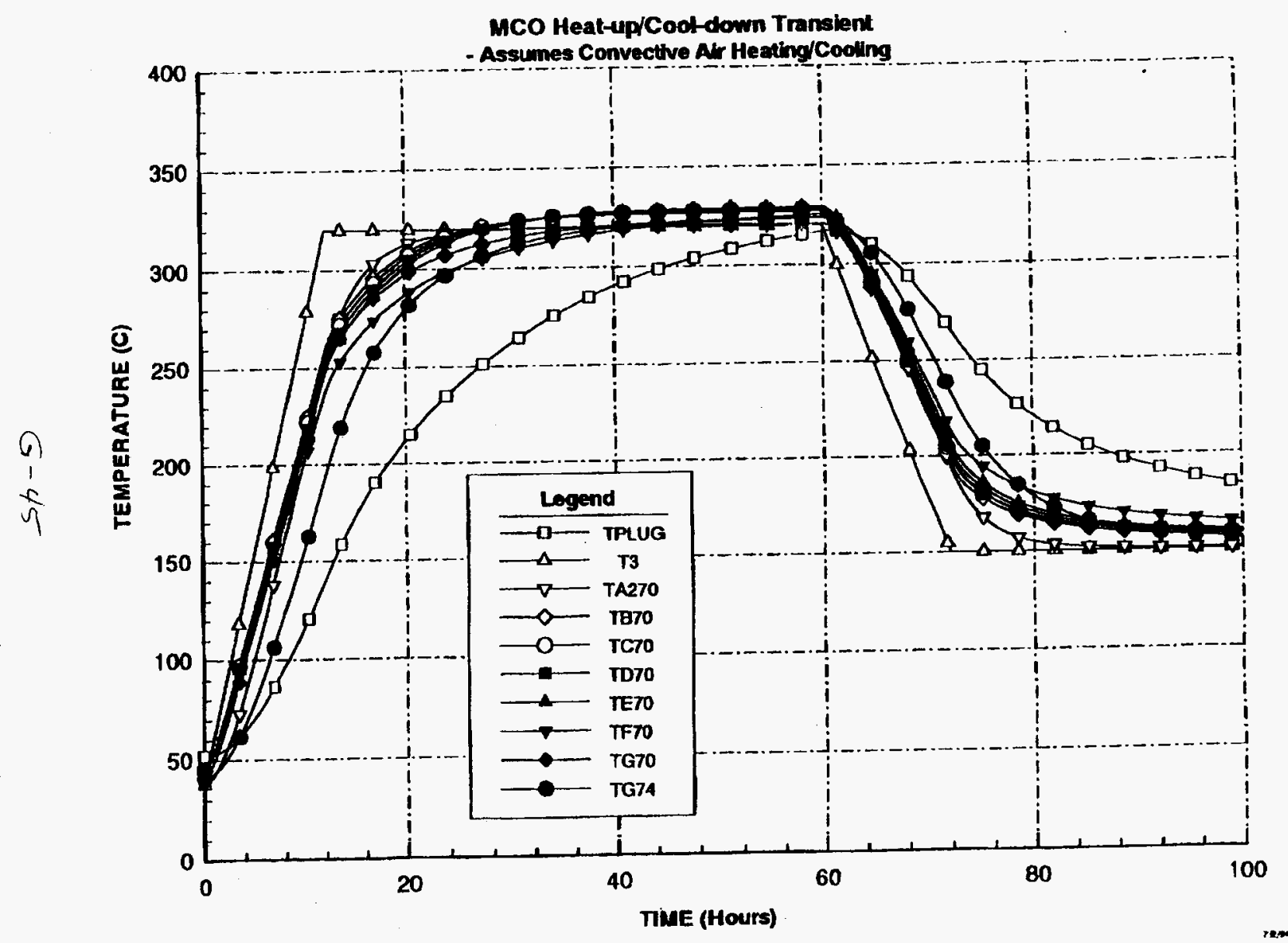




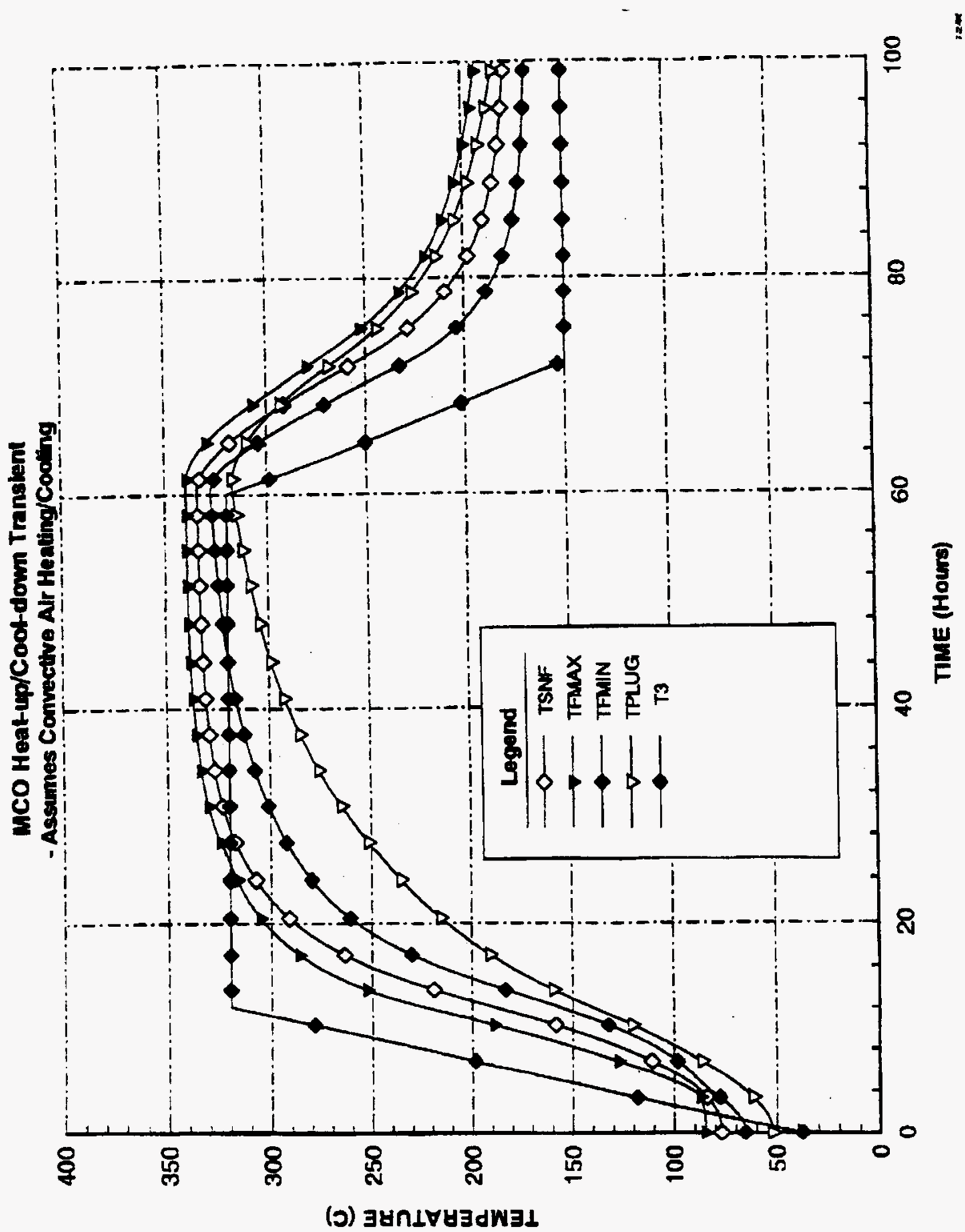


WHC-SD-SNF-CDR-007, REV. 0

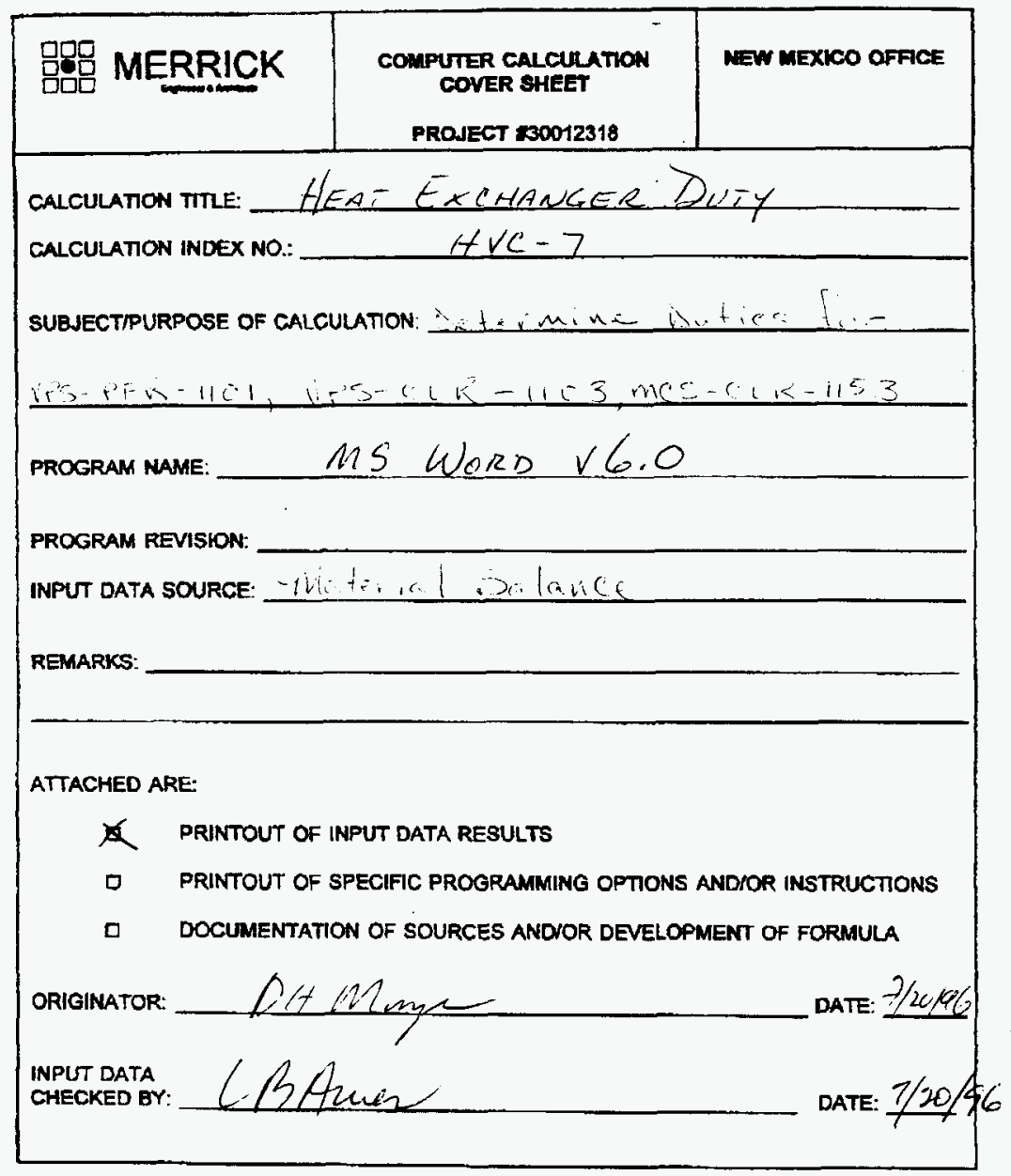

$G-47$ 


\section{Vacuum Pumping System Coolers}

$C_{p}$ Air $@$ Ambient Conditions $=0.0181 \mathrm{BTU} / \mathrm{Ft}^{3}$

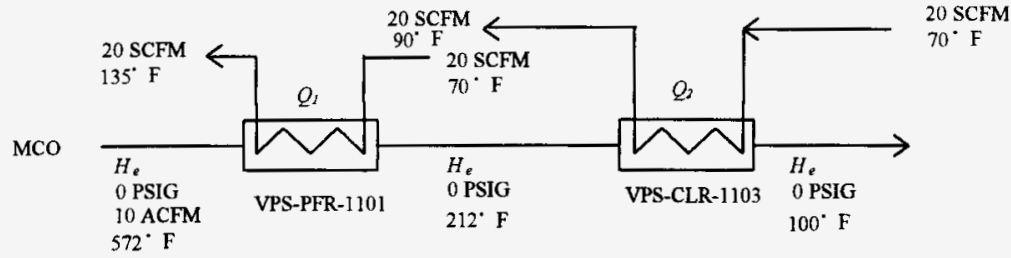

Mass Flow $H_{e}$

$\frac{10 \mathrm{Ft}^{3}}{\operatorname{Min}} X \frac{68460}{572460} \frac{0.0103 \#}{\mathrm{Ft}^{3}}=0.0527 \# / \mathrm{Min} H_{e}$

$H_{c}$ Heat Load $Q_{1}$

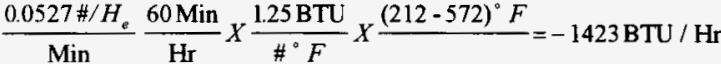

$H_{e}$ Heat Load $Q_{2}$

$\frac{0.0527 \# / H_{e}}{\operatorname{Min}} X \frac{60 \mathrm{Min}}{\mathrm{Hr}} X \frac{1.25 \mathrm{BTU}}{\#^{\circ} F} X \frac{(100-212)^{\circ} F}{{ }^{\circ}}-443 \mathrm{BTU} / \mathrm{Hr}$

20 SCFM each for Air Cool in

$\frac{0.0181 \mathrm{BTU}}{\mathrm{Ft}^{3}{ }^{\circ} \mathrm{F}} X \frac{20 \mathrm{Ft}^{3}}{\mathrm{Min}} X \frac{60 \mathrm{Min}}{\mathrm{Hr}}=21.72 \mathrm{BTU} / \mathrm{Hr}{ }^{\circ} \mathrm{F}$

$Q_{1}$ Load $\frac{1423 \mathrm{BTU}}{\mathrm{Hr}} X \frac{\mathrm{Hr}^{\circ} \mathrm{F}}{2172 \mathrm{BTU}}=69^{\circ} F$ Temp. Rise

$Q_{2}$ Load $\frac{443 \mathrm{BTU}}{\mathrm{Hr}} X \frac{\mathrm{Hr}^{\circ} F}{2172 \mathrm{BTU}}=20.4^{\circ} F$ Temp. Rise 


\section{MCO Cooling System Cooler \\ MCS-CLR-1153}

Assume $82,000 \mathrm{Btu} / \mathrm{hr}$ max duty which is the rate of heat transfer for an MCO temperature change of $50^{\circ} \mathrm{C}$ per hour.

CASE \#1: High Temp Case $572^{\circ}$ F Air @ 750 ACFM

$$
\begin{gathered}
\frac{750 \mathrm{Ft}^{3}}{m} \frac{(68 \times 460)}{(572+460)} \frac{0.075^{\#}}{\mathrm{Ft}^{3}} \frac{60 \mathrm{~m}}{\mathrm{Hr}}=1726 \# / \mathrm{Hr} \\
\frac{82,000 \mathrm{Btu}}{\mathrm{Hr}} \frac{\mathrm{Hr}}{1,726 \# .241 \mathrm{Btu}} \frac{\#^{\circ} \mathrm{F}}{}=197^{\circ} \mathrm{F}
\end{gathered}
$$

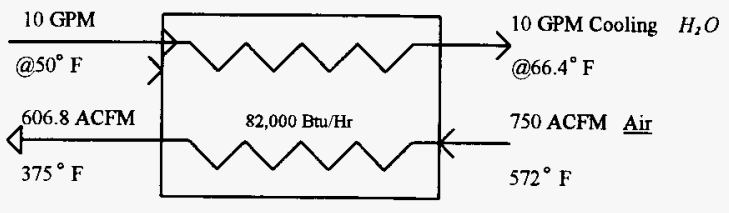

$$
\begin{aligned}
& \Delta T H_{2} O=\frac{82,000 \mathrm{BTU}}{\mathrm{Hr}} \frac{\#^{\circ} \mathrm{F}}{1 \mathrm{BTU}} X \frac{}{10 \mathrm{GPM}} X \frac{\mathrm{GPM}}{500 \# / \mathrm{Hr}}=16.4^{\circ} \mathrm{F} \\
& 750 \mathrm{ACFM} \frac{375+460}{572+460}=606.8 \mathrm{ACFM} 3375^{\circ} \mathrm{F}
\end{aligned}
$$


CASE \#2: Low Temp Case $300^{\circ} \mathrm{F}$ Air@750 ACFM

$$
\begin{aligned}
& \frac{750 \mathrm{Ft}^{3}}{m} \frac{68+460}{300+400} \frac{0.075^{\sharp}}{\mathrm{Ft}^{3}} \frac{60 \mathrm{~m}}{\mathrm{Hr}}=2.345 \# / \mathrm{Hr} \\
& \frac{82,000 \mathrm{Btu}}{\mathrm{Hr}} \frac{\mathrm{Hr}}{2.345^{*}} \frac{\#{ }^{\circ} \mathrm{F}}{0.241 \mathrm{Btu}}=145^{\circ} \mathrm{F} \\
& \frac{750 \mathrm{Ft}^{3}}{n} \frac{155+460}{300+460}=606 \mathrm{ACFM}(a) 155^{\circ} \mathrm{F}
\end{aligned}
$$

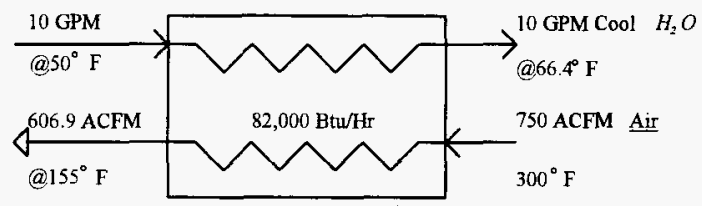




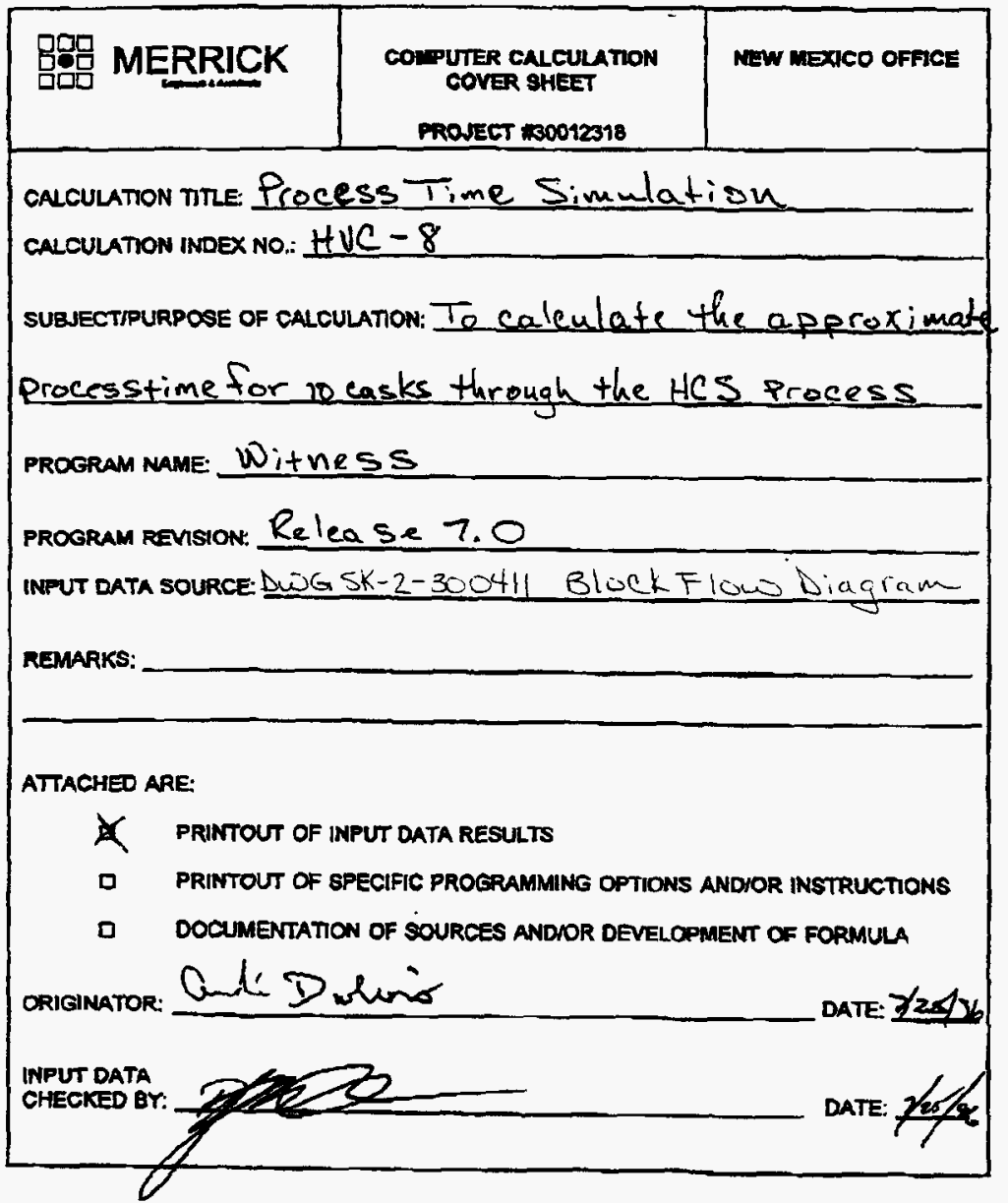




\section{INTRODUCTION:}

The following report summarizes results taken from a simulated run of a model created in WITNESS Release 7.0. WITNESS can simulate real events through random number generation and will create reports documenting inputs into the model as well as results from the actual run. The following statistics report details the activity of each block during the simulated run. The following summary report describes the characteristics of each block and thus how they behaved in the model. Any questions regarding report interpretation should be directed at Merrick.

\section{PURPOSE:}

The purpose of this model is to calculate the approximate process time for 10 casks through a hot conditioning process.

\section{ASSUMPTIONS:}

1. All information for the model, except for shift times, was supplied by the block flow diagram, Figure 2.1-1, Hot Conditioning Model Operating Sequence. This included identification of each process, order of processes, process times, decision blocks, and percentages on yes/no decisions.

2. Decision blocks that did not indicate a percentage were assigned a 50/50 chance yes or no.

3. Only one item could be processed for each cycle.

4. Shift times were input to the model. It was assumed seven days on, no days off. Each day on consisted of three 8 hour shifts.

5. No statistical or deterministic breakdowns were included in the model.

6. The model had an infinite amount of labor available, i.e. tasks were not resource constrained.

7. Ten (10) complete operations would be modeled.

8. An allowance of $\mathbf{2 4}$ hours was placed in the model between cycle completion and restart to allow for any clean up or preparatory activities.

\section{CONCLUSION:}

The model determined it would take approximately 115.52 hours to process each cask through the entire process. To process ten casks consecutively, approximately $1,410.30$ hours would be required; this is roughly 59 days, or 8.5 weeks. Of course, these results are largely based on the above assumptions. Consideration should be given that these assumptions are valid.

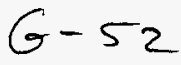


STATISTICS REPORT:

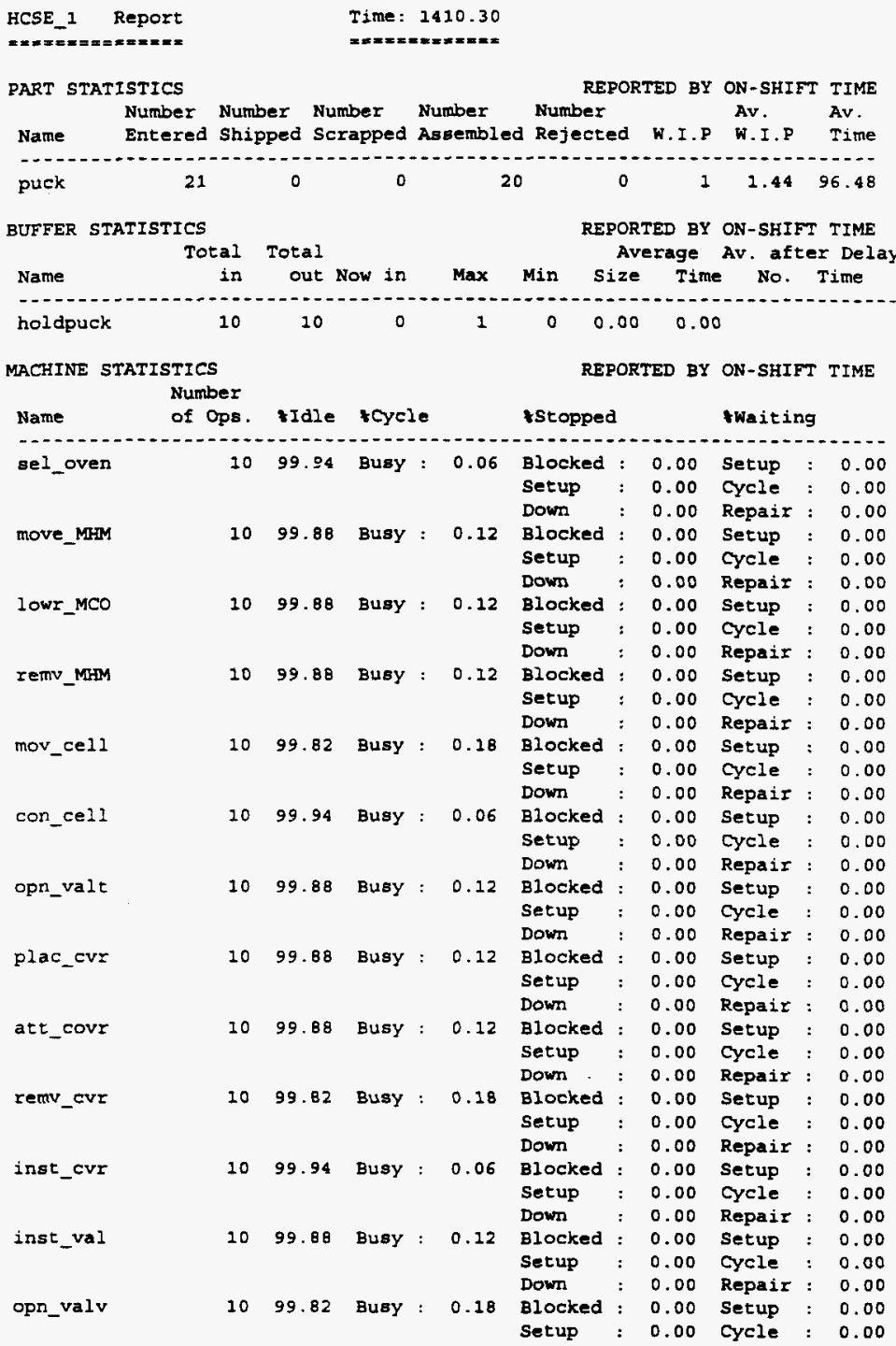




\begin{tabular}{|c|c|c|c|c|c|c|c|c|c|c|c|}
\hline & Number & & & & & & - & & & & \\
\hline Name & of Ops. & tIdle & tcycl & & & Istopped & & & twaitis & & \\
\hline & & & & & & Ra- & & & & & \\
\hline & & & & & & Down & : & 0.00 & Repair & : & 0.00 \\
\hline evac_MCO & 10 & 99.76 & Busy & $:$ & 0.24 & Blocked & : & 0.00 & Setup & $:$ & 0.00 \\
\hline & & & & & & Setup & : & 0.00 & Cycle & : & 0.00 \\
\hline & & & & & & Down & : & 0.00 & Repair & $:$ & 0.00 \\
\hline Eill_MCO & 10 & 99.94 & Buby & $:$ & 0.06 & Blocked & : & 0.00 & Setup & : & 0.00 \\
\hline & & & & & & Setup & : & 0.00 & Cycle & : & 0.00 \\
\hline & & & & & & Down & : & 0.00 & Repair & : & 0.00 \\
\hline leak_chk & 12 & 99.79 & Busy & : & 0.21 & Blocked & : & 0.00 & Setup & : & 0.00 \\
\hline & & & & & & Setup & $:$ & 0.00 & oycle & : & 0.00 \\
\hline & & & & & & Down & $:$ & 0.00 & Repair & : & 0.00 \\
\hline passleak & 12 & 99.99 & Busy & $:$ & 0.01 & Blocked & : & 0.00 & Setup & $:$ & 0.00 \\
\hline & & & & & & Setup & : & 0.00 & cycle & : & 0.00 \\
\hline & & & & & & Down & $:$ & 0.00 & Repair & : & 0.00 \\
\hline cls_valv & 2 & 99.99 & Busy & : & 0.01 & Blocked & : & 0.00 & Setup & : & 0.00 \\
\hline & & & & & & Setup & : & 0.00 & cycle & : & 0.00 \\
\hline & & & & & & Down & : & 0.00 & Repair & : & 0.00 \\
\hline disc_vIv & 2 & 99.99 & Busy & : & 0.01 & Blocked & $:$ & 0.00 & Setup & $:$ & 0.00 \\
\hline & & & & & & setup & $:$ & 0.00 & Cycle & : & 0.00 \\
\hline & & & & & & Down & : & 0.00 & Repair & : & 0.00 \\
\hline cln_port & 2 & 99.99 & Busy & : & 0.01 & Blocked & : & 0.00 & Setup & : & 0.00 \\
\hline & & & & & & setup & $:$ & 0.00 & Cycle & $:$ & 0.00 \\
\hline & & & & & & Down & : & 0.00 & Repair & : & 0.00 \\
\hline inst_vlv & 2 & 99.99 & Busy & $:$ & 0.01 & Blocked & : & 0.00 & Setup & : & 0.00 \\
\hline & & & & & & Setup & : & 0.00 & oycle & : & 0.00 \\
\hline & & & & & & Down & : & 0.00 & Repair & : & 0.00 \\
\hline insl_cvr & 10 & 99.94 & Busy & $:$ & 0.06 & Blocked & : & 0.00 & Setup & $:$ & 0.00 \\
\hline & & & & & & Setup & : & 0.00 & Oycle & : & 0.00 \\
\hline & & & & & & Down & $:$ & 0.00 & Repair & : & 0.00 \\
\hline cls_valt & 10 & 99.94 & Busy & : & 0.06 & Blocked & $:$ & 0.00 & Setup & : & 0.00 \\
\hline & & & & & & Setup & : & 0.00 & Cycle & : & 0.00 \\
\hline & & & & & & Down & : & 0.00 & Repaix & $:$ & 0.00 \\
\hline het_cyel & 10 & 91.49 & Busy & : & 0.51 & Blocked & $:$ & 0.00 & Setup & $:$ & 0.00 \\
\hline & & & & & & Setup & : & 0.00 & Oycle & $:$ & 0.00 \\
\hline & & & & & & Down & $:$ & 0.00 & Repair & $:$ & 0.00 \\
\hline surv_cel & 10 & 99.65 & Busy & : & 0.35 & Blocked & : & 0.00 & Setup & : & 0.00 \\
\hline & & & & & & Setup & $:$ & 0.00 & Cycle & $:$ & 0.00 \\
\hline & & & & & & Down & $:$ & 0.00 & Repair & $:$ & 0.00 \\
\hline discproc & 10 & 99.94 & Busy & : & 0.06 & Blacked & $:$ & 0.00 & Setup & : & 0.00 \\
\hline & & & & & & Setup & : & 0.00 & Cycle & : & 0.00 \\
\hline & & & & & & Down & $:$ & 0.00 & Repair & : & 0.00 \\
\hline remvproc & 10 & 99.82 & Busy & $:$ & 0.18 & Blocked & $:$ & 0.00 & Setup & : & 0.00 \\
\hline & & & & & & Setup & : & 0.00 & Cycle & : & 0.00 \\
\hline & & & & & & Down & $:$ & 0.00 & Repait & : & 0.00 \\
\hline purg_vac & 10 & 65.96 & Busy & $:$ & 34.04 & Blocked & $:$ & 0.00 & Setup & : & 0.00 \\
\hline & & & & & & Setup & : & 0.00 & Cycle & : & 0.00 \\
\hline & & & & & & Down & $:$ & 0.00 & Repair & : & 0.00 \\
\hline perftest & 11 & 98.44 & Busy & $:$ & 1.56 & Blocked & : & 0.00 & Setup & : & 0.00 \\
\hline & & & & & & setup & $:$ & 0.00 & Cycle & : & 0.00 \\
\hline & & & & & & Down & $:$ & 0.00 & Repair & : & 0.00 \\
\hline accept & 11 & 99.99 & Busy & $:$ & 0.01 & Blocked & : & 0.00 & Setup & : & 0.00 \\
\hline & & & & & & Setup & : & 0.00 & cycle & : & 0.00 \\
\hline & & & & & & Down & $:$ & 0.00 & Repair & : & 0.00 \\
\hline add_purg & 1 & 98.30 & Busy & $:$ & 1.70 & Blocked & : & 0.00 & Setup & $:$ & 0.00 \\
\hline & & & & & & setup & : & 0.00 & Cycle & $:$ & 0.00 \\
\hline & & & & & & Down & : & 0.00 & Repair & : & 0.00 \\
\hline coolpass & 10 & 91.49 & Busy & : & B. 51 & Blocked & $:$ & 0.00 & Setup & $:$ & 0.00 \\
\hline & & & & & & Setup & : & 0.00 & Cycle & : & 0.00 \\
\hline
\end{tabular}




\begin{tabular}{|c|c|c|c|c|c|c|c|c|c|c|c|}
\hline & Number & & & & & & & & & & \\
\hline Name & of Ops. & idle & tCycl & & & Istopped & & & twaitir & & \\
\hline- & $=-$ & & & & $=$ & & & -- & $=$ & & \\
\hline & & & & & & Down & : & 0.00 & Repair & : & 0.00 \\
\hline eva eMco & 10 & 99.76 & Busy & $:$ & 0.24 & Blocked & : & 0.00 & Setup & : & 0.00 \\
\hline & & & & & & Setup & : & 0.00 & Cycle & : & 0.00 \\
\hline & & & & & & Down & : & 0.00 & Repair & $:$ & 0.00 \\
\hline pass_gas & 10 & 99.94 & Busy & $:$ & 0.06 & Blocked & : & 0.00 & Setup & $:$ & 0.00 \\
\hline & & & & & & Setup & : & 0.00 & Sycle & : & 0.00 \\
\hline & & & & & & Down & : & 0.00 & Repair & : & 0.00 \\
\hline pass_per & 10 & 91.49 & Busy & $:$ & 8.51 & Blocked & : & 0.00 & Setup & : & 0.00 \\
\hline & & & & & & Setup & $:$ & 0.00 & Cycle & : & 0.00 \\
\hline & & & & & & Down & : & 0.00 & Repair & 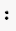 & 0.00 \\
\hline cooldown & 10 & 92.91 & Busy & $:$ & 7.09 & Blocked & : & 0.00 & Setup & : & 0.00 \\
\hline & & & & & & setup & : & 0.00 & Cycle & : & 0.00 \\
\hline & & & & & & Down & : & 0.00 & Repaix & : & 0.00 \\
\hline evacMCO2 & 10 & 99.76 & Busy & : & 0.24 & Blocked & : & 0.00 & Setup & : & 0.00 \\
\hline & & & & & & setup & : & 0.00 & cycle & : & 0.00 \\
\hline & & & & & & Down & : & 0.00 & Repair & 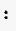 & 0.00 \\
\hline fill_He & 10 & 99.94 & Busy & : & 0.06 & Blocked & : & 0.00 & Setup & : & 0.00 \\
\hline & & & & & & Setup & : & 0.00 & Gyele & : & 0.00 \\
\hline & & & & & & Down & : & 0.00 & Repair & : & 0.00 \\
\hline move_in & 10 & 99.82 & Bugy & : & 0.18 & Blocked & : & 0.00 & setup & : & 0.00 \\
\hline & & & & & & setup & : & 0.00 & Cycle & : & 0.00 \\
\hline & & & & & & Down & : & 0.00 & Repair & $:$ & 0.00 \\
\hline conn_cel & 10 & 99.94 & Busy & $:$ & 0.06 & Blocked & : & 0.00 & Setup & : & 0.00 \\
\hline & & & & & & setup & $:$ & 0.00 & cycle & : & 0.00 \\
\hline & & & & & & Down & : & 0.00 & Repair & $:$ & 0.00 \\
\hline openvalt & 10 & 99.88 & Busy & : & 0.12 & Blocked & : & 0.00 & setup & : & 0.00 \\
\hline & & & & & & setup & : & 0.00 & cycle & : & 0.00 \\
\hline & & & & & & Down & : & 0.00 & Repair & : & 0.00 \\
\hline remvcovr & 10 & 99.94 & Busy & : & 0.06 & Blocked & : & 0.00 & Setup & : & 0.00 \\
\hline & & & & & & Setup & : & 0.00 & Cycle & : & 0.00 \\
\hline & & & & & & Down & : & 0.00 & Repair & $:$ & 0.00 \\
\hline clos_MCO & 10 & 99.82 & Busy & : & 0.18 & Blocked & : & 0.00 & Setup & : & 0.00 \\
\hline & & & & & & setup & : & 0.00 & Cycle & : & 0.00 \\
\hline & & & & & & Down & : & 0.00 & Repair & : & 0.00 \\
\hline weldcovr & 10 & 99.88 & Busy & $:$ & 0.12 & Blocked & : & 0.00 & Setup & : & 0.00 \\
\hline & & & & & & Setup & : & 0.00 & Cycle & : & 0.00 \\
\hline & & & & & & Down & : & 0.00 & Repait & : & 0.00 \\
\hline instweld & 10 & 99.76 & Busy & : & 0.24 & Blocked & ; & 0.00 & Setup & : & 0.00 \\
\hline & & & & & & Setup & ; & 0.00 & Oycle & : & 0.00 \\
\hline & & & & & & Down & : & 0.00 & RepaiI & : & 0.00 \\
\hline weldroot & 10 & 99.65 & Busy & $:$ & 0.35 & Blocked & : & 0.00 & Setup & : & 0.00 \\
\hline & & & & & & setup & : & 0.00 & Cycle & : & 0.00 \\
\hline & & & & & & Down & $\vdots$ & 0.00 & RepaiI & : & 0.00 \\
\hline vis_insp & 14 & 99.83 & Busy & $:$ & 0.17 & Blocked & : & 0.00 & Setup & : & 0.00 \\
\hline & & & & & & Setup & : & 0.00 & Cycle & : & 0.00 \\
\hline & & & & & & Down & : & 0.00 & Repaiz & : & 0.00 \\
\hline clenweld & 14 & 99.83 & Busy & : & 0.17 & Blocked & : & 0.00 & setup & : & 0.00 \\
\hline & & & & & & Setup & $:$ & 0.00 & Cycle & $:$ & 0.00 \\
\hline & & & & & & Down & : & 0.00 & Repais & : & 0.00 \\
\hline accept2 & 14 & 99.98 & Busy & $:$ & 0.02 & Blocked & : & 0.00 & Setup & : & 0.00 \\
\hline & & & & & & setup & $:$ & 0.00 & Cycle & : & 0.00 \\
\hline & & & & & & Down & : & 0.00 & Repair & : & 0.00 \\
\hline grind & 4 & 99.93 & Busy & $:$ & 0.07 & B1ocked & : & 0.00 & setup & : & 0.00 \\
\hline & & & & & & setup & $:$ & 0.00 & Cycle & : & 0.00 \\
\hline & & & & & & Down & : & 0.00 & Repair & : & 0.00 \\
\hline rep_weld & 4 & 99.93 & Busy & : & 0.07 & Blocked & : & 0.00 & Setup & : & 0.00 \\
\hline & & & & & & setup & : & 0.00 & Cycle & : & 0.00 \\
\hline
\end{tabular}


Number

\begin{tabular}{|c|c|c|c|c|c|c|c|c|c|c|c|}
\hline Name & of Ops. & tIdle & tcycl & & & IStopped & & & Waitin & & \\
\hline & & & & & & Down & $\therefore$ & 0.00 & Repair & : & 0.00 \\
\hline soak & 15 & 99.82 & Busy & $:$ & 0.18 & Blocked & : & 0.00 & Setup & $:$ & 0.00 \\
\hline & & & & & & Setup & : & 0.00 & cycle & $:$ & 0.00 \\
\hline & & & & & & Down & : & 0.00 & Repair & : & 0.00 \\
\hline aply_die & 15 & 99.82 & Busy & : & 0.18 & Blocked & : & 0.00 & Setup & $:$ & 0.00 \\
\hline & & & & & & Setup & : & 0.00 & Cycle & : & 0.00 \\
\hline & & & & & & Down & : & 0.00 & Repair & : & 0.00 \\
\hline remv_die & 25 & 99.73 & Busy & $:$ & 0.27 & Blocked & : & 0.00 & Setup & $:$ & 0.00 \\
\hline & & & & & & Setup & $:$ & 0.00 & Cycle & : & 0.00 \\
\hline & & & & & & Down & $:$ & 0.00 & Repair & : & 0.00 \\
\hline aply_dev & 15 & 99.82 & Busy & $:$ & 0.18 & Blocked & $:$ & 0.00 & Setup & : & 0.00 \\
\hline & & & & & & Setup & : & 0.00 & Cycle & $:$ & 0.00 \\
\hline & & & & & & Down & : & 0.00 & Repair & : & 0.00 \\
\hline bleed & 15 & 99.82 & Busy & : & 0.18 & Blocked & $:$ & 0.00 & setup & $:$ & 0.00 \\
\hline & & & & & & Setup & $:$ & 0.00 & Cycle & $:$ & 0.00 \\
\hline & & & & & & Down & $:$ & 0.00 & Repair & $:$ & 0.00 \\
\hline inspect & 15 & 99.91 & Busy & : & 0.09 & Blocked & $:$ & 0.00 & Setup & : & 0.00 \\
\hline & & & & & & Setup & $:$ & 0.00 & Cycle & : & 0.00 \\
\hline & & & & & & Down & $:$ & 0.00 & Repair & : & 0.00 \\
\hline cleen & 15 & 99.82 & Busy & : & 0.18 & Blocked & : & 0.00 & Setup & : & 0.00 \\
\hline & & & & & & Setup & : & 0.00 & Cycle & $:$ & 0.00 \\
\hline & & & & & & Down & $:$ & 0.00 & Repair & $:$ & 0.00 \\
\hline accept 3 & 15 & 99.98 & Busy & : & 0.02 & Blocked & : & 0.00 & Setup & : & 0.00 \\
\hline & & & & & & Setup & : & 0.00 & Cycle & : & 0.00 \\
\hline & & & & & & Down & $:$ & 0.00 & Repair & : & 0.00 \\
\hline grind2 & 5 & 99.91 & Busy & : & 0.09 & Blocked & $:$ & 0.00 & Setup & $:$ & 0.00 \\
\hline & & & & & & Setup & : & 0.00 & Cycle & $:$ & 0.00 \\
\hline & & & & & & Down & : & 0.00 & Repair & $:$ & 0.00 \\
\hline reprweld & 5 & 99.91 & Busy & : & 0.09 & Blocked & : & 0.00 & Setup & $:$ & 0.00 \\
\hline & & & & & & Setup & : & 0.00 & Cycle & $:$ & 0.00 \\
\hline & & & & & & Down & : & 0.00 & Repair & $:$ & 0.00 \\
\hline makecovr & 10 & 99.65 & Busy & : & 0.35 & Blocked & : & 0.00 & setup & : & 0.00 \\
\hline & & & & & & Setup & : & 0.00 & Cycle & : & 0.00 \\
\hline & & & & & & Down & : & 0.00 & Repair & : & 0.00 \\
\hline visinsp2 & 10 & 99.88 & Busy & $:$ & 0.12 & Blocked & $:$ & 0.00 & setup & : & 0.00 \\
\hline & & & & & & Serup & : & 0.00 & Cycle & $:$ & 0.00 \\
\hline & & & & & & Down & : & 0.00 & Repair & : & 0.00 \\
\hline accept 4 & 10 & 99.99 & Busy & $:$ & 0.01 & Blocked & : & 0.00 & Setup & : & 0.00 \\
\hline & & & & & & Setup & : & 0.00 & Cycle & : & 0.00 \\
\hline & & & & & & Down & : & 0.00 & Repair & : & 0.00 \\
\hline covipass & 10 & 99.65 & Busy & $:$ & 0.35 & Blocked & : & 0.00 & Setup & $:$ & 0.00 \\
\hline & & & & & & Setup & : & 0.00 & Cycle & : & 0.00 \\
\hline & & & & & & Down & : & 0.00 & Repair & $:$ & 0.00 \\
\hline grind 3 & 0 & 100.00 & Busy & $:$ & 0.00 & Blocked & : & 0.00 & setup & : & 0.00 \\
\hline & & & & & & setup & : & 0.00 & cycle & : & 0.00 \\
\hline & & & & & & Down & : & 0.00 & Repair & : & 0.00 \\
\hline repweld & 0 & 100.00 & Busy & $:$ & 0.00 & Blocked & : & 0.00 & Setup & : & 0.00 \\
\hline & & & & & & Setup & : & 0.00 & Cycle & : & 0.00 \\
\hline & & & & & & Down & : & 0.00 & Repair & : & 0.00 \\
\hline visingp3 & 11 & 99.87 & Busy & $:$ & 0.13 & Blocked & $:$ & 0.00 & setup & : & 0.00 \\
\hline & & & & & & Setup & $:$ & 0.00 & Cycle & : & 0.00 \\
\hline & & & & & & Down & : & 0.00 & Repair & : & 0.00 \\
\hline accepts & 11 & 99.99 & Busy & $:$ & 0.01 & Blocked & $:$ & 0.00 & Setup & : & 0.00 \\
\hline & & & & & & Setup & : & 0.00 & Cycle & $:$ & 0.00 \\
\hline & & & & & & Down & : & 0.00 & Repair & : & 0.00 \\
\hline grind4 & 1 & 99.98 & Busy & $:$ & 0.02 & Blocked & : & 0.00 & Setup & : & 0.00 \\
\hline & & & & & & Setup & : & 0.00 & Cycle & : & 0.00 \\
\hline
\end{tabular}




\begin{tabular}{|c|c|c|c|c|c|c|c|c|c|c|c|}
\hline & Number & & & & & & & & & & \\
\hline Name & of Ops. & *Idle & Cycl & & & \& stoppec & & & tWaitir & & \\
\hline & & & & & & & & & & & \\
\hline & & & & & & Down & : & 0.00 & Repair & : & 0.00 \\
\hline repaweld & 1 & 99.98 & Busy & : & 0.02 & Blocked & : & 0.00 & setup & : & 0.00 \\
\hline & & & & & & Setup & : & 0.00 & cycle & : & 0.00 \\
\hline & & & & & & Down & : & 0.00 & Repair & : & 0.00 \\
\hline corpass2 & 10 & 99.65 & Busy & $:$ & 0.35 & Blocked & : & 0.00 & setup & : & 0.00 \\
\hline & & & & & & Setup & : & 0.00 & cycle & : & 0.00 \\
\hline & & & & & & Down & : & 0.00 & Repair & : & 0.00 \\
\hline visinep4 & 10 & 99.88 & Busy & $:$ & 0.12 & Blocked & : & 0.00 & setup & : & 0.00 \\
\hline & & & & & & setup & : & 0.00 & cycle & : & 0.00 \\
\hline & & & & & & Down & : & 0.00 & Repair & : & 0.00 \\
\hline accept 6 & 10 & 99.99 & Busy & : & 0.01 & Blocked & : & 0.00 & setup & : & 0.00 \\
\hline & & & & & & Setup & : & 0.00 & Cycle & : & 0.00 \\
\hline & & & & & & Down & ; & 0.00 & Repair & : & 0.00 \\
\hline grind5 & 0 & 100.00 & Busy & $:$ & 0.00 & Blocked & : & 0.00 & setup & $:$ & 0.00 \\
\hline & & & & & & setup & : & 0.00 & cycle & : & 0.00 \\
\hline & & & & & & Down & $:$ & 0.00 & Repair & : & 0.00 \\
\hline reparwld & 0 & 100.00 & Busy & $:$ & 0.00 & Blocked & : & 0.00 & setup & : & 0.00 \\
\hline & & & & & & secup & : & 0.00 & cycle & : & 0.00 \\
\hline & & & & & & Down & : & 0.00 & Repair & : & 0.00 \\
\hline cvIpass3 & 10 & 99.65 & Busy & : & 0.35 & Blocked & : & 0.00 & setup & : & 0.00 \\
\hline & & & & & & Setup & : & 0.00 & Cycle & : & 0.00 \\
\hline & & & & & & Down & : & 0.00 & Repair & $:$ & 0.00 \\
\hline visingp5 & 13 & 99.85 & Busy & $:$ & 0.15 & Blocked & : & 0.00 & setup & : & 0.00 \\
\hline & & & & & & Setup & : & 0.00 & Cyele & : & 0.00 \\
\hline & & & & & & Down & : & 0.00 & Repair & : & 0.00 \\
\hline accept 7 & 13 & 99.98 & Busy & $:$ & 0.02 & Blocked & : & 0.00 & setup & : & 0.00 \\
\hline & & & & & & Setup & : & 0.00 & Cycle & : & 0.00 \\
\hline & & & & & & Down & : & 0.00 & Repair & : & 0.00 \\
\hline grind6 & 3 & 99.95 & Busy & $:$ & 0.05 & Blocked & : & 0.00 & Setup & : & 0.00 \\
\hline & & & & & & Setup & : & 0.00 & Cycle & : & 0.00 \\
\hline & & & & & & Down & : & 0.00 & Repair & : & 0.00 \\
\hline repr_wd & 3 & 99.95 & Busy & : & 0.05 & Blocked & : & 0.00 & Setup & : & 0.00 \\
\hline & & & & & & Setup & : & 0.00 & Cycle & : & 0.00 \\
\hline & & & & & & Down & : & 0.00 & Repair & : & 0.00 \\
\hline cvIpass4 & 10 & 99.65 & Busy & $:$ & 0.35 & Blocked & : & 0.00 & Setup & : & 0.00 \\
\hline & & & & & & Setup & : & 0.00 & Cycle & : & 0.00 \\
\hline & & & & & & Down & : & 0.00 & Repair & : & 0.00 \\
\hline visinsp6 & 10 & 99.88 & Busy & : & 0.12 & Blocked & : & 0.00 & Setup & : & 0.00 \\
\hline & & & & & & Setup & : & 0.00 & Cycle & : & 0.00 \\
\hline & & & & & & Down & : & 0.00 & Repair & : & 0.00 \\
\hline accept 8 & 10 & 99.99 & Busy & : & 0.01 & Blocked & : & 0.00 & Setup & : & 0.00 \\
\hline & & & & & & Setup & : & 0.00 & Cycle & : & 0.00 \\
\hline & & & & & & Down & : & 0.00 & Repair & : & 0.00 \\
\hline grind7 & 0 & 100.00 & Busy & : & 0.00 & Blocked & : & 0.00 & Setup & : & 0.00 \\
\hline & & & & & & setup & : & 0.00 & Cycle & : & 0.00 \\
\hline & & & & & & Down & : & 0.00 & Repair & : & 0.00 \\
\hline repr_wld & 0 & 100.00 & Busy & : & 0.00 & Blocked & : & 0.00 & Setup & : & 0.00 \\
\hline & & & & & & Setup & : & 0.00 & Cycle & : & 0.00 \\
\hline & & & & & & Down & : & 0.00 & Repair & : & 0.00 \\
\hline apply_ci & 12 & 99.86 & Busy & $:$ & 0.14 & Blocked & : & 0.00 & Setup & : & 0.00 \\
\hline & & & & & & Setup & : & 0.00 & Cycle & : & 0.00 \\
\hline & & & & & & Down & : & 0.00 & Repair & : & 0.00 \\
\hline soke & 12 & 99.86 & Busy & : & 0.14 & Blocked & : & 0.00 & setup & : & 0.00 \\
\hline & & & & & & setup & : & 0.00 & Cycle & : & 0.00 \\
\hline & & & & & & Down & : & 0.00 & Repair & : & 0.00 \\
\hline mov_di & 12 & 99.79 & Busy & : & 0.21 & Blocked & : & 0.00 & setup & : & 0.00 \\
\hline & & & & & & Sefup & : & 0.00 & cycle & : & 0.00 \\
\hline
\end{tabular}




\begin{tabular}{|c|c|c|c|c|c|c|c|c|c|c|c|}
\hline Name & $\begin{array}{l}\text { Number } \\
\text { of Ops. }\end{array}$ & tIdle & $+0 y c 1$ & & & IStopped & & & tWaitis & & \\
\hline & 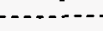 & & & & & & & $-\infty$ & $--\infty$ & & \\
\hline & & & & & & Down & $:$ & 0.00 & Repaix & : & 0.00 \\
\hline aplydevl & 12 & 99.86 & Busy & : & 0.14 & Blocked & : & 0.00 & setup & : & 0.00 \\
\hline & & & & & & Setup & : & 0.00 & Cycle & : & 0.00 \\
\hline & & & & & & Down & : & 0.00 & Repair & : & 0.00 \\
\hline bleed2 & 12 & 99.86 & Busy & $:$ & 0.14 & Blocked & : & 0.00 & Setup & : & 0.00 \\
\hline & & & & & & Setup & : & 0.00 & cycle & : & 0.00 \\
\hline & & & & & & Down & : & 0.00 & Repair & : & 0.00 \\
\hline inspect 2 & 12 & 99.93 & Bugy & : & 0.07 & Blocked & : & 0.00 & Setup & : & 0.00 \\
\hline & & & & & & Setup & : & 0.00 & Cycle & : & 0.00 \\
\hline & & & & & & Down & : & 0.00 & Repair & : & 0.00 \\
\hline cleen 2 & 12 & 99.86 & Busy & : & 0.14 & Blocked & : & 0.00 & setup & : & 0.00 \\
\hline & & & & & & Setup & : & 0.00 & Cycle & : & 0.00 \\
\hline & & & & & & Down & : & 0.00 & Repair & : & 0.00 \\
\hline accept9 & 22 & 99.99 & Buвy & : & 0.01 & Blocked & : & 0.00 & setup & : & 0.00 \\
\hline & & & & & & Setup & : & 0.00 & Cycle & : & 0.00 \\
\hline & & & & & & Down & : & 0.00 & Repair & : & 0.00 \\
\hline grinde & 2 & 99.96 & Bugy & : & 0.04 & Blocked & : & 0.00 & Setup & : & 0.00 \\
\hline & & & & & & Setup & : & 0.00 & Cycle & : & 0.00 \\
\hline & & & & & & Down & : & 0.00 & Repair & : & 0.00 \\
\hline repr_wel & 2 & 99.96 & Busy & : & 0.04 & Blocked & : & 0.00 & Setup & : & 0.00 \\
\hline & & & & & & Setup & : & 0.00 & Cycle & $:$ & 0.00 \\
\hline & & & & & & Down & : & 0.00 & Repair & : & 0.00 \\
\hline remvtrap & 10 & 99.29 & Busy & $:$ & 0.71 & Blocked & : & 0.00 & setup & : & 0.00 \\
\hline & & & & & & Setup & : & 0.00 & Cycle & : & 0.00 \\
\hline & & & & & & Down & : & 0.00 & Repair & : & 0.00 \\
\hline new_trap & 10 & 99.29 & Busy & : & 0.71 & Blocked & : & 0.00 & Setup & $:$ & 0.00 \\
\hline & & & & & & Setup & : & 0.00 & cycle & : & 0.00 \\
\hline & & & & & & Down & : & 0.00 & Repair & : & 0.00 \\
\hline wast drum & 10 & 99.65 & Buвy & : & 0.35 & Blocked & : & 0.00 & setup & : & 0.00 \\
\hline & & & & & & Setup & : & 0.00 & Cycle & : & 0.00 \\
\hline & & & & & & Down & : & 0.00 & Repair & : & 0.00 \\
\hline remvshed & 10 & 99.88 & Busy & : & 0.12 & Blocked & : & 0.00 & Setup & : & 0.00 \\
\hline & & & & & & Setup & : & 0.00 & Cycle & : & 0.00 \\
\hline & & & & & & Down & : & 0.00 & Repair & : & 0.00 \\
\hline hot_trap & 10 & 99.88 & Busy & : & 0.12 & Blocked & : & 0.00 & Setup & : & 0.00 \\
\hline & & & & & & Setup & : & 0.00 & Cycle & : & 0.00 \\
\hline & & & & & & Down & : & 0.00 & Repaix & : & 0.00 \\
\hline topshiel & 10 & 99.88 & Busy & : & 0.12 & Blocked & : & 0.00 & Setup & : & 0.00 \\
\hline & & & & & & Setup & : & 0.00 & cycle & : & 0.00 \\
\hline & & & & & & Down & : & 0.00 & Repair & : & 0.00 \\
\hline tie_bag & 10 & 99.82 & Busy & : & 0.18 & Blocked & : & 0.00 & Setup & : & 0.00 \\
\hline & & & & & & Setup & : & 0.00 & Cycle & : & 0.00 \\
\hline & & & & & & Down & : & 0.00 & Repair & : & 0.00 \\
\hline closdrum & 10 & 99.88 & Busy & $:$ & 0.12 & Blocked & : & 0.00 & Setup & : & 0.00 \\
\hline & & & & & & Setup & : & 0.00 & cycle & : & 0.00 \\
\hline & & & & & & Down & : & 0.00 & Repair & : & 0.00 \\
\hline undocovr & 10 & 99.88 & Busy & : & 0.12 & Blocked & : & 0.00 & Setup & : & 0.00 \\
\hline & & & & & & Setup & : & 0.00 & Cycle & : & 0.00 \\
\hline & & & & & & Down & : & 0.00 & Repair & : & 0.00 \\
\hline rem_covr & 10 & 99.94 & Busy & : & 0.06 & Blocked & : & 0.00 & Setup & : & 0.00 \\
\hline & & & & & & Setup & : & 0.00 & Cycle & : & 0.00 \\
\hline & & & & & & Down & : & 0.00 & Repair & : & 0.00 \\
\hline closvalt & 10 & 99.94 & Busy & : & 0.06 & Blocked & : & 0.00 & Setup & : & 0.00 \\
\hline & & & & & & setup & : & 0.00 & Cycle & : & 0.00 \\
\hline & & & & & & Down & ; & 0.00 & Repaix & : & 0.00 \\
\hline survcell & 10 & 99.65 & Bugy & : & 0.35 & Blocked & : & 0.00 & Setup & : & 0.00 \\
\hline & & & & & & setup & & 0.00 & Cycle & : & 0.00 \\
\hline
\end{tabular}




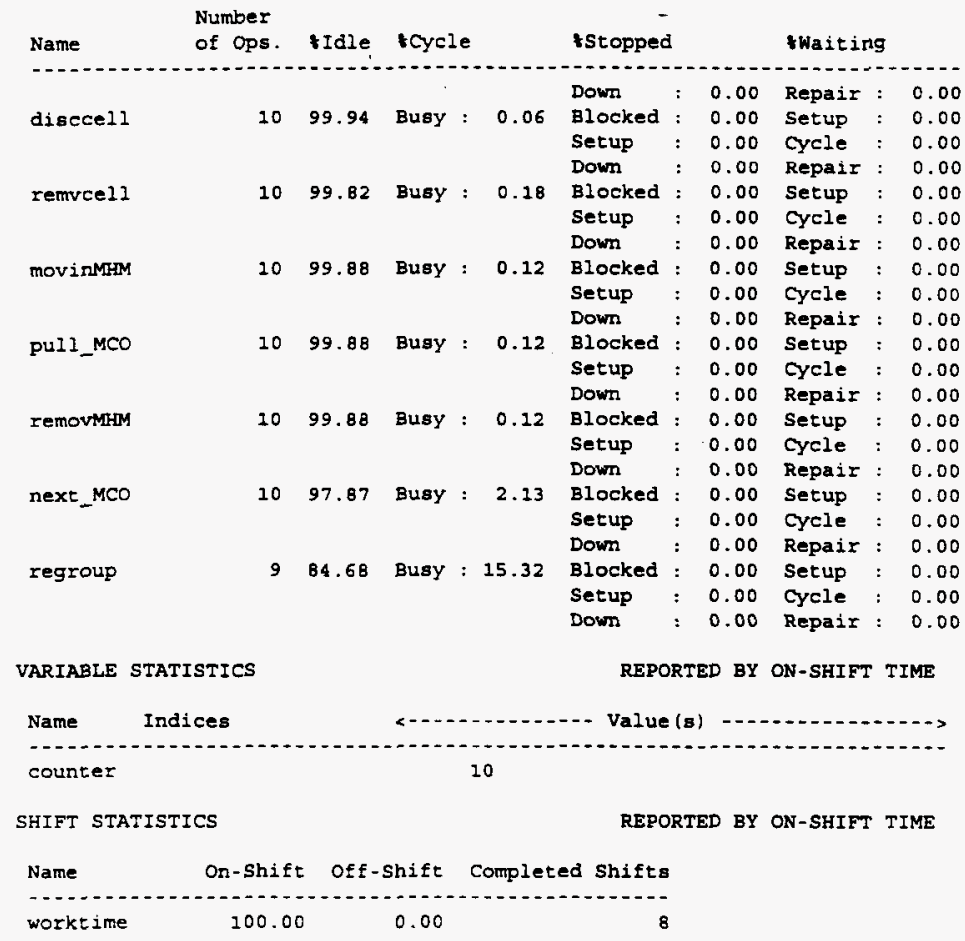

\section{SUMMARY REPORT:}

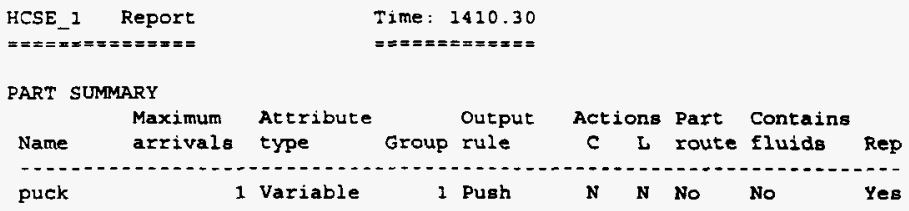

BUFFER SUMMARY

\begin{tabular}{|c|c|c|c|c|}
\hline & & & output & <-Actions-> \\
\hline Name & Qty Delay & Capacity Input & Dirn. Rule & I o Min Max Rep \\
\hline holdpuck & 1 None & 1000 Rear & Front First & N N N \\
\hline
\end{tabular}


MACHINE SUMMARY

\begin{tabular}{|c|c|c|c|c|c|c|c|c|c|c|c|c|}
\hline Name & Qty & TYpe & Lab & Down & $\begin{array}{l}\text { Input } \\
\text { rule }\end{array}$ & $\begin{array}{l}\text { output } \\
\text { rule }\end{array}$ & AC & $\begin{array}{c}\text { ctio } \\
\text { F B }\end{array}$ & $\begin{array}{l}\text { ons } \\
B R\end{array}$ & $\begin{array}{c}\text { Fluid } \\
F\end{array}$ & $\begin{array}{c}\text { rules } \\
\mathbf{E}\end{array}$ & Ref \\
\hline & & & & & & & & & & & & \\
\hline sel_oven & 1 & Single & N N & $\mathbf{N}$ & Pull & Push & $\mathbf{N}$ & $\mathbf{N} \mathbf{N}$ & $N$ & $\mathbf{N}$ & $\mathrm{N}$ & I \\
\hline move_MHM & 1 & Single & $\mathbf{N}$ & $\mathbf{N}$ & Wait & Push & $\mathbf{N}$ & $\mathbf{N} \mathbf{N}$ & $\mathbf{N}$ & $\mathbf{N}$ & $\mathbf{N}$ & $I$ \\
\hline lowr_MCO & 1 & Single & N 1 & $\mathbf{N}$ & Wait & Push & $\mathbf{N}$ & $\mathbf{N} \mathbf{N}$ & $N$ & $\mathbf{N}$ & $\mathbf{N}$ & $I$ \\
\hline remv_MHM & 1 & Single & $\mathbf{N}$ & $\mathbf{N}$ & Wait & Push & N & N N & $N$ & $\mathbf{N}$ & $\mathbf{N}$ & $I$ \\
\hline mov_cell & 1 & Single & N & $\mathbf{N}$ & Wait & Push & N & $N N$ & $N$ & N & $N$ & $I$ \\
\hline con_cell & 1 & single & $N \mathbf{N}$ & $\mathbf{N}$ & Wait & Push & $\mathbf{N}$ & $\mathbf{N} \mathrm{N}$ & $\mathrm{N}$ & $\mathrm{N}$ & $\mathbf{N}$ & $I$ \\
\hline opn_valt & 1 & single & $\mathrm{N} N$ & $\mathbf{N}$ & Wait & Push & $\mathbf{N}$ & $\mathbf{N} \mathbf{N}$ & $N$ & $\mathbf{N}$ & $\mathrm{N}$ & $I$ \\
\hline plac_cvr & 1 & Single & N $\mathbf{1}$ & $\mathbf{N}$ & Wait & Push & $\mathbf{N}$ & $\mathbf{N} \mathbf{N}$ & $\mathbf{N}$ & $\mathbf{N}$ & $\mathbf{N}$ & $I$ \\
\hline att_covr & 1 & single & $\mathrm{N} N$ & $\mathbf{N}$ & Wait & Push & $\mathbf{N}$ & $\mathbf{N} \mathbf{N}$ & $\mathrm{N}$ & $\mathbf{N}$ & $\mathbf{N}$ & $I$ \\
\hline remv_cur & 1 & single & N N & $\mathbf{N}$ & Wait & Push & $\mathbf{N}$ & $\mathbf{N} \mathbf{N}$ & $\mathrm{N}$ & $\mathbf{N}$ & $\mathbf{N}$ & I \\
\hline inst_cvr & 1 & Single & N N & $\mathbf{N}$ & Wait & Push & $\mathbf{N}$ & $\mathbf{N} \mathbf{N}$ & $\mathrm{N}$ & $N$ & $\mathbf{N}$ & $I$ \\
\hline inst val & 1 & single & N $N$ & $\mathbf{N}$ & Wait & Push & $\mathbf{N}$ & $\mathbf{N} \mathbf{N}$ & $\mathrm{N}$ & $\mathbf{N}$ & $\mathbf{N}$ & I \\
\hline opn_valv & 1 & Single & N $\mathbf{N}$ & $\mathbf{N}$ & Wait & Push & $\mathbf{N}$ & $N \mathbf{N}$ & $\mathrm{N}$ & $\mathbf{N}$ & $\mathbf{N}$ & $I$ \\
\hline evac_MCO & 1 & Single & N 1 & $\mathbf{N}$ & Wait & Push & $\mathbf{N}$ & $\mathrm{N} N$ & $\mathrm{~N}$ & $\mathbf{N}$ & $\mathbf{N}$ & I \\
\hline E111_MCO & 1 & Single & N & $\mathrm{N}$ & Nait & Push & $\mathbf{N}$ & $\mathbf{N} \mathbf{N}$ & $\mathrm{N}$ & $\mathbf{N}$ & $\mathbf{N}$ & I \\
\hline leak_chk & 1 & Single & $N \mathbf{N}$ & $\mathbf{N}$ & Wait & Push & $\mathbf{N}$ & $\mathbf{N} \mathbf{N}$ & $N$ & $\mathbf{N}$ & $\mathrm{N}$ & $I$ \\
\hline passleak & 1 & Single & N $\mathbf{N}$ & $\mathbf{N}$ & Wait & Percent & $\mathbf{N}$ & N N & $\mathrm{N}$ & $\mathbf{N}$ & $\mathbf{N}$ & I \\
\hline cle_valv & 1 & Single & N N & $N$ & Wait & Push & $\mathbf{N}$ & N N & $N$ & $\mathbf{N}$ & $\mathrm{N}$ & I \\
\hline disc_vlv & 1 & Single & N 1 & $\mathbf{N}$ & Wait & Push & $\mathbf{N}$ & $N \mathbf{N}$ & $N$ & $\mathrm{~N}$ & $\mathbf{N}$ & $I$ \\
\hline cln_port & 1 & Single & N & $\mathbf{N}$ & Wait & Push & $\mathbf{N}$ & $\mathrm{NN}$ & $\mathrm{N}$ & $\mathbf{N}$ & $\mathbf{N}$ & I \\
\hline Inst_vlv & 1 & Single & N N & N & Wait & Push & $\mathbf{N}$ & $N \mathrm{~N}$ & $\mathrm{~N}$ & $\mathbf{N}$ & $\mathbf{N}$ & I \\
\hline insl_cvr & 1 & Single & N 1 & $\mathbf{N}$ & Wait & Push & $\mathbf{N}$ & $\mathbf{N} \mathbf{N}$ & $\mathbf{N}$ & $\mathbf{N}$ & $\mathbf{N}$ & I \\
\hline cls_valt & 1 & Production & N N & $\mathbf{N}$ & Waذt & Sequence & $\mathbf{N}$ & $N \mathrm{~N}$ & $\mathrm{~N}$ & $\mathbf{N}$ & $\mathbf{N}$ & I \\
\hline het_cycl & 1 & single & $\mathbf{N} \mathbf{N}$ & $\mathbf{N}$ & Wait & Push & $\mathbf{N}$ & $N \mathbf{N}$ & $\mathrm{N}$ & $\mathbf{N}$ & $\mathbf{N}$ & I \\
\hline surv_ce1 & 1 & Single & N & $\mathbf{N}$ & Wait & Push & $\mathbf{N}$ & $\mathbf{N}$ & $N$ & $\mathbf{N}$ & $\mathbf{N}$ & I \\
\hline discproc & 1 & Single & N N & $\mathrm{N}$ & Wait & Push & $\mathbf{N}$ & $N N$ & $\mathrm{~N}$ & $\mathbf{N}$ & $\mathbf{N}$ & I \\
\hline remvproc & 1 & Single & N N & $\mathrm{N}$ & Wait & Pugh & $\mathbf{N}$ & $N \mathrm{~N}$ & $\mathrm{~N}$ & $\mathbf{N}$ & $\mathbf{N}$ & I \\
\hline purg_vac & 1 & Assembly & N 1 & $\mathbf{N}$ & Wait & Pugh & $\mathrm{N}$ & $N \mathrm{~N}$ & $\mathrm{~N}$ & $\mathbf{N}$ & $\mathbf{N}$ & I \\
\hline perftest & 1 & Single & $\mathrm{NH}$ & $\mathbf{N}$ & Wait & Push & $\mathrm{N}$ & $\mathrm{N} \mathrm{N}$ & $\mathbf{N}$ & $\mathbf{N}$ & $\mathbf{N}$ & I \\
\hline accept & 1 & Single & N & $\mathbf{N}$ & Wait & Percent & $\mathbf{N}$ & $N N$ & $\mathrm{~N}$ & $\mathbf{N}$ & $\mathbf{N}$ & I \\
\hline add_purg & 1 & Single & N $\mathbf{N}$ & N & Wait & Push & $\mathbf{N}$ & $\mathbf{N} \mathbf{N}$ & $\mathrm{N}$ & $\mathbf{N}$ & $\mathbf{N}$ & I \\
\hline coolpass & 1 & Single & N $\mathrm{r}$ & $\mathbf{N}$ & Wait & Push & $\mathbf{N}$ & $N \mathrm{~N}$ & $\mathrm{~N}$ & $\mathbf{N}$ & $\mathbf{N}$ & I \\
\hline evacmco & 1 & Single & N 1 & $\mathbf{N}$ & Wait & Push & $\mathbf{N}$ & $\mathbf{N}$ & $N$ & $\mathbf{N}$ & $\mathbf{N}$ & I \\
\hline pass_gas & 1 & Single & N 1 & $\mathbf{N}$ & Wait & Push & $\mathrm{N}$ & $\mathbf{N} \mathbf{N}$ & $\mathrm{N}$ & $\mathbf{N}$ & $\mathbf{N}$ & I \\
\hline pass_per & 1 & Single & N $N$ & $\mathbf{N}$ & Wait & Push & $\mathbf{N}$ & $\mathbf{N} \mathbf{N}$ & $\mathrm{N}$ & $\mathbf{N}$ & $\mathbf{N}$ & I \\
\hline cooldown & 1 & Single & $\mathrm{N} N$ & $\mathbf{N}$ & Wait & Push & $\mathbf{N}$ & $\mathbf{N} \mathbf{N}$ & $n$ & $\mathbf{N}$ & $\mathbf{N}$ & I \\
\hline evacMCO2 & 1 & single & N N & $\mathbf{N}$ & Wait & Push & $\mathbf{N}$ & $N \mathbf{N}$ & $\mathrm{N}$ & $\mathbf{N}$ & $\mathbf{N}$ & I \\
\hline Eill_He & 1 & Single & N $N$ & $\mathbf{N}$ & Wait & Push & $\mathbf{N}$ & $\mathbf{N} \mathbf{N}$ & $\mathrm{N}$ & $\mathbf{N}$ & $\mathbf{N}$ & $I$ \\
\hline move_in & 1 & single & N N & $\mathbf{N}$ & Wait & Push & $\mathrm{N}$ & $N$ N & $N$ & $\mathbf{N}$ & $\mathbf{N}$ & I \\
\hline conn_cel & 1 & Single & N N & $\mathbf{N}$ & Wait & Push & $\mathrm{N}$ & N N & $N$ & $\mathbf{N}$ & $\mathbf{N}$ & I \\
\hline openvalt & 1 & Single & N N & $\mathbf{N}$ & Wait & Pugh & N & $\mathbf{N} \mathbf{N}$ & $N$ & $\mathbf{N}$ & $\mathbf{N}$ & I \\
\hline remvcovr & 1 & single & $\mathbf{N} \mathbf{N}$ & $\mathbf{N}$ & Wait & Push & $\mathbf{N}$ & N N & 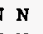 & $\mathbf{N}$ & $\mathbf{N}$ & I \\
\hline Clos_MCO & 1 & Single & $\mathbf{N} \mathbf{N}$ & $\mathbf{N}$ & Wait & Puah & $\mathbf{N}$ & $\mathbf{N} \mathbf{N}$ & $N$ & $\mathbf{N}$ & $\mathbf{N}$ & I \\
\hline weldcovr & 1 & Single & $\mathbf{N}$ & $\mathbf{N}$ & Wait & Push & $\mathbf{N}$ & $\mathbf{N} \mathbf{N}$ & $N$ & $\mathbf{N}$ & $\mathbf{N}$ & I \\
\hline instweld & 1 & single & $\mathbf{N} \mathbf{N}$ & $\mathbf{N}$ & Wait & Push & $\mathbf{N}$ & $\mathrm{N} \mathrm{N}$ & $\mathrm{n}$ & $\mathbf{N}$ & $\mathbf{N}$ & I \\
\hline weldroot & 1 & single & N N & $\mathbf{N}$ & Wait & Pugh & $\mathbf{N}$ & $N \mathbf{N}$ & $N$ & $\mathbf{N}$ & $\mathbf{N}$ & I \\
\hline vis_insp & 1 & single & N N & $\mathbf{N}$ & Wait & Push & $\mathbf{N}$ & $\mathbf{N} \mathbf{N}$ & $\mathrm{N}$ & $\mathbf{N}$ & $\mathbf{N}$ & I \\
\hline clenweld & 1 & Single & N $\mathbf{N}$ & $\mathbf{N}$ & Wait & Push & $\mathbf{N}$ & $\mathbf{N} \mathbf{N}$ & $\mathrm{N}$ & $\mathbf{N}$ & $\mathbf{N}$ & I \\
\hline accept 2 & 1 & Single & N 1 & $\mathbf{N}$ & Wait & Percent: & $N$ & $\mathbf{N ~ N}$ & $N$ & $\mathbf{N}$ & $\mathbf{N}$ & I \\
\hline grind & 1 & Single & N & $\mathbf{N}$ & Wait & Push & $\mathbf{N}$ & $\mathbf{N} \mathbf{N}$ & $N$ & $\mathbf{N}$ & $\mathbf{N}$ & I \\
\hline rep_weld & 1 & single & N N & $\mathbf{N}$ & Wait & Push & $\mathbf{N}$ & $\mathbf{N} \mathbf{N}$ & $\mathrm{N}$ & $\mathbf{N}$ & $\mathbf{N}$ & I \\
\hline soak & 1 & single & N & $\mathbf{N}$ & Wait & Puah & $\mathbf{N}$ & $\mathbf{N} \mathbf{N}$ & $\mathrm{N}$ & $\mathbf{N}$ & $\mathbf{N}$ & 1 \\
\hline aply_die & 1 & Single & N $\mathbf{L}$ & $\mathbf{N}$ & Wait & Push & N & $N \mathrm{~N}$ & $N$ & $\mathbf{N}$ & $\mathbf{N}$ & I \\
\hline remv_die & 1 & Single & N 1 & $\mathbf{N}$ & Wait & Puah & $N$ & $\mathrm{~N} \mathrm{~N}$ & $\mathrm{~N}$ & $N$ & $\mathbf{N}$ & I \\
\hline aply_dev & 1 & Single & $\mathbf{N}$ & $\mathbf{N}$ & Wait & Push & $\mathbf{N}$ & $N \mathrm{~N}$ & $\mathrm{~N}$ & $\mathbf{N}$ & $N$ & I \\
\hline bleed & 1 & Single & $N$ & $\mathbf{N}$ & Wait & Pugh & $\mathbf{N}$ & $\mathrm{NN}$ & $\mathrm{n}$ & $\mathbf{N}$ & $\mathbf{N}$ & I \\
\hline
\end{tabular}




\begin{tabular}{|c|c|c|c|c|c|c|c|c|c|c|c|c|c|}
\hline $\begin{array}{l}\text { Lab } \\
\text { Name }\end{array}$ & $\begin{array}{l}\text { Inpu } \\
\text { Qty }\end{array}$ & $\begin{array}{l}\text { ut } \\
\text { Type }\end{array}$ & $\mathrm{R}$ & $c^{A}$ & sown & $\begin{array}{l}\text { ns Fluid } \\
\text { rule }\end{array}$ & $\begin{array}{l}\text { rules } \\
\text { rule }\end{array}$ & $\begin{array}{l}- \\
\mathbf{s}\end{array}$ & F $B$ & B $R$ & $\boldsymbol{F}$ & $E$ & Rep \\
\hline & & & & & & & & & & & & & . \\
\hline inspect & 1 & single & $\mathbf{N}$ & $\mathbf{N}$ & $\mathbf{N}$ & Wait & Push & N & $\mathbf{N} \mathbf{N}$ & $\mathbf{N}$ & $\mathbf{N}$ & $\mathbf{N}$ & $I$ \\
\hline cleen & 1 & Single & $\mathbf{N}$ & $\mathbf{N}$ & N & Wait & Push & N & $\mathbf{N} \mathbf{N}$ & N & $\mathbf{N}$ & $\mathbf{N}$ & $I$ \\
\hline accept 3 & 1 & Single & $\mathbf{N}$ & $\mathbf{N}$ & $\mathbf{N}$ & Wait & Percent & N & $\mathbf{N} \mathbf{N}$ & $\mathrm{N}$ & $\mathbf{N}$ & $\mathbf{N}$ & $I$ \\
\hline grind 2 & 1 & single & $\mathbf{N}$ & $\mathbf{N}$ & $\mathbf{N}$ & wait & Push & N & $\mathbf{N} \mathbf{N}$ & $\mathbf{N}$ & $\mathbf{N}$ & $\mathbf{N}$ & $I$ \\
\hline reprweld & 1 & Single & $\mathbf{N}$ & $\mathbf{N}$ & $\mathrm{N}$ & Wait & Push & $\mathbf{N}$ & $\mathbf{N} \mathbf{N}$ & $N$ & $\mathbf{N}$ & $\mathbf{N}$ & $I$ \\
\hline makecovz & 1 & Single & $\mathbf{N}$ & $\mathbf{N}$ & $\mathbf{N}$ & Wait & Push & $\mathbf{N}$ & $N \mathbf{N}$ & $\mathbf{N}$ & $\mathbf{N}$ & $\mathbf{N}$ & $I$ \\
\hline visinsp2 & 1 & Single & $\mathbf{N}$ & N & $\mathbf{N}$ & Wait & Push & N & $\mathrm{N} N$ & $\mathrm{~N}$ & $\mathbf{N}$ & $\mathbf{N}$ & I \\
\hline accept 4 & 1 & Single & $\mathbf{N}$ & $\mathbf{N}$ & $\mathbf{N}$ & Wait & Percent & $\mathbf{N}$ & $\mathbf{N} \mathbf{N}$ & $\mathrm{N}$ & $\mathbf{N}$ & $\mathbf{N}$ & I \\
\hline covrpass & 1 & Single & $\mathbf{N}$ & $\mathbf{N}$ & $\mathrm{N}$ & Wait & Push & $\mathbf{N}$ & $\mathbf{N} \mathbf{N}$ & $N$ & $N$ & N & I \\
\hline grind 3 & 1 & Single & $\mathbf{N}$ & $\mathbf{N}$ & $\mathbf{N}$ & Wait & Push & $\mathbf{N}$ & $\mathbf{N} \mathbf{N}$ & $N$ & $\mathbf{N}$ & $\mathbf{N}$ & I \\
\hline repweld & 1 & single & $\mathbf{N}$ & $\mathbf{N}$ & $\mathbf{N}$ & Wait & Push & $\mathbf{N}$ & $\mathbf{N} \mathbf{N}$ & $\mathbf{N}$ & $\mathbf{N}$ & $\mathbf{N}$ & $I$ \\
\hline visinsp3 & 1 & single & $\mathbf{N}$ & $N$ & $\mathbf{N}$ & wait & Push & $\mathbf{N}$ & $\mathbf{N} \mathbf{N}$ & $N$ & $\mathbf{N}$ & $\mathbf{N}$ & I \\
\hline accept 5 & 1 & Single & $\mathbf{N}$ & $\mathbf{N}$ & $\mathrm{N}$ & Wait & Percent & $N$ & $N \mathrm{~N}$ & $\mathrm{~N}$ & $\mathbf{N}$ & $\mathbf{N}$ & I \\
\hline grind4 & 1 & Single & $\mathbf{N}$ & $\mathbf{N}$ & $\mathbf{N}$ & Wait & Push & $\mathbf{N}$ & $\mathbf{N} \mathbf{N}$ & $\mathbf{N}$ & $\mathbf{N}$ & $\mathbf{N}$ & I \\
\hline repaweld & $I$ & Single & $N$ & N & $\mathbf{N}$ & Wait & Puah & $\mathbf{N}$ & N $\mathbf{N}$ & $\mathrm{N}$ & $\mathbf{N}$ & $\mathbf{N}$ & I \\
\hline corpass2 & 1 & single & $\mathbf{N}$ & $\mathbf{N}$ & $\mathbf{N}$ & Wait & Push & $\mathbf{N}$ & $N \mathbf{N}$ & $\mathrm{N}$ & $\mathbf{N}$ & $\mathbf{N}$ & I \\
\hline visinsp4 & 1 & Single & $\mathbf{N}$ & $\mathbf{N}$ & $\mathbf{N}$ & Wait & Push & $\mathbf{N}$ & $\mathrm{N} \mathrm{N}$ & $\mathrm{N}$ & $\mathbf{N}$ & $\mathbf{N}$ & I \\
\hline accept 6 & 1 & Single & $N$ & $\mathrm{~N}$ & $\mathbf{N}$ & wait & Percent & $\mathbf{N}$ & N $\mathbf{N}$ & $\mathrm{N}$ & $\mathbf{N}$ & $\mathbf{N}$ & I \\
\hline grind5 & 1 & Single & $\mathbf{N}$ & $\mathbf{N}$ & $\mathbf{N}$ & Wait & Push & $\mathbf{N}$ & $\mathbf{N} \mathbf{N}$ & $\mathbf{N}$ & $\mathbf{N}$ & $\mathbf{N}$ & I \\
\hline reparwld & 1 & Single & $\mathbf{N}$ & $\mathbf{N}$ & $\mathbf{N}$ & Wait & Push & $\mathbf{N}$ & $N N$ & $\mathrm{~N}$ & $\mathbf{N}$ & $\mathbf{N}$ & I \\
\hline cvrpass 3 & 1 & Single & $\mathbf{N}$ & $\mathbf{N}$ & $\mathbf{N}$ & Wait & Push & $\mathbf{N}$ & N N & $N$ & $\mathbf{N}$ & $\mathbf{N}$ & I \\
\hline visinsp 5 & 1 & Single & $\mathbf{N}$ & $\mathbf{N}$ & $\mathbf{N}$ & Wait & Push & $\mathbf{N}$ & $\mathbf{N} \mathbf{N}$ & $\mathrm{N}$ & $\mathbf{N}$ & $\mathbf{N}$ & I \\
\hline accept 7 & 1 & Single & $\mathbf{N}$ & $\mathbf{N}$ & $\mathbf{N}$ & Wait & Percent & $\mathbf{N}$ & $\mathbf{N}$ & $\mathrm{N}$ & $\mathbf{N}$ & $\mathbf{N}$ & $I$ \\
\hline grind6 & 1 & Single & $\mathbf{N}$ & $\mathbf{N}$ & $\mathbf{N}$ & Wait & Push & $\mathbf{N}$ & $\mathbf{N} \mathbf{N}$ & $N$ & $\mathbf{N}$ & $\mathbf{N}$ & I \\
\hline repr_wd & 1 & Single & $\mathbf{N}$ & $\mathbf{N}$ & $\mathbf{N}$ & Wait & Push & N & $\mathbf{N} \mathbf{N}$ & $\mathrm{N}$ & $\mathbf{N}$ & $\mathbf{N}$ & I \\
\hline cvrpass4 & 1 & Single & $\mathbf{N}$ & $\mathbf{N}$ & $\mathbf{N}$ & Wait & Push & N & $\mathrm{N} \mathrm{N}$ & $\mathrm{N}$ & $\mathbf{N}$ & $\mathbf{N}$ & I \\
\hline visinsp 6 & 1 & Single & $\mathbf{N}$ & $\mathbf{N}$ & N & Wait & Push & N & $\mathbf{N} \mathbf{N}$ & $\mathrm{N}$ & $\mathbf{N}$ & $\mathbf{N}$ & I \\
\hline accept & 1 & Single & $\mathbf{N}$ & $\mathrm{N}$ & $\mathbf{N}$ & Wait & Percent & $\mathbf{N}$ & $\mathrm{N} \mathbf{N}$ & $\mathbf{N}$ & $\mathrm{N}$ & $\mathbf{N}$ & I \\
\hline grind 7 & 2 & Single & $\mathbf{N}$ & $\mathbf{N}$ & $\mathrm{N}$ & Wait & Push & $\mathbf{N}$ & N N & $N$ & $\mathbf{N}$ & $\mathbf{N}$ & I \\
\hline repr_wld & $I$ & Single & $\mathbf{N}$ & If & $\mathbf{N}$ & wait & Push & $\mathbf{N}$ & $N \mathrm{~N}$ & $\mathrm{~N}$ & $\mathbf{N}$ & $\mathbf{N}$ & I \\
\hline apply_di & 1 & Single & $\mathbf{N}$ & $\mathbf{N}$ & $\mathbf{N}$ & Wait & Push & N & $\mathrm{N} \mathrm{N}$ & $\mathrm{N}$ & $\mathrm{N}$ & $\mathbf{N}$ & I \\
\hline soke & 1 & Single & $\mathbf{N}$ & $\mathbf{N}$ & $\mathbf{N}$ & Wait & Push & N & $\mathrm{N} \mathrm{N}$ & $\mathrm{N}$ & $\mathbf{N}$ & $\mathbf{N}$ & I \\
\hline remov_di & 1 & Single & $\mathbf{N}$ & $N$ & $N$ & Wait & Push & $\mathbf{N}$ & $\mathbf{N} \mathbf{N}$ & $\mathrm{N}$ & $\mathbf{N}$ & $\mathbf{N}$ & I \\
\hline aplydevl & 1 & Single & $\mathbf{N}$ & $\mathbf{N}$ & $\mathbf{N}$ & Wait & Push & $\mathbf{N}$ & $\mathbf{N} \mathbf{N}$ & $N \mathbb{N}$ & $\mathbf{N}$ & $\mathbf{N}$ & I \\
\hline bleed2 & 1 & Single & $\mathbf{N}$ & $\mathbf{N}$ & $\mathbf{N}$ & Wait & Push & N & $N \mathrm{~N}$ & $\mathrm{~N}$ & $\mathbf{N}$ & $\mathbf{N}$ & $I$ \\
\hline inspect 2 & 1 & Single & $\mathbf{N}$ & $\mathrm{N}$ & $\mathbf{N}$ & Wait & Push & $\mathbf{N}$ & $\mathrm{N} N$ & N & $\mathbf{N}$ & $\mathbf{N}$ & I \\
\hline cleen2 & 1 & Single & $\mathbf{N}$ & $\mathbf{N}$ & $\mathbf{N}$ & Wait & Push & N & $\mathbf{N} \mathbf{N}$ & $\mathbf{N}$ & $\mathbf{N}$ & $\mathbf{N}$ & I \\
\hline accept 9 & 1 & Single & $\mathbf{N}$ & $\mathbf{N}$ & $\mathbf{N}$ & Wait & Percent & $\mathbf{N}$ & $N \mathbf{N}$ & $\mathrm{N}$ & $\mathbf{N}$ & $\mathbf{N}$ & I \\
\hline grind8 & 1 & Single & $\mathbf{N}$ & $\mathbf{N}$ & $\mathbf{N}$ & Wait & Push & N & $\mathrm{N} N$ & $\mathrm{~N}$ & $N$ & $\mathbf{N}$ & I \\
\hline repr_wel & 1 & Single & $\mathbf{N}$ & $\mathbf{N}$ & $\mathbf{N}$ & Wait & Push & N & $\mathbf{N} \mathbf{N}$ & $\mathrm{N}$ & $\mathbf{N}$ & $\mathbf{N}$ & I \\
\hline remvtrap & 1 & Production & $\mathbf{N}$ & $\mathbf{N}$ & $\mathbf{N}$ & Wait & Sequence & $\mathbf{N}$ & $\mathbf{N} \mathbf{N}$ & $\mathrm{N}$ & $\mathbf{N}$ & $\mathbf{N}$ & I \\
\hline new_trap & 1 & Single & $N$ & $\mathbf{N}$ & $\mathbf{N}$ & Wait & Push & N 1 & $\mathbf{N} \mathbf{N}$ & $\mathrm{N}$ & $\mathbf{N}$ & $\mathbf{N}$ & I \\
\hline wastdrum & 1 & Single & $\mathbf{N}$ & $\mathbf{N}$ & $\mathbf{N}$ & Wait & Push & N 1 & N N & $\mathrm{N}$ & $\mathbf{N}$ & $\mathbf{N}$ & I \\
\hline remvshed & 1 & Single & $\mathbf{N}$ & $\mathbf{N}$ & $\mathbf{N}$ & Wait & Push & $\mathbf{N}$ & $N \mathrm{~N}$ & $N$ & $\mathbf{N}$ & $\mathbf{N}$ & $\mathbf{I}$ \\
\hline hot_trap & 1 & Single & $\mathbf{N}$ & $\mathbf{N}$ & $\mathbf{N}$ & Wait & Push & $\mathbf{N}$ & $N \mathrm{~N}$ & $\mathrm{~N}$ & $\mathbf{N}$ & $\mathbf{N}$ & I \\
\hline topshiel & 1 & Single & $\mathbf{N}$ & $\mathbf{N}$ & $\mathbf{N}$ & Mait & Push & $\mathbf{N}$ & N N & $\mathrm{N}$ & $N$ & $\mathbf{N}$ & I \\
\hline tie_bag & 1 & Single & $\mathbf{N}$ & $\mathbf{N}$ & $\mathbf{N}$ & Wait & Push & $\mathbf{N}$ & $N \mathrm{~N}$ & $\mathrm{~N}$ & $\mathbf{N}$ & $\mathbf{N}$ & I \\
\hline closdrum & 1 & Single & $\mathbf{N}$ & $\mathbf{N}$ & $\mathbf{N}$ & Wait & Puah & $\mathbf{N}$ & N N & $\mathrm{N}$ & $\mathbf{N}$ & $\mathbf{N}$ & I \\
\hline undocovx & 1 & Ascembly & $\mathbf{N}$ & $\mathbf{N}$ & $\mathbf{N}$ & Wait & Push & $\mathbf{N}$ & $\mathrm{N} \mathbf{N}$ & $\mathrm{N}$ & $\mathbf{N}$ & $\mathbf{N}$ & I \\
\hline rem_covr & 1 & Single & $\mathbf{N}$ & $\mathbf{N}$ & $\mathbf{N}$ & Wait & Pugh & $\mathbf{N}$ & $\mathbf{N} \mathbf{N}$ & $\mathrm{N}$ & $\mathbf{N}$ & $\mathbf{N}$ & I \\
\hline closvalt & 1 & Single & $\mathbf{N}$ & $\mathbf{N}$ & $\mathbf{N}$ & wait & Push & N & $\mathbf{N ~ N}$ & $\mathrm{N}$ & $\mathbf{N}$ & $\mathbf{N}$ & I \\
\hline survcell & 1 & Single & $\mathbf{N}$ & $\mathbf{N}$ & $\mathbf{N}$ & Wait & Push & $\mathrm{N}$ & $\mathbf{N} \mathrm{N}$ & $\mathrm{N}$ & $\mathbf{N}$ & $\mathbf{N}$ & $I$ \\
\hline disccell & 1 & Single & $\mathbf{N}$ & $\mathrm{N}$ & $\mathbf{N}$ & Wait & Push & $\mathbf{N}$ & N N & $\mathrm{N}$ & $\mathbf{N}$ & $\mathbf{N}$ & I \\
\hline remvce 11 & 1 & Single & $\mathbf{N}$ & $\mathbf{N}$ & $\mathbf{N}$ & Wait & Push & $\mathbf{N}$ & $\mathbf{N} \mathbf{N}$ & $\mathbf{N}$ & $\mathbf{N}$ & $\mathbf{N}$ & I \\
\hline movinMHM & 1 & Single & $\mathbf{N}$ & $\mathbf{N}$ & $\mathbf{N}$ & Wait & Push & $\mathbf{N}$ & $\mathbf{N} \mathbf{N}$ & $\mathrm{N}$ & $\mathbf{N}$ & $\mathrm{N}$ & I \\
\hline pul1_mco & 1 & single & $\mathbf{N}$ & $\mathbf{N}$ & $\mathbf{N}$ & Wait & Push & $\mathbf{N}$ & $\mathbf{N} \mathbf{N}$ & $\mathrm{N}$ & $\mathbf{N}$ & $\mathbf{N}$ & $I$ \\
\hline removMIM & 1 & single & $\mathbf{N}$ & $\mathbf{N}$ & $\mathbf{N}$ & Wait & Push & $\mathbf{N}$ & $\mathbf{N} \mathbf{N}$ & $\mathbf{N}$ & $\mathbf{N}$ & $\mathbf{N}$ & I \\
\hline
\end{tabular}




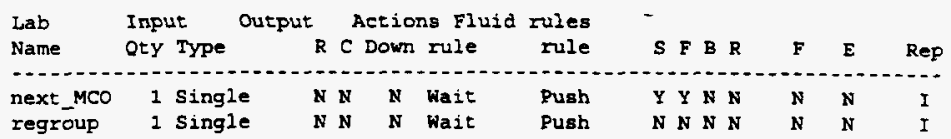

VARIABLE SUMMARY

\begin{tabular}{lc} 
Name & Quantity Type \\
\hline counter & 1 Integer
\end{tabular}

SHIFT SUMMARY

$\begin{array}{lcccccc} & & & & & & \\ \text { Actions }\end{array}$


WHC-SD-SNF-CDR-007, Revision 0

\section{APPENDICES}

\section{APPENDIX H}

\section{VENDOR CUT SHEETS}


WHC-SD-SNF-CDR-007, Revision 0

\section{APPENDIX H - VENDOR CUT SHEETS}

1.0 STACK MONITORING
A-1000
Beta/Gamma Particulate Monitor (1)
A-2000 Beta/Gamma Particulate Monitor (1)
A-3000
Alpha/Beta Particulate Monitor (1)
A-3001
Tritium Monitor (1)
A-4000
Alpha/Beta Particulate Monitor (1)
A-4001
Tritium Monitor (1)
A-5000
Record Air Sampler (1)
A-6000
Record Air Sampler (1)

2.0 CHILLED WATER SYSTEM
CHW-ACC-2021
CHW-CH-2026
Expansion Tank (1)
CHW-P-2022
Chiller (1)
CHW-P-2023
Chilled Water Circulation Pump (1)
Chilled Water Circulation Pump (1)

3.0 COOLING WATER SYSTEM
CW-ACC-2011
Expansion Tank (1)
CW-HX-2014
CW-P-2012
Glycol/Cooling Water Exchanger (1)
CW-P-2013
Cooling Water Pump (1)
Cooling Water Pump (1)

4.0 MCO COOLING SYSTEM

MCS-CLR-1X53 MCO Chiller Exchanger (6)

5.0 PROCESS ENCLOSURE

Remote Manipulator (1)

Weld Head (1)

6.0 PROCESS HEATING SYSTEM

PHS-BLO- $1 \times 51$

PHS-HCL-1X52

Heat/Cool Blower (6)

MCO Heating Element (6) 
WHC-SD-SNF-CDR-007, Revision 0

\section{APPENDIX H - VENDOR CUT SHEETS}

7.0 PROCESS VENT

PV-BLO-2043

PV-BLO-2046

PV-F-1X71

PV-F-2041

PV-F-2042

PV-F-2044

PV-F-2045
Vent Blower (1)

Vent Blower (1)

MCO Vault Filter (6)

Main Vent HEPA - First Stage (1)

Main Vent HEPA - Second Stage (1)

Main Vent HEPA - First Stage (1)

Main Vent HEPA - Second Stage (1)

8.0 VACUUM SYSTEM

VAC-VAC-3041

VAC-VAC-3042

Main Dewar Vacuum Pump (1)

Main Dewar Boost Pump (1)

9.0 VACUUM PUMPING SYSTEM

VPS-BLO-1X06

VPS-CLR-1X03

VPS-F-1X02

VPS-F-1X07

VPS-HCL-1X05

VPS-VAC-1X04
He Recirculation Blower (6)

MCO Vent Cooler (6)

MCO Vent HEPA (6)

MCO Vent HEPA (6)

MCO Vent Heater (6)

MCO Vent Vacuum/Circulation Pump (6) 
Particulate, Model PING-3B

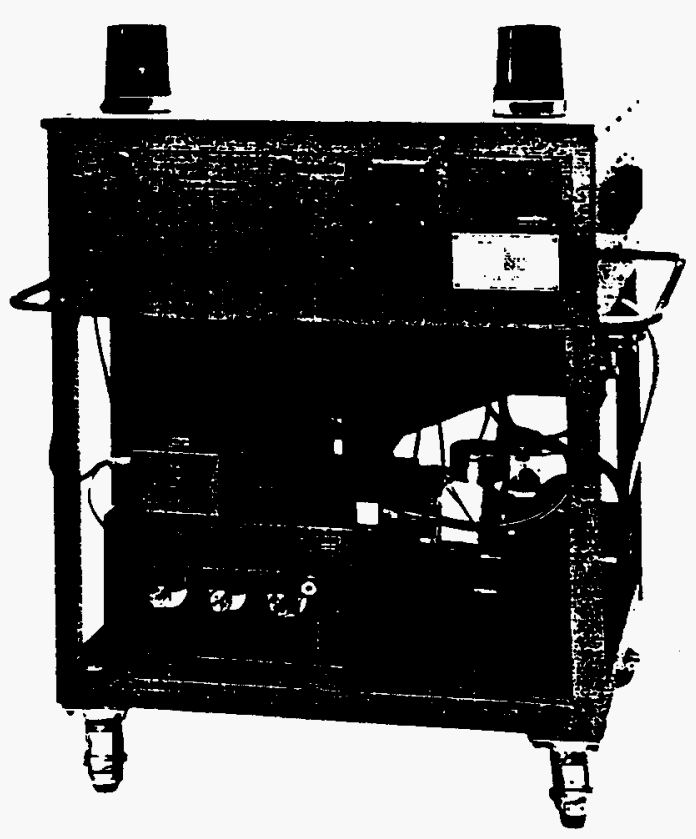

- SELF-CONTAINED MICROCOMPUTER-BASED UNIT FOR MONITORING STACK EFFLUENTS AND WORK AREAS

- FEATURES INCLUDE: DIGITAL DISPLAYS, BACKGROUND SUBTRACTION, HIGH AC. CURACY AND SENSITIVITY, SIMPLE TO OPERATE AND MAINTAIN

\section{a division of \\ Eberline \\ $\mathrm{H}-\mathrm{I}$ \\ $\$ 54,700$ ea

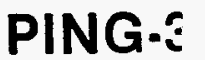 \\ MNC 22}


Model PING-3B

Particulate, lodine and Noble Gas Air Monitoring System

\section{GENERAL DESCRIPTION}

The Model PING-3B is a microcomputerbased, cart-mounted monitor designed to measure the alrborne concentration or stack emission rates of radioactive particulates, iodine, and noble gas. The PING-3B is simple to operate and maintain, containing all of the necessary hardware and programming (software) to be a complete, stand-atone system. A simple, easy-to-understand keyboard allows the operator to input operating parameters and request current or historical data for hard-

\section{SPECIFICATIONS}

\section{MICROCOMPUTER}

The microcomputer handles eight channels: one each for particulate and lodine, two for noble gas, and four for background subtraction.

It accumulates count data from each detec. tor, then computes count rates and subtracts user specified background factors.

It determines, annunciates and logs status changes to the printer for each channel and controls the alarm lights and check sources. The microcomputer computes and retains the average net count rate in history files for each channel. The PING-3B automatically prints (logs) the one-hour history files for any or all channels at a user-specified time each day and can optionally $\log$ the ten-minute files every four hours. Files are maintained for the past 24 one-minute, ten-minute, one-hour and one-day averages.

History file information can be averaged, integrated or differentiated. In addition, the microcomputer can calculate the average release rate for a history file based on discharge rate when the PING-3B is used as a stack or duct monitor.

Battery-backed random access memory enables the PING-3B to retain channel parameter files in the event of a complete loss of power to the system.

Continuous flow and pressure inputs from a new solid-state flow sensor combined with new programming enables the PING-3B to perform derived concentration calculations on fixed filter channels resulting in readouts in units of $\mu \mathrm{Cl} / \mathrm{cm}^{2}$. Absolute pressure copy output. The microcomputer monitors the operation of the detectors and pumping system, does all of the calculations, and communicates with the keyboard, printer, digital displays and alarm system. Eberline's data acquisition methods and background subtraction features yield the lowest possible minimum detectable concentration from a given detector configuration. The PING-3B is ideal for backfltting at existing nuclear power plants that have analog systems.

measurements made by the flow sensor allow the microcomputer to correct the noble gas readings for variations in chamber pressures.

\section{SECURITY}

Two key-locked switches provide security against unauthorized operation or modification of channel parameter files. The first key is necessary to allow operator commands such as "pump on" and "check source". The second, and highest priority key, is necessary to alter data base parameters such as alarm setpoints.

\section{DISPLAY}

The Eberline Display III is a LED (light emitting diode) display module used to display current channel readings. The format of the data display is three significant mantissa diglts with a $11 / 2$-digit exponent $(10 \pm$ " maximum). An array of six lights display the current status of the selected channel. The channel to be displayed is selected by a thumbwheel switch located on the display panel.

\section{PRINTER}

The printer assembly consists of a new and highly reliable 40 column, $7 \times 7$ dot matrix printer. This printer has been used worldwide in retail, financial and industrial terminals, and has a mean printhead life expectancy of $1.5 \times 10^{4}$ characters. The new printer is one of the most significant improvements incorporated into the PING.3B. As part of Eberline's continuing effort to support current PING-3 users, the new printer is now available in a PING-3 printer upgrade kit. 


\section{KEYBOARD}

The keyboard is the communication point from man to machine and has two subgroups. One group is the instruction pad, and the second group is the data pad. All commands begin with a single entry via the instruction pad, followed by an entry or entries via the data pad, and are terminated with the ENTER key. Numerical data (calibration constants, etc.) are entered in fixed decimal point scientific notation. The instruction and data pads are color-keyed according to function.

\section{SAMPLER AND DETECTOR SUBSYSTEM}

An Eberline Model SA-13 sampler assembly contains sample chambers for particulate, iodine, and noble gas in one compact, $4 \pi$ lead-shielded assembly ( 3 inches of lead shielding with 1 inch of lead between detectors).

\section{PARTICULATE}

Fixed Filter: 47-mm-diameter, Millipore SM is recommended. (Part No. FIFP1/9)

Detector: Eberline Model RDA-3A, beta scintillation detector with the Model RDS-1 solid. state alpha detector for radon background subtraction.

Nominal Sensithity (at flow rates of $60 \mathrm{Lmin}$ ): "'r $\mathrm{Cs}=5 \mathrm{cpm} / \mathrm{h}$ for $1 \times 10^{-11} \mu \mathrm{Cl} / \mathrm{cm}^{2}$, $\omega \mathrm{Sr}-\omega y=8.8 \mathrm{cpm} / \mathrm{h}$ for $1 \times 10^{-11} \mu \mathrm{Cl} / \mathrm{cm}^{2}$, "TC $=3.8 \mathrm{cpm} / \mathrm{h}$ for $1 \times 10^{-11}{ }_{\mu} \mathrm{Cl} / \mathrm{cm}^{2}$.

Range: Approximately $10^{-11}$ to $10^{-1} \mu \mathrm{Cl} / \mathrm{cm}^{3}$.

Background: Approximately $25 \mathrm{cpm}$ (depending on geographic location) plus $10 \mathrm{cpm}$ per $\mathrm{mR} / \mathrm{h}$ of external 's'Cs field. Both fixed and "live" background sources may be specified for background subtraction. A solid-state alpha detector provides a means of subtracting out the bulld-up of radon daughters on the filter, and the area monitor provides a means of subtracting gamma background. The noble gas effect can be subtracted via a portion of the low-range gas channel data.

\section{IODINE}

Cartridge: 2 -inch-diameter $\times 3 / 4$-inch-thick metal-cased cartridge containing TEDA im. pregnated charcoal (Part No. FIFC1/4). Silver zeolite cartridges are available upon request.

Detector: Eberline Model RDA-2A 2-inchdiameter $\times$ 2-inch-thick Nal(TI) crystal with a 20'Am seed embedded for automatic gain stabilization for drift-free pulse-height analysis.
Nominal Sensitivity: ${ }^{13} /=3.5 \mathrm{cpm} / \mathrm{h}$ for $1 \times$ $10^{-11} \mu \mathrm{Cl} / \mathrm{cm}^{\prime}$ at a flow rate of $60 \mathrm{Lmin}$.

Range: Approximately $10^{-11}$ to $10^{-1}{ }_{\mu} \mathrm{Ci} / \mathrm{cm}^{2}$.

Background: Approximately $45 \mathrm{cpm}$ (depen. ding on geographic location) plus $15 \mathrm{cpm}$ per $m R / h$ of external "Cs gamma which is sub. tracted via the adjacent energy window.

\section{NOBLE GAS (LOW RANGE)}

Volume: 2.65 inches in diameter $\times 3$ inches deep (270 $\mathrm{cm}^{4}$ volume). Approximately $35 \mathrm{~cm}^{3}$ of the volume is taken up by the noble gas detector.

Detector: Eberline Model RDA-3A beta scin. tiliation detector, 2-inch-diameter $\times 0.010$. inch-thick plastic with $1.6 \mathrm{mg} / \mathrm{cm}^{2}$ aluminized Mylare window.

Nominal Sonaltivity:

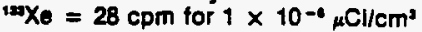
at 14.7 psia;

${ }^{m} \mathrm{Kr}=41 \mathrm{cpm}$ for $1 \times 10^{-\bullet} \mu \mathrm{Ci} / \mathrm{cm}^{\prime}$ at 14.7 psia.

Range: Approximately $10^{-7}$ to $10^{-2} \mu \mathrm{Cl} / \mathrm{cm}^{3}$ "'Je equivalent.

Background: Approximately $25 \mathrm{cpm}$ (depen. ding on geographic location) plus $10 \mathrm{cpm}$ per $\mathrm{mR} / \mathrm{h}$ of external ${ }^{12} \mathrm{Cs}$ field. An empirically determined fraction of the background channel is subtracted to correct for gamma background.

\section{NOBLE GAS (INTERMEDIATE RANGE)}

Volume: 2.65 inches in diameter $\times 3$ inches deep ( $270 \mathrm{~cm}^{2}$ volume). This is the same volume viewed by the low range noble gas detector. (Approximately $35 \mathrm{~cm}^{3}$ of the volume is taken up by the noble gas detector.)

Detector: Eberline energy-compensated Geiger-Mueller (G-M) tube.

Nominal Sensitivity:

${ }^{120} \mathrm{Xe}_{\mathrm{e}}=0.41 \mathrm{cpm}$ for $1 \gamma^{\mathrm{Bq}} \mathrm{MeV} / \mathrm{cm}^{\mathrm{s}}$ $\left(5.99 \times 10^{-4} \mu \mathrm{Cl} / \mathrm{cm}^{2}\right)$ at 14.7 psia,

${ }^{a K r}=0.61 \mathrm{cpm}$ for $1 \gamma \mathrm{Bq} \mathrm{MeV} / \mathrm{cm}^{2}$ $\left(0.01 \mu \mathrm{Cl} / \mathrm{cm}^{2}\right)$ at $14.7 \mathrm{psia}$.

Ranga: Approximately $10^{-3}$ to $10^{2} \mu \mathrm{Ci} / \mathrm{cm}^{3}$ 13'Xe equivalent (1 to $10^{\circ} \gamma \mathrm{Bq} \mathrm{MeV} / \mathrm{cm}$ ).

Background: Approximately $0.5 \mathrm{cpm}$ (depending on geographic location) plus $1 \mathrm{cpm}$ per mPh of external "co fieid. An empirically determined fraction of the background channel (identical detector in the lead shield) is subtracted for background compensation. 


\section{AREA MONITOR}

A gamma-detecting area radiation monitor which has a range of 0.01 to $100 \mathrm{mR} / \mathrm{h}$ is included.

\section{MISCELLANEOUS SPECIFICATIONS}

Electronics: Eberline Interface Boxes, Models IB-2, IB-3C and IB-4A, which contain the detector high voltage, signal amplifier and line driver, and provide the function of inter. facing the detectors to the microcomputer.

Battery Backup: The PING-38 contains a battery which powers the electronics for 8 hours in the event of a loss of external power. This insures against information being lost from the microcomputer's memory.

Check Source: There is a motor-driven check source assembly for each of the monitoring channels, except the intermediate range noble gas. Installed sources include a $30 \mu \mathrm{Ci}$ '" $\mathrm{Cs}$ for particulate and low range noble gas, a $0.5 \mu \mathrm{Ci}{ }^{12} \mathrm{Ba}$ for iodine, and a $0.3 \mu \mathrm{Ci}$ " $\mathrm{Sr}$ - " $\mathrm{Y}$ for area monitor (NRC license required). The check source is completely shielded from the detector in a retracted position, and it is actuated either individually or as a group by keyboard request.

Analog Signal Input: The acquisition of an analog signal may be desirable in the event the PING-3B is used as a stack monitor. A signal can be acquired which is representative of the stack flow rate and used in computations of radioactive effluent release rates. Six channels of analog input are standard with the PING-3B. Two of the channels are used for input of absolute and differential pressure from the installed transducers with four channels of input available for other signals.

\section{PUMP AND FLOW INDICATION SYSTEM}

Pump: Eberline Model RAP-3 with adjustable, regulated flow to a maximum flow of $110 \mathrm{~L} / \mathrm{min}$. Recommended sample flow rate is $60 \mathrm{~L} / \mathrm{min}$.

Flow indicator. The flow measurement system consists of absolute and differential pressure transducers which pass information to the microcomputer. This information is then used to caiculate the mass flow rate of the monitored air stream. The current sample flow rate in liters per minute may be viewed at any time by selecting Channel 15 on the digital display. Channel 14 displays the sample pressure. This solid-state flow transducer system is used to correct problems defined by NRC Notice $82-49$.

\section{MECHANICAL SPECIFICATIONS}

Size: 36.4 inches wide ( 44.5 inches with handles) $\times 31.75$ inches deep $\times 50.1$ inches high $(0.92 \mathrm{~m} \times 0.81 \mathrm{~m} \times 1.27 \mathrm{~m})$ with casters.

Weight: Approximately 1500 pounds $(682 \mathrm{~kg}$ ). Oporating Tomperature: 32 of to $122^{\circ} \mathrm{F}\left(0^{\circ} \mathrm{C}\right.$ to $\left.50^{\circ} \mathrm{C}\right)$.

Power Requirements: $115 \mathrm{Vac}, 60 \mathrm{~Hz}$ at $15 \mathrm{~A}$ maximum (typically $7 \mathrm{~A}$ running). (220 Vac, $50 \mathrm{~Hz}$ optional.)

Iniet and Outlef Connections: 1-inch-o.d. $(2.54 \mathrm{~cm})$ tube-compression fittings.

\section{OPTIONS}

High Range Noble Gas Dotuctor

An Eberline Model SA.9 detector assembly providing 3 inches $(7.6 \mathrm{~cm})$ of lead shielding in a $4 \pi$ configuration.

Detector: Energy-compensated G-M tube with $0.7 \mu \mathrm{Cl}{ }^{\infty} \mathrm{Sr}-{ }^{-\infty} \gamma$ check source which views a portion of a 1-inch stainless-steel pipe running through the SA-9.

Nominal Sensitlvity: $0.01 \mathrm{cpm}$ per $r \mathrm{~Bq}$ MeV/cm at 14.7 psia

Renge: 1 to $10^{4} \mu \mathrm{Cl} / \mathrm{cm}^{\prime}{ }^{29} \mathrm{Xe}$ equivalent.

Background: Background compensation is accomplished by automatically subtracting a portion of the signal from the G-M detector imbedded in the SA-13 lead block.

\section{Analog Signal Output Option}

Occasionally it is desirable to output a signal which is representative of the radiation level sensed by a particular detector in the PING-3B. This is made possible by the analog output option which is a 4 to $20 \mathrm{~mA}$ current loop. Any or all of the measurement channels may have this option except Channel 2 (alpha particulate).

\section{Manual Purga/Grab Sample Option}

This option provides the necessary valves and plumbing to allow purging the PING-3B and to allow grab sampling. All valves included in this option are manualiy-operated, stainiesssteel valves.

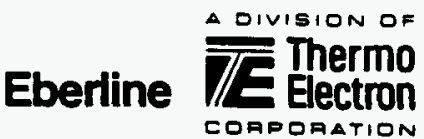

P.O. $80 \times 2108$

Santa Fe. New Mexico 87504-2108

(505) 471.3232 TWX: 910-885-0578 


\section{Model PING.3}

\section{Particulate, lodine, and Noble Gas Air Monitoring System}

\author{
02-03 MORNAL \\ 01 IODINE \\ $02 \mathrm{CPH}$ \\ O3 CAL.CONSTANT +1.00E+UI \\ O4 BKG 1 CHE 0 \\ O5 BKG\$ 1 FACTOR +0.00E+OOO \\ 06 BKG42 CHE 0 \\ 07 BKG+2 FACTOR +0.0OE+riC \\ 08 FIX.BKG.5L8. +0.DOE+10J \\ 09 HI ALF SET +1.00E +CE \\ 10 ALT ALI SET $\bullet 1$. DUEtije \\ 11 TN ALN $(z / \pi)+1.00 E+01$ \\ 16 LOGGED? 10 \\ 19 SAM.FLO.CH. 0

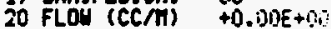 \\ 21 REL.FLO.CH. 00 \\ 22 REL.FLO.FAC. +0.00E +10 ? \\ lodine Channel Parameter File \\ (shown bofore edit)
}

\author{
02-03 NORMAL \\ DI IOOINE \\ 02 UCI \\ 03 CAL.CONSTANT +2.00E-05 \\ OL BKG 1 CHF \\ O5 EKG 1 FACTOR +D. DOUE+DI! \\ U6 BKG $\$ 2$ CH: \\ 07 BKGะ2 FACTOR +0. VIJE + 90 \\ 08 FIX.BKG. SUB. +0.60E+ (III) \\ 09 HI ALH SET +4.00E+104 \\ 10 ALT ALH SET +1. CUEE+OA \\ 11 TN ALH $(2 / \mathrm{M})+1.00 E+190$ \\ 16 LOGGED? 10 \\ 19 SAT.FLO.CH. 15 \\ 20 SAM.FLO.FAC. +1. OOE+OC \\ 21 REL.FLO.CH. 00 \\ 22 REL.FLO.FAC. +O. $\$ U U E+00$
}

lodine Channel Paramoter Flle (shown after edit)

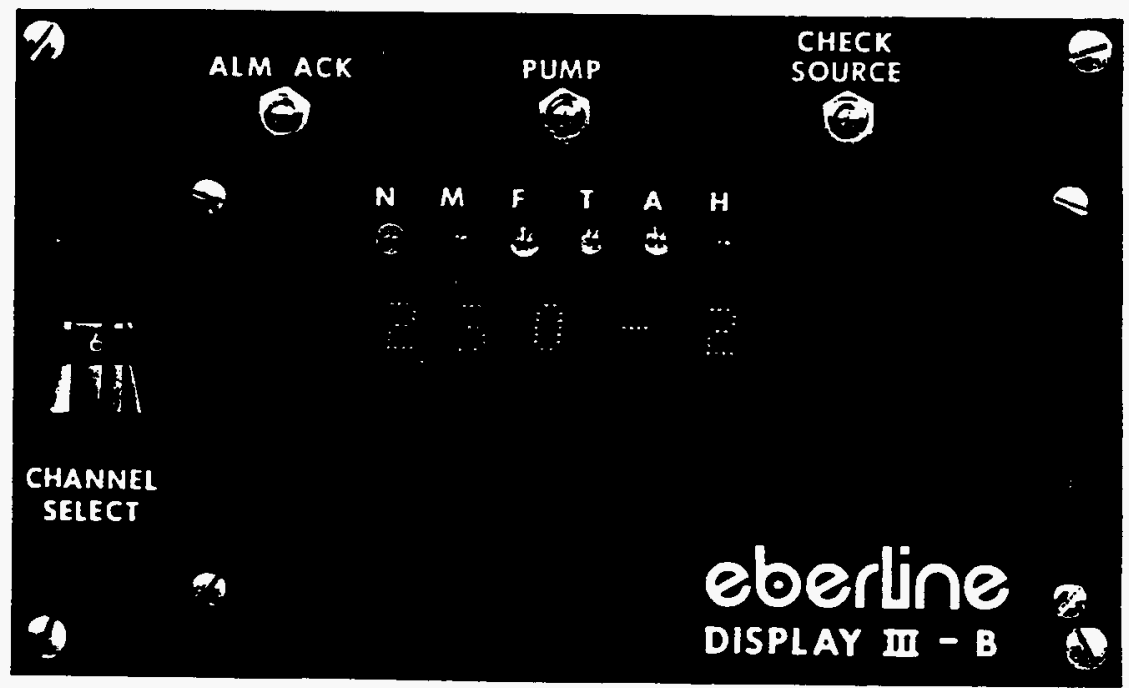

Visual Display

$\mathrm{H}-5$ 
WHC-SD-SNF-CDR- 00 ; REV. 0

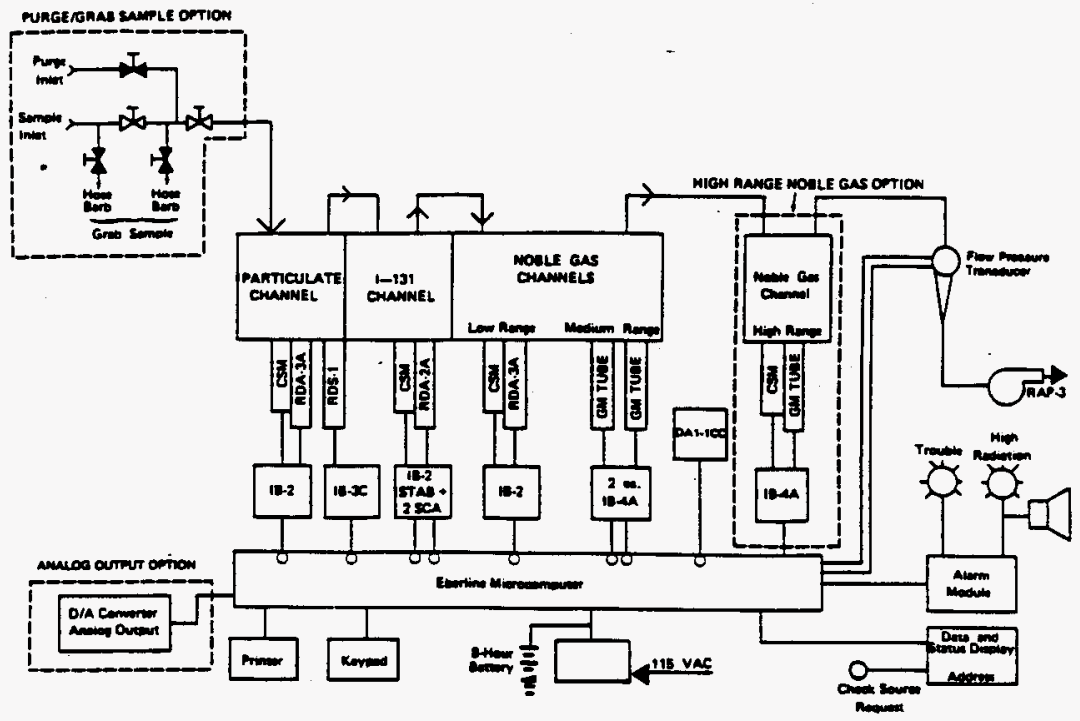

PING.3B Functlonal Diagram

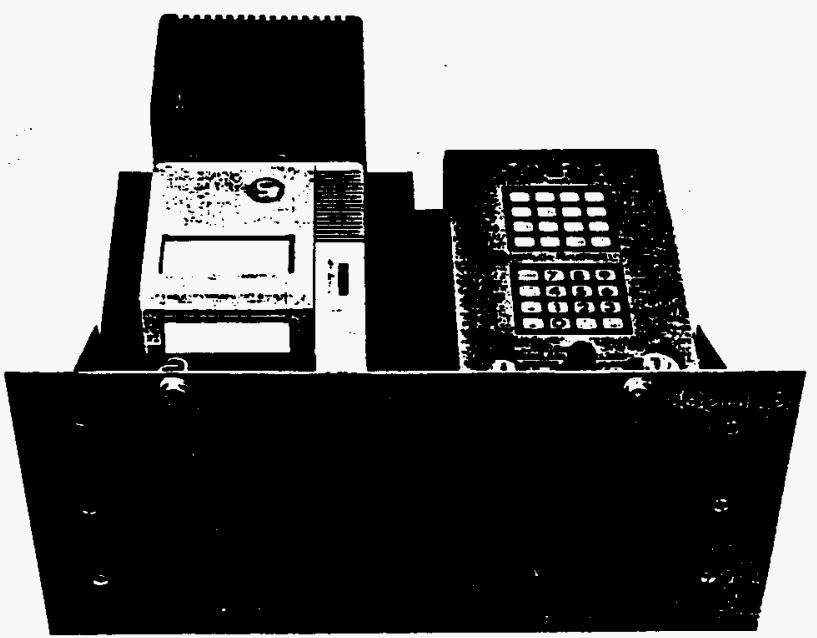

PING-3B Printer and Keyboard

$$
H-6
$$


WI-SD-SNF-CDR-007, REV. 0

\section{Alpha Particulate Sampler Assembly}

\section{Model SA-4A}

$A-3000$
$A-4000$
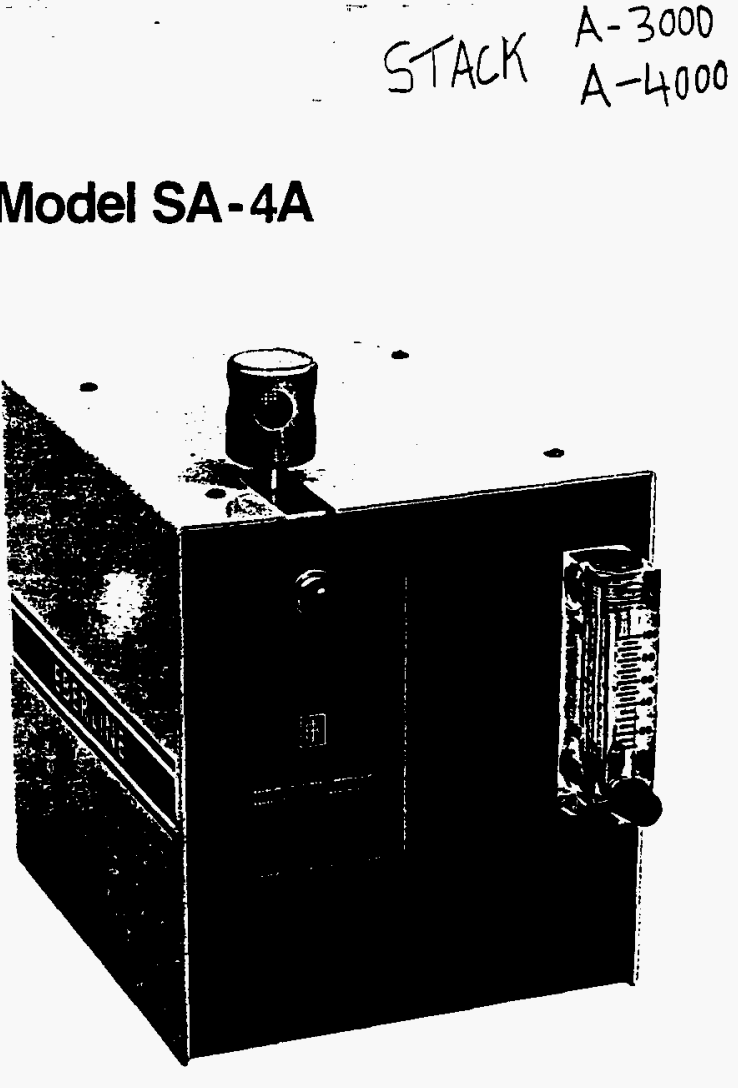

COLLECTS AND MEASURES AIRBORNE ALPHA

PARTICULATE

SOLID-STATE DETECTOR

- MAY BE USED TO MEASURE SPECIFIC ALPHA EMITTERS

(for example ${ }^{239} \mathrm{Pu},{ }^{230} \mathrm{Th},{ }^{234} \mathrm{U}$, or $235 \mathrm{U}$ ) WITH RADON

DAUGHTER BACKGROUND SUBTRACTION

- MAY BE USED TO MEASURE RADON DAUGHTERS

INDIVIDUALLY OR AS A GROSS MEASUREMENT

$\square$ DESIGNED TO INTERFACE WITH DIGITAL OR ANALOG SYSTEMS 


\section{Model SA-4A, Alpha Particulate Sampler'Assembly}

\section{GENERAL DESCRIPTION}

The Model SA-4A Sampler Assembly utilizes a particulate filter for sample collection and a solid-state aipha detector for determination of the alpha activity on that filter. The detector is followed by an amplifier and up to three pulse-height-analyzer (PHA) modules. The PHA modules provide the SA-4A with the capability of monitoring for specific alphaemitting isotopes as well as performing the func-

\section{SPECIFICATIONS}

Detector: Silicon-diffused junction type (cleanable) with $490 \mathrm{~mm}^{2}$ area.

Filter: 47-mm-diameter; Millipore SM or equivalent recommended.

Counting Etficiency: Approximately 20 percent of $2 \pi$ from a plated $47 \cdot \mathrm{mm}$-diameter $239 \mathrm{Pu}$ source in the filter holder.

Amplifier: Charge-sensitive input allowing very high input sensitivity with excellent noise rejection followed by a dc-coupled amplifier fed back for stability and control. Overall sensitivity is adjustable by an internal gain control.

Pulse-Height Analysis (PHA): Normally supplied with two, but will accept three pulse-height analyzer modules. Each pulse-height analyzer contains adjustable threshold and window levels. A window switch may be selected "out" for gross counting. Output process line signal drivers are capable of driving 2000 teet of twisted-pair cable. tions of signal processing and line driving for interfacing the SA-4A to a digital or analog system.

The filter is $\mathbf{4 7} \mathrm{mm}$ in diameter and is easily accessible through the front door of the unit. A flowmeter (10 to $100 \mathrm{~L} / \mathrm{min}$ ) is provided. The SA-4A is also available with a wall-mounting bracket.

Power Requirement: $12 \mathrm{Vdc}$ at $150 \mathrm{~mA}$ (add $50 \mathrm{~mA}$ for each additional PHA card); mating power connector is Amphenol No. 165-14.

Temperature: Operational from $+20^{\circ} \mathrm{F}$ to $+130^{\circ} \mathrm{F}$ $\left(-7^{\circ} \mathrm{C}\right.$ to $\left.+55^{\circ} \mathrm{C}\right)$; total change in system gain is less than \pm 5 percent.

Size: 6.2 inches wide $\times 10$ inches deep $\times 8.1$ inches high (15.6 cm $\times 25.4 \mathrm{~cm} \times 20.6 \mathrm{~cm})$.

Weight: 8.8 pounds ( $4 \mathrm{~kg}$ ).

The intake has a screen-protected cap with four ports for approximately 1 in 2 total open area. The cap is easily removed for attachment of a .5-inchi.d. hose for remote sampling. The outlet has a hose barb for $5 / 16$-inch-i.d. hose.

\section{Eberline}

A subsidiary of Thermo Instrument Systems Ine.

P.O. Box 2108

Sente Fe, New Mexico 87504-2108

(505) 47l-3232 TLX: 66-0438 EIC SFE 


\section{Alpha Air} Monitor

\section{ALPHA6 Product Family}
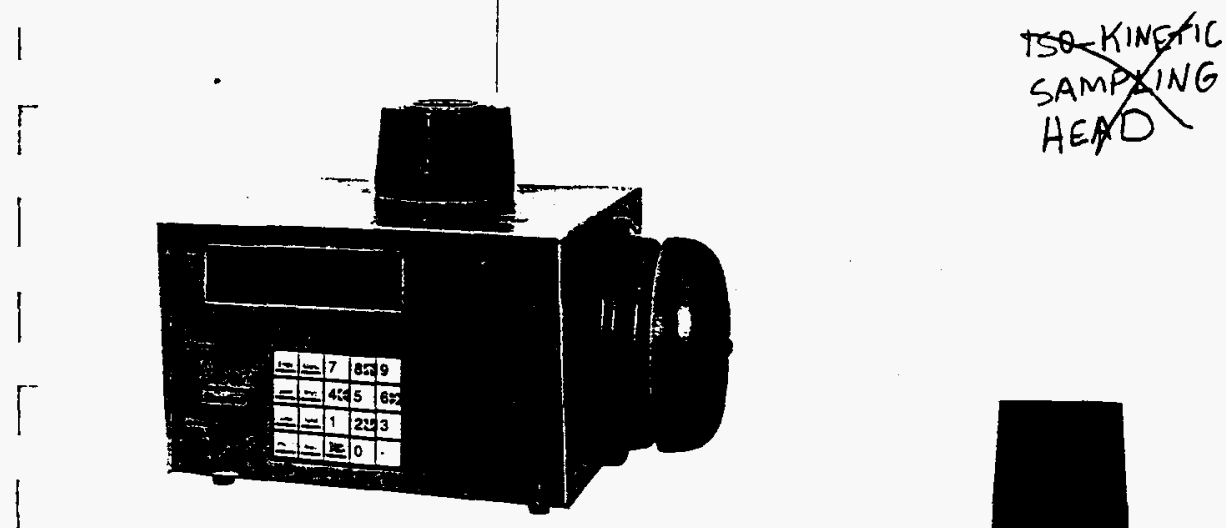

ALPHAGA-1

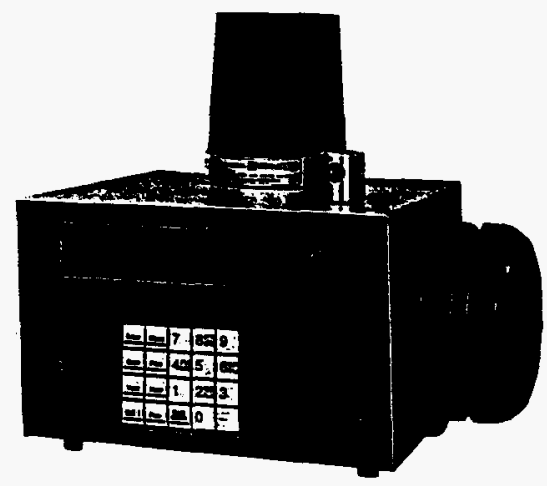

ALPHA6-1

- CONTINUOUS AIRBORNE ALPHA MONITOR

- USER CONFIGURABLE FOR SPECIFIC ISOTOPE IDENTIFICATION

- 256 CHANNEL ANALYZER FOR RADON-THORON DISCRIMINATION

- TWO RS-232C COMMUNICATION PORTS FOR HARDCOPY PRINTOUT AND COMMUNICATION WITH PERIPHERAL DEVICES

- A VARIETY OF CONFIgURAT̈IONS INCLUDING STANDALONE, REMOTE DETECTOR HEAD, INLINE DETECTOR HEAD, AND NETWORKED SYSTEMS.

$\begin{array}{cc}\text { Eberline } \\ \substack{\text { A subsidiary of } \\ \text { Systems Inder }} & \$ 5,000 \text { ea } \\ H-9 & \text { ALPHA6 }\end{array}$




\section{ALPHA6 Product Family}

\section{GENERAL DESCRIPTION}

The ALPHA6 product family utilizes a solid-state detector and a 256 channel analyzer to measure aipha particles of specific energies while minimizing interference from radon - thoron alpha emitters. This increases the sensitivity over conventional one or two channel pulse height analyzer techniques so that 4 DAC hours of 230Pu may be detected in a typical radon-thoron background.

The instrument incorporates micro-computer technology which greatly enhances the measurement and data storage capabilities. Multiple historical files are maintained so that the most information possibie may be obtained from the data.

A mass air flow measuring system provides flow and flowrate information which is used in computing the concentration level. The combination of the mass flow system and the 256 channel analyzer ensures that the best measurement at the highest possible sensitivity can be made.

Two PS-232C communication ports are provided so that data and information may be output to a printer for hardcopy data retention or so that a communication link may be made between the ALPHAG and a terminal or other computer. Information and setup parameters may be transferred bi-directionally.

A local keypad is included so that calibration parameters and alarm settings may be input at the unit.

Both visual and audible alarms are actuated when the user adjustable alarm setpoints are exceeded. Alarm relay contacts are located on the back panel of the instrument and the alarm status is transmit. ted over communication lines if a printer or other peripheral is connected.

A failure warning is given if a major fault is detected, such as loss of the detector signal, loss of the air flow or failure of the real time clock. A local visual and audible alarm is activated and a normally activated relay drops out to provide an external alarm. In addition the alarm condition is transmitted over the communication lines to a printer or other peripheral.

The instrument is designed to be used with either an external air pump, such as the Eberline Model RAP-1, or with a house vacuum system.

\section{CONFIGUAATIONS}

The ALPHA6 family of alpha air monitors inciude a variety of configurations to help meet user specific system requirements. The ALPHA6 family includes:

ALPHA6-1 Alpha air monitor utilizing the traditional top entry air intake stack identical to the ALPHA-5A and AMS-3A.

\footnotetext{
ALPHAGA-1 Alpha air monitor utilizing the radial entry head. The radial entry head is designed to maximize particulate collection efficiency, meeting the requirements of DOE Order 5480.11 .
}

ACS-1 Alpha cam system features a centralized electronics mounted in $19^{n}$ instrument racks and utilizing remote detector heads located in the work place. A central computer is used for all display and control functions. Each rack unit will hold up to six alpha cam channels.

ALPHA6A OPT1 Remote detector using the radial entry head.

ALPHAGA OPT4 Remote detector using an inline configuration for stack and duct monitoring applications. 


\section{SPECIFICATIONS}

Detector: Diffused junction, solid state, $490 \mathrm{sq} . \mathrm{mm}$ (25 $\mathrm{mm}$ dia) active area. The gross efficiency is approximately $25 \%(4 \pi)$. The efficiency to $239 \mathrm{Pu}$ is approximately $17 \%(4 \pi)$.

Filter: $47 \mathrm{~mm}$ Millipore SM or Fluoropore FS. Deposition area is restricted to center one inch diameter.

Readout: The readout is a dot matrix liquid crystal display system. Various forms of information are presented, including time and date, ajr flow rate, energy spectra, and the measured values (cpm. concentration, elc.) and the alarm points. A strip chart recorder type of presentation is also available with selectabie time units of seconds, minutes, hours and days. The display provides prompts to guide the user through the menus and historical data files.

Equation Specification: Five user definable variables are available for configuration of the ALPHAG to a specific application. Default equations are defined for measurement of 230 Pu but may be changed by the user to configure the instrument for the measurement of different isotopes.

Alarm Determination: Up to six comparisons on the user variables may be made to determine alarms and/or failures. Default settings determine 239Pu alarms but these settings may be changed to reflect changes in equation definitions.

Clock: A real time clock is provided so that all alarms and data can be time stamped.

High Alarm: The computer in the ALPHA6 provides optimum high alarm determinations. Slow, possibly statistical, count rate increases are examined at length while faster increases give an immediate alarm.

Alarm Outputs: High alarm and failure alarm outputs are available. Relay contacts rated for $1 \mathrm{~A}, 115$ vac are supplied. The failure alarm is normally energized. Locally, a strobe light and bell are provided.

Air Fiow Alarm: Provides an alert if the flow rate deviates from the nominal range.

Computer Output: Dual RS-232C communication ports are provided so that the information may be transferred to a printer and so that communications may be established with other peripherals. ALPHA6 OPT2 provides an RS-232/RS-485 converter for networking a group of aipha air monitors to a central computer.

Historical Files: Included are files on energy regions, air flow volume, computed values of counts per minute, total computed counts, concentration, etc. Each file kept includes the most recent 64 seconds, 64 minutes, 64 hours and 64 days.
Battery Backup: Used to maintain the real time clock, operational configuration, and historical files in case of a power failure.

Local Keyboard: For local entry of calibration and alarm parameters and for the selection of information to be displayed. A security code entry is required before operational parameters can be changed.

Subtraction Function: Provides automatic background subtraction. The amount of subtraction is determined by the percentage of the RaA and ThC peaks which scatters into the region of the isotope of interest. This percentage varies due to the changing amount of dust loading on the filter medium. The equation automatically corrects for this changing percentage of scatter.

Air Flow System: The air flow system incorporates a mass flow measurement. Flow data is incorporated in the concentration calculations.

Sensitivity: 4 DAC-hours of 239Pu can be detected with an average radon-thoron background.

Max. Count Rate: Approximately $500,000 \mathrm{cpm}$

Max. Channel Counts: Greater than 4,000,000 counts per channel

Temperature: Operation from $32^{\circ} \mathrm{F}$ to $104^{\circ} \mathrm{F}\left(0^{\circ} \mathrm{C}\right.$ to $\left.40^{\circ} \mathrm{C}\right)$

Power: 120 or 240 Vac, $50 / 60 \mathrm{~Hz}$, at $0.5 \mathrm{~A}$. AFI fitter incorporated for line noise rejection.

Size: ALPHA6-1 Approximately 13.75 inches high $\times 12.5$ inches deep $\times 15$ inches wide $(35 \mathrm{~cm} \times 32 \mathrm{~cm}$ $\times 38 \mathrm{~cm}$ )

ALPHA6A-1 10.5 inches high $\times 15$ inches wide $\times 12.5$ inches deep $(27$ $\mathrm{cm} \times 38 \mathrm{~cm} \times 32 \mathrm{~cm}$ )

Weight:
ALPHA6-1
15.4 pounds $(7 \mathrm{~kg})$
ALPHAGA-1
14.6 pounds $(6.6 \mathrm{~kg})$

Software Options: Alternate programs are available which default to measure uranium isotopes, thorium and radon daughter products. Contact the factory for current list of alternate programs available. 


\section{CENTRAL COMPUTER SOFTWARE}

Central computer sottware is available for systems using either individual alpha cams such as the ALPHAGA- 1 or the ACS-1 Alpha Cam System. This software provides the operator with centralized data acquisition and control over all alpha cams connected to the central PC. The central computer software utilizes a pull down menu system and provides the following functions:

Edit Allows the user to display and modity the configuration of all alpha cams in the systern. The operator may modify alarm setpoints, variable equations and regions of interest.
Readings Provides floor plans and group displays which are configured by trie user.

Data This menu item allows the operator to display historical and current data. History files are displayed in either tabular or graphical modes. A graph of the current alpha spectrum can also be displayed.

Utilities Counter control provides stop, start and reset commands. A clock set function is also provided.

\title{
TYPICAL SYSTEM CONFIGURATIONS
}

Individual Alpha Air Monttors

\author{
RS-485 Network
}

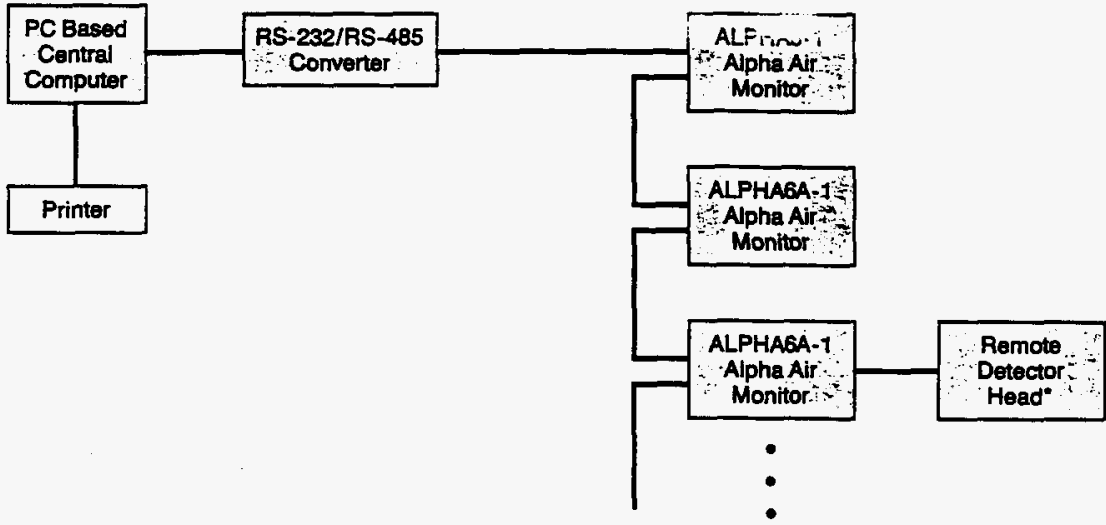

ACS-1 Alpha Cam

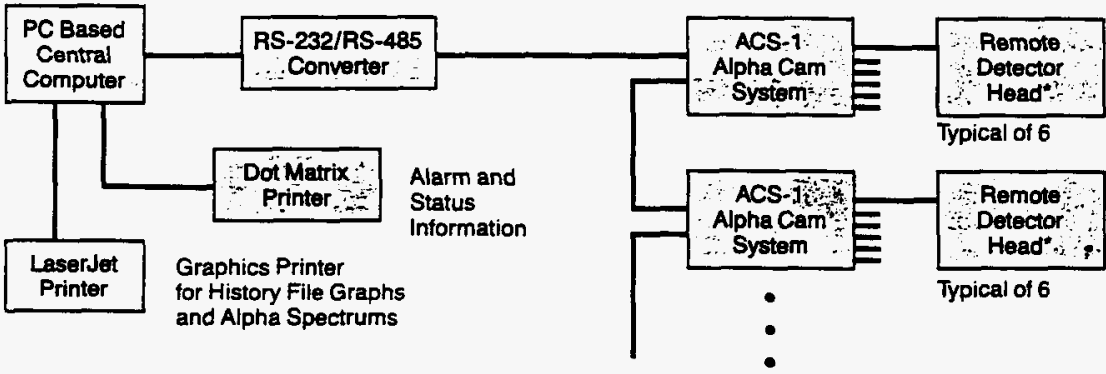

-Either Remote Detector Head Style May Be Used. 

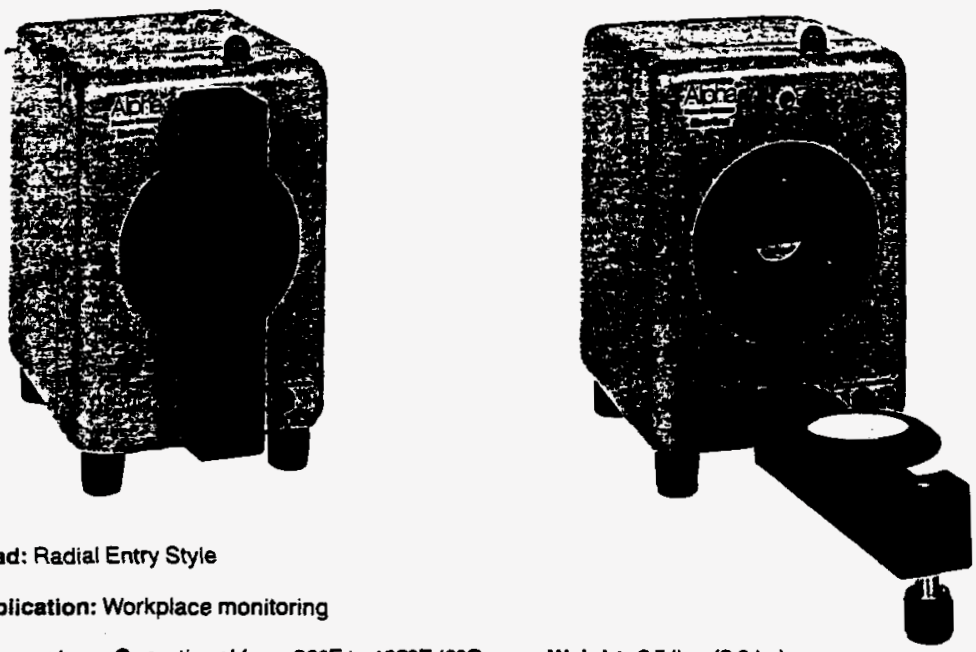

Head: Radial Entry Style

Application: Workplace monitoring

Temperature: Operational from $32^{\circ} \mathrm{F}$ to $122^{\circ} \mathrm{F}\left(0^{\circ} \mathrm{C}\right.$ to $50^{\circ} \mathrm{C}$ )

Humidity: $0-100 \%$ relative humidity

Size: 8.3 inches high $\times 7.9$ inches deep $\times 5.5$ inches wide $(21 \mathrm{~cm} \times 20 \mathrm{~cm} \times 14 \mathrm{~cm})$
Weight: $6.5 \mathrm{lbs} .(2.9 \mathrm{~kg})$

Distance from Electronics: Up to 500 cable feet (152.4 m)

Seal: Dust tight

\section{ALPHA6A OPT4}

Remote Inline Detector Head

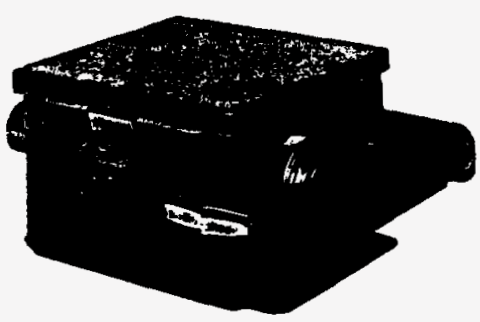

Head: Inline Entry Style

Application: Duct and Stack Monitoring

Temperature: Operational from $32^{\circ} \mathrm{F}$ to $122^{\circ} \mathrm{F}\left(0^{\circ} \mathrm{C}\right.$ to $50^{\circ} \mathrm{C}$ )

Humidity: $0.100 \%$ relative humidity

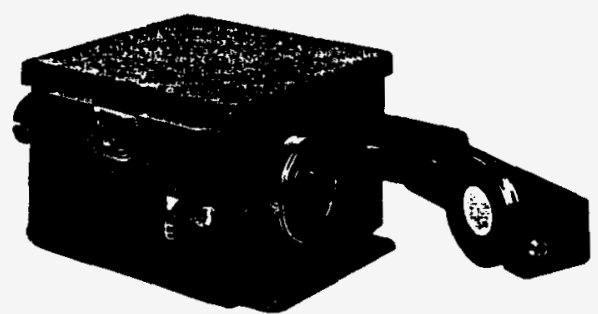

Size: 7.5 inches high $\times 4.4$ inches deep $\times 10.25$ inches wide

Weight: 11.25 lbs. $(5.1 \mathrm{~kg})$

Distance from Electronics: Up to 500 cable feet (152.4 m)

Seal: Dust tight

$-13$ 


\section{FIXED MONITORS 300 AND 400 SERIES \\ FEATURES}

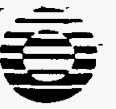

DVEFHOFF

$A-3001$

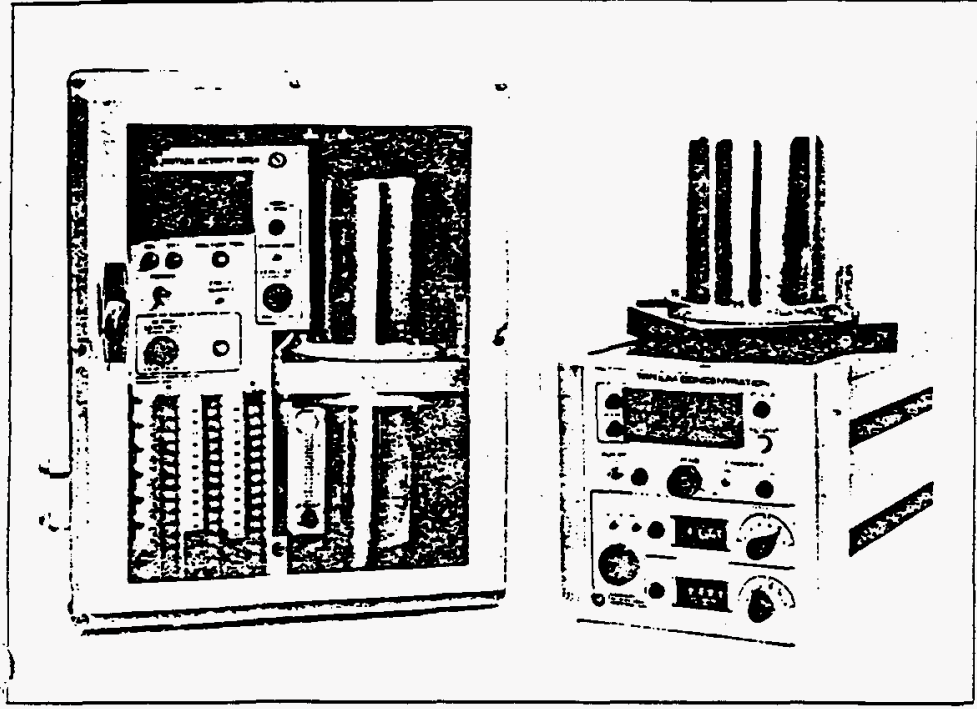

$A-4001$

\section{FIXED, RACKMOUNT AND BENCHTOP MONITORS}

These are line powered. and continuousty operating monitors. They can be built with a large number of combinations of FEATURES and OPTIONS. to suit the end user's particular needs. They are designed around three primary product FEATURES:

- RANGe of MEASUREMENT

- IONIZATION CHAMBERS

- PANEL METERS.

Each leature is designated by an aipha/numerical code.

Fixed monitors are classilied into four basie groups.

$31 \times$ series - single range, with single ionization chamber

$32 X$ series - single range, with dual ionization chamber

$41 X$ series - multi range, with single ionization chamber

$\$ 6,000$ each "TRITIUM MaviTOR"

$42 X$ series - multi range, with dual ionization chamber 


\section{RANGE OF MEASUREMENT}

Instruments are available in both single and multi range versions.

The single range instruments are lower in cost. and are recommended when a very wide range of measurement is not required.

- Single Range (3xX)

Applications which require a limited range of measurement can be mel by a single range instrument. designaled as "three hundred" series. Range of measurement can extend from three decades to five or even six decades.

Multi Range (4XX)

Multi range instruments are used for applications where a much wider range of measurement is required. These instruments are designated as "four hundred" series, either automatically (AR) or manually (MR) ranged versions. The most common are instruments with ive ranges, however. any range from three to seven can be supplied. For example, to obtain a measurement span of eight decades, a four hundred series tritium monitor equipped with a three digit panel meler has to be provided with five automatically switched ranges.

\section{PANEL METERS}

Instruments can be supplied with either digtal or analog displays. Either linear or logarithmic scales can be supplied for analog meter faces.

Digital Panel Meters (DP)

These meters are available in $3 \frac{1}{2}$ and $4 \frac{1}{2}$ digit versions. Single range monitors (300 series) can be equipped with either type of meter, depending upon the measurement range required. Multiranging monitors ( 400 series) are normally equipped with $3 \frac{1}{2}$ digit panel meters. since the overail measurement range is determined equally by the number of automatically switched ranges as by the digit range of the panel meter itself.

Analog Panel Meters (AP)

Analog panel meters are available with either logarithmic (LOG) or linear (LIN) scales. A logarithmic scale can cover up to six decades for a 300 series insirument, or up 10 nine or even ten decades for the 400 series instruments.

\section{IONIZATION CHAMBERS}

Moninors are available with etther single or dual chambers. The chambers can be mounted inside the electronics cabinet (ICI), or they can be located remotely (ICR).

lonization chambers are available in a large number of sizes and configurations, each specificaliy designed tor a paticular purpose. A more general description of the nalure and design of ionizalion chambers is lound on a separate sheet.

Single lonization Chamber $(X 1 X)$

Dual lonization Chamber $(X 2 X)$

The middle number in the alpha/numeric code designation for the description of the basic fealures of an instrument refer to the number of the ionization chambers to form part of the iritium monitor.

Monitors are generally supplied with either single chambers, or with dual chambers, atthough there are occasional applications where clusters of three or more chambers can be used.

Mounting of lonization Chambers

Internal lonization Chambers (ICI)

Remote lonization Chambers (ICR)

Mounting of the ionization chamber assembly can be internal to the main electronics cabinet. This ionization chamber assembly can also be mounted remolely at distances up to several hundred leet from the main electronics cabinet.

Chamber mounting must be selected at the time of speciłying the instrument.

$$
\mathrm{H}-15
$$


gation provided here is of a general nature. and serves to provide an overvew of some of the key imance characteristics to be found in OVERHOFF TECHNOLOGY CORPORATION tritium monitors.

\section{MEASUREMENT}

Tritium monitors with ionization chambers are capable of an extremely wide range of measurement. Sorne OTC tritium monitors are capable of measuring activities as low as $0.01 \mu \mathrm{Ci} / \mathrm{m}^{3}$, other versions of the instruments are designed tor high level measurements, approaching pure streams of tritum.

\section{RADON (ALPHA) PULSE SUPPRESSION (APS)}

OTC tritium monitors can be suppled with proprietary circuitry which suppresses response to naturally occurring spurious background, thereby extending low level Iritum measurement well below environmental radon or cosmic ray background.

\section{STABILITY AND NOISE}

The zero stability of an instrument is guaranteed to be equal or better than the smaliest required increment of measurement. For those instruments which are furnished with a digitai panel meter, the short term drit or noise is equal to, or less than, the least signiticant digit of the meter.

\section{DRIFT. ENVIRONMENTAL (AZ)}

Longterm dritt can be caused by iritium plate-out, as well as by changes in the electronics. Auto zeroing circuitry can be incorporated in order to eliminale all longterm electronic drit. Special chamber configuration such as heated or gridded chambers are recommended for prevention of elfects from tritium plate-out.

\section{RESPONSE RATE}

measurement signal level and the rate (tume constant) are inherently interrelated. High

burement levels demand tast response, whereas low measurement levels dernand long lime -instants, in order to smooth out noise and to provide a stable display. To accommodale this contradictory requirement, three distinct time constants have been incorporated into the instruments. 2 second tor measurement above $1000 \mu \mathrm{Cl} / \mathrm{m} 3$

5 to 10 second lime consiant for measurement of $80.1000 \mu \mathrm{Ci} / \mathrm{m} 3$

20 seconds or more for measurement below $80 \mu \mathrm{Ci} / \mathrm{m} 3$

Time constants usually switch automatically, although manual switching as well as different break points are available.

\section{ALARM SYSTEM. SIGNAL}

Dual independent alarms are standard. Single, tripie and quadruple independent alarm systems can be provided

\section{SET POINT}

Setting the level at which the alarm is actialed is accomplished with the use of tront panel mounted potentiometers. Multi-ranging models also include tront panel mounted rotary RANGE selector swiches. thereby permiting set point adjusiment over the entire range of operation of the instrument.

\section{MODE SELECT, CONFIGURATIONS}

Front panel switch for operator selection ol several atarm modes

Non-latching

Alarm active above sel point: ceases below set point

Latching

Alarm triggered above set point: remains active until reset. Can only be reset ather the measurement : receded below the set point value.

howiedge

mi alarm which continues active once tripped. but which can be deactivaled by means of a push

bumon at any time, regardless of set point levet status

$$
1+-16
$$




\section{INDICATORS AND ACTUATORS}

Incandescent or solid state lamps for atarm stalus and ac power stalus. Piezoelectric acoustic signailers with steady or pulsating tones.

\section{INTERFACE}

24 pin receptacle, located on the rear of the cabinet for interface connections for remole display, control and atarms, process controls or for interconnection to digital computer systems

\section{ENVIRONMENTAL}

\section{Temperature}

operating $0^{\circ} \mathrm{C}$ to $+50^{\circ} \mathrm{C}$

storage $-40^{\circ} \mathrm{C}$ to $+70^{\circ} \mathrm{C}$

Humidity

0-95\% RH

Shock

$5 \mathrm{G}$. all directions

PHYSICAL

\section{Power}

$115 / 230 \mathrm{~V}$ ac. $50 / 60 \mathrm{~Hz}$

Enclosure

$19^{\prime}$ rack mounting

$13^{\prime}$ deep

heights from $5.25^{\prime \prime}$ to $10.50^{\prime \prime}$

other cabinets available

Weights

cabinets-under $10 \mathrm{~kg}$

ionization chambers 10 to $50 \mathrm{~kg}$, depending on number, size and shielding 


\section{Environmental Air Sampler}

Model RAS-2

A-5000

A -6000

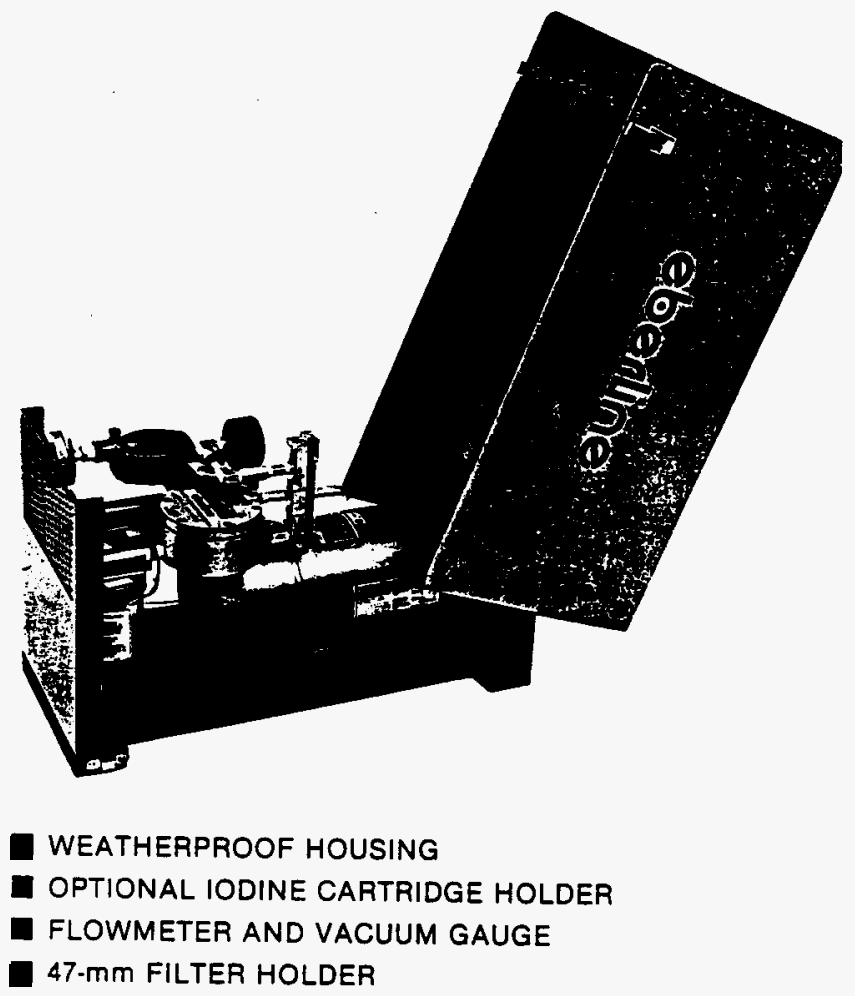

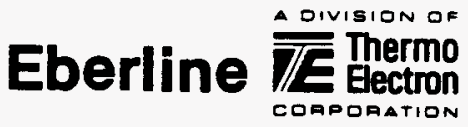 \\ $\$ 1,250$ eq \\ RAS-2 \\ $H-18$




\section{Model RAS-2, Environmental Air Sampler}

\section{GENERAL DESCRIPTION}

The Model RAS-2 is a weatherproof air particulate sampler containing an oil-free vacuum pump, motor, airflow regulator, flowmeter, vacuum gauge, and filter holder. The system is mounted in a weatherproof enclosure (WPH.1) permitting exterior use. The pump and motor provide enough heat to prevent winter freeze-up, and an exhaust fan provides summer cooling.

The Eberline airflow regulator is designed to maintain a constant pressure drop across an in-line orifice by controlling a variable bypass valve into the pump. The orifice is adjustable, permitting flow rate adjustment from near

zero up to the maximum pump flow capacity.

\section{SPECIFICATIONS}

Pump Type: Oll-tree, carbon vane

Motor: $1 / 4 \mathrm{HP}, 115 \mathrm{~V}, 60 \mathrm{~Hz}, 5 \mathrm{~A}$

(220 V, $50 \mathrm{~Hz}$ optional)

Vacuum: 26 inches $\mathrm{Hg}$ at sea level

Flow Rates: See figure

Filter Holder: Model FH-1, 47-mm

Flowmeter: 0 to $100 \mathrm{Lmin}$ (0 to $3.5 \mathrm{ft}^{2} / \mathrm{min}$ )

Vacuum Gauge: 0 to 30 inches $\mathrm{Hg}$

(0 to $760 \mathrm{~mm} \mathrm{Hg}$ )

Size: 23 inches long $\times 9.25$ inches wide $\times 13$ inches high $(58 \mathrm{~cm} \times 23.5 \mathrm{~cm} \times 33 \mathrm{~cm})$.

Welght: 60 pounds (27 kg)

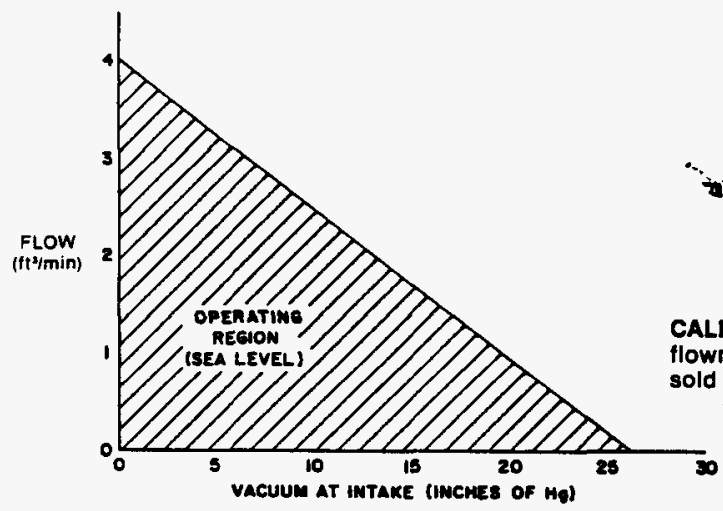

This flow control system permits the pump to operate at a minimum pressure drop at all times, which provides cooler pump operation to extend the lifetime.

It should be noted that when pressure varies, the flow through an orifice with a constant pressure drop will vary as the square root of the ratio of the absolute pressure. Therefore, if filter loading creates a pressure drop of 50 percent of the original pressure, the flow referenced to atmosphere will decrease by 30 percent.

\section{AVAILABLE ACCESSORIES}

lodine Cartridge Holder: Model ICH-1

lodine Cartridge: Part No. IC-1

Caltbration Adapter: Model ZP10758009

(Mates FH-1 to 1-inch NPT)

Filter Paper: Part No. FIFP8, 47-mm

\section{Calibration}

The unit is supplied with a three-point fiow calibration using a mass flowmeter.

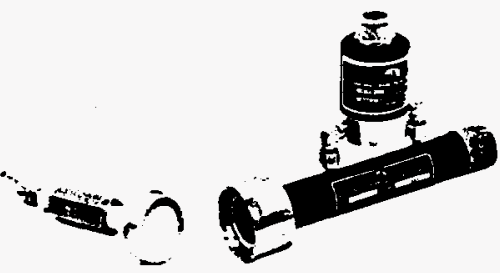

CALIBRATION ADAPTER (Shown with mass flowmeter attached) mass flowmeters are not sold by Eberline.

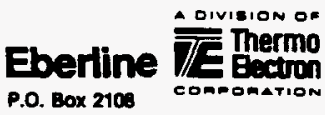

Santa Fo, New Mexico 875042108 (505) 4713222 TWX: $810-0950378$ 


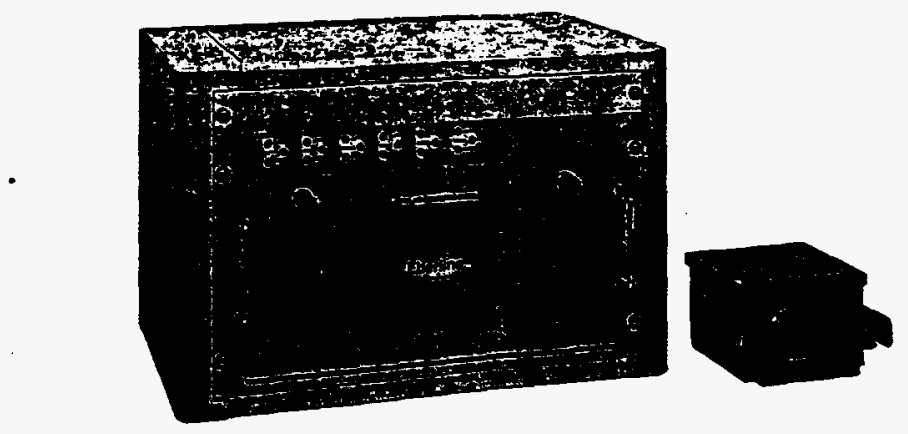

\section{ACS-1 Alpha Cam System}

The Alpha Cam System (ACS-1) is based on the Eberline Model Alpha6 Alpha Air Monitor, with appropriate design changes to create the most efficient system configuration. The most significant difference is the use of rack mounted electronics modules. Up to six Alpha6 electronics modules can be placed in one $19^{\prime \prime}$ rack. The electronics module is the heart of the Alpha6 monitor and includes the pulse processing electronics, MCA (multi channel analyzer) and the microprocessor based computer system used to analyze the spectral data and control the monitor. In order to conserve valuable floor space, improve the man-machine interface and cut the costs of multiple individual displays, a PC based system controller for readout and control of the monitoring system is used. The individual displays, typical on the individual Alpha6 cams, have been removed and the $P C$ will provide this function for the whole system. The PC also provides data archiving/report generation capability. The electronics modules pertorm the monitoring and alarm functions independent of the central computer. Therefore, a loss of the central computer does not compromise the safety function of the ACS-1.

The ACS-1 is comprised of two modules: the electronics module and the remote detector head assembly. These modules are connected by a single multi-conductor cable. The detector head assembly may be located up to $500 \mathrm{~h}$ away from the electronics module. Either the radial entry or inline versions of the remote detector head can be used.

\section{Electronics Module}

The electronics modules are contained within the $19^{\prime \prime}$ rack mount enclosure which is capabie of hoiding up to six electronics modules. The electronics module is the standard computer board used in the Alpha6 Alpha Air Monitor. In this configuration the liquid crystal display and keypad typical to each monitor are removed in favor of a single PC based display/control system for all monitors. The display and keypad is not necessary on each monitor when all the monitors and the display/control computer system are all located together. The display/control computer system provides an enhanced operator interface by displaying the current status and reading of all monitors in the system on one display.

Contained within the electronics modules are the power supply, the multi-channel analyzer and electronic subsystems to support the flowmeter, communications and various alarms. Each electronics module has an RS-232/RS-485 converter for communications to the system display/control computer. Two relay outputs are provided for each module. One relay is provided for alarm and a second relay for failure conditions. Each relay has a set of DPDT contacts.

The rack unit has indicating LEDs for each channel. The LEDs provide indication of normal, alarm and failure status for each channel. Each channel also includes a connector for a portable terminal accessible just behind the hinged tront of the panel. The portable terminal allows the operator to take a given channel out of the system for periodic maintenance and calibration.

\section{Eberline}

A subsidiary of Thermo Instrument Systeros Inc.

P. O. Box 2108

Santo Fe, New Mexico 87554-2108

(505) 471-3232 FAX: (505) 473-9221 
WHC-SD-SNF-CDR-007, Rev. $0^{-}$

Pages $\mathrm{H}-21$ through $\mathrm{H}-28$ have been removed from the document due to copyright and/or trademark restrictions. The information on these pages can be obtained from the project manager. 


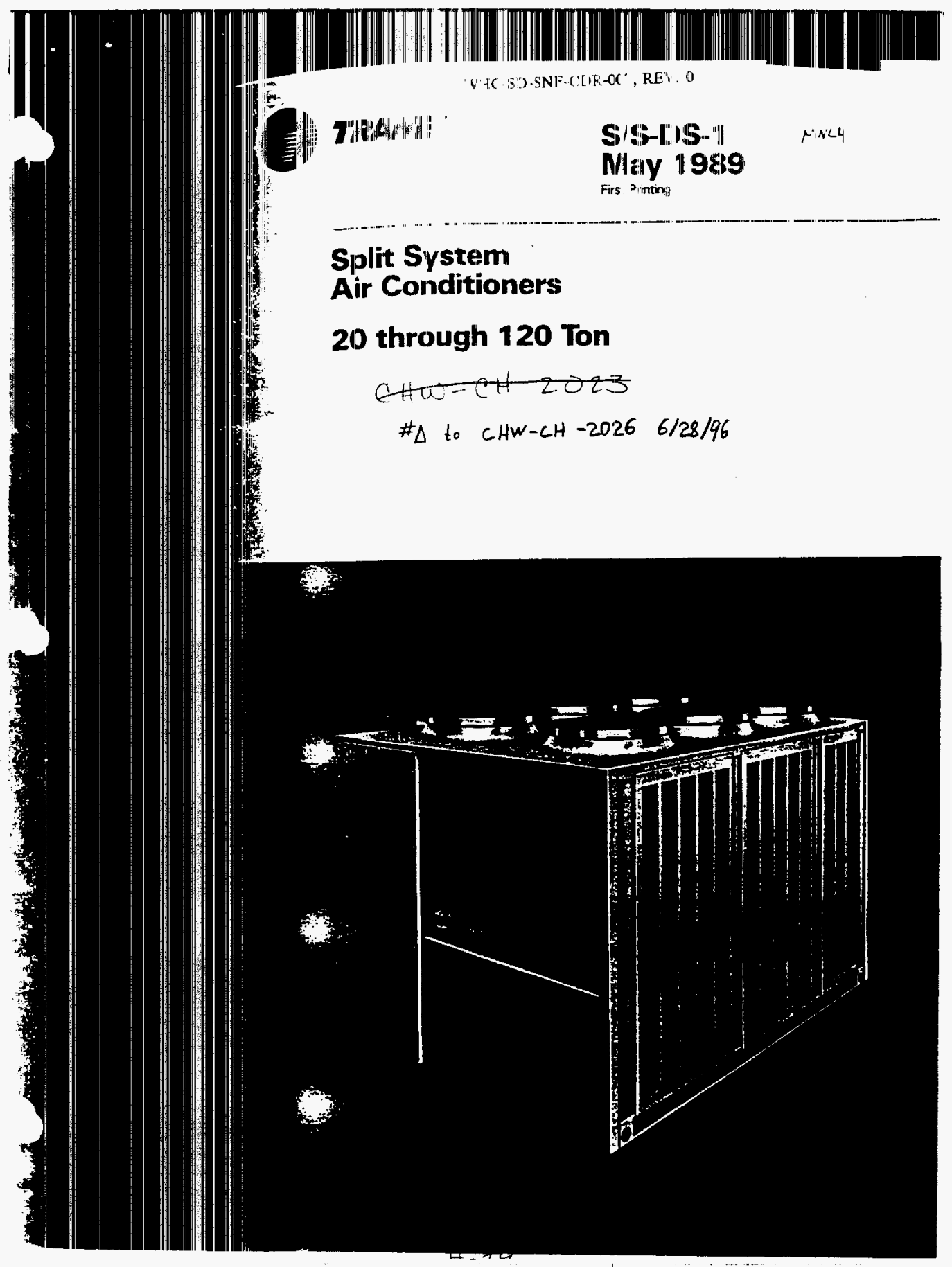




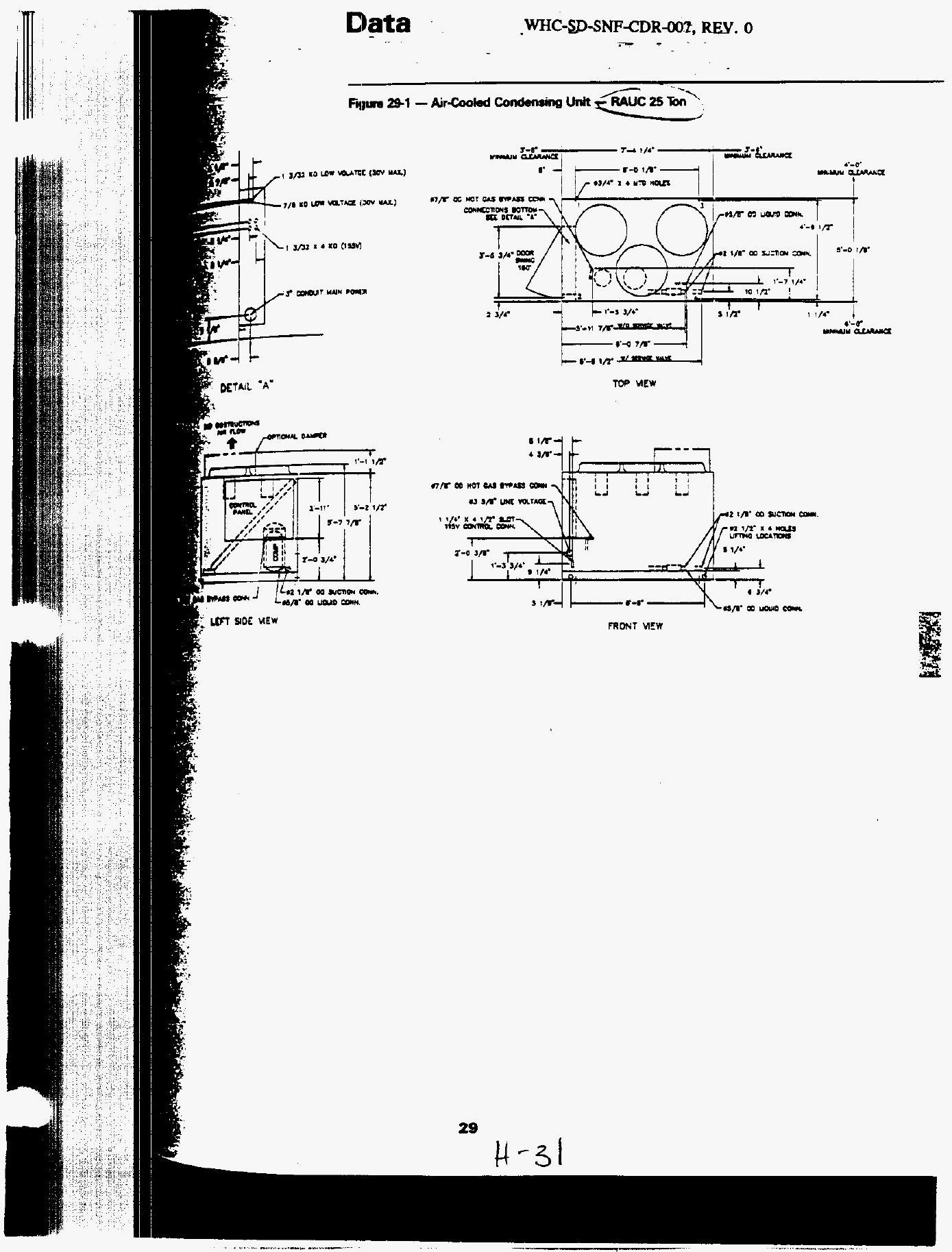




\section{Dimensional Data}

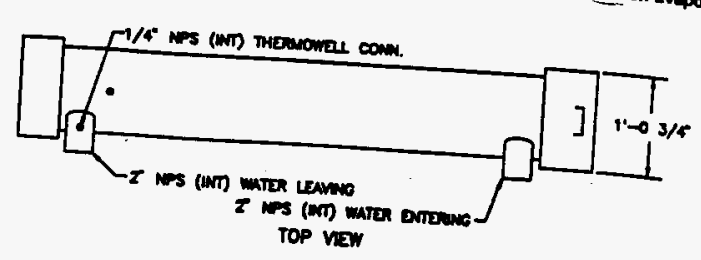

Fioure 36-1 - 20 and 25-Ton Eveporrtor Orilles

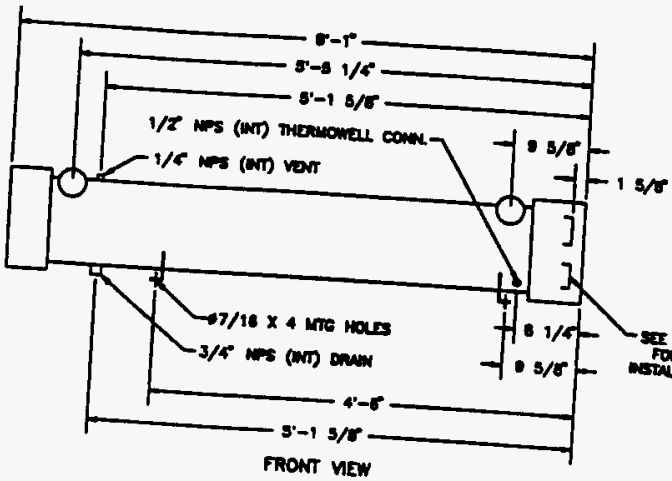

NOTES: Rich soe val

1. Dungsionul rolounce is $\pm 1 / 6$.

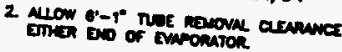

Eveporator Finge Combetion

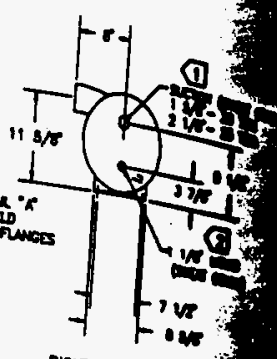


WHC-SD-SNF-CDR-007, Rev. $0^{-}$

Pages $\mathrm{H}-35$ through $\mathrm{H}-42$ have been removed from the document due to copyright and/or trademark restrictions. The information on these pages can be obtained from the project manager. 
PENTAD ASSOCIATES, INC.

WHC-SD-SNF-CDR-007, $\overline{\mathrm{REV}}$. 0- $\quad \because-$

T950 S. Lincoin, Suite 102

Litieter, $0060122-2713$

$308794-4802$

PAx IMNBWT2AT

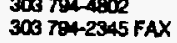

DRE: June 14, 1996

To: Santa Fe Engineering $\quad \mathrm{U} J-\mathrm{H}-2 \mathrm{O}$

8w1t: Dave Munger

In t: 505-983-5608

Frou: Pan standley

8.: Heat Exchanger ct-x=2013

Plate \& Prame/ Haxchanger sizings

Total pages (inalvaing oover): 6

Mases:

Dave, please find the following sizings, per our conversation.

$\mathrm{H}-43$ 


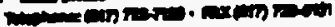

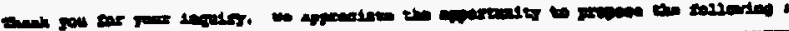

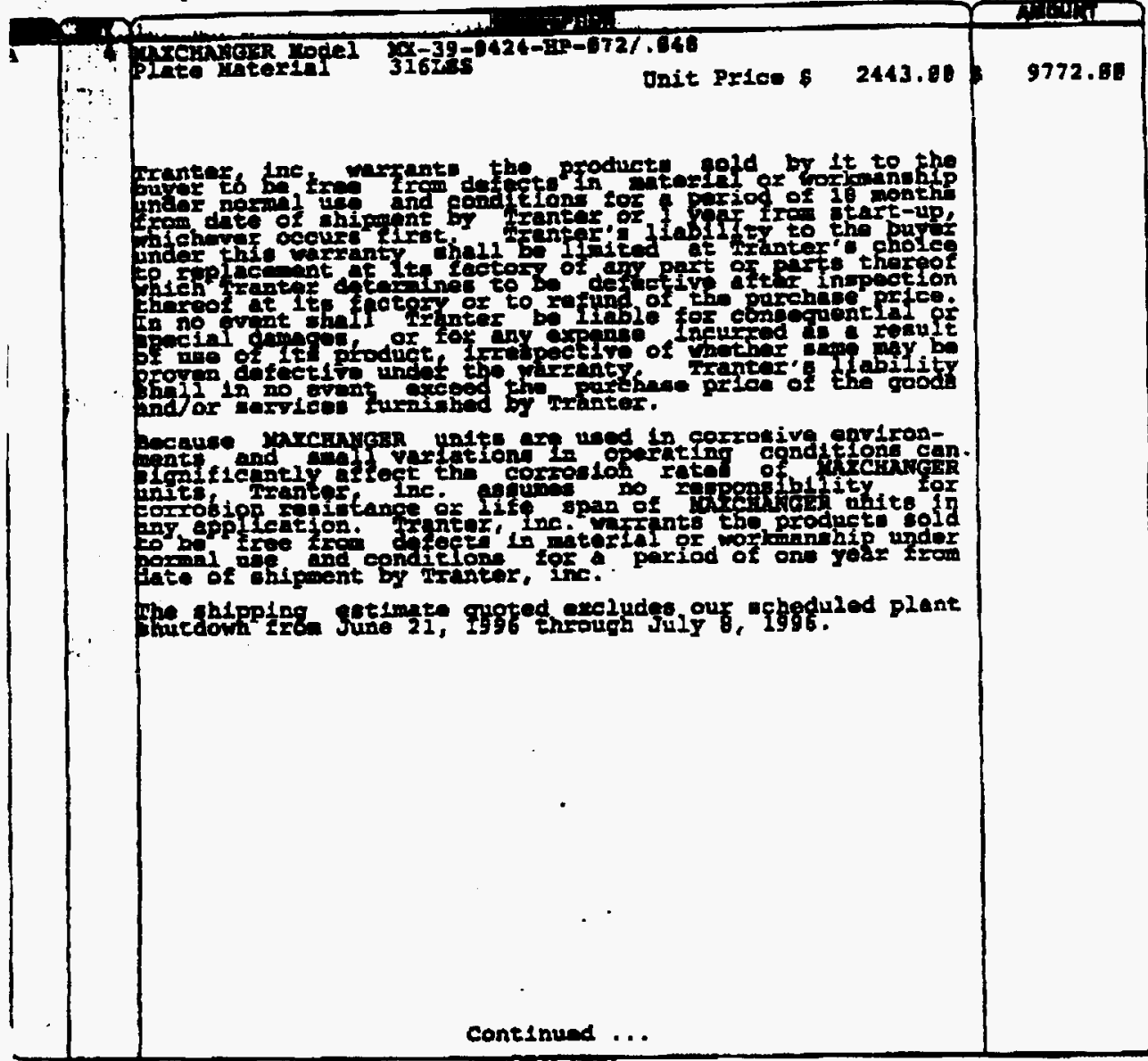


WHC-SD-SNF-CDR-007, REV 0

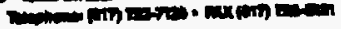

\begin{tabular}{|c|c|c|c|c|}
\hline 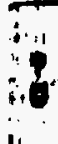 & DAve yonregs & 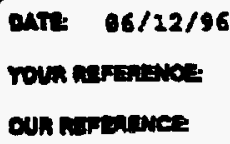 & mat & 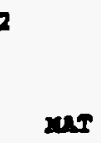 \\
\hline
\end{tabular}

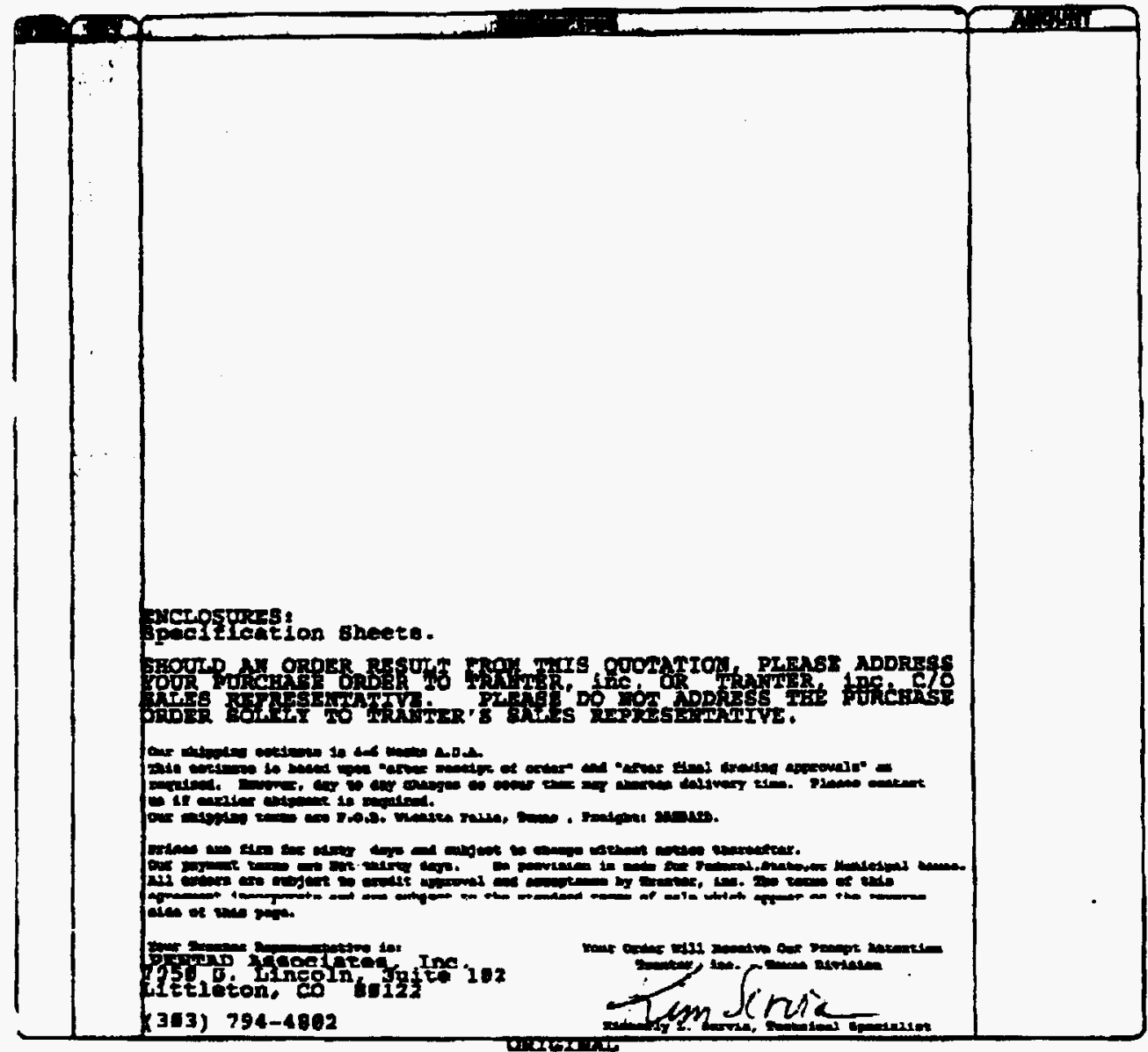

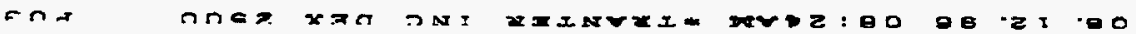

$$
H-45
$$




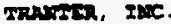

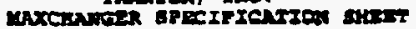

$\begin{array}{ll}\text { Defe } & 06 / 12 / 96 \\ \text { Custoner : DAYz minosh }\end{array}$

Ras rusbar: 3243

Hode1: $2 x-39-0424-19-072,0.048$

Total onder

unse in paraliel is

onter in sexies it/A

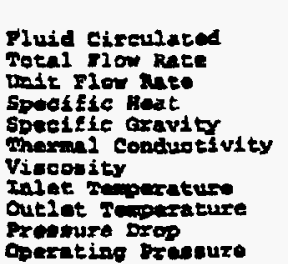

teet Dreharaged

- corterivestros

no. eode ntan? Deteg bonase Tese raeverre

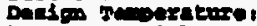
bate wet watent :

\section{Bet side}

inn.

63.0000 ore

15.7600

1. 0000

0.9995

0.3374

1.2335

57.6000

50.0000

0.8661

60.0000

oris

Exp/ (T) Des I)

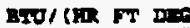

ep ar are $\mathrm{xt}$

DI

Dix $\mathrm{P}$

25I reted

ISTC

239631.6 teference : $13343-18 k$ Roo

Iterg thraber, $\mathrm{A}$
Flow Ddr: Comente Flow

soent Pese Irenefer ares: $256.750 \mathrm{Ft}$

Todosteien : 14s

Rave Arxomeneat: 2 \& 1

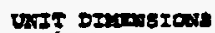

videh: $4.00^{\circ}$

Lamer : $24.00^{\circ}$

natebe: $6.56^{\circ}$

nostele: $1.50^{\circ}, 1.50^{\circ}$

- antarirs

yieces and Pdtting , 316L ss

- Irnars

Your Tranter reprenentetive is:

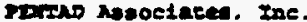

7950 6. Idneola, suite 102

Lierieted, co s0122

(303) $794-4802$

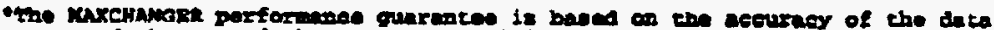

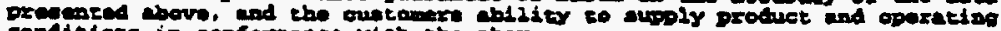

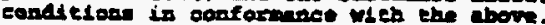



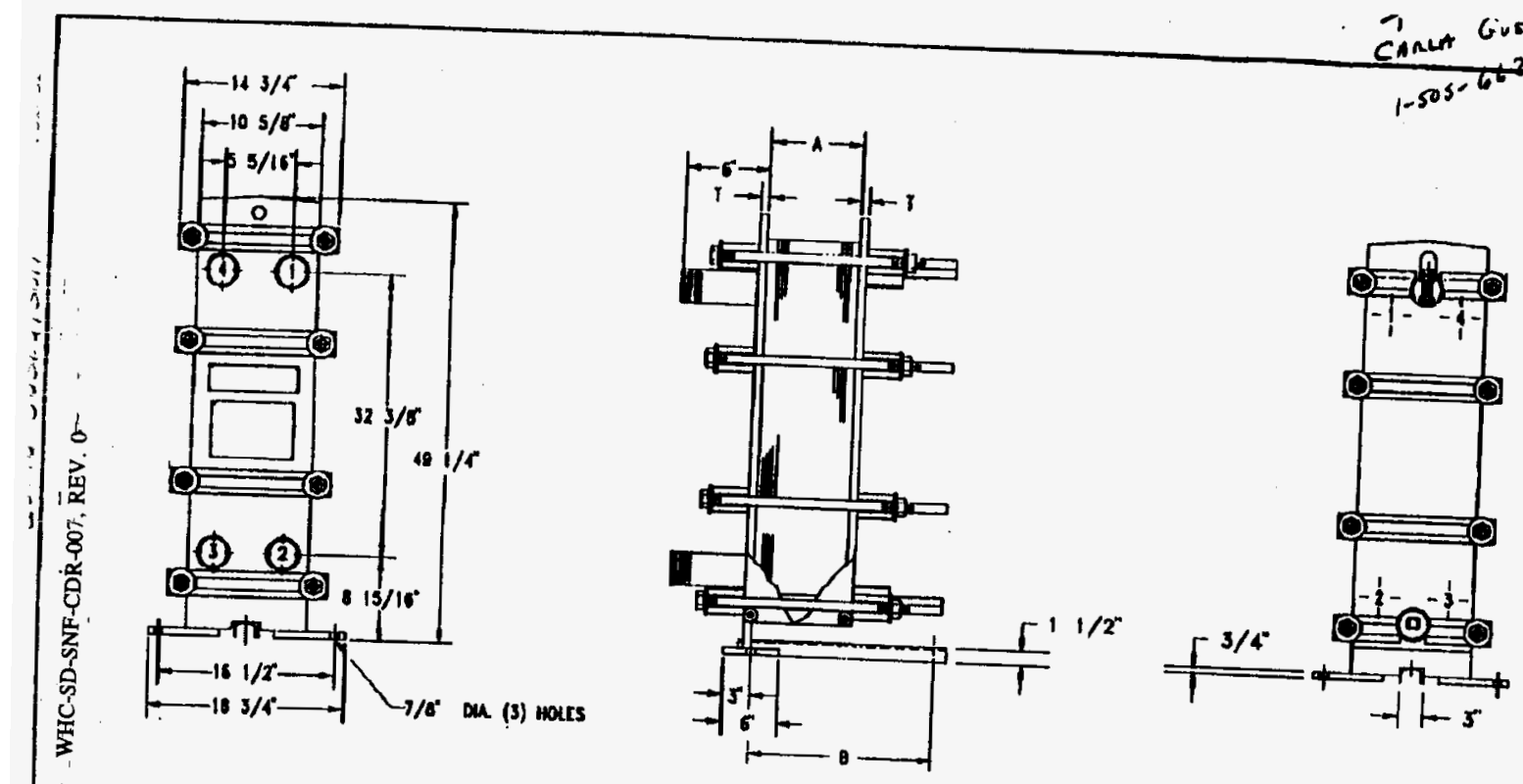

TRANTER, he. - TEXA8 DIVI8ION menur Fon, Toxer U.8A 


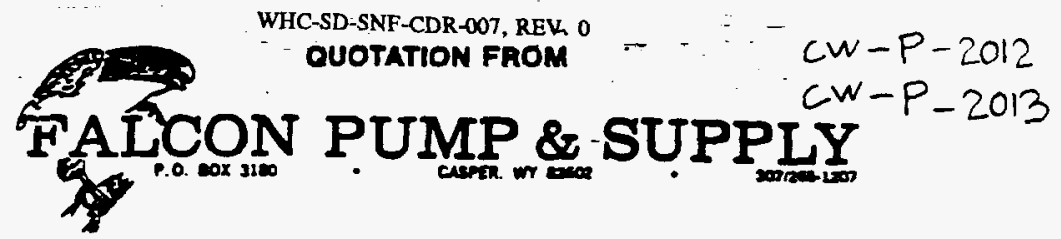

rone Loeatton

WHeAe this

cuote

emenareo
$60 \times 3100$

BOX 1317

1424 WEST CEDAR

BOX 1351
PHONE 307.265-1207

307.225200

30300-2500

$701.572-6012$

To:

Merrick

CASPER, WYOMING 20602
AOCK SPAINGS, WYOMING
OENVER, COLORADO
WL223
WLUSTON, NORTH DAKOTA SEDO1

Date: 2-26-96

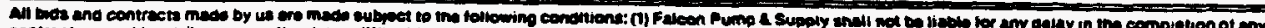

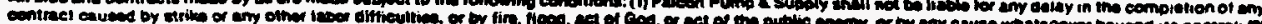

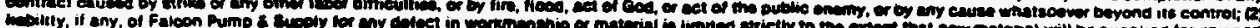

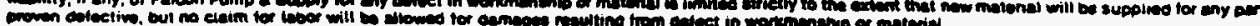

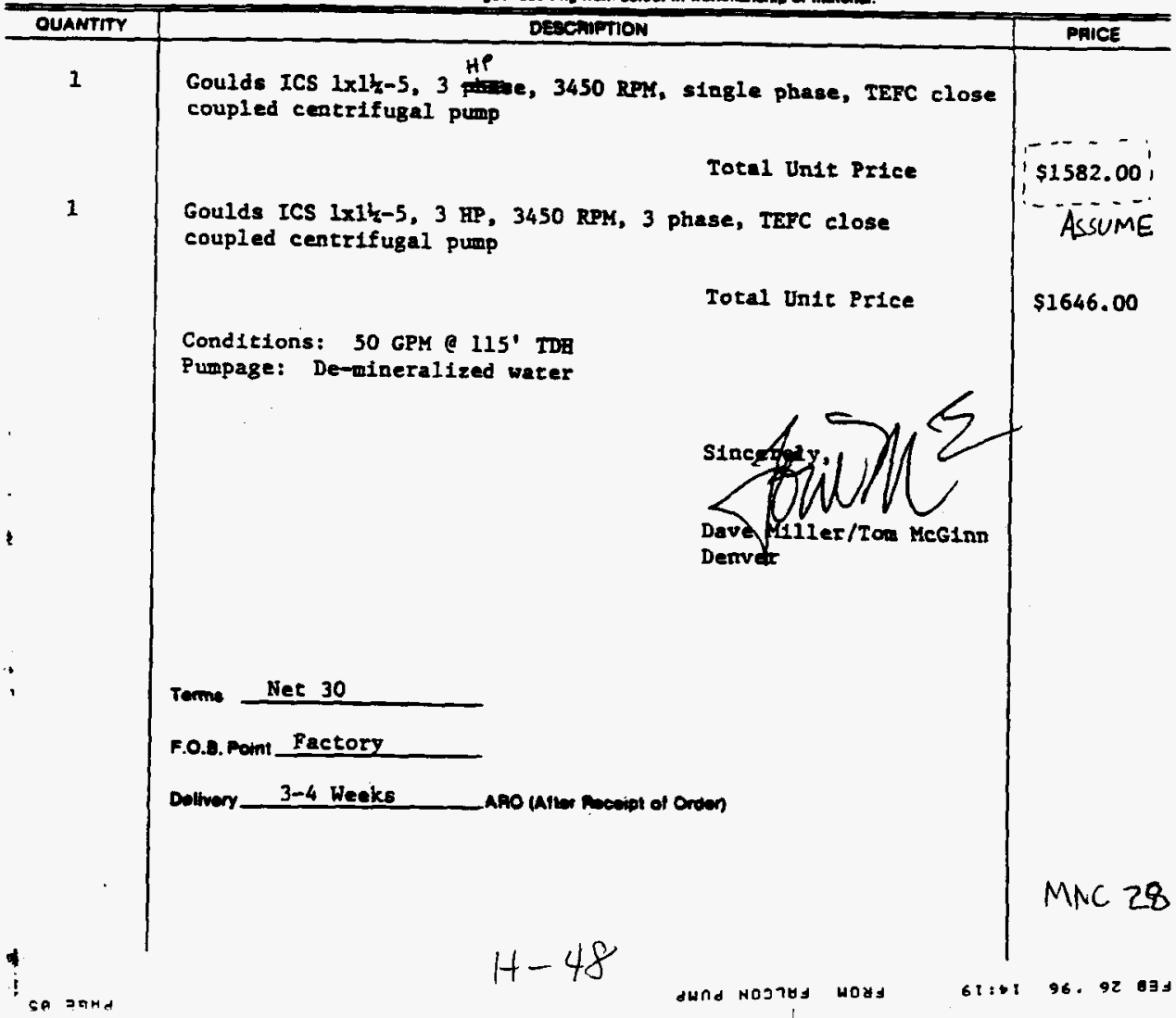




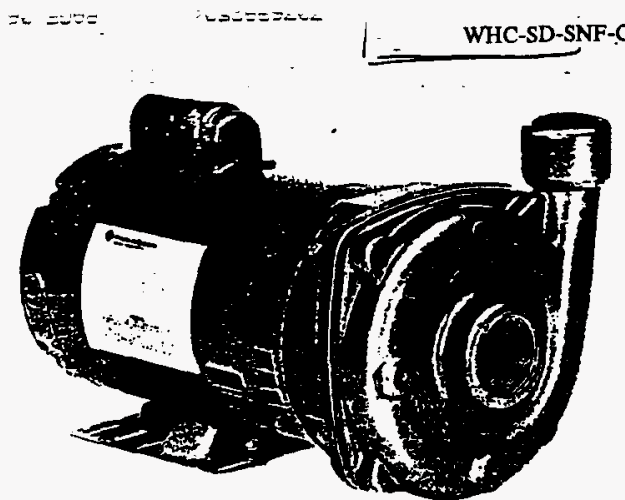

$\because \therefore, \quad=-\quad \Xi-\quad==0$ 


\section{ENGINEERING DATA

\begin{tabular}{|c|c|c|}
\hline \multirow{2}{*}{ Clockwise Rotalion Viewed trom Drive End } & \multirow{2}{*}{$\begin{array}{c}\text { Installation } \\
\text { Drawing No. } \\
119-64\end{array}$} & $\begin{array}{l}\text { Certified for constnuction purpases } \\
\square \text { for Approval } \square \text { for Record }\end{array}$ \\
\hline & & By \\
\hline
\end{tabular}
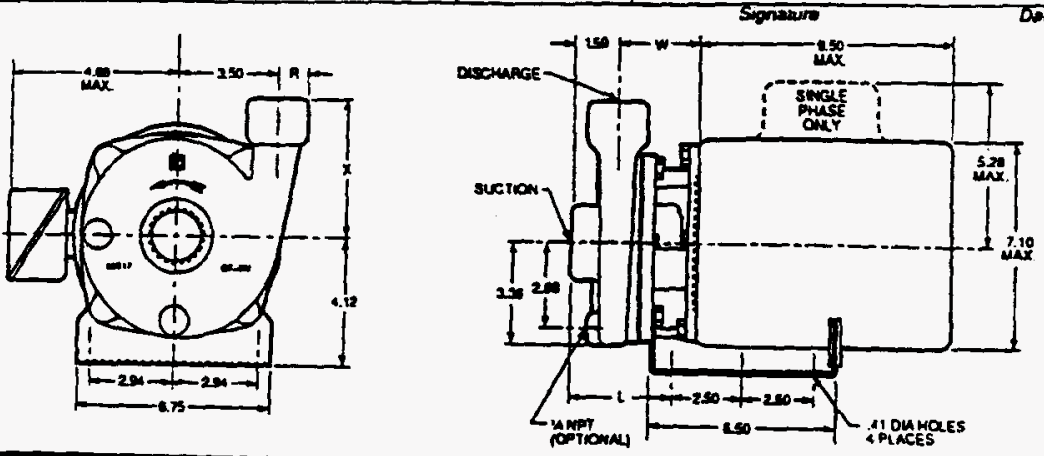

\section{WEIGHTS \& DIMENSIONS - DETERMINED BY PUMP}

\begin{tabular}{|c|c|c|c|c|c|c|c|}
\hline Pump & $\begin{array}{l}\text { Suet. } \\
\text { NPT }\end{array}$ & $\begin{array}{c}\text { Disth. } \\
\text { MPT }\end{array}$ & L & n & $w$ & $x$ & ina. \\
\hline $1 \times 1 \%-5$ & $1 \%$ & 1 & 3.75 & 94 & 3.01 & 1.62 & 17 \\
\hline $11 / 4 \times 1 \times-5$ & Ix & $3 x$ & 3.52 & 1.06 & 2.80 & 4.56 & 17 \\
\hline
\end{tabular}

1. Pumps will be shioped with top vertical discharge as standard, for other orientations, momove casing botts.

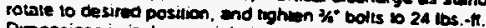

2 Dimensions in inches, waight in pounds.

3 Not to be used lor consinucton purposes uniess ce titied.

4 Molor dimensions may vary with motor manutacturers.

- Weight is liquid end only. For complete pump. add waight of epplienble molor shown below.

\section{AVAILABLE MOTOR WEIGHTS}

\begin{tabular}{|c|c|c|c|c|}
\hline \multirow{3}{*}{ HP } & \multicolumn{4}{|c|}{ Meror weingus in Pounds } \\
\hline & \multicolumn{2}{|c|}{1 Phase } & \multicolumn{2}{|c|}{3 Phase } \\
\hline & oop & JEPC & DBP & TEF \\
\hline 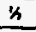 & 19 & 20 & 19 & 20 \\
\hline
\end{tabular}




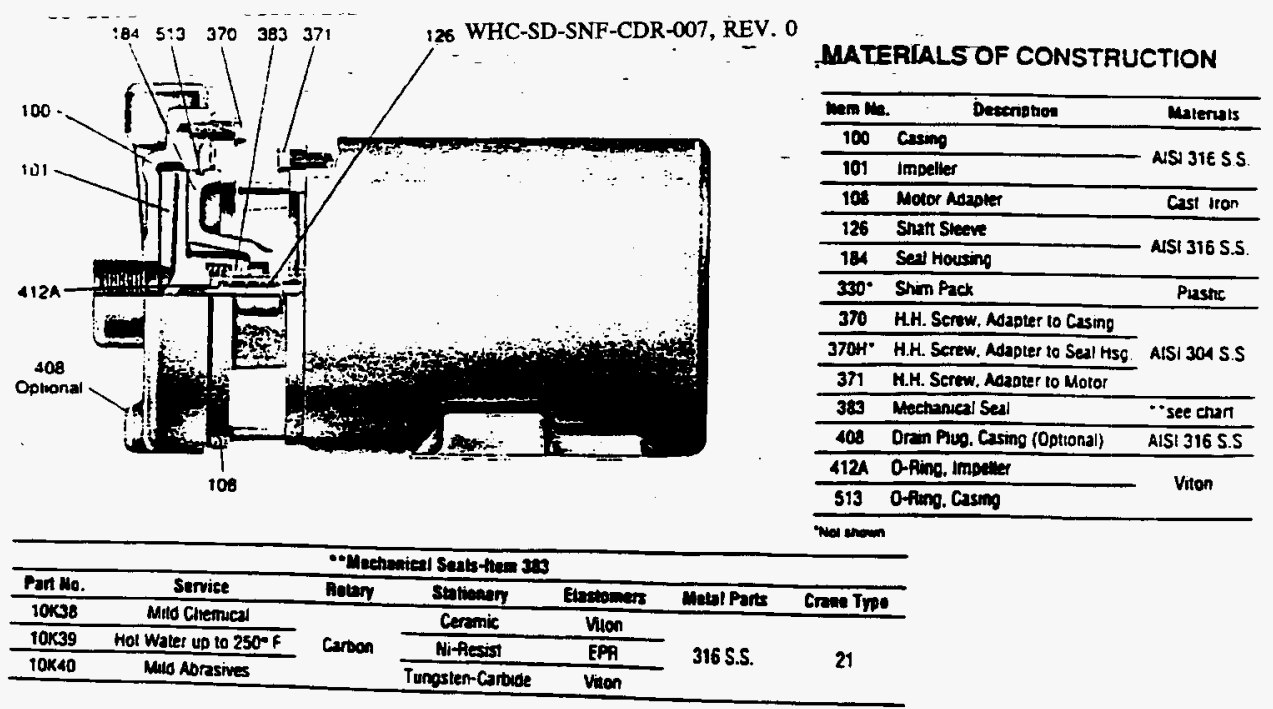

\section{PUMP DIMENSIONS \& WEIGHTS}
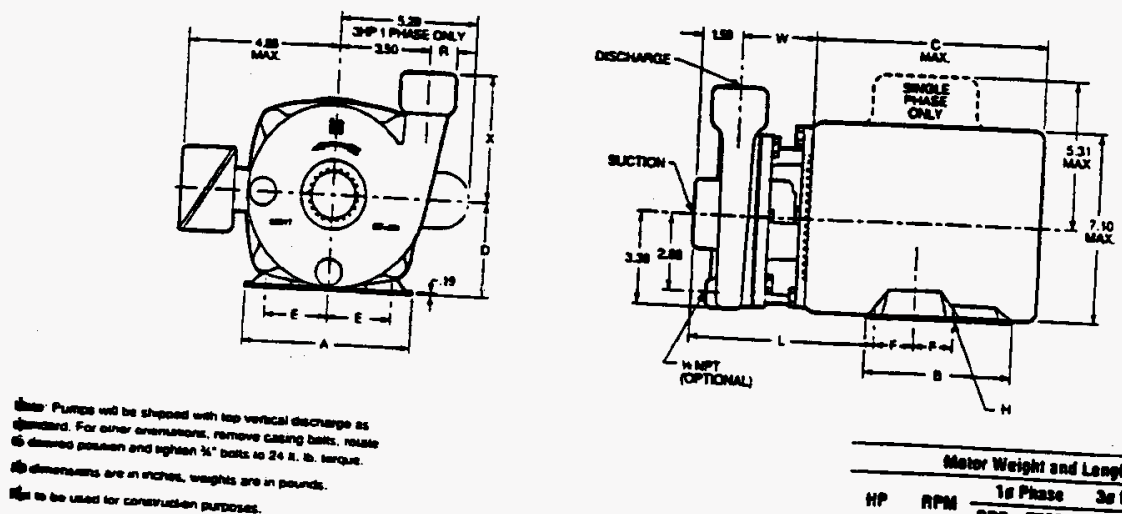

\begin{tabular}{|c|c|c|c|c|c|c|}
\hline \multicolumn{7}{|c|}{ Clover Woight and Leng"h } \\
\hline \multirow{2}{*}{ H } & \multirow{2}{*}{ Arm } & \multicolumn{2}{|c|}{ If Pinse } & \multicolumn{2}{|c|}{$21 \mathrm{Phate}$} & \multirow{2}{*}{ mis. } \\
\hline & & ODP & TEE & 000 & Irfc & \\
\hline \multirow{2}{*}{$1 / h$} & 1750 & 19 & 20 & 19 & 20 & 2.5 \\
\hline & 3500 & 19 & $=$ & 20 & $=$ & 10.34 \\
\hline $2 / 4$ & 3500 & 22 & 24 & $\underline{2}$ & 21 & 1034 \\
\hline 1 & 3500 & 25 & 26 & 24 & 23 & $10 \pi$ \\
\hline $1 \%$ & 3500 & 31 & 35 & 27 & 21 & 11.21 \\
\hline 2 & 3500 & 36 & 39 & 39 & 33 & 11.72 \\
\hline 3 & 3500 & 40 & 45 & 42 & 37 & 13.34 \\
\hline
\end{tabular}

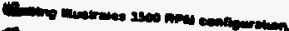

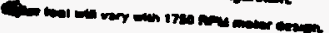

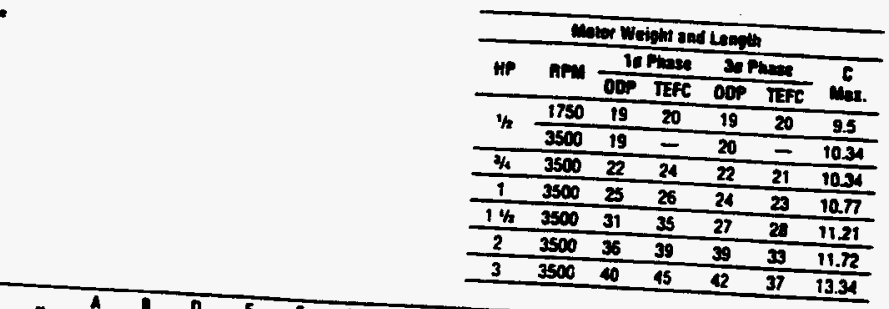

\begin{tabular}{|c|c|c|c|c|c|c|c|c|c|c|c|c|c|c|c|c|c|c|c|c|}
\hline $1 \% / 4-5$ & $1 \%$ & 1 & 94 & 3.01 & 4.62 & & & & $600 \pi$ & & & & & & & $750 \mathrm{nr}$ & & & & $m$ \\
\hline$x>x-5$ & $1 \%$ & $1 \%$ & 1.06 & 2.83 & 456 & $\frac{6.50}{6.50}$ & $\frac{5.80}{5.80}$ & $\frac{350}{3.50}$ & $\frac{2.44}{2.44}$ & $\frac{150}{150}$ & $\frac{7.35}{7.22}$ & $34 x 80$ & 6.75 & 65 & 4.12 & 294 & 25 & 3.75 & 41 & 17 \\
\hline
\end{tabular}

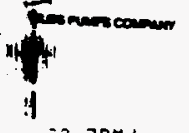




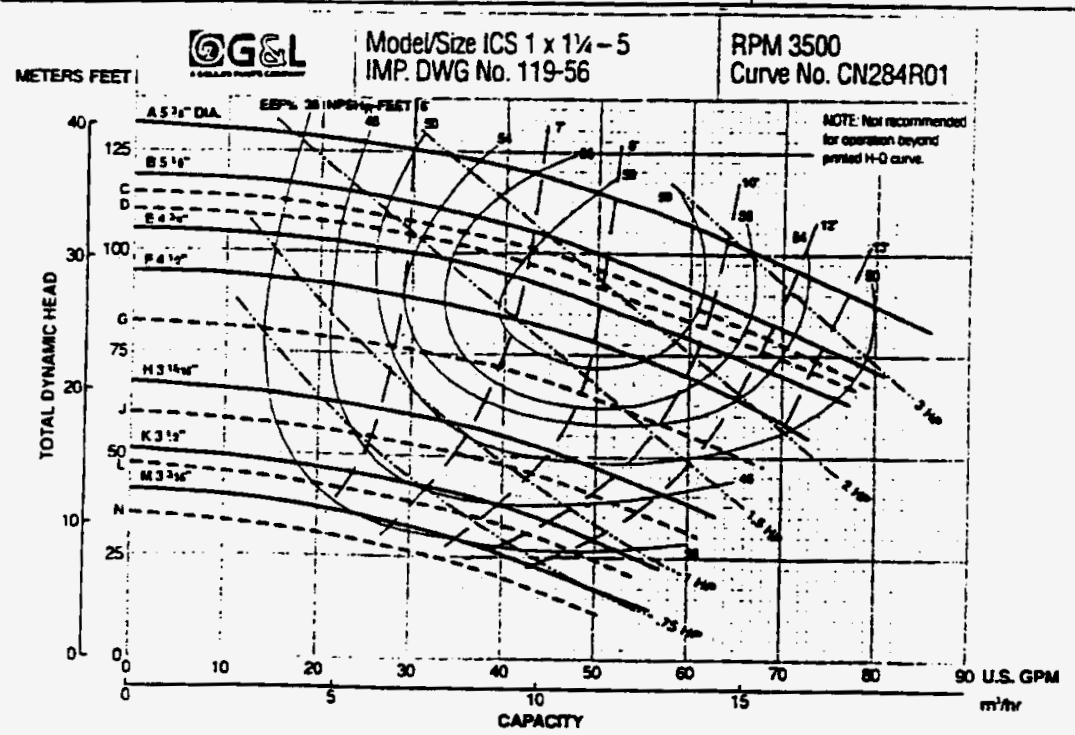

Page:

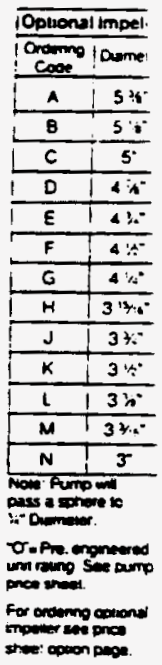

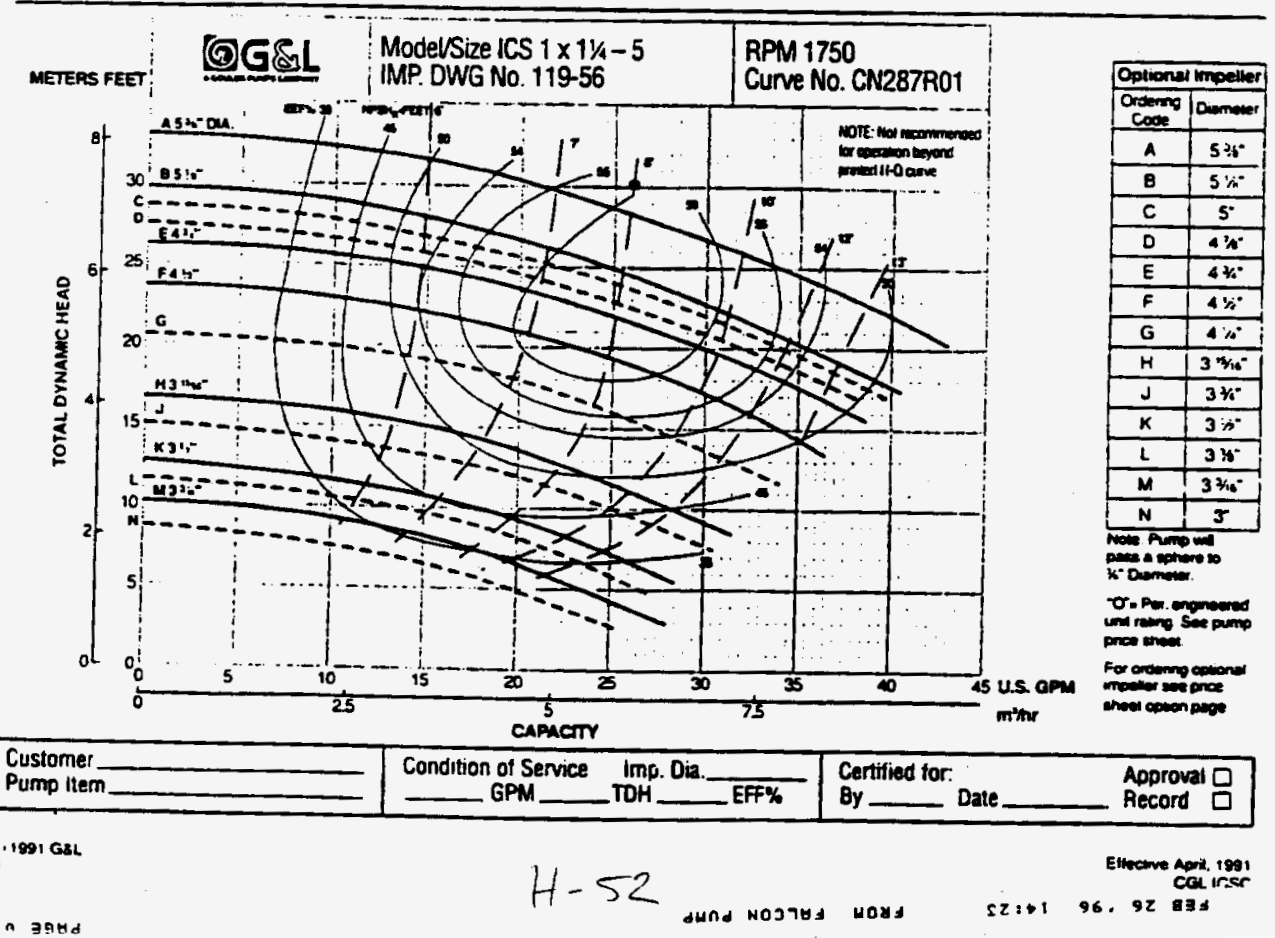


June 14, 1996

Subject: Santa Fe Ingineering Marlo of 96230370

Dear Mr. Hunger,

Marlo is plessed to provide budget prieing per your request:

Dty.

Description

Het

Bach

Extended

Airtight Eousing $w /$ Transitions and

(1) 8w8-12-5611F-1-K-.5-n-W

$\$ 5,073$

$\$ 5,073$

All prtcing 18 F.o.s. High Ridge, wo, freight collect.

Matarials of construction:

Fins: $\quad .010^{n}$ thk. 304 otainless gteel

Tubes: $\quad .049^{n}$ wall, .625" 0.D. 304 statnless steel

Hoaders: $\quad$ schio 304 stainless steel pipe

Connections: $\quad I^{\prime \prime}$ Class 150304 steinless steel pipe flanges

Casing: min. 16 ga. 304 gtainless gteel

Insulation: TBD

Assembly consists of ar airtight coil assembly with upstream and dowatream transitions from square coil face to round pipe flanges. Intire assenbly will be copered with insulation.

Coll connections will be same end.

If you have any questions, please call ne at (314)677-7731 ext.38. Thank you for the opportun1ty.

Best Regards,<smiles>CC1CCCC1</smiles><smiles>CCC(C)C(C)C(C)C(C)C</smiles>

Chris Lohnenn 
WHC-SD-SNF-CDR-007, REV. 0

MARLO (A), P.O. BOX 17t, HYGH RIDAE, Mo. 03048-0171

EPAESENTED BY

Arto $(3+4) 677-6000$

P.O. Box 171

High Ridge, Miseouri 63049-0171

ENTRY DATE $=06-14-1996$

CUSTOMER ID $=$ SANTA FE ENOINERINO

TAE MLMBER =

PROARAM ID = WATER COIL RATING / VERSION 9.8

APPLICATION $=$ COOLINO

\section{DESCRIPTION}

Air flow

(ACFM)

LVE. atr DB $/$ wB (F)

Total capact ty (BTUH)

Serns. capacity (BTUH)

Ent / Lvg water (F)

water flow (OAL/MIN)

water velocity(FT/8EC)

water pres. arop (FT)

Face velocity(FT/MIN)

std. air P.O. (IH.WO)

Altitude

(FEET)
RATING

750
$302.0,0.0$
$194.9,0.0$
99,898
99,898
$50.0 / 79.4$
8.0
2.7
4.2
727.8
0.61
440

COIL DESCRIPTION

$\begin{array}{ll}\text { FIN HEICHT } & =12.375 \\ \text { FINNED LENGTH } & =12.000 \\ \text { FACE AREA } & =1.0 \\ \text { TUBE MATERIAL } & =88304 \\ \text { TUEE THICKNESS } & =.049 \\ \text { FIN MATERIAL } & =83 \\ \text { FIN THICKNESS }=.01 \\ \text { TUFGLATORS }=N \\ \text { INSIDE FOULING }=0 \\ \text { OUTSIDE FOULINO }=0 \\ \text { FLUID TYPE = WATER } \\ \text { GLY COWCENTRATION } 0 \times\end{array}$

IARLO MOOEL: : BW 8- 12.0-5611F- $1.0-H / V-0.50-R / L$ B/W

COIL RATED IN ACCORDAMCE WITH CURRENT EDITION OF ARI STANDARD 410.

- oenotes Variable(s) outside the certification range. 
WHC-SD-SN

MARLO (R), P.O. BOX 171, HIOH RIDQE, MO. O3049-0171

TEPRESENTED BY

AarTo (314) $677-6000$

P.O. Box 179

High Ridge, Missourt 69049-0171

ENTAY DATE $=08-14-1996$

CUSTOMER ID = SANTA FE ENOIHERINO

TAQ NUMEER $=$

PROGRAM ID = WATER COIL RATINO / VERSION 9.8

APPLICATION $=$ COOLINO

\section{DEBCRIPTICN}

Air frow

Ent. air DB / we

Lvg. atr DB / we

Total capacity (BTI

sens. cepact ty (BTUH)

Ent / Lva water (F)

water flow (GAL/MIN)

wazor velocity(FT/SEC)

wator pres, drop (FT)

Face velocity(FT/MIN)

9td. atr P.D. (IN.WE)

Aiti tude (FEET)

RATING

572.07000
$182.0 / 0.0$
101.342
$104.3+2$
$50.0 / 90.5$
8.0
2.7
4.2
727.3
0.97
440

COIL DESGRIPTION

\begin{tabular}{|c|c|}
\hline 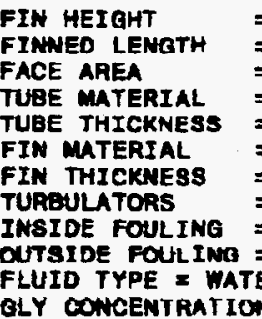 & $\begin{array}{l}=12.375 \\
=12.000 \\
=1.0 \\
=\text { s8904 } \\
=.049 \\
=\mathbf{3 5} \\
=.01 \\
=N \\
=0 \\
=0\end{array}$ \\
\hline
\end{tabular}

AARLO MODELE: BW B- 12.0-5611F- $1.0-H / V-0.50-R / L$ B/W

COIL RATED IN ACCOPDANCE WITH CUARENT EDITION OF ARI 8TANDARD 410.

- denOtes VARIABLE(S) OUTSIDE THE certification RaNGe. 
The TITAN III is a dexterous and powerful telerobotic manipulator system that is available in a variety of configurations for use in extreme environments.

\section{TITAN III S}

Ideally suited for applications using remotely operated vehicles and manned submersibles at depths to 21,000 fsw $(6,500 \mathrm{~m})$.

\section{TITAN III T}

Designed for nuclear inspection and maintenance, environmental restoration, sorting and characterization, disassembly and dismantlement, and explosive ordnance disposal.

\section{$\checkmark$ TITAN III VME}

For customers developing applications in the VME environment, this system provides a shared memory interface to the user's VME control system.

\section{GECALSTHOM}

SCHILING ROBOTIC SYSTEMS

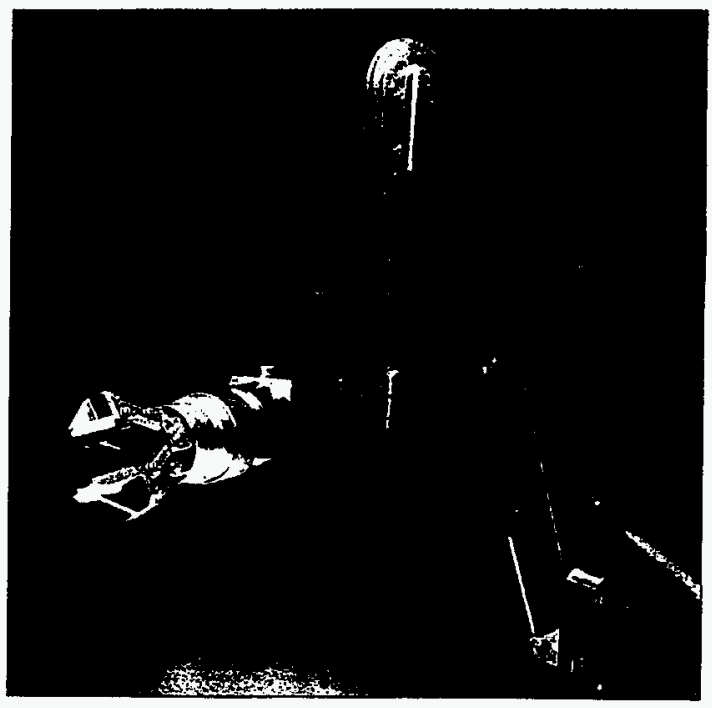

To meet the critical demands of remote manipulation in hostile environments, Schilling Robotic Systems, Inc. (SRS) offers the TITAN III, a dexterous, servo-hydraulic, telerobotic manipulator system with six degrees of freedom. The TITAN, now in its third generation, has a proven track record of reliability and unequaled performance in some of the most demanding environments in the worid. SRS's six-degree-of-freedom miniature replica master arm ensures comfortable and intuitive manipulator operation.

Constructed primarily of 6-4 titanium, the TITAN III is capable of lifting $250 \mathrm{lb}(113.4 \mathrm{~kg})$ at ils full reach of 75.4 inches $(191.5 \mathrm{~cm})$.

The variety of possible remote work tasks requires the manipulator to be highly reliable, field maintainable, and adaptable. SRS's experience in cesigning and manufacturing telerobotic manipulators for extreme environments has resulted in a range of standard configurations and options to meet a variety of requirements.

SRS offers the Timn III $S$ for manipulative tasks that require high dexterity and a large payload capacity, and that must be performed in hostile subsea environments of up to $21,000 \mathrm{fsw}(6,500 \mathrm{~m})$. The TITAN III $S$ is ideally suited for use on remotely operated vehicles (ROVs) and mamned submersibles. It comes equipped with everything necessary for installation on hydraulically powered vehicles, inciuding a slave arn. compact master controller with 


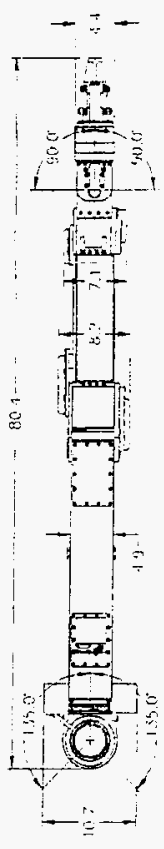

Top View

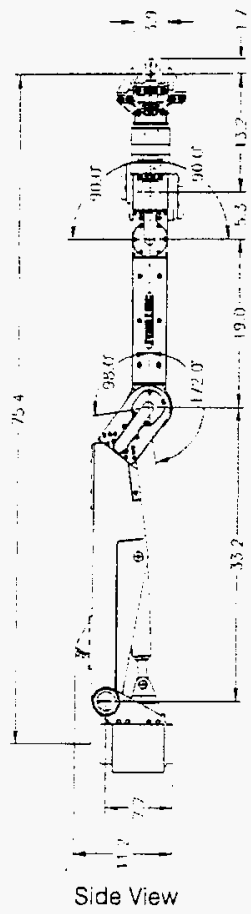

Side View
TITAN III slave arm dimensions (inches)

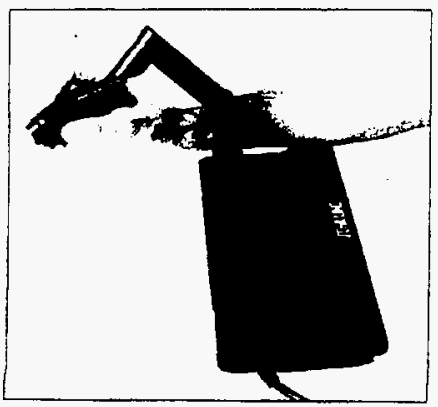

TITAN III compact master controller with replica master arm replica master arm, control electronics, slave controller with submersible enclosure, electrical interface cables, and hydraulic compensator. Available options include software for robotic control and Cartesian control.

The TITAN III T offers the same performance and dexterity as the TITAN III $S$, but is designed for land-based applications or shallow water applications up to a depth of 100 feet $(30.5 \mathrm{~m})$. Typical applications include commercial nuclear inspection and maintenance, environmental restoration, hazardous material sorting and characterization, disassembly and dismantlement, and explosive ordnance disposal. With the addition of a user-supplied hydraulic power unit, the TITAN III T is shipped ready for operation, with a slave arm, compact master controller with replica master arm, control electronics, slave controller with enclosure, and electrical interface cables. Available options include radiation hardening (for applications exceeding $1 \times 10^{2} \mathrm{rad}$ gamma cumulative dosage), tool interchange, Cartesian control, and robotic control.

For customers developing applications in the VME environment, SRS offers the TITAN III VME, which provides a VME interface to the user's host control system. This configuration provides a shared memory interface to the user's VME control environment. The system includes a slave arm, electrical interface cables, slave arm data acquisition electronics, and a VME interface card. Options include a force/torque sensor, tool interchange, and Magellar (a 3-D, graphics-based, integrated telerobotic controller that simplifies remote operation in both structured and unstructured environments).

\section{GENERAL DESCRIPTION}

Mode of operation ......................... Closed-loop position control input device ............... Six-degree-of-freedom replica master arm Degrees of freedom ......................................... Six plus grip

\section{SLAVE ARM SPECIFICATIONS}

Maximum reach ..................................... $75.4 \mathrm{in.}(191.5 \mathrm{~cm})$ Weight in air ........................................ $223 \mathrm{lb}(101.2 \mathrm{~kg})$ Weight in sea water ................................... $168 \mathrm{lb}(76.2 \mathrm{~kg})$ Maximum lift capacity at full reach ................. $250 \mathrm{lb}(113.4 \mathrm{~kg})$ Maximum grip opening (standard gripper) .......... 3.9 in. (10 cm) Maximum grip force Maximum wrist torque ......................... 1.200 in-lb (135.5 N-m) Wrist rotate, slaved .......................................... 360 degrees Wris: rotate, continuous ......................................... $0-35 \mathrm{rpm}$

\section{MASTER CONTROULER DIMENSIONS}

Length ................................................... $19.1 \mathrm{in.}(48.5 \mathrm{~cm})$

Width...... $6.0 \mathrm{in} .(15.25 \mathrm{~cm})$

Height 2.7 in $(6.9 \mathrm{~cm})$

Weight $9.5 \mathrm{lb}(4.3 \mathrm{~kg})$ 
SLAVE ARM FUNCTIONS

\begin{tabular}{llcl} 
Function & $\begin{array}{c}\text { Actuator } \\
\text { Type }\end{array}$ & $\begin{array}{c}\text { Torque or force } \\
\text { 3000 psi }\end{array}$ & $\begin{array}{c}\text { Mechanical } \\
\text { Range }\end{array}$ \\
\hline Azimuth & Rotary & $18,000 \mathrm{in}-1 \mathrm{~b}(2,032 \mathrm{~N}-\mathrm{m})$ & $270^{\circ}$ \\
\hline Shoulder pitch & Lineer & $21,400 \mathrm{in}-1 \mathrm{~b}(2,416 \mathrm{~N}-\mathrm{m})$ & $120^{\circ}$ \\
\hline Elbow pitch & Rotary & $9,200 \mathrm{in}-\mathrm{lb}(1,038 \mathrm{~N}-\mathrm{m})$ & $270^{\circ}$ \\
\hline Wrist pitch & Rotary & $4,000 \mathrm{in}-\mathrm{lb}(451.6 \mathrm{~N}-\mathrm{m})$ & $180^{\circ}$ \\
\hline Wrist yaw & Rotary & $4,000 \mathrm{in}-\mathrm{lb}(451.6 \mathrm{~N}-\mathrm{m})$ & $180^{\circ}$ \\
\hline Wrist rotate & Gerotor & $1,200 \mathrm{in}-\mathrm{lb}(135.5 \mathrm{~N}-\mathrm{m})$ & $360^{\circ}$ \\
\hline Jaw & Linear & $1,000 \mathrm{bb}(4,448 \mathrm{~N})$ & $\begin{array}{r}3.9 \mathrm{in} . \\
(10 \mathrm{~cm})\end{array}$ \\
\hline
\end{tabular}

\section{HYDRAUUC REQUIREMENTS}

Fluid type: Petroleum-based or water/glycol-based hydraulic fluid (non-corrosive)

Viscosity

$10-200 \mathrm{cSt}$

Flow ....................................... 1.5-5.0 gpm (5.7-19.0 $1 / \mathrm{min})$

Pressure .................................................... 3,000 psi (200 bar)

\section{ELECTRICAL AND TELEMETRY REQUIREMENTS}

100-240 VAC $50 / 60 \mathrm{~Hz}$ standard. Consult SRS for other power options.

Standard with RS-422/485 half-duplex, optional RS-422/485 fullduplex or RS-232 communication protocol.

Consult SRS for other telemetry options.

\section{OPTIONS}

Robotic software
$\begin{aligned} & \text { Than ill } \\ & \text { Radiation hardening }\end{aligned}$

- The TITAN III VME ises Magellan robotic soliware.

"The TITAN !H S standard desth ratirg s up :0 $5.000 \mathrm{fsw}(1.524 \mathrm{~m}$ )

Magellan is a 3-D, graphics-based, integrated telerobotic controller. Magellan simplifies complex tasks by providing computer-aided teleoperation through modeling, motion planning, and manipulator control. With features such as simple and efficient trajectory specification, real-time graphical display, and collision-free path planning, Magellan provides a cost-effective solution to complex remote manipulation problems.

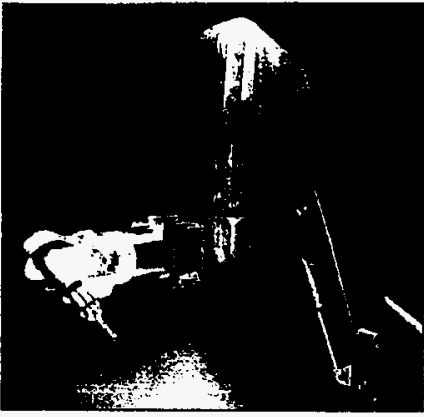

TITAN III T shown with interchangeable rotary power tool, 90" drill head

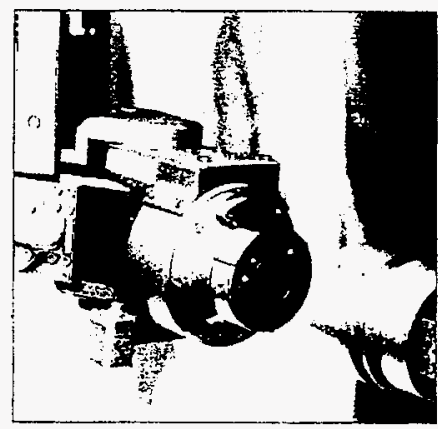

TITAN III tool interchange coupling

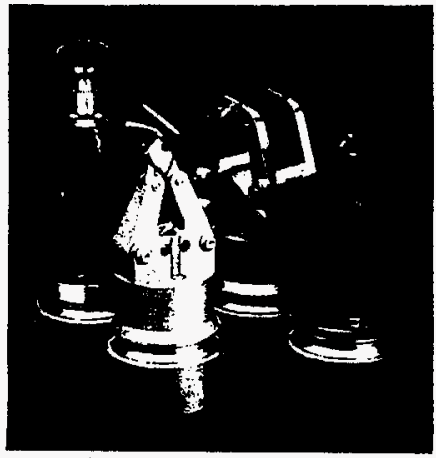

Examples of interchangeoble electric and hydraulic toois 


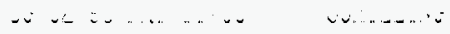

WHC-SD-SNF-CDR-007, REV, 0

\section{Titan III}

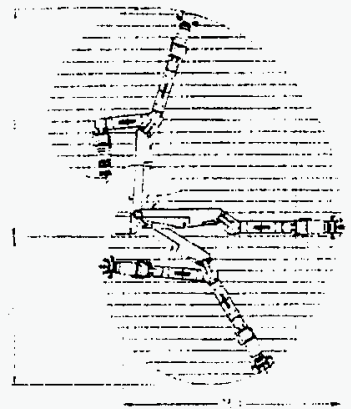

Elevation View ( 1 square $=4$ inches)

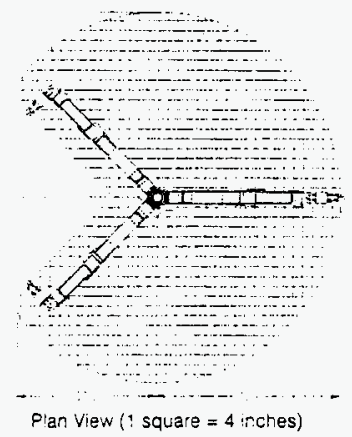

TITAN III range of motion (inches)

\section{TITAN III FEATURES}

- Corrosion-resistant titanium construction

- Miniature replica master arm

- Wide range of motion

- High payload-to-weight ratio

- Rugged and reliable

\section{GEC ALSTHOM}

SCHILLING ROBOTIC SYSTEMS 1632 Da Vinci Court

Davis, California 95616. USA Phone: (916) 753-6718

Fax: $(916) 753-8092$

\section{ACCESSORIES}

- Hydraliia power unit (TiTAN III TNME only)

- interchangeable tocls (consult SRS for specifications)

- Hydrauíi nose kits

- Spares kits

- Mounting pedestal

\section{FOR MORE INFORMATION}

Contact SRS tor configuration detail sheets and technical application guides for the TITAN III S, T, and VME.

\section{Descriftions and specifications are subject to change withotit notice Contaw Schilling Robotic Systems. Inc. for the latest information. C 1995 Schilling Robotic Systems, Inc.}

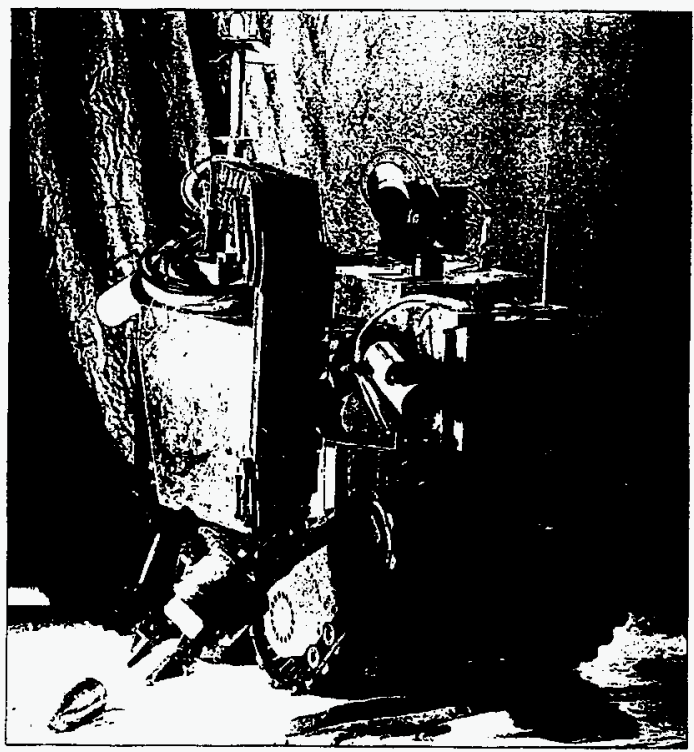

Developed by Battelle, the Remote Ordnance Neutralization System (RONS) is a stair-elimbing, off-rood vehicle with robotic control, fiber optic communication system, remote operator control system, and a TITAN telerobotic manipulator systern 
D

WHC-SD-SNF-CDR:-007, REV. 0

Model ' $F$ ' GTAW Weld Head.

P/N 282-1100-103

\section{Features}

- Precision electromechanical servos for Travel, AVC, Wire Feed and Oscillator.

- Patented, zero backlash traction drive travel carriage. No expensive gear drive travel mechanisms.

- Fast mounting to all Dimetrics circular and flat track without latch rolter change-out.

- Weld head mounted jog and sequence controls.

- Optional front-end/wire manipulator configurations for most any application.

- 'A' single wire, one direction

- 'C' dual wire, bi-directional

- ' $D$ ' deep groove, bi-directional

- Optional remote video viewing front-ends with mechanized torch tilt and wire manipulation.

\section{Specifications}

Travel Speed:

Jog Speed:

Oscillation Trave!:

Afc Voltage Control Stroke:

Wire Size:

Wire Spool Weight/Size:

Wire Feed Rate:

Wire Positioning:

Welding Torch Rating:

Axial Clearance:

Radial Clearance:

Head Weight:

Cable Length/Weight:

- 1.00 Cross.Seam with 1.00* Oscillation Stroke plus $1.00^{\circ}$ Stide Travel - 2.25" Vertical Stide Adjurtment

$20 \mathrm{lbs}$. (less cable)

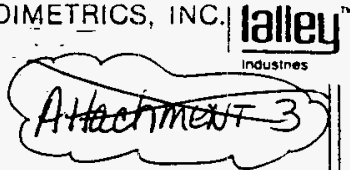

$0.201 \mathrm{Pm}$ Process Eudlosu 40 IPM Deld Head

$2.00^{\circ}$

$1.50^{\circ} \cdots$

$.030^{\prime \prime}$ to $.063^{\prime \prime}$ diameter

2 lbs., 4" diameter std.

0.99 IPM

Up/Down +1.3/8* In/Out $+1.15^{\circ}$

300 AMPS, $100 \%$ duty cycle $17.75^{\circ}$ (minimum)

4.75* (minimum)

35', $40 \mathrm{lbs}$.

Model ' $F$ ' Weld Head pictured with ' $C$ ' Front-End

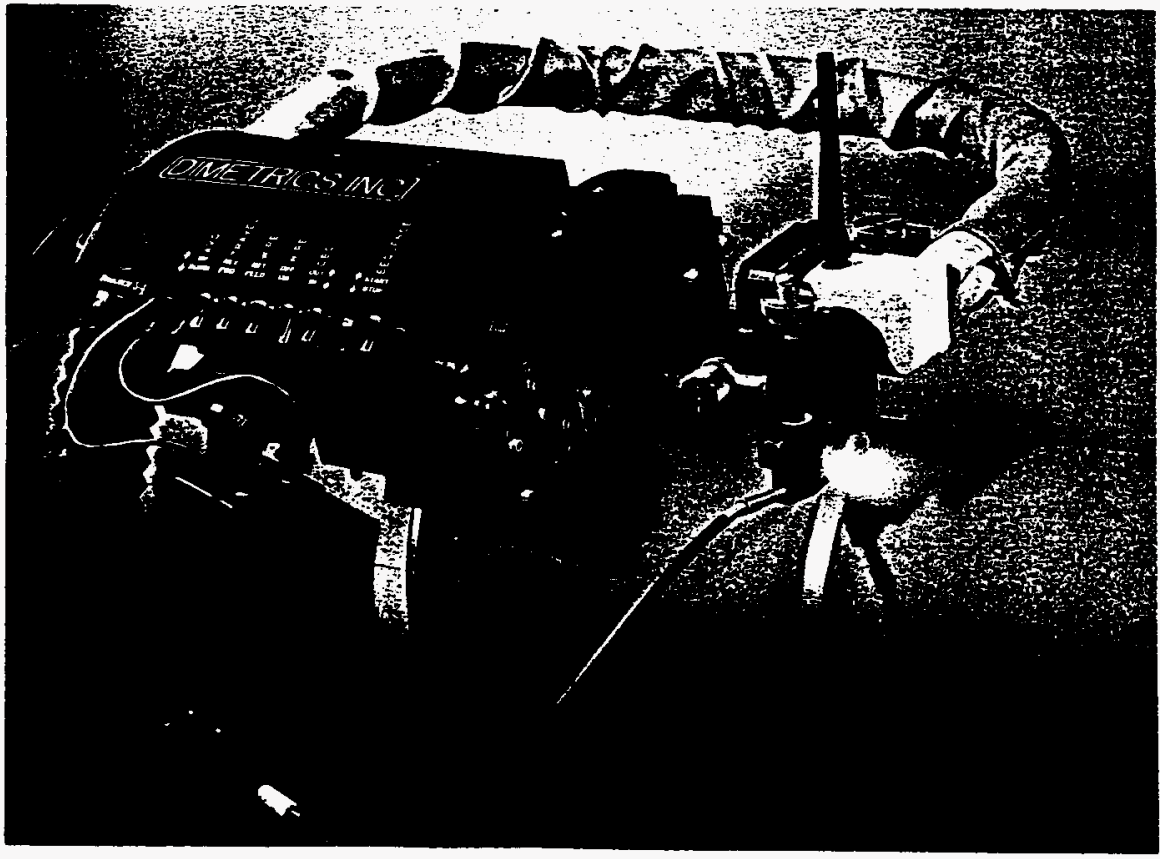

August 1993

$$
H-59
$$


WHC-SD-SNF-CDR-007, REV. 0

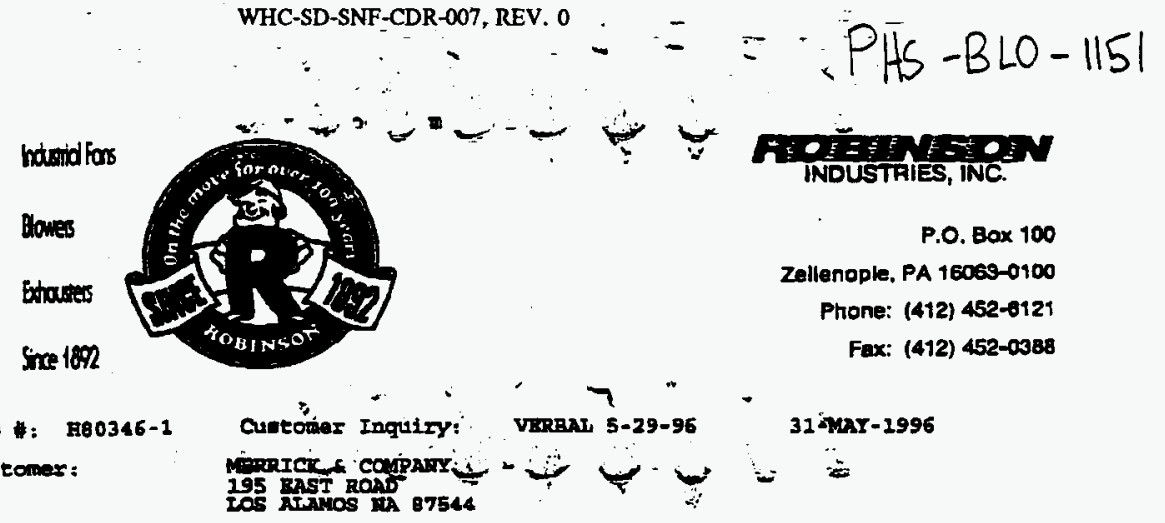

Attention: CARTA GUSTAPASON (FAX:505-662-3851) (PFETE:505-662-0606 BXT.3143)

Fon Appleation: $\quad$ I. D. FAr

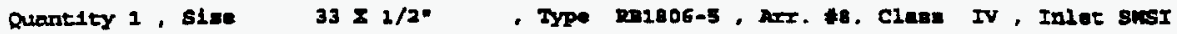

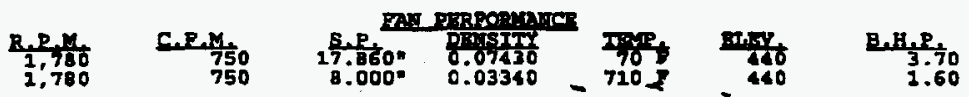

Note: Bvase required to metet pertormance.

irice complete wth checked Acegagarieg

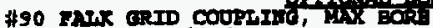

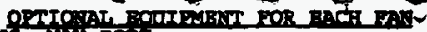

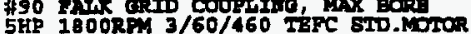

$9498 \mathrm{Fa}$. Fan

$\$ 232$ Ba. Fan

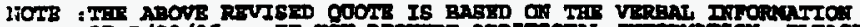

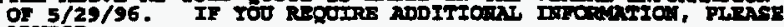
Aovis?.

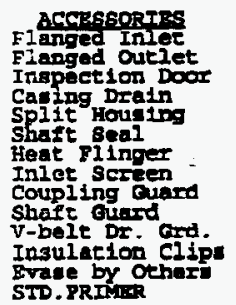

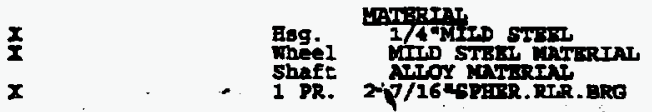

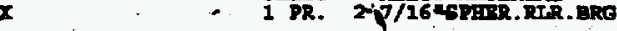

$\therefore \quad \therefore$ melght $\quad \therefore 1,462$ 1be.

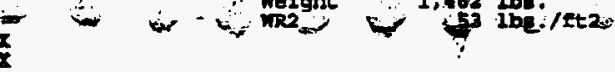

STD. PRIMLA

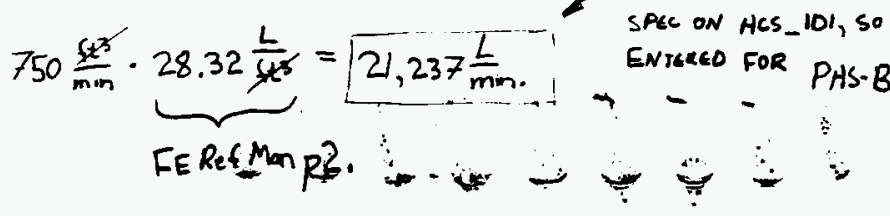

- Cusesr to 21,000 LPM

$\mathbf{x}$

BLO-115!

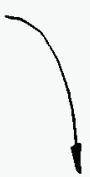

MNC 10

$H-60$

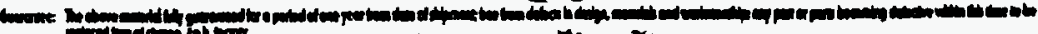

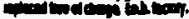


WHC-SD-SNF-CDR-007, Rev. 0
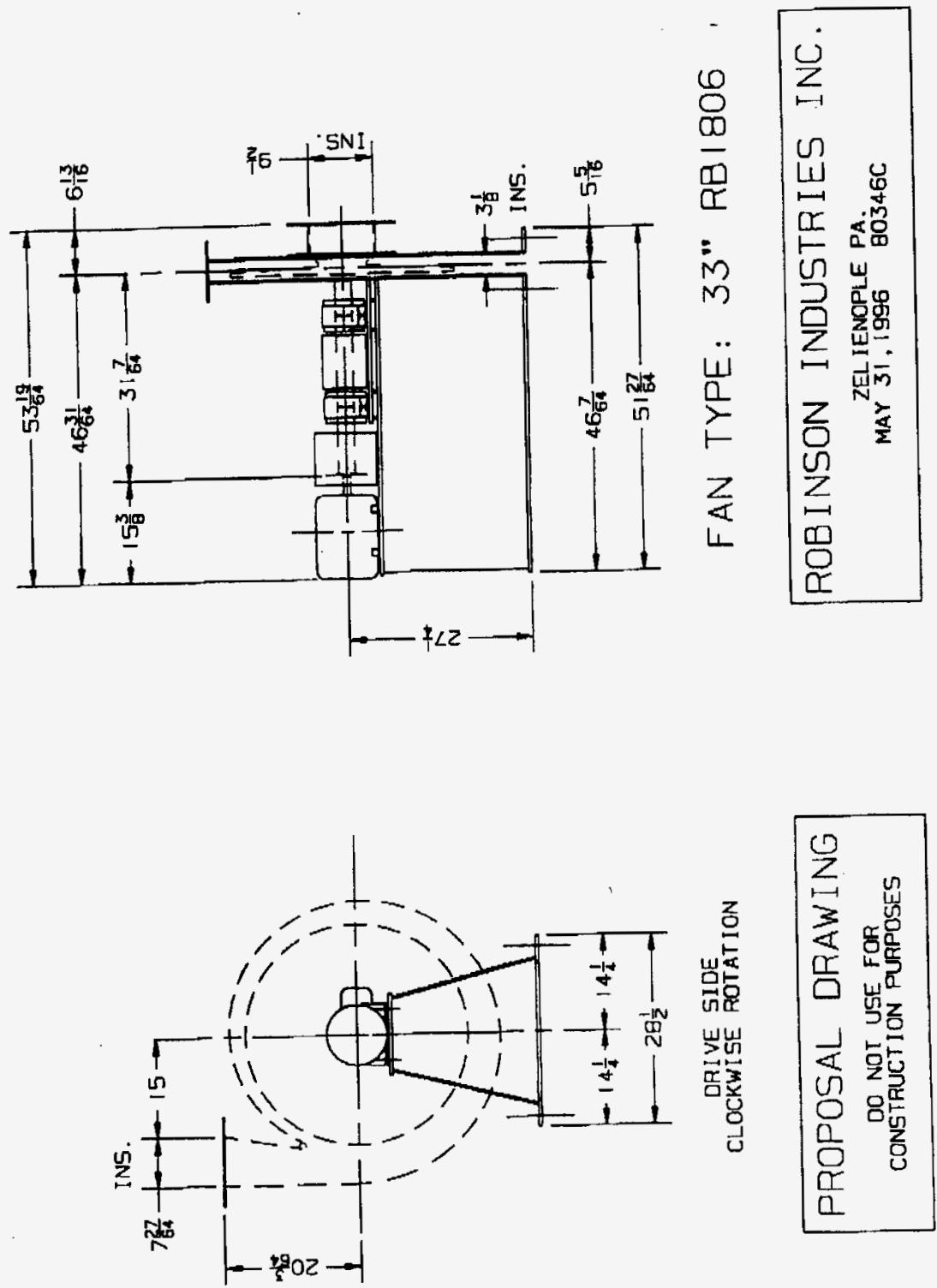

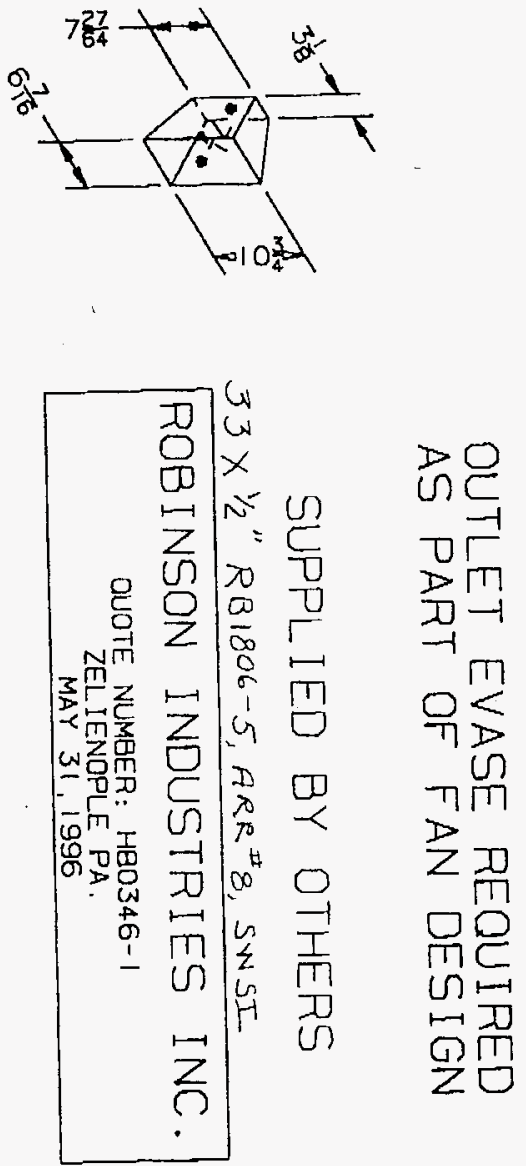
ROBINSON INDUSTAIES INC.

FAN: $33 \times 0.5$ AB1806-5 SWSI FOR: MEARICK \& COMPANY
SPEED: 1780 APM

TEMP: 710 DEG. $F$

DENSITY: 0.0334 \#/FT3
DATE: $5-30-199$

QUOTE \#: HE0346-1

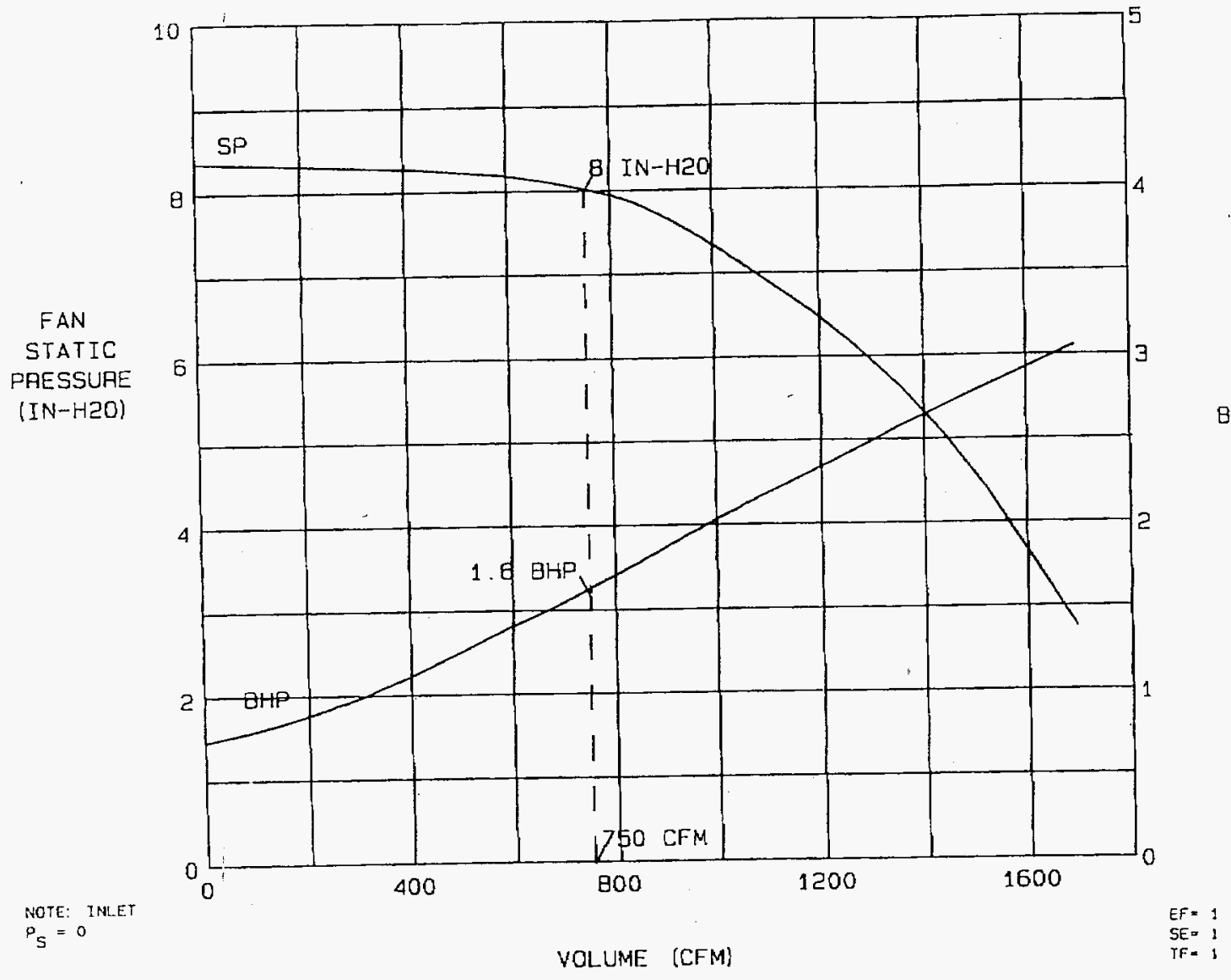


WHC-SD-SNF-CDR-007, REV. 0

ROBINSON INDUSTRIES, INC.

DATE: $05 / 31 / 1996$

SOUND PROGRAM OUTPUT

QUOTE \# : $\$ 380346-1$

CUSTOMER : MERRICK \& $C O$.

$\begin{array}{lllllll}\text { DESIGN } & : \text { RBI806-5 } & \text { DIAMETER: } 33.00000 & \text { INCH } & \text { RPM: } 1780.00 & \text { WIDTH: SWSI } \\ \text { CFM } & : & 750.00 & \text { PS-INCHES H20: } & \text { B.00000 ELADES: } 18 & & \end{array}$

NEAR FIELD APEROXIMATELY

3 FEET

SOUND POWER LEVEL RATINGS SHOWN ARE DECIBELE REFERRED TO 1O-12 WATT AND

OBTAINED IN ACCORDANCE WITH AMCA STANDARD 300.

RELATED AIR PERFORMANCE RATINGS PER AMCA STANDAFD 210.

PWL FOR EACI BAND AND DEA ARE CALCULATED PER AMCA STANDARD 301.

IEVELS SFOWN DO NOT INCLUDE MOTOR OR OTUER AUXIIIARY EQUIPMENT.

OPERATING CONDITIONS OTHER THAN THE ABOVE STATED VALUES CAN RESULT IN SIGNIFICANTLY DIFEERENT SOUND IEVELS.

DATA IS FOR USE BY A SYSTEM ACOUSTICAL DESIGN ENGINEER FOR EVALUATION OF THE FAN SINGUTARIY AND WITHIN A SYSTEM.

BECAUSE OF THE INEINITE VARIATIONS IN SYSTEM ARRANGEMENTS

AND THE MANY FACTORS WHICH AFEECT SOUND FRESSURE LEVELS,

IT IS THE DESIGNERS RESPONSIRILITY TO PROPERLY APPLY THIS DATA BASED

ON HIS KNOWLEDGE OF THE SYSTEM. SOMS GUIDELINES FOR USE OF THIS DATA ARE:

FOR "NEAR FIELD" COMPUTER DATA TO APPIY TO DUCTED INLET AND OUTLET

INSTALIATIONS, ANY OPENING IN THE DUCT,MUST $5 E$ A MINIMUM OF 100 EEET REMOTE OF THE FAN,

OPENINGS WITHIN THIS RANGE ARE ASSUMED TO EMIT A SOUNE PRESSURE EQIJAI

TO THE SOUND POWER IEVEL. THIS ALSO APPLIES TO UNTTREATED INLET \& OUTTEET

EXPANSION JOINTS. NOTE THAT FOR DUCTED INLEY/OUTLET THE DUCTWORK THICKNESS AND

DENSITY MUST EQUAL THAT OF THE FAN HOUSING TO ACHIEVE THE SOUND LEVELS NOTED.

NEAR FIEID.-A HEMISPHERICAI SPACE WHERE SOUND PRESSURZ WAVES FROM ONE

RADIATING SUREACE TEND TO INTEREERE WITH WAVES GENERATED BY OTHER SURFACES.

NEAR FIELD BOUNDARY, DISTANCE FROM RADIATING SURFACE, IS REIATED

TO THE WAVELENGTH OF THE LOWEST FREQUENCY AND OVERALL SIZE OF SOURCE.

FREE EIELD--AREA BEYOND NEAR FIELD, WITH NO OBSTRUCTIONS, WEERE SOUND

FRESSURE LEVELS DECAY 6 DB FOR EACH DOUBLING OF DISTANCE FROM NEAR FIEID.

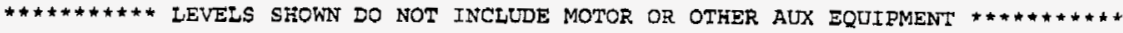

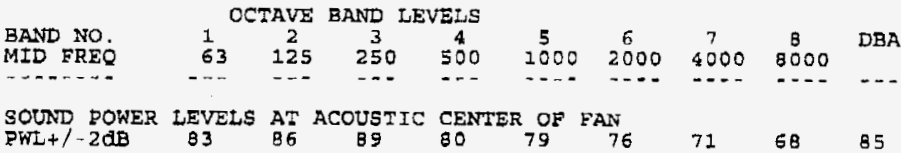

FOR DUCTED INLET AND OUTLET INSTAILATION

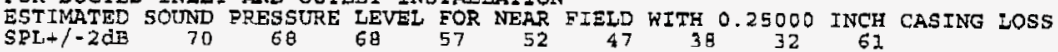

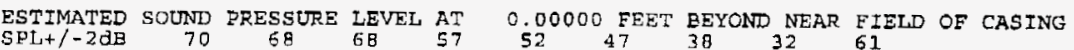

$\begin{array}{cccc}S \mathrm{PL}+1-2 \mathrm{~dB} & 70 & 68 & 6 \mathrm{~B}\end{array}$

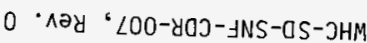




\section{WATLOW HEATERS}

\section{Tubular and}

\section{Process Assemblies}

\section{Circulation Heaters}

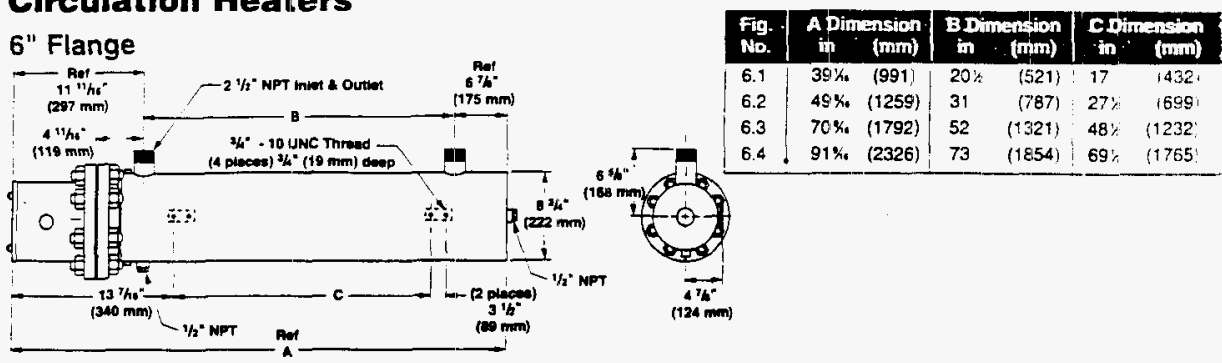

\section{6" $150 \mathrm{lb}$ ANSI Flange-WATROD Element}

\begin{tabular}{|c|c|c|c|c|c|c|c|c|c|c|c|}
\hline \multirow{2}{*}{$\begin{array}{l}\text { WATROD } \\
\text { Description }\end{array}$} & \multirow[b]{2}{*}{$k W$} & \multirow[b]{2}{*}{$\begin{array}{l}\text { Fig } \\
\text { No. }\end{array}$} & \multicolumn{6}{|c|}{ Code No. } & 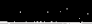 & & \multirow{2}{*}{$\begin{array}{l}\text { Est. Ship } \\
\text { Weight } \\
\text { los }(\mathrm{kg})\end{array}$} \\
\hline & & & $\begin{array}{l}\text { 240vac } \\
\text { 1-Phase }\end{array}$ & $\mid \begin{array}{c}\text { No of } \\
\text { Circults }\end{array}$ & $\begin{array}{l}\text { 240VAC } \\
\text { 3-Phase }\end{array}$ & $\begin{array}{l}\text { No of } \\
\text { Circuits }\end{array}$ & $\begin{array}{l}\text { 480VAC } \\
\text { 1-Phase }\end{array}$ & $\begin{array}{c}\text { Na. of } \\
\text { Circuits }\end{array}$ & $\begin{array}{l}\text { 4BOUAC } \\
\text { 3Phase }\end{array}$ & $\begin{array}{l}\text { Wo.pl } \\
\text { Circults }\end{array}$ & \\
\hline
\end{tabular}

\section{Application: Clean Water}

\begin{tabular}{|c|c|c|c|c|c|c|c|c|c|c|c|}
\hline \multirow[t]{2}{*}{$\begin{array}{l}60 \mathrm{~W} / \mathrm{m}^{2} \\
\text { Steal Tank } \\
\text { 12-Coppar } \\
\text { (9.3 W/cm })\end{array}$} & $\begin{array}{l}24 \\
36 \\
48 \\
60\end{array}$ & \begin{tabular}{l|}
6.1 \\
6.1 \\
6.2 \\
6.2 \\
\end{tabular} & $\begin{array}{l}\text { CFPC715G10 } \\
\text { CFPC721G10 }\end{array}$ & $\begin{array}{l}2 \\
3\end{array}$ & $\begin{array}{l}\text { CFPC715G3 } \\
\text { CFPC721C3 } \\
\text { CFPCT2SA3 } \\
\text { CFPC732G3 }\end{array}$ & $\begin{array}{l}2 \\
2 \\
4 \\
4\end{array}$ & $\begin{array}{l}\text { CFPC715G11 } \\
\text { CFPC72tG11 } \\
\text { CPPC726A1T } \\
\text { CPPC732G11 }\end{array}$ & $\begin{array}{l}1 \\
2 \\
2 \\
2\end{array}$ & $\begin{array}{l}\text { CFPC715G5 } \\
\text { CFPC721G5 } \\
\text { CFPC726R5 } \\
\text { CFPC732G5 }\end{array}$ & $\begin{array}{l}1 \\
1 \\
2 \\
2\end{array}$ & \begin{tabular}{|rr}
212 & $(97)$ \\
217 & $(99)$ \\
222 & $(101)$ \\
226 & $(103)$
\end{tabular} \\
\hline & $\begin{array}{r}72 \\
100 \\
120 \\
\end{array}$ & $\begin{array}{l}6.3 \\
6.3 \\
6.4 \\
\end{array}$ & & & CFPC737R3 & 4 & & & $\begin{array}{l}\text { CFPCT37R5 } \\
\text { CFPCTSOAS } \\
\text { CFPCTEOCS }\end{array}$ & $\begin{array}{l}2 \\
2 \\
4\end{array}$ & $\begin{array}{ll}290 & (132) \\
298 & (136) \\
360 & (164) \\
\end{array}$ \\
\hline $\begin{array}{l}60 \text { W/in2 } \\
\text { Steel Tank } \\
\text { 15-Copper } \\
\left(9.3 \mathrm{~W}_{\mathrm{cm}} \mathrm{cm}^{2}\right)\end{array}$ & $\begin{array}{r}90 \\
125 \\
150\end{array}$ & $\begin{array}{l}6.3 \\
6.3 \\
6.4\end{array}$ & & & CFPCTs7R3X & 5 & & & $\begin{array}{l}\text { CFPC737R5X } \\
\text { CFPC750R5X } \\
\text { CFPC760G5X }\end{array}$ & $\begin{array}{l}5 \\
5 \\
5\end{array}$ & $\begin{array}{ll}296 & (134) \\
306 & (139) \\
370 & (168)\end{array}$ \\
\hline
\end{tabular}

Application: Deionized Water, Demineralized Water

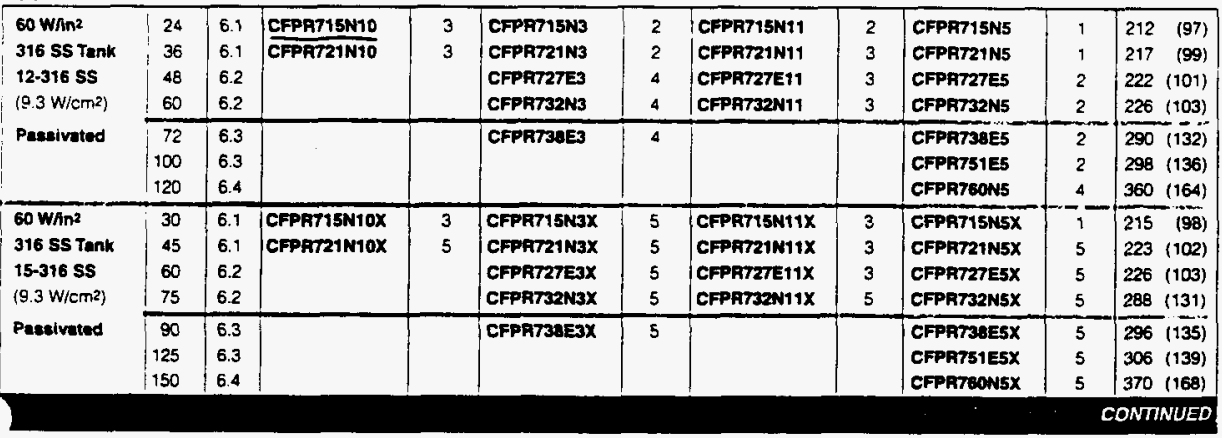

Alt circulation heaters are Assembly Stock

unless oinerwise noted.

Availability

(2) Standard

Aseembly Stock: 5-7 working days 
$t r=60-20436,204 t$
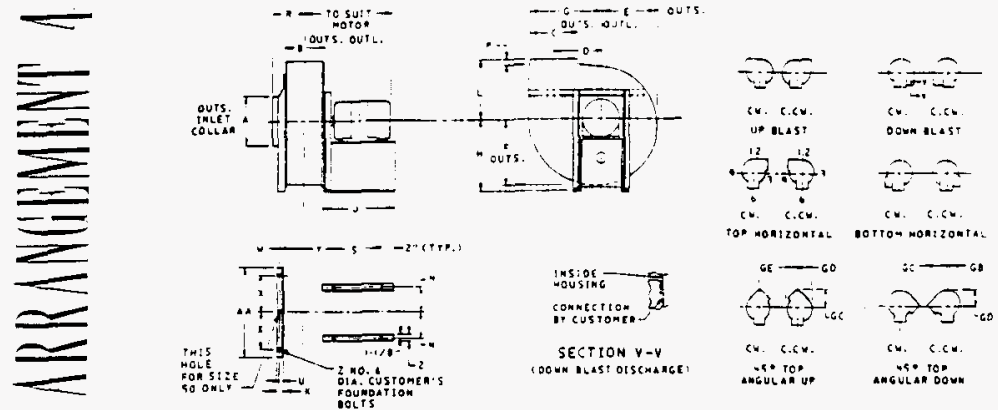

NOTES

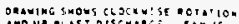

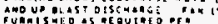

ondecos

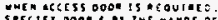

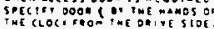

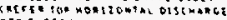

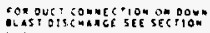

co. c.ce cy c.co.

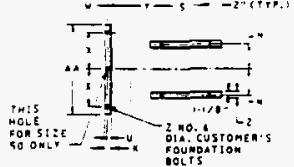

top monizonial

rion monizomita

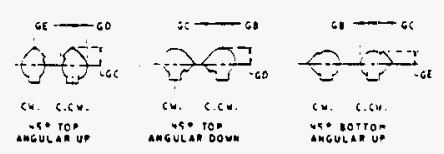

FOUNOATION PLAN

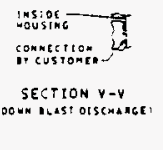

angulat dor

ningurition

\begin{tabular}{|c|c|c|c|c|c|c|c|c|c|c|c|c|c|c|c|c|c|c|c|c|c|c|c|c|c|c|c|c|c|}
\hline & \multicolumn{2}{|c|}{ moters } & & & & & & & & \multicolumn{3}{|c|}{$\mathrm{H}$} & \multirow[b]{2}{*}{1} & \multirow[b]{2}{*}{ K } & \multirow[b]{2}{*}{$i$} & \multirow[b]{2}{*}{ \# } & \multirow[b]{2}{*}{$P$} & \multirow[b]{2}{*}{ R } & \multirow[b]{2}{*}{3} & \multirow[b]{2}{*}{0} & \multirow[b]{2}{*}{$n$} & \multirow[b]{2}{*}{$\mathbf{x}$} & \multirow[b]{2}{*}{1} & \multirow[b]{2}{*}{$I$} & \multirow[b]{2}{*}{ Ad } & \multirow[b]{2}{*}{ GB } & \multirow[b]{2}{*}{ Gt } & \multirow[b]{2}{*}{ CD } & \multirow[b]{2}{*}{ 65 } \\
\hline S12r & Min & Mat & $A$ & E & $c$ & $D$ & I & F & E & \begin{tabular}{|l} 
48. 06. TH. \\
TRU. IAD
\end{tabular} & BAU & $\mathrm{BH}$ & & & & & & & & & & & & & & & & & \\
\hline 17 & 56 & 184! & 74 & 6 & $2 x$ & $n$ & $\mathbf{a x}_{\mathbf{k}}$ & $9 x$ & $100 \%$ & 14 & 14 & 14 & $15 n x$ & 2. & *h & 74 & 14 & $6 \%$ & $11 \%$ & in & 14 & 74 & 5 & 65 & 16x) & $Y_{H_{1}}$ & $10 y_{3}$ & $10 \mathrm{~h}$ & $B$ \\
\hline 210 & 56 & 2151 & 9 & 74 & BX & $81 K_{16}$ & $10 \%$ & $111 k_{k}$ & 11 & 168 & 164 & $15 \%$ & $15 \%_{11}$ & 2 & $11 x_{3}$ & $7 x$ & 14 & $\theta x$ & $111 \%$ & 15 & 4\% & 74 & $5 \%$ & $6 \mathrm{~h}$ & 19K & IIh & $17 x$ & $127 / \mathrm{m}$ & $10 \mathrm{j}_{\mathrm{H}}$ \\
\hline 25 & 56 & $215 t$ & $10 x$ & 3 & 104 & 19K & 124 & $13 \%$ & $15 \%$ & $16 \%$ & 16\% & 19 & $151 \%$ & 2 & 1214 & $7 \%$ & $1 k$ & $\pi$ & $11+4,4$ & 14 & $5 \%$ & 74 & $6 k_{1}$ & 54 & $22 x$ & 13k & 2034 & $i+y_{4}$ & $11 \%$ \\
\hline 301 & $162 \pi$ & $215 i$ & $: 2 \%$ & $10 \mathrm{~K}$ & 123 & $12 \%$ & $14 x$ & $16 \%$ & $18 \%$ & 18 & 18 & $20 \%$ & $\mathrm{~T}_{\mathrm{Th}}$ & 2 & $15 x$ & $8 x$ & 13. & th & $13 \%$ & 1k & $G_{4}$ & ex, & $7 t_{h t}$ & $6 \mathrm{~h}$ & $20 \%$ & $15 \times k$ & $2314 \mathrm{H}$ & $19 x_{k}$ & $64 \psi_{4}$ \\
\hline 35 & 1825 & $215 \pi$ & $10 / \mathrm{h}$ & $11 \%$ & 1016 & $10 \%$ & $17 \%$ & 194, & $21 \%$ & $21 \%$ & 213 & 246 & $17 h_{1}$ & 2 & 18 & $8 x$ & 14 & 93. & $13 \%$ & in & 1 & Bx & $n_{h}$ & $6 x$ & 30 & $10 \mathrm{H}_{4}$ & 26 & $20 k_{6}$ & $196 / 6$ \\
\hline 401 & $182 \pi$ & $25+1$ & 17 & $13 \mathrm{X}$ & $16 \mathrm{x}$ & 16\%: & $19 k$ & 22 & $21 \%$ & 24 & 24 & 27 & $\mathbf{L S k}_{\mathrm{k}}$ & 24 & 204 & Hx & 13 & $10 x$ & $15 \%$ & 18 & $8 H_{4}$ & 9.7. & $s^{\prime} y_{n}$ & $6 x$ & $3 x$ & 2016 & $314_{4}$ & $231 \mathrm{hs}$ & $15 x$ \\
\hline 451 & 25AT & $25 \Delta t$ & $19 \mathrm{k}$ & 15 & 163 & $16 \%$ & $21 \%$ & 20140 & $27 x$ & $25 \%$ & $30 \%$ & $30 \times$ & $19 K_{u}$ & $v_{\mathrm{f}}$ & 23 & $5 x$ & 2 & III & $15 K_{m}$ & 15 & $v_{1}$ & 㬐 & $3 \%$ & $6 x$ & $3 \mathrm{~m}$ & $23 x$ & $351_{k}$ & $x y$ & 23 \\
\hline 50 & $28 \mathrm{TI}$ & 3261 & $2: 4$ & $16 x$ & $20 x_{k}$ & $20 \%$ & $24 \%$ & .276 & $301 y_{k}$ & 30th & $30 \mathrm{n}$ & 344 & $22 \%$ & $2 \pi$ & 25 & $9 \pi$ & 2 & $12 \mu_{6}$ & $2 \pi x_{n}$ & 11 & $9+h_{n}$ & 184 & $10 \%$ & $T_{\mathbf{X}}$ & 415 & $8 x$ & 3913,4 & $20 x$ & $23 k$ \\
\hline
\end{tabular}

\begin{tabular}{|c|c|c|c|c|c|c|c|c|c|c|c|c|c|c|}
\hline FAN SIZE & & 17 & 21 & 25 & 30 & 35 & 40 & 45 & 50 & 55 & 60 & 70 & 80 & 90 \\
\hline 4 Arr: 94 & $0.0 . P$. & 184 & 184 & 215 & 215 & 215 & .286 & 286 & 326 & 326 & 326 & 445 & 445 & 445 \\
\hline $1 \rightarrow \quad$ Rivot Base & T.E.F.C. & 184 & 184 & 215 & 215 & 215 & 256 & 256 & 286 & 286 & 286 & 405 & 445 & 445 \\
\hline Arr. $9+4$ & $0 . D . P$. & 256 & 284 & 284 & 324 & 364 & 364 & 365 & 404 & 405 & 444 & - & - & - \\
\hline Channel Base & T.E.F.C. & 256 & 284 & 284 & 324 & 364 & 364 & 365 & 404 & 405 & 444 & - & - & - \\
\hline$=$ Arr 10 & $\begin{array}{l}\text { O.D.P. } \\
\text { ANO } \\
\text { T.E.F. }\end{array}$ & 145 & 145 & 145 & 145 & 215 & 215 & 256 & 286 & 286 & 286 & - & - & - \\
\hline
\end{tabular}

\begin{tabular}{|c|c|c|c|c|c|c|c|}
\hline SIzE & & ARR. No. 1 & ARR. No. 4 & ARR. No. B & ARR. No. 9 & ARR. No. 10 & $\begin{array}{l}\text { ARP Na. } 9 \\
\text { CHANNEL }\end{array}$ \\
\hline $17=$ & $\vdots$ & 130 & 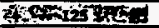 & 180 & $y+x=530 x+4$ & 155 & 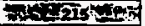 \\
\hline$\therefore 21$ & $:$ & 200 & $12 \mathrm{Ra} 0 \mathrm{x}=\mathrm{N}$ & 250 & 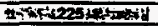 & 225 & 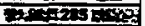 \\
\hline 25 & $i$ & 250 & $2 x+a+2 x y=1$ & 275 & 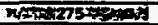 & 275 & Dangersis \\
\hline 30 & $\therefore$ & 385 & 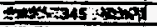 & 425 & 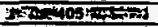 & 405 & $5 x+153 x-1$ \\
\hline 35 & $\dot{-}$ & 550 & 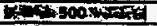 & 500 & Dorbound & 575 & 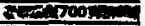 \\
\hline 40 & $-\alpha$ & 700 & 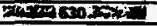 & 770 & 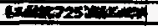 & 725 & $4-200$ \\
\hline-45 & $\bar{i}$ & 910 & 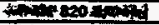 & 1000 & $n=2041: 9$ & 935 & $7100 x \operatorname{los}$ \\
\hline 50 & $\div$ & 1100 & 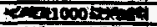 & 1200 & 71902 & 1130 & 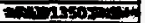 \\
\hline 55 & $:$ & 1350 & $+23000=1$ & 1500 & Con & $13 \mathrm{BO}$ & $2 \times 2$ con $=$ \\
\hline 50 & & 1500 & $20,3=7$ & 1650 & 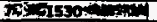 & 1530 & 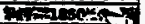 \\
\hline 70 & & 2500 & 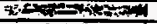 & 2700 & 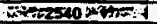 & $=$ & 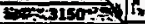 \\
\hline 80 & & 3300 & 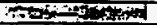 & 3500 & $=43345 \div 574$ & - & Ex+my \\
\hline 90 & 1 & 3700 & 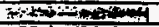 & 4000 & 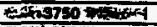 & $=$ & $A x^{2}+x^{2}=$ \\
\hline 100 & & 4400 & 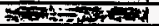 & - & 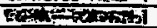 & - & 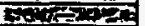 \\
\hline 310 & & 5000 & 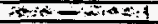 & $=$ & 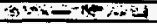 & $=$ & Everis $46 \alpha^{2}$ \\
\hline 120 & & 6000 & 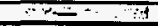 & 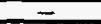 & $\cdots-1-1=1$ & $=$ & 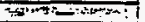 \\
\hline
\end{tabular}

$$
H-66
$$

62 BUFFALO FORGE COMPANY I BUFFALO. NEW YORK DL MNL 12 
WHC-SD-SNF-CDR-DCT, REV. O

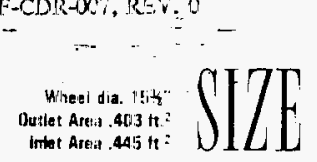

\begin{tabular}{|c|c|c|c|c|c|c|c|c|c|c|c|c|c|c|c|c|c|c|c|}
\hline CAP. & 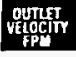 & \multicolumn{2}{|c|}{ IN S.P. } & \multicolumn{2}{|c|}{ 1\%" S.P. } & \multicolumn{2}{|c|}{ RPW SP. } & \multicolumn{2}{|c|}{$24 "$ S.P. } & \multicolumn{2}{|c|}{$3^{*} \mathrm{SP}$} & \multicolumn{2}{|c|}{ 3K" s.p. } & \multicolumn{2}{|c|}{ AF" S.P. } & \multicolumn{2}{|c|}{$\begin{array}{l}\text { 4H" S.P. } \\
\text { RPH }\end{array}$} & \multicolumn{2}{|c|}{ DPU S.P. } \\
\hline $\begin{array}{l}400 \\
490 \\
980 \\
670 \\
760\end{array}$ & $\begin{array}{l}993 \\
1216 \\
1439 \\
1653 \\
1866\end{array}$ & $\begin{array}{r}893 \\
926 \\
966 \\
1013 \\
1065\end{array}$ & $\begin{array}{l}0.11 \\
0.13 \\
0.16 \\
0.20 \\
0.24\end{array}$ & $\begin{array}{l}1071 \\
1093 \\
1125 \\
164 \\
1209\end{array}$ & $\begin{array}{l}0.16 \\
020 \\
0.24 \\
0.23 \\
0.33\end{array}$ & $\begin{array}{l}1226 \\
1242 \\
1267 \\
1300 \\
1338\end{array}$ & $\begin{array}{l}0.22 \\
0.27 \\
0.31 \\
0.36 \\
0.42\end{array}$ & $\begin{array}{l}1360 \\
1377 \\
1396 \\
1424 \\
1457\end{array}$ & $\begin{array}{l}0.29 \\
0.34 \\
0.39 \\
0.45 \\
0.52\end{array}$ & $\begin{array}{l}1495 \\
1502 \\
1517 \\
1539 \\
1569\end{array}$ & $\begin{array}{l}0.35 \\
0.45 \\
0.4 \\
0.54 \\
0.61\end{array}$ & 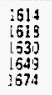 & $\begin{array}{l}0.43 \\
0.49 \\
0.56 \\
0.63 \\
0.71\end{array}$ & $\begin{array}{l}1725 \\
1727 \\
1735 \\
1752 \\
1773\end{array}$ & $\begin{array}{l}051 \\
057 \\
065 \\
0.73 \\
082\end{array}$ & $\begin{array}{l}1830 \\
1831 \\
1837 \\
1850 \\
1870\end{array}$ & $\begin{array}{l}059 \\
0.60 \\
074 \\
0.93 \\
0.92\end{array}$ & $\begin{array}{l}1990 \\
1929 \\
1993 \\
1944 \\
1961\end{array}$ & $\begin{array}{l}0.67 \\
0.75 \\
0.83 \\
0.93 \\
1.03\end{array}$ \\
\hline $\begin{array}{r}850 \\
940 \\
1030 \\
1120 \\
1210\end{array}$ & $\begin{array}{l}2109 \\
2333 \\
2556 \\
2779 \\
3002\end{array}$ & $\begin{array}{l}11208 \\
178 \\
1739 \\
1301 \\
3365\end{array}$ & $\begin{array}{l}0.29 \\
0.34 \\
0.41 \\
0.48 \\
0.56\end{array}$ & $\begin{array}{l}1257 \\
1310 \\
1365 \\
4242 \\
1482\end{array}$ & $\begin{array}{l}0.38 \\
0.45 \\
0.52 \\
0.560 \\
0.69\end{array}$ & $\begin{array}{l}1391 \\
1429 \\
1480 \\
1553 \\
1589\end{array}$ & $\begin{array}{l}0.499 \\
0.56 \\
0.54 \\
0.72 \\
0.82\end{array}$ & $\begin{array}{l}1499 \\
1539 \\
1586 \\
1696 \\
1689\end{array}$ & $\begin{array}{l}0.59 \\
0.67 \\
0.76 \\
0.85 \\
0.96\end{array}$ & $\begin{array}{l}1604 \\
11643 \\
1686 \\
1733 \\
1783\end{array}$ & $\begin{array}{l}0.70 \\
0.78 \\
0.88 \\
0.98 \\
.1 .10\end{array}$ & $\begin{array}{r}7055 \\
3481 \\
178 \\
1325 \\
1872\end{array}$ & $\begin{array}{l}0.80 \\
0.90 \\
1.00 \\
1.12 \\
1.24\end{array}$ & $\begin{array}{l}180202 \\
1895 \\
1872 \\
1913 \\
1958\end{array}$ & $\begin{array}{l}091 \\
1.02 \\
1.13 \\
1.25 \\
1.38\end{array}$ & $\begin{array}{r}18929 \\
1995 \\
1959 \\
19988 \\
2040\end{array}$ & $\begin{array}{r}03 \\
1.1 \\
1 . x \\
1 . x \\
1.3\end{array}$ & $\begin{array}{l}2982 \\
2011 \\
2043 \\
2080 \\
2119\end{array}$ & $\begin{array}{l}1.14 \\
1.26 \\
1.39 \\
1.53 \\
.1 .68\end{array}$ \\
\hline $\begin{array}{l}1300 \\
1390\end{array}$ & $\begin{array}{l}3226 \\
3449\end{array}$ & $\begin{array}{l}1431 \\
1498\end{array}$ & $\begin{array}{l}0.56 \\
0.76\end{array}$ & $\begin{array}{l}1544 \\
1607\end{array}$ & $\begin{array}{l}0.79 \\
0.91\end{array}$ & $\begin{array}{l}1647 \\
1707\end{array}$ & $\begin{array}{l}0.93 \\
1.05\end{array}$ & $\begin{array}{l}1743 \\
1800\end{array}$ & $\begin{array}{l}1.08 \\
1.20\end{array}$ & $\begin{array}{l}1885 \\
1889\end{array}$ & 122 & $\begin{array}{l}1921 \\
1927\end{array}$ & 4.37 & $\begin{array}{l}1958 \\
2005 \\
2054\end{array}$ & $\begin{array}{l}1.50 \\
1.53 \\
1.68\end{array}$ & $\begin{array}{l}2040 \\
2085 \\
2137\end{array}$ & $\begin{array}{l}1.33 \\
1.68\end{array}$ & $\begin{array}{l}2119 \\
2162\end{array}$ & $\begin{array}{l}.1 .68 \\
1.84\end{array}$ \\
\hline $\begin{array}{l}1480 \\
1570\end{array}$ & $\begin{array}{l}3672 \\
3896\end{array}$ & $\begin{array}{l}1566 \\
1635\end{array}$ & $\begin{array}{l}0.88 \\
1.01\end{array}$ & $\begin{array}{l}1671 \\
1736\end{array}$ & $\begin{array}{l}1.03 \\
1.17\end{array}$ & $\begin{array}{l}17768 \\
7830\end{array}$ & $\begin{array}{l}1.19 \\
1.33\end{array}$ & $\begin{array}{l}1859 \\
1919\end{array}$ & $\begin{array}{l}1.25 \\
1.50\end{array}$ & $\begin{array}{l}1945 \\
2003\end{array}$ & 151 & $\begin{array}{l}19621 \\
2027\end{array}$ & 1.67 & $\begin{array}{l}2106 \\
2160\end{array}$ & 1.84 & $\begin{array}{l}2182 \\
2182\end{array}$ & 201 & $\begin{array}{l}2208 \\
2256 \\
2305\end{array}$ & $\begin{array}{l}201 \\
219\end{array}$ \\
\hline 1660 & 4119 & 1705 & 1.16 & 1803 & 1.32 & 1894 & 1.49 & 1980 & 1.67 & 2062 & 1.85 & 2140 & 2.03 & 2215 & 225 & $\begin{array}{l}2234 \\
2287\end{array}$ & $\begin{array}{l}220 \\
2\end{array}$ & $\begin{array}{l}2306 \\
2358\end{array}$ & $\begin{array}{l}2.38 \\
2.59\end{array}$ \\
\hline $\begin{array}{l}1750 \\
1840\end{array}$ & $\begin{array}{l}4342 \\
4566\end{array}$ & $\begin{array}{l}1776 \\
1847\end{array}$ & $\begin{array}{l}1.32 \\
1.50\end{array}$ & $\begin{array}{l}1870 \\
1939\end{array}$ & $\begin{array}{l}1.49 \\
1.68\end{array}$ & $\begin{array}{r}1959 \\
2025\end{array}$ & $\begin{array}{l}1.67 \\
1.86\end{array}$ & $\begin{array}{l}2043 \\
2106\end{array}$ & $\begin{array}{l}1.85 \\
2.05\end{array}$ & $\frac{2122}{2184}$ & $\begin{array}{l}2.04 \\
2.24\end{array}$ & $\begin{array}{l}2: 9 \mathrm{gc} \\
2258\end{array}$ & $\frac{2.23}{2.44}$ & $\begin{array}{l}2272 \\
2330\end{array}$ & $\frac{242}{2.64}$ & $\begin{array}{l}2343 \\
2399\end{array}$ & $\begin{array}{l}251 \\
251\end{array}$ & $\begin{array}{l}241 ! \\
2467\end{array}$ & $\begin{array}{l}281 \\
305\end{array}$ \\
\hline $\begin{array}{l}1930 \\
2020\end{array}$ & $\begin{array}{l}8789 \\
5019\end{array}$ & 1919 & 1.69 & 2008 & 1.88 & 2092 & 2.07 & 2171 & 2.27 & 2247 & 2.47 & 2320 & 2.67 & $\begin{array}{l}2390 \\
2390\end{array}$ & 288 & $\begin{array}{l}2457 \\
245\end{array}$ & 309 & $\begin{array}{l}2467 \\
2523\end{array}$ & 3.30 \\
\hline 2310 & 5236 & 2065 . & 2.13 & 2148 & 2.33 & 22159 & $\begin{array}{l}2.30 \\
2.54\end{array}$ & 2303 & $\begin{array}{l}2.50 \\
275\end{array}$ & $\begin{array}{l}2311 \\
2375\end{array}$ & 2.97 & $\begin{array}{l}2382 \\
2445\end{array}$ & $\begin{array}{l}2.92 \\
3.19\end{array}$ & $\begin{array}{l}2250 \\
2512\end{array}$ & 341.313 & $\begin{array}{l}2516 \\
2577\end{array}$ & $\begin{array}{l}3.55 \\
3.65\end{array}$ & $\begin{array}{l}2581 \\
2640\end{array}$ & $\begin{array}{r}3.57 \\
3.85\end{array}$ \\
\hline $\begin{array}{r}2200 \\
200\end{array}$ & $\begin{array}{l}5459 \\
5689\end{array}$ & $\begin{array}{l}2138 \\
2212\end{array}$ & 2.37 & 2220 & 2.59 & 2297 & 2.80 & 2370 & 3.02 & 2441 & 325 & 2509 & 3.47 & 2574 & $370^{\circ}$ & 2638 & 3.93 & & 4.17 \\
\hline & 5906 & 2287 & $\begin{array}{l}2.64 \\
2.92\end{array}$ & 2363 & 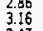 & $\begin{array}{l}22666 \\
2436\end{array}$ & $\begin{array}{l}3.99 \\
3.39\end{array}$ & $\begin{array}{l}22958 \\
2507\end{array}$ & $\begin{array}{l}3.31 \\
1.63\end{array}$ & $\begin{array}{l}2507 \\
2574\end{array}$ & $\begin{array}{l}3.55 \\
3.86\end{array}$ & $\begin{array}{l}2573 \\
2539\end{array}$ & $\begin{array}{l}3.78 \\
4.11\end{array}$ & $\begin{array}{l}25688 \\
2702\end{array}$ & $\begin{array}{l}4.02 \\
435\end{array}$ & $\begin{array}{l}27000 \\
2763\end{array}$ & $\begin{array}{l}4 \frac{x}{4} \\
40\end{array}$ & $\begin{array}{l}27653 \\
2823\end{array}$ & $\begin{array}{l}4.50 \\
4.85\end{array}$ \\
\hline $\begin{array}{l}2470 \\
2560\end{array}$ & $\begin{array}{l}6129 \\
6352\end{array}$ & $\begin{array}{l}2361 \\
2436\end{array}$ & $\begin{array}{l}3.23 \\
3.56\end{array}$ & $\begin{array}{l}22836 \\
2509\end{array}$ & 3.87 & $\begin{array}{l}2507 \\
2579\end{array}$ & $\begin{array}{l}3.72 \\
4.06\end{array}$ & $\begin{array}{l}2576 \\
2655\end{array}$ & $\begin{array}{l}3.96 \\
431\end{array}$ & $\begin{array}{l}2642 \\
2710\end{array}$ & 420 & $\begin{array}{l}2705 \\
27775\end{array}$ & $\begin{array}{l}4.45 \\
887\end{array}$ & 2757 & 4.70 & 2827 & 4.5 & 2885 & 5.22 \\
\hline & & & & & & & & & & & & & 4.82 & 2835 & 208 & & 3.34 & 2949 & $6 !$ \\
\hline
\end{tabular}

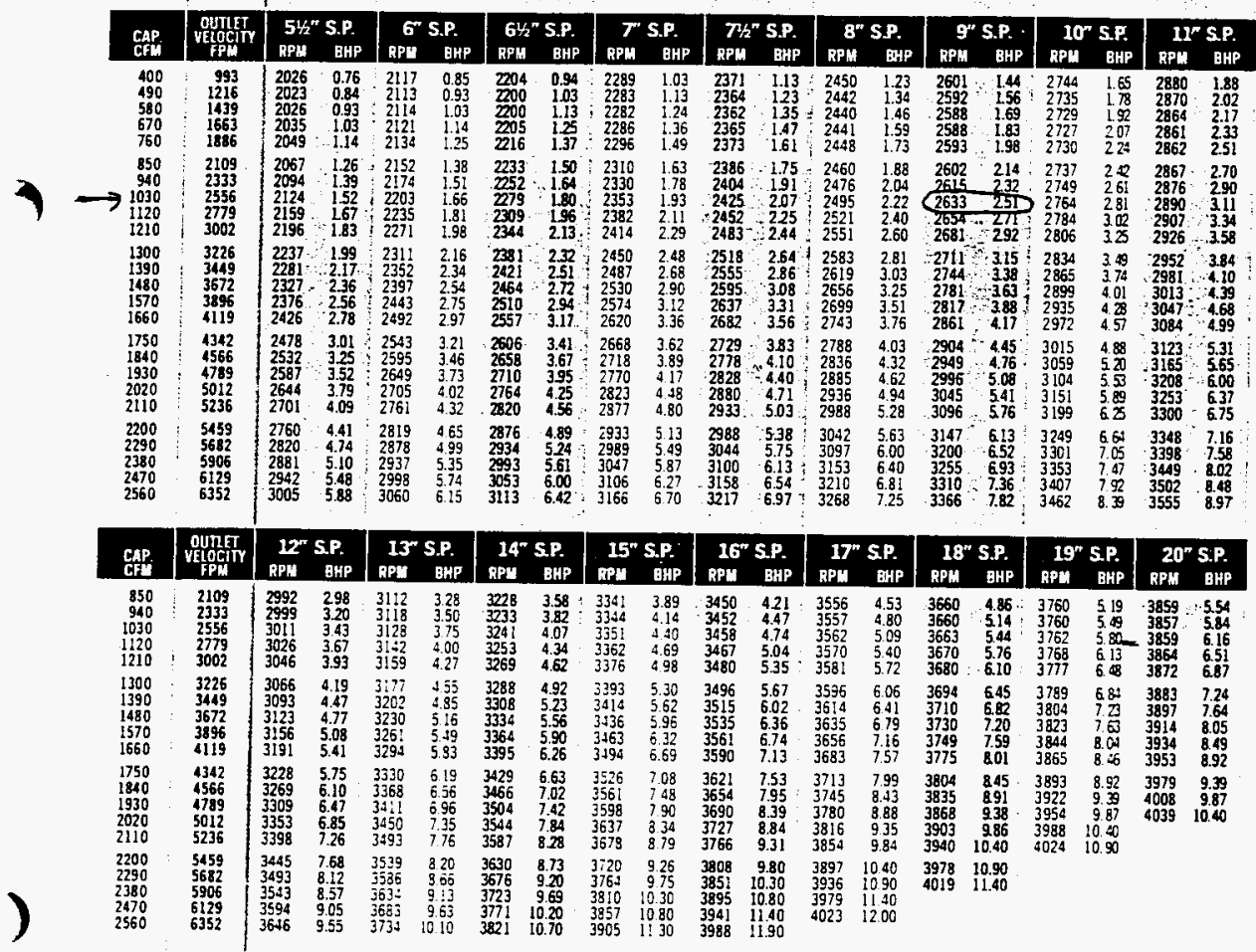

Raungs are at $70=F$ and 29.92 " beromeler.

BHP does not include drive losses.

The perlormance shom is tar standard MW. AW and OW bans with inlet and outlet duc

High speed ratings may require special construction. Contact Bulfalo Forge Company. 


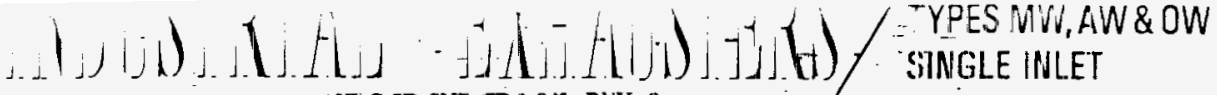 NF C-SD-SNF-CD 2-0 \%, RI:V 0}

IFFECIAL REAIOVIXBLE SLFF CLL COINSTRUETIISN

S" ANDARI SI:RCILL SDNS RIJCHON
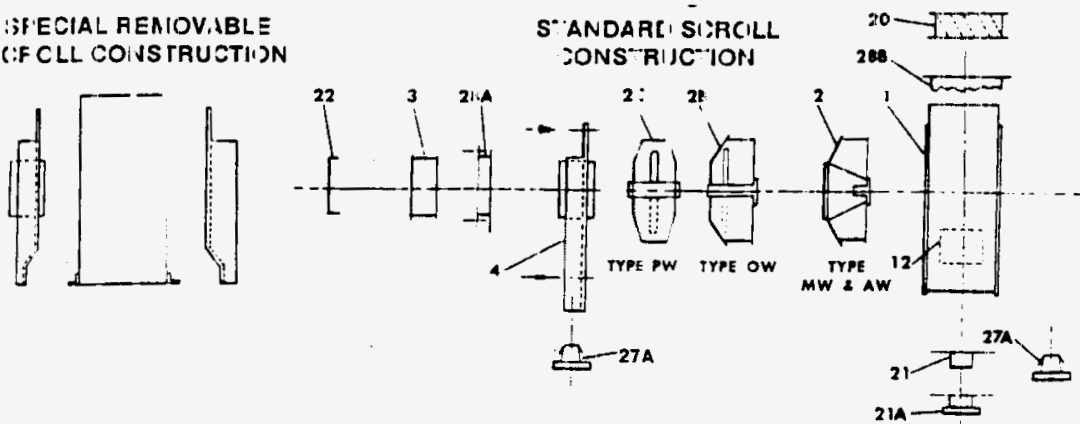

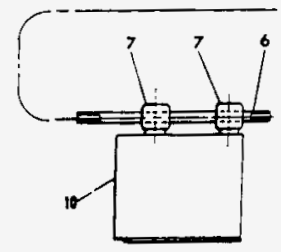

ARR. NO. 1
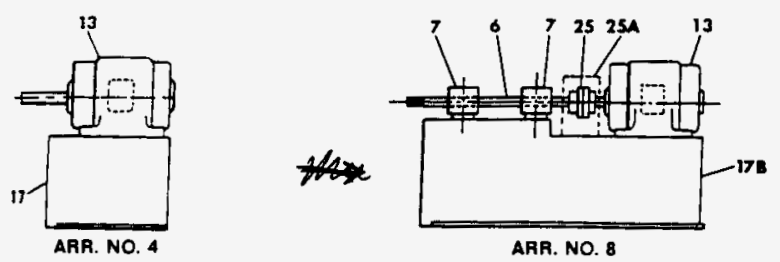

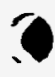

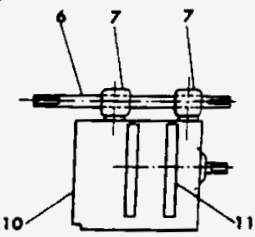

ARR. NO. 9

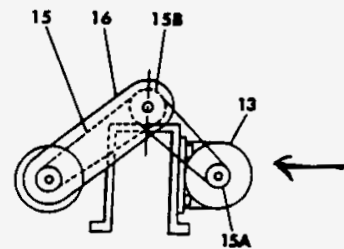

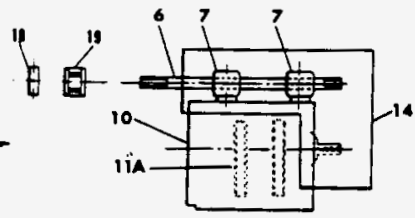

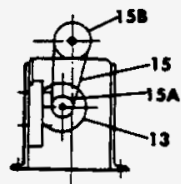

ARR. NO. 10

\section{FAN PARTS LIST}

1 . Housing

2 - Backplate and Flange Wheel

28 - Open Wheel

2C. Paddle (Blast) Wheel

3 - Inlet Cone

4 - Inlet Stand

6 - Shaft

7 - Pillow Block Bearing

10 - Bearing Base

11 - Arr. 9 Motor Stide Rails

11 A - Arr. 10 Motor Stide Rails

12 - Standard or Raised Access Door
13 - Motor
14 - Combination All-Weather Cover and
Belt Guard
15 - V-Belts
$15 A$ - Motor Sheave
158 - Fan Sheave
16 - Belt Guard
17 - Arr. 4 Motor Base
178 - Arr. 8 Motor and Bearing Base

18 - Shaft Seal

19 - Heat Slinger

20 - Outlet Damper

21 - Standard Drain, 1/2 Tapped Pipe Coupling

21 A - Flanged Type Drain

22 - inlet Screen

25 - Coupling

25A - Coupline Guard

27A - Rail Type Vibration Isolators

28A - tnlet Flange

$28 B$ - Outlet Flange 


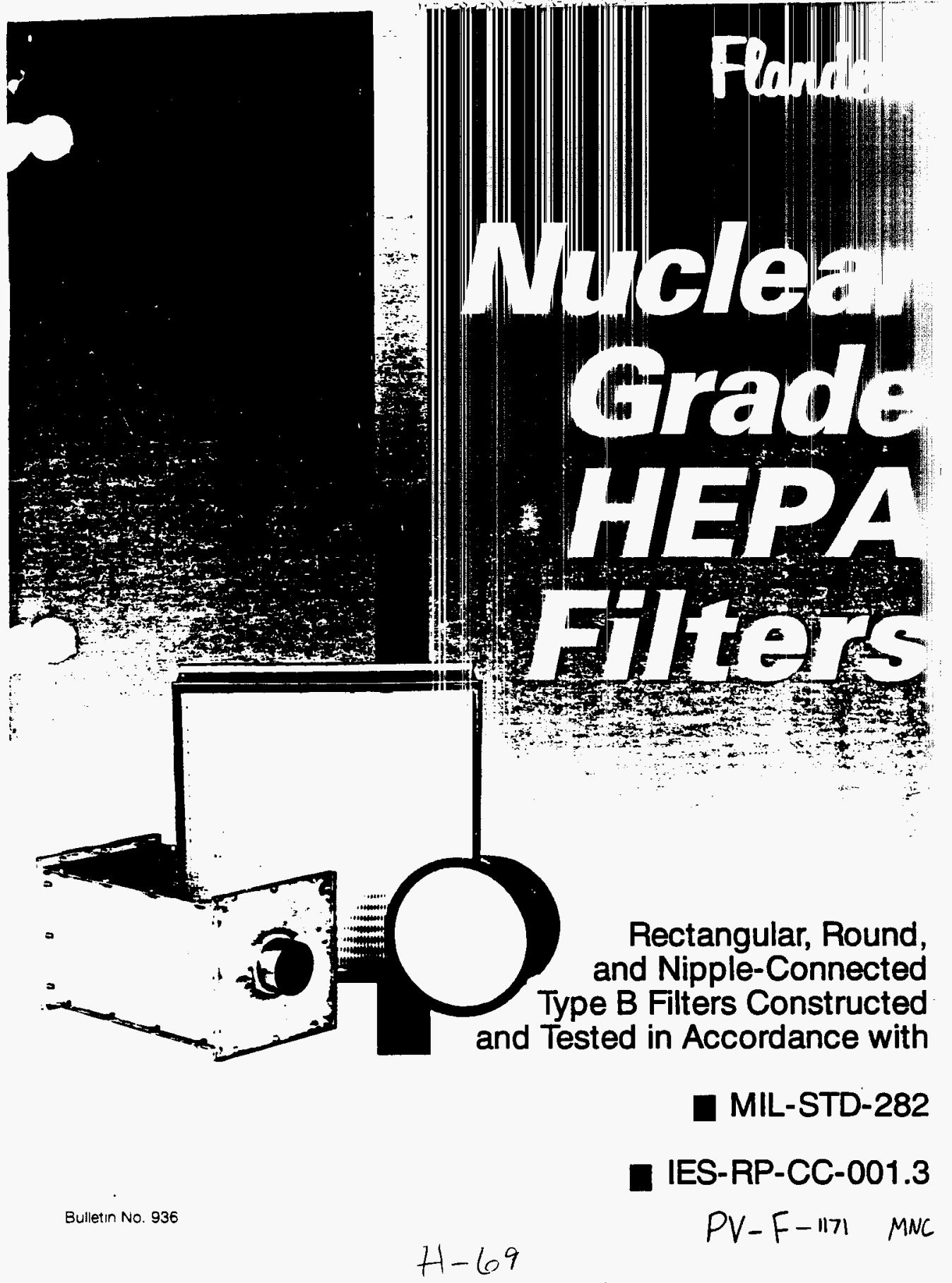




\section{Filter Design and Construction}

\section{Filter Media}

The filter media in Flanders' Nuclear Grade filter elements is all glass (boron silicate microfiber) and contains a waterprooting binder which adds strength under both wet and dry conditions. Flanders manufactures its own filter media to meet or exceed the requirements of MIL-F-51079 (latest issue). Flanders '007' media is currently listed on the U.S. Army Qualified Products List QPL 51079. OPL-approved media which have been treated for resistance to aibome acids are also available: consult the factory.

\section{Sealants}

Fire-retarciant urethanes are used to bond the fither element to its integral trame. PUREFORM'm filters are made using solid urethane whereas separator-type fitiers are made with either polyurethane foam or solid urethane.

\begin{abstract}
Frame Material
Plywood - Fire-retardant plywood frames are ideal for many applications, and are easier to incinerate or breakdown for disposal than are metal trame filters. However, they are not recommended for use in systems having high-moisture content, since they may wap or support biological growth. Stainiess steel frame materials should be used in moisture-faden applications.
\end{abstract}

Stainless Steel - Type 409 and Type 304 stainless steel frames are also available. The 14-gauge Type 409 stainless steel is used in place of the cadmium-plated and chromized steel that was used in the past. However, since the Type 409 material has a low resistance to caustic atmospheres, the Type 304 stainless steel is recommended for those applications.
The Separatorless PUREFORM ${ }^{\text {TM }}$ Filter

Flanders has developed unique processes for the production of seltsupporting, self-separating PUREFORM ${ }^{m}$ titer elements, which offer higher dustholding capacity, and thus longer service lite, than separator-type filters. The higher capacity of separatorless filters is due not only to greater surface area, but also to the thickness of the medium - a fact often overlooked. (See chart, below right.)

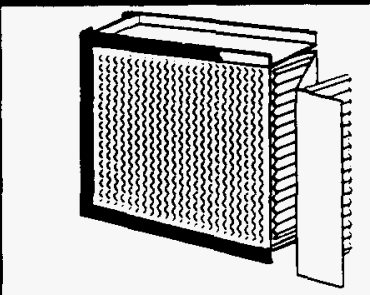

Seperator-type filters use paper or aluminum foil to separate the folds of the fitter eloment.
Flanders PUREFORMTw filtert use a formed, corrugeted eiement that is self-eupporting.

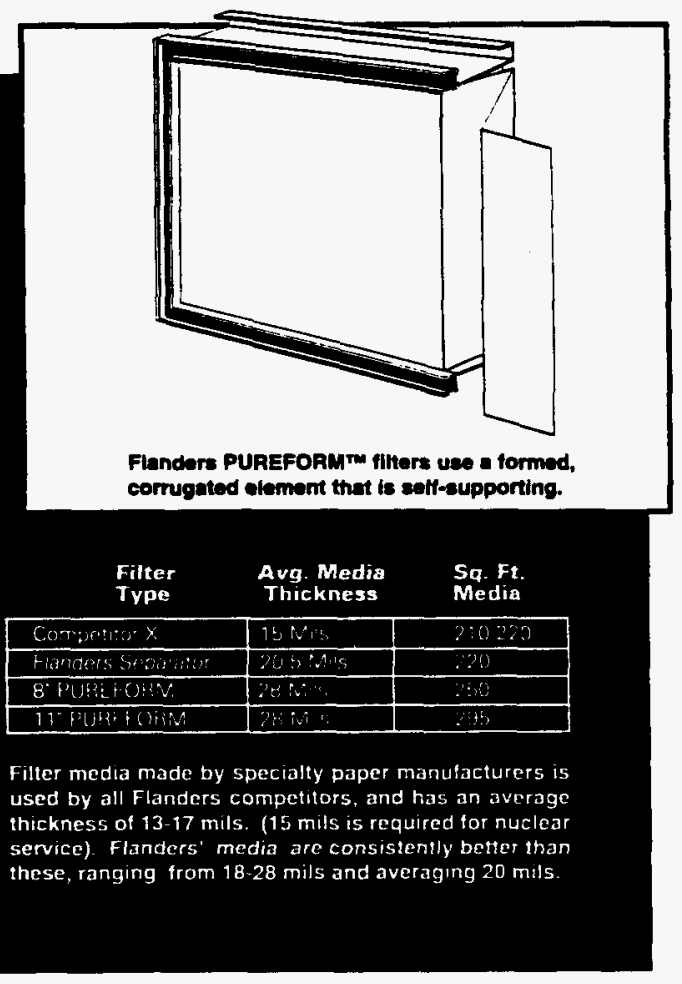

Filter media made by specialty paper manufacturers is used by all Flanders competitors, and has an average thickness of 13-17 mils. (15 mils is required for nuclear senvice). Flanders' media are consistently better than these, ranging from 18.28 mils and averaging 20 mils. 


\section{Frame Style}

The technique that will be used to seal the filter in service determines the selection of the filter frame style. (See illustrations below.)

Fluid Seal - Metal frame fluid seal filters have a tabricated channel ( $2 / 4^{*}$ wide $\times 3 / 4^{*}$ deep) located on one face. Wood frame fitters have a routed channel ( $3 / 4^{\prime \prime}$ wide $x^{3 / 4}$ " deep) on one face.

Gasket Seal - Metal trame gasketed filters are provided with one or two flanges for the placement of the gasket as specificed by the customer. On wooden frames, the gasket is simply applied to the edge of the frame.

\section{Fluid or Gasket Seal}

Fluid Sea/ - BLU-JEL Seal was developed by Flanders' and is the standard fluid seal material. Service temperatures are to $392^{\circ} \mathrm{F}$. For complete information on BLU-JEL ${ }^{\oplus}$ Seal, soe Det Shee 86010.

Gasket Sea/ - Gasketed filters for normal service are supplied with either closed cell sponge neoprene or rnhrtastic silicone sponge gaskets $\left(1 / 4^{\prime \prime} \times \%^{\prime \prime}\right)$. High- temperature fitters require the special sealants described on page 8. Spuctity upstream, downstream, or both for the location of the gasket or fluid saal in the model number.

\section{Faceguards}

The standard faceguard is $4 \times 4$ mesh, 23-gauge, weided and galvanized dipped steel. Type 304 stainless steel taceguards ( 4 × 4 mesh, 17-gauge woven wire per ASTM A276) are also available for highly-corrosive atmospheres. Faceguards protect the media, but are not a guarantee against damage due to mishandling. Specify foceguard lacetion as upstreem, downstream or both.

\section{Underwriters Laboratory, UL 586}

To be listed under UL 586, filters must be submitted to Underwriters Laboratories for extensive testing including spot ftame, and environmental exposure to heated air. A UL 586 listing is accepted by the DOE as meeting the Heated Air requirement for Nuclear Grade fitters.

UL labeis are aptional. Some high-temperature tihers, and filters larger than $24^{\circ} \times 30^{\circ} \times 11 \mathrm{~K}$;, are not eligible for UL 586 .

\section{The Fluid Seal}

\section{The Gasket Seal}

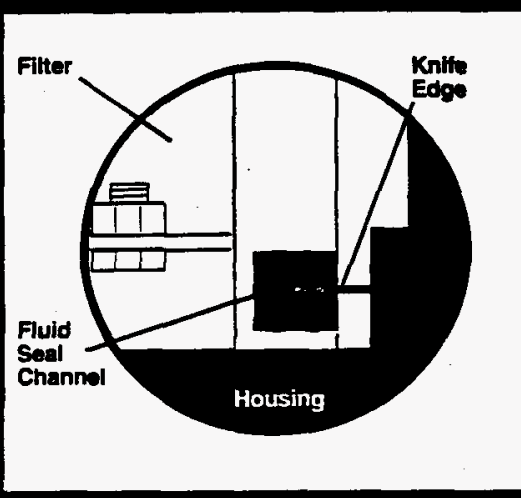

A knife edge in the filter housing mates into a fluidtilled channel provided on the tilter. Flanders invented the fluid seal in response to requirements for an absolute seal in the most critial applications. In most cases, fluid seal filters are also easier and quicker to change out than gasketed filters.

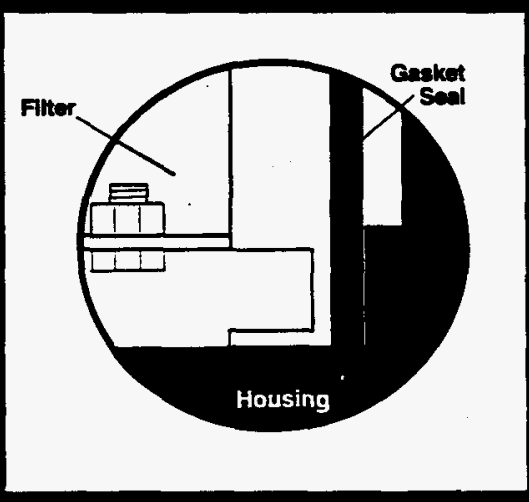

A filter clamping mechanism is typically used to maintain sealing pressure on gasketed filters. Gasket seais have a tendency to develop bypass leaks, primarily because of compression set. 


\section{Nipple-Connected Filters}

Nipple-Connected Nuclear Grade filters are available

with one (N1) or two (N2) pipe connections, and with

separatorless PUREFORM ${ }^{\mathrm{m}}$ or separator-type filter

elements. Frame materials are Type 304 or Type 409

stainless steel and 3/4" fire-retardant plywood. Only

gasket seals are available. High temperature models are

also available (see page 8 for information on sealants).

Nipple-Connected filters are made of the same materials

specified in MIL-F-51068 but are not tested for rough

handling, wet over-pressure, or heated air as called out

by that specification. Flanders performs acceptance

testing of penetration and resistance only.

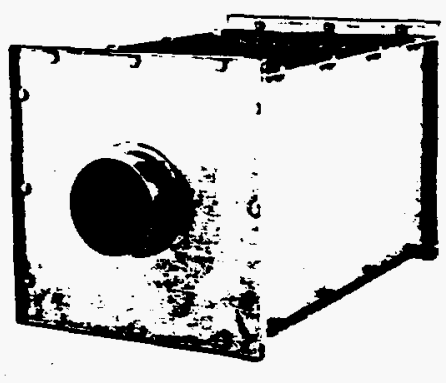

N1 Filters

TPPICAL METAL FRME FLTEA WUSTMATED

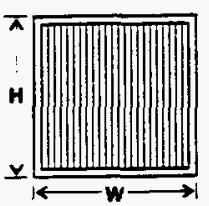

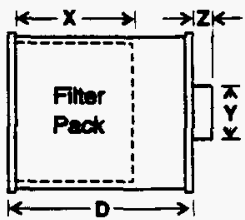

N2 Filters

Mncal wOOD FAME FLTEN WLSTRATEO

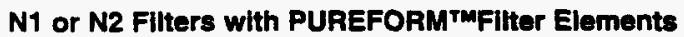

\begin{tabular}{|c|c|c|c|c|c|c|c|c|}
\hline \multirow{2}{*}{$\begin{array}{c}\text { SIZE } \\
\text { DESIGNATOH }\end{array}$} & \multicolumn{6}{|c|}{ DIMENSIONS } & \multirow{2}{*}{$\begin{array}{l}\text { NOMINAL } \\
\text { AATED } \\
\text { CAPACITY }\end{array}$} & \multirow{2}{*}{$\begin{array}{l}\text { MAXIMUM } \\
\text { INITIAL } \\
\text { RESISTANCE }\end{array}$} \\
\hline & $H$ & $w$ & $D$ & $x$ & $r$ & $z$ & & \\
\hline BB-D & $8^{\circ}$ & 8 & $10^{\circ}$ & $4^{*}$ & 5 & $11^{\prime}$ & 35 & \\
\hline CC-D & 12 & 12 & 12 & $4^{*}$ & $4^{\circ}$ & $11 k^{\circ}$ & 100 & \\
\hline CC-F & $12^{\circ}$ & 12 & $16^{\circ}$ & $8^{\circ}$ & $4^{*}$ & $11 / 2^{*}$ & 100 & $1.0^{*}$ พ.g. \\
\hline GG-D & $24^{*}$ & 24" & 12 & 4 & $12^{*}$ & $4^{\circ}$ & 500 & \\
\hline GG-F & $24^{\circ}$ & $24^{\circ}$ & 16 & $8^{\circ}$ & 12 & 4" & 1000 & \\
\hline
\end{tabular}

N1 or N2 Filters with Separator-Type Filter Elements

\begin{tabular}{|c|c|c|c|c|c|c|c|c|}
\hline $\begin{array}{c}\text { SIZE } \\
\text { DESIGNATOR }\end{array}$ & $H$ & $w$ & $\begin{array}{l}M E \\
D\end{array}$ & IONS & $\mathbf{Y}$ & $z$ & $\begin{array}{l}\text { NOMINAL } \\
\text { RATED } \\
\text { CAPACITY }\end{array}$ & $\begin{array}{c}\text { MAXIMUM } \\
\text { INITIAL } \\
\text { RESISTANCE }\end{array}$ \\
\hline BB-A & $\mathbf{B}^{\mathbf{0}}$ & 8 & $\boldsymbol{B}^{\circ}$ & $31 / m^{\circ}$ & 2 & $1 \frac{1}{2}$ & 20 & \\
\hline BB-D & $\theta^{n}$ & 8 & 10 & $5 \%$ & 5 & $11 / 2 "$ & 35 & \\
\hline CCD & $12^{*}$ & $12^{\circ}$ & 12 & $5 \% 0^{\circ}$ & $4^{\circ}$ & $11 / 2$ & 100 & $1.0^{\circ}$ w.o. \\
\hline CC-F & $12^{\circ}$ & $12^{*}$ & $16^{\circ}$ & $111 / h^{\prime \prime}$ & 4 & $11^{\prime}$ & 160 & . \\
\hline GG-D & $24^{\circ}$ & $24^{\circ}$ & $12^{\circ}$ & $5 \%$ & $12^{n}$ & $4^{\circ}$ & 500 & \\
\hline QGFF & $24^{\circ}$ & $24^{\circ}$ & $16^{\circ}$ & $111 / 2$ & 12 & $4^{\circ}$ & 1000 & \\
\hline
\end{tabular}

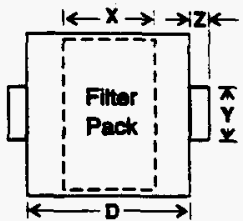

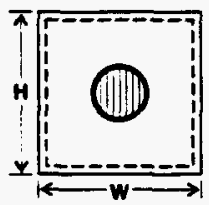

NOTE:

When ordering an N1 Finer, the customer must specily the location of the nipplo (i.e. upstream or downstream).

When ordering N1 or N2 fithers, the customer should spectiy the size of the nipple connections $\|$ Flandors' etandard sizes (see the $r$ dimensions) ane not eppropriate. 


\section{Roughing Prefilter}

a frames of N1 filters may be extended 2" in depth .u accomodate a roughing prefilter. Indicare the 'P' prefix in the model number to specity this option. Also requires a special size designator. Contact the factory for details.

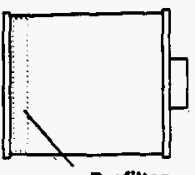

Prefliter
Prefiltar

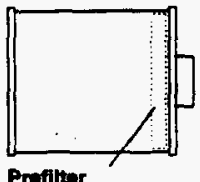

\section{Gasket Seal (N1 Filters Only)}

Gaskets are made from $1 / 4^{\prime \prime} \times 3 / 4^{\prime}$ closed cell neoprene material. Specity location of the gasket as upstream, downstream, or both, in the fither modal number.

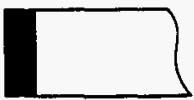

Wood Frame

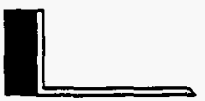

Motal Frame

\section{Drilled Flanges (N1 Filters Only)}

itandard ftanges may be located on the fitter face or on se nipple connection. These flanges may be drilled in a standard pattem or according to customer specifications. Flanders' standard patterns are as follows: $5 / 16^{\prime \prime}$ diameter holes equally spaced and certered on the filter face flange; or 5/16" diameter holes equally speced and centered on the nipple-connected flange.

\section{Nipple-Connected Fienos}
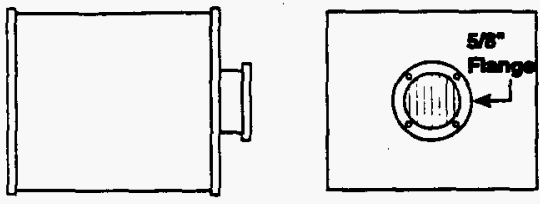

Fitur Foed Flang
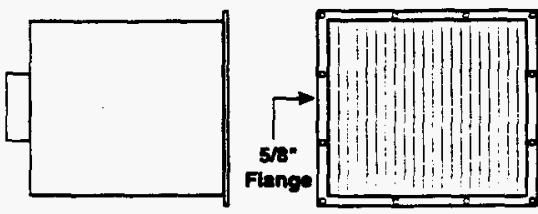

\section{Slip-On Flanges}

Optional 150 pound slip-on flanges for pipe-nipple connections shall be available in carbon steel or Type 304 stainless steel, and in any standard IPS size.

Other sizes and types of flanges are available per customer specifications.

\section{Faceguards} (N1 and N2 Filters) Standard taceguard material is $4 \times 4$ mesh, 23-gauge, welded and gatvanized dipped

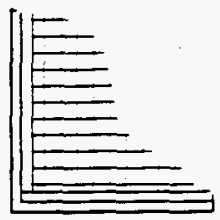
steel. Type 304 stainless steel faceguards (4X4 mesh, 17-gauge woven wire per ASTM-A276) are aiso avaitable for highly corrosive atmospheres. Specity faceguard location as upstream, downstream, or both.

\section{UL 586 (N1 and N2 Filters)}

To be listed under UL586, filters must be submitted to Underwriters Laboratories for extensive lesting including spot ftame and exposure to heated air. A UL586 listing is accepted by the DOE as meeting the Heated Air Requirement for Nuclear Grade titers. UL labels are optional and are applied onty it specified by the buyer.

NOTE: Hoh-dompersure evelumb are not ellgible for ULse6.

\section{Model Numbers and Specification}

To write a specification for NippleConnected Filters, use the Designating Chart on Page 15 to determine the model number. Fill in the numbered locations in the Suggested Specification Text on Page 16 with the model number and other appropriate selections from the fisting at the bottom of the page. 


$$
\begin{aligned}
& F V-F-2041=-2014 \quad \text { WHC-SD-SNF-CDR-007, REV. } 0 \\
& P V-F=2-042,-2045
\end{aligned}
$$

\section{Containment Housings With In-Place Test}

In-place testing of hepa filters and adsorbers is required for nuclear systems and should be considered for any HVAC system where toxic particulates or gases are present in the effluent airstream. The testing of adsorbers usually presents no problem since the freon that is used to challenge the system passes through particulate filters. Hepa filter banks require distance both up and downstream of the bank to successfully introduce and disperse challenge aerosol on the upstream side and to mix potential leakage into the airstream (i.e. for an efficiency test) on the downstream side. This is not always a practical solution in complex fitter trains. Flanders offers a complete built-in test system that can be installed between successive filter banks. Regardless of the number of filters in parallel or in series, the maximum distance between successive banks utilized by test apparatus is $28^{\prime \prime}$. (See below) (Refer to Flanders Bulletin No. 381C for further information.)

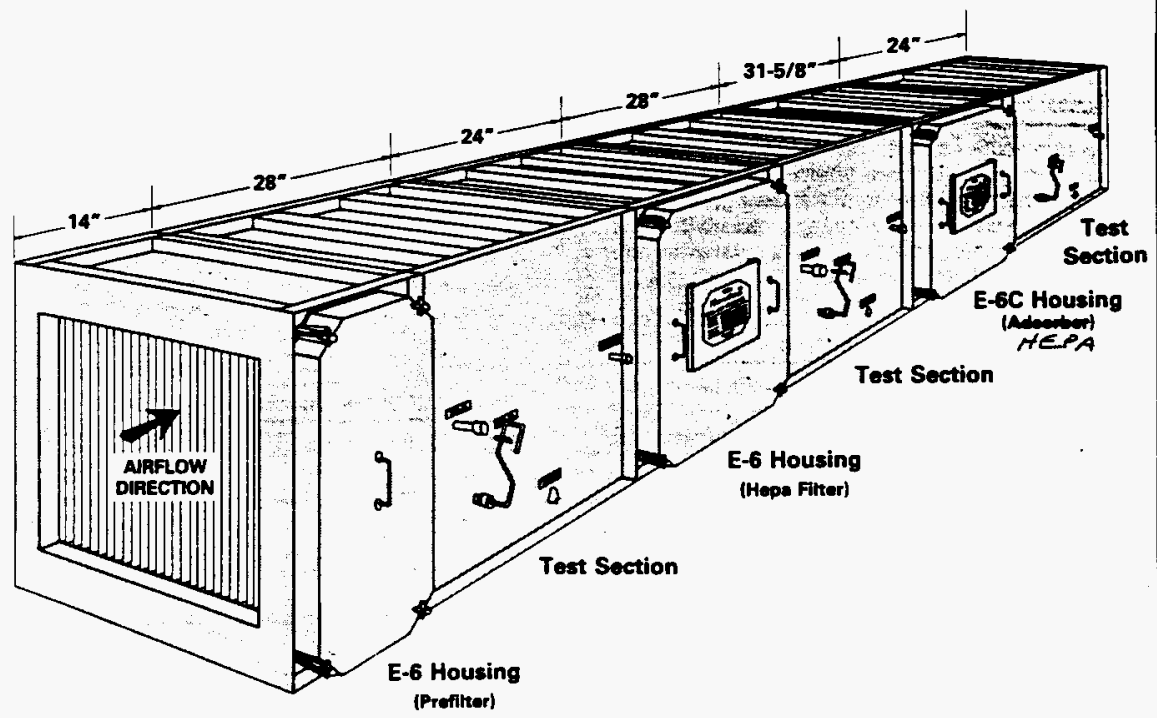

Fig. 6 - E-6 filter Housings with in-Place Test Sections $D^{C}$ MNC 13

CFLANDERS FILTERS, INC., 1988 FOREMOST PAODUCERS OF HEPA FILTERS AND SYSTEMS FOR SCIENCE AND INDUSTRY STNCE - 1990

$$
H-74
$$




\section{E-6 (Hepa Filter) Housing Component and Designating Code}

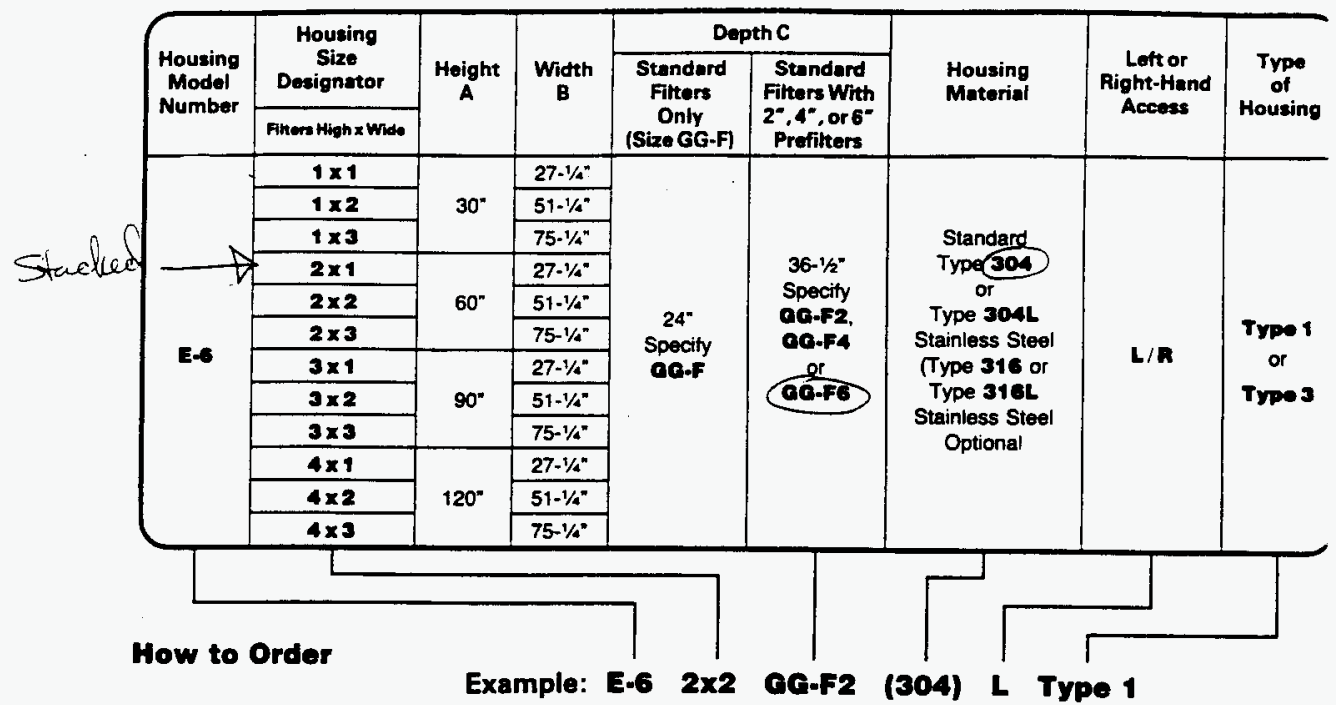

\begin{tabular}{|c|c|c|c|c|c|}
\hline \multicolumn{6}{|c|}{ Additional E-6 Information } \\
\hline \multirow{2}{*}{$\begin{array}{c}\text { Housing } \\
\text { Size } \\
\text { Designator }\end{array}$} & \multicolumn{3}{|c|}{ Nominal Capacity (CFM) } & \multicolumn{2}{|c|}{ Approximate Woight (in lbs.) } \\
\hline & $\begin{array}{l}1000 \mathrm{CFM} \\
\text { Per Fitter }\end{array}$ & $\begin{array}{l}1200 \mathrm{CFM} \\
\text { Per Filter }\end{array}$ & $\begin{array}{l}1500 \mathrm{CFM} \\
\text { Par Fitter }\end{array}$ & $\begin{array}{c}\text { GG-F } \\
\text { Housing }\end{array}$ & $\begin{array}{c}\text { GG-F2 } \\
\text { Housing }\end{array}$ \\
\hline $1 \times 1$ & 1000 & 1200 & 1500 & 180 & 300 \\
\hline $1 \times 2$ & 2000 & 2400 & 3000 & 270 & 375 \\
\hline $1 \times 3$ & 3000 & 3600 & 4500 & 335 & 480 \\
\hline $2 \times 1$ & 2000 & 2400 & 3000 & 360 & 600 \\
\hline $2 \times 2$ & 4000 & 4800 & 6000 & 540 & 750 \\
\hline $2 \times 3$ & 6000 & 7200 & 9000 & 670 & 960 \\
\hline $3 \times 1$ & 3000 & 3600 & 4500 & 540 & 900 \\
\hline $3 \times 2$ & 6000 & 7200 & 9000 & 810 & 1125 \\
\hline $3 \times 3$ & 9000 & 10800 & 13500 & 1005 & 1400 \\
\hline $4 \times 1$ & 4000 & 4800 & 6000 & 720 & 1200 \\
\hline $4 \times 2$ & 8000 & 9600 & 12000 & 1080 & 1500 \\
\hline $4 \times 3$ & 12000 & 14400 & 18000 & 1340 & 1920 \\
\hline
\end{tabular}

Type of Door Arrangement

Type 1 Housing: The standard E-6 Housing has an access door on one side and a flanged plate on the other. The owner must specity loft or right-hand access.

Type 3 Housing: This housing consists of two side-by-side housings in parallel. Access to the fitters and operation of the fitter removal rod is from both sides of the parallel housings (see page 8.)

Special E-6 Housings are oveilable upon special requent. 
SAIC ACCOPTANCE TEST PROCEDURE

\begin{tabular}{|c|c|c|}
\hline Project No:: $\quad 9277-01$ (486.1) & LESAT/950460 & \multirow{2}{*}{$\begin{array}{l}\text { EGS-TR-927701-104 } \\
\text { Revision Orig } \\
\text { Attachment: } \frac{1}{1} \text { Part: } 2 \\
\text { Page 1 of } 1\end{array}$} \\
\hline $\begin{array}{l}\text { Item Description: Bubbletight Damper } \\
\text { Model/Par No.: BTR-92 and BTO-92 }\end{array}$ & $\begin{array}{l}\text { OEM: } \frac{\text { Ruskin }}{\text { Dwg./Spec: } 50-001162-01 \mathrm{D} \text { thru }-13 \mathrm{D}}\end{array}$ & \\
\hline $\begin{array}{l}\text { Safety Function: Maintain structural } \\
\text { after a seismic event }\end{array}$ & on ontilin & Prep. by \\
\hline
\end{tabular}

1. Product İdentification

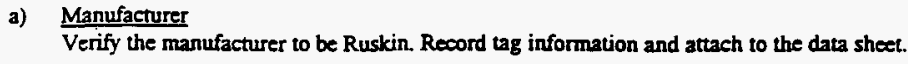
b) Model Number
Verify the model number to be as specified on Figures 1-1 through 1-13.

2. Physical Attributes
a) Dimensions
Verify dimersions are per the customer-approved drawing (Reference Figures 1-1 through 1-13) by review of the Ruskin Final Inspection Reports.
b) Configuration
Verify the damper is free from damage and is of the configuration depicted in Figures 1-1 through 1-13 or the
customer-approved drawings. Record the curtomer-approved drawing number, including revision, on the data sheet

\section{Mechanical Attributes}

a) Material

Verify the material used for construction of the dampers bas been independently tested and accepted by SAICIEGS.

The following materials shall be verified:
Frame
Blade Stiffeners
Flange
Actuator Bracket
Blade
Weld Wire

Verify all welders performing welding on the damper are cerified to ASME Section DX.

4. Electrical Attributes

Verified per Paragraph 5.

5. Operability

a) Proper Operation

Verify each damper operates as required by Ruskin Procedures Nos. 092395 and 092295. 




Figure 1-5 


\section{How to order your Turbo Mini or Turbo Cart lumping Station}

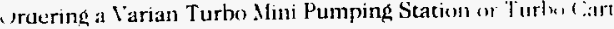
Pumping Station is quick and easy. Please usc the enclunct ()rder Form and this Price List to configure and price vint turbo pumping system

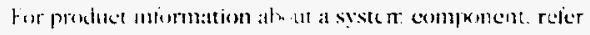

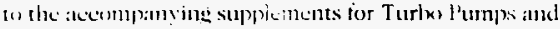

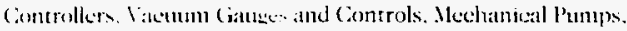

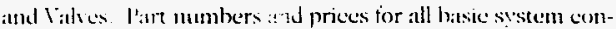
figurations and customer-sedectable optons and acessories are loc:ated in this l'ries list. It what you want is not here, please

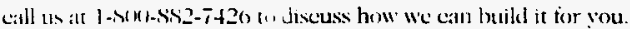

\section{\begin{tabular}{|l|l|}
\hline Turbo Cart Pumping Station & Turbo Mini Pumping Station \\
\hline
\end{tabular}}

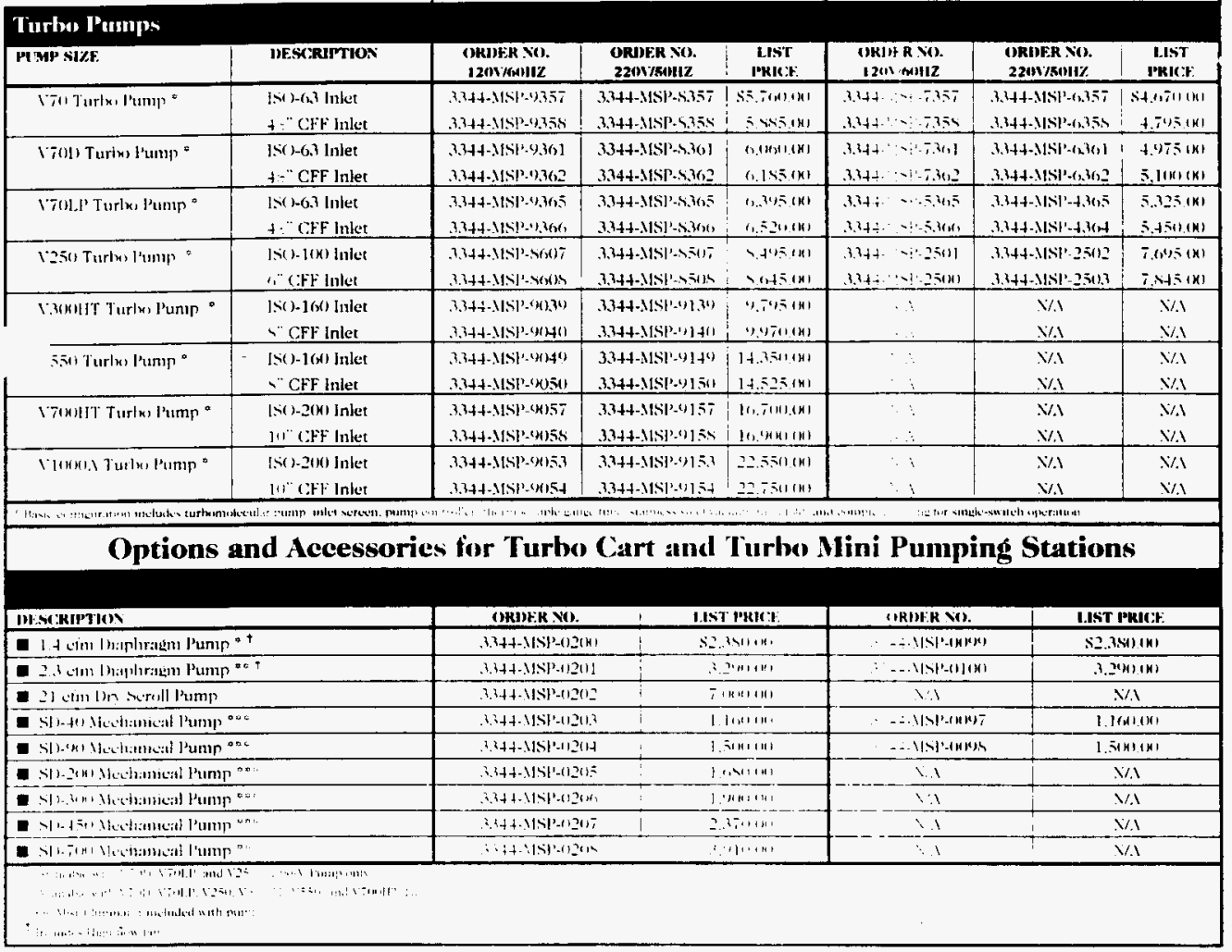

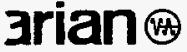

\section{vacuum producis}

Iarian I:acum J'roducts

121 Harnell Ivertuc

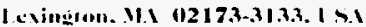

$H-78$

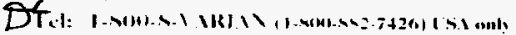

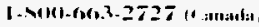

1: $12:(1.17),(61) .54 .75$ 


\begin{tabular}{|c|c|c|c|}
\hline$|x| \leq 0$ & 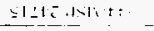 & nns & 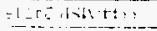 \\
\hline$|n|=E I$ & 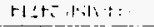 & $111 \leq 21$ & 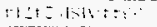 \\
\hline (A) 61 & 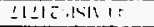 & $111=1$ & 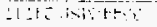 \\
\hline In) $\doteq 1$ & 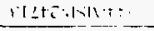 & $111 \leq+1$ & latedita \\
\hline$|n| \leq 11$ & alsteksia & $1 n !=11$ & $\because 2 t+N$ N t : \\
\hline$|n| \leq i||$ & $1125 x, 412$ & $111=111$ & $\therefore: 1-1: 4:+4$ \\
\hline$|N| \leq 4$ & 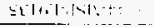 & $1111=4$ & 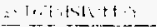 \\
\hline $111,11 \leq 1$ & 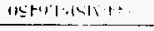 & $1+\cdots 1=1$ & toldstio \\
\hline $\mid H, 1) \leq$ & 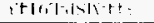 & 11111 & $+11, \ldots$ ix: $1+t+:$ \\
\hline inul. & 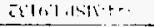 & IHrins & - $11,1911-+4:$ \\
\hline$|x| \leq \backslash 4$ & Entolisutr. & $111)=2 x$ & $=111,1,1 \times 12+511$ \\
\hline$(n) \leq 1 \leq$ & 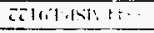 & $(11) \leq 11$ & 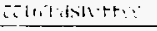 \\
\hline WIStll I & 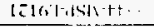 & (3) 5411 & 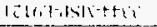 \\
\hline$|M| \leq 11 \mid T$ & melfolisilet. & $14 \leq 1,11$ & 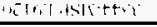 \\
\hline$|n| \leq Z \mid$ & otminalsitt: : & $|x|=2 \mid$ & 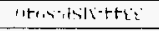 \\
\hline INIUEI & axterdsint+ & $x(1) \leq 1$ & (x) \\
\hline (x) & loterisiditt: & 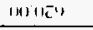 & Hathatiottis \\
\hline$|n| \leq 1 \leq$ & 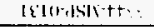 & 1115:5 & 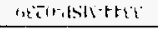 \\
\hline$|n| \leq 0,1$ & mal-mintr: & $(x) \subseteq(x)$ & 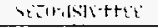 \\
\hline$(x)(1,1,5)$ & 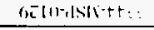 & (x) & 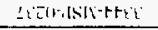 \\
\hline (ntries) & selardsistt: & $\left.131 x^{-1}\right)$ & 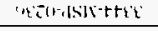 \\
\hline Ixtocs: & 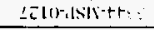 & moros' & 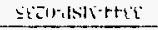 \\
\hline$(x)(u \leq t$ & "glatelsintti: & $13(1)(1) \leq t$ & tichralsi-tte \\
\hline$(x) 11 ! 2$ & 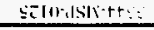 & $1 x 0010 \leq-2$ & 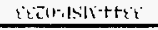 \\
\hline$|x| \leq \leq 1$ & 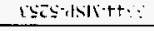 & misest & $t=こ=i N d-t+2 i$ \\
\hline$|n| \leq S$ & zEs-ant+a & $16) \leq t^{\circ}$ & 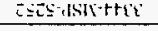 \\
\hline$M A=Z$ & ISÉtsilett: : & $M=E E$ & 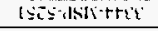 \\
\hline$|x| \leq i t$ & tento-dslitta: & mosict & 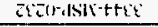 \\
\hline$(n) \leq 1 t$ & 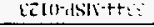 & $(x) \leq 1 t$ & 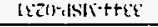 \\
\hline$(x) \subseteq 40$ & zelordsinta: & Ixts $=8 t^{\circ}$ & mę)-ASIL-t+C \\
\hline$|x| \leq 2$ & 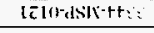 & $1 x) \leq 16$ & föcu-dSIS-t+tic \\
\hline$|x| \leq 24$ & (6) & $|x| \leq 16$ & 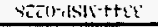 \\
\hline twitil & bllodsingt+a & initic & sétr-asli-ttat \\
\hline InI But & Sillo-4silett: & $(H)(x)$ & "get)-rSIT-t+es \\
\hline 1915i: & Illordsilett: & (x) & 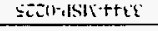 \\
\hline inilis: & 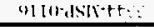 & MQES: & tEGIn-ISIS-ttic \\
\hline$\left.(N 1 \leq)^{4}\right)$ & $\sum$ IIII-4III-t+: : & (HISN) & 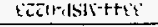 \\
\hline$\left.(x)^{10}\right)(-1)$ & tllo-dsilit+a & $(x) \cdot 9+6$ & $\operatorname{cac}(0-$ dSIN-t+is \\
\hline (1) $4=5$ & E้ll1-dSIS+tt & IMSSE & LECb-asintted \\
\hline (n) $1=15$ & Ellordsitrta & MULIS & 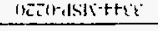 \\
\hline $1 \times 151=$ & $11111-4 \leq 16-t+\because:$ & MISYEZ & GLEOLISISt+tis \\
\hline$(n) \leq(4)$ & 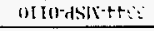 & (N)SK) & SIZOO-ISIN-t+i: \\
\hline (n) $=2 t$ & (x)lo-dsilitti: & $100 \leq-1$ & 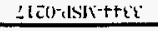 \\
\hline$(n) \omega$ & oles-dsintti: & 10096 & gIt-dSIV+tte \\
\hline (n) it & stre-dsirtti: & 0086 & SIRS-dSIV-t+ce \\
\hline 1xitis & hl6s-ash+tai & 0086 & tIES-dSIIVtte \\
\hline$(x) \in S$ & Elcsisistti: & 10168 & kLES-dNIV-ttic \\
\hline$(x) \leq 5$ & cIre-asinttis & $001 \leq 8$ & zIES-dSW-ttes \\
\hline (n) is & $118 \mathrm{~s}-\mathrm{dSIN}+t i 2$ & 99198 & IIES-dSIV+tec \\
\hline InI $\leq t=$ & solo-dsinttii & $00 \leq 5$ & $9120-\mathrm{dSI})^{-5 t+C}$ \\
\hline iks sit & 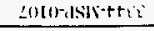 & (M) $\leq L t$ & $\$ 120-d S J-t+2 \varepsilon$ \\
\hline$(x) \leq l \vec{c}$ & molu-disinttaz & intete & taco-ANin-ttic \\
\hline$(x)+\infty)$ & 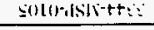 & $(n)+(x) \vec{c}$ & $i \mathrm{~L}\left(0-\mathrm{dSN}-5+\mathrm{Cl}^{2}\right.$ \\
\hline (n) $\leq \bar{c}$ & folo-dsil-t+s & $10 \div 26$ & zIzO-ASII-ttiz \\
\hline$|k| \leq \leq t$ & zolor-asinttiz & 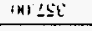 & I Izorasis-ttit \\
\hline$(k) \leq E)$ & zolirasist+a & $x \rightarrow \leq E$ & olatodsi-ttit \\
\hline$|k| \leq x S$ & Ioll-asilttis & 1n1 $\leq \leq \mathrm{ES}$ & menodsirttic \\
\hline F) Iमd INI & ox y:taryo & f.JPLA ISI7 & Ox нanlus? \\
\hline
\end{tabular}

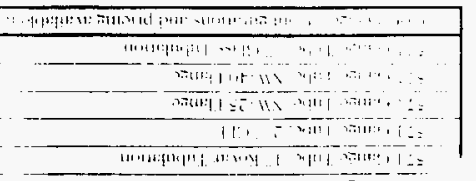


WHC-SD-SNF-CDR-007, REV. 0

\section{(inuge Controller (Options (eontimued)}

\section{of ST:RIMT)}

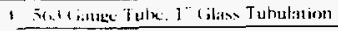

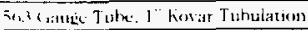

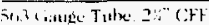

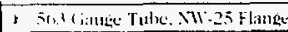

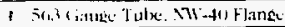

" Thet range Tulke. 1" (ilass Tubulation

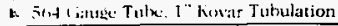

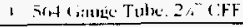

1 ind 1,inge Tulx N11:-25 Flange

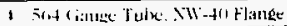

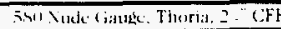

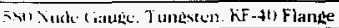

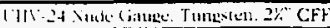

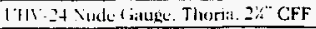

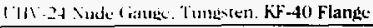

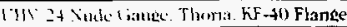

Inthin, andmi:

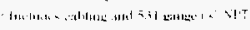

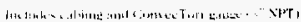

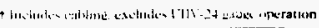

\section{Turbo Pump Accessories}

\section{Divis:kilption}

Vuning lowices

- Emerecacy Vint

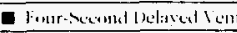

- Viltmatihle Vulnt

tirc Amuling

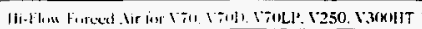

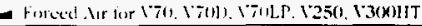

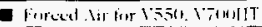

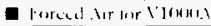

Wuler Comition:

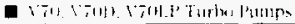

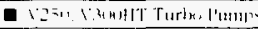

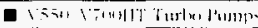

Ilemeler lisumals

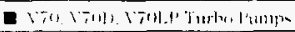

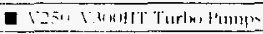

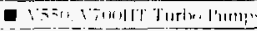

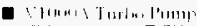

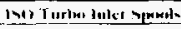

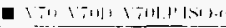

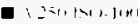

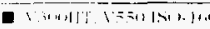

-

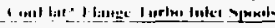

-

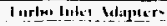

- !
Turbo Cart Pumping station

Turbo-Mini Pumping Station

\begin{tabular}{|c|c|c|c|}
\hline ORINER YO. & L.IST PRICY: & ARIDEA VII. & LIST PRICE: \\
\hline $3.3+4-115]^{\prime}-34601$ & $51251 k+$ & $\therefore+4-1151$ '-24ro,l & $5 ! 35(N)$ \\
\hline $3.4+4.35 t^{2}-24002$ & 1.35131 & 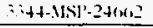 & $1.15(N)$ \\
\hline $3,44-115 \mathrm{P}-2400.3$ & $\ln 5(\mathrm{n})$ & $\left.\therefore+4-x_{1}\right)^{2}-2+\left(x_{1}, 3\right.$ & $1(1,5.1 M)$ \\
\hline $3.34+-115 P^{3}-2+1004$ & $1+21 n 1$ & $\therefore+4 t-1$ ISI. 24004 & $1+2(x)$ \\
\hline $3.7+4-\left.115\right|^{2}-24065$ & $155.1 x_{1}$ & $\because 4 t-\left.11 S\right|^{\prime}-2+16,5$ & $15 \bar{S}(\mathrm{~N})$ \\
\hline $33+4-M[S P \cdot 2500]$ & 15.5141 & $\therefore+4+\left\{1 \leqslant k^{\prime}-251\right)(1\}$ & $15.5(n)$ \\
\hline $3344-115 P-250(12$ & 195 (n) & $\therefore+4.1961 \cdot 25(\mathrm{Cl} 2$ & 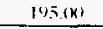 \\
\hline $3,34+-11 S P+25(x)\}$ & $225(x)$ & $\therefore 44-1 / 51235(x) 3$ & 225141 \\
\hline 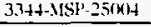 & $2(x)(x)$ & $\therefore 34-1151$-25CKH & $2015(x)$ \\
\hline $334+-11 S^{\prime}-25005$ & $215(H)$ & $\because 44-1151$-25IKL & $215(x)$ \\
\hline $3.34+4.151^{2}-515012$ & $3+5.611$ & $\therefore+4+11+1)-51502$ & $3451 \mathrm{~K} !$ \\
\hline $3.3+4-M I S P-51505$ & อลร. & $\therefore 4+4.11818-51515$ & $275(x)$ \\
\hline $3.344-115 \mathrm{I}^{2}-\mathbf{5}(\mathrm{x}) \mathrm{s}$ & $3+5.061$ & $\therefore 4+-.11 \mathrm{il} \cdot 5 \mathrm{Km}$ & $.345,0 \mathrm{MH}$ \\
\hline $.33+4-155 P-5 n=$ & $3(x+1)(k)$ & $\therefore+4-3|S|$ S $-5(x)$ & $3(1,1)(x)$ \\
\hline $3344-15 P-06511$ & +1111111 & $\therefore+4-11 S)^{\prime} \cdot\left(n_{1}, 511\right.$ & $+111 .(n)$ \\
\hline $3.344-315[-605] 2$ & $f f(1) ; k\}$ & $\because 7+-31590,-60517$ & \\
\hline
\end{tabular}

BEST AVALABLE COPY 


\section{Turbo Cart Pumping̣ Stationt

\section{Vialucs}

IDES:KIPTICS

\section{tou Inler Vulve.}

or V71. V7011. Y701.1 Gar and Mini Stationn

- 26" CFF IU/ ) limeir (inte Valke

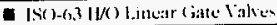

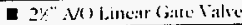

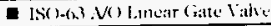

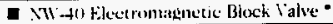

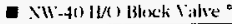

- NII-411.VI Block Valve "

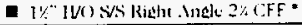

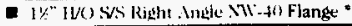

- $1 \% "$ NU) SA Risht Angle $2 \%$ (CFF*

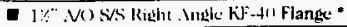

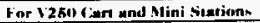

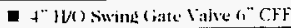

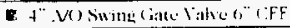

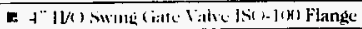

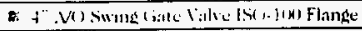

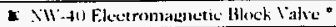

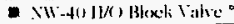

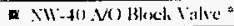

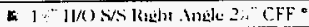

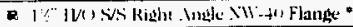

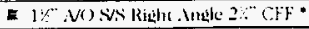

- 1\%*No SS Righ . Ungle KF.tu Flange * or VMMUlT. VERIl Ciart Station.

b" lUf ) swing (iate Valwe s" CFF

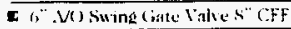

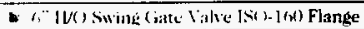

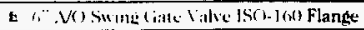

55 $>11-40$ Flectromanetic Bloch Valve *

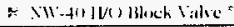

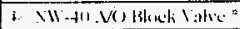

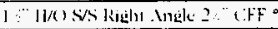

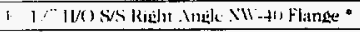

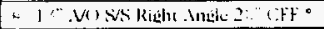

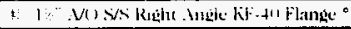

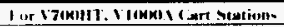

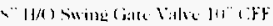

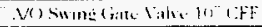

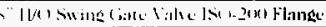

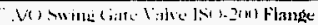

fareline tulues

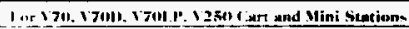

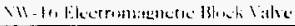

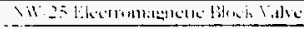

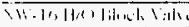

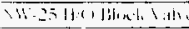

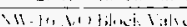

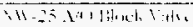

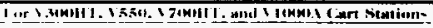

113. finlede

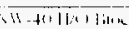

11- +1, in fine

\begin{tabular}{l}
$\mid$ \\
\hline \\
\\
\end{tabular}

ORILER NO.

L.1NT PRICE

(IRI) N NO.

LIST PRIC:

$.3,44-.15 S^{2}-0261$

$3.344-195 P^{2}-0262$

$\therefore 1.45$ in

$3.34+-115 P^{2}-(1) 2(2,3$

3344-.14SP-1)26-

$3344-14 S P-1) 205$

$.3 .34+-1.15 P-10260$

$3.3+4+.11 S P-11267$

$33+4-195 P-0265$

$3.344-915 P^{-1}-(1269)$

$.374-16 \mathrm{SP}^{\mathrm{P}}-(1) 270$

$3.344-14 S P-() 27]$

$3.2(k+)$

$1,525 \ln (4$

J. $3(x) 130$

51500

$205, k)$

.45100

$415(4)$

$315 \mathrm{int}$

$3.344+\mathrm{MSP}-(0272$

475 int

$.3,3+4-115$ SP $^{2}(127.3$

$3.34+.3 \mathrm{SP}-(1)-24$

$3.34+-115 t^{2}-112-5$

$\left.3,3^{2}+4-1 / 5 p^{2}-1\right) 270$

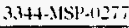

$3.3+4 \cdot 1 / 5 T^{2}-11275$

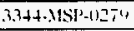

$3.3+4-3151-412 \times 11$

.3.344-1151'-(1)2>1

$3.344-115 P-1) 252$

$+$

-1.

$+$

$(3-4+1) \div 3(1145$

$-4+3\left(3^{2}+01+6\right.$

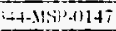

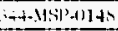

$3+4-1351^{2}-(1) 140$

$24+-115] \cdot(1] 53$

$30+4-19 S\left[\begin{array}{l}3 \\ 3\end{array}(15]\right.$

$3+4-1451^{3}-(1152$

$4+.3151+1153$

$\because+4-115 P^{2}-0154$

$3+4+2151+1155$

$\$ 1.425(x)$

$1.2(x)(x)$

$1.525(x)$

$1.3(x)(k)$

sis $(x)$

$26.5(x)$

$3+5 .(x)$

$415 .(x)$

4(H):4)

$.33+4-1355-(1) 243$

$334+-\left.115\right|_{1}-1254$

$33+4-1181 \cdot(1245$

$3.34+-118131956$

$3.3+4-1151$-1335:

$3,3+4-\left.115\right|^{3}-13246$

$1,690(1)$

$\therefore+4+-14 S T^{-}-1272$

$\therefore+4+-11<1^{2}+037.3$

1.75041

$\therefore+4-115)^{2}-11254$

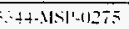

3)5.(x)

$3.3+4-1158 \cdot(1349$

1.

$4+-.4151-112 \pi$

$455(x)$

$4+-.115 l^{\prime}-(1277$

$4+-11 S J^{\prime}-112 \pi s$

$\therefore+4-11 \leqslant 11-(1)=20$

$4(x),(x)$

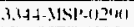

$3.344-115[10(12)$

$++-14 \times 1)-(12 \leqslant 0)$

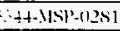

$\left.\therefore+4-(4)^{3}-1\right) 2 \times 2$

1.050 .00

3.4+4-1156t-1121]

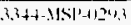

z. J(n) $(x)$

$2.375(1)$

2.31414)

$2.45 \sin$

1.115.14\%

स1, है।

54.

i, $1=1 \mu_{1}$

ज11, 311

3itinger

11.

(x)11+11?

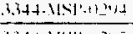

$.134+-\left.115\right|^{3}-41^{2}=$

$3,3+4.115$

$3,3+4-8|5|^{3}+112,1$

tonin

ing

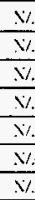

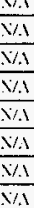

$\mathrm{v} / \mathrm{i}$

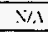

is

1.75 .00

1.h.5(I) (n)

[075(N)

Slsiki

$205 .(x)$

$3+5 .(x)$

$+1.5(M)$

, $(05 .(x)$

$\operatorname{tin}(x)$

$f(x)(0)$

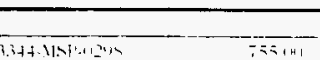

$34+9 \times 190$

$3.4+1-1 / 49$

$\frac{3+4 ! 31}{3.4+11<1}$

a.t+-iki

$2+95+141$

305144

3. 3514

vis

\begin{tabular}{|c|c|}
\hline$\forall /$ & $\mathrm{NA}$ \\
\hline$\forall / 1$ & $6 / 1$ \\
\hline 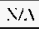 & $\mathrm{B}$ \\
\hline$\times / 1$ & $\%$ \\
\hline
\end{tabular}

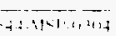

A- IVI

$\therefore i+14=1$

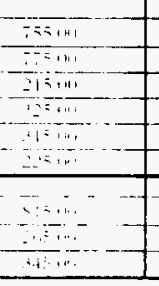

$+4-1151,-111 x$

TลS (x)

Xix

$+7-21 \mathrm{Sr}-11157$

$\mathrm{N} / \mathrm{s}$

$4+1610-1115$

?]र(n)

is

$\frac{\mathrm{X}}{415+14}$

Na

in

in

$\mathrm{N} / \mathrm{i}$ 


\section{Turbo-V550}

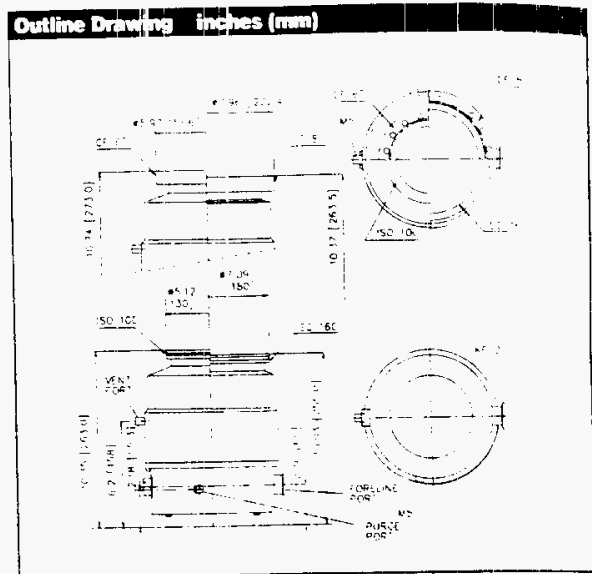

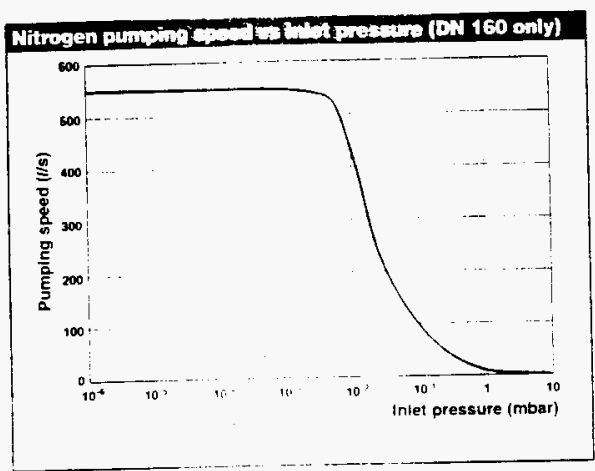

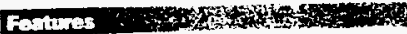

- Macrotorr stages

- Excellent reliability

- High compression ...... riani gases

- Standard gas purgi :

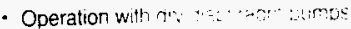

- Installation rr. ari . i...

- Ceramic bearing:

- Maintenancér.

\section{Compression ratio vs foreline pressure}

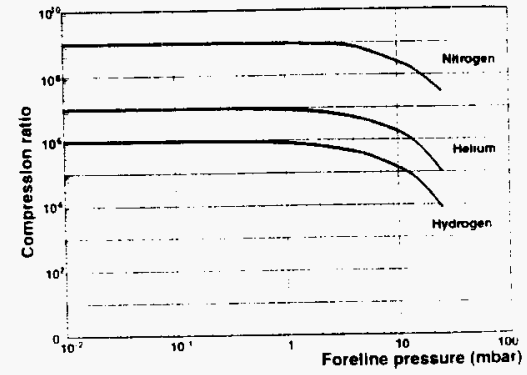

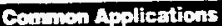

- HV and UHV technology

- Semiconductor process technology

- Physicsiresearch/accelerators

- Eiectron microscopy

- Industrial applications

- Mass spectrometry

MNL 14

$$
H-83
$$

BEST AVAILABLE COPY 


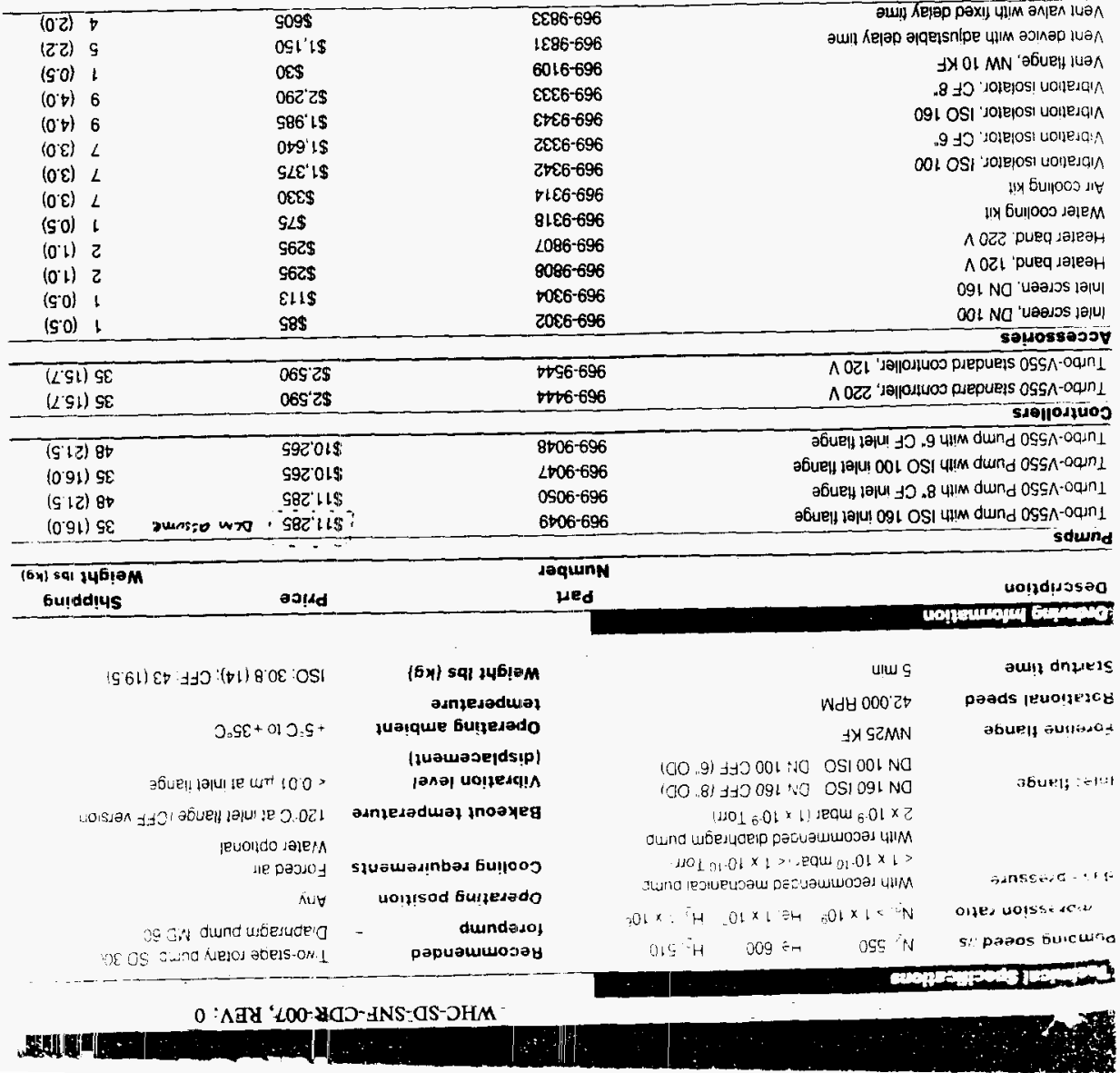



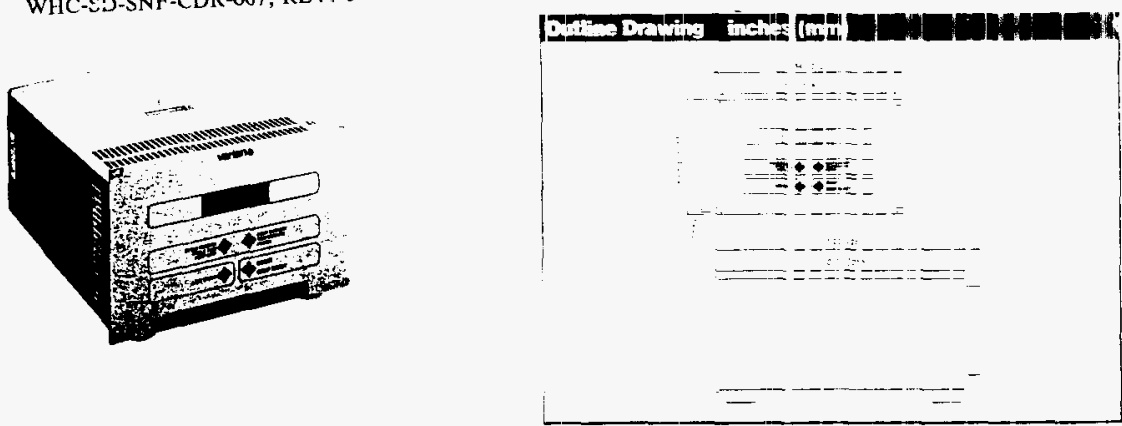

These Turbo-V controllers are microprocessor-controlked trequency converters with self diagnostic and protection features that ensure the highest degree of reliability. The compact, $1 / 2$ rack unit has a multifunction alphanumeric display for pump status and error code diagnostics. The tront panel display is a two-line dot matrix LCD display with back lighting. It displays rotational speed as the pump starts up and indicates when full speed is reached. At any time during the operation of the pump, the speed, current,

power, and bearing temperature can be displayed. Additionally. the microprocessor acts as a pump cycle log, and can display the number of vacuum cycles, the cycle time for the current cycle. and the total operating hours on the pump. Remote operation can be accomplished with logic level contact closures, and with optional computer interfaces.

PCB controllers are available on request.

Technical Specifications

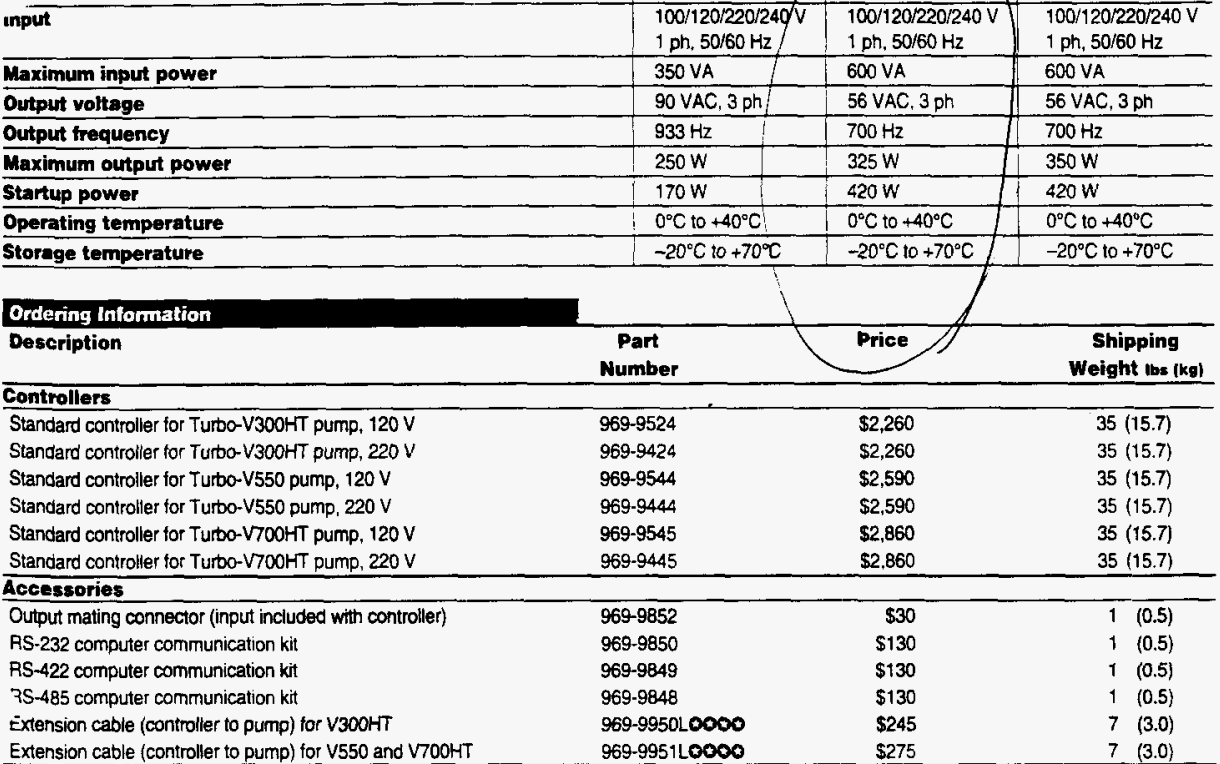

0000 insert the length in centimelers (example: cable $1.5 \mathrm{~m}$ long is $969-9950 \mathrm{~L} 0150$ ). If the hwo connectors only are required, order 969-9950L0000 or 969-9951L0000 $H-85$ 


\section{High-Vacuuın Pirani Gauge}

[IEN

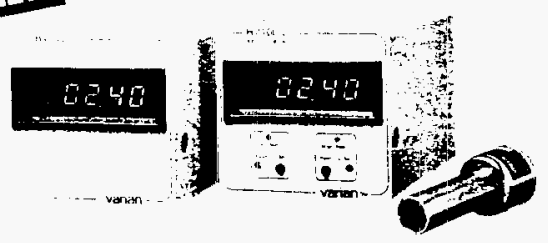

The Varlan HV100 and HV100-2 High-Vacuum Pirani Gauges; measure pressure from $0.011 \mathrm{mTorr}$ to $100 \mathrm{mTorr}\left(10^{-5} \mathrm{mbar}\right.$ to $0.1 \mathrm{mbar}$ and ofier precision and high resolution digital electronics. These compact instruments use a newly-developed Pirani sensor which incorporates an upscale trim to improve tracking abilty. The extremely rugged sensor is constructed with a solid, inert. noble metal that mantains its calibration over long penods.

Lower operating temperatures in the Pirani-type gauge tube (half that of thermopile gaugesi enhances stability as does a unique temperature compensation network. The gauge tubes are matched so they can be replaced without recalibration

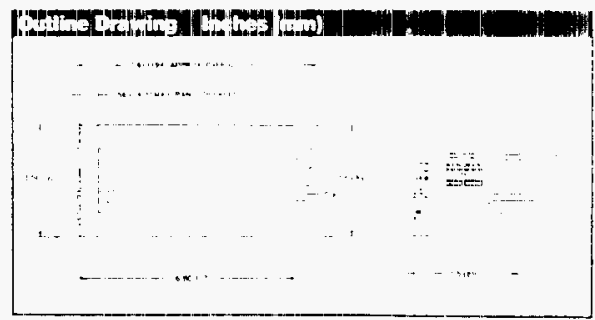

The Varian HV100-2 dual set cont controller operates two inde. pendently-set control relays. Set point values are easily adjusted from the front panel and displeved on the meter. Pusin-10-set switches simplify display of the Eet point pressures, without interrupting the pressure measure-ent circuit. Felays have 3 -ampere. Form $C$ contacts. Sirrew term - als for the relay outputs are provided on the rear panel.

Both the indicator and the co:-: cliter instruments teature simple. single-hole installation The ne..: panel mounting system makes panel mounting as easy as tu-n a screw. Gauge tubes have $3 / 4$ inch O.D. tubulation that fits siandard quick connects.

\section{Fertumes Bandits}

- Linear anaiog output 0 to $1.0 \mathrm{VDC}$

- Ideal for analytical as well $a s$ industrial uses

- Optional duai set ponıs

- Process control with \pm 0.02 Torr $\left( \pm 2 \times 10^{-5}\right.$ mbar $)$ set point reliability

- 115 VAC. $50 / 60 \mathrm{~Hz}: 230$ VAC

- Line regulation

- Temperature compensation

- Digital LED display
- Meets all power requireme- $s$ at no additional cost

- Change in line voltage proc_zes less than $1 \%$ change in reading

- Changes in ambient tempe $z$ :ure between $0^{\circ} \mathrm{C}$ and $50^{\circ} \mathrm{C}$ change the reading less the $e^{-} 0.004 \mathrm{mTorr}\left(5 \times 10^{-6} \mathrm{mbar}\right)$ per ${ }^{\circ} \mathrm{C}$ at hard vacuum

- Crisp. clear, and sharp pressure reading 


\section{(1)}

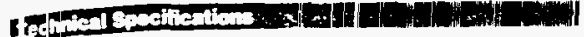

je

001 nTorr to $100 \mathrm{miT}$.

(. $0^{5}$ mba: to $01 \mathrm{mbar}$;

fesilution

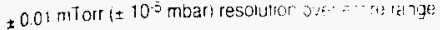

Accuracy

Eerter than $5 \%$ of value or $\pm 0.03 \mathrm{mT}$ or : $: \div 10$ mba

whiclever is larger

Sot Point Range

Adju:tiable over $100 \%$ of range

Set Point Repeatability

$\pm 0 .(12$ mTorr

(ะ $2 \times 10^{-5}$ mbar)

Recorder Output

0 to 1.000 VDC

\section{Catibration}

For accurate and easy calibration, an optional vacuum gauge calibrator is available. See Ordering iniormation for part numbers.
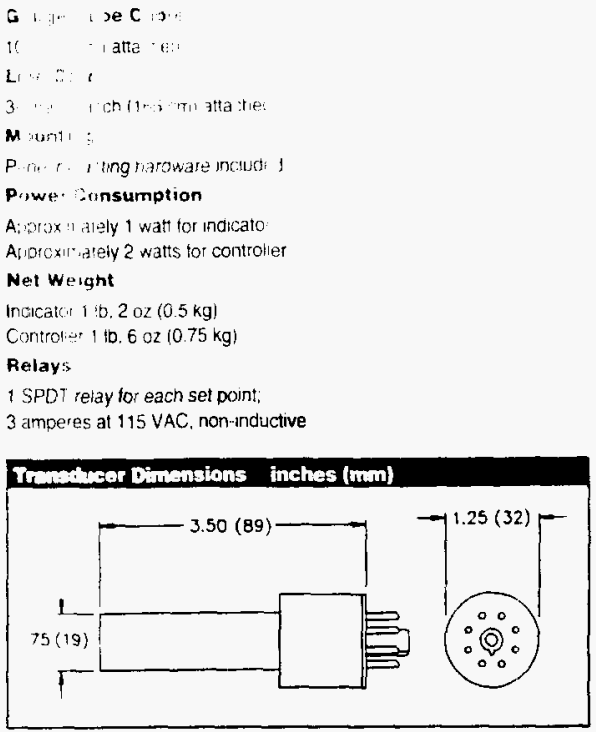

\section{Ordering Information}

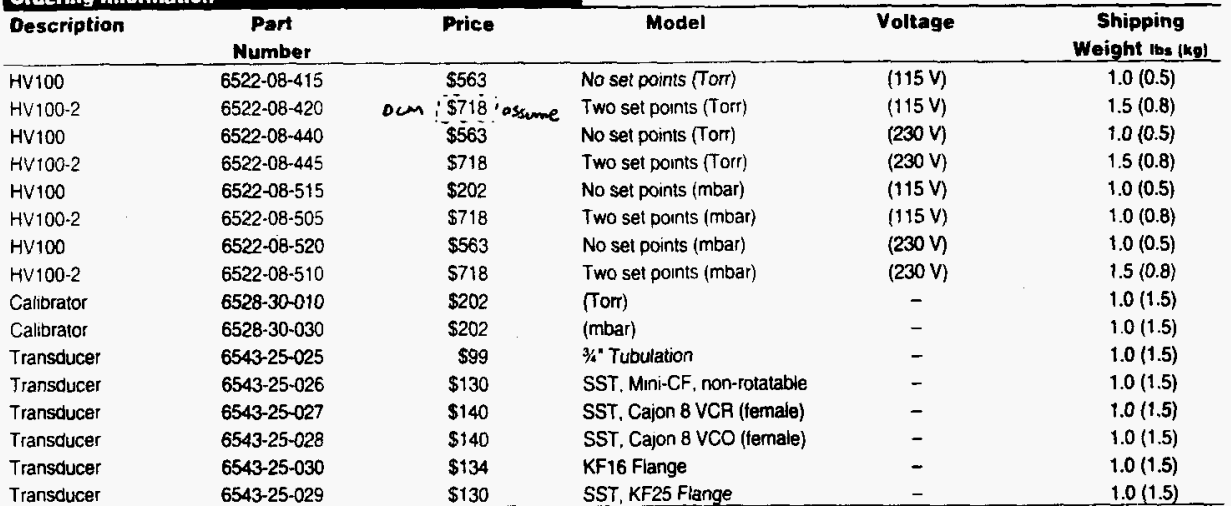

NOTE - Special-length cable quotations are available upon request. 


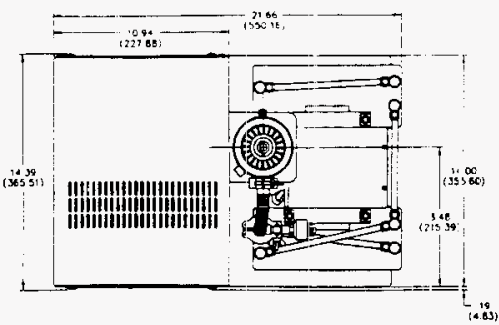

Turbo Pump Mini Station
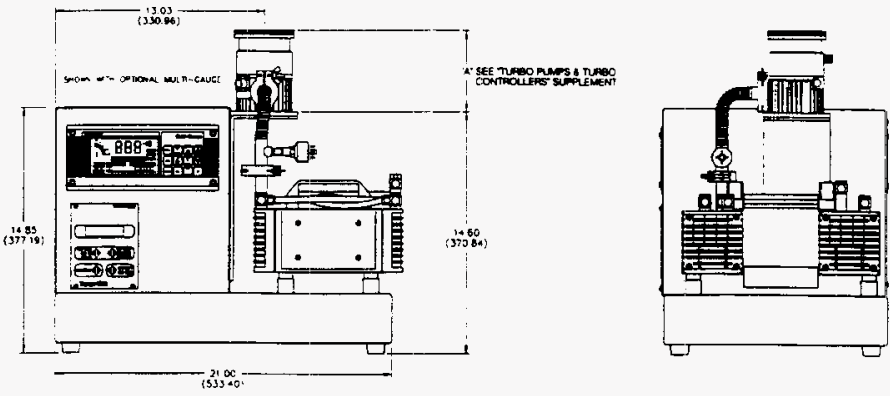

2

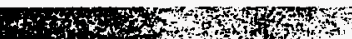

से 3

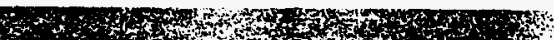

\section{Turbo Cart Pumping Station}
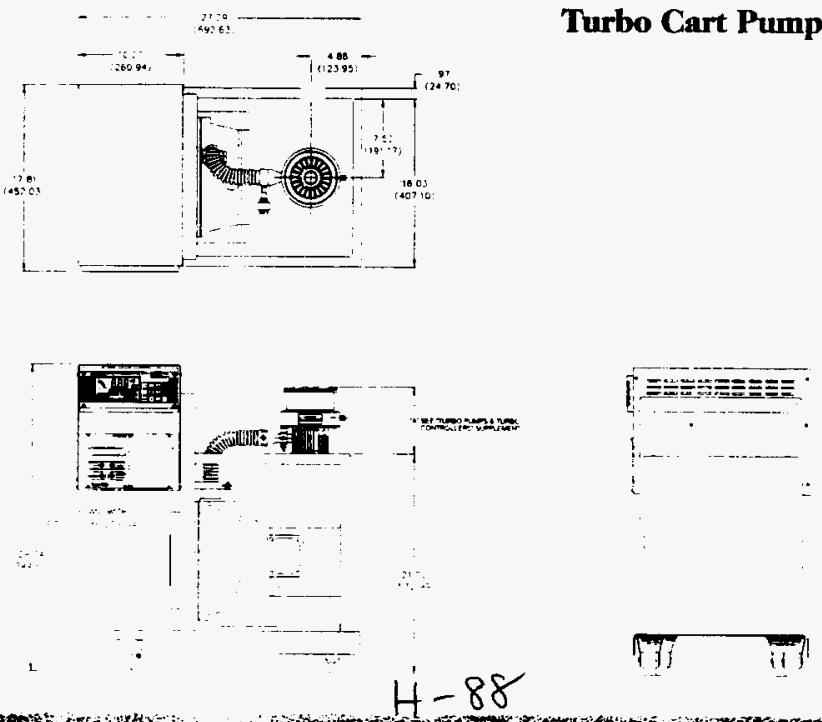


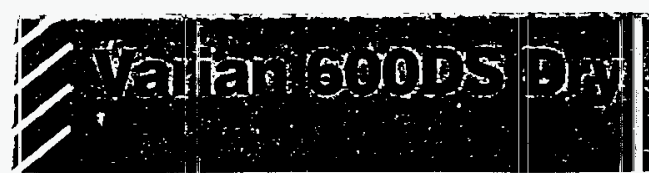

WFEC-SD-SNF- ZDR-007, REV. I

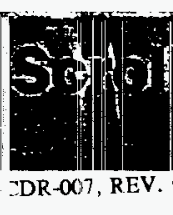

ariar's New 600DS Dry Scroll Pump ncorporate a technology that prodices clean, cost effective, and re table vacul.m pumping performance

Unlike many other types of dry pumps. the 600DS hial both a low ultimate pressure and high pumping speei. With a base pressure in the $10^{-3}$ Torr rangre and supe ior pumping efficiency, it is suitable for applications whe $e$ oil-sealed inechanical pumps have typically been usec .

In the oil-free scroll pump, crescent-shaped pockets ale formed and bounded by the meshed scrolls. As the orbiting scroll moves within the fixed scroll, these poikets progressively decrease in volume as they move the: pumped gases in the spiral path from iniet to discharge. Sealing is accomplished with PTFE seals against anodized aluminum walls eliminating the need for oil sealing.

Because the scroll pump is intrinsically clean, there is no risk of contaminating your vacuum system. Conventional oil sealed mechanical pumps require costly accessories (traps and baffles) to achieve only marginal levels of cleanliness. Since no oils are required, the costs of purchasing and disposal of them are eliminated. Few moving parts and carefully chosen materials of construction minimize pump failures, with typical maintenance intervals of twelve to eighteen months. All these factors contribute to low cost of ownership and maximum uptime.

Varian's 600DS Dry Scroll pump is the preferred choice for your clean high vacuum requirements.

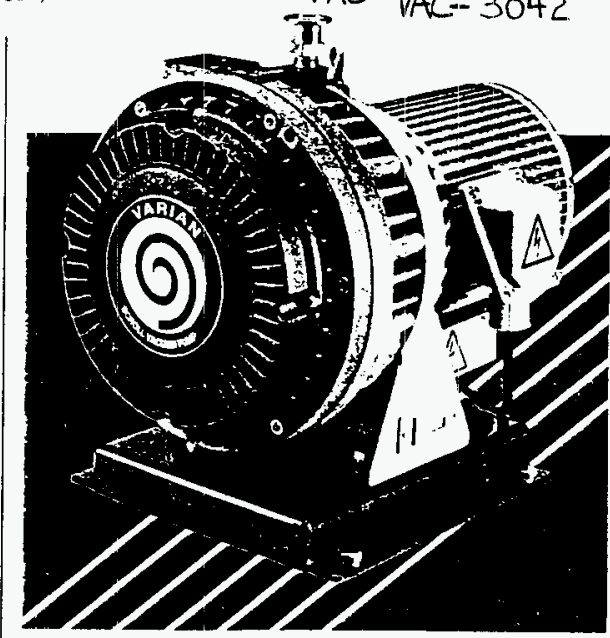
VAC - VAC- 3042

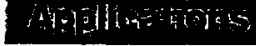

- Turbo. Ion, or Cryo Pumped Systems

- Load Lock Chambers

- Leak Detection Systems

- Optics

- Research

- Any application where the presence of hydrocarbons is detrmental to your process

\section{Bentitis:}

- Scroll Design

- Low ultimate pressure

- High speed

- Compact size

- Quiet, low vibration operation

- Two moving parts

- Long service life

Oil Free

- No risk of oil contamination

a No expensive traps or filters

I No costs of buying and disposing of oil

Carefully chosen construction materials

a Long life operation

a Low maintenance

$H-89$. Low cost of ownership

MNC 16 


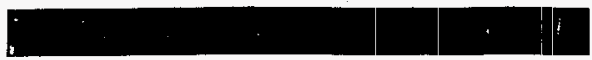

- Free Air Displacement

WHC-SD-SNF-CDR-007, REV. 0

$60 \mathrm{~Hz} \quad 600 \mathrm{l} / \mathrm{m} 121 \mathrm{cfm}$

$50 \mathrm{~Hz} \quad 500 \mathrm{~lm} 117.5 \mathrm{~cm}$

- Pumping Speed

$60 \mathrm{~Hz} \quad 500 \mathrm{l} / \mathrm{m}(17.5 \mathrm{cfm})$

$50 \mathrm{~Hz} \quad 420 \mathrm{l} / \mathrm{m}(15 \mathrm{cfm})$

n Utimate Total Pressure

$<10^{-2}$ Tort (mbar)

Inlet Connection

NW-40

- Outlet Connection

NW-25

\section{Motor Rating}

$0.8 \mathrm{hp}(0.6 \mathrm{~kW})$

- Electrical Supply

1 Phase $/ 60 \mathrm{~Hz} / 120 \mathrm{~V}$

3 Phase $/ 60 \mathrm{~Hz} / 208 \mathrm{~V}$

noise Level at 1 meter

60 dBA

- Operating Range

$40^{\circ} \mathrm{F}$ to $105^{\circ} \mathrm{F}\left(5^{\circ} \mathrm{C}\right.$ to $\left.40^{\circ} \mathrm{C}\right)$

Weight

$88.2 \mathrm{lb}(40.0 \mathrm{~kg})$

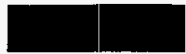

Varian Pait No. 600 DS- 16120

\section{Description}

Model $600 \mathrm{DS}$ with base, hour meter. and direct drive motor $1 \mathrm{ph} / 60 \mathrm{~Hz} / 120 \mathrm{~V}$

$600 \mathrm{DS}-36208$ Model 600DS with base, hour meter. and direct drive motor $(3 \mathrm{ph} / 60 \mathrm{~Hz} / 208 \mathrm{~V}$

600DS-KIMI 600DS-KIMA

Minor Maintenance Kit Major Maintenance Kit
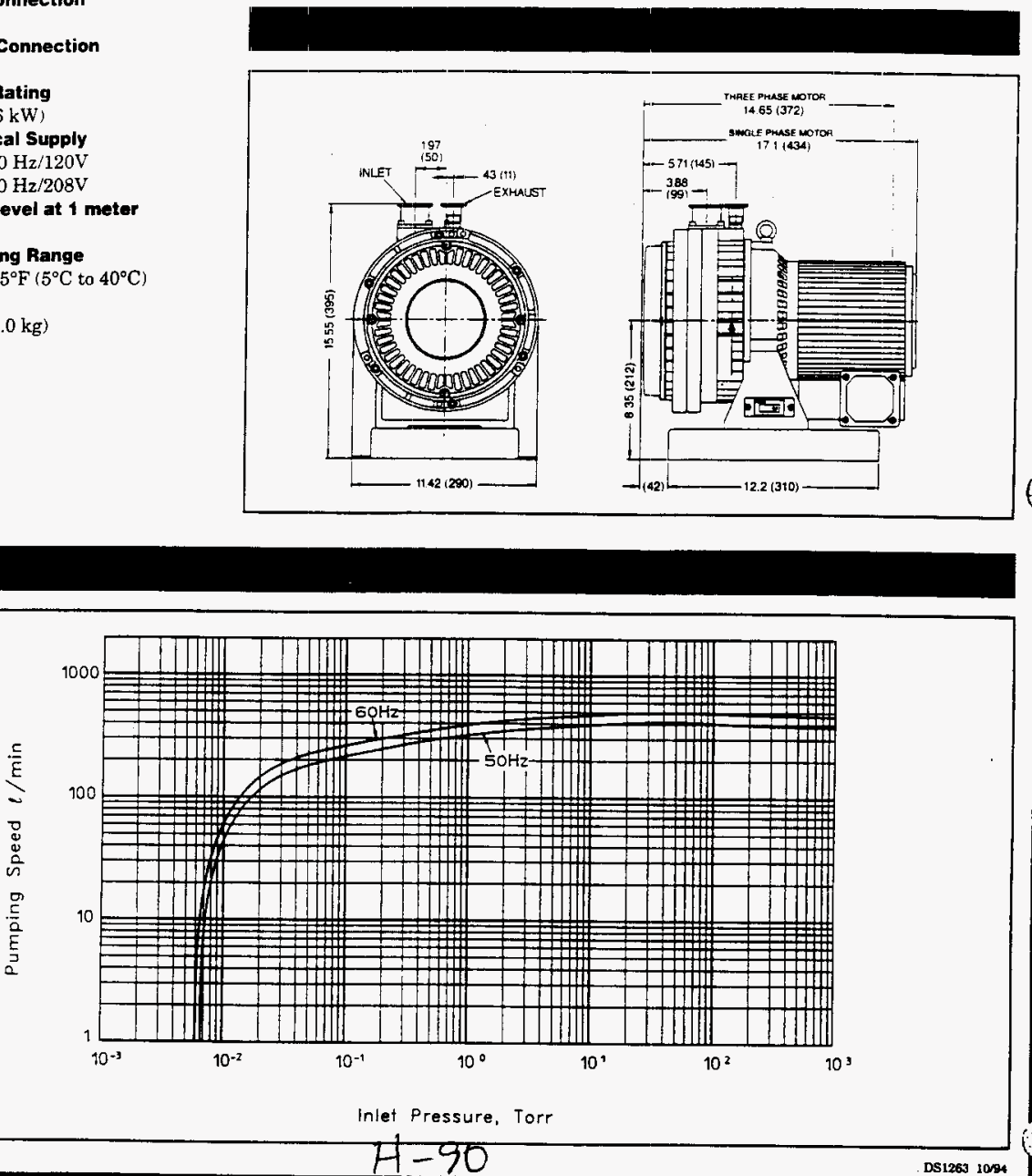


\section{Magnetic-Drive Regenerative Blower}

$$
\begin{gathered}
\text { VPS-BSLO-1106 } \\
\$ 24,068 \\
\text { MNC } 36
\end{gathered}
$$

FEATUR:S

- Hermetically designed seal-less blower

- Continuous operation

- Manufactured in the USA

- Maximum flow: 29 SCFM

- Maximum pressure: 25" WG

- Maximum vacuum: $23^{\prime \prime}$ WG

- 0.25 HP TEFC motor standard

- Disconnect motor without disassembling from piping

- Blower construction - cast aluminum housing. impeller and flanges; permanently sealed ball bearings in housing

- Motor construction - permanently sealed ball bearings

- Quiet operation within OSHA standards

\section{OPTIONS}

- Explosion-proof motors

- $50 \mathrm{~Hz}$ motor

- International voltages

- Corrosion resistant surface treatments

\section{ACCESSORIES}

- Moisture separators

- Explosion-proof motor starters

- Inline \& inlet filters

- Vacuum \& pressure gauges

- Reliet valves

- External mufflers

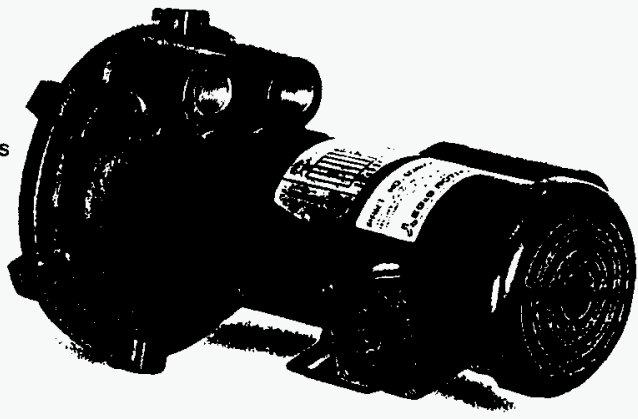

\section{BLOWER PERFORMANCE}

AIR FLOW RATE (MI/MIN)
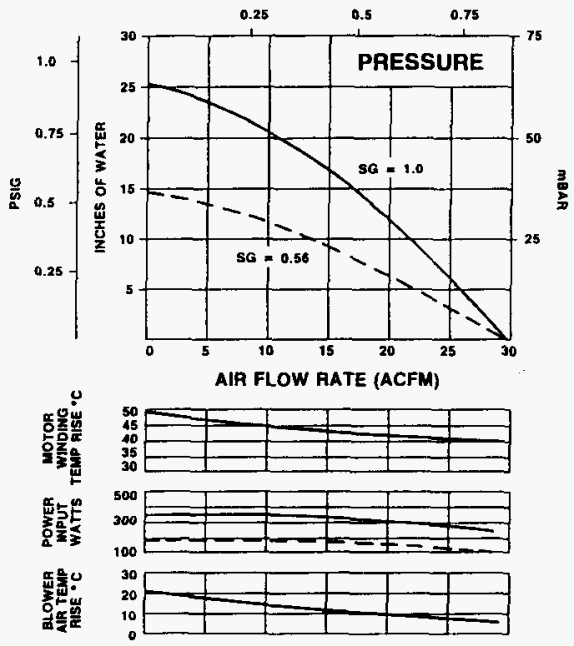

AIR FLOW RATE (M3/MIN)

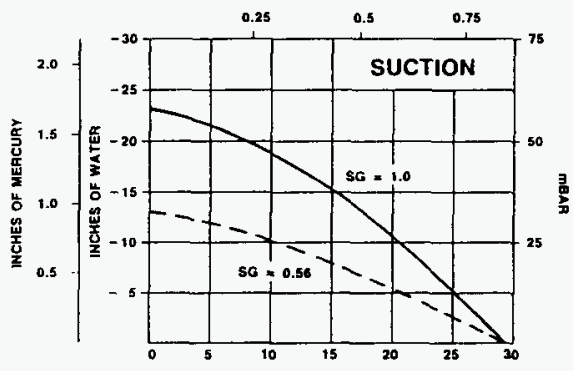

AIR FLOW RATE (ACFM)

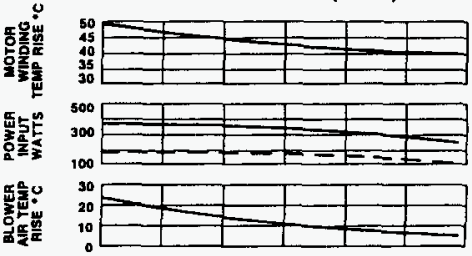




\section{101 \\ Magnetic-Drive Regenerative Blower}
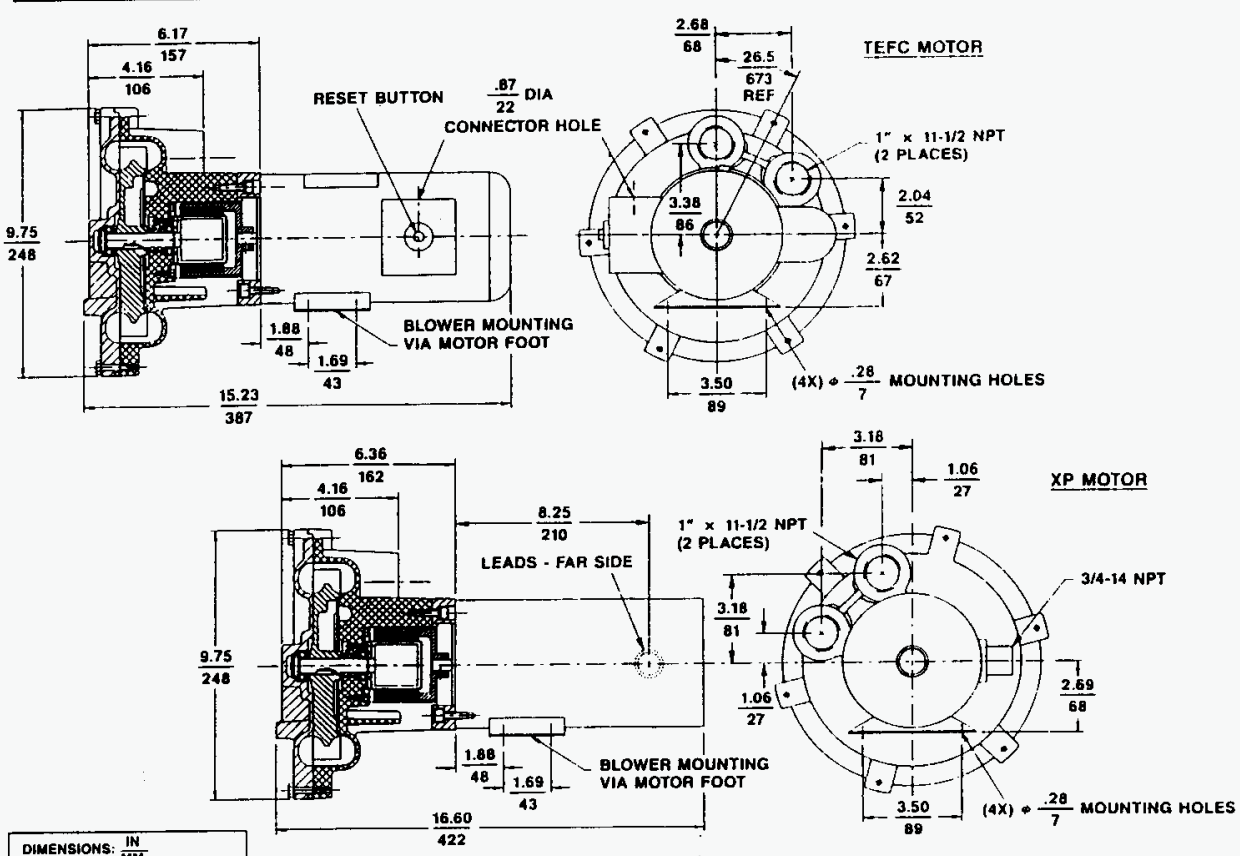

TOLERANCES: $. X X \pm \frac{.08}{2}$

(UNLESS OTHEAWISE NOTED)

A 0.87 DIAM ON TEFC MOTOR, 0.75" NPT ON XP MOTOR

\section{SPECIFICATIONS}

\begin{tabular}{|l|c|c|}
\hline MODEL & MD101CB4 & MD101CC4 \\
\hline Part No. & 038014 & X38271 \\
\hline Motor Enclosure Type & TEFC & 0.25 \\
\hline Horsepower & 0.25 & Single $-60 \mathrm{~Hz}$ \\
\hline Phase - Frequency & Single $-60 \mathrm{~Hz}$ & 115 \\
\hline Voltage & 115 & 2.6 \\
\hline Motor Nameplate Amps & 2.0 & 2.2 \\
\hline Maximum Blower Amps & 2.2 & 7.2 \\
\hline Inrush Amps & 7.2 & 00 \\
\hline Starter Size & 00 & 1.0 \\
\hline Service Factor & 1.0 & Automatic \\
\hline Thermal Protection & Automatic, Manual Feset & Sealed, Ball \\
\hline Bearing Type & Sealed, Ball & 40 (18) \\
\hline Shipping Weight Ibs (kg) & 40 (18) & \\
\hline
\end{tabular}


WHC-SD-SN $=C \mathrm{JT}-\mathrm{CO} \%$, REV. 0

Trivisy, Ix:.

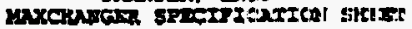

\begin{tabular}{|c|c|c|}
\hline & ó & Item Mnis \\
\hline
\end{tabular}

num mare: 3263

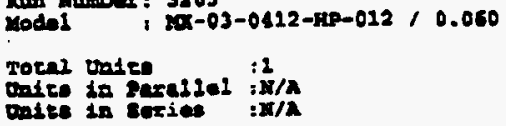

net stat rision:

43. 8000

43.5000

1.2400

0.0002

0.0900

0.0223

222.0000

60.0000

0.0042

24.7000

eso. 1
Thenusein , Tho

Rave Arrangewer: $2 \times 2$
Flow DLE: councer low

potal dene grunefor dras: 2.8 so $\mathrm{mr}$

ROO
- cold side

Ges $2.0000 \quad$ bes

orer 1.0000 ors

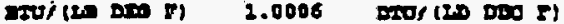

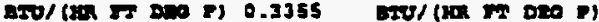

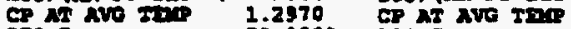

E... 50.0000 bor

Die 5 31.1366 bes

ger zoend 0.0524 per zaeal

Pate जxd/a Iorne

notet all is at gat ant

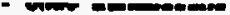

wien $1.00^{\circ}$

zanoth : $22.00^{\circ}$

Belane : 1.70

Mesice : 0.74., 0.740

- mamerera

Deses and Fiefinge : 3162 as

Bingengen

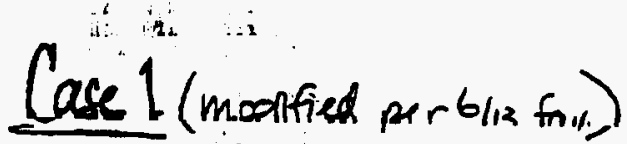

Your stanter noprementate it:

bring Magelpes. Ins.

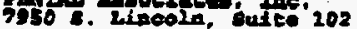

wetereten, 60 b02z2

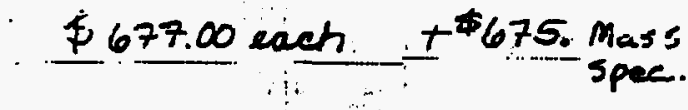

(303) 784-1802

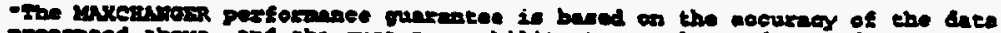

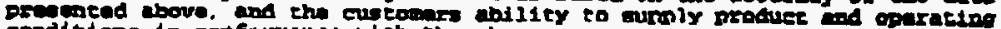
conditlon in codfermanes with the ibove.

* single wall construction

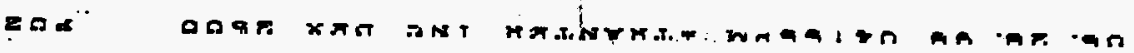
$4-93$ 


\section{$P R \cap P \cap S A 1$}

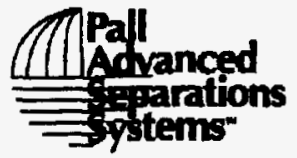

July 25,1996

Sants Fe Engineering

2204 Brothen Rond

Senta Fo, NM 87505

Atteation: Dave Munger

Reference: Pull Proposd Number BP7ACDR96-129PDI

Der Mr. Munger:

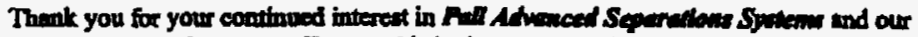

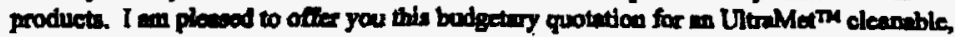
Metel HEPA rued filter.

\section{Dosurtine Conditing:}

The filter is designed for the following condition:

Fluid:

Nitrogen or Helium

Flow:

20 ACFM

Temperature:

0 - $575 \%$

Prearer:

$5 \mathrm{TOR} \rightarrow 10 \mathrm{PSIO}$

Dapheront Davedition:

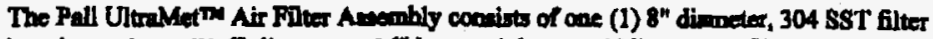

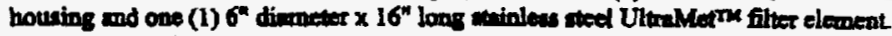

The filter aswembly is a completely closed canister that is weldod parmmeatly stut. The cenister is a cylindrical filter veasel with wold cep ends and $1^{n} 150$ i ANSI flanged iniet and outlet congections locatied on the couter line of the vesel.

$$
4-94
$$




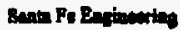

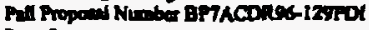

Ruge 2

\section{Eudeng:}

Pricen for the Pall UltonMetrs Air Filter Asembly as described beroln are:

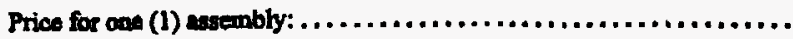

Price auch for three (3) assemblies: ............................

\section{Detturg:}

Eritimatod delivery time is $12-16$ weeks aftur recelpt of a purchane order.

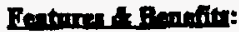

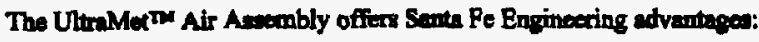

\section{Bing 8trougth:}

All strinlese stoel, tully welded construction.

\section{Cleamable:}

The filters con be cleaned uning a variety of mochanical methods.

\section{High Temperature Capability:}

Surtained operution at $750^{\circ} \mathrm{F}$ with intermittent temperature excursion to $1100^{\circ} \mathrm{F}$.

\section{Snity:}

The strength and durablity of UltraMetim Air filters provide modimum protection to people and the environement.

$$
H-95
$$




\title{
PROPOSAI
}

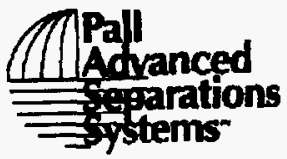

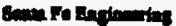

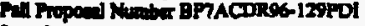 \\ Pus
}

\section{Condinian:}

Pall Corparation is a 900+ million doller, multi-netiond company and the watd's largest producer of fine parous medin fittere. In onder to necoed, it is evandil thet wo provide better value to our customer. We are dedicated to this poliey, which is why Pell's filtar sales are four to five timas that of our neares competitor. We look formend to the opportumity of working with you to provide a clemble, high efficienoy metal filter system. If you have any questions, or require finther waictence, please do not besitute to contact me directly at (607) 753-6041 Ext. 1680 or Teree Rush of Kyner Compeny, Inc.

Very trily yours,

\section{Pall Afrancad Sepandows Syatews}

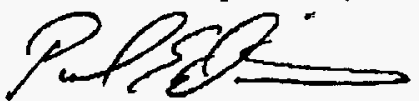

Paul Dittunen

Senior Applications Engineer

CC: Kyser Company, Inc.

$\$ 821$ Midway Purk Blvd. Ne/Suite K

Alboquenque, NM 87109

Contact: Teroe Rush

Phone: (505) 343-9355

Fax: (505) 345-0885 


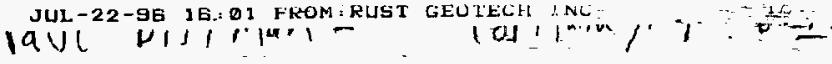

\section{ULTRAMET AIR FILTER GRADES AND THEIR CHARACTERISTICS}
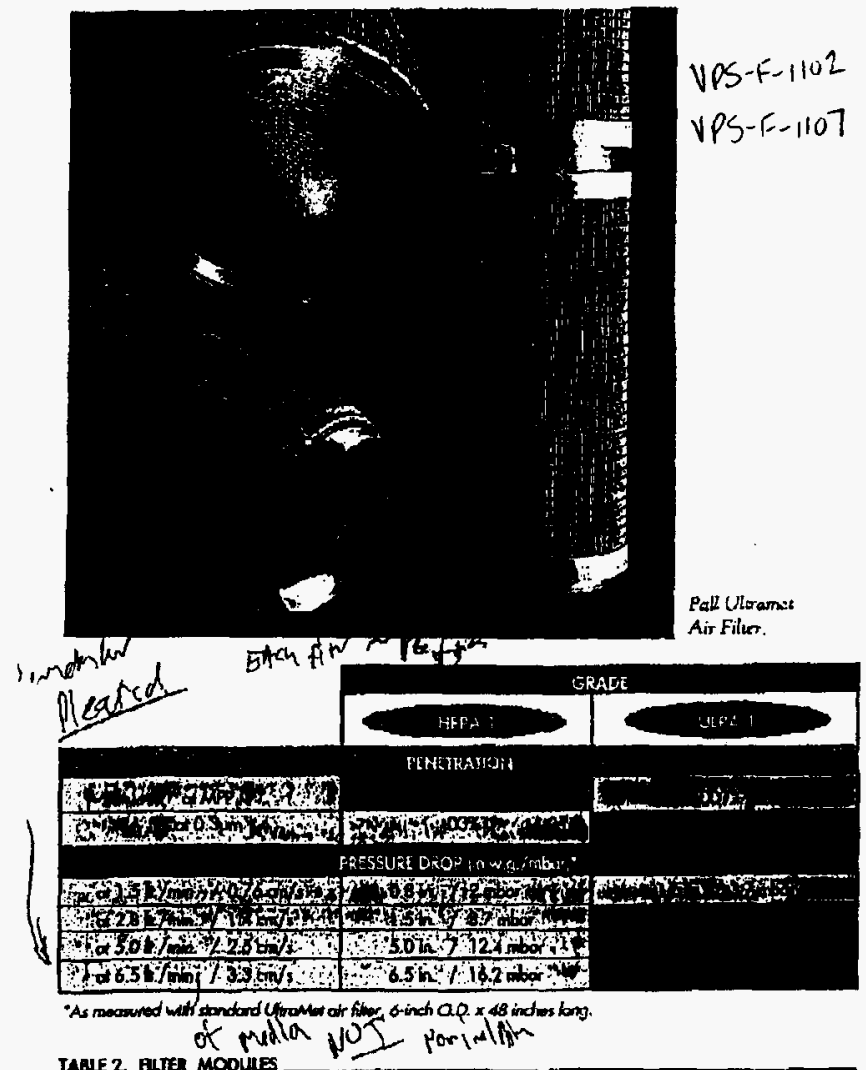

TARE 2 RLIER MOOULP

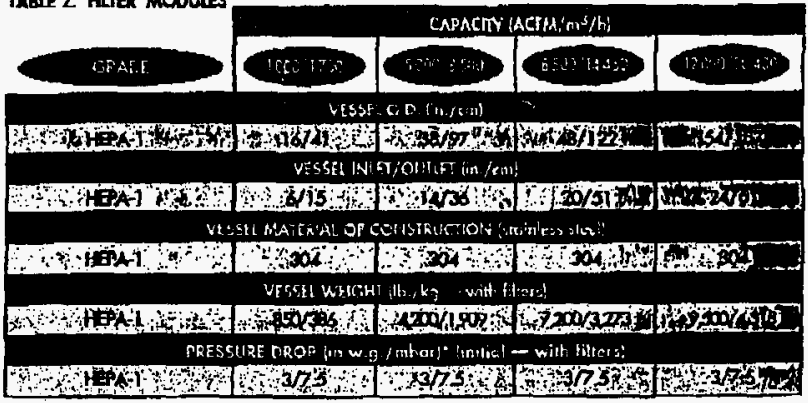

Resistance to Heated Air: Resiscant to air at $750^{\circ} \mathrm{F}$ for 48 hours or at $1000^{\circ} \mathrm{F}$ for 5 uxinutes per UL-586 and MIL F51068:

Spot Flame Resistance: No suscained flame on duwnstream side and no transmitral of flane to outside surlaces per ANSIBI32.1.

Resiscance to Moisture and Overpressure: No permanent damage after 10 yoi water differendial for 1 hour.

Resiscance to Rough Handling: No damage per MIL.STD.282, method 105.9.

Qualin Assurance: To ASME-NQA-1. 1989 and in accondance with MIL-F. 51079. Certifiat or ISO9001.

Dust Capacity: 29 grn/fr. ${ }^{2}$ per ASHRAE 52.76.

$$
\begin{aligned}
& \text { Anticion lin }= \\
& \text { Fpua Roto ins } \\
& \text { त्र } 1811
\end{aligned}
$$




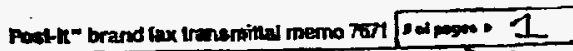
WRAL PA MaNSRE PACL DITIAA RUST SEO ग70-248-6040 $607-858-574$
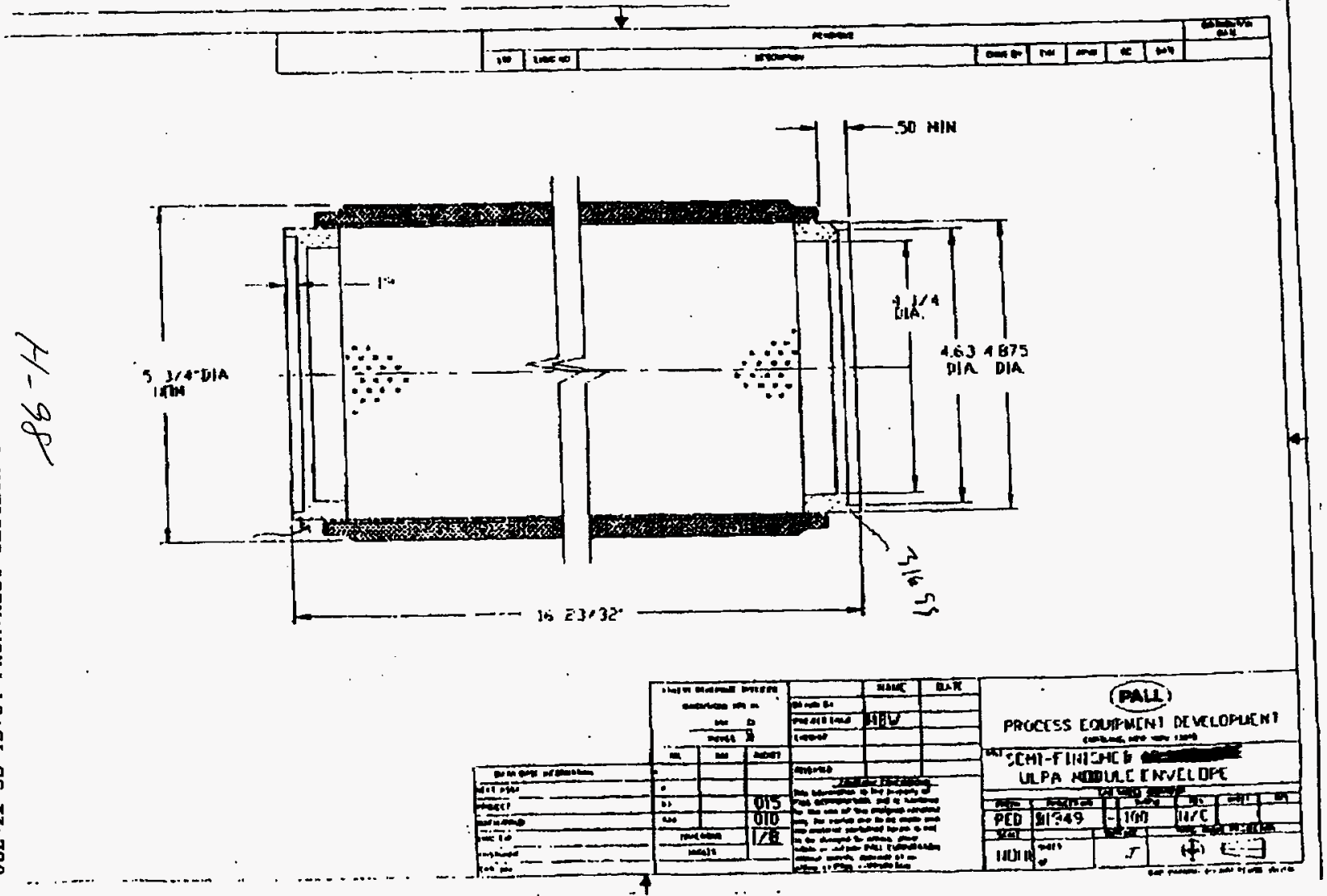
JUL- 22 - 16 日 162 FROM IRUST GEOTECH J NL

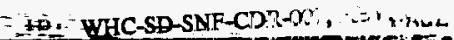






\section{Small capacity/low flow gas heaters}

\begin{tabular}{l}
\hline Stainless steel \\
\hline 0.5 to $3 \mathrm{~kW}$ \\
\hline 120 and $240 \mathrm{~V}, 1$ phase \\
\hline Incoloy ${ }^{\circ}$ sheath $\left(50 \mathrm{~W} / \mathrm{in}^{2}\right)$ \\
\hline Type GCHIS-C \\
\hline
\end{tabular}

\section{Application}

For heating gases, especially efficient for low flow rate/high temperature applications. (Outlet temperatures to $600^{\circ} \mathrm{F}$ )

\section{Features}

Compact, rugeed design permits easy installation.

Overtemperature protection. Type K thermocouple located inside heating element sheath.

Accurate process control by means of Type $J$ thermocouple installed in the heater outlet.

Controls - ordered separately. Consult Controls Section of this catalog.

High temporature insulation and insulation jacket.

304 Stainlese steel construction of all wetted parts except Incoloy sheath.

Speclal voltage and wattage ratings avallable. Contact your locail Chroma. lox representative.

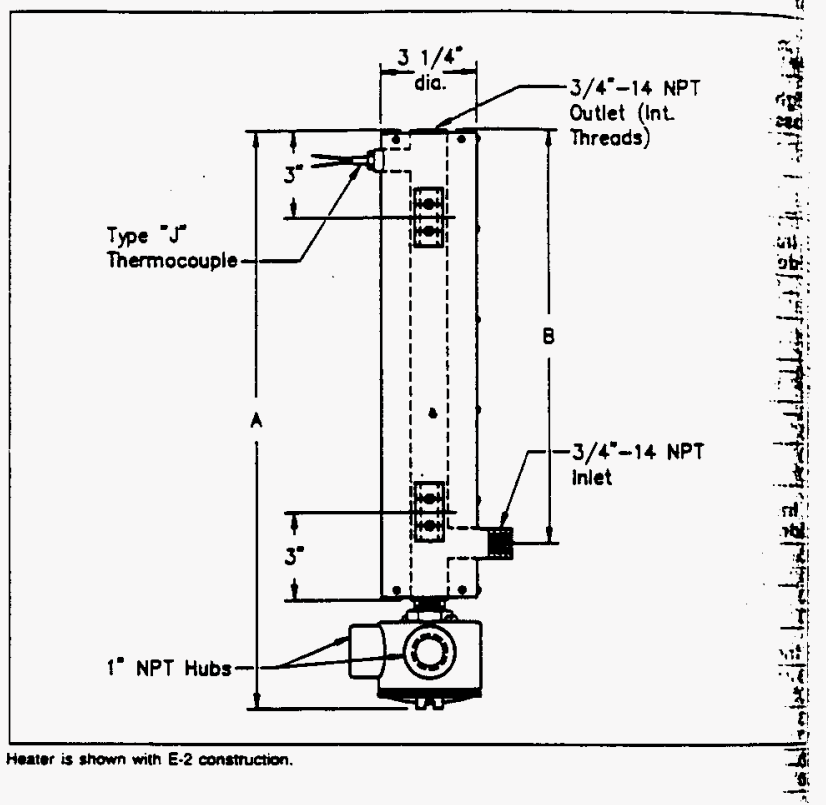

E-1 Cen. purpose enciosure

E-2 Moisture binhtexplosion-nuistent exelor?

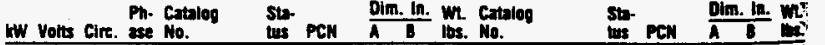

Stainless steel vessel construction/incoloy sheath element (50 W/in')

\begin{tabular}{|c|c|c|c|c|c|c|c|c|c|c|c|}
\hline $\begin{array}{l}0.5120 \\
0.5240\end{array}$ & $\begin{array}{l}1 \\
1\end{array}$ & 1 & $\begin{array}{l}\text { GCH } \\
\text { GCH }\end{array}$ & NS & $\begin{array}{l}12448314 \\
02449114\end{array}$ & $\begin{array}{l}8 \\
8\end{array}$ & $\begin{array}{l}3 \\
3\end{array}$ & $\begin{array}{l}\text { GCHIS-COSE2 } \\
\text { GCHIS-COSE2 }\end{array}$ & NS & $\begin{array}{ll}024555 & 14 \\
024553 & 14\end{array}$ & $\begin{array}{l}8 \\
8\end{array}$ \\
\hline $\begin{array}{l}1.0120 \\
1.0240\end{array}$ & 1 & & & NS & $\begin{array}{l}02450420 \\
02451220\end{array}$ & 14 & 4 & & $\begin{array}{l}\text { NS } \\
\text { NS }\end{array}$ & 57120 & 14 \\
\hline & 1 & & & $\begin{array}{l}\text { NS } \\
\text { NS }\end{array}$ & 203932 & $\begin{array}{l}26 \\
26\end{array}$ & 5 & & $\begin{array}{l}\mathrm{NS} \\
\mathrm{MS}\end{array}$ & $00 \frac{32}{32}$ & 20 \\
\hline 30240 & 1 & & GCHIS-C3O & NS & 02454744 & 38 & 6 & GCHIS-C30E2 & NS & 02461944 & 38 \\
\hline
\end{tabular}

$\$ 6765 \stackrel{\circ 0}{=}$ 


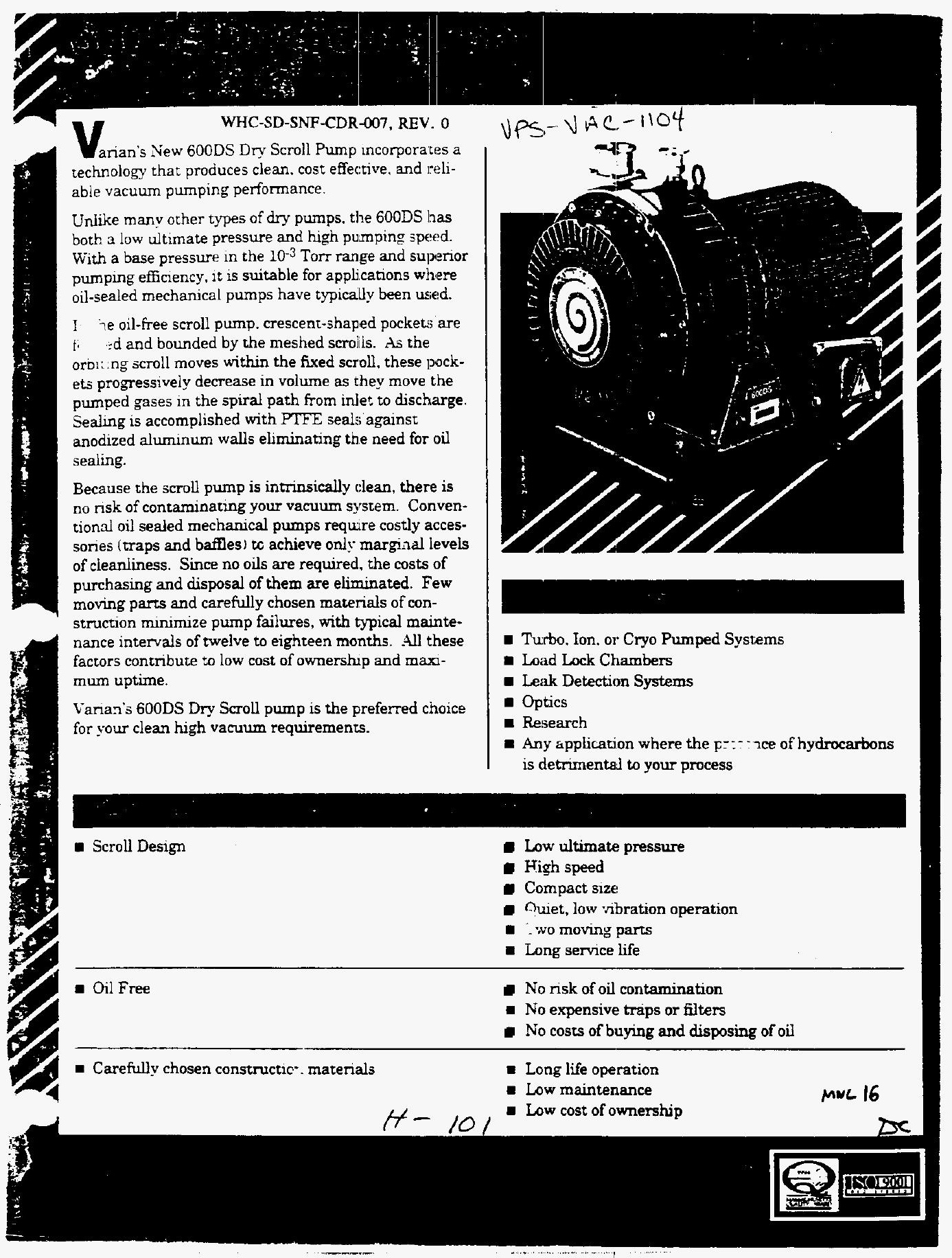


- Free Air Displacement

$60 \mathrm{~Hz} \quad 600 \mathrm{~lm}(2 \mathrm{~m} \mathrm{cfm})$

$50 \mathrm{~Hz} \quad 500 \mathrm{l} / \mathrm{m}(17.5 \mathrm{~cm})$

- Pumping Speed

$60 \mathrm{~Hz} \quad 500 \mathrm{l} / \mathrm{m}(17.5 \mathrm{cfm})$

$50 \mathrm{~Hz} \quad 420 \mathrm{Lm}(15 \mathrm{cfm})$

- Uitimate Total Pressure

$<10^{-2}$ Torr (mbar)

Inlet Connection

NW-40

- Outlet Connection

NW-25

- Motor Rating

$0.8 \mathrm{hp}(0.6 \mathrm{~kW})$

- Electrical Supply

1 Phase, $50 / 60 \mathrm{~Hz}, 100 / 115 / 200 / 230 \mathrm{~V}$

3 Phase, $50 / 60 \mathrm{~Hz}, 200 / 208 / 230 / 380 / 415 / 460 \mathrm{~V}$

- Noise Level at 1 meter

$60 \mathrm{dBA}$

- Operating Range

$40^{\circ} \mathrm{F}$ to $105^{\circ} \mathrm{F}\left(5^{\circ} \mathrm{C}\right.$ to $\left.40^{\circ} \mathrm{C}\right)$

\section{- Weight}

$88.2 \mathrm{lb}(40.0 \mathrm{~kg}) 1$ Phase

$100 \mathrm{lb}(45.0 \mathrm{~kg}) 3$ Phase
WHC-SD-SNF-CDR-007, REV. 0

Varian Part No. 600DS-1UNTV

600DS-3UNTV

600DS-KMI 600DS-KMA

\section{Description}

Model 600DS with base, hour meter, and direct drive, 1 phase motor

Model 600DS with base, hour meter. and direct drive, 3 phase motor

Minor Maintenance Kit

Factory Rebuild Service
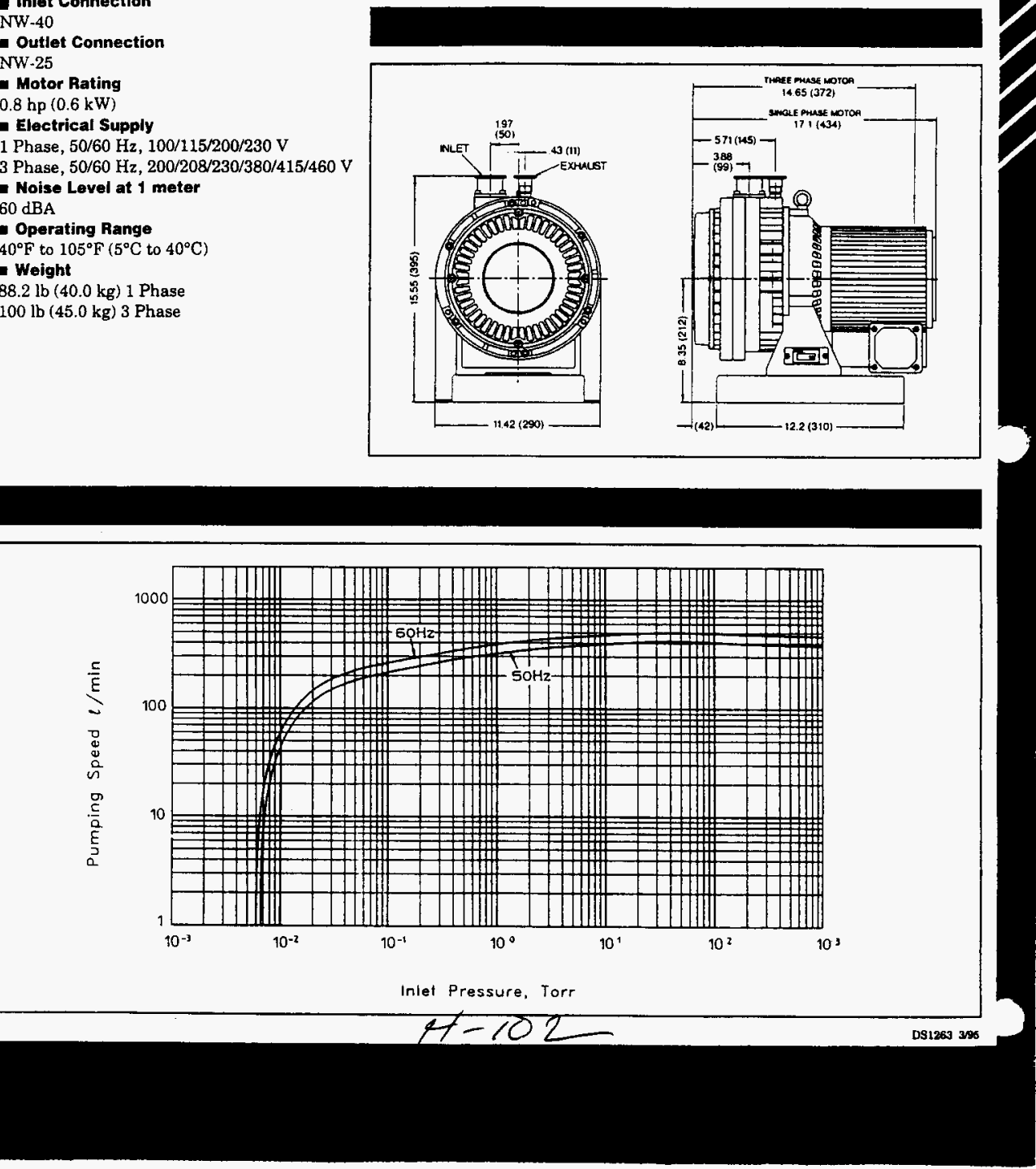
WHC-SD-SNF-CDR-007, Revision 0

\section{APPENDICES}

\section{APPENDIX I}

\section{CRITERIA EVALUATION}


WHC-SD-SNF-CDR-007, Revision 0

\title{
APPENDIX I - CRITERIA EVALUATION
}

\author{
CRITERIA EVALUATION \\ for the \\ HOT CONDITIONING SYSTEM EQUIPMENT \\ of the \\ K-BASIN SPENT NUCLEAR FUEL STORAGE PROJECT
}

\subsection{INTRODUCTION}

Spent Nuclear Fuel (SNF) is to be conditioned by heating it in an inert (helium) environment, by holding the heated SNF under vacuum until the outgassing rate diminishes, and by controlled oxidation of exposed uranium surfaces. The process will be accomplished by placing a container full of SNF, called the Multiple Canister Overpack (MCO), into an oven where it will be connected to a process system that can circulate gas through the $M C O$ and that can draw a vacuum within the MCO while the oven heats the MCO. The hot conditioning process development and the hot conditioning process equipment design, fabrication, and installation are the scope of the Hot Conditioning System Equipment (HCSE) project.

The HCSE is to be housed in an addition to the Canister Storage Building (CSB) called the Hot Conditioning System Annex (HCSA). The HCSA is to be coupled to the CSB such that it is completely open to the storage area of the CSB. This arrangement allows the MCO Handling Machine (MHM) to perform transactions of MCOs between storage tubes that are below the CSB floor and the HCSE ovens that must also be in tubes below the floor for proper MHM interfacing. The HCSA is a separate project from the HCSE being performed through an addition of scope to the CSB project.

The interface of the HCSE/HCSACSB projects determines many of the criteria for the HCSE. The CSB utilizes work performed previously as part of the Hanford Waste Vitrification Project (HWVP). The building foundation was completed previously and the final design of the CSB building has been issued for review in preparation of an immediate construction start. Therefore, the structural and nuclear material release confinement criteria have been selected and implemented for the CSB. Since there will be no wall between the HCSA and the CSB, the same criteria must also apply to the HCSA.

The CSB facility design is based on the fact that the MCO will always be secondarily contained within very rigid/protected structures (below ground storage holes with thick concrete plug caps or within the MHM) that will be 
WHC-SD-SNF-CDR-007, Revision 0

\section{APPENDIX I - CRITERIA EVALUATION}

qualified to perform the confinement functions required during and after credible Design Basis Accidents (DBAs). Therefore, the CSB design does not include a building shell and ventilation system designed to perform nuclear material confinement. It follows that the HCSA will not have a building shell or ventilation system designed to perform nuclear material confinement.

Given that the HCSE does have the potential of accidentally releasing radioactive material through a variety of scenarios caused by a variety of initiators, the responsibility of providing nuclear material confinement systems to adequately protect site workers and the off-site public lies with the HCSE project. This fact is fundamental to the selection and interpretation of appropriate criteria given below.

\subsection{FACILITY CLASSIFICATION CRITERIA}

\subsection{DOE STD -1027 "Hazard Categorization"}

The design of a nuclear facility necessarily requires iteration between design concept development, hazards analysis, and criteria definition in order to arrive at a consistent package of criteria and designs. This is because the hazard classification ( $\mathrm{HC}$ ), which is key to the criteria selection and design characteristics of the facility can be controlled to some extent by controlling the amount and form of the radioactive materials that will be present in the process systems and facility. The performance categorization (PC) of structures, systems, and components (SSCs) that are subjected to postulated Design Basis Accidents (DBAs) is dependent on the HC. And, the determination of whether an SSC is "safety class" or "safety significant" is determined by the off-site consequences (safety class) or on-site consequences (safety significant) of releases that might occur as a result of DBAs. However, calculation of consequences requires some model of the facility and the processes that it contains. PC and safety class determinations imply certain structural loads and analysis, as well as special design features to assure that there are no single point failure modes for an SSC.

DOE Order 5480.23, "Nuclear Safety Analysis Reports", requires that a Hazard Categorization (HC) be performed for planned nuclear facilities for the purpose of establishing a graded approach to safety analysis. Ratings range from HC-1 for the most hazardous rating to $\mathrm{HC}-4$ for non-nuclear facilities. The methodology for assessing the Hazard Category is given in DOE STD-1027-92, "Hazard Categorization and Accident Analysis Techniques for Compliance with DOE Order 5480.23, Nuclear Safety Analysis Reports". 
WHC-SD-SNF-CDR-007, Revision 0

\section{APPENDIX I - CRITERIA EVALUATION}

In a general sense DOE STD - 1027 defines HC's as follows:

- HC-3: Hazard analysis shows potential for only significant localized consequences. Facilities with quantities of radioactive materials in excess of the Table A-1 values which would yield a does of 10 Rem in $24 \mathrm{hrs}$ at $30 \mathrm{~m}$ using the calculation method given in Attachment 1.

- HC-2: Hazard analysis shows potential for significant on-site consequences. The facility has potential for nuclear criticality events or has quantities of material at risk and energy sources to effect a release that would exceed 1 Rem exposure at a distance of $100 \mathrm{~m}$. using the calculation method given in Attachment 1 , or would require on-site emergency planning activities.

- HC-1: Hazard analysis shows the potential for significant off-site consequences. Category A reactors ( $>20 \mathrm{MW}$ ) and facilities designated by PSO.

The STD - 1027 methodology is based on comparing the radiological inventory of a facility to HC Threshold Quantities (TQ) provided in Attachment 1. The Standard recognizes that large quantities of radioactive materials may be present in a facility, but in a containment vessel, distribution, or a form that precludes release, and which need not be counted in the inventory when making the $\mathrm{HC}$ determination.

- "The concept of independent facility segments should be applied where facility features preclude bringing material together or causing harmful interaction among from a common severe phenomena. ... It is not desirable to estimate the potential consequences from an inventory of hazardous materials when facility features would preclude bringing this material together. Therefore, the standard permits the concept of facility segmentation provided the hazardous material in one segment could not interact with hazardous materials in other segments."

- "Additionaliy, material contained in DOT Type B shipping containers (with or without overpack) may also be excluded from summation of a facilities radioactive inventory."

- "Alternatively, for facilities initially classified as Hazard Category 2, if release fractions can be shown to be significantly different than these values based on physical and chemical form and available dispersive energy sources, the threshold inventory values for Category 2 in Table A-1 may be divided by the ratio of the maximum potential release fraction to that found on Page A-9. All 
WHC-SD-SNF-CDR-007, Revision 0

\section{APPENDIX I - CRITERIA EVALUATION}

assumptions which are used to reduce the inventory at risk should be supported in the Hazards Analysis."

Facilities that have radioactive material inventories in excess of the HC-2 may be derated to $\mathrm{HC}-3$ by demonstrating that hazardous material is not part of the at-risk inventory and by scaling the threshold values according to the ratio of realistic facility specific release fractions to the release fractions that were assumed when the thresholds were established. A discussion of the TQ assumptions and calculations is given in Attachment 1 of the standard.

At this time, the Hazards Analysis for the HCSE/HCSA is being performed. Preliminary results suggest that the MCO package will limit the postulated releases to levels that will not exceed the site boundary (public exposure) limitation. The hazard category rating for the HCSE/HCSA facility and process support systems that confine releases will be HC-2 (the same as the CSB). The HCSA and HCSE will not have any safety class SSCs. The consequences for onsite workers are expected to exceed the limits that are allowed without mitigating SSCs. Therefore, the HCSAJHCSE projects will provide safety significant SSCs to confine releases.

Given that the CSB/HCSA structures are not designed to confine releases and the CSB/HCSA facilities do not include HEPA filtered exhaust, the HCSE must provide the safety significant SSCs.

\subsection{DOE STD - 1021 "Performance Categorization"}

DOE Order 5480.28, "Natural Phenomena Hazards Mitigation" establishes a graded approach for determining the Performance Categorization PC of a Structure, System, and Component (SSC) when subjected to Natural Phenomena Hazards based on the probability that SSC response will exceed a safety limitation. Guidance for PC determination is given in DOE STD 1021-93, "Natural Phenomena Hazards Performance Categorization Criteria for Structures, Systems, and Components". Figure 1-1 of STD 1021 summarizes the guidance. It can be seen from the table that SSCs that perform a safety significant function in an $\mathrm{HC}-2$ facility will be rated $\mathrm{PC}-2$.

PC-2 rating does not require dynamic finite element analysis. The critical facility static analysis methodology given in. UBC may be applied to HCSE safety significant SSCs such as the process piping, HEPA filters, and so forth. 
WHC-SD-SNF-CDR-007, Revision 0

\section{APPENDIX I - CRITERIA EVALUATION}

\subsection{DOE 6430.1A "Facility Type Categorization"}

The HCSA will be a "Nuclear Facility" as defined in the glossary of terms (page 21). Therefore, it will be a "Special Facility" as defined by paragraph 1300-1.1. The criteria given in Division 13 "Special Facilities" are the basic guidance for the design of the HCSA and HCSE. Application of Division 13 requirements invoives the general requirements given in Section 1300 as well as the requirements given in the applicable facility type section. In addition, the special facilities specific paragraphs labeled - 99.0 in the Discipline Divisions apply.

The HCSA will be classified as an Irradiated Fissile Material Storage Facility (IFMSF) because it is an extension of the CSB. As discussed above, the HCSE is an active process that could have accidental releases with a higher frequency than a passive SNF storage facility and some amount of radioactivity may be withdrawn from the $\mathrm{MCO}$ as a normal process practice. The inclusion of this HCSE process within the IFMSF does not alter the CSB criteria so long as the HCSE includes its own confinement SSCs.

\subsection{CFR Part 72 "Licensing Requirements for the Independent Storage of Spent Nuclear Fuel And High Level Radioactive Waste"}

The DOE has stated that it generally desires to comply with the requirements of the CFRs except for the licensing requirements, except for those instances and facility types that are not specifically addressed by the CFRs, and except for those instances where the DOE policies are more conservative than the requirements of the CFRs.

The CSB will comply with the requirements of 10 CFR Part 72 because it is an SNF Storage Facility. The HCSA, as an extension of the CSB will also be considered to be an Independent Spent Fuel Storage Installation. This classification is dependent upon designing the HCSE so that it confines nuclear releases without reliance on the facility structure and systems.

\subsection{DESIGN CRITERIA}

\subsection{DOE Orders \& Standards}

Table 3.1 contains a list of DOE Orders and DOE Technical Standards that contain provisions that will effect features of the design of the HCSE/HCSA or that may effect the contents of the engineering/design documentation of the HCSE/HCSA. 
WHC-SD-SNF-CDR-007, Revision 0

\section{APPENDIXI - CRITERIA EVALUATION}

A brief description of each of the entries is as follows:

5400.1 General Environmental Protection Program -This order states that environmental notification and reporting requirements are to be determined on a facility case by case basis. It requires an annual environmental report which is essentially an emissions summary. It requires that an environmental protection plan and an environmental monitoring plan be developed and approved. These should be approved before authorization to initiate operations is given. The HCSE design will have to include appropriate monitoring capability. 
WHC-SD-SNF-CDR-007, Revision 0

\section{APPENDIXI - CRITERIA EVALUATION}

\section{DOE ORDERS SUMMARY}

\begin{tabular}{|c|c|}
\hline $\begin{array}{l}\text { ORDER } \\
\text { STANDARD }\end{array}$ & TITLE \\
\hline 5400.1 & General Environmental Protection Program \\
\hline 5400.3 & Hazardous and Radioactive Mixed Waste Program \\
\hline 5400.4 & $\begin{array}{l}\text { Comprehensive Environmental Response, Compensation, and Liability Act } \\
\text { Requirements }\end{array}$ \\
\hline 5400.5 & Radiation Protection of the Public and the Environment \\
\hline $5440.1 \mathrm{E}$ & National Environmental Policy Act Compliance Program \\
\hline $5480.1 B$ & Environment, Safety, and Health Program \\
\hline 5480.4 & Environmental Protection, Safety and Health Protection Standards \\
\hline N5480.6 & DOE RadCon Manual \\
\hline $5480.7 \mathrm{~A}$ & Fire Protection \\
\hline 5480.9 & Construction Safety and Health Program \\
\hline 5480.19 & Conduct of Operations Requirements for DOE Facillites \\
\hline 5480.21 & Unreviewed Safety Questions \\
\hline 5480.22 & Technical Safety Requirements \\
\hline 5480.23 & Nuclear Safety Analysis Reports \\
\hline 5480.28 & Natural Phenomena Hazards Mitigation \\
\hline 5481.31 & Start-Up and Restart of Nuclear Facilities \\
\hline 5483.1A & $\begin{array}{l}\text { Occupational Safety and Health Program for DOE Contractor Employees at } \\
\text { Government-Owned Contractor-Operated Facilities }\end{array}$ \\
\hline $5700.6 \mathrm{C}$ & Quality Assurance \\
\hline $5820.2 A$ & Radioactive Waste Management \\
\hline $6430.1 A$ & General Design Criteria \\
\hline STD-0101 & $\begin{array}{l}\text { Compilation of Nuclear Safety Criteria for Potential Application to DOE Non-Reactor } \\
\text { Nuclear Facilities }\end{array}$ \\
\hline STD-1020 & Natural Phenomena Hazards Design and Evaluation Criteria for DOE Facilities \\
\hline STD-1021 & $\begin{array}{l}\text { Natural Phenomena Hazards Performance Categorization Guidelines for Structures, } \\
\text { Systems, and Components }\end{array}$ \\
\hline STD-1022 & Natural Phenomena Hazards Site Characterization \\
\hline
\end{tabular}


WHC-SD-SNF-CDR-007, Revision 0

\section{APPENDIX I - CRITERIA EVALUATION}

\section{DOE ORDERS SUMMARY}

(Continued)

\begin{tabular}{|l|l|}
\hline $\begin{array}{c}\text { ORDER/ } \\
\text { STANDARD }\end{array}$ & \multicolumn{1}{|c|}{ TITLE } \\
\hline STD-1024 & Guidelines for Use of Probabilistic Seismic Hazard Curves at DOE Sites \\
\hline 10CFR835 & Radiation Exposure \\
\hline STD-1027 & $\begin{array}{l}\text { Guidance For Preliminary Hazard Classification and Accident Analysis Techniques for } \\
\text { Compliance With DOE Order 5480.23, Safety Analysis Reports }\end{array}$ \\
\hline STD-1044 & Guide To Good Practices for Equipment and Piping Labeling \\
\hline STD-3003 & Backup Power Sources for DOE Facilities \\
\hline STD-3006 & Planning and Conduct of Operations Readiness Reviews \\
\hline STD-3009 & Preparation Guide for U.S. DOE Nonreactor Nuclear Facility Safety Analysis Reports \\
\hline STD-3011 & $\begin{array}{l}\text { Guidance for Preparation of DOE 5480.22 (TSR) and DOE 5480.23 (SAR) } \\
\text { Implementation Plans }\end{array}$ \\
\hline 40CFR61 & National Standards for Hazardous Air Pollutants \\
\hline
\end{tabular}

5400. 3 Hazardous and Radioactive Mixed Waste Program - This order implements RCRA in the DOE. The HCSE will generate radioactive waste that is regulated by RCRA.

5400.4 CERCLA Requirements -This order implements CERCLA in the DOE.

5400.5 Radiation Protection of the Public and the Environment -Sets limits on public doses from normal operations. Defines Derived Air Concentrations and implements ICRP recommendations.

5440.1E NEPA Compliance Program - This order implements the National Environmental Protection Act in the DOE.

5480.1B Environment, Safety, and Health Program - This document is the master document for the 5480 series of orders.

5480.4 Environmental Protection, Safety, and Health Protection Standards - This order implements a list of regulations that apply to private organizations but which are not automatically applied to the DOE. The order is a useful reference list. 
WHC-SD-SNF-CDR-007, Revision 0

\section{APPENDIX I - CRITERIA EVALUATION}

N5480.6 DOE RadCon Manual - This notice sets policy regarding radiation exposure limits, radiation area practices, and ALARA considerations. A design program is required to achieve the ALARA goal. The guideline also contains criteria governing the design of change rooms and other radiation/radioactive material control procedures. One key criteria is the ALARA design guideline of $500 \mathrm{mR}$ per year for an individual radiation exposure.

5480.7A Fire Protection - This order relates to nuclear facility safety issues such as fire protection of safety class equipment, fire hazards analysis for the design basis fire to be included in the SAR, seismic criteria for the fire protection system, and life safety codes. It sets forth provisions that address loss limitation for government owned facilities.

5480.9 Construction Safety and Health Program -This order implements requirements found in the OSHA regulations regarding construction safety. Construction specifications should reference appropriate requirements.

10CFR835 Radiation Protection for Occupational Workers - This document sets the requirements for radiation exposure protection and As Low As Reasonably Achievable (ALARA) policy.

5480.19 Conduct of Operations Requirements for DOE Facilities - Safety of workers and protection of the public requires that nuclear facilities be formally managed by operations program that meets high standards of discipline. In the nuclear power, NRC, arena the conduct of operations requirements are specified by the ICRP. This order implements similar requirements for facilities regulated by the DOE. It addresses operations procedures, shift changes, operator training, and so forth. The design team will write a draft Conduct of Operations Plan.

5480.21 Unresolved Safety Questions - Is analogous to 10CFR50.59. Establishes a process for changing the operating basis of the facility. Allows the operator the freedom to perform experiments and investigate safety issues that may arise while conducting day-to-day operations.

5480.22 Technical Safety Requirements - This order requires that safety limits for the operating parameters of the facility be established, that procedures for assuring that these limits are not exceeded be established, that these parameters be monitored, and that response actions for conditions outside the safety limits be determined.

5480.23 Nuclear Safety Analysis Reports - This order applies in its entirety because it is the document that defines the requirements for safety analysis and SARs, which are the means of demonstrating that the public/environment and workers are adequately protected. The major impacts on the project are: 
WHC-SD-SNF-CDR-007, Revision 0

\section{APPENDIX I - CRITERIA EVALUATION}

- A preliminary hazards analysis is to be performed and published. Guidance is given by DOE STD - 1027.

- A Preliminary Safety Analysis Report (PSAR) is to be developed. The PSAR is developed simultaneously with design. There is iteration between safety analysis and design. The PSAR analysis demonstrates that the design adequately mitigates the consequences of the hazards presented in the hazards analysis.

- A Final Safety Analysis Report is to be developed. This report updates the PSAR to reflect the as-built facilities and incorporates the operating procedures for the facilities.

5480.28 Natural Phenomena Hazards Mitigation - Establishes requirements for response of facilities to natural phenomena such as earthquakes and high winds.

5480.31 Start-up and Restart of Nuclear Facilities - Sets out requirements for authorization to initiate operations utilizing nuclear materials. Essentially this document establishes the DOE "licensing" criteria. The Operating Readiness Plan should be written by the design team. Th plan should follow the guidance of DOESTD-3006.

5483.1A Occupational Safety and Health Program for DOE Contractor Employees at Government Owned Contractor Operated Facilities - This order implements OSHA within the DOE.

5700.6C Quality Assurance - The document is analogous to ASME NQA-1 which is a nuclear facilities quality assurance guideline required by DOE 6430.1A.

5820.2A Radioactive Waste Management - This order requires a waste management plan, has D\&D requirements, and sets waste characterization standards, as well as establishing numerous other requirements that affect both the design and operations of the CVDS.

6430.1A General Design Criteria - This order sets forth design criteria for all engineering disciplines as well as special facility and special equipment criteria. Facility type classification and safety class SSC requirements are discussed in Section 2..3 above.

Key confinement system ventilation requirements are referred to ERDA 76-21 and to the 1550-99 paragraphs of the order. The key statement comes from 1550-99.0.1 "General Ventilation and Off-Gas Criteria", "These criteria cover ventilation and off-gas systems, or portions of them, that are identified as safety class items in accordance with Section 1300-3.2, Safety Class Items ${ }^{n}$. Strictly 
WHC-SD-SNF-CDR-007, Revision 0

\section{APPENDIX I - CRITERIA EVALUATION}

speaking, this means that the HCSE will not be governed by these requirements. None-the-less the principles stated in 1550-99.0.2 “Confinement Ventilation Systems" should be followed as a matter of good engineering practice (except where specific references to the safety class characteristics of the system are made). The basic design concepts for exhaust filtration performance and stack design should be derived from safety analysis that analyzes the consequences of credible release scenarios.

Section 1300-12 "Human Factors Engineering" requires that a human factors program plan be written. The level of detail of the plan is to be determined by the complexity or safety issues associated with human activities. The subsections also set out extensive human factors requirements for human factors engineering. The HCSE should write and implement a plan given that the radiation exposure during operations can severely restrict the staff planning.

STD-0101 Compilation of Nuclear Safety Criteria For Potential Application to DOE Non-Reactor Nuclear Facilities - This STD contains a listing of references.

STD-1020 Natural Phenomena Hazards Design and Evaluation Criteria for DOE Facilities - This standard gives guidance regarding the selection of design criteria to be applied to nuclear facilities structural design.

STD-1021 Natural Phenomena Hazards Performance Categorization Guidelines For Structures, Systems and Components - See discussion in Section 2.2.

STD - 1022 Natural Phenomena Hazards Site Characterization - The site NPHs have been characterized by the CSB project.

STD - 1024 Guidelines For Use of Probabilistic Seismic Hazard Curves at DOE Sites - Seismic hazards risks have been established for the HCSAHCSE by the CSB project.

STD-1027 Guidance for Preliminary Hazard Classification and Accident Analysis Techniques for Compliance With DOE Order 5480.23, Safety Analysis Reports See discussion in Section 2.1.

STD - 1044 Guide To Good Practices For Equipment and Pipe Labeling - Sets forth guidelines for labeling equipment and piping. 
WHC-SD-SNF-CDR-007, Revision 0

\section{APPENDIX I - CRITERIA EVALUATION}

STD -3003 Backup Power Sources For DOE Facilities - There is no safety requirement for backup power in the CVDS. The guidance in the is guideline will be followed if backup power is included for other reasons.

STD-3006 Planning and Conduct of Operations Readiness Reviews - ORR planning will be addressed by the design team in order to assure that the necessary engineering documentation is ready for the ORR.

STD-3009 Preparation Guide for U.S. DOE Nonreactor Nuclear Facility Safety Analysis Reports - See discussion in Section 2.1.

STD-3011 Guidance for Preparation of DOE 5480.22 (TSR) and DOE 5480.23 (SAR) Implementation Plans - These plans will be drafted as part of the design process.

40CFR61 National Standards for Hazardous Air Pollutants - This sets forth restrictions on the release amount of those materials considered by this standard to be hazardous air pollutants.

\subsection{NRC Equivalency}

Report WHC-SD-SNF-DB-003 Rev. 1, December, 1995, “Spent Nuclear Fuel Project Path Forward Additional NRC Requirements" addresses implementation of the U.S. DOE K Basin Spent Nuclear Fuel Regulatory Policy (August 4, 1995) to achieve "nuclear safety equivalency" to comparable U.S. Nuclear Regulatory Commission (NRC) licensed facilities. This report addresses the project as a whole. Each of the components of the project is required to look at application for the specific features of the components. This section addresses application of each of the 29 items in WHC-SD-SNF-DB-003 to the HCSE as follows:

1. Fire Protection - Assure that DOE 5480.7A and DOE 6480.1A are equivalent to 10 CFR50, Appendix R. There are significant hazards associated with the event that a hot MCO undergoing conditioning might be suddenly flooded with fire water. A Fire Hazards Analysis will be performed to determine appropriate fire protection system features.

2. Response to Natural Phenomena Hazards. The key difference is that NRC includes a Design Basis Tornado and missile analysis. The CSB and HCSA will not be designed to perform nuclear release confinement in the event that they are struck by a tornado. The HCSE will contain appropriate tornado resistant features (such as oven instaliation in 
WHC-SD-SNF-CDR-007, Revision 0

\section{APPENDIX 1 - CRITERIA EVALUATION}

covered pits) and will also rely on probabilistic arguments for specific targets.

3. In the event that safety class 1 electrical equipment may be required and may be exposed to harsh environments ..... The HCSE will not have any safety class 1 SSCs requiring power to function.

4. SAR should include a loss of power accident analysis. The HCSE will be designed to shut down safely to a passive condition in the event of a (Loss Of operating Power) LOOP incident. LOOP analysis is standard practice in DOE 5480.23 safety analysis as prepared according to DOESTD-3009.

5. Incorporate the requirements of IEEE Std 484-1987 into the design and installation of safety class 1 batteries. The HCSE will not have safety class 1 batteries.

6. Incorporate the requirements of IEEE Std 535-1986 into the design and installation of safety class 1 batteries. The HCSE will not have safety class 1 batteries

7. Incorporate the requirements of IEEE Std 603-1991 into the design of safety class 1 instrumentation and control systems. The HCSE will not have any safety class 1 instrumentation and control systems.

8. Incorporate the requirements of ANSI/ANS 8.31986 for criticality alarm systems. The HCSE will not have a criticality alarm system because criticality cannot occur in the process.

9. Human factors planning and engineering. See discussion about the DOE 6430.1A human factors engineering requirements in Section 3.1 above.

10. Use Regulatory Guide 1.26 to assist in assigning appropriate code class to ASME Section III systems and components. The HCSE will not contain any ASME Section III systems or components.

11. Regulatory Guides 1.84 and 1.85 regarding application of ASME Section III requirements to safety class 1 systems. The HCSE will not have any safely class 1 systems. 
WHC-SD-SNF-CDR-007, Revision 0

\section{APPENDIX I - CRITERIA EVALUATION}

12. Ensure that the requirements of ANSI/ANS 509 and ANSI/ANS 510 are incorporated into HEPA filter design. The NRC and DOE 6430.1A requirements are the same. HEPA filter systems will be designed and specified according to these standards.

13. Incorporate the requirements of ANSI/ANS 57.1 and 57.2. Does not apply to the HCSE since there will be no cranes.

14. Incorporate the applicable design requirements of Generic Letters 88-14, $89-10$, and $89-13$ into safety class 1 .... The HCSE will not have safety class 1 SSCs.

15. 10 CFR Part 21 requires that manufacturers of procured items report defects in items or services for safety class 1 SSC procurement. Standard clause in WHC procurement terms and conditions. The HCSE will not have any safety class 1 SSCs.

16. 10 CFR 830.120 Quality Assurance program requirements. The normal DOE requirements for compliance with DOE $5700.6 \mathrm{c}$ exceeds this requirement. The HCSE will follow the DOE requirements.

17. 10 CFR 50.55 requires reporting of unusual occurrences during construction. This is covered by WHC-CM-1-5. The HCSE will observe this requirement.

18. 10 CFR 50, Appendix B Quality Assurance Criteria for Nuclear Power Plants and Fuel Reprocessing Plants. The normal DOE requirements for compliance with DOE $5700.6 \mathrm{c}$ exceeds this requirement. The HCSE will follow the DOE requirements.

19. Institute a process to identify safety class 1 equipment that has been identified in the commercial nuclear power industry via IE Bulletins and Notices as being potentially defective. The HCSE will not have safety class 1 SSCs.

20. 10 CFR Part 20 - incorporate control devices for access to high radiation areas that conform to the requirements of Section 20.161. The requirement will be met by means of installing shielding and by use of a process enclosure that prevents access to the high radiation area above an oven when the top is open. 
WHC-SD-SNF-CDR-007, Revision 0

\section{APPENDIX I - CRITERIA EVALUATION}

21. Apply the radiological exposure criteria of Section $72.104 \ldots$... The criteria will be met.

22. Use a deterministic value of 5 Rem in safety analyses for the onsite worker at the boundary of the controlled area (100 $\mathrm{m}$ from the CVDS release point) for design basis accidents to assist in determining safety class 2 and 3 SSCs (refs. 10 CFR 72.106, .100, .126). 10 CFR Part 72 requirements do not apply to the CVDS. The DOE orders and STDs do not address safety class 2 and 3 specifically. Equivalently they discuss SSCs that are safety significant to encompass those systems that protect on site workers and plant workers. The DOE criteria is 1 Rem at $100 \mathrm{~m}$., which is more restrictive than the referenced NRC requirement.

23. Implementation of RG 8.8, Information Relative to Ensuring That Occupational Radiation exposures at Nuclear Power Stations Will Be As Low As Reasonably Achievable, Rev. 3, DOE requirements from DOE N 5480.6 are more restrictive than RG 8.8. The design process will include a thorough ALARA analysis that estimates exposure considering source trems, work sequences, and anticipated repair requirements.

24. Implementation of RG 3.26, Standard Format and Content of Safety Analysis Reports for Fuel Reprocessing Plants or RG 3.48 Standard Format and Content of Safety Analysis Reports for an Independent Spent Fuel Storage Installation (Dry Storage). The safety analysis methodology established for the CSB will be implemented. The HCSE project will assume that the CSB project has complied with this requirement.

25. Review the monitoring requirements of 10 CFR 20, 10 CFR 70.59, 10 CFR50, Appendix A. These are analogous to DOE 6430.1A 1589-99.0.1. which is a project requirement stated above in DOE 6430.1A review in Section 3.1.

26. The 10 CFR 50, Appendix 50 general design criteria are adequately covered by the application of the criteria in this report.

27. Incorporate a criticality safety value of keff $=0.95$. The CVDS cannot achieve a critical condition. Criticality analysis for the configuration of the SNF inside the MCO will be the responsibility of the MCO design team. No criticality analysis will performed as part of the HCSE project.

28. Review ANSI/ANS 57.9 and RG 3.60 which set forth ISFSI design criteria. These criteria affect the CSB and HCSA projects but do not effect the HCSE project. 
WHC-SD-SNF-CDR-007, Revision 0

\section{APPENDIX I - CRITERIA EVALUATION}

29. Identify items that are "important to safety" in accordance with $10 \mathrm{CFR}$ 72.3 and impose safety class 1 requirements. Items that are important to safety will be identified and treated as safety significant items.

\subsection{NUCLEAR SAFETY PROGRAM CRITERIA}

Key nuclear facility safety documents that define public safety aspects of the HCSE/HCSA criteria are discussed in Sections 2.1 through 2.4 above. The key DOE Orders are the DOE 5480 series and the DOE 5400 and 5500 series.

Worker radiation exposure safety within the plant is defined by 10 CFR 835 and DOE N5480.6. Of these the DOE N5480.6 is the most comprehensive and restrictive document. A program to achieve radiation exposure As Low As Reasonably Achievable with an administrative control limitation of $500 \mathrm{mrem}$ per year per person is the stated whole body exposure criteria in DOE N 5480.6. 10 CFR 835 defines the DOE ALARA design target as 1.0 Rem per year. The HCSE design criteria will the DOE N 5480.6 value $(500$ mrem) unless features required to achieve this value prove to be unreasonable (in which case the 10 CFR 835 of 1.0 rem will be observed).

\subsection{OPERATIONS PREPARATIONS CRITERIA}

The transition from the end of the HCSE construction project to operations will be an Operations Readiness Review. This review will be conducted according to the requirements of DOE 5480. 31. The ORR will determine that the facility is complete, that the safety analysis is complete, that TSRs (DOE 5480.22 and DOE STD -3011, 1082) are complete, that appropriate design criteria have been established and met, that systems operations tests are complete, that personnel training has been completed (DOE STD 1005, 1007, 1008, 1009, 1011, 1056, $1060,1070,1074,1078$ ) that safety programs are operational (DOE STD 1082), that an appropriate conduct of operations program (DOE 5480.19 and DOE STD - 1032, 1033, 1034, 1035, 1036, 1037, 1038, 1039, 1040, 1041, 1042, $1043,1045,1051,1054,1055)$ is in place, that emergency preparedness programs are appropriate. In order to activate the transition and ORR plan is required per DOE STD-3006. This plan shouid be developed in parallel with the detailed design. 
WHC-SD-SNF-CDR-007, Revision 0

\section{APPENDIX I - CRITERIA EVALUATION}

Addendum 1 - Listing of Reference Criteria Documents 


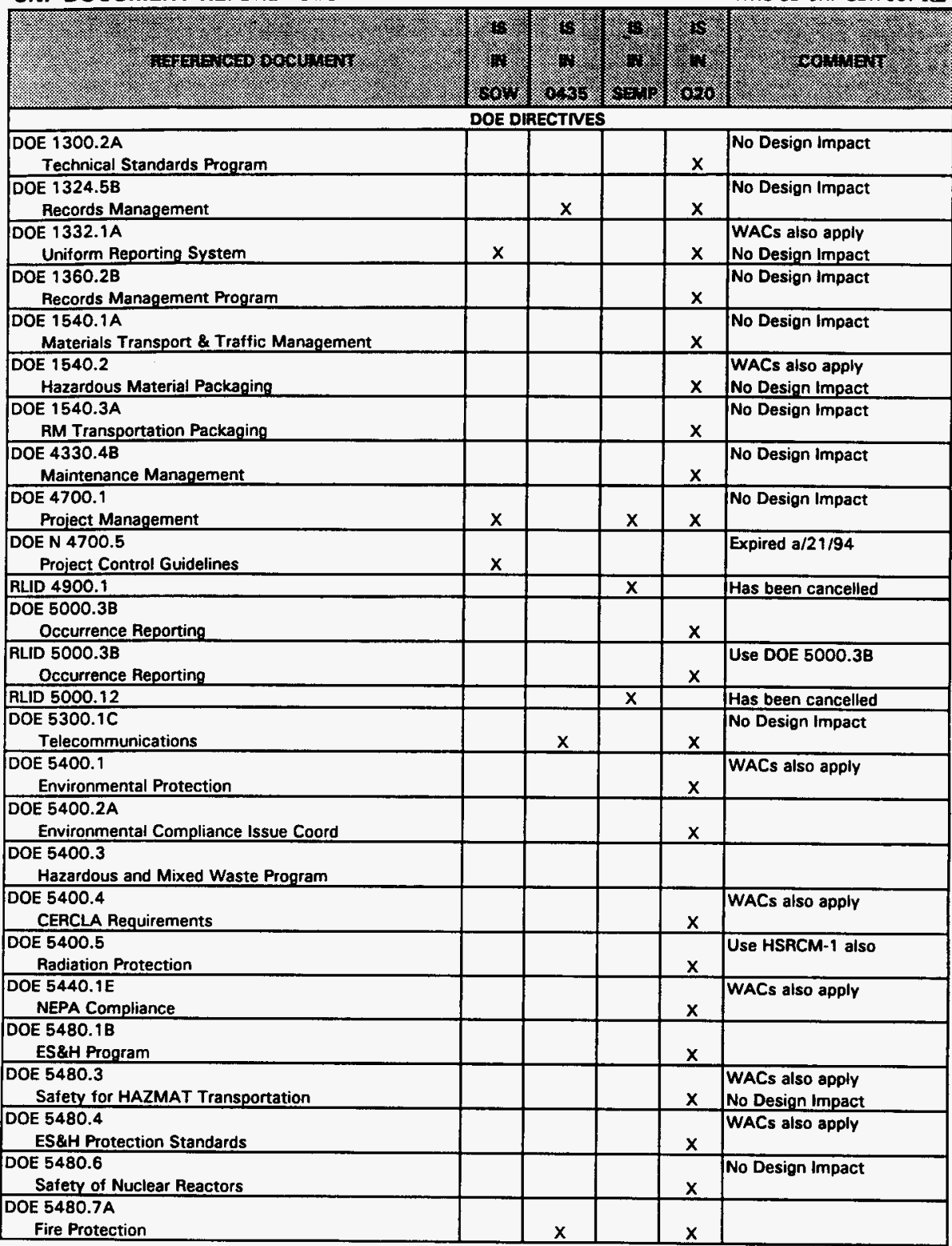


SNF DOCUMENT REFERENCES

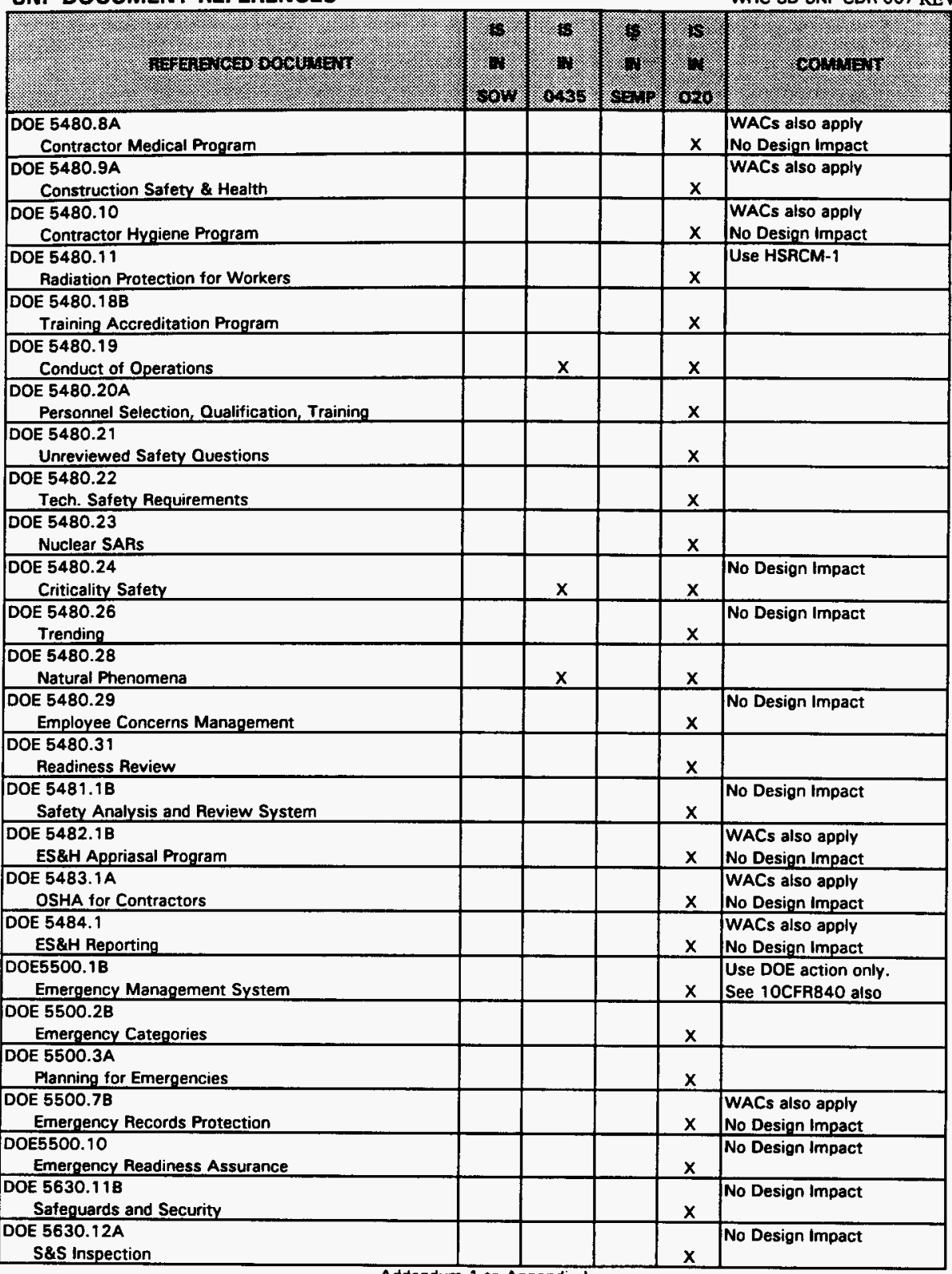


WHC-SD-SNF-COR-007 REV. 0

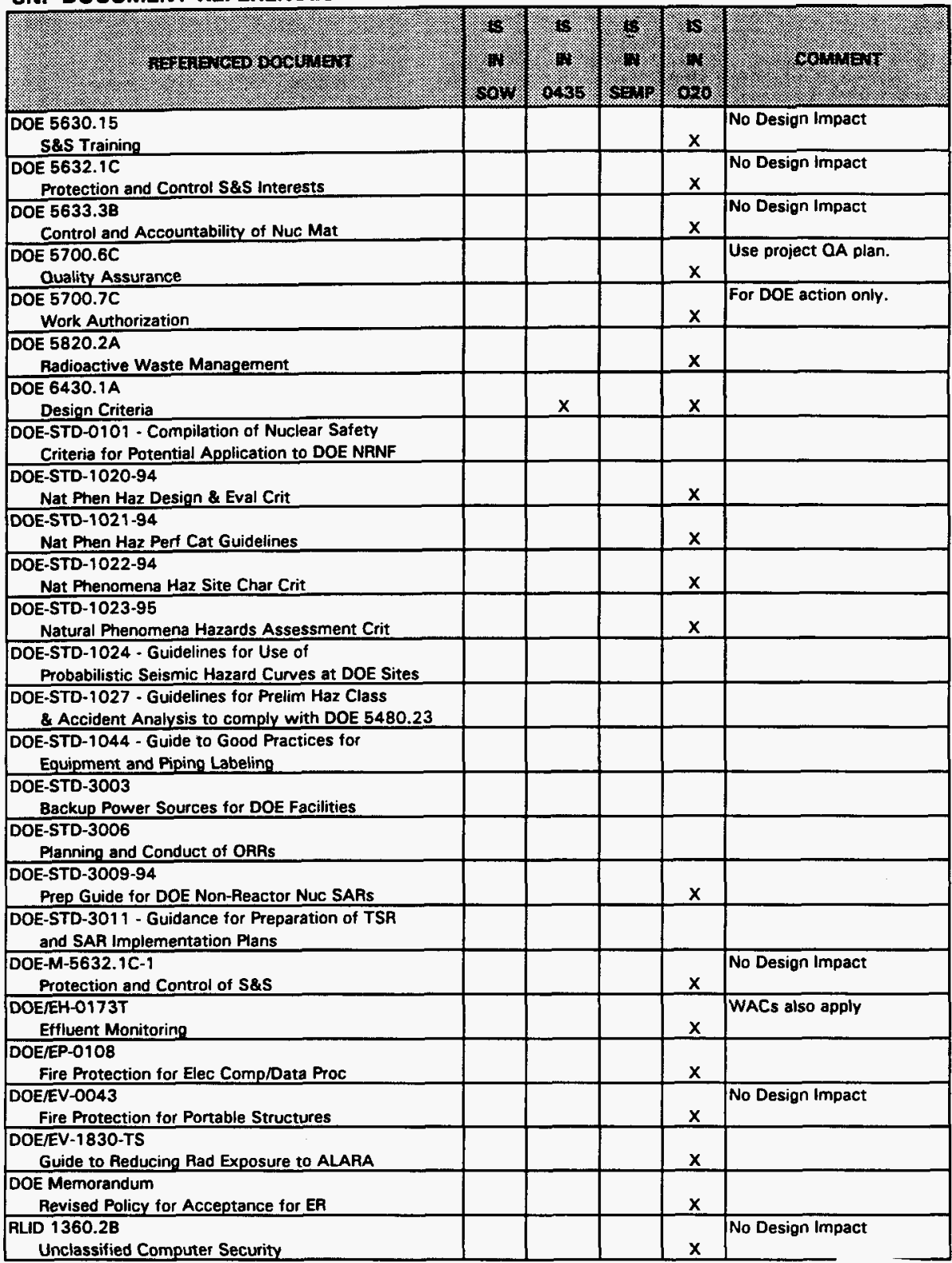


SNF DOCUMENT REFERENCES

\begin{tabular}{|c|c|c|c|c|c|}
\hline 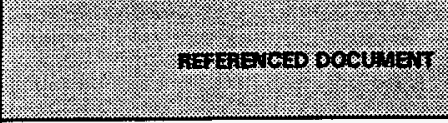 & 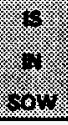 & 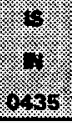 & 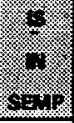 & 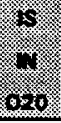 & 86, $1+21 \%$ \\
\hline $\begin{array}{l}\text { RLID } 5480.7 \\
\text { Fire Protection } \\
\end{array}$ & & & & $\mathbf{x}$ & \\
\hline $\begin{array}{l}\text { RLID } 5480.29 \\
\text { RL Employee Concerns Program } \\
\end{array}$ & & & & $\underline{x}$ & No Design Impact \\
\hline $\begin{array}{l}\text { RLID } 5480.31 \\
\text { Startup and Restart of Nuclear Facilities }\end{array}$ & & & & $\mathbf{x}$ & \\
\hline $\begin{array}{l}\text { RLID 5484.1A } \\
\text { EPS\&H Reporting Requirements } \\
\end{array}$ & & & & $\underline{x}$ & No Design Impact \\
\hline $\begin{array}{l}\text { DOE/RL 92-36 } \\
\text { Hoisting \& Rigping } \\
\end{array}$ & & $\underline{x}$ & & $\mathbf{x}$ & \\
\hline $\begin{array}{l}\text { DOE/RL 93-102 } \\
\text { Fy } 95 \text { Mission PIan }\end{array}$ & & & & $\underline{x}$ & No Design Impact \\
\hline \begin{tabular}{|l} 
DOE/RL-XX \\
Systems Engineering Criteria \\
\end{tabular} & & & $\mathbf{x}$ & & $\begin{array}{l}\text { Document not approved } \\
\text { by RL. }\end{array}$ \\
\hline \begin{tabular}{|l|} 
DOE-STD-1073-93 \\
Guide for Configuration Management
\end{tabular} & & & $\underline{x}$ & & For guidance only. \\
\hline $\begin{array}{c}\text { SEN } 15-90 \\
\text { NEPA } \\
\end{array}$ & & & & $\mathbf{x}$ & WACs also apply \\
\hline $\begin{array}{r}\text { SEN 35-91 } \\
\text { Nuclear Safety Policy } \\
\end{array}$ & & & & $\mathbf{x}$ & Use HSRCM-1 \\
\hline $\begin{array}{r}\text { HPS-SDC-4, 1, Rev } 12 \\
\text { Facility Design Loads }\end{array}$ & & $\mathbf{x}$ & & $\underline{x}$ & $\begin{array}{l}\text { Applicable Seismic } \\
\text { Criteria }\end{array}$ \\
\hline $\begin{array}{c}\text { HPS-SDC- } 5.1 \\
\text { HVAC }\end{array}$ & & & & $\mathbf{x}$ & \\
\hline $\begin{array}{l}\text { SNF-RD-PM-001 } \\
\text { SNF Program Requirements } \\
\end{array}$ & & & & $x$ & \\
\hline \multicolumn{6}{|c|}{ WASHINGTON ADMINISTRATIVE CODES } \\
\hline $\begin{array}{r}51-13 \\
\text { Energy Code }\end{array}$ & & $\mathbf{x}$ & & $\underline{x}$ & \\
\hline $\begin{array}{l}173-160 \\
\text { Construction and Maint of Wells }\end{array}$ & & & & $\mathbf{x}$ & \\
\hline $\begin{array}{l}\text { 173-201A } \\
\text { Water Quality Standards }\end{array}$ & & & & $\mathbf{x}$ & \\
\hline $\begin{array}{l}173.200 \\
\text { Water Quality for Ground Water }\end{array}$ & & & & $x$ & \\
\hline $\begin{array}{l}\text { 173-216 } \\
\text { Waste Discharge Permit Program }\end{array}$ & & & & $\underline{x}$ & \\
\hline $\begin{array}{l}\begin{array}{l}173-218 \\
\text { Underground Injection }\end{array} \\
\end{array}$ & & & & $\mathbf{x}$ & \\
\hline $\begin{array}{r}\begin{array}{r}173-224 \\
\text { Water Discharge Permit Fees }\end{array} \\
\end{array}$ & & & & $\mathbf{x}$ & \\
\hline $\begin{array}{l}173-240 \\
\text { Sub of Plans for Waste Water Fac }\end{array}$ & & & & $\underline{x}$ & \\
\hline $\begin{array}{l}\begin{array}{l}173-303 \\
\text { Dangerous Waste Regulations }\end{array} \\
\end{array}$ & & $x$ & & $\mathbf{x}$ & \\
\hline $\begin{array}{l}\text { 173-304 } \\
\text { Min Stds for Solid Waste Handling }\end{array}$ & & & & $\mathbf{x}$ & \\
\hline $\begin{array}{r}173-307 \\
\text { Plans } \\
\end{array}$ & & & & $\mathbf{x}$ & \\
\hline $\begin{array}{l}173-340 \\
\text { Model Toxic Control Regs }\end{array}$ & & & & $\underline{x}$ & \\
\hline
\end{tabular}


SNF DOCUMENT REFERENCES

\begin{tabular}{|c|c|c|c|c|c|}
\hline 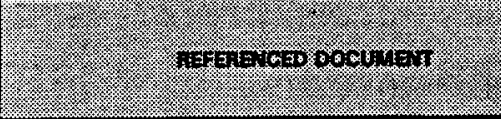 & (1) & 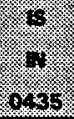 & 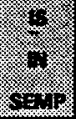 & 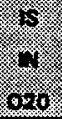 & 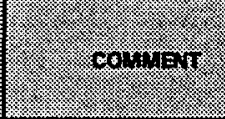 \\
\hline $\begin{array}{r}173-360 \\
\text { UST Regulations }\end{array}$ & & & & $x$ & \\
\hline $\begin{array}{r}173-400 \\
\text { General Regulations for Air Poll Sources }\end{array}$ & & & & $\underline{x}$ & \\
\hline $\begin{aligned} \begin{array}{l}173-401 \\
\text { Operating Permit Regulations }\end{array} \\
\end{aligned}$ & & & & $x$ & \\
\hline \begin{tabular}{|l}
$173-460$ \\
Controls for Toxic Air \\
\end{tabular} & & & & $x$ & \\
\hline $\begin{array}{l}173-480 \\
\text { Air Quality for Radionuclides }\end{array}$ & & & & $x$ & \\
\hline $\begin{array}{l}197.11 \\
\text { SEPA Rules } \\
\end{array}$ & & & & $\underline{x}$ & \\
\hline \begin{tabular}{|l}
$246-247$ \\
Radioactive Air Emmissions \\
\end{tabular} & & & & $\mathbf{x}$ & \\
\hline $\begin{array}{r}246-247-050 \\
\text { Pegistration } \\
\end{array}$ & & & & $x$ & \\
\hline \begin{tabular}{|r|}
$246-247-060$ \\
Air Emissions Permit \\
\end{tabular} & & & & $x$ & \\
\hline $\begin{array}{r}246-249 \\
\text { Rad Waste, Use of Commercial Site } \\
\end{array}$ & & & & $\mathbf{x}$ & \\
\hline \begin{tabular}{|l|}
$246-272$ \\
On Site Sewage Systems
\end{tabular} & & & & $x$ & \\
\hline $\begin{array}{l}246-290 \\
\text { Public Water Supplies } \\
\end{array}$ & & & & $x$ & \\
\hline $\begin{array}{l}\text { 402-40 } \\
\text { Wash Std for Prot Against Radiation }\end{array}$ & & & & $x$ & \\
\hline $\begin{array}{l}446-50 \\
\text { Transportation of Hazardous Wastes }\end{array}$ & & & & $\mathbf{x}$ & \\
\hline Tri Party Agreement & & & & $\mathrm{x}$ & \\
\hline & CODE C & WASH & NGTON & & \\
\hline $\begin{array}{l}36.58 \\
\text { Washington Solid Waste Disposal }\end{array}$ & & & & $\mathrm{X}$ & \\
\hline $\begin{array}{l}36.94 \\
\text { Sewage, Water and Drainage Systems }\end{array}$ & & & & $x$ & \\
\hline \begin{tabular}{|l|}
$43.21 \mathrm{C}$ \\
State Environmental Policy \\
\end{tabular} & & & & $x$ & Implemented by WAC \\
\hline $\begin{array}{l}43.200 \\
\text { Washington Radioactive Waste Act }\end{array}$ & & & & $x$ & \\
\hline $\begin{array}{l}46.37 \\
\text { Vehicle Lighting and Other Equipment }\end{array}$ & & & & $x$ & \\
\hline $\begin{array}{l}46.44 \\
\text { Size, Weight, Load } \\
\end{array}$ & & & & $x$ & \\
\hline $\begin{array}{l}49.17 \\
\text { Wash Industrial Safety and Health Act }\end{array}$ & & & & $\underline{x}$ & \\
\hline $\begin{array}{l}70.94 \\
\text { Washington Clean Air Act }\end{array}$ & & & & $x$ & implemented by WAC \\
\hline $\begin{array}{l}70.95 \\
\text { Solid Waste Management Reduction \& Recycle }\end{array}$ & & & & $\mathbf{x}$ & \\
\hline $\begin{array}{l}70.96 \\
\text { Washington Underground Storage Tanks }\end{array}$ & & & & $x$ & \\
\hline $\begin{array}{l}\text { 70.105 } \\
\text { Hazardous Waste Management }\end{array}$ & & & & $x$ & Implemented by WAC \\
\hline
\end{tabular}

WHC-SD-SNF-CDR-007 REV, 0 0 
SNF DOCUMENTT REFERENCES

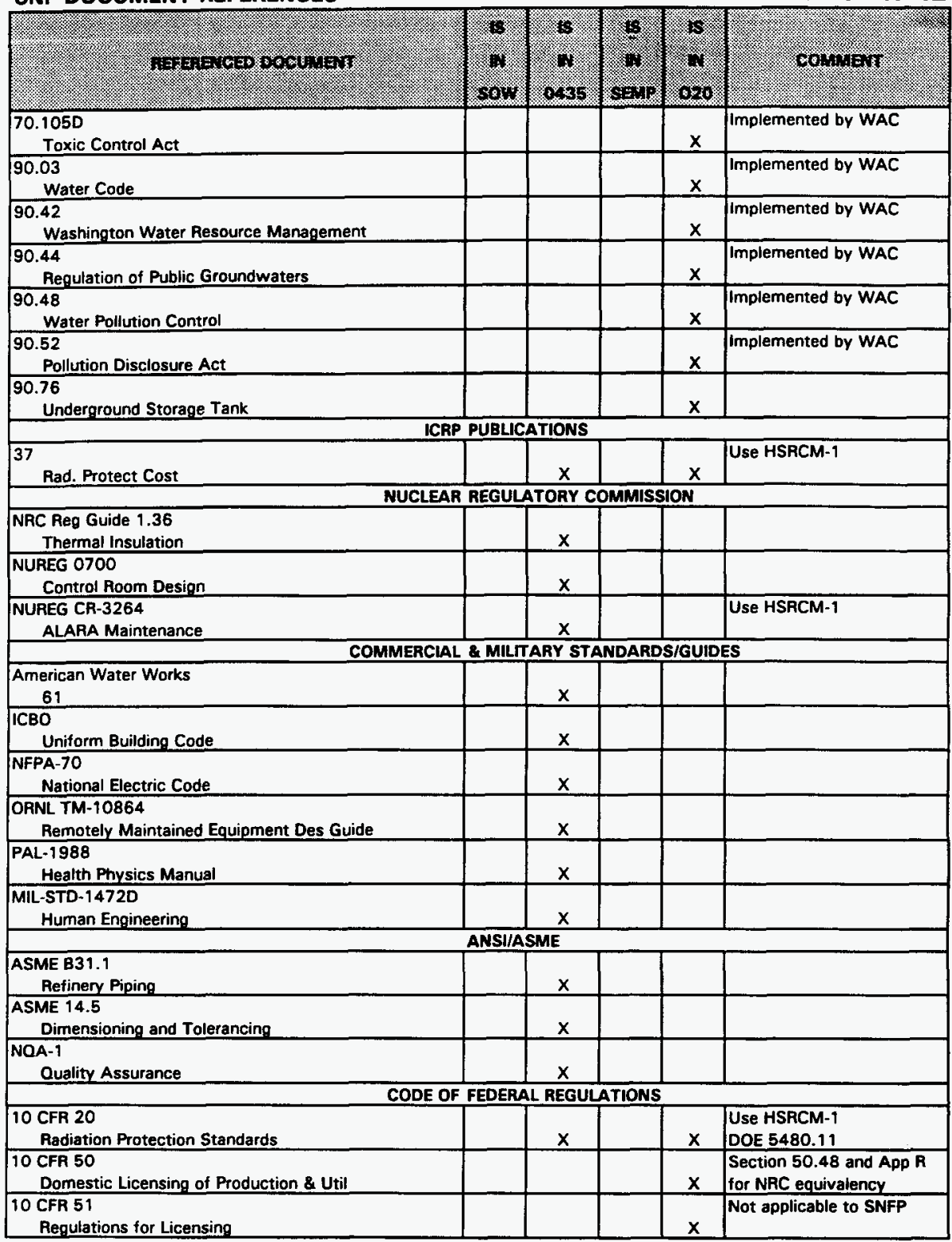


SNF DOCUMENT REFERENCES

\begin{tabular}{|c|c|c|c|c|c|}
\hline 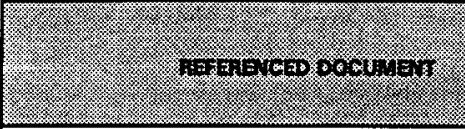 & 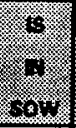 & 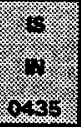 & 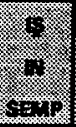 & 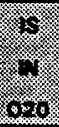 & 30 \\
\hline $\begin{array}{l}10 \text { CFR } 71 \\
\text { Radioactive Pkg \& Trans } \\
\end{array}$ & & & & $x$ & Use 49 CFRs \\
\hline $\begin{aligned} 10 \text { CFR } 72 \\
\text { Licensing of Spent Fuel Storage }\end{aligned}$ & & $x$ & & $x$ & Not Applicable \\
\hline $\begin{array}{l}10 \text { CFR } 73 \\
\text { Protection of Plants and Materials } \\
\end{array}$ & & & & $x$ & Use RLID 5632.1B \\
\hline $\begin{array}{l}10 \text { CFR } 436 \\
\text { Life Cycle Cost Methods \& Procedures }\end{array}$ & & & & $x$ & Not Applicable \\
\hline $\begin{array}{r}10 \text { CFR } 830.120 \\
\text { Quality Assurance } \\
\end{array}$ & $x$ & $x$ & & $x$ & \\
\hline \begin{tabular}{|l|}
10 CFR 835 \\
Padiation Protection \\
\end{tabular} & & $x$ & & $x$ & \\
\hline $\begin{array}{l}10 \text { CFR } 840 \\
\text { Extraordinary Nuclear Occurances }\end{array}$ & & & & & \\
\hline $\begin{array}{l}10 \text { CFR } 961 \\
\text { Std Contact for Disposal of SNF \& HLRW }\end{array}$ & & & & $x$ & \\
\hline $\begin{array}{l}10 \text { CFR } 1021 \\
\text { NEPA } \\
\end{array}$ & & & & $x$ & Use WAC \\
\hline $\begin{array}{r}29 \text { CFR } 1910 \\
\text { OSHA ACt } \\
\end{array}$ & & $x$ & & $x$ & \\
\hline \begin{tabular}{|l|}
29 CFR 1926 \\
Construction OSHA
\end{tabular} & & & & $\mathbf{x}$ & \\
\hline $\begin{array}{l}40 \text { CFR } 6.3029 \\
\text { Fish } 8 \text { Wildlife } \\
\end{array}$ & & & & $x$ & \\
\hline \begin{tabular}{|l|}
40 CFR 52 \\
Ambient Air Quality
\end{tabular} & & & & $x$ & Use WAC \\
\hline $\begin{array}{l}40 \text { CFR } 58 \\
\text { Ambient Air Quality Surveillance } \\
\end{array}$ & & & & $x$ & \\
\hline $\begin{array}{l}40 \text { CFR } 60 \\
\text { Stds of Perf for New Stationary Sources } \\
\end{array}$ & & & & $x$ & \\
\hline $\begin{array}{l}40 \text { CFR } 61 \\
\text { Haz Air Emissions Stds } \\
\end{array}$ & & & & $x$ & Use WAC \\
\hline $\begin{array}{l}40 \text { CFR } 122 \\
\text { Permitting Regmnts for Land Disp Fac }\end{array}$ & & & & $\underline{x}$ & \\
\hline \begin{tabular}{|l|l|}
40 CFR 124 \\
Procedures for Decision Making \\
\end{tabular} & & & & $x$ & \\
\hline $\begin{array}{l}40 \text { CFR } 125 \\
\text { Criteria and Stds for NPOES } \\
\end{array}$ & & & & $\mathbf{x}$ & \\
\hline $\begin{array}{l}40 \text { CFR } 191 \\
\text { Management \& Disposal of SNF \& TRW }\end{array}$ & & & & $x$ & \\
\hline \begin{tabular}{|l|}
40 CFR 240 \\
Guidelines for Thermal Processing \\
\end{tabular} & & & & $x$ & \\
\hline $\begin{array}{l}40 \text { CFR } 241 \\
\text { Guidelines for Land Disp of Solid Waste }\end{array}$ & & & & $\mathbf{x}$ & \\
\hline $\begin{array}{l}40 \text { CFR } 260 \\
\text { Haz Waste Management System } \\
\end{array}$ & & & & $x$ & \\
\hline $\begin{array}{l}40 \text { CFR } 261 \\
\text { Identification and Licensing } \\
\end{array}$ & & & & $x$ & \\
\hline $\begin{array}{l}40 \text { CFR } 262 \\
\text { Stds Applicable to Generators } \\
\end{array}$ & & & & $x$ & Use WAC \\
\hline $\begin{array}{l}40 \text { CFR } 264 \\
\text { Stds for Owners \& Operators }\end{array}$ & & & & $x$ & WACs also apply \\
\hline
\end{tabular}

WHC-SD-SNF-CDR-007 REV. 0 
WHC-SD-SNF-CDR-007 REV. 0

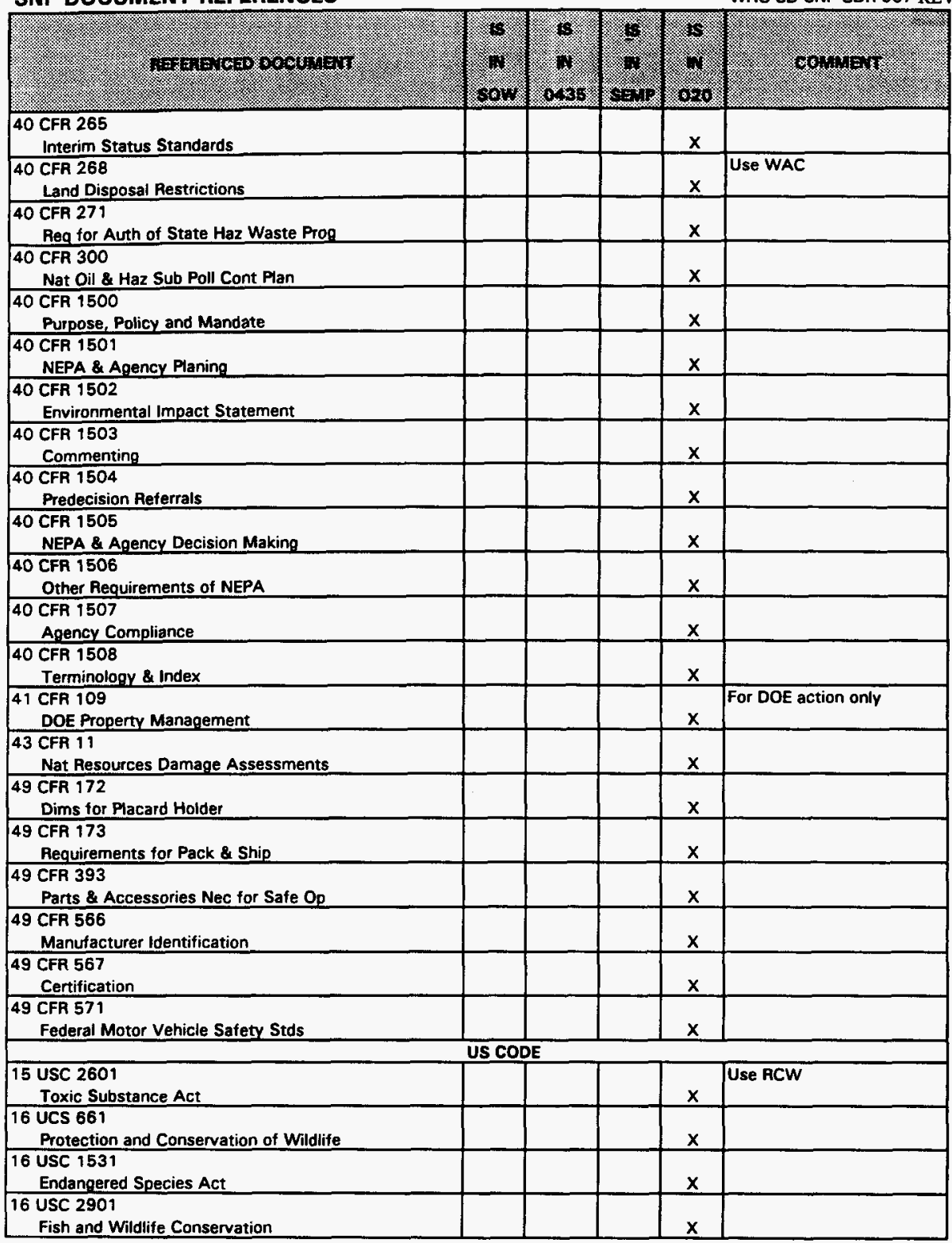


SNF DOCUMENT REFERENCES

WHC-SD-SNF-CDR-0O7 REV. 0

\begin{tabular}{|c|c|c|c|c|c|}
\hline 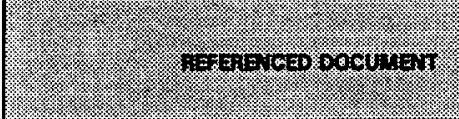 & 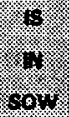 & 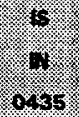 & 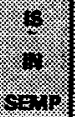 & 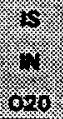 & (6) \\
\hline $\begin{aligned} 25 & \text { USC } 3001 \\
& \text { Native American Graves Protection }\end{aligned}$ & & & & $x$ & \\
\hline $\begin{array}{l}29 \text { USC } 651 \\
\text { Occupational Safety \& Health }\end{array}$ & & & & $x$ & \\
\hline $\begin{array}{l}33 \text { USC } 1251 \\
\text { Clean Water Act }\end{array}$ & & & & $x$ & \\
\hline \begin{tabular}{|c|}
42 USC 1996 \\
American Indian Relipious Freedom
\end{tabular} & & & & $x$ & \\
\hline $\begin{array}{l}42 \text { USC } 2011 \\
\text { Atomic Energy Act } \\
\end{array}$ & & & & $x$ & \\
\hline \begin{tabular}{|l|}
42 USC 4321 \\
NEPA of 1969 \\
\end{tabular} & & & & $x$ & Use RCW \\
\hline $\begin{array}{l}42 \text { USC } 5801 \\
\text { Energy Reorganization Act of } 1974\end{array}$ & & & & $x$ & \\
\hline $\begin{array}{l}42 \text { USC } 6901 \\
\text { Solid Waste Disposal } \\
\end{array}$ & & & & $x$ & \\
\hline $\begin{aligned} 42 \text { USC } 7101 \\
\text { DOE Organization Act }\end{aligned}$ & & & & $x$ & \\
\hline $\begin{array}{l}42 \text { USC } 7401 \\
\text { Clean Air Act }\end{array}$ & $x$ & & & $x$ & Use RCW \\
\hline $\begin{array}{l}42 \text { USC } 9601 \\
\text { Compensation and Liability }\end{array}$ & & & & $x$ & \\
\hline $\begin{array}{l}42 \text { USC } 10101 \\
\text { Nuclear Waste Policy Act }\end{array}$ & & & & $x$ & \\
\hline $\begin{array}{l}49 \text { USC } 1802 \\
\text { Haz Materials Transportation Act }\end{array}$ & & & & $x$ & \\
\hline \begin{tabular}{|l}
46 FR 42237 \\
Responses to Environmental Stds \\
\end{tabular} & & & & $x$ & \\
\hline $\begin{array}{l}\text { EO-12316 } \\
\text { Responses to Environmental Stds }\end{array}$ & & & & $x$ & 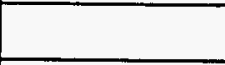 \\
\hline $\begin{array}{l}\text { PL } 92-500 \\
\text { Clean Water Act } \\
\end{array}$ & & & & $x$ & Use RCW \\
\hline $\begin{array}{l}\text { PL } \begin{array}{l}101+189 \\
\text { Defense Authorization Act }\end{array} \\
\end{array}$ & & & & $x$ & Implemented by CFRs \\
\hline & DOCUM & ENTS & & & \\
\hline $\begin{array}{l}\text { HSRCM-1 } \\
\text { Hanford Rad Control Manual }\end{array}$ & & $x$ & & $x$ & \\
\hline $\begin{array}{l}\text { CM } 1 \\
\text { Company Policies and Charters }\end{array}$ & & & & $x$ & \\
\hline $\begin{array}{l}\text { CM 1-3 } \\
\text { Requirements \& Procedures }\end{array}$ & & & & $\mathrm{x}$ & \\
\hline $\begin{array}{l}\text { CM } 1-5 \\
\text { Std Operating Procedures }\end{array}$ & & & & $\mathrm{x}$ & \\
\hline $\begin{array}{r}\text { CM } 1-8 \\
\text { Work Management } \\
\end{array}$ & & & & $x$ & \\
\hline $\begin{array}{l}\text { CM 2-2 } \\
\text { Material Management Manual }\end{array}$ & & & & $x$ & \\
\hline $\begin{array}{l}\text { CM } 2-3 \\
\quad \text { Property Management Manual }\end{array}$ & & & & $x$ & \\
\hline $\begin{array}{l}\text { CM } 2-5 \\
\text { Management Control System }\end{array}$ & & & $x$ & $x$ & \\
\hline
\end{tabular}


SNF DOCUMENT REFERENCES

WHC-SD-SNF-CDR-007 REV. 0

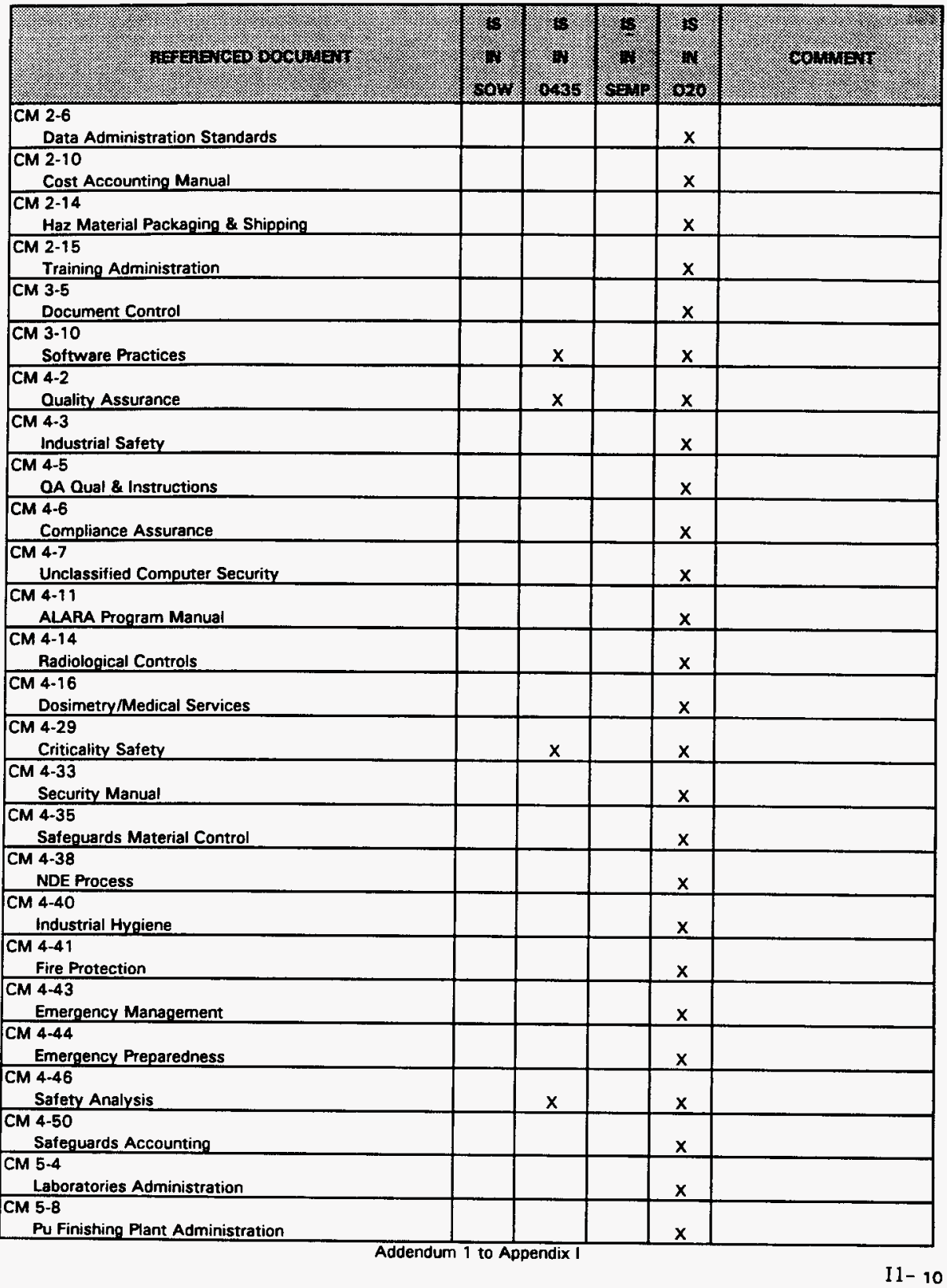


SNF DOCUMENT REFERENCES

WHC-SD-SNF-CDR-007 REV. 0

\begin{tabular}{|c|c|c|c|c|c|}
\hline 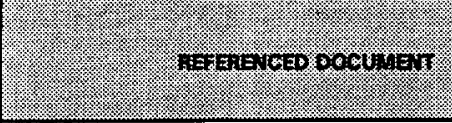 & 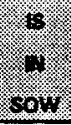 & 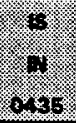 & 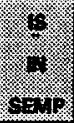 & 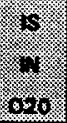 & 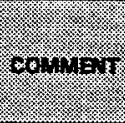 \\
\hline $\begin{array}{l}\text { CM 5-9 } \\
\text { PUAEXNUO3 Plant Administration } \\
\end{array}$ & & & & $x$ & \\
\hline $\begin{array}{l}\text { CM 5-13 } \\
\text { K Basins Policy Manual }\end{array}$ & & & & $x$ & \\
\hline $\begin{array}{l}\text { CM 5-16 } \\
\text { Solid Waste Management } \\
\end{array}$ & & & & $x$ & \\
\hline $\begin{array}{l}\text { CM 5-34 } \\
\text { SWD Operations Administration }\end{array}$ & & & & $x$ & \\
\hline \begin{tabular}{|l} 
CM 6-1 \\
Standard Engineering Practices \\
\end{tabular} & & $x$ & $x$ & $x$ & \\
\hline \begin{tabular}{|l|} 
CM $6-2$ \\
Project Management \\
\end{tabular} & & & & $\underline{x}$ & \\
\hline $\begin{array}{l}\text { CM } 6-3 \\
\text { Drafting Standards }\end{array}$ & & & & $\mathrm{x}$ & \\
\hline $\begin{array}{l}\text { CM 6-10 } \\
\text { Welding Manual }\end{array}$ & & & & $x$ & \\
\hline $\begin{array}{r}\text { CM } 6-12 \\
\text { Project Procedures } \\
\end{array}$ & & & & $x$ & \\
\hline $\begin{array}{l}\text { CM 7-4 } \\
\text { Environmental Monitoring } \\
\end{array}$ & & & & $x$ & \\
\hline $\begin{array}{l}\text { CM 7-5 } \\
\text { Environmental Compliance }\end{array}$ & & $x$ & & $x$ & \\
\hline \begin{tabular}{|l} 
CM $8-7$ \\
Operations Support Systems \\
\end{tabular} & & & & $x$ & \\
\hline $\begin{array}{l}\text { CM 8-9 } \\
\text { Workmanship Standards } \\
\end{array}$ & & & & $x$ & \\
\hline $\begin{array}{l}\text { EP-0009 } \\
\text { Acronyms and Abbreviations }\end{array}$ & & & & $x$ & \\
\hline $\begin{array}{l}\text { EP-0063-4 } \\
\text { Solid Waste Acceptance } \\
\end{array}$ & & & & $x$ & \\
\hline $\begin{array}{r}\text { EP-0231-5 } \\
\text { Surplus Facilities Program Plan }\end{array}$ & & & & $x$ & \\
\hline $\begin{array}{l}\text { EP-0496 } \\
\text { Pollution Prevention Implementation }\end{array}$ & & & & $x$ & \\
\hline $\begin{aligned} & \text { EP-0722 } \\
& \text { Systems Engineering F\&Rs }\end{aligned}$ & & & & $x$ & \\
\hline $\begin{array}{l}\text { EP-0779 } \\
\text { Architecture Systhesis Basis }\end{array}$ & & & & $x$ & \\
\hline $\begin{array}{l}\text { EP-0830 } \\
\text { SNF Path Forward } \\
\end{array}$ & & & $x$ & & \\
\hline $\begin{array}{l}\text { GG-DWG-01 } \\
\text { Preperation \& Control of Drawings }\end{array}$ & & & & $x$ & \\
\hline $\begin{array}{l}\text { GG-DWG-02 } \\
\text { Layering Conventions }\end{array}$ & & & & $x$ & \\
\hline $\begin{array}{l}\text { GG-DWG-03 } \\
\text { Drawing index }\end{array}$ & $x$ & $x$ & & & \\
\hline $\begin{array}{l}\text { GG-DWG-04 } \\
\text { Parts List/BOM } \\
\end{array}$ & $x$ & $\underline{x}$ & & & \\
\hline $\begin{array}{l}\text { GH-CLIM-01 } \\
\text { Hanford Site Climate Data }\end{array}$ & & $x$ & & & \\
\hline $\begin{array}{l}\text { IP-0117 } \\
\text { Procedure Development }\end{array}$ & & & & $x$ & \\
\hline
\end{tabular}


SNF DOCUMENT REFERENCES

WHC-SD-SNF-CDR-007 REV. 0

\begin{tabular}{|c|c|c|c|c|c|}
\hline flif & 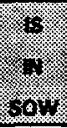 & 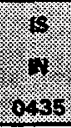 & $\frac{1}{3}$ & 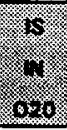 & $00011=11$ \\
\hline $\begin{array}{l}\text { IP-0240 } \\
\text { Operating Procedures Format }\end{array}$ & & & & $\underline{x}$ & \\
\hline $\begin{array}{r}1 P-0263 \\
\text { Bldg Emergency Plan East Patrol Bidg }\end{array}$ & & & & $x$ & \\
\hline $\begin{aligned} \mathrm{P}-0382 \\
\text { Nuclear Materials Safeguards Users }\end{aligned}$ & & & & $x$ & \\
\hline $\begin{array}{l}\text { IP-0565 } \\
\text { Safeguards Disk Procedures } \\
\end{array}$ & & & & $\underline{x}$ & \\
\hline $\begin{array}{l}\text { PP-0821 } \\
\text { Plutonium Operation Admin }\end{array}$ & & & & $x$ & Not applicable to SNFP \\
\hline \begin{tabular}{|l|} 
P-0836 \\
Order Compliance Admin \\
\end{tabular} & & & & $\underline{x}$ & Not applicable to SNFP \\
\hline $\begin{array}{l}\mid \text { IP-1026 } \\
\text { Engineering Practice Guidelines }\end{array}$ & & & & $x$ & \\
\hline $\begin{array}{l}\text { IP-1043 } \\
\text { Occupational ALARA Program }\end{array}$ & & & & $x$ & Use HSRCM-1 \\
\hline $\begin{array}{l}\text { IP-1117 } \\
\text { Systems Engineering Manual }\end{array}$ & & & $x$ & & \\
\hline \begin{tabular}{|l|} 
IP-1140 \\
Procedure Development \& Control Services \\
\end{tabular} & & & & $x$ & \\
\hline \begin{tabular}{|l|} 
S-0436 \\
$\quad$ Performance Specifications \\
\end{tabular} & $\underline{x}$ & & & & \\
\hline $\begin{aligned} \text { SD-GN-DGS-30011 } \\
\text { Radiological Design Guide } \\
\end{aligned}$ & & $x$ & & $x$ & \\
\hline $\begin{array}{l}\text { SD-GN-ER-1006 } \\
\text { K-Basin Floor Loads }\end{array}$ & & & & $x$ & \\
\hline $\begin{array}{l}\text { SD-GN-ES-30006 } \\
\text { Criteria for Uniform Bolting Preloads }\end{array}$ & & & & $x$ & \\
\hline $\begin{array}{l}\text { SD-MA-SPP-001 } \\
\text { Welding Procedure Supporting Document }\end{array}$ & & & & $x$ & \\
\hline $\begin{array}{l}\text { SD-NR-SA-024 } \\
\text { Structural Feasibility of Consolidation }\end{array}$ & & & & $x$ & \\
\hline $\begin{array}{l}\text { SD-SNF-CM-001 } \\
\text { SNF Configuration Management }\end{array}$ & & & $x$ & & \\
\hline $\begin{array}{l}\text { SD-SNF-CM-003 } \\
\text { SNF interface Control }\end{array}$ & & & $x$ & & \\
\hline $\begin{array}{l}\text { SD-SNF-DB-004 } \\
\text { SNF Project Seismic Design Criteria, NRC Eg }\end{array}$ & & & & & \\
\hline $\begin{array}{l}\text { SD-SNF-DGS-001 } \\
\text { K-Basin Design Guidelines }\end{array}$ & & & & $x$ & \\
\hline $\begin{array}{l}\text { SD-SNF-SD-002 } \\
\text { SNF Technical Baseline }\end{array}$ & & & $x$ & & \\
\hline $\begin{array}{l}\begin{array}{l}\text { SD-SNF-SD-003 } \\
\text { SNF Technical Baseline }\end{array} \\
\end{array}$ & & & $\underline{x}$ & & \\
\hline $\begin{array}{l}\text { SD-SNF-SEMP-001 } \\
\text { SNF Engr Management Plan } \\
\end{array}$ & $x$ & & & & \\
\hline $\begin{array}{l}\text { SD-SNF-TT-009 } \\
\text { Design Basis Feed Preparation }\end{array}$ & & & & $x$ & \\
\hline $\begin{array}{l}\text { SD-SNF-TI-012 } \\
\text { Underwater Fuel Survey }\end{array}$ & & & & $x$ & \\
\hline $\begin{array}{l}\text { SD-WM-OSA-006 } \\
\text { OSAS for KE \& KW Basins }\end{array}$ & & & & $x$ & \\
\hline
\end{tabular}

Addendum 1 to Appendix 1 
SNF DOCUMENT REFERENCES

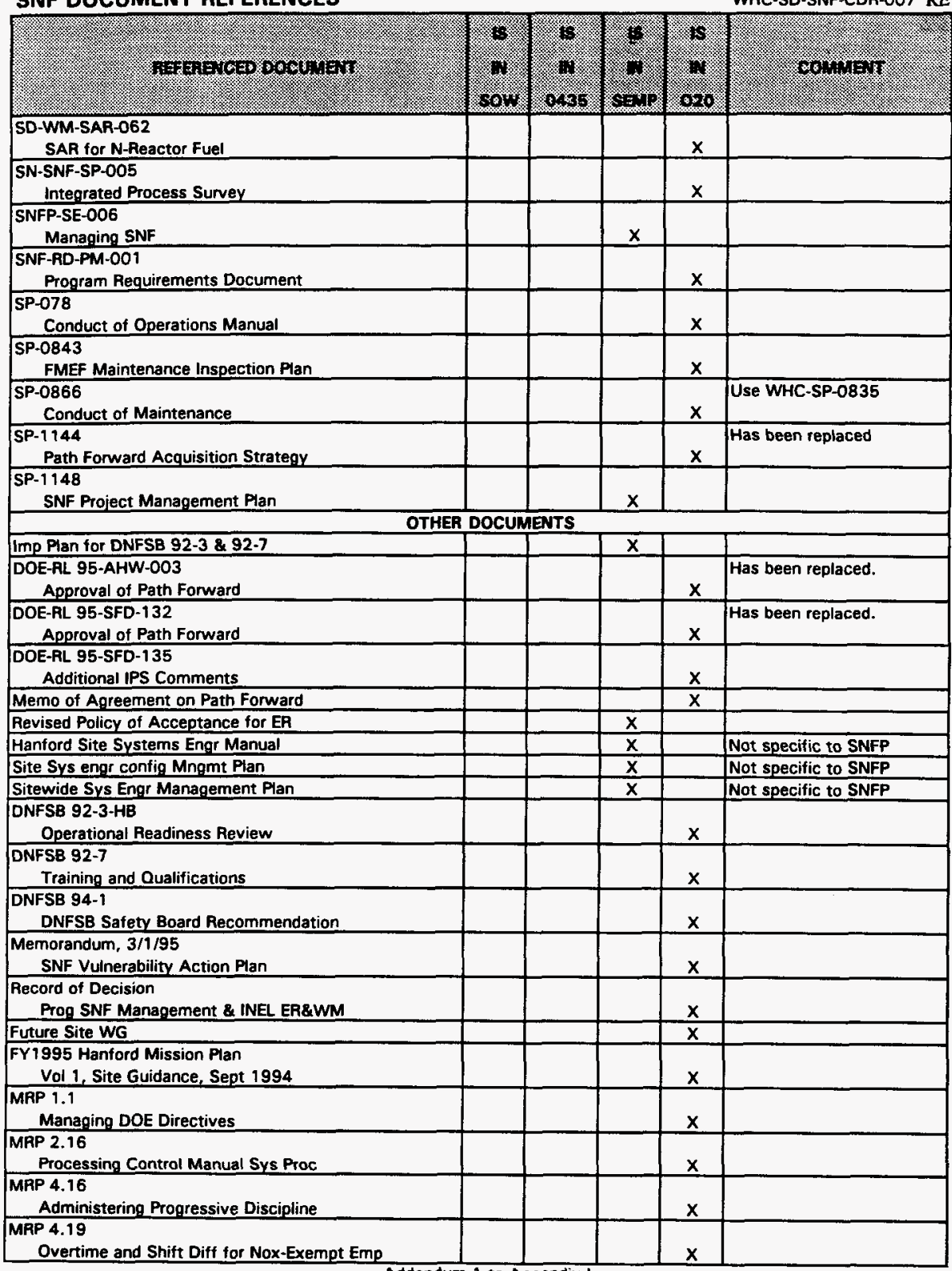




\begin{tabular}{|c|c|c|c|c|c|}
\hline 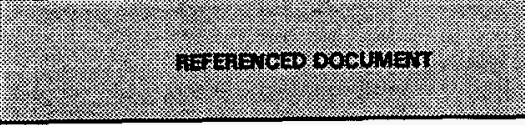 & 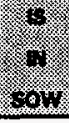 & $\frac{1 \%}{4}$ & 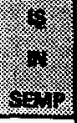 & 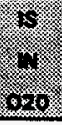 & $89+1=1$ \\
\hline $\begin{array}{l}\text { MRP } 5.44 \\
\text { Waste Minimization Program }\end{array}$ & & & & $\mathrm{x}$ & \\
\hline $\begin{array}{l}\text { MRP } 6.15 \\
\text { Facility Shutdown, Standby \& Transfer }\end{array}$ & & & & $\underline{x}$ & \\
\hline $\begin{array}{l}\text { WHC } 9553866 \text {-Letter } \\
\text { Rec Ingegrated Process Strategy }\end{array}$ & & & & $x$ & \\
\hline $\begin{array}{l}\text { DOE EM-36, } 9 \text { Nov } 94 \\
\text { Lytle Ltr to Grumbly, App of Path Forward }\end{array}$ & & & & $\underline{x}$ & Not specific to SNFP \\
\hline \multicolumn{6}{|c|}{ ADDITIONAL NRC REQUIREMENTS } \\
\hline $\begin{array}{c}\text { WHC-SD-SNF-DB-002, Table 5.b } \\
\text { As relates to fire protection }\end{array}$ & & & & & $\begin{array}{l}\text { Confirm Safety Class } \\
\text { Fire Protection not req. }\end{array}$ \\
\hline $\begin{array}{l}10 \text { CFR Part } 50.48 \& \text { Appendix } R \\
\text { As relates to fire protection }\end{array}$ & & & & & $\begin{array}{l}\text { Confirm Safety Class } \\
\text { Fire Protection not req. }\end{array}$ \\
\hline $\begin{array}{l}10 \text { CFR Part } 72.122(\mathrm{C}) \\
\text { As relates to fire protection }\end{array}$ & & & & & $\begin{array}{l}\text { Confirm Safety Class } \\
\text { Fire Protection not req. }\end{array}$ \\
\hline $\begin{array}{l}\text { Regulatory Guide } 1.76 \\
\text { As relates to tornado criteria } \\
\end{array}$ & & & & & $\begin{array}{l}\text { Not applicable, No } \\
\text { Safety Class equipment }\end{array}$ \\
\hline $\begin{array}{l}\text { SECY-93-087 } \\
\text { As relates to tornado criteria }\end{array}$ & & & & & $\begin{array}{l}\text { Not applicable, No } \\
\text { Safety Class equipment }\end{array}$ \\
\hline $\begin{array}{l}\text { NUREG/CR-4461 } \\
\text { As relates to tornado criteria }\end{array}$ & & & & & $\begin{array}{l}\text { Not applicable, No } \\
\text { Safety Class equipment }\end{array}$ \\
\hline $\begin{array}{l}\text { Potential Revisions to SAP-3.5.1.4, Rev } 2 \\
\text { As relates to tornado criteria } \\
\end{array}$ & & & & & $\begin{array}{l}\text { Not applicable, No } \\
\text { Safety Class equipment }\end{array}$ \\
\hline $\begin{array}{c}\text { WHC-SD-SNF-DB-002, Table 5.C } \\
\text { As relates to tornado criteria }\end{array}$ & & & & & $\begin{array}{l}\text { Not applicable, No } \\
\text { Safety Class equipment }\end{array}$ \\
\hline $\begin{array}{l}10 \text { CFR Part } 72.24 \\
\text { As relates to tornado criteria }\end{array}$ & & & & & $\begin{array}{l}\text { Not applicable, No } \\
\text { Safety Class equipment }\end{array}$ \\
\hline $\begin{array}{l}10 \text { CFR Part } 72.122 \\
\text { As relates to tomado criteria } \\
\end{array}$ & & & & & $\begin{array}{l}\text { Not applicable, No } \\
\text { Safety Class equipment }\end{array}$ \\
\hline $\begin{array}{l}\text { Reg Guide } 1.97 \text {, Section } 50.49(\mathrm{e})(5) \\
\text { As relates to Safety Class } 1 \text { electrical equipment }\end{array}$ & & & & & $\begin{array}{l}\text { Not applicable, No } \\
\text { Safety Class equipment }\end{array}$ \\
\hline $\begin{array}{l}\text { Reg Guide } 1.89 \text {, Section } 50.49 \text { (1) (1-4) } \\
\text { As relates to Safety Class } 1 \text { electrical equipment }\end{array}$ & & & & & $\begin{array}{l}\text { Not applicable, No } \\
\text { Safety Class equipment }\end{array}$ \\
\hline $\begin{array}{l}10 \text { CFR Section } 50.63 \text {, } \\
\text { Loss of all alternating current power }\end{array}$ & & & & & Evaluate loss of ac power \\
\hline \multicolumn{6}{|l|}{$\begin{array}{l}\text { IEEE Std } 484-1987 \\
\text { As relates to safety class } 1 \text { batteries }\end{array}$} \\
\hline \multicolumn{6}{|l|}{$\begin{array}{l}\text { IEEE Std } 535-1986 \\
\text { As relates to safety class } 1 \text { batteries }\end{array}$} \\
\hline $\begin{array}{l}\text { IEEE Std 603-1991 } \\
\text { As relates to safety class } 1 \text { 18C systems }\end{array}$ & & & & & $\begin{array}{l}\text { Not applicable, No } \\
\text { Safety Class equipment }\end{array}$ \\
\hline $\begin{array}{l}\text { ANSI/ANS-8.3-1986 } \\
\text { As relates to criticality alarm systems }\end{array}$ & & & & & $\begin{array}{l}\text { Confirm that criticality } \\
\text { is not possible }\end{array}$ \\
\hline $\begin{array}{l}\text { NUREG - } 0700 \\
\text { Review against DOE6430.1A }\end{array}$ & & & & & Review \\
\hline $\begin{array}{l}\text { SRP } 18.1 \\
\text { Review against DOE6430.1A }\end{array}$ & & & & & Review \\
\hline $\begin{array}{l}\text { Reg Guide } 1.26 \text { - As relates to assigning appropriate } \\
\text { ASME Section III code classes }\end{array}$ & & & & & Use to assist \\
\hline $\begin{array}{l}\text { Reg Guide } 1.84 \text { and } 1.85 \text { - As relates to assigning } \\
\text { ASME Section III for Safety Class I cases }\end{array}$ & & & & & $\begin{array}{l}\text { Not applicable, No } \\
\text { Safety Class equipment }\end{array}$ \\
\hline
\end{tabular}




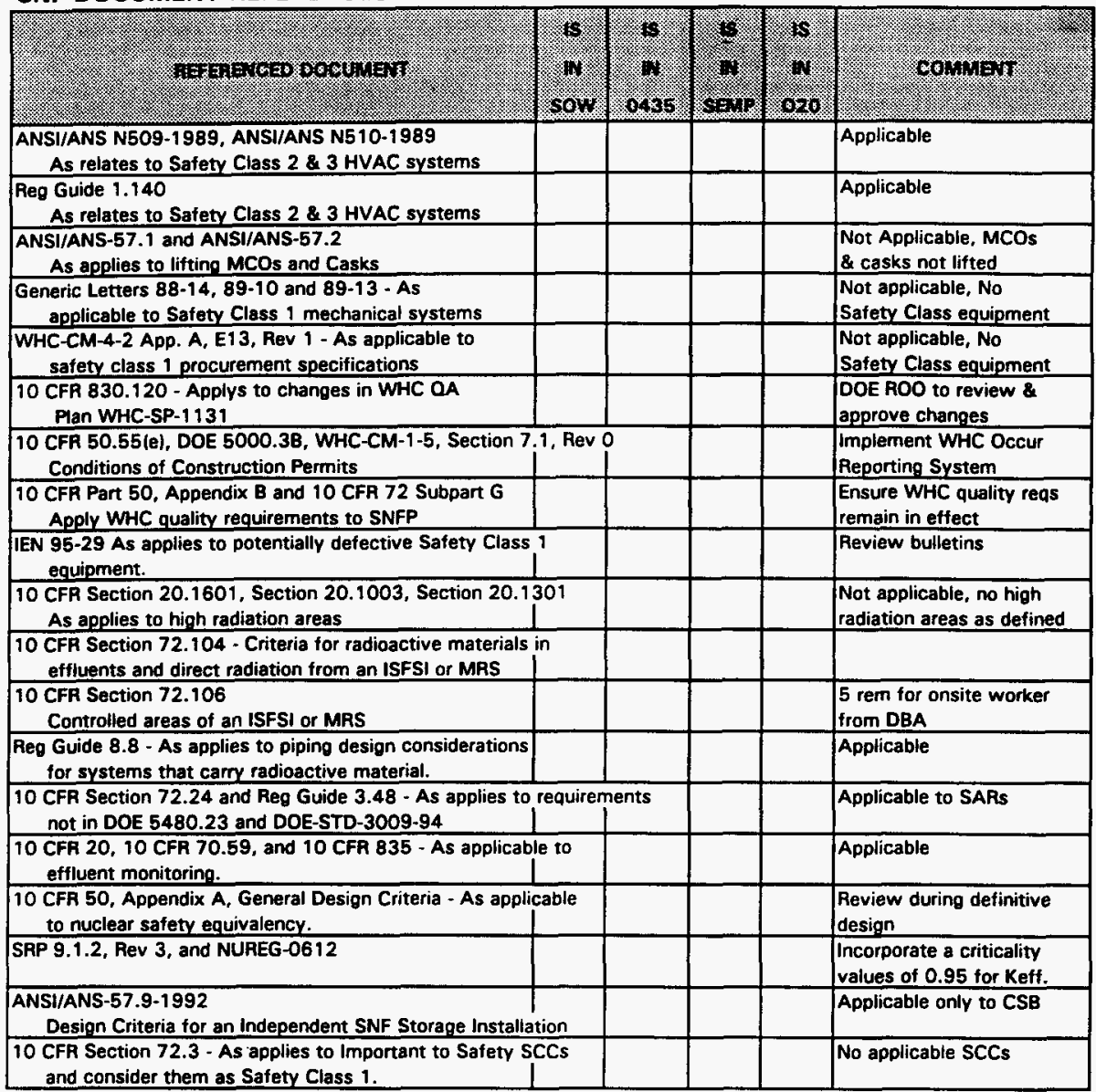


WHC-SD-SNF-CDR-007, Revision 0

\section{APPENDICES}

\section{APPENDIX $J$}

OPERABILITY AND MAINTAINABILITY ASSESSMENT 
WHC-SD-SNF-CDR-007, Revision 0

\title{
APPENDIX $\mathrm{J}$ - OPERABILITY AND MAINTAINABILITY ASSESSMENT
}

\author{
OPERABILITY AND MAINTAINABILITY ASSESSMENT \\ for the \\ HOT CONDITIONING SYSTEM EQUIPMENT \\ of the
}

K-BASIN SPENT NUCLEAR FUEL STORAGE PROJECT

\subsection{INTRODUCTION}

The purpose of this report is to assess the operability and maintainability of the Hot Conditioning System Equipment Of The K-Basin Spent Nuclear Fuel Storage Project as described in the Conceptual Design Report.

Beyond ensuring that the process system will perform its intended function, a key design criteria is ensuring that the mechanical and electrical systems can operate reliably under expected conditions with acceptable maintenance requirements while simplifying operation and maintenance by site operations personnel. Operability and maintainability are important to the efficient performance of process functions within predetermined schedules, determining manpower requirements, controlling maintenance costs, and keeping worker radiation exposure ALARA.

The level of development of the design in progress affects the type of assessment that can be performed and the format of its results. A design in a conceptual phase necessarily lacks details to permit a detailed assessment. Instead, the most important potential findings of a conceptual design review would be conclusions that particular design concepts are flawed to the point that detailed design could not be expected to be satisfactory. This would require a change to the design concept.

The next most important findings of an operability and maintainability review of a conceptual design are identification of the most significant challenges facing the detailed designer in achieving a satisfactory final product. Next, any other observations made while looking for the above two types of findings should be mentioned. Finally, specific recommendations are offered.

This report will follow the format described above. 
WHC-SD-SNF-CDR-007, Revision 0

\section{APPENDIX J - OPERABILITY AND MAINTAINABILITY ASSESSMENT}

\subsection{SUMMARY OF RESULTS}

There are no apparent conceptual design flaws which would threaten the beginning of detailed design. Overall design, layout, and equipment selection present no major operability or maintainability concerns. Three areas of significant challenge for detailed design are identified and two recommendations offered as well as a number of specific observations. Subsequently, the findings of this group were independently reviewed by others with similar background in the nuclear power industry.

\subsection{DISCUSSION}

\subsection{Methodology}

The review was conducted in several steps. Copies of the Conceptual Design Report were distributed for familiarization to four reviewers with operations and maintenance experience. An initial meeting was held for preliminary discussions, question and direction. Next, the experts reviewed the material individually and accumulated comments. A final group discussion developed consensus and provided the material for the report.

\subsection{Conceptual Flaws}

The most important conclusion reached was that there are no apparent conceptual design flaws which would threaten the beginning of detailed design. Overall design, layout, and equipment selection present no major concerns. Despite the design challenges discussed below, we believe that the correct concepts are being employed and a satisfactory detailed design can be completed.

\subsection{Challenges for Detailed Design}

3.3.1 The detail design of the process enclosure will be a challenge. This tool will be critical. It will have many moving parts e.g. welding support and remote, leak-tight connections exposed to thermal cycling. There are similar design requirements (and even more challenging) at the Defense Waste Processing Facility (DWPF) at Savannah River which should provide valuable insight during the detailed design. 
WHC-SD-SNF-CDR-007, Revision 0

A Failure Modes and Effects Analysis should be done in parallel with the design process as a design tool to determine critical parts, potential failures/maintenance, spares etc. See Section 3.5.1

3.3.2 Welding the top hat will be time consuming. The welding equipment will need to be specially designed, and will be complex. Considerable attention must be paid to reliable operation while allowing for variations to be expected over the population of MCOs to be processed. The complexity will provide a great deal of opportunity for maintenance problems. See Section 3.5.2

3.3.3 The detailed designer must look carefully at radiation monitoring equipment for potential impact on operation and maintenance. Scheduled preventative maintenance should be performed on this equipment to assure that they continue to function as they are intended.

\subsection{Other Observations}

In searching for flaws in design concept and major design challenges, we have made the following observations.

3.4.1 The detailed designer needs to look carefully at the placement of critical valves and at the detailed maintenance that may need to be performed to make sure that they function correctly (e.g. pressure actuated relief valves), facilitate testing requirements and don't leak under any potential operating conditions.

3.4.2 Make sure that the process enclosure can handle inspection and cleaning fluid waste such as dye penetrant if used.

3.4.3 Consider the potential impact on maintenance requirements of off normal operations e.g. over temperature, over pressurization. This could result from a control system failure or a failure of a component such as chilled water pump.

3.4.4 Consider the implications of failures of support systems such as chilled water, service air, electrical, HVAC.

3.4.5 Section 2.2.1.2.1 of the Conceptual Design notes that since Report VPS VAC 1104 will operate at low capacity this will likely 
WHC-SD-SNF-CDR-007, Revision 0

\section{APPENDIX $\mathrm{J}$ - OPERABILITY AND MAINTAINABILITY ASSESSMENT}

ensure that design life will be achieved. Operation at low capacity can actually shorten the life of some equipment (e.g. diesels, some centrifugal pumps etc.)

3.4.6 Make sure that maintenance of control system components such as sensors, detectors, and transmitters is addressed in the detailed design including replacement, adjustment, and calibration.

3.4.7 Consider cooler failure and potential impact on other system equipment.

3.4.8 Consider the life expectancy and potential for maintenance of electric heaters to be specified.

3.4.9 Ensure that the detailed design properly addresses process monitoring instrumentation such as $\mathrm{O}_{2}$ from the standpoint of replacement, maintenance and calibration. Ensure that their potential impact on production and safety in the event of failure or off normal operation is addressed.

3.4.10 Make sure that equipment critical to production and safety is identified so that it will be appropriately maintained to ensure function e.g. purge valves, purge supplies, blower seal system, over pressure protections, detectors etc.

3.4.11 Consider the implications of bellows failure in the oven.

3.4.12 What is the reliability history for turbomolecular pumps. What is impact of failure? What are failure modes and mitigating maintenance requirements?

3.4.13 Is the service air system critical to safety and production? If so, performance monitoring may be needed. To preclude equipment damage or personnel injury, ensure the system fails safe on loss of electrical power or pneumatics.

3.4.14 Make sure that the trench covers can be removed easily e.g. crane or lifting device located to support removal.

3.4.15 Make sure that equipment in trench is arranged to facilitate maintenance including valves, piping, instrumentation etc. 
WHC-SD-SNF-CDR-007, Revision 0

\section{APPENDIX J - OPERABILITY AND MAINTAINABILITY ASSESSMENT}

3.4.16 Make sure that the remote maintenance tooling in the process enclosure addresses the potential for fastener or "pop up" spring failure.

3.4.17 Remote maintenance tooling in the process enclosure should address over torque potential where applicable.

3.4.18 Make sure that lifting devices, lighting, power and service air required to easily conduct maintenance is addressed in the detailed design.

3.4.19 Avoid the possibility of water in the trench. If this cannot be done, provide means to keep it out of the oven pit

3.4.20 Make provisions for cleaning the weld surfaces following each grinding step.

3.4.21 Although it is outside of the scope of this review, the design constraints on the MCO filter may be difficult to meet. This interface could affect the detailed design if it is changed.

\subsection{Recommendations}

3.5.1 Prepare and maintain a Failure Modes and Effects Analysis during the detailed design. This would provide:

- means to identify systems critical to safety

- means to identify systems critical to production

- upset conditions which might impact equipment operations or maintenance

- identification of scheduled and preventative maintenance which would be required

- identification emergency procedures required

- means to estimate maintenance staffing

- means to estimate dose resulting from maintenance

- identify critical spares needed

- identify additional process monitoring which would facilitate/delete maintenance

There are a number of software tools available that can be used to guide the above work. These tools provide a structured, consistent 
WHC-SD-SNF-CDR-007, Revision 0

\section{APPENDIX J - OPERABILITY AND MAINTAINABILITY ASSESSMENT}

methodology for conducting a maintenance and operability review. They also provide documentation of the maintenance and operability program basis.

3.5.2 Consider welding plugs in the MCO ports to seal them rather than using a "top hat" cover.

Advantages:

Speed: Steam generator tube plug welding experience suggests a single pass tig weld, which could be autogenous (no filler) if designed properly. Production rates of one tube per hour including plug installation, weld and inspection are typical in steam generators.

Tooling aiready developed: Steam generator tooling could be adapted to support this application.

Inspection: Steam generators tube plugging operations use only visual inspection with good results.

Repair is straight forward: Tooling already exists to remove a plug weld and re-install. In steam generators this can be done repeatedly.

Potential cost impact.

Disadvantage:

Would need to address stacking and future drying if required i.e. how to make new connections after welding in/removing plug. 
WHC-SD-SNF-CDR-007, Revision 0

APPENDIX J - OPERABILITY AND MAINTAINABILITY ASSESSMENT

\section{REFERENCES}

1) Hot Conditioning System Equipment, K-Basin Spent Nuclear Fuel Storage Project, 90\% Submittal, Conceptual Design Report 
WHC-SD-SNF-CDR-007, Revision 0

\section{APPENDICES}

\section{APPENDIX K}

\section{COMMENT DATABASE}




\section{Project 2318: Hot Conditioning CDR Comments}

PART 1: COMMENTS I DISPOSITION

WHC-SD-SNF-CDR-007 Rev 0

\begin{tabular}{|c|c|c|c|c|c|}
\hline REVIEWER & COMMENT * & COMMENT & HOLD POINT & DISPOSITION & STATUS \\
\hline $\begin{array}{l}\text { Alaconis, W.C. } \\
90 \% \text { CDR }\end{array}$ & 1 & $\begin{array}{l}\text { All comments from the } 60 \% \text { CDR Review must be } \\
\text { answered. To date no response has been given. }\end{array}$ & no & $\begin{array}{l}\text { All responses to the } 60 \% \text { design comments will be included in the } \\
\text { final CDR. }\end{array}$ & closed \\
\hline $\begin{array}{l}\text { Alaconis, W.C. } \\
90 \% \text { CDR }\end{array}$ & 2 & $\begin{array}{l}\text { The concept of manipulator assisted operation is } \\
\text { acceptable, however the ability of the manipulator to } \\
\text { preform all of the required functions within the process } \\
\text { enclosure needs to be demonstrated with in the time } \\
\text { estimates of the CDR. }\end{array}$ & no & $\begin{array}{l}\text { The time estimates in the CDR are based upon experience. A time } \\
\text { and motion study for the process enclosure will be an element of } \\
\text { the definitive design }\end{array}$ & DD \\
\hline $\begin{array}{l}\text { Alaconis, W.C. } \\
90 \% \text { CDR }\end{array}$ & 3 & $\begin{array}{l}\text { The dose estimate assumed a solid 1" steel process } \\
\text { enclosure to develop the calculations (no viewing } \\
\text { window). The process enclosure must have a shielded } \\
\text { window to allow the operator } 100 \% \text { visual observation of } \\
\text { the manipulator movement and function. The video } \\
\text { viewing will not provide the 3D reference required. }\end{array}$ & no & The process enclosure design will incorporate a shtelded window. & closed \\
\hline $\begin{array}{l}\text { Alaconis, W.C. } \\
90 \% \text { CDR }\end{array}$ & 4 & $\begin{array}{l}\text { If the HCSE does nat plan on using the hellum and } \\
\text { nitrogen banks that are being Installed by Fluor Daniel in } \\
\text { the CSB design, then section } 4 \text { (interfaces) lacks the } \\
\text { appropriate requirements for siting HCS gas trucks around } \\
\text { the facility. Civil and structural interfaces will be required } \\
\text { with FD. }\end{array}$ & no & $\begin{array}{l}\text { The HCSE design team will provide any clvil/structural design } \\
\text { required for these tanks. The lanks will be provided after the CSB } \\
\text { construction is complete. }\end{array}$ & closed \\
\hline $\begin{array}{l}\text { Alaconis, W.C. } \\
90 \% \text { CDR }\end{array}$ & 5 & $\begin{array}{l}\text { The current dose estimates of } 5 \mathrm{mRern} / \mathrm{hr} \text { while manning } \\
\text { the manipulator operating station is not acceptable. This } \\
\text { exceeds } 10 \text { CFR } 835 \text { requirements of } 20 \% \text { of } 835.202 \text {. } \\
\text { Based on available data there will be approximately } 2300 \\
\text { operating hours per year on the process enclosure shared } \\
\text { by two operators per shift. At } 384 \text { hours }(2300 / 6) \text { per } \\
\text { operator per year a dose rate of less than } 2.6 \mathrm{mRem} / \mathrm{hr} \text { at } \\
\text { the operating station will result in } 1 \mathrm{R} \text { per operator. As a } \\
\text { result of this data a final design estimate of less than } 2.5 \\
\text { mRem/hr would be expected. }\end{array}$ & no & $\begin{array}{l}\text { Agree. Wili incorporate during definitive design. See Curt Miska } \\
\text { comments } 12-15 \text { and resolution. }\end{array}$ & DD \\
\hline
\end{tabular}

\section{APPENDIX K: HOT CONDITIONING COMMENT DATABASE}

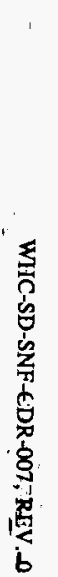
(1) 


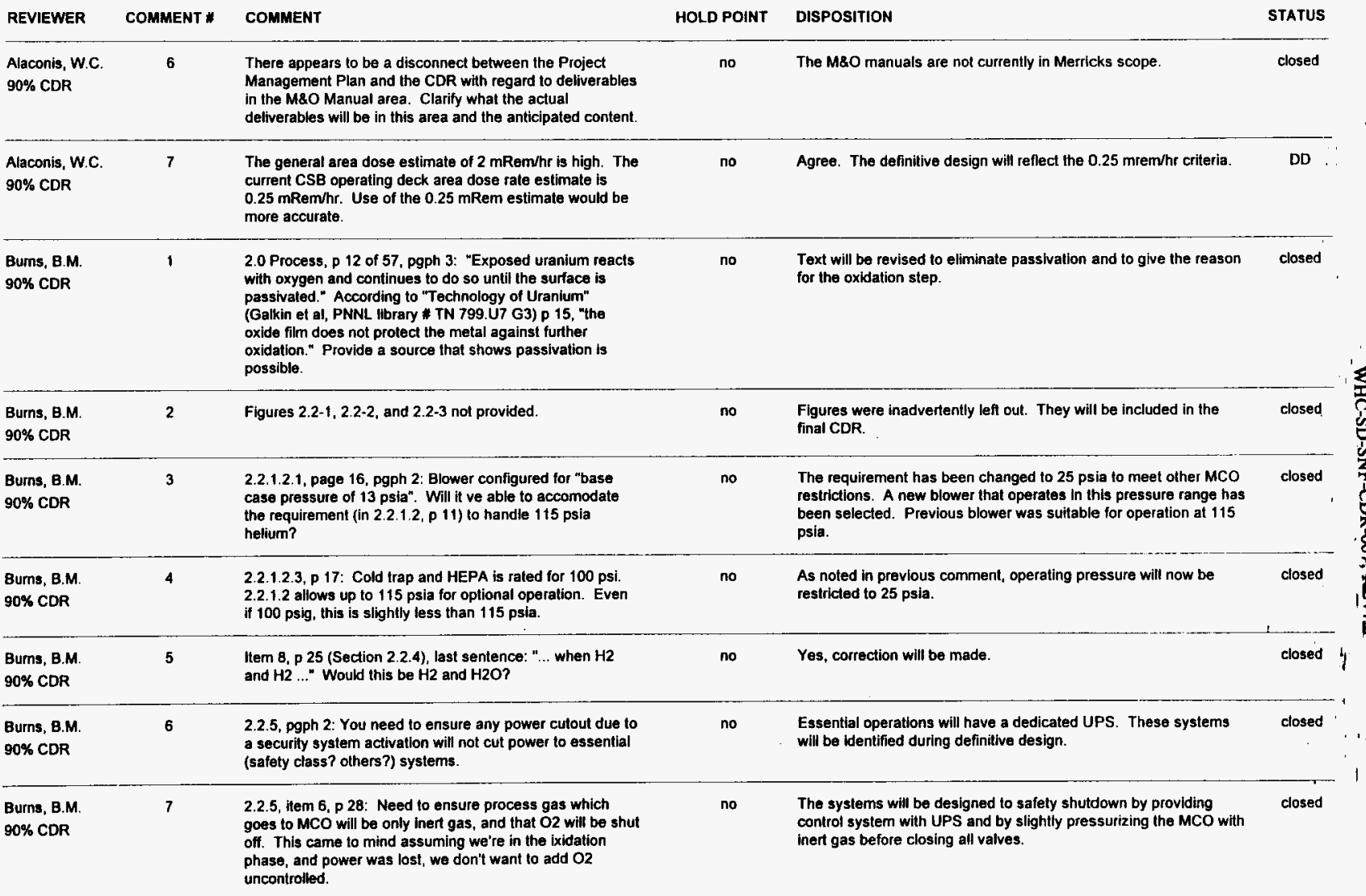




\begin{tabular}{|c|c|c|c|c|c|}
\hline REVIEWER & COMMENT \# & COMMENT & HOLD POINT & DISPOSITION & STATUS \\
\hline $\begin{array}{l}\text { Cowan, R.G. } \\
90 \% \text { CDR }\end{array}$ & 1 & $\begin{array}{l}\text { Several interfaces with the MCO are very simitar to those } \\
\text { at CSB such as receiving venting and should have } \\
\text { common solutions The design should attempt to use the } \\
\text { same approach where possible during detaifed design. }\end{array}$ & no & $\begin{array}{l}\text { The interfaces with the MCO are similar for the CSB, CVDS and } \\
\text { HCSE. The interfaces are being coordinated with these teams and } \\
\text { with the MCO design leam. }\end{array}$ & closed \\
\hline $\begin{array}{l}\text { Cowan, R.G. } \\
\text { 90\% CDR }\end{array}$ & 2 & $\begin{array}{l}\text { Port covers should be removed at CSB receiving and } \\
\text { should not be handled at HVC. }\end{array}$ & & $\begin{array}{l}\text { Handling will be required if it is determined that closure of the MCO } \\
\text { will be accomplished by seal welding the port covers at the } \\
\text { completion of the hot conditioning process. }\end{array}$ & closed. \\
\hline $\begin{array}{l}\text { Cowan, R.G. } \\
\text { 90\% CDR }\end{array}$ & 3 & $\begin{array}{l}\text { Single pass air flow directly around the MCO should be } \\
\text { used in place of water cooled circulating air. Allhough this } \\
\text { may indrease the stack flow, it would be a much easier } \\
\text { system to maintain and get through safety. }\end{array}$ & no & $\begin{array}{l}\text { A water study will be included in the CDR to address this issue. } \\
\text { Final resolution will be during definitive design. }\end{array}$ & DD . \\
\hline $\begin{array}{l}\text { Cowan, R.G. } \\
90 \% \text { CDR }\end{array}$ & 4 & Design for premixed oxygen/argon from cylinders outside. & no & $\begin{array}{l}\text { We will be using premixed oxygen as you suggest. The present } \\
\text { design will accommodale that mode. }\end{array}$ & closed \\
\hline $\begin{array}{l}\text { Cowan, R.G. } \\
90 \% \text { CDR }\end{array}$ & 6 & $\begin{array}{l}\text { Welding in the oven is not a good idea as this will ask each } \\
\text { station to do too many functions. One pit designed for } \\
\text { welding will work better even with the extra transfer. With } \\
\text { a good design on shielding, it should be possible to do a } \\
\text { manual selup and automatic wald for closure. }\end{array}$ & no & $\begin{array}{l}\text { Welding will be done with a welding machine operated from the } \\
\text { process enclosure. The process enclosure will service all slx } \\
\text { process pits with one welder. Welding at the pit does not alter the } \\
\text { oven design. If welding were to be moved to the seventh pit with a } \\
\text { dedicated welder, the process enclosure would still be needed for } \\
\text { weld inspection and repair so it would still have multiple functions } \\
\text { to perform. }\end{array}$ & closed \\
\hline $\begin{array}{l}\text { Cowan, R.G. } \\
90 \% \text { CDR }\end{array}$ & 7 & $\begin{array}{l}\text { The proposed vacuum bottle with air heating seems a } \\
\text { good concept but if the MCO group will comment to the } \\
\text { stepped design for the MCO, it should be possible to } \\
\text { support both the MCO and vacuum bottle from a top } \\
\text { flange. This has a lot of advantages for process (the MCO } \\
\text { top would not move during heating) and shielding. (I did } \\
\text { not see how the shielding would work as shown.) }\end{array}$ & no & $\begin{array}{l}\text { The new design from the MCO contractor accommodates top } \\
\text { support of the MCO. }\end{array}$ & closed \\
\hline
\end{tabular}




\begin{tabular}{|c|c|c|c|c|c|}
\hline REVIEWER & COMMENT * & COMMENT & HOLO POINT & DISPOSITION & STATUS \\
\hline $\begin{array}{l}\text { Goldman, L. } \\
\text { 90\% CDR }\end{array}$ & 1 & $\begin{array}{l}\text { Using refrigerants containing halogens around the MCO } \\
\text { during HCS operations makes me nervous. They could } \\
\text { get inside and react or break down at temperature to an } \\
\text { acid forming gas such as HC1, a diatomic halogen, or } \\
\text { poison gasses. If we had an unexplained loss of } \\
\text { refrigerant, freon or suva for example, who could say it did } \\
\text { not end up inside the MCO and reacted inside the heated } \\
\text { MCO. Then that MCO would be put up as a "suspect", as } \\
\text { potentially damaged from the halogens. }\end{array}$ & no & $\begin{array}{l}\text { The cooling for the MCO vacuum system is with ambient air which } \\
\text { leaves no possibility for halogen entry. As currently conceived, the } \\
\text { air would be cooled by a closed water loop and the water would be } \\
\text { cooled by a refrigerent loop. }\end{array}$ & closed \\
\hline $\begin{array}{l}\text { Goldman, L. } \\
\text { 90\% CDR }\end{array}$ & 2 & $\begin{array}{l}\text { I wasn't close enough to see the PFD. Was the residual } \\
\text { gas analyser going to be used to measure the input and } \\
\text { output streams of the MCO to determine take up of oxygen } \\
\text { by the inside of the MCO? Was the output gas stream } \\
\text { containing water and } \mathrm{H} 2 \text { going to be measured as it comes } \\
\text { out of the MCO? Or how was O2 take up inside the MCO } \\
\text { going to be determined? }\end{array}$ & no & $\begin{array}{l}\text { The oxygen analyzer is on the MCO gas circulation line. We can } \\
\text { determine the } \mathrm{O} 2 \text { uptake within the MCO by knowing the } \\
\text { circulation rate and taking interval samples. }\end{array}$ & closed \\
\hline $\begin{array}{l}\text { Kurtz, J. } \\
\text { 90\% CDR }\end{array}$ & 1 & $\begin{array}{l}\text { Not much in detail about rad monitoring equipment for the } \\
\text { workplace. Just a small section in the ALARA section. We } \\
\text { will require the use of CAMS and other monitoring that } \\
\text { requires power, etc. Request you expand on this or say } \\
\text { they will leave it up to us. }\end{array}$ & no & $\begin{array}{l}\text { Specific details of the radiation monitoring system will be } \\
\text { developed during detail design of the HCSA in accordance with the } \\
\text { criteria for the CSB. }\end{array}$ & closed \\
\hline $\begin{array}{l}\text { Kurtz, J. } \\
90 \% \text { CDR }\end{array}$ & 2 & $\begin{array}{l}\text { Looking over the exhaust instrumentation, I get the feeling } \\
\text { that it may be excessive. I thing you need to have the } \\
\text { environmental folks look this over. When the CSB was } \\
\text { reviewed, some of that was bound to be excessive and } \\
\text { expensive. }\end{array}$ & no & $\begin{array}{l}\text { For the conceptual design effort a conservative stack monitoring } \\
\text { system was selected. The system may be simplified during } \\
\text { definitive design. }\end{array}$ & DD \\
\hline $\begin{array}{l}\text { Kurtz, J. } \\
90 \% \text { CDR }\end{array}$ & 3 & $\begin{array}{l}\text { If we want to use the PING-3B Particulate/lodine and } \\
\text { Noble gas monitors in the exhaust, I have two brand new, } \\
\text { never been used PING-1A units that were purchased for } \\
\text { encapsulation. These are equivalent to the } 3 B \text { 's except for } \\
\text { the dual range noble gas. We spent about } 30 \mathrm{~K} \text { on each of } \\
\text { these. }\end{array}$ & no & $\begin{array}{l}\text { Use of the new PING-1A units will be evaluated during definitive } \\
\text { design. This cost reduction opportunity is appreciated. }\end{array}$ & i closed \\
\hline $\begin{array}{l}\text { Kuntz, J. } \\
90 \% \text { CDR }\end{array}$ & 4 & $\begin{array}{l}\text { Looking at these PINGs and our requirements, I'm not sure } \\
\text { that these will do the job. The lodine channels on these } \\
\text { units are designed to measure l-131 activity. The I-129 we } \\
\text { are going to try to measure only emits a } 40 \mathrm{KeV} \text { gamma. } \\
\text { Theoretically, the unit can be adjusted to see this but } \\
\text { would be at the extreme low end and would be subject to } \\
\text { noise problems. The current best technology for } \\
\text { monitoring I-129 involves sampling with cartridge filter and } \\
\text { laboralory analysis with low energy gamma detector. }\end{array}$ & no & $\begin{array}{l}\text { The application of these units will be investigated during definitive } \\
\text { design. The lodine trap will use the same cartridge filter so lodine } \\
\text { release is not anticipated to be a problem. }\end{array}$ & DD \\
\hline
\end{tabular}




\begin{tabular}{|c|c|c|c|c|c|c|}
\hline $\begin{array}{l}\text { Lucas, C. } \\
90 \% \text { CDR }\end{array}$ & 1 & $\begin{array}{l}\text { Section 2.9:1) Waste items described in the earlier } \\
\text { sections include; dye penatrant, cleaning fluid, developer, } \\
\text { bulfer pads, etc. Non of these items are described in the } \\
\text { solid waste section.2) Other disposal requirements need to } \\
\text { be listed besides WAC 173-303 such as WHC EP 0063 } \\
\text { Solid Waste Acceptance Criteria.3) Alt of the potential } \\
\text { streams appear to be workable, I am assuming that the } \\
\text { project will develop an approved disposal path and } \\
\text { summary as a part of the design so we don't design a } \\
\text { waste stream that is cost prohibitive to operate (cartridge } \\
\text { filters). For example, having units to be disposed actually } \\
\text { sized to fit in an existing approved container (get } \\
\text { packaging folks on board), and handling methods that do } \\
\text { not require high dose expenditure.4) I would recommend } \\
\text { getting Acceptance Services representation in on this early } \\
\text { to make the road smoother. }\end{array}$ & no & $\begin{array}{l}\text { We will comply with your suggesstion with the details to be worked } \\
\text { out during definitive design. }\end{array}$ & closed & . \\
\hline $\begin{array}{l}\text { Merkling. T. } \\
\text { 60\% CDR }\end{array}$ & 1 & $\begin{array}{l}\text { Overhead/Drawing of Cell. This drawing shows a CAM } \\
\text { (Continuous Air Monitor) located in the center of the cell. } \\
\text { Comment: 1) Ensure that the CAM is of proper sensitivity } \\
\text { to detect the nuclides of interest, 2) Ensure that the } \\
\text { location of the detector/sample hose is such that the } \\
\text { nuclides of interest are correctly monitored, 3) Ensure that } \\
\text { calibration/maintenance of the CAM can be performed with } \\
\text { exposure to radiation ALARA. }\end{array}$ & no & $\begin{array}{l}\text { CAM will be specified during definitive design in accordance with } \\
\text { these recommendations. It is anticipated that maintenance can be } \\
\text { performed while process enclosure is in cold status, i.e. not } \\
\text { servicing any of the process pits. }\end{array}$ & Do & $\sum_{\substack{n \\
\vdots}}^{\frac{1}{2}}$ \\
\hline $\begin{array}{l}\text { Merkling, } T \text {. } \\
60 \% \text { CDR }\end{array}$ & 2 & $\begin{array}{l}\text { Hot Treatment Process Operations Cell Functional } \\
\text { Description. Steps that involve manned entry for survey of } \\
\text { the cell (steps 24, 58). Comment: From a logistics } \\
\text { standpoint of making an entry into a confined/contaminated } \\
\text { space from a radiologically clean space, recommend that } \\
\text { surveys for radiological contamination be performed using } \\
\text { the remote manipulator with sample smears being passed } \\
\text { out through the pass-through box for analysis. Try to } \\
\text { ellminate manned entry until all salety conditions are } \\
\text { delermined remotely. }\end{array}$ & no & $\begin{array}{l}\text { Recommendations will be incorporated in the definitive destgn of } \\
\text { process enclosure. }\end{array}$ & $\mathrm{DD}$ & is \\
\hline $\begin{array}{l}\text { Merkling. T. } \\
60 \% \text { CDR }\end{array}$ & 3 & $\begin{array}{l}\text { Shielding and Radlation Exposure Analysis. Comment: } \\
\text { Consider an ALARA analysis for mainlenance/calibration } \\
\text { activities in addition to the ALARA analysis already } \\
\text { performed for normal HVC work tasks. }\end{array}$ & no & $\begin{array}{l}\text { There is no maintenance/calibration activity antlcipated when the } \\
\text { MCO is present. Traps and HEPA filters are installed in the trench } \\
\text { to assure that process equipment is not contaminaled. } \\
\text { Maintenance on equipment should be cold. }\end{array}$ & closed & 1 \\
\hline
\end{tabular}




\begin{tabular}{|c|c|c|c|c|c|}
\hline REVIEWER & COMMENT * & COMMENT & HOLD POINT & DISPOSITION & STATUS \\
\hline $\begin{array}{l}\text { Merkling, T. } \\
60 \% \text { CDR }\end{array}$ & 4 & $\begin{array}{l}\text { Shielding and Radiation Exposure Analysis. Comment: } \\
\text { Section } 2.0 \text {, To avoid the pitfall of using a single exposure } \\
\text { number (single numbers give the impression that they are } \\
\text { exact), recommend using a range for estimaling the total } \\
\text { exposure. This range would be based on the task times } \\
\text { spent in the radiation field. Suggest getting wilh SNF } \\
\text { Operations and fine-tuning the duration times for the } \\
\text { activities and calculating a range of times (say for } \\
\text { "Undoing Top Fasteners, instead of exactly } 10 \text { minutes, } \\
\text { give a range of } 6 \text { to } 12 \text { minutes or whatever is agreed by } \\
\text { yourself and operations). }\end{array}$ & no & $\begin{array}{l}\text { Yes. We are working on a PERT analysis of the production } \\
\text { schedule. This will be delivered during detailed design. }\end{array}$ & DD \\
\hline $\begin{array}{l}\text { Merkling, T. } \\
60 \% \text { CDR }\end{array}$ & 5 & $\begin{array}{l}\text { Shielding and Radiation Exposure Analysis. Comment: } \\
\text { Section 3.1. The sentence "The workforce and time } \\
\text { required to perform each exposure activity was estimated" } \\
\text { needs to be changed to reflect a combined effort belween } \\
\text { the ALARA analysis engineer and SNF Operations arriving } \\
\text { at an accurate time delermination (see above comment). }\end{array}$ & no & $\begin{array}{l}\text { Operations input has been requested. The final analysis that } \\
\text { incorporated the malntenance estimates will be completed during } \\
\text { definitive design. }\end{array}$ & DD \\
\hline $\begin{array}{l}\text { Merkling, T. } \\
60 \% \text { COR }\end{array}$ & 6 & $\begin{array}{l}\text { ALARA Implementation Plan. Comment: Reference } \\
\text { currentcorrect documents. 1) Third paragraph, delete first } \\
\text { sentence (10 CFR } 835 \text { is the design authority). 2) Third } \\
\text { paragraph, third sentence, Add "design objective for" } \\
\text { belween ".,"federal law governing" and "radiation } \\
\text { exposure,."." 3) same sentence, delete "in." 4) Delete } \\
\text { "DOE Order 5480.11, Radiation Protection for } \\
\text { Occupational Workers," and replace with "DOE Notice } \\
441.1, \text { Radiological Protection for DOE Activities." 5) } \\
\text { Section } 2.0, \text { a) Replace DOE Order } 5480.11 \text { with DOE } \\
\text { Notice } 441.1, \text { b) Replace WHC-CM-4-11 with "WHC-IP. } \\
\text { 1043, WHC Occupational ALARA Program, c) Replace } \\
\text { "DOE N5480.6" with "DOE/EH-0256T." }\end{array}$ & no & $\begin{array}{l}\text { Comments incorporaled into the } 90 \% \text { Conceptual ALARA } \\
\text { implementation Plan. }\end{array}$ & closed \\
\hline $\begin{array}{l}\text { Merkling, T. } \\
60 \% \text { CDR }\end{array}$ & 7 & $\begin{array}{l}\text { ALARA Implementation Plan. Comment: Section } 3.0 \text { A } 3 . \\
\text { When the hole cover is pulled, is there a possibllity for } \\
\text { additional radiation exposure from gamma scatter or } \\
\text { "ceiling shine"? If an analysis shows that this is possible, } \\
\text { then it needs to be included in the exposure estimate. }\end{array}$ & no & Sky shine analysis will be incorporated in detailed design. & DD \\
\hline
\end{tabular}




\begin{tabular}{|c|c|c|c|c|c|}
\hline $\begin{array}{l}\text { Merkling, T. } \\
60 \% \text { CDR }\end{array}$ & 8 & $\begin{array}{l}\text { ALARA Implementation Plan. Comment: Section 4.D.3. } \\
\text { This section states that "the design will include Continuous } \\
\text { Air Monitors (CAMs) capable of detecting,..." To meet } 10 \\
\text { CFR } 835 \text { and HSRCM requirements, an analysis would } \\
\text { need to be performed that shows the potential of airborne } \\
\text { activity during normal activities. Also, an engineering } \\
\text { study (smoke test) should be performed to ensure correct } \\
\text { location(s) of the sampler/monitor. }\end{array}$ & no & $\begin{array}{l}\text { Will incorporate in detailed design and in startup testing (smoke } \\
\text { tests, etc.) }\end{array}$ & DD \\
\hline $\begin{array}{l}\text { Merkling, T. } \\
60 \% \text { CDR }\end{array}$ & 9 & $\begin{array}{l}\text { Operations and Maintenance Philosophy. Comment: } \\
\text { Administrative limit. 1) Section 1.0.B. Change the } \\
\text { sentence to allow the facility to manage the administrative } \\
\text { levels accordingly. Suggest "The radiation doses of a } \\
\text { given worker will be administratively controlled per HSRCM- } \\
\text { 1." 2) Section 4.0, first paragraph, first sentence. Change } \\
\text { this sentence to read "The administrative radiation } \\
\text { exposure limitation for the HVCE will be per the HSRCM-1." }\end{array}$ & no & $\begin{array}{l}\text { Comments incorporaled into the } 90 \% \text { Conceptual O/M Philosophy } \\
\text { section. }\end{array}$ & closed. \\
\hline
\end{tabular}




\begin{tabular}{|c|c|c|c|c|c|}
\hline $\begin{array}{l}\text { Merkling, T. } \\
60 \% \text { CDR }\end{array}$ & 10 & 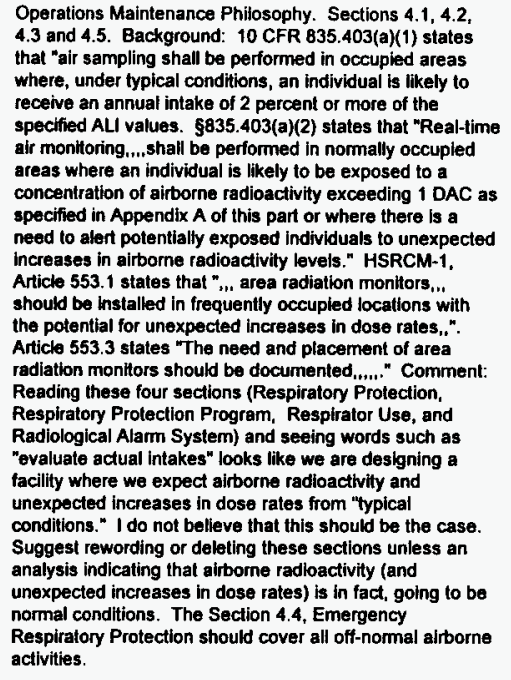 & no & $\begin{array}{l}\text { Comments incorporated into the } 90 \% \text { Conceplual OM Philosophy } \\
\text { section. }\end{array}$ & closed \\
\hline $\begin{array}{l}\text { Merkling, T. } \\
60 \% \text { CDR }\end{array}$ & 11 & $\begin{array}{l}\text { Section } 4.0 \text { Radiation Safety and Exposure Control. } \\
\text { General Comment: Replace all references to } 10 \text { CFR } 20 \\
\text { and } 10 \text { CFR } 72 \text { with appropriate DOE references } 10 \text { CFR } \\
835 \text { and DOE Order } 5400.5 \text {. Quote these appropriale } \\
\text { rules and requirements as needed. }\end{array}$ & no & $\begin{array}{l}\text { Suggested revisions will be incorporated into the Final Conceptual } \\
\text { design report. }\end{array}$ & - closed \\
\hline
\end{tabular}




\begin{tabular}{|c|c|c|c|c|c|}
\hline REVIEWER & COMMENT : & COMMENT & HOLD POINT & DISPOSITION & STATUS \\
\hline $\begin{array}{l}\text { Merkling, T. } \\
\text { 90\% CDR }\end{array}$ & 1 & $\begin{array}{l}\text { Definitions, Cold Trap; } 2.1 \text { Sequence Description, ID } 25.0 \text {; } \\
\text { Comment: I } 129 \text { is a concern because like tritium it is an } \\
\text { internal exposure problem and a low energy beta emitter } \\
\text { (0.15 Mev) which cannol be detected using normally used } \\
\text { portable RC survey instruments. Since it cannot be easily } \\
\text { detected and it is most likely present, a characterization } \\
\text { study needs to be performed to ratio Cs } 137 \text { and I } 129 \text {. } \\
\text { Once this ratio is known, any detected Cs } 137 \text { can be } \\
\text { coupled with the known I } 129 / C s \text { t } 137 \text { ratio and } \\
\text { accountability can be taken for all radionuclides of } \\
\text { concern. }\end{array}$ & no & $\begin{array}{l}\text { The ratio of Cs-137 to } 1-129 \text { is known for the design basis fuel and } \\
\text { is documented in the WHC design basis feed document. Draft } \\
\text { environmental permitting documentation indicates that mitigation (I- } \\
129 \text { removal) is not required. The design of the Cold Trap will } \\
\text { continue in detailed design, and the need for } 1-129 \text { removal will be } \\
\text { reassessed al that time. }\end{array}$ & DO \\
\hline $\begin{array}{l}\text { Merkling, T. } \\
\text { 90\% CDR }\end{array}$ & 2 & $\begin{array}{l}\text { 2.1. Sequence Description, ID 5.0, 2.8. Comment: How } \\
\text { does the enclosure seal to the floor? If there is no seal, or } \\
\text { the seal depends on the ventilation system to provide a } \\
\text { continuous positive air flow, then this design idea needs to } \\
\text { match the expectations of the Safety Analysis and possible } \\
\text { accident scenarios to prevent potential radiological } \\
\text { releases under all possible accident scenarios. }\end{array}$ & no & It is anticipated that an Inflatable seal will be provided. & closed \\
\hline $\begin{array}{l}\text { Merkling, T. } \\
90 \% \text { CDR }\end{array}$ & 3 & $\begin{array}{l}\text { 2.1, Sequence Description, Section } 2.1 \text { ID } 8.0, \text { ID } 105 \text {, ID } \\
\text { 106; Section } 2.8 \text { ID } 8.0,9.0 \text {; and Drawing SK2-2-300420 } \\
\text { Comment: Per the Definitions on page v, this annular } \\
\text { space cover has been eliminated. If this is true, reference } \\
\text { to the cover here and throughout the document needs to } \\
\text { also be eliminated. }\end{array}$ & no & $\begin{array}{l}\text { The baseline design still requires the annular space cover but it is } \\
\text { anticipated that it will be eliminated during detalfed design. The } \\
\text { definition will be revised. }\end{array}$ & closed \\
\hline $\begin{array}{l}\text { Merkling. T. } \\
90 \% \text { CDR }\end{array}$ & 4 & $\begin{array}{l}2.1, \text { Sequence Description, ID } 52 \text { - } 69 \text { : Comment: Is a } \\
\text { visual/dye penetrant inspection sufficient for a Safely Class } \\
\text { application? }\end{array}$ & no & $\begin{array}{l}\text { The new weld lest proposal from the MCO designers specifies } \\
\text { ultrasonic testing. This will be incorporated in the delail design of } \\
\text { the HCSE. }\end{array}$ & closed \\
\hline $\begin{array}{l}\text { Morkling, T. } \\
90 \% \text { CDR }\end{array}$ & 5 & $\begin{array}{l}\text { Section 2.2.1.2.1, Gas Pumps: Comment: Delete "high- } \\
\text { lovel" from last sentence of first paragraph. The vacuum } \\
\text { pump should be radiologically surveyed first and then } \\
\text { disposed of as needed based on the survey. This is in } \\
\text { keeping with the statement on page } 57 \text { of } 57 \text { of the same } \\
\text { section. "failed equipment will be disposed of appropriately } \\
\text { based on a radiation survey." }\end{array}$ & no & Text changed as recommended. & closed \\
\hline $\begin{array}{l}\text { Merkling, T. } \\
90 \% \text { CDR }\end{array}$ & 6 & $\begin{array}{l}\text { Section 2.2.4.9: Comment: Change } \mathrm{l}-131 \text { to } \mathrm{I}-129 \text {, and } \mathrm{Kr} \text { - } \\
86 \mathrm{Kto} \mathrm{Kr}-85 \text {. This is in keeping with decay patterns and } \\
\text { what is noted in drawing } 300413 \text {. If spontaneous fission is } \\
\text { a problem, then } 1-131 \text { could be present. As noted in } \\
\text { comment } 1 \text {, a characterization study needs to be } \\
\text { performed to calculate the amounts of each of these } \\
\text { isotopes. }\end{array}$ & no & Text changed as recommended. & closed \\
\hline
\end{tabular}




\begin{tabular}{|c|c|c|c|c|c|}
\hline REVIEWER & COMMENT * & COMMENT & HOLD POINT & DISPOSITION & status \\
\hline $\begin{array}{l}\text { Merkling. T. } \\
90 \% \text { CDR }\end{array}$ & 7 & $\begin{array}{l}\text { Section 2.3.1, Reference Standards; Comment: Add } 40 \\
\text { CFR 61, (NESHAPs for radiological discharge points) }\end{array}$ & no & Suggested addition will be made. & closed \\
\hline $\begin{array}{l}\text { Merkling. T. } \\
\text { 90\% CDR }\end{array}$ & 8 & $\begin{array}{l}\text { Section } 2.3 .4 \text { Equipment Description and } 3.2 .8 \text { I \& C } \\
\text { interface "The HCSE will provide the stack monitoring } \\
\text { system." Comment: Effluent moniloring and controls shall } \\
\text { comply with the requirements of } 40 \text { CFR } 61 \text { and the DOE } \\
5400 \text { series. Before the type of monitoring equipment is } \\
\text { decided/designed, the project needs to analyze for what it } \\
\text { is monitoring (isotope(s) and amount discharged from } \\
\text { normal operations as well as releases from DBAs). These } \\
\text { evaluations are usually a part of the SAR. This } \\
\text { characterization study estimates/calculates the total } \\
\text { amount of radionuclides/isolopes transportable to } \\
\text { upstream and downstream of the MCO filters and process } \\
\text { filters (normal operations and DBA with Safety Class SSCs } \\
\text { [i.e. MCO]). This information can then be used to calculate } \\
\text { if a monitoring or onfy a sampling system is needed, and } \\
\text { depending on what is needed, what type and sensitivity of } \\
\text { instrumentation/sampler will be needed. Without this } \\
\text { information, it is hard to design a stack } \\
\text { monitoring/sampling system. }\end{array}$ & no & $\begin{array}{l}\text { A conservative stack monitoring system is shown in the CDR. The } \\
\text { design will be revisited during definitive design with these } \\
\text { considerations in mind. }\end{array}$ & closed \\
\hline $\begin{array}{l}\text { Merkling. T. } \\
90 \% \text { CDR }\end{array}$ & 9 & $\begin{array}{l}\text { Section 2.6.2.1, page } 39 \text { of } 57 \text {, second paragraph, "The } \\
\text { wetted surfaces..... reach the pump." Comment: Delete } \\
\text { "and gasses" as the gasses will pass through the system } \\
\text { and up the stack. Delete "Kr" as this is a gas, and delete } \\
\text { "Xe" as this isotope is not present. Consider adding "Co" } \\
\text { as this isotope was mentioned earlier in the report. }\end{array}$ & no & Suggested changes will be made. & closed \\
\hline $\begin{array}{l}\text { Merkling. T. } \\
\text { 90\% CDR }\end{array}$ & 10 & $\begin{array}{l}\text { Section 2.8.2 Comment: Delete "high." } 10 \text { CFR } 835 \text { has a } \\
\text { specific definition for high radiation area with rigid access } \\
\text { controls. }\end{array}$ & no & Suggested changes will be made. & closed \\
\hline $\begin{array}{l}\text { Merkling, } \mathrm{T} \text {. } \\
90 \% \text { CDR }\end{array}$ & 11 & $\begin{array}{l}\text { Section 2.8, Process step F12; Comment: Is the location } \\
\text { of the TV camera and equipment considered for ease of } \\
\text { maintenance for ALARA considerations? }\end{array}$ & no & $\begin{array}{l}\text { ALARA will be considered in the selection of the camera location } \\
\text { during definitive design. }\end{array}$ & closed \\
\hline
\end{tabular}




\begin{tabular}{|c|c|c|c|c|c|}
\hline REVIEWER & COMMENT * & COMMENT & HOLD POINT & DISPOSITION & STATUS \\
\hline $\begin{array}{l}\text { Merkling. T. } \\
\text { 90\% CDR }\end{array}$ & 13 & $\begin{array}{l}\text { Appendix B, } 1.0 \text { Introduction, second paragraph; } \\
\text { Comment: Add narrative to address the minimization of } \\
\text { radiation exposure. See narrative in Section 3B, page } 5 \text { of } \\
8 \text { of this same section for example. }\end{array}$ & no & Comment incorporated. & closed \\
\hline $\begin{array}{l}\text { Merkling. T. } \\
90 \% \text { CDR }\end{array}$ & 14 & $\begin{array}{l}\text { Appendix B, 4.0, B; Comment: What is the source of this } \\
\text { "shall" statement of not exceeding } 5.0 \mathrm{mrem} / \mathrm{hr} \text { ? }\end{array}$ & no & $\begin{array}{l}\text { Document revised to reflect } 2.5 \text { mrem/hr criteria. This is per } 10 \\
\text { CFR 835.202. }\end{array}$ & closed \\
\hline $\begin{array}{l}\text { Merkling, T. } \\
\text { 90\% CDR }\end{array}$ & 15 & $\begin{array}{l}\text { Appendix } C \text {, Section 3.3, page } 3 \text { of } 9 \text {; Comment: This } \\
\text { exposure result should inchude exposure from maintenance } \\
\text { activities as mentioned in Appendix B, section } 2 \text { C, "The } \\
\text { delermination should include normal operations and } \\
\text { maintenance/repair exposure. }\end{array}$ & no & $\begin{array}{l}\text { Agree. An estimate of maintenance related exposure will be added } \\
\text { to this analysis during definitive design. }\end{array}$ & DD \\
\hline $\begin{array}{l}\text { Merkling, } T \text {. } \\
\text { 90\% CDR }\end{array}$ & 16 & $\begin{array}{l}\text { The information listed in the vendor section for the stack } \\
\text { iso-kinetic sampling head is incorrect. The Eberline } \\
\text { ALPHA6 is an in-line sampler head which draws a sample } \\
\text { from an iso-kinetic sampling array or a shrouded probe (it } \\
\text { is not the probe itseli). The project still needs a sampling } \\
\text { probe (iso-kinetic or shrouded). }\end{array}$ & no & Probe wilt be specified in definitive design stage. & closed \\
\hline $\begin{array}{l}\text { Merkling, T. } \\
90 \% \mathrm{CDR}\end{array}$ & 17 & $\begin{array}{l}\text { Drawing SK-300413; Comment: Delete "Xe-127" from the } \\
\text { drawing, this isotope is not available. }\end{array}$ & no & Wo will delete Xe-127 from SK-300413 & closed \\
\hline $\begin{array}{l}\text { Merkling, } T \text {. } \\
\text { 90\% CDR }\end{array}$ & 18 & $\begin{array}{l}\text { Drawing SK-300413; Comment: The Eberline PING-3B is } \\
\text { listed as the instrument offered to detect lodine } 129 . \text { I do } \\
\text { not believe that this PING-38 instrument is sensittve } \\
\text { enough to delect the low levels of this low energy beta } \\
\text { emitter. It was designed to delect the higher energy } 1-131 \\
\text { in higher concentrations than } 1-129 \text {. }\end{array}$ & no & $\begin{array}{l}\text { We will review the Eberine PING-3B capability regarding } 1-129 \text { and } \\
\text { make any changes necessary during definitive design. }\end{array}$ & closed \\
\hline $\begin{array}{l}\text { Merkling, T. } \\
90 \% \text { CDR }\end{array}$ & 19 & $\begin{array}{l}\text { Drawing SK-2-300417; Comment: The drawing gives the } \\
\text { look of concrete over the trench [ } 31.72 \text { inches to the left } \\
\text { and } 81.63 \text { inches to the right of the } 3 \text { inch steel plate over } \\
\text { the center of the MCO]. The narrative in appendlx B, page } \\
4 \text { of } 8 \text { states that the trench will be covered with steel to } \\
\text { provide shielding. Which is correct? Change the drawing } \\
\text { to reflect the steel over the trench. Also, there is possible } \\
\text { scatter through the } 10 \text { inch push-through HEPA filters as } \\
\text { the filters are configured in the drawing. }\end{array}$ & no & $\begin{array}{l}\text { The trench cover will be fabricated of steel. Drawing will be } \\
\text { clarified. }\end{array}$ & closed \\
\hline
\end{tabular}




\begin{tabular}{|c|c|c|c|c|c|}
\hline REVIEWER & COMMENT * & COMMENT & HOLD POINT & DISPOSITION & STATUS \\
\hline $\begin{array}{l}\text { Miska, C.R. } \\
60 \% \text { CDR }\end{array}$ & 1 & $\begin{array}{l}\text { Please provide clear descriptive names for major pieces of } \\
\text { equipment to eliminate confusion. Also consider providing } \\
\text { a numbering scheme that would coordinate the block flow } \\
\text { diagram with the process sequence etc. }\end{array}$ & no & $\begin{array}{l}\text { A section that defines names for major pieces of equipment has } \\
\text { been added to the CDR and these names are used throughout. } \\
\text { Coordination of block flow diagrams with process sequence will be } \\
\text { done during definitive design. }\end{array}$ & DD \\
\hline $\begin{array}{l}\text { Miska, C.R. } \\
60 \% \text { CDR }\end{array}$ & 2 & $\begin{array}{l}\text { The process sequence appears to be founded on the } \\
\text { assumption that the MCO will have some TBD valves } \\
\text { rather than the existing MCO design with quick disconnect } \\
\text { fittings. While I do nol like the MCO "quick" disconnect } \\
\text { fitting the HCS } 90 \% \text { conceptual design will need to be } \\
\text { baselined to the MCO design. Unless "we" can decide on } \\
\text { a specific alternative and convince the MCO team and } \\
\text { others to design, test, and implement this concept, then we } \\
\text { must baseline to the existing MCO design. }\end{array}$ & no & $\begin{array}{l}\text { Agree. } \\
\text { Will reissue the sequence to allow for remote connection of quick } \\
\text { disconnects. }\end{array}$ & closed. \\
\hline $\begin{array}{l}\text { Miska, C.R. } \\
60 \% \text { CDR }\end{array}$ & 3 & $\begin{array}{l}\text { The HCS design needs to provide HEPA filtration on the } \\
\text { supply lines to the MCO. Note the Lab has recently } \\
\text { experienced a contamination spread (Cs ?) whlle they } \\
\text { were working on a furnace gas supply line outside of the } \\
\text { hot cell. }\end{array}$ & no & $\begin{array}{l}\text { Mas been incorporated in } 90 \% \text { conceptual design. Added VPS-F- } \\
1107 \text { on the dip-tube side of the MCO. }\end{array}$ & closed \\
\hline $\begin{array}{l}\text { Miska, C.R. } \\
60 \% \text { CDR }\end{array}$ & 4 & $\begin{array}{l}\text { The RGA instrument should be able to sample the offgas } \\
\text { prlor to the gas cooler/cold trap. I suggest that the RGA be } \\
\text { set up to sample the gas from several locations, (supply } \\
\text { gas, offgas prior to cooler, after cooler, and just prior to } \\
\text { return to the MCO). It is suggested that this instrument be } \\
\text { for process control purposes, and that a reliable simple to } \\
\text { calibrate flammable gas analyzer etc be use to detemine } \\
\text { that H2 concentration limits are met. This simple reliable } \\
\text { instrument could then ensure compliance with safety limits. }\end{array}$ & no & Issue will be subject of study during definitive design. & DD \\
\hline $\begin{array}{l}\text { Miska, C.R. } \\
60 \% \text { CDR }\end{array}$ & 5 & $\begin{array}{l}\text { The Shield Plug will likely need to bo held in place in the } \\
\text { MCO vaull cover prior to lifting the vault cover. This detail } \\
\text { should be developed in such a way as to essentially } \\
\text { preclude damage to the MHM / Vault Cover / Process } \\
\text { Enclosure trom improper operation of the restraint. A likely } \\
\text { solution would be to design this restraint as part of the pick } \\
\text { point for tipping the MCO vault cover. If this was done } \\
\text { then the cover would be constrained when lifted and the } \\
\text { restraint would atways be removed prior to process } \\
\text { enclosure removal. }\end{array}$ & no & Agree. Will includa in definitive design of process plt cover. & DD \\
\hline
\end{tabular}




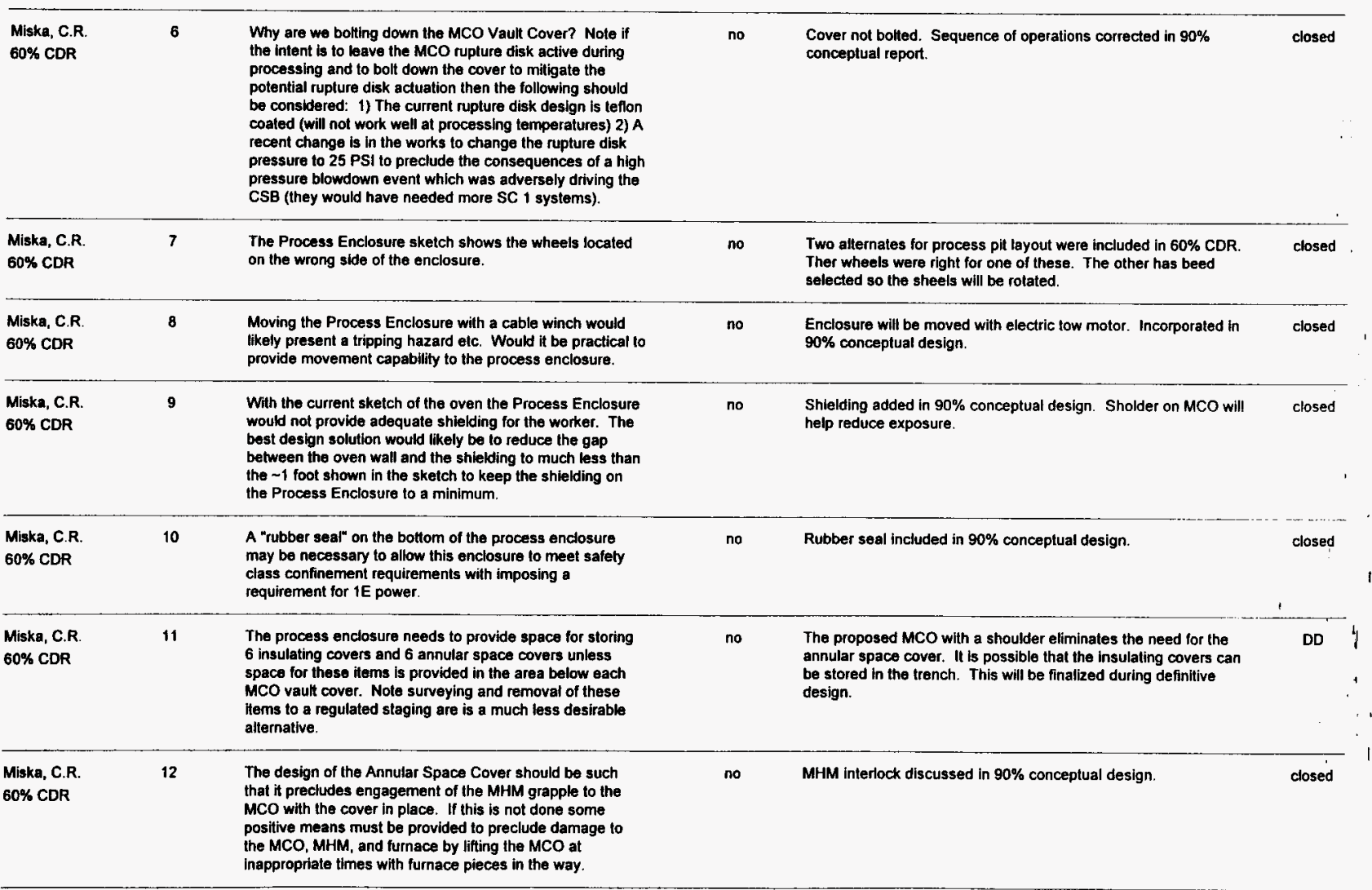




\begin{tabular}{|c|c|c|c|c|c|}
\hline REVIEWER & COMMENT * & COMMENT & HOLD POINT & DISPOSITION & STATUS \\
\hline $\begin{array}{l}\text { Miska, C.R. } \\
\text { 60\% CDR }\end{array}$ & 13 & $\begin{array}{l}\text { Step } 24 \text { survey cell should be revised.Routine manned } \\
\text { entry into the process enclosure should be avoided } \\
\text { whenever possible, especially when surface contamination } \\
\text { status is unknown. A manipulator survey of key locations } \\
\text { is suggested instead, possibly with pass through of survey } \\
\text { swipes for counting. }\end{array}$ & no & Revised in $90 \%$ conceptual report. & closed \\
\hline $\begin{array}{l}\text { Miska, C.R. } \\
60 \% \text { CDR }\end{array}$ & 14 & $\begin{array}{l}\text { Slep \# } 28 \text { and } 29 \text { may need to be switched to determine if } \\
\text { the dryness criteria is met at the end of the } 300 \text { degree } \\
\text { hold step. Note I do not think that this type of test would } \\
\text { be meaningiul. }\end{array}$ & no & Revised in $90 \%$ conceptual report. & closed \\
\hline $\begin{array}{l}\text { Miska, C.R. } \\
60 \% \text { CDR }\end{array}$ & 15 & $\begin{array}{l}\text { Step * 25The Process Enclosure needs to be raised } \\
\text { before the power is disconnected. }\end{array}$ & no & Agree. Changed in $90 \%$ conceptual report. & closed \\
\hline $\begin{array}{l}\text { Miska, C.R. } \\
\text { 60\% CDR }\end{array}$ & 16 & $\begin{array}{l}\text { Consider attaching the Process Enclosure to the Process } \\
\text { Module with an umbilical cord to allow its activities to be } \\
\text { surveiled by the PLC control system. }\end{array}$ & no & Agree. Will be incorporated in definitive design. & DD \\
\hline $\begin{array}{l}\text { Miska, C.R. } \\
\text { 60\% CDR }\end{array}$ & 17 & $\begin{array}{l}\text { Consider locating a fumace PLC "idiot"/status light where it } \\
\text { can be observed by the operalor moving and operating the } \\
\text { Process Enclosure. These "idiot"/status lights would show } \\
\text { what part of the process each individual station was in, and } \\
\text { would help preclude an operator from inadvertently } \\
\text { performing actions on the wrong MCO (station } 2 \text { instead of } \\
\text { station } 3 \text { for example). Also it would likely be helpful for } \\
\text { the DCS to have a similar "status board" screen with the } \\
\text { status of each of the } 6 \text { process stations shown pictorially at } \\
\text { the same time. }\end{array}$ & no & $\begin{array}{l}\text { Will be incorporated in detailed design. } \\
\text { Agree this and several other visual aids will be provided to the } \\
\text { operators. As these are developed they will be added to the } \\
\text { appropriale P\&IDs during definilive design. } \\
\text { The technology exists to mount a system monitor on the Process } \\
\text { Enclosure with communicatlons being either a cable or infrared. } \\
\text { The Process Enclosure operator should have access to the same } \\
\text { data as the control room operators. }\end{array}$ & DD \\
\hline $\begin{array}{l}\text { Miska, C.R. } \\
60 \% \text { CDR }\end{array}$ & 18 & $\begin{array}{l}\text { Step } 34 \text { Why is the MCO backfilled to a slight negative } \\
\text { pressure, this is not consistent with the performance } \\
\text { specification. }\end{array}$ & no & Will be corrected to agree with the performance spec. & closed \\
\hline
\end{tabular}




\section{REVIEWER \\ COMMENT}

Miska, C.R

19

$60 \%$ CDR
Waler should not be used as a coolant for the process offgas unless it is demonstrated that it is impractical to use other design solutions such as an air to air heat exchanger. Note this also applies to a lesser degree to the process heating/cooling system. Use of water as a coolant in an offgas system localed directly above a 300 degree $C$ MCO filled with damaged reactive fuel would raise safety questions that would fikely have adverse cost and schedule impacts. Water cooling of the offgas hydraulically above the MCO provides the potential for "steam explosions", tuel ignition, and resultant loss of MCO boundary scenario's that would be very difficult to address.
A study is being developed to be included in the final CDR. The study will address the issue of water use in the HCSE. The waler coolers, for the heating/cooling system, with features to prevent water entering the process equipment will be retained for the $90 \%$ CDR

\begin{tabular}{|c|c|c|c|c|c|}
\hline $\begin{array}{l}\text { Miska, C.R. } \\
60 \% \text { CDR }\end{array}$ & 20 & $\begin{array}{l}\text { I would like to seo heal transfer calculations that show that } \\
\text { the cooldown of the MCO head occurs in a reasonable } \\
\text { time while complying with the } 100 \text { degree } C \text { maximum } \\
\text { MCO temperature diference limit called out in the } \\
\text { performance specification. }\end{array}$ & no & $\begin{array}{l}\text { The calculation in the } 60 \% \text { CDR has been replaced by a } \\
\text { calculation from Q-Matrix that addresses this issue. The heating } \\
\text { and cooling profile must be optimized during definitive design to } \\
\text { meet this restriction. }\end{array}$ & DD \\
\hline $\begin{array}{l}\text { Miska, C.R. } \\
60 \% \text { CDR }\end{array}$ & 21 & $\begin{array}{l}\text { The Vacuum pump down calculation needs to be redone. } \\
\text { Pumping down the MCO to } 10 \text { torr will take longer than the } \\
\text { time indicated to transfer the free space gas volume of the } \\
\text { MCO. }\end{array}$ & no & $\begin{array}{l}\text { This calculation is incorrect and has been redone using the method } \\
\text { described in Perry's Chemical Engineers Handbook, } 2 \mathrm{E} \text { With this } \\
\text { calculation, the evacuation time has been increased by a factor of } \\
6 \text { to } 7 \text {. }\end{array}$ & closed \\
\hline $\begin{array}{l}\text { Miska, C.R. } \\
60 \% \text { CDR }\end{array}$ & 22 & $\begin{array}{l}\text { Review of the Heat transfer calculations by WHC indicates } \\
\text { that the calcuiated inside heat transfer coeficient } \mathrm{HI}=1 \text {, } \\
\text { and the calculated outside heat transfer coefficient } \mathrm{Ho}=5 \\
\text { are likely conservative. However a concern was expressed } \\
\text { that the spiral flow envisioned to enhance the outside } \\
\text { coefficient likely could not be maintained over the full MCO } \\
\text { height with only a 1" gap. }\end{array}$ & no & $\begin{array}{l}\text { Recent information from Q-Matrix indicates that the heat transfer } \\
\text { calculations are not conservative as they show heat up in } 20 \text { hours } \\
+ \text { rather than } 12 \text { and the } 100 \text { degree temperalure difference across } \\
\text { the MCO is exceeded. Additlonal calculations are required during } \\
\text { definitive design. }\end{array}$ & DD \\
\hline
\end{tabular}




\begin{tabular}{|c|c|c|c|c|c|}
\hline REVIEWER & COMMENT * & COMMENT & HOLD POINT & DISPOSITION & STATUS \\
\hline $\begin{array}{l}\text { Miska, C.R. } \\
90 \% \text { CDR }\end{array}$ & 2 & $\begin{array}{l}\text { Sec } 1.0 \text { page } 1 \text {, Appendix E page } 1 \text {, and throughout } \\
\text { documentPlease use the issued revision } 0 \text { of HCS } \\
\text { performance specification, not the old draft revision } A \text {. } \\
\text { Please discuss with WHC if specific information in rev } A \text { is } \\
\text { required that is not in rev } 0 \text {. (search doc for rev A)Note } \\
\text { revision } 1 \text { of this document will be issued in the near future } \\
\text { and direction will likely be given to use revision } 1 \text { for the } \\
\text { detailed design phase. }\end{array}$ & no & Document will be revised to reference Rev 0 . & closed \\
\hline $\begin{array}{l}\text { Miska, C.R. } \\
\text { 90\% CDR }\end{array}$ & 3 & $\begin{array}{l}\text { Sec } 1.0 \text { Page 3Statement that "the proposed HCSE } \\
\text { conceptual design should be able to achieve whatever } \\
\text { degassing specification is established by simply heating } \\
\text { and pumping longer" is not totally valid. Some of the } \\
\text { residual moisture (contributor to pressure in sealed interim } \\
\text { storage) after hot conditioning is expected to be tightly } \\
\text { associated with fuel oxides and sludge, thus holding at the } \\
\text { design lemp can not remove all residual moisture from } \\
\text { oxides. However if oxide inventory is low enough then } \\
\text { maximum residual moisture criteria can be met. Suggest } \\
\text { deletion of sentence from report. }\end{array}$ & no & Sentence will be deleted as suggested. & closed \\
\hline $\begin{array}{l}\text { Miska, C.R. } \\
\text { 90\% CDR }\end{array}$ & 4 & $\begin{array}{l}\text { Section } 2.0 \text { pages } 6,7,8,9,47,48,49,50,53,54 \ldots \\
\text { Please revise document to show weld inspection technique } \\
\text { more suitable for remote implementation (radiograph or } X \text { - } \\
\text { ray for example). Alternately for COR indicate in text that } \\
\text { more approprlate weld inspection techniques would greatly } \\
\text { improve the probability that the process enclosure will } \\
\text { meet duty cycle requirements (will not adversely constrain } \\
\text { throughput). Note i believe DP inspection was chosen at } \\
\text { CVD because that weld design would not allow full } \\
\text { penetration inspection. }\end{array}$ & no & $\begin{array}{l}\text { The new weld test proposal from the MCO designers specifies } \\
\text { ultrasonic testing. This will be incorporated in the detail design if } \\
\text { the HCSE. }\end{array}$ & closed \\
\hline
\end{tabular}




\section{REVIEWER COMMENT * COMMENT}

\begin{tabular}{ll}
\hline Miska, C.R. & Section 2.0, page $11,2.2 .1 .2$ (Pressurized Operation), also \\
40\% CDR & pepage 3 interface MCO7The process can should not be \\
designed to support operation at 115 PSIA. The MCO \\
performance specification/ interface agreements etc \\
require it to be maintained below 75 PSID when the \\
temperature is above 200 deg C. Note also that this \\
pressure is above the current rupture disk design pressure \\
(25 PSI) and thus would require covering or replacing the \\
ruplure disk. Note the heating and cooling durations in the \\
performance specification are not hard and fast design \\
criteria but targets. We should have sufficient excess \\
design capacity that pressurized heatup and cool down \\
should not be required (especially after eliminating the cool \\
down durations for that would have been required for \\
hands on access to the MCO.Note also if the lobe rotor \\
pump is a high capacity pump this raises difficulty in \\
dealing with particulate entrainment, and line break \\
accident analysis. Note if gas circulation pump is required \\
to guarantee vacuum pump life a more appropriate gas \\
circulation device should be chosen (regenerative blower \\
for example). A lobe rotor would not be dependable in this \\
service, (it would fail catastrophically the first time flow was \\
Inadvertently isolated or restricted).
\end{tabular}

Miska, C.R. $\quad 6 \quad 2.0$, Page 12, Third full paragraphSecond sentence is

$90 \%$ CDR incorrect "Exposed uranium reacts with oxygen and continues to do so until the surface is passivaled." This is incorrect because the uranium will continue to oxidize as long as oxidant is present. This is true in part because urantum does not form a true "passive" surface upon oxidation (the oxidation products begin to spall after reaching a certain thickness).

\section{HOLD POINT DISPOSITION}

STATUS

no

The process will be designed to support operation at 25 psia rather than 115 psia. A magnetically coupled regenerative blower as recommended is now shown in the CDR. A pumping study will be performed during definitive design to select the most appropriate pump for this service. no

\section{Replace first two sentences with the following:}

During the lower temperature partial exidation phase of the hot

conditioning process, low concentrations of oxygen are introduced

into the MCO in an inert cartier gas stream. This low concentration

of oxygen consumes highly readtive sites on the fuel inside the

$M C O$, reducing the chemical reactivity of the damaged fuel matrix

that may be present in the MCO. Not the reactive sites consumed by this partial oxidation may include small fuel fragments, high surface area uranium hydride particles, and high surface area uranium metal particles created by the thermal decomposition of uranium hydride.

\begin{tabular}{ll}
\hline Miska, C.R. & 7 \\
90\% CDR & 2.0, Page 32, 2.4 .2 and 2.4 .5$, Why is it advantageous to \\
use a radio modem to communicate between the PMC and \\
the PLC (why is mobility required). Also what is the PMC \\
(I did not see it defined in the control section). Note the \\
salety analyst are already complaining about nol liking \\
reliance on PLC to respond appropriately. The potential for \\
radio interference just adds fuel to that potential \\
disagreement. (NOTE PUREX had big problems with \\
portable 2 way radio's disrupting sensitive equipment like \\
central fire atarm dispatch panels).
\end{tabular}

During the oral presentation it was incorrectly stated that a radio modem would be used for data communication between the process module and the control room. This circuit is hard wired only used between the moblle process enclosure and the control room and only to monitor inputs such as temperalure and other parameters which are not used in closed loop contral circuits. If any of the control circuits and signals are multiplexed over $\mathbf{2}$ conductors. A radio modem is communication via radio modem is erratic, this would not impact

closed

,

.


REVIEWER

\begin{tabular}{|c|c|c|c|c|c|}
\hline $\begin{array}{l}\text { Miska, C.R. } \\
90 \% \text { CDR }\end{array}$ & 8 & $\begin{array}{l}\text { 2.0, page } 43 \text {, Instrument Air SystemWhy are we providing } \\
\text { instrument air, has it been decided that the CSB instrument } \\
\text { air supply will not be used? }\end{array}$ & no & $\begin{array}{l}\text { The instrument air system in the CSB is at the opposite end of the } \\
\text { buithing. In order to minimize the impact of the HCSE on the CSB } \\
\text { design, it will not be extended to the HCSE. }\end{array}$ & closed \\
\hline $\begin{array}{l}\text { Miska, C.R. } \\
\text { 90\% CDR }\end{array}$ & 9 & $\begin{array}{l}\text { 3.0, Page } 1 \text { and } 2 \text { HCSE layout More detail is needed than } \\
\text { is provided on the referenced drawing SK-2-3-00421. For } \\
\text { example the exact size of the shuttle table pit needs to be } \\
\text { specified. Note also that the dimension from the centerline } \\
\text { of the nearest obstruction east-west and north south is not } \\
\text { as shown on SK-2-3-00421 (greater clearance is } \\
\text { required). Also floor elevations need to be specilied unless } \\
\text { the entire floor is at one elevation. Nole recessing the skid } \\
\text { and waste storage area may be highly desirable lo prevent } \\
\text { water from process cooling leaks, or fire protection } \\
\text { systems from reaching the furnace or CSB vault tubes. }\end{array}$ & no & $\begin{array}{l}\text { Shuttle table no longer required as } M H M \text { box beam has been } \\
\text { raised to } 9 \mathrm{ft} \text { and process enclosure will be less than } 9 \mathrm{ft} \text {. It has } \\
\text { been determined that the floor will not be recessed since loakage } \\
\text { from process cooling water will be contained by the process } \\
\text { module and no fire protection water is required. }\end{array}$ & closed \\
\hline $\begin{array}{l}\text { Miska, C.R. } \\
90 \% \text { CDR }\end{array}$ & 10 & $\begin{array}{l}\text { 3.0, page 3, Trench Coverl strongly suggest specifying the } \\
\text { design of the trench to HCSA designer such that a trench } \\
\text { cover greater than } 6^{*} \text { could be supported (design strong } \\
\text { enough to support a stepped cover extending below lip for } \\
\text { trench cover). I recommend designing to support } \\
\text { something like 8-10 inches of steel. This would allow the } \\
\text { HCSE designers more flexibility to go above } 6 \text { inches for } \\
\text { trench covers if required for any reason. }\end{array}$ & no & Trench will be specified as suggested. & closed \\
\hline $\begin{array}{l}\text { Miska, C.R. } \\
\text { 90\% CDR }\end{array}$ & 11 & $\begin{array}{l}\text { Appendix (general)Please tab each appendix separately. } \\
\text { Also please try to arrange the vendor cul sheels in a } \\
\text { logical order if possible (group like equipment l.e. hepa } \\
\text { filters) }\end{array}$ & no & $\begin{array}{l}\text { Appendices will be tabbed as suggested and cut sheets will be } \\
\text { organized alphabetically by equipment number. }\end{array}$ & closed \\
\hline
\end{tabular}




\section{REVIEWER}

COMMENT

\section{2}

Miska, C.R $90 \%$ CDR

12

Ap

Appendix, SK-2-300418 (Oven Assembly)As shown the

Appendix, SK-2-300418 (Oven Assembly)As shown the
furnace shielding will likely result in much more than the $5 \mathrm{mR} / \mathrm{Hr}$ operator dose rate discussed in the shielding section. As shown in the sketch a "direct view" of unshielded side of MCO would exist it looks like this would result in effectively less than 2 inches of shielding with process pit covers up and enclosure in place. This will grocess pit covers up and enclosure in place. This estimate the potential dose to worker at the enclosure using a factor of 4 reduction in dose per inch of steel, a factor of 4 reduction to correct the dose from 1 meter to the dose at 2 meters, and the saleguards dose of $-65 \mathrm{R} / \mathrm{hr}$ from the side of an unshielded MCO at 1 meter (for average fuel not peek shlelding design fuel), I end up with a dose rate to the operator at the process enclosure greater than $1,000 \mathrm{mR} / \mathrm{Hr}$. This is three orders of magnitude greater than what the design dose rate should be. Even if concrete is added to the open space to the left be. Even if concrete is added to the open space to the left still exist due to the direct beam from the heating/cooling piping on the right side of drawing SK 2-300418. It is very likely that this type of shielding design deficlency could not be adequately address with the process enclosure, and thus must be addressed via the design of the hurnace. Note to ensure the success of the HCS project it is essential to be confidant that the pit dimensions and "fioor loadings" given to the HCSA designers will be adequate to provide for this necessary shlelding close to the MCO. It is suggested that the steel top shield shown on the sketch be greatly increased in diameter to eliminate any "direct view" of the unshieided portion of the MCO.
HOLD POINT DISPOSITION no

These comments address the shielding analysis and the resulting dose rates above the oven and at the process enclosure. The dose rates calculated for the currently envisioned oven and process enclosure are "ballpark" accurate. I agree that a more detailed (Monte-Carlo) analysis chould be performed (Comment 15) given the complex geometries involved. I also agree that more shielding can, and chould, be incorporated into the oven/process enclosure design to lower the currenly projected dose rate at the operator station and the general area dose rate above the oven. This will be incorporated during the definitive design. Various design options will be evaluated and a rough contour dose rate map (with and without the process enclosure) will be provided to demonstrate the feasibility of the design. The oven and shielding desing as currently depicted does leave a small unshleided solid angle to the process enclosure. The will be fixed by adding shlelding to either the oven or process enclosure - the closer to the source, the better. The shielding and dose rate lssures are manageable and will be addressed and resolved during definitive design. Text of Exposure Analysis has been revised to reflect options for reducing dose rales.
Appendix B, Alara Implementation Plan, page 6, Item B Criteria "Maximum exposure shall not exceed $5.0 \mathrm{mR} / \mathrm{H}$. on the average." is inappropriate for application as an acceptable design dose for personnel performing routine (extended duration work) at a remole operator console. that the impact of the viewing window was ignored, and that the dose calculations were likely highly optimistic (at best). Note this is especially inappropriate when it is considered 


\begin{tabular}{|c|c|c|c|c|c|}
\hline $\begin{array}{l}\text { Miska, C.R. } \\
\text { 90\% CDR }\end{array}$ & 14 & $\begin{array}{l}\text { Appendix C ShieldingSee above comments on non- } \\
\text { conservative shielding estimation. Also the duration } \\
\text { allowance for work by the process enclosure may be non } \\
\text { conservative (I would not be surprised if the enclosure is in } \\
\text { use nearly all the time that the MHM is nol in it's way), thus } \\
\text { the dose rate that the worker receives from this source } \\
\text { must be minimized. As discussed above this may best be } \\
\text { done by optimizing the design of the furnace. Nole } \\
\text { assuming adding shielding will just be added to the } \\
\text { process enclosure will grealty increase the floor loading. } \\
\text { and will likely require abandoning the viewing window } \\
\text { (which I am not willing to give up). Note the reflected dose } \\
\text { when the process pit covers are open must also be } \\
\text { considered. This bounce could greatly increase the } \\
\text { "background dose" potentially invelidating the .25 mi/Hr } \\
\text { shielding design criteria for the CSB as well as edding } \\
\text { significantly to the worker dose. }\end{array}$ & no & See response to comment 12 . & DD \\
\hline $\begin{array}{l}\text { Miska, C.R. } \\
\text { 90\% CDR }\end{array}$ & 15 & $\begin{array}{l}\text { Appendix C ShleldingProvide a rough dose conlour map } \\
\text { around the furnace (with or without the shielding of the } \\
\text { process enclosure). I see view angles that are not } \\
\text { consistent with the } 5 \mathrm{mr} \text { dose at the process enclosure or } \\
\text { the design maximum of } 5 \mathrm{mr} \text {. Note WHC is pursuing } \\
\text { getting this type of dose contour map via Jay Lan but this } \\
\text { will require } \sim 2 \text { months to complete. }\end{array}$ & no & See response to comment 12 . & DD \\
\hline $\begin{array}{l}\text { Miska, C.R. } \\
90 \% \text { CDR }\end{array}$ & 16 & $\begin{array}{l}\text { Appendix D, OSM Philosophy. Page 1: I do not believe } \\
\text { that the CSB is designed for workers to routinely wear } \\
\text { protective clothing. I believe that the CSB and thus the } \\
\text { Annex should be designed for access in street clothes, } \\
\text { with temporary RCA's being used as necessary. }\end{array}$ & no & Agree. Document has been revised. & closed \\
\hline $\begin{array}{l}\text { Miska, C.R. } \\
90 \% \text { COR }\end{array}$ & 17 & $\begin{array}{l}\text { Appendix D, O\&M Philosophy, Page 1, CThe expected } \\
\text { process duration for HCS is } 2 \text { years with a } 10 \text { year design } \\
\text { life for the equipment and } 75 \text { year design life for the annex. } \\
\text { Note this was not clear in the equipment performance } \\
\text { specification, but will be clariffed in REV } 1 \text { of the } \\
\text { equipment performance specification. }\end{array}$ & no & Comment incorporated. & closed \\
\hline
\end{tabular}




\begin{tabular}{|c|c|c|c|c|c|}
\hline REVIEWER & COMMENT * & COMMENT & HOLD POINT & DISPOSITION & STATUS \\
\hline $\begin{array}{l}\text { Miska, C.R. } \\
90 \% \text { CDR }\end{array}$ & 18 & $\begin{array}{l}\text { Appendix D, O\&M Philosophy, Page 2, Equipment } \\
\text { SelectionAdd new item, components potentially requiring } \\
\text { maintenance or replacement in the furnace should be } \\
\text { designed for remote manipulator replacernent if at all } \\
\text { possible. Note contact maintenance of this equipment } \\
\text { would only be possible after removal of the MCO, and } \\
\text { shielding or removal of accumulated dose contributors } \\
\text { (contaminated process lines, cold traps, and filters). }\end{array}$ & no & Comment incorporated. & closed \\
\hline $\begin{array}{l}\text { Miska, C.R. } \\
\text { SO\% CDR }\end{array}$ & 19 & $\begin{array}{l}\text { Appendix D, O\&M Philosophy, Page 3This page contains } \\
\text { several "relics" from CVD that must be eliminated including } \\
\text { "Drying Bays", "internal Washdown", "baged connectlons", } \\
\text { "Hood". }\end{array}$ & no & Agree. Document has been revised. & closed \\
\hline $\begin{array}{l}\text { Miska, C.R. } \\
\text { 90\% CDR }\end{array}$ & 20 & $\begin{array}{l}\text { Appendix D, O\&M Philosophy, Page } 384 \text {, Radiation } \\
\text { Safety, second sentence in sectionChange to read: } \\
\text { Preliminary estimates suggest that this can be achieved } \\
\text { through a combination of shielding near the MCO integral } \\
\text { to the furnace assembly, and shielding provided by the } \\
\text { process pit covers or process enclosure. }\end{array}$ & no & Comment incorporated & closed \\
\hline $\begin{array}{l}\text { Miska, C.R. } \\
\text { 90\% CDR }\end{array}$ & 21 & $\begin{array}{l}\text { Appendix D, OAM Philosophy, Page 6, ShieldingSection } \\
\text { on normally Occupied areas implies a dose rate in these } \\
\text { areas of } 1 / 2 \mathrm{mR} / \mathrm{Hr} \text {. This is not consistent with the } 2 \\
\text { mR/Hr used in appendix C. I believe that the "background } \\
\text { dose" through the shielding with the trench covers down } \\
\text { should be }<1 / 2 \mathrm{mR/Hr} \text {. }\end{array}$ & no & $\begin{array}{l}\text { Agree. Shielding design will relect criteria of } 0.25 \text { rem/hr. See } \\
\text { Watt Alaconis comment } * 7 \text { and resolution. }\end{array}$ & closed \\
\hline $\begin{array}{l}\text { Miska, C.R. } \\
\text { 90\% CDR }\end{array}$ & 22 & $\begin{array}{l}\text { Appendix D, O\&M Philosophy, Page } 8 \text {, Second } \\
\text { Sentence The stalement that "the facility will be considered } \\
\text { an HC-3 facility..." is not consistent with the HC-2 indicated } \\
\text { on page } 1 \text { of the introduction to the } 90 \% \text { CDR. At this } \\
\text { point in time I tend to agree with assuming HC-2 } \\
\text { designation. }\end{array}$ & no & Document revised to reflect $\mathrm{HC}-2$ designation. & closed \\
\hline
\end{tabular}


Miska, C.R.

90\% CDR

23

Appendix E, Understanding of Performance

SpecificationThis section needs to provide clear indication

where the process design is nol consistent with the

performance specification. An example is page 2 " $\mathrm{Cool}$

Down for Connection" rather than repeat the performance

specification this appendix should indicale that this

process step is not needed because remote connection will

be uttilized. It should also be noted that this change will

reduce the heating duration as well and will thus

beneficially affect the process cycle time. Another

example of what should have been present in this section

is the proposed deviation from the firm requirement to

eliminate reacthe flulds (water) for heating or cooling the

MCO process offgas. This type of presentation would allow

formally accept the proposal to eliminate the cool down

step from the performance specification, and modify future

revisions of the performance specification accordingly.

This type of candid evaluation of compliance with the

performance specification will also encourage thorough

consideration of the issue, and help clarify where deviation

from the performance specification is not allowed.

Examples of areas where deviation of from the

performance specification are proposed by the equipment

designer which will not be allowed Include exceeding the

MCO design pressure limit of 75 PSIA at temperatures

above $\mathbf{2 0 0}$ degrees $\mathrm{C}$, and the use of water for cooling the

process offgas.
Section will be revised to reflect current design in terms of initial cooldown, use of water, etc. A study on the use of water for cooling will be included in the final CDR. 


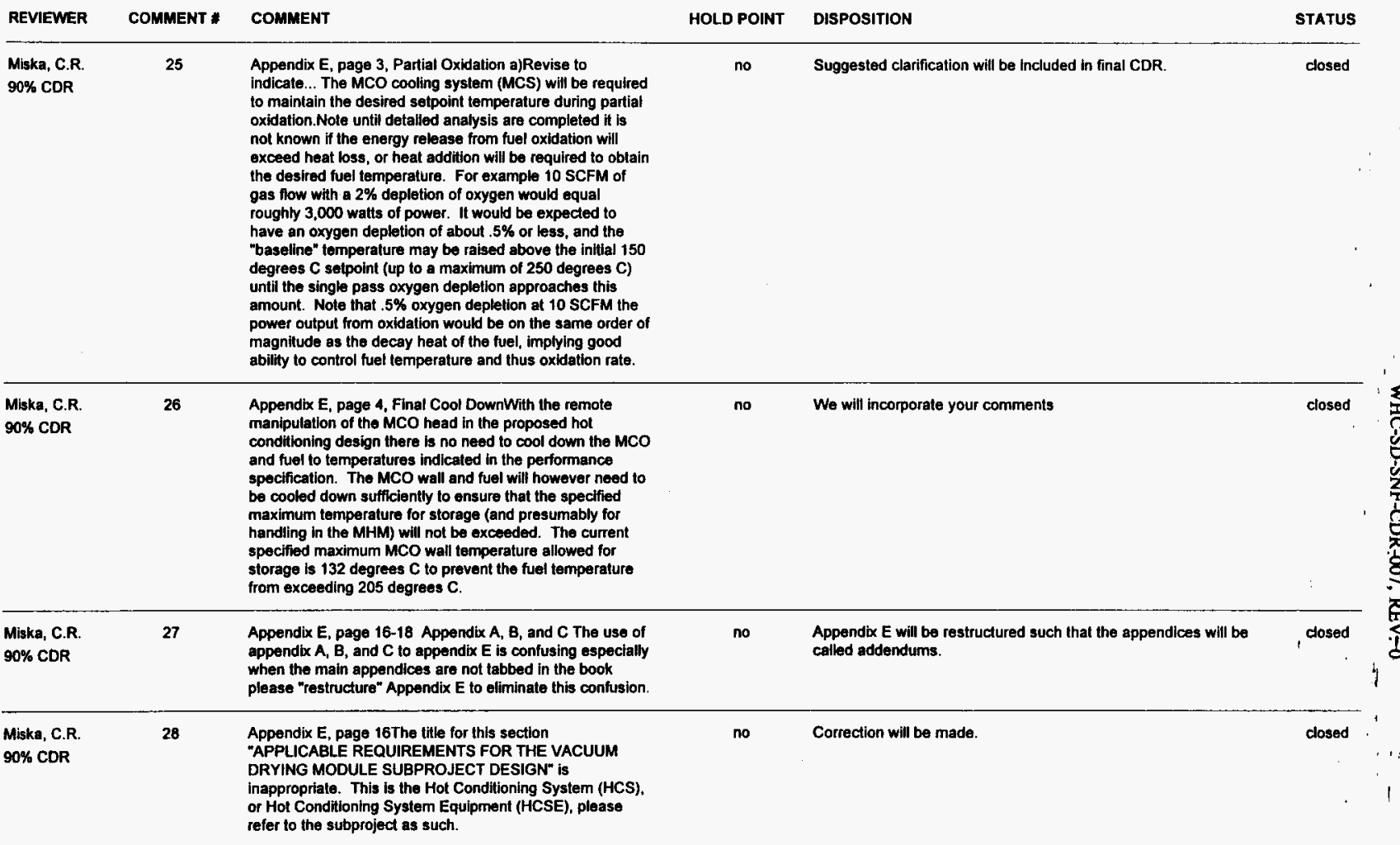




\begin{tabular}{|c|c|c|c|c|c|}
\hline REVIEWER & COMMENT * & COMMENT & HOLD POINT & DISPOSITION & STATUS \\
\hline $\begin{array}{l}\text { Miska, C.R. } \\
90 \% \text { CDR }\end{array}$ & 29 & $\begin{array}{l}\text { Appendix GPlease try lo tabulate and organize the } \\
\text { information in this appendix better (a table of contents and } \\
\text { page numbering for this appendix may be required). } \\
\text { Obviously this is a detail issue that is nice but not } \\
\text { mandatory, however breaking the appendix into logical } \\
\text { tabbed sections as a minimum would help. }\end{array}$ & no & $\begin{array}{l}\text { We concur. A calculation log will be generated and added to } \\
\text { Appendix } \mathbf{G} \text {. }\end{array}$ & closed \\
\hline $\begin{array}{l}\text { Miska, C.R. } \\
\text { 90\% CDR }\end{array}$ & 30 & $\begin{array}{l}\text { Appendix } G \text {, last two pages (PART I/E-9)it is not clear } \\
\text { what the purpose is for these two page, or even if they } \\
\text { belong in this document. }\end{array}$ & no & This was not intended. These pages will be removed. & closed \\
\hline $\begin{array}{l}\text { Miska, C.R. } \\
\text { 90\% CDR }\end{array}$ & 31 & $\begin{array}{l}\text { Appendix } H, \text { Vendot cut sheetslt appears that a divider is } \\
\text { needed prior to vendor cul sheeis. My book goes directly } \\
\text { from appendix } G \text { to the vendor cut sheets (Appendix } H \text { ). }\end{array}$ & no & Tabbed divider will be added. & closed \\
\hline $\begin{array}{l}\text { Miska, C.R. } \\
\text { 90\% CDR }\end{array}$ & 32 & $\begin{array}{l}\text { Appendix } H \text {, Vendor cut sheetsPlease try to tabulate and } \\
\text { organize the information in this appendix better (a table of } \\
\text { contenis and page numbering for this appendix may be } \\
\text { required). Obviously this is a detail issue that is nice but } \\
\text { not mandatory, however grouping like information, and } \\
\text { breaking the appendix into logical tabbed sections as a } \\
\text { minimum would help. }\end{array}$ & no & $\begin{array}{l}\text { Information in Appendix } \mathrm{H} \text { will be organized alphabetically by } \\
\text { equipment number. }\end{array}$ & closed \\
\hline $\begin{array}{l}\text { Miska, C.R. } \\
90 \% \text { CDR }\end{array}$ & 33 & $\begin{array}{l}\text { Appendix } \mathrm{H} \text {, Vendor cut sheetsPlease Include vendor } \\
\text { information for the manipulator proposed for the process } \\
\text { enclosure. This is a vital piece of equipment which } \\
\text { requires additional information to allow fail evaluation of its } \\
\text { capabilities (for those of us who are not familiar with this } \\
\text { particular manipulalor). }\end{array}$ & no & We will incorporate your comments in the final CDR. & closed \\
\hline $\begin{array}{l}\text { Miska, C.R. } \\
90 \% \text { CDR }\end{array}$ & 34.1 & $\begin{array}{l}\text { Comments 34.1-34.6 refer to Appendix H, Vendor cut } \\
\text { sheets, and P\&ID SK-2-3004 } 3 \text { Flanders G-1 Bag In/Bag } \\
\text { Out housings. } \\
\text { 1) Per the P\&ID these filters are localed inside the irench } \\
\text { (changed remotely with the manipulator). Having been } \\
\text { involved with the change out of several of these filters in } \\
\text { the past I am not sure that they are suitable for remote } \\
\text { operation without significant modification. }\end{array}$ & no & $\begin{array}{l}\text { Filters in trench will be custom design to facilitate manipulator } \\
\text { change out. }\end{array}$ & closed \\
\hline
\end{tabular}




\begin{tabular}{|c|c|c|c|c|c|}
\hline REVIEWER & COMMENT * & COMMENT & HOLD POINT & DISPOSITION & STATUS \\
\hline $\begin{array}{l}\text { Miska, C.R. } \\
90 \% \text { CDR }\end{array}$ & 34.2 & $\begin{array}{l}\text { 2) A fluid seal filter is unacceptable for this application } \\
\text { unless the equipment designer can provide a bullet proof } \\
\text { case that it is incredible (< } 1 \mathrm{EE}-6 \text { ) for significant quantities } \\
\text { of seal fluid (grease) to leave the filter system and enter } \\
\text { the MCO. Note }<100 \text { grams of organic would significantly } \\
\text { degrade the capacily of the MCO to contain the hydrogen } \\
\text { pressure from radiolysis of retained water in interim } \\
\text { storage. Further more if these seal fluids contain halogens } \\
\text { they could potentially degrade the performance of the } \\
\text { MCO (Safety Class } 1 \text { boundary). }\end{array}$ & & Seal fluids will be eliminated. & closed \\
\hline $\begin{array}{l}\text { Miska, C.R. } \\
90 \% \text { CDR }\end{array}$ & 34.3 & $\begin{array}{l}\text { 3) Restriction on high lemperature: The vendor cut sheet } \\
\text { Bulletin No. } 936 \text { page } 3 \text { indicates that maximum } \\
\text { temperature for the fluld seat is } 392 \text { degrees F ( } 200 \\
\text { degrees C). It is questionable if the G-t gasket seal } \\
\text { housing could be made to work (would pass DOP testing) } \\
\text { if the fitters are installed remotely, and are themally } \\
\text { cycled. Note that the Flanders cut sheets also Indicate } \\
\text { that for high temperature application special sealants are } \\
\text { required for gasket sealed units, and refers to page } 8 \\
\text { which is not included in this appendix. }\end{array}$ & no & Metal filters will not have the temperature restrictlons. & closed \\
\hline $\begin{array}{l}\text { Miska, C.R. } \\
\text { 90\% COR }\end{array}$ & 34.4 & $\begin{array}{l}\text { 4) Restriction on high pressure: The vendor cut sheets } \\
\text { indicate that the G1 housing with a high pressure design is } \\
\text { only good for } 15 \text { PSI positive or } 14.7 \text { PSI negative. This } \\
\text { would not be compatible with a pressurized circulation } \\
\text { system, and would could not be taken credit for unless all } \\
\text { credible system pressurization mechanisms are eliminated. }\end{array}$ & no & Custom designed metal filters will not have this pressure restriction. & closed \\
\hline $\begin{array}{l}\text { Miska, C.R. } \\
\text { 90\% CDR }\end{array}$ & 34.5 & $\begin{array}{l}\text { 5) Room for the filter housings: It is not clear that there is } \\
\text { room in the process trench or furnace pit as it is being } \\
\text { designed to install these filter housings and filters. }\end{array}$ & no & $\begin{array}{l}\text { Metal filters will be custom designed for this application to fit in the } \\
\text { available space. }\end{array}$ & closed \\
\hline $\begin{array}{l}\text { Miska, C.R. } \\
\text { 90\% CDR }\end{array}$ & 34.6 & $\begin{array}{l}\text { 6) Suggested approach for this filter: It is suggested that } \\
\text { further investigation be performed to identify sultable } \\
\text { filters for this difficult application. One potential worth } \\
\text { considering is PALL sintered metal filters, for example at } \\
\text { one time the Hanford site HWWP project was planning on } \\
\text { using Pall Sintered metal media for testable HEPA } \\
\text { filtration. I know that PALL makes sintered stainless steel } \\
\text { HEPA media in various configurations, the question is do } \\
\text { they have a housing configuration that is suitable (testable). }\end{array}$ & no & Metal filters will be specified in the final CDR. & closed \\
\hline
\end{tabular}




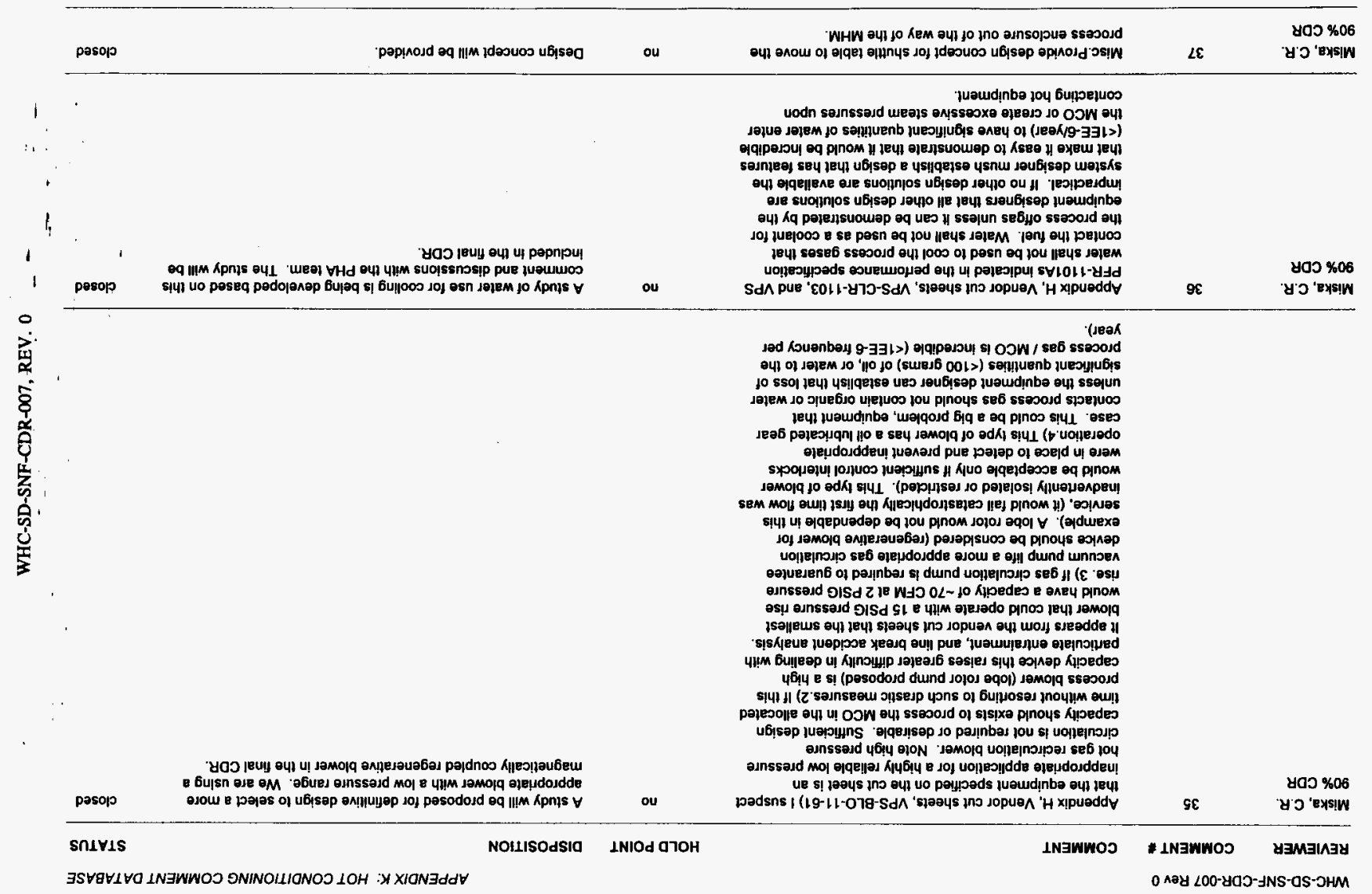




\begin{tabular}{|c|c|c|c|c|c|c|}
\hline REVIEWER & COMMENT * & COMMENT & HOLD POINT & DISPOSITION & STATUS & \\
\hline $\begin{array}{l}\text { Miska, C.R. } \\
\text { 90\% CDR }\end{array}$ & 38.1 & $\begin{array}{l}\text { Misc. It is strongly suggested that if the offgas piping } \\
\text { associated with the furnace are intolerant of rotational } \\
\text { misalignment that a feature be provided by the HCS } \\
\text { equipment designer to reestablish proper MCO rotational } \\
\text { position. This could be done by providing a "tumtable" in } \\
\text { the welding pit for occasional "off normal" use. Alternately } \\
\text { a turntable could be incorporated into the furnace. }\end{array}$ & no & $\begin{array}{l}\text { A turn-table is being developed for the top of the oven to properly } \\
\text { orient the MCO. }\end{array}$ & closed & \\
\hline $\begin{array}{l}\text { Miska, C.R. } \\
90 \% \text { CDR }\end{array}$ & 38.2 & $\begin{array}{l}\text { SK-2-300413 P\&IDThe RGA instrument for gas sampling } \\
\text { needs to measure both the supply and exhaust gas } \\
\text { concentration, potentially even before and after the gas } \\
\text { cooler and traps if we can get significant mass removal } \\
\text { (condensation). }\end{array}$ & no & (re RGA sampling points) We will comply with your suggestion. & closed & \\
\hline $\begin{array}{l}\text { Miska, C.R. } \\
90 \% \text { CDR }\end{array}$ & 39 & $\begin{array}{l}\text { SK-300413 P\&IDThe oxygen supply system needs some } \\
\text { more thought and work in the detaited design phase. The } \\
\text { story for pre dilution of the oxygen (buy at < } 20 \% \text { oxygen in } \\
\text { inert gas) add at limited rate (orlfice) to recirculating offgas, } \\
\text { with measurement to confirm that oxygen concentration } \\
\text { does not exceed maximum allowable concentration). }\end{array}$ & no & $\begin{array}{l}\text { The gas supply system will be revised to provide these monitoring } \\
\text { points. }\end{array}$ & DD & i \\
\hline $\begin{array}{l}\text { Miska, C.R. } \\
90 \% \text { CDR }\end{array}$ & 40 & $\begin{array}{l}\text { SK-300d } 13 \text { P\&IDThe design should reflect a high } \\
\text { reliability flammable gas monitor or similar device to } \\
\text { ensure that the offgas concentration is maintained low } \\
\text { enough to preclude combustion (easy to use / malntain } \\
\text { safety significant system). }\end{array}$ & no & Study in detailed design. & DD & 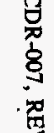 \\
\hline $\begin{array}{l}\text { O'Neill, C. } \\
90 \% \text { CDR }\end{array}$ & 1 & $\begin{array}{l}\text { The COR should include either a HVAC Zone drawing or a } \\
\text { descriplion of the zones. }\end{array}$ & no & $\begin{array}{l}\text { The interior of the MCO and connected process equipment will be } \\
\text { Zone 1. The process pit, trench, and process enclosure will be }\end{array}$ & closed & 10 \\
\hline & & & & $\begin{array}{l}\text { Zone } 2 \text { and everything else will be the same zone as the CSB. } \\
\text { The text will be modified to reflect this. }\end{array}$ & 1 & 1 \\
\hline $\begin{array}{l}\text { O'Neill, C. } \\
90 \% \text { CDR }\end{array}$ & 2 & $\begin{array}{l}\text { Section 2.9. Solid Waste. This section identifies "Getter } \\
\text { and Charcoal Beds" as being a solid waste. The design } \\
\text { does not include any of these items. }\end{array}$ & no & $\begin{array}{l}\text { Depending upon the final selection of the stack monitoring system, } \\
\text { these materials could be in it. }\end{array}$ & closed & , \\
\hline $\begin{array}{l}\text { O'Neill, C. } \\
90 \% \text { CDR }\end{array}$ & 3 & $\begin{array}{l}\text { Section } 2.9, \text { Solid Waste. The filters from VPS-F-1102 and } \\
\text { VPS-F-1107 are not listed here as waste. }\end{array}$ & no & $\begin{array}{l}\text { VPS-F-1102 has been eliminated and is part of VPS-PFR-1101. } \\
\text { This fitter as well as VPS-F-1107 will be listed in the solid waste } \\
\text { section. }\end{array}$ & closed & 1 \\
\hline $\begin{array}{l}\text { O'Neill, C. } \\
90 \% \text { CDR }\end{array}$ & 4 & $\begin{array}{l}\text { Section 3.3.2.2, stack description. The location of the } \\
\text { stack should be coordinaled with the CSB sub-project to } \\
\text { make sure there is no conflicts. }\end{array}$ & no & $\begin{array}{l}\text { The suggested coordination is planned with the HCSA and CSB } \\
\text { design teams. }\end{array}$ & closed & \\
\hline
\end{tabular}




\begin{tabular}{|c|c|c|c|c|c|}
\hline REVIEWER & COMMENT * & COMMENT & HOLD POINT & DISPOSITION & STATUS \\
\hline $\begin{array}{l}\text { O'Neill, C. } \\
90 \% \text { CDR }\end{array}$ & 5 & $\begin{array}{l}\text { Drawing SK-2-300413, Sh.2. Fillers VPS-F-1102 and VPS- } \\
F-1107 \text {, this type of fiter cannot withstand high pressures. } \\
\text { If the high pressure option that is mentioned in the CDR is } \\
\text { proposed than a different type of filter (metal) will have to } \\
\text { be used. }\end{array}$ & no & $\begin{array}{l}\text { These filters, Flanders G-1 can be procured in ASME rated } \\
\text { enclosures capable of withstanding } 100 \text { psi according to the } \\
\text { supplier we spoke to. That is one of the chief reasons these units } \\
\text { were specified. Subsequent changes to the design however have } \\
\text { removed the requirement for this high pressure and the filters will } \\
\text { be reevaluated for } 25 \text { psi service. }\end{array}$ & DD \\
\hline $\begin{array}{l}\text { O'Neill, C. } \\
90 \% \text { CDR }\end{array}$ & 6 & $\begin{array}{l}\text { Drawing SK-2-300413, sh.2. Fitter VPS-F-1102, this type } \\
\text { of filter cannot withstand high temperature. A metal filter } \\
\text { should be used in this location. }\end{array}$ & no & Filter VPS-F-1102 is now anlicipated to be a metal fitter. & closed \\
\hline $\begin{array}{l}\text { O'Nelli, C. } \\
90 \% \text { CDR }\end{array}$ & 7 & $\begin{array}{l}\text { Drawing SK-2-300421. The equipment shown on this } \\
\text { drawing does not match the equipment shown on SK-2- } \\
300413 \text {, sh.3. The layout of the equipment should include } \\
\text { the ductwork to make sure there is space for the } \\
\text { installation. }\end{array}$ & no & $\begin{array}{l}\text { Space in the area of the Service Module does not appear to be a } \\
\text { problem so the effort was not expended to show the ductwork } \\
\text { during conceptual design. The ductwork will be designed early in } \\
\text { the definitive design effort. }\end{array}$ & DD \\
\hline $\begin{array}{l}\text { Ruff, E.S. } \\
90 \% \text { CDR }\end{array}$ & 1 & $\begin{array}{l}\text { General Note 1: I would like to compliment Paul Smith, } \\
\text { Martin Muller, and Lauren Ames on their presentation. The } \\
\text { design review was conducted in a very thorough and } \\
\text { professional manner. They have generated a good } \\
\text { conceptual design study for the hot conditioning project. }\end{array}$ & no & Your compliment is certainly appreciated. & closed \\
\hline $\begin{array}{l}\text { Ruff, E.S. } \\
90 \% \text { CDR }\end{array}$ & 2 & $\begin{array}{l}\text { General Note 2: I don't have a copy of the conceptual } \\
\text { design document, so, I am unable to give page number } \\
\text { references for my comments. Comments in this RCR are } \\
\text { based on the oral presentation given in the design review } \\
\text { meeting. }\end{array}$ & no & Noted, no action required. & closed \\
\hline $\begin{array}{l}\text { Rutt, E.S. } \\
\text { 90\% CDR }\end{array}$ & 3 & $\begin{array}{l}\text { A significant amount of time is consumed during heat-up of } \\
\text { the MCO because internal differential temperatures are } \\
\text { limited to } 50 \text { deg. C.Suggestion: It would probably be } \\
\text { worth additional analysis time to try and develop a rational } \\
\text { for increasing the allowable temperature differential. }\end{array}$ & no & $\begin{array}{l}\text { Agreed, subsequent calculations have identified the } 100 \mathrm{C} \\
\text { temperalure requirements for all components of the MCO to be an } \\
\text { even more restrictive parameter. }\end{array}$ & closed \\
\hline $\begin{array}{l}\text { Ruff, E.S. } \\
\text { 90\% CDR }\end{array}$ & 4 & $\begin{array}{l}\text { I agree with a comment made during the meeting that } \\
\text { suggested the following: If possible, use a direct } \\
\text { refrigerant coil for cooling. not water heat exchangers. }\end{array}$ & no & $\begin{array}{l}\text { A water study that addresses this issue will be included as an } \\
\text { appendix in the CDR. }\end{array}$ & DD. \\
\hline $\begin{array}{l}\text { Ruff, E.S. } \\
\text { 90\% CDR }\end{array}$ & 5 & $\begin{array}{l}\text { Observation: In performing grinding operations to affect } \\
\text { weld repairs, you will also need a vacuum-type syslem to } \\
\text { clean up the grinding swart (dust). }\end{array}$ & no & $\begin{array}{l}\text { Good catch, the required functions fo the process enclosure will be } \\
\text { expanded to include cleanup of the grinding swarf. Perhaps a } \\
\text { vacuum tube that follows the grinder. }\end{array}$ & closed \\
\hline
\end{tabular}




\section{REVIEWER COMMENT * COMMENT}

Ruff, E.S.
90\% CDR $\quad \begin{aligned} & \text { Regarding MCO orientation for process connection } \\ & \text { operations:I believe that it is wishful thinking to expect all } \\ & \text { of the MCO vessels to maintain an unchanging rotational } \\ & \text { orientation from CSB to K-Basin, to Cold Vacuum Drying } \\ & \text { Facility, to CSB storage tube, to Hot Conditioning } \\ & \text { Furnace. Hence, some sort of rotational alignment } \\ & \text { capability for the MCO must be present in the Hot } \\ & \text { Conditioning Faclity. As mentioned in the meeting, this } \\ & \text { could be a lurntable with "high temperalure bearings" in the } \\ & \text { conditioning furnace. Atternatively, a "room temperature" } \\ & \text { turnlable could be placed in the spare furnace well. }\end{aligned}$

Ruff, E.S.
90\% COR $\quad \begin{aligned} & \text { Comments on Process Enclosure shielding:Observation 1: } \\ & \text { Based on the table of radiation exposures presented in the } \\ & \text { design document, most of the hot conditioning dose is } \\ & \text { laken by the operator in the Process Enclosure } \\ & \text { module.Observation 2: It was stated that the Process } \\ & \text { Enclosure was designed primarily as a "constraining } \\ & \text { device" to prevent the operator from getting direct radiation } \\ & \text { shine from the open end of the furnace well. To whit, the } \\ & \text { Process Enclosure structure is conceptually designed } \\ & \text { using 1" plate sleel, with no built-in shielding. }\end{aligned}$

Ruff, E.S. $\quad 7.2 \quad$ (Continued, No. 7 above.)l recommend that the Process

90\% CDR Enclosure front wall, front window, and floor be designed

using as much operator shielding as weightloed limits

allow. In addition, overhead shielding and back-wall

shielding for the Process Enclosure should also be added

to protect the operator from reflected radiation shine.

\begin{tabular}{|c|c|c|}
\hline $\begin{array}{l}\text { Smith, C.M. } \\
90 \% \text { CDR }\end{array}$ & 1 & Sec 1.0, Page 1 of 3 Add CVDS to acryonym list. \\
\hline $\begin{array}{l}\text { Smith, C.M. } \\
90 \% \text { CDR }\end{array}$ & 2 & $\begin{array}{l}\text { Sec 1.0, Page } 1 \text { of } 3 \text { How does processing the SNF Into } \\
\text { MCO's fit into the final disposition of this materiar? What } \\
\text { the overall milestone/decision that this processing } \\
\text { supports? }\end{array}$ \\
\hline $\begin{array}{l}\text { Smith, C.M. } \\
90 \% \text { CDR }\end{array}$ & 3 & $\begin{array}{l}\text { Sec 2.0, Page } 12 \text { of } 57 \text { \$2.2.1.2.2 To further heat } \\
\text { transfertheat-up, or cool down have you considered pre- } \\
\text { heating the Helium purge gas going into the MCO? } \\
\text { WhyMhy not? }\end{array}$ \\
\hline
\end{tabular}

\section{HOLD POINT DISPOSITION}

STATUS

no

The oven top is being revised to include a tapered roller bearing

closed plate that will allow rotational orientation of the MCO. 


\begin{tabular}{|c|c|c|c|c|c|}
\hline $\begin{array}{l}\text { Smith, C.M. } \\
90 \% \text { CDR }\end{array}$ & 4 & $\begin{array}{l}\text { Appendix H Need Table of Contents listing the vendor } \\
\text { cut sheets for somewhat easier reference. Note: The } \\
\text { scroll pump looks like an excellent choice for a vacuum } \\
\text { pump. Varian is reputable. }\end{array}$ & no & $\begin{array}{l}\text { An index to the vendor cut sheets will be provided and the cut } \\
\text { sheets will be organized alphabetically by the equipment plece } \\
\text { number. }\end{array}$ & closed \\
\hline
\end{tabular}

\begin{tabular}{|c|c|c|c|c|c|c|}
\hline $\begin{array}{l}\text { Smith, C.M. } \\
90 \% \text { CDR }\end{array}$ & 5 & $\begin{array}{l}\text { Sec } 2.0, \text { Page 14, } 21,25 \\
\text { Supplement supplied? }\end{array}$ & Where are the figures? & no & $\begin{array}{l}\text { The figures were inadvertently left out of the } 90 \% \text { design package. } \\
\text { They were transmitted following the meeting and will be included in } \\
\text { the final CDR. }\end{array}$ & closed \\
\hline
\end{tabular}

Smith, C.M.
$90 \%$ CDR $\quad \begin{aligned} & \text { Sec 2.2.3 Page } 20 \text { of } 57 \text { Has any energy recovery been } \\ & \text { considered such as using counterflow heat exchanges to } \\ & \text { preheat gas going to the heaters from another MCO that is } \\ & \text { being cooled? }\end{aligned}$

Each MCO stands alone. While this idea would certainly conserve

energy it has not been implemented for two reasons: (1) MCO cycle times will be unpredictable so there is no assurance that
another will be in the proper point of its process sequence to take advantage of energy savings and (2) the cost of the complex valving and ducting would exceed the savings in energy that would result. Another concern would be cross contamination should one MCO system have a leakage problem.

\begin{tabular}{|c|c|c|}
\hline $\begin{array}{l}\text { Smith, C.M. } \\
90 \% \text { CDR }\end{array}$ & 7 & $\begin{array}{l}\text { Sec 2.2.4 Page } 24 \text { of } 57 \text { This sequence of operation } \\
\text { does not reference any of the SK-2-300XXX drawings and } \\
\text { should! The process (written) is very difficult to follow } \\
\text { wilhout reference to the sketches. Add "See SK-2-300413, } \\
\text { sheet, Appendix A." }\end{array}$ \\
\hline
\end{tabular}

Meferences to the P\&iDs whl be included in the text.

no

References to the P\&IDs will be included in the text.

closed sheet, Appendix A."

\begin{tabular}{|c|c|c|c|c|c|}
\hline $\begin{array}{l}\text { Smith, C.M. } \\
90 \% \text { CDR }\end{array}$ & 8 & $\begin{array}{l}\text { Sec 2.2.4, Page } 26 \text { of } 57 \text { paragraph \#13 Another major } \\
\text { process after welding is the inspection of the weld. }\end{array}$ & no & Weld inspection will be added. & closed \\
\hline $\begin{array}{l}\text { Smith, C.M. } \\
90 \% \text { CDR }\end{array}$ & 9 & $\begin{array}{l}\text { Sec 2.3.3 Page } 30 \text { of } 57 \text { Variable speed fan divers are } \\
\text { used for the varying flow rates needed for processing a } \\
\text { range of cells at one time. Good design! }\end{array}$ & no & Compliment appreciated, no action required. & closed \\
\hline $\begin{array}{l}\text { Smith, C.M. } \\
90 \% \text { CDR }\end{array}$ & 10 & $\begin{array}{l}\text { Sec } 2.5 \text { Page } 35 \text { of } 57 \text { (last paragraph) A varlety of } \\
\text { welding processes shoud be explored. Perhaps the robot } \\
\text { can do the welding. If this is chosen, extra care in } \\
\text { selecting a robot and controller/computer that is compatible } \\
\text { with the electrical and RF spikes that are produced during } \\
\text { welding processes is needed. Some robotic systems } \\
\text { (ASEA) are designed specifically for welding operations. } \\
\text { However, here there is a size constraint. }\end{array}$ & no & $\begin{array}{l}\text { A welder has been proposed by WMC for this purpose, see Section } \\
H \text { for the vendor cut sheet. The specific design will be selected } \\
\text { during definitlve design after the MCO design has been finalized. }\end{array}$ & DD \\
\hline $\begin{array}{l}\text { Smith, C.M. } \\
90 \% \text { CDR }\end{array}$ & 11 & $\begin{array}{l}\text { Sec } 2.7 .1 \text { Page } 41 \text { of } 57 \text { Chilled water system. Does } \\
\text { the process actually use a glycole mixture that can be } \\
\text { chilled below freezing? Will that be needed to achleve the } \\
\text { final cool-down cycle? }\end{array}$ & no & $\begin{array}{l}\text { An unspecified refrigerent is proposed for the chilled water system. } \\
\text { A closed loop water system is proposed for inside the HCSA. A } \\
\text { water vs air cooling study will be included in the CDR. }\end{array}$ & $\mathrm{DD}$ \\
\hline
\end{tabular}




\begin{tabular}{|c|c|c|c|c|c|}
\hline $\begin{array}{l}\text { Smith, C.M. } \\
90 \% \text { CDR }\end{array}$ & 12 & $\begin{array}{l}\text { Appendix H Vendor Cut Sheets) Also SK2-2-300420 } \\
\text { Heat exchanges, pumps, heaters, chillers, filters etc., but } \\
\text { not much for the process enclosure. Information needed } \\
\text { on the manipulator, detectors, and other bought-out-parts } \\
\text { (BOP). (wheel drivers etc). }\end{array}$ & no & $\begin{array}{l}\text { Cut sheets for the maniputator and weider have been included in } \\
\text { the CDR. No wheel drivers are proposed, a motorized hand cart } \\
\text { will be used to push/pull the enclosure into position. }\end{array}$ & closed \\
\hline $\begin{array}{l}\text { Smith, C.M. } \\
90 \% \text { CDR }\end{array}$ & 13 & $\begin{array}{l}\text { Page } 52 \text { of } 57 \text { (Function F1) I would suggest rails for the } \\
X \text {-direction and some small amount of Y-positioning within } \\
\text { the enclosure. Standard train rails and wheels could } \\
\text { handle the large load and do not require custom } \\
\text { fabrication. (Advantage for transporting to the facility on } \\
\text { train rails ???) }\end{array}$ & no & $\begin{array}{l}\text { One requirement from the MHM subproject is that there be no } \\
\text { projections above floor level. Sunken rails are not desirable since } \\
\text { they would have a greater impact on the HCSA design and could } \\
\text { be contamination traps. }\end{array}$ & closed \\
\hline $\begin{array}{l}\text { Smith, C.M. } \\
90 \% \text { CDR }\end{array}$ & 14 & $\begin{array}{l}\text { Page 52, F1 At the end of travel for stowage either a } \\
\text { turntable could be used of a platform that supports the ralis } \\
\text { and moves cross-ways on another set of rails (low profile). } \\
\text { With the amount of utilities required at the process } \\
\text { enclosure on overhead utility should probably be supplied. }\end{array}$ & no & $\begin{array}{l}\text { Subsequent to the } 90 \% \text { design presentation the MHM design team } \\
\text { raised the box beam to } 9 \text { feet so it can now pass over the process } \\
\text { enclosure which will be lowered to less than } 9 \text { feet. The need for } \\
\text { the shuttle table is gone. }\end{array}$ & closed \\
\hline $\begin{array}{l}\text { Smith, C.M. } \\
90 \% \text { CDR }\end{array}$ & 15 & $\begin{array}{l}\text { Page 52, F2 The whole enclosure doesn't need to lift, } \\
\text { only a skirt is necessary if at all. Perhaps the seal could } \\
\text { drag on the floor. }\end{array}$ & no & $\begin{array}{l}\text { This suggestion will be incorported into the design of the process } \\
\text { enclosure during definitive design. Currently an inflatable seal is } \\
\text { proposed so contact with the floor during transportation won't be } \\
\text { necessary. Absence of contarnination on the interior of the } \\
\text { enclosure will be verified before the floor seal is broken. }\end{array}$ & $\mathrm{DD}$ \\
\hline $\begin{array}{l}\text { Smith, C.M. } \\
90 \% \text { CDR }\end{array}$ & 16 & $\begin{array}{l}\text { Page 52, F3 It was suggested at the design review that } \\
\text { a hydraulic actuator for the pit cover could be installed into } \\
\text { the floor. That would be great. I suggest that you look } \\
\text { carefully at utilizing a } 4 \text {-bar mechanism type of hing for this } \\
\text { cover to minimize the swing spare required inside the } \\
\text { process enclosure. Note in the figure beside that the lid } \\
\text { top swings from posittons } 1-6 \text { to open. ( a drawing, not } \\
\text { included here, was attached to this comment). }\end{array}$ & no & $\begin{array}{l}\text { This concept will be evaluated further in definitive design, it } \\
\text { certainly shows promise. }\end{array}$ & $\mathrm{DD}$ \\
\hline $\begin{array}{l}\text { Smith, C.M. } \\
90 \% \text { CDR }\end{array}$ & 17 & $\begin{array}{l}\text { Page 53, F6 Having stereo vision on the manipulator } \\
\text { near the tool interchange plate is probably more } \\
\text { advantageous than a force feedback system. Though the } \\
\text { operator can usually view directly and look at the overview } \\
\text { monitor, the additional view (especially in stereo) will } \\
\text { provide very meaningful assistance. If a force feedback } \\
\text { system is implemented, it should reflect the world or tool } \\
\text { orientation force reflections rather than the Individual joint } \\
\text { forces. Joint forces are meaningless to a remote operatorl }\end{array}$ & no & $\begin{array}{l}\text { Your comments are appreciated and will passed on to the definitive } \\
\text { design team who will be dealing with the details of the manipulator } \\
\text { system. }\end{array}$ & DD \\
\hline
\end{tabular}




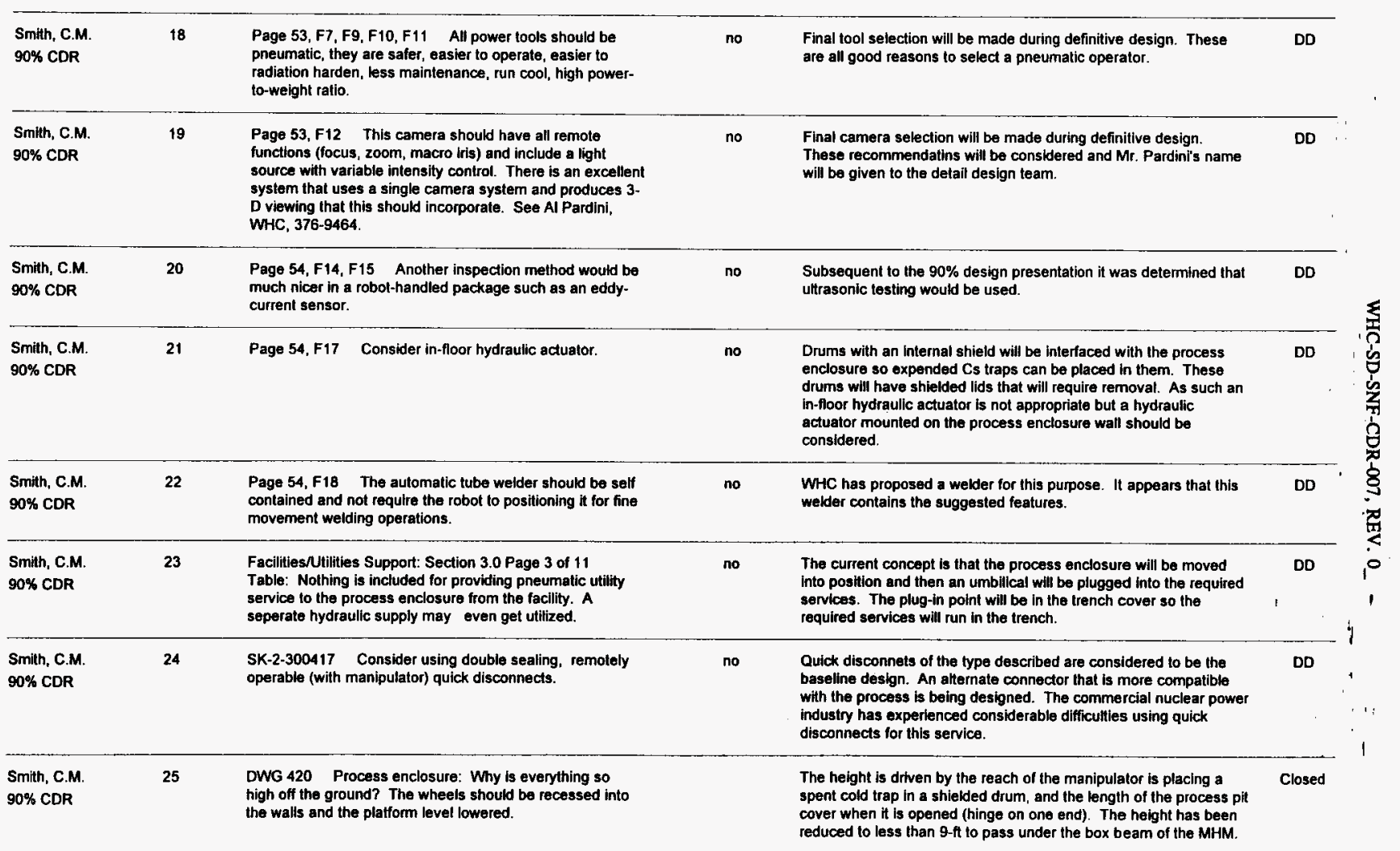




\begin{tabular}{|c|c|c|c|c|c|}
\hline $\begin{array}{l}\text { Smith, D. } \\
\text { 90\% CDR }\end{array}$ & 1 & $\begin{array}{l}\text { Introduction page } 1 \text {. The last paragraph states that the } \\
\text { process will be designated as a Hazard Category } 2 \\
\text { system. Hazard Categories are specified for facilities, not } \\
\text { for systems. DOE-STD-1027 allows for facility } \\
\text { segmentation in determining Hazard Category but the } \\
\text { HCSA does not meet the criteria for being designated } \\
\text { seperately from the CSB. This report should note that the } \\
\text { HCSA is part of the CSB and the CSB is HC-2. A final } \\
\text { hazard category for the CSB will need to consider the } \\
\text { impacts of HCS accidents. }\end{array}$ & no & The introduction will be revised to reflect this comment. & closed \\
\hline $\begin{array}{l}\text { Smith, D. } \\
\text { 9o\% CDR }\end{array}$ & 2 & $\begin{array}{l}\text { Appendix E. The functions and requirements should } \\
\text { identify the acceptance criteria for the SNF. The } \\
\text { confinement function of the HCS should be better defined. } \\
\text { For example, the allowable leak rate at the design } \\
\text { pressure should be identified since the design does not } \\
\text { provide active confinement during accident conditions. } \\
\text { The ventilation flow needs to be specified based upon } \\
\text { routine and upset conditions during the HCS process. The } \\
\text { design pressure and temperature for the confinement } \\
\text { needs to be specified based on potential accidents (to be } \\
\text { addressed in the PSE). The design needs to provide the } \\
\text { capability to periodically lest the confinement function and } \\
\text { replace seals when required. The HEPA fitters need to be } \\
\text { designed for accident temperatures and pressures. }\end{array}$ & no & $\begin{array}{l}\text { Appendix E only addresses the performance specification, the } \\
\text { functions and requirements were not available until loo late in the } \\
\text { CDR development phase to include and evaluation of them in the } \\
\text { CDR. The F\&R evaluation report will be written early in the } \\
\text { definitive design phase and these commenls will be incorporated } \\
\text { into it. }\end{array}$ & DD \\
\hline $\begin{array}{l}\text { Smith, D. } \\
90 \% \text { CDR }\end{array}$ & 3 & $\begin{array}{l}\text { Appendix } E \text {. WHC-CM-4-46 should be specified to } \\
\text { identify the design requirements for safety class and } \\
\text { significant items. Also it appears strange to have } \\
\text { appendices in an appendix (Appendices A, B, and C to } \\
\text { appendix E). }\end{array}$ & no & $\begin{array}{l}\text { We will revisa the final CDR to incorporate your first comment. For } \\
\text { the second comment, the performance specification report was } \\
\text { written as a standalone document and then Incorporated Into the } \\
\text { CDR thus leading to the appendix in an appendix dilema. The } \\
\text { Appendix E attachments are now referred to as addendums. }\end{array}$ & closed \\
\hline $\begin{array}{l}\text { Zaman, A.A. } \\
60 \% \text { CDR }\end{array}$ & 1 & $\begin{array}{l}\text { Section 2.0: "Cool Down Process Connection." There will } \\
\text { be some free water left over from the CVC process. } \\
\text { Speclfy or estimate the amount and list whether this free } \\
\text { waler demands consideration for further action } \\
\text { (conditioning) in the Hot Conditioning Facility. }\end{array}$ & no & $\begin{array}{l}\text { It would be expected that only a small amount of free water would } \\
\text { remain after cold vacuum drying. however, it would not be } \\
\text { improbable that several MCO's would contain a greater tolal water } \\
\text { inventory after cold vacuum drying than is allowed by the Hot } \\
\text { Conditioning Product criteria ( } 1.67 \mathrm{Kg} \text { per WHC-SD-SNF-OCD- } \\
\text { o01). It should be noted that this question is really "what is the } \\
\text { Cold Vacuum Drying product criteria." This question should be } \\
\text { directed to the CVD project. (They are preparing a CVD product } \\
\text { criteria document.) }\end{array}$ & closed \\
\hline
\end{tabular}




\begin{tabular}{|c|c|c|}
\hline $\begin{array}{l}\text { Zaman, A.A. } \\
60 \% \text { CDR }\end{array}$ & 2 & $\begin{array}{l}\text { Section 2.0: Some of the temperatures of the defined } \\
\text { conceptual processes are potentially close to the ignition } \\
\text { point for pyrophoric materials in fuels (eg U-hydrides). } \\
\text { Data on ignition point and process temperature selection } \\
\text { based on the minimum risk for ignition needs to be } \\
\text { established and justified. }\end{array}$ \\
\hline
\end{tabular}

no

This is not an equipment conceptual design question, this is a question pertaining to the overall SNF process strategy selection. Please refer to the IPS training strategy document "Integrated Process strategy for K Basin Spent Nuclear Fuel", WHC-SD-SNFSP-005, to obtain this type of background information.

Zaman, A.A.
60\% CDR

$60 \%$ CDR need to be explored due to the recent study (TH-021) on Hydrogen detonation.

no

The strategy to preclude Hydrogen burn events provided in the Hot Conditioning Performance Specification, being implemented by the design is to provide a system design that will ensure both an inert environment in the MCO, and dllute hydrogen concentrations to less than $2 \%(<1 / 2$ of the lower flammable limit in air.) This is a very conservative approach and should keep the probability of hydrogen ignition very low.

Zaman, A.A.
$60 \%$ CDR

4

Section 5.0: A - The requirements for participation of the Spend Nuclear Fuels Safety (Independent) need to be laic out. This is in view of the requirements in DOE $\mathbf{5 4 8 0 . 5}$ (contractual obligation), DOE 4700 and SNF Safety Management Plan. no

This is not a valid comment agatnst the equipment conceptual design. This is a Hot Conditioning Project Management documen question. If after reviewing this document questions still remaln, you should contact Rick Bradshaw for further clarification.
Section 5.0: B - The DOE Order lists ASMEIANSI B31.3

Zaman, A.A.

$60 \%$ CDR
5 under the "Reference Standard and Guides."
Page 8 of the Performance Specification Report, DOE 6430 A General Design Crtteria, does idicate that ASME B31.1 Chemical Plant and Petroleum Refinery Piping standard and the Industrial Vacuum Practices are considered to be supplemental standards that will be used for the HVCE design.

\begin{tabular}{|c|c|c|c|c|c|}
\hline $\begin{array}{l}\text { Zaman, A.A. } \\
60 \% \text { CDR }\end{array}$ & 6 & $\begin{array}{l}\text { Section 5.0: B - There are additional divisions/sections that } \\
\text { are important for design consideration of the Hot } \\
\text { Conditioning Facilities. Example, 0110-6.1 Fire } \\
\text { Protection. 0110-99.0, 1319, etc. Add these sections. } \\
\text { Add generic statement regarding other than listed } \\
\text { reference. }\end{array}$ & no & $\begin{array}{l}\text { The criterla evalualon document developed for the Hot Vacuum } \\
\text { Conditioning equipment goes into the applicability of the general } \\
\text { design crlterla in more extensive delall. This document will be } \\
\text { Included in the final CDR. }\end{array}$ & closed \\
\hline $\begin{array}{l}\text { Zaman, A.A. } \\
60 \% \text { CDR }\end{array}$ & 7 & $\begin{array}{l}\text { The most important manual is SHC-CM-4-46. It provides } \\
\text { guidance on safety analysis (hazards, acident, risk, etc) } \\
\text { and safety classification. }\end{array}$ & no & $\begin{array}{l}\text { Further review of the HCS performance specification will be done } \\
\text { during the detailed design and this potential reference requlrement } \\
\text { will be discussed with the HCS design authority to determine } \\
\text { whether it is applicable to the HCS equipment design. }\end{array}$ & closed \\
\hline $\begin{array}{l}\text { Zaman, A.A. } \\
60 \% \text { CDR }\end{array}$ & 8 & Consider reference to DOE $5480.24,5480.31,5480.22$, etc. & no & See response to comment $\# 7$ above. & closed \\
\hline $\begin{array}{l}\text { Zaman, A.A. } \\
60 \% \text { CDR }\end{array}$ & $\mathbf{9}$ & $\begin{array}{l}\text { Does this facility need to be designed and operaled to } \\
\text { NRC equivalency? }\end{array}$ & no & $\begin{array}{l}\text { This is the equipment conceptual design, not the facility design. } \\
\text { Please refer facility design questions to the facility designer (Fluor). }\end{array}$ & closed \\
\hline
\end{tabular}




\begin{tabular}{|c|c|c|c|c|c|}
\hline $\begin{array}{l}\text { Zaman, A.A. } \\
60 \% \text { CDR }\end{array}$ & 10 & $\begin{array}{l}\text { Depending on the Safety Classification of the Hot } \\
\text { Conditioning Facilities, the configuration in drawings may } \\
\text { change (eg SC1 will require redundant and single failure } \\
\text { proof power supplies/utilities for safe shut down.) }\end{array}$ & no & Comment noted. No reply needed. & closed \\
\hline $\begin{array}{l}\text { Zaman, A.A. } \\
90 \% \text { CDR }\end{array}$ & 1 & $\begin{array}{l}\text { The Preliminary Safety analysis/evaluation needs to be } \\
\text { performed to ldentify the safely class and safety significant } \\
\text { structure, systems and components per DOE } 5480.23 \\
\text { (section 9.0) DOE } 6430.1 A(110-5.2) \text { and WHC-CM-4-46. } \\
\text { The information in the drawing needs to be checked } \\
\text { against the accident mitigation function specified In the } \\
\text { safety analysis. This could not be done. }\end{array}$ & no & $\begin{array}{l}\text { A PSAR is being developed in parallel with the CDR. No safety } \\
\text { class systems have been idenlified. The design leam will continue } \\
\text { to work with the safety team to define mitigation measures. }\end{array}$ & closed \\
\hline $\begin{array}{l}\text { Zaman, A.A. } \\
90 \% \text { CDR }\end{array}$ & 2 & $\begin{array}{l}\text { a) We recommend that the } 90 \% \text { design report should add } \\
\text { discussion on the NRC equivalency issue and impact on } \\
\text { the process and facilities design. } \\
\text { b) Has the evaluation (by NEC experts) been done to see } \\
\text { where the identification and application of NFPA Article } \\
504 \text { ("Intrinsically Safe" systems) apply? }\end{array}$ & no & $\begin{array}{l}\text { NRC equivalency is addressed in the criteria evaluation that wil be } \\
\text { in the final CDR. NFPA evaluation will be conducted during } \\
\text { definitive design. }\end{array}$ & DD \\
\hline $\begin{array}{l}\text { Zaman, A.A. } \\
90 \% \text { CDR }\end{array}$ & 3 & $\begin{array}{l}\text { The discussion in the text/report is based on the nomnal } \\
\text { operations. Need to cover off-nomal, accident and design } \\
\text { basis accident. }\end{array}$ & no & $\begin{array}{l}\text { These operations are addressed by the PHA which is being } \\
\text { developed by WHC. Results of the hazards anatysts qre being } \\
\text { incorporated into the design. }\end{array}$ & Ċūsè̃ \\
\hline $\begin{array}{l}\text { Zaman, A.A. } \\
\text { 90\% CDR }\end{array}$ & 4 & $\begin{array}{l}\text { Introduction, p 1, pgph 2: The conditions of high pH in } \\
\text { (borated water) and high temperature may produce } \\
\text { corrosion of the stainless steel. What is the potential } \\
\text { generation of hydrogen gas by this process? Refer to } \\
\text { NRC notice } 96-34 \text {. (Need a copy? Call me at (509) 376- } \\
\text { 1692.) }\end{array}$ & no & $\begin{array}{l}\text { When the MCO reaches the HCSE process only chemically bound } \\
\text { water (no free water) will be present, so corrosion of the SST is not } \\
\text { anticipated to be a problem. Hydrogen gas will be generated when } \\
\text { the uranlum and zirconium hydrides decompose. These will be } \\
\text { removed before a flammable concentration is reached. The } \\
\text { process gases will be continuously monitored for hydrogen. }\end{array}$ & closed \\
\hline $\begin{array}{l}\text { Zaman, A.A. } \\
\text { 90\% CDR }\end{array}$ & 5 & $\begin{array}{l}\text { Introduction, p 3, pgph 1: Hot conditioning facility will need } \\
\text { to work with the specified design life of } 6 \text { yr for the CSB- } \\
\text { MHM. Suggest clarification. }\end{array}$ & no & $\begin{array}{l}\text { The hot conditioning program is scheduled to be complete in two } \\
\text { years. }\end{array}$ & closed \\
\hline $\begin{array}{l}\text { Zaman, A.A. } \\
90 \% \text { CDR }\end{array}$ & 6 & $\begin{array}{l}\text { Section 2.1, pgph 4: The CSB MHM is designed to }-27 \mathrm{~F} \text { to } \\
115 \mathrm{~F} \text { ambient temperature. The interfaces with the hot } \\
\text { conditioning oven need to be identified and ensured } \\
\text { compliance. }\end{array}$ & no & $\begin{array}{l}\text { Maximum MCO storage tube temperatures are expected to be } \\
\text { about } 150 \text { degrees-C. which is the temperature of the MCO's when } \\
\text { they are removed from the oven. the MHM design conditions are } \\
\text { being re-evaluated by the MHM design team to assure } \\
\text { compatibility. }\end{array}$ & closed \\
\hline
\end{tabular}




\begin{tabular}{|c|c|c|c|c|c|}
\hline REVIEWER & COMMENT * & COMMENT & HOLD POINT & DISPOSITION & STATUS \\
\hline $\begin{array}{l}\text { Zaman, A.A. } \\
90 \% \text { CDR }\end{array}$ & 7 & $\begin{array}{l}\text { Section } 2.0 \text { Process, ID } 47.0 \text { : Visual inspection of a weld } \\
\text { in a safely class MCO boundary may not be acceptable. } \\
\text { ASME sec. III and applicable V and IX may need to be } \\
\text { researched to determine the minimum requirements } \\
\text { (radiography, ultrasonic, etc.) }\end{array}$ & no & $\begin{array}{l}\text { This will be revised to reflect ultrasonic weld testing rather than dye } \\
\text { penetrant. }\end{array}$ & closed \\
\hline $\begin{array}{l}\text { Zaman, A.A. } \\
\text { 90\% CDR }\end{array}$ & 8 & $\begin{array}{l}\text { Section } 2.2 .1 .2 .1 \text { : The Helium blower VPS-BLO-1106 is } \\
\text { identified as "spark proof". The right terminology from } \\
\text { NEC (NFPA 70, article 504) is "intrinsically safe" and may } \\
\text { go beyond the blower itself to its electrical/grounding/piping } \\
\text { systems. If intent is to prevent hydrogen tgnition } \\
\text { ("classified hazardous location"), correct the terminology. }\end{array}$ & no & The text has been revised. & closed \\
\hline $\begin{array}{l}\text { Zaman, A.A. } \\
90 \% \text { CDR }\end{array}$ & 9 & $\begin{array}{l}\text { Section } 2.2 .1 .2 .3 \text { : Since the MCO is designed to } 150 \text { psig. } \\
\text { the pressure rating of the system (specifically components } \\
\text { close to the MCO) be safe at that rating. The text calls out } \\
100 \text { psi ("a" or " } \mathrm{g} \text { "). }\end{array}$ & no & $\begin{array}{l}\text { Although the MCO is designed for } 150 \mathrm{psig} \text {, it is configured with a } \\
\text { ruplure disc with a } 25 \text { psig rating. This rating sets the design } \\
\text { pressure of the components withtn the system. The HCSE system } \\
\text { will also include rupture discs. }\end{array}$ & closed \\
\hline $\begin{array}{l}\text { Zaman, A.A. } \\
90 \% \text { CDR }\end{array}$ & 10 & $\begin{array}{l}\text { Section 2.2.1.2.4: a) If the PLC are relied on the sutomatic } \\
\text { shut-down for the design basis accident (impacting off-site } \\
\text { public) then their qualification requirements are going to be } \\
\text { stringent (IEEE } 323,344 \text {, etc) and costly. This needs to be } \\
\text { addressed clearly here. }\end{array}$ & no & $\begin{array}{l}\text { The interlocks prevent subsequent actions from taking place if the } \\
\text { interlock logic is not satisfied. This is not automatic shutdown. } \\
\text { The PLC may require operator interventlon if all interlocks or } \\
\text { sequences have not been satisfied. The system will shut down in } \\
\text { a passively sale configuration. }\end{array}$ & closed. \\
\hline $\begin{array}{l}\text { Zaman, A.A. } \\
90 \% \text { COR }\end{array}$ & 11 & $\begin{array}{l}\text { Section 2.2.2.3.1: Was the induction electrical heating } \\
\text { considered for the process? }\end{array}$ & no & $\begin{array}{l}\text { Induction heating was considered but abandoned in favor of } \\
\text { convective heating as the convectlve heating system can be used } \\
\text { for both heating and cooling. Induction heating would not be able } \\
\text { to assure that the MCO would not heat up faster than } 50 \text { degrees- } \\
\text { Chr or that all parts of the MCO would be within } 100 \text { degrees-C of } \\
\text { the same temperature. }\end{array}$ & closed \\
\hline $\begin{array}{l}\text { Zaman, A.A. } \\
\text { 90\% CDR }\end{array}$ & 12 & $\begin{array}{l}\text { SK-2-300412: The pump VPS-VAC-1104 needs to be } \\
\text { slected such that operator or maintenance error does not } \\
\text { help reverse the flow and pressurize the MCO. } \\
\text { Hazards/Accident assessment wilt need to adress such } \\
\text { accident and mitigation features. }\end{array}$ & no & $\begin{array}{l}\text { This particular vacuum pump, of scroll design, will not pump gas if } \\
\text { operated backwards. It is also connected to a single phase power } \\
\text { source which will not reverse if connected incorrectly. }\end{array}$ & closed \\
\hline $\begin{array}{l}\text { Zaman, A.A. } \\
90 \% \text { CDR }\end{array}$ & 13 & $\begin{array}{l}\text { Section 2.2.2.3.2: Missing content? If so, please provide } \\
\text { write-up. }\end{array}$ & no & Inclusion of this section was not intended. Text will be corrected. & closed \\
\hline $\begin{array}{l}\text { Zaman, A.A. } \\
90 \% \text { CDR }\end{array}$ & 14 & $\begin{array}{l}\text { Section 2.2.3.1.3.1: The water cooling system may need } \\
\text { detection device for radioactivity that are polentially } \\
\text { available from accumulation over time from MCO } \\
\text { surface/leakage, etc. }\end{array}$ & no & $\begin{array}{l}\text { There is an air barrier between the water system and the MCO. } \\
\text { thus no contamination or buildup over time is expected. }\end{array}$ & closed \\
\hline
\end{tabular}




\begin{tabular}{|c|c|c|c|c|c|}
\hline REVIEWER & COMMENT * & COMMENT & HOLD POINT & DISPOSITION & STATUS \\
\hline $\begin{array}{l}\text { Zaman, A.A. } \\
90 \% \text { CDR }\end{array}$ & 15 & $\begin{array}{l}\text { Section 2.2.4, item 3: Assign identification number to the } \\
\text { block valve. }\end{array}$ & no & $\begin{array}{l}\text { Assignment of valve ID numbers will be done at the definitative } \\
\text { design stage. }\end{array}$ & DD \\
\hline $\begin{array}{l}\text { Zaman, A.A } \\
\text { 90\% CDR }\end{array}$ & 16 & $\begin{array}{l}\text { Section 2.3.1: The codes need to be iffentified along with } \\
\text { date/revision, eg ASME AG-1, ASME N509, etc. }\end{array}$ & no & $\begin{array}{l}\text { The exact standards that apply will be specified during definitive } \\
\text { design. }\end{array}$ & DD \\
\hline $\begin{array}{l}\text { Zaman, A.A. } \\
90 \% \text { CDR }\end{array}$ & 17 & $\begin{array}{l}\text { Section 2.7.4: The air recelver if supporting a safety class } \\
\text { of safety signiticant function may need to have capacity } \\
\text { and classification thereto. }\end{array}$ & no & $\begin{array}{l}\text { This receiver is a utility and not meant to support a safety class } \\
\text { function. It will be U stamped to assure design and construction in } \\
\text { accordance with ASME Section 8, Dhision } 1 \text {. }\end{array}$ & closed \\
\hline $\begin{array}{l}\text { Zaman, AA. } \\
\text { 90\% CDR }\end{array}$ & 18 & $\begin{array}{l}\text { Section 2.9: The Facility has the responsiblity to } \\
\text { characterize their waste before shipment to storage (ie } \\
\text { burial ground, WRAP, etc) This function is missing from } \\
\text { the document. }\end{array}$ & no & $\begin{array}{l}\text { Characterization of the waste will be done in the waste storage } \\
\text { room. The text will be revised to reflect this function. }\end{array}$ & closed \\
\hline $\begin{array}{l}\text { Zaman, A.A. } \\
90 \% \text { COR }\end{array}$ & 19 & $\begin{array}{l}\text { Section 4.0, Interface \#MHM3: Current direction is to allow } \\
\text { a mazimum clearance of about seven feet between the } \\
\text { floor and the MHM superstructure. Thsi won't allow the 11'- } \\
9^{\prime \prime} \text { Process Enclosure (SK2-2-300420) to passunder the } \\
\text { MHiM. (Contact Gerry Bazinet of CSB for current } \\
\text { informalion.) }\end{array}$ & no & $\begin{array}{l}\text { The configuration shown in the } 90 \% \text { CDR anticipated that the } \\
\text { enclosure would be moved out of the path of the MHM rather than } \\
\text { pass under it. Subsequently it has been delermined that the MHM } \\
\text { box beam will be raised to } 9 \text { ft and the process enclosure height } \\
\text { will be reduced to } 8 \text { ft } 6 \text { in. }\end{array}$ & closed \\
\hline $\begin{array}{l}\text { Zaman, A.A. } \\
90 \% \text { CDR }\end{array}$ & 20 & $\begin{array}{l}\text { SK-2-300413, sheet 2: To keep the MCO isolated, a } \\
\text { HEPA filter may be needed between the block valve and } \\
\text { the device VPS-PFR-1101. }\end{array}$ & no & Drawing will be revised to incorporate the fitter as recommended. & closed \\
\hline
\end{tabular}




\section{Project 2318: Hot Conditioning CDR Comments}

WHC-SD-SNF-CDR-007 Rev 0

\begin{tabular}{|c|c|c|c|}
\hline NAME & PHONE & ORGIGROUP & LOCATION \\
\hline Alaconis, W.C. & (509) $376-9390$ & SNF Operations & MO-285 \\
\hline Burns, B.M. & & WHC & WHC 78 \\
\hline Cowan, R.G. & & WHC 335 & \\
\hline Goldman, L. & (509) 373-6371 & Process Engineering & MO-285 \\
\hline Kurtz, J. & & & \\
\hline Lucas, C. & (509) 373-1006 & OPS Analysis/ Waste Handling & $M O-102$ \\
\hline Merkling. $\mathrm{T}$. & (509) 373-9412 & Nuclear Safety & 2751E \\
\hline Miska, C.R. & (509) $376-7103$ & SNF Engineering & $\mathrm{MO}-285$ \\
\hline O'Neill, C. & (509) 373-7642 & ICF Kaiser & 2261 STEVENS \\
\hline Ruff, E.S. & (509) 376-1943 & Mech Eng ICFKH & H5-70 \\
\hline Smith, C.M. & (509) 375-3915 & PNNL & 2400 STVCN \\
\hline Smith, D. & (509) $372-3623$ & SAR Engineering & 2751E/C-101 \\
\hline Zaman, A.A. & (509) $376-1692$ & & $B 1-10$ \\
\hline
\end{tabular}


WHC-SD-SNF-CDR-007, Revision 0

\section{APPENDICES}

\section{APPENDIX L}

\section{USE OF COOLING WATER IN THE HOT CONDITIONING SYSTEM}




\author{
USE OF COOLING WATER \\ IN THE HOT CONDITIONING SYSTEM
}

\title{
1.0 STATEMENT OF PROBLEM
}

The proposed process for removing chemically bound water and hydrides from the SNF in the MCOs following the cold vacuum drying process involves heating the SNF to temperatures of 300 to $350^{\circ} \mathrm{C}$ and drawing a vacuum. This heating is followed by cooling the SNF back to $150^{\circ} \mathrm{C}$ for oxidation of exposed uranium surfaces. The process involves cooling in the following process streams.

\section{A. Helium Recirculating Through MCO}

During both the heatup and cool down phases, helium is continuously recirculated through the MCO to: a) enhance the heat transfer rate; b) allow monitoring of the heatup/cool down rate; and c) allow monitoring of the released gases. Two cooling steps are proposed for this stream.

1. Cold Trap - The released gases could include cesium so a cold trap will be provided before the gases exit the trench in order to remove the cesium before it can plate out in the above grade piping where it would result in unacceptable radiation exposure to personnel in the HCSA. In this case, cold is a relative term, the gases must be cooled to approximately $100^{\circ} \mathrm{C}$.

2. Prior to Vacuum Pump/Recirculation Pump - The vacuum pump that has been selected for the HCSE has a temperature limitation of $40^{\circ} \mathrm{C}$ for the gases entering the pump. The recirculation pump can be specified for higher temperatures so it does not present the limitation that the vacuum pump does.

\section{B. Cooling Air Recirculating Through Oven}

It is proposed to cool the MCO from approximately $300^{\circ} \mathrm{C}$ to $150^{\circ} \mathrm{C}$ by recirculating air through the annular space between the $\mathrm{MCO}$ and the oven. The heat that is transferred to this air must subsequently be removed before the air is returned to the oven.

The most straight forward means of cooling the gases is to use a refrigerant system to chill water which can then be used to cool the process gases. The downside to this approach is threefold: 1) if liquid water is introduced into an MCO that has been heated to $300^{\circ} \mathrm{C}$, a steam explosion could occur; 2) if water 


\section{APPENDIX L - USE OF COOLING WATER IN THE HOT CONDITIONING SYSTEM}

is introduced into an oven that has been heated to $300^{\circ} \mathrm{C}$, a steam explosion could occur; and 3 ) water in a cool MCO would require that the hot conditioning process be repeated.

To preclude water being introduced into an MCO which is in a storage vault, water is not allowed within the CSB storage vaults. The fire hazards analysis for the CSB has determined that a water-based fire protection system is not required in any areas where MCOs are handled.

\subsection{PURPOSE}

The purpose of this study is to identify and evaluate possible options for cooling the MCO and the recirculating helium loop in the HCSE process.

\subsection{CRITERIA}

If water is to be introduced into the HCSE process, then the following criteria for protection of the MCO must be satisfied. Intrinsically safe mechanisms must be designed into the cooling system such that water is excluded from the MCO during normal operations, abnormal operations, and accident conditions such as breaching of a process line or vessel. This includes both the MCOs in storage tubes in the CSB and MCOs in the HCSE process pits. Additionally, the water must be controlled such that it is not allowed to be in contact with hot surfaces such as the interior of the oven. Any system that violates these criteria is not acceptable if air from the HCSA is used for cooling then, whatever system is chosen should have minimal impact on the HCSA air and heat balance. The selected system should also conform to the HCSA criteria for constant velocity in the exhaust stack which has been set in the project's application for an air emissions permit.

\subsection{COOLING SYSTEM ALTERNATIVES}

The cooling methods/alternatives that have been identified and that are evaluated in this study to provide the required cooling are: a) Draw air from HCSA; b) use the 150 cfm of ventilation air from the process pit; c) cool with a gaseous coolant such as carbon dioxide or helium and d) use a chilled water system with intrinsically safe features. 


\section{APPENDIX L - USE OF COOLING WATER IN THE HOT CONDITIONING SYSTEM}

\section{Alternative A - Draw Air From HCSA}

Air drawn from the HCSA through a HEPA filter would be used as required and then exhausted through HEPA filtration and into the HCSA stack. The MCO oven represents from 90 to 95 percent of the cooling load while the helium circulation loop represents the remaining 5 to 10 percent of the load. The volumetric intake air rate to the MCO is $750 \mathrm{cfm}$ and the combined rate to both of the circulating heat exchangers is $75 \mathrm{cfm}$.

Prior to its introduction to the MCO oven, the air would be blended with circulating hot air to achieve the programmed inlet temperature, thus the amount of air required from the HCSA would vary, increasing with time, as the programmed inlet temperature dropped through the cooling period. The exhaust temperature would reach a maximum of approximately $260^{\circ} \mathrm{C}$ at the initial stages of cooling. This hot air would be combined with the ventilation air from the other MCO pits and be sent to the stack at a maximum of $100^{\circ} \mathrm{C}$ if two MCOs were being cooled simultaneously.

Air piped to the helium cooling loop would be at the free air temperature of $20^{\circ} \mathrm{C}$. Compared to the volumetric requirement for the MCO oven, the amount of air required for helium loop cooling would be approximately 5 percent of that required for the MCO oven. Exhaust air from the helium loop cooling would be combined with the exhaust from the MCO oven before being sent through a HEPA and up the HCSA stack.

\section{Alternative B - Use the $150 \mathrm{cfm}$ of Ventilation Air discharging from the Process} Pit Ventilation

A constant $150 \mathrm{cfm}$ of process pit ventilation air would be used for both MCO cooling and helium exchanger cooling. This air, having passed through a HEPA filter, would be heated to a temperature of approximately $25^{\circ} \mathrm{C}$, having picked up approximately 5 degrees from heat losses around the top of the MCO. As in the previous alternative, it would be blended with circulating air to achieve the programmed inlet temperature for MCO cooling. The cooling air to the MCO oven may have to be operated at a higher temperature than what is called for by the cooling temperature profile, owing to the $150 \mathrm{cfm}$ restriction. This would have the effect of increasing the time required for cooling. This option should be studied further.

Approximately 5 percent of the pit ventilation exhaust would be piped to fin tube exchangers in the helium circulation loop. After picking up heat there the exhaust would then join the exhaust from the MCO oven to be directed through HEPA filtration and up the stack. The combined exhaust ventilation air from all of the MCO process pits would result in a stack temperature of up to $100^{\circ} \mathrm{C}$. 


\section{APPENDIX L - USE OF COOLING WATER IN THE HOT CONDITIONING SYSTEM}

\section{Altemative C - Cool With a Gaseous Coolant Such as Carbon Dioxide or Helium}

Candidate gaseous cooling media includes helium, nitrogen and carbon dioxide. All gasses would be handled under pressure to increase the heat capacity per unit volume. While helium and nitrogen would remain as gas, carbon dioxide could be used above its critical point as a supercritical fluid. In this state, the distinction between gas and liquid is lost and the fluid would exhibit the properties of liquid $\mathrm{CO}_{2}$. The triple points of helium and nitrogen are at too high a pressure for their use as supercritical fluids to be practical.

The cooling media would be chilled using a conventional refrigerant expansion system. The cold gas would be then moved to the process heat exchangers where it would pick up heat from the circulating load. In the case of the MCO oven, this would be the load of the air circulating at $750 \mathrm{cfm}$ and in the case of the helium loop, this load would come from the helium.

\section{Alternative D - Use a Chilled Water System With Intrinsically Safe Features}

Intrinsic safety can be achieved for cooling the MCO oven air with chilled water. The chilled water would receive heat from the exhaust MCO oven air. Water entry to the MCO oven could be prevented by piping the circulating air stream through a 125-gallon knockout pot that separates and collects any water leakage, thereby preventing entry to the MCO oven area. The water would be confined to the process module, well away from the oven. The cooling water loop would be contained by double walled piping to preclude water leaks from reaching the HCSA floor. The process module would be designed to contain any leakage from the exchangers as well as to serve as a drain for the outer pipe of the cooling water loop.

Intrinsic safety through indirect heat exchange between the helium circulation $100 \mathrm{p}$ and chilled water is another matter. No intrinsically safe system for heat exchange in this application can be foreseen. All indirect heat exchange methods can have safeguards but not intrinsic safety. For this reason, water cannot be considered for helium circulation cooling.

\subsection{EVALUATION OF ALTERNATIVES}

\section{Alternative A - Draw Air From HCSA}

When air is drawn from the HCSA, it must be made up by the building's HVAC system to maintain proper air balance in the annex. With variable flow of air drawn from the annex, the amount of which depends upon the cooling 


\section{APPENDIX L - USE OF COOLING WATER IN THE HOT CONDITIONING SYSTEM}

requirements of the $\mathrm{MCO}$, increased control sophistication and increased capacity must be provided. This added complexity to HCSA ventilation requirements makes this option undesirable. The flow through the HCSA stack would also be variable.

\section{Alternative B - Use the $150 \mathrm{cfm}$ of Ventilation Air discharging from the Process PitVentilation}

The HCSA ventilation system is designed to deliver $150 \mathrm{cfm}$ to each of six MCO vaults, the use of this air for ventilating the vaults does not preclude its use in subsequent cooling applications. While not as effective as chilled water in being able to achieve patterned temperature control of the MCO, it can be done if some extension of the time required for cool down is permitted. For heat transfer from the helium circulation loop only, no reduction in the time required for cooling would be apparent.

\section{Alternative C - Cool With a Gaseous Coolant Such as Carbon Dioxide or Helium}

Chilling through the use of high pressure gasses or supercritical fluids requires attention to pressure containment of these fluids. While adequate heat transfer can be obtained, the fluid system needed to accommodate this consists of extra pressure rated pumps or blowers, receivers and piping. Careful attention must be paid to the installation of this equipment, making sure that all leaks are stopped. The consequences of small leaks is the loss of potentially expensive heat transfer fluid, while massive leakage of materials such as nitrogen and more importantly $\mathrm{CO}_{2}$ could result in the displacement of air, thereby creating an atmosphere adjacent to the leakage which cannot be safely occupied.

\section{Alternative D - Use a Chilled Water System With Intrinsically Safe Features}

Intrinsic safety, excluding water from the area of the MCO oven can be assured through the installation of a knockout interceptor in the air line. Since intrinsic safety cannot be obtained for the circulating helium stream, air cooling using the heat capacity of a portion of the MCO pit exhaust is suggested.

\subsection{RECOMMENDATIONS}

Our conceptual design shows and thereby recommends a combination of Alternative $B$ and Alternative $D$. This system combines the practicality of controllable temperature obtainable with mechanical refrigeration with the availability of ample heat capacity for helium cooling from MCO pit exhaust air. 


\section{APPENDIX L - USE OF COOLING WATER IN THE HOT CONDITIONING SYSTEM}

Prior to implementation of this method, a complete heat balance will be conducted using any revised cooling temperature profile which may result as a result of the recently completed Q-Matrix study and the effect the $100^{\circ} \mathrm{C}$ temperature flux specification has upon the programmed cooling temperature profile. 


\begin{tabular}{|c|c|c|c|c|c|}
\hline \multicolumn{6}{|c|}{ DISTRIBUTION SHEET } \\
\hline \multirow{2}{*}{$\begin{array}{l}\text { To } \\
\text { Distribution }\end{array}$} & \multirow{2}{*}{\multicolumn{2}{|c|}{$\begin{array}{l}\text { From } \\
\text { Process Engineering }\end{array}$}} & & \multicolumn{2}{|c|}{ Page 1 of 2} \\
\hline & & & & \multicolumn{2}{|c|}{ Date $7 / 30 / 96$} \\
\hline \multirow{2}{*}{\multicolumn{4}{|c|}{$\begin{array}{l}\text { Project Title/Work Order } \\
\text { Hot Conditioning Equip }\end{array}$}} & \multirow{2}{*}{\multicolumn{2}{|c|}{\begin{tabular}{|l|} 
EDT No. 616136 \\
ECN No. N/A
\end{tabular}}} \\
\hline & & & & & \\
\hline Name & MSIN & $\begin{array}{c}\text { Text } \\
\text { With All } \\
\text { Attach. }\end{array}$ & Text Only & $\begin{array}{l}\text { Attach./ } \\
\text { Appendix } \\
\text { Only }\end{array}$ & $\begin{array}{l}\text { EDT/ECN } \\
\text { Only }\end{array}$ \\
\hline $\begin{array}{l}\text { C. J. Alderman } \\
\text { G. D. Bazinet } \\
\text { K. H. Bergsman } \\
\text { F. W. Bradshaw } \\
\text { B. S. Carlisle } \\
\text { D. M. Chenault } \\
\text { R. G. Cowan } \\
\text { R. } \text { Cramond } \\
\text { J. B. Crystal } \\
\text { G. E. Culley } \\
\text { W. P. Dana } \\
\text { B. L. Debban } \\
\text { D. R. Duncan } \\
\text { B. R. Fillion } \\
\text { J. R. Frederickson } \\
\text { J. T. Fulton } \\
\text { W. D. Gallo } \\
\text { E. W. Gerber } \\
\text { T. G. Ghigleri. } \\
\text { L. H. Goldmann } \\
\text { J. J. Irwin } \\
\text { A. T. Kee } \\
\text { M. K. Mahaffey } \\
\text { R. L. McCormack } \\
\text { T. D. Merkling } \\
\text { C. R. Miska } \\
\text { C. T. O'Neill } \\
\text { A. L. Pajunen } \\
\text { D. R. Precechtel. } \\
\text { G. W. Reddick } \\
\text { M. A. Reilly } \\
\text { J. I. Rivera } \\
\text { J. P. Schmidt } \\
\text { P. A. Scott } \\
\text { D. L. Sherrell } \\
\text { E. J. Shen } \\
\text { P. K. Shen } \\
\text { S. M. Short } \\
\text { D. W. Siddoway } \\
\text { D. W. Smith } \\
\text { K. E. Smith } \\
\text { J. A. Swenson } \\
\end{array}$ & $\begin{array}{l}\text { R3-48 } \\
\text { B4-55 } \\
\text { R3-48 } \\
\text { R3-85 } \\
\text { R3-85 } \\
\text { R3-86 } \\
\text { R3-86 } \\
\text { R3-86 } \\
\text { X3-85 } \\
\text { R3-86 } \\
\text { G3-05 } \\
\text { R3-85 } \\
\text { R3-86 } \\
\text { E6-03 } \\
\text { R3-86 } \\
\text { T3-28 } \\
\text { R3-85 } \\
\text { R3-86 } \\
\text { R3-86 } \\
\text { R3-86 } \\
\text { R3-85 } \\
\text { R3-86 } \\
\text { B4-55 } \\
\text { R3-86 } \\
\text { B1-10 } \\
\text { R3-86 } \\
\text { G3-17 } \\
\text { R3-86 } \\
\text { R3-48 } \\
\text { H5-49 } \\
\text { R3-86 } \\
\text { R3-86 } \\
\text { X3-78 } \\
\text { R3-87 } \\
\text { R3-86 } \\
\text { R3-86 } \\
\text { R3-87 } \\
\text { R3-87 } \\
\text { X3-71 } \\
\text { R3-11 } \\
\text { R3-85 } \\
\text { R3-11 }\end{array}$ & s & & & $\begin{array}{l}x \\
x \\
x \\
x \\
x \\
x \\
x \\
x \\
x \\
x \\
x \\
x \\
x \\
x \\
x \\
x \\
x \\
x \\
x \\
x \\
x \\
x \\
x \\
x \\
x \\
x \\
x \\
x \\
x \\
x \\
x \\
x \\
x \\
x \\
x \\
x \\
x \\
x \\
x \\
x \\
x \\
x\end{array}$ \\
\hline
\end{tabular}




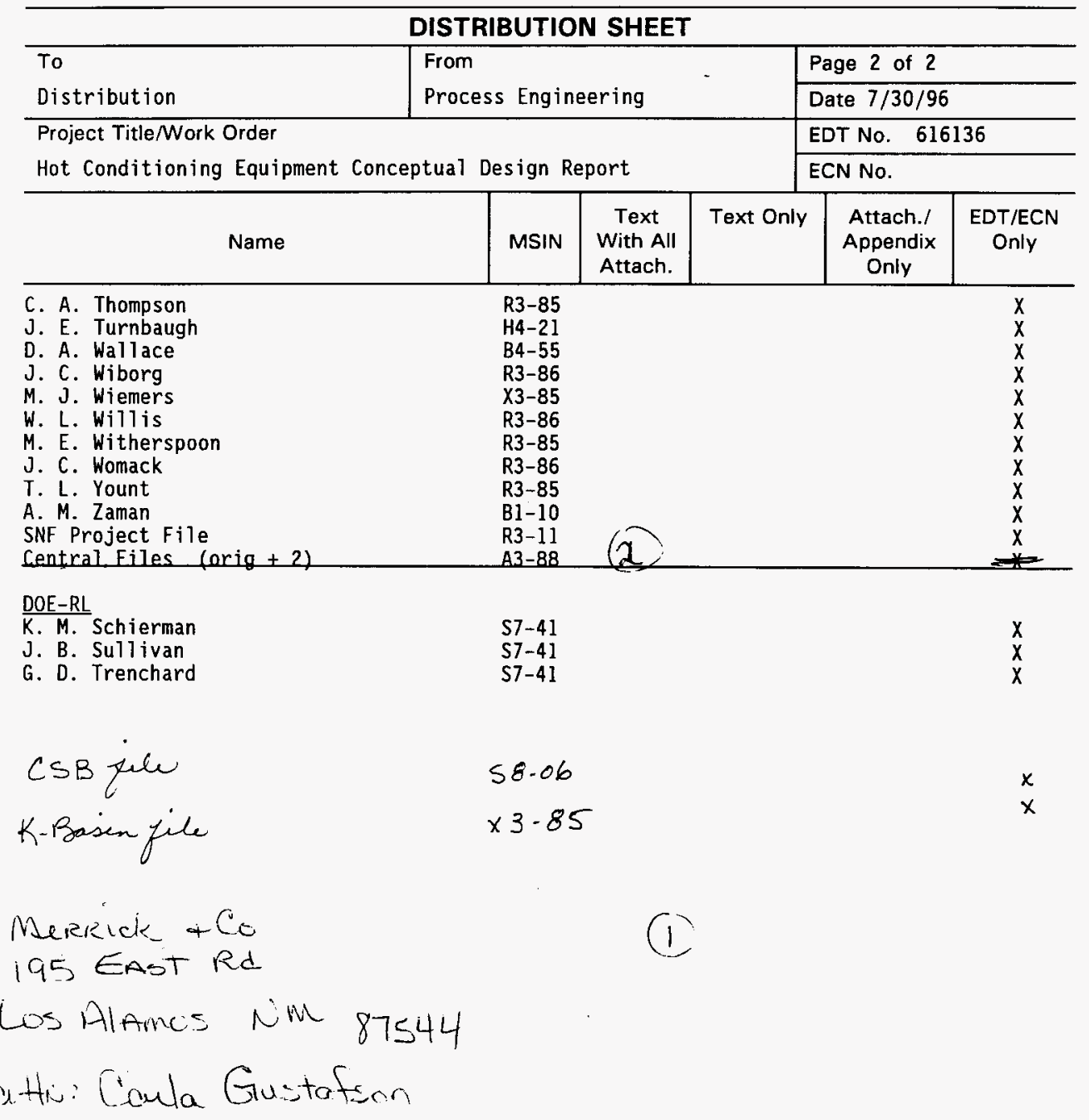

A-6000-135 (01/93) WEF067 